Universidade de São Paulo

Escola de Engenharia de São Carlos

Departamento de Engenharia Elétrica

JOSAPHAT RICARDO RIBEIRO GOUVEIA JÚNIOR

\title{
Bifurcações da Região de Estabilidade Induzidas por Bifurcações Locais do Tipo Hopf
}





\section{JOSAPHAT RICARDO RIBEIRO GOUVEIA JÚNIOR}

\section{Bifurcações da Região de Estabilidade Induzidas por Bifurcações Locais do Tipo Hopf}

Tese de Doutorado apresentada à Escola de Engenharia de São Carlos da Universidade de São Paulo como parte dos requisitos para obtenção do título de Doutor em Ciências, Programa de Engenharia Elétrica.

Área de Concentração: Sistemas Elétricos de Potência.

Orientador:Prof. Dr. Luís F. C. Alberto

São Carlos

2015 
AUTORIZO A REPRODUÇÃO TOTAL OU PARCIAL DESTE TRABALHO, POR QUALQUER MEIO CONVENCIONAL OU ELETRÔNICO, PARA FINS DE ESTUDO E PESQUISA, DESDE QUE CITADA A FONTE.

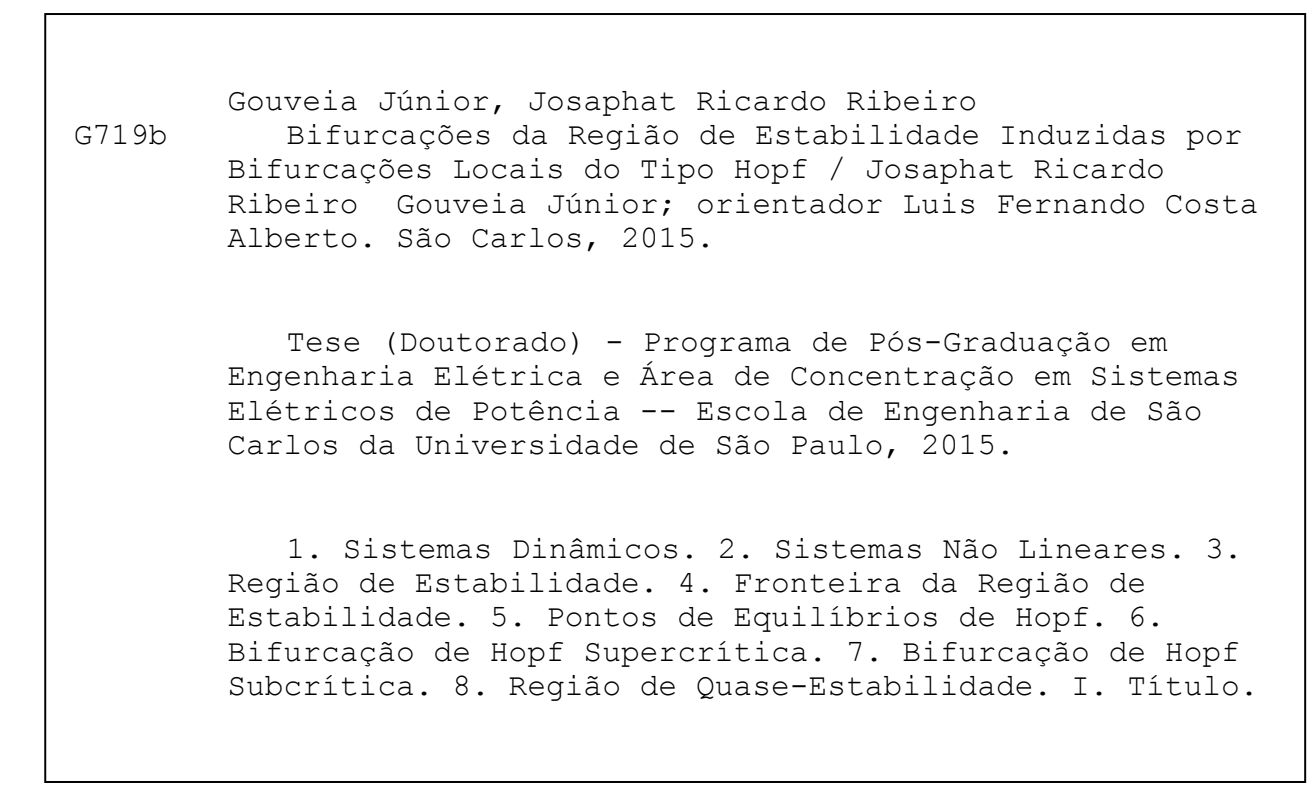


FOLHA DE IULGAMENTO

Candidato: Bacharel JOSAPHAT RICARDO RIBEIRO GOUVEIA JUNIOR.

Título da tese: "Bifurcações da região de estabilidade induzidas por bifurcações locais do tipo hopf".

Data da defesa: 19/03/2015

Comissāo Julgadora:

Resultado: Prof. Associado Luís Fernando Costa Alberto (Orientador) Aprova do
(Escola de Engenharia de São Carlos/EESC)

Profa. Associada Maria do Carmo Carbinatto apronado (Instituto de Ciências Matemáticas e de Computação/ICMC)

\section{Dr. Elbert Einstein Nehrer Macau}

(Instituto Nacional de Pesquisas Espaciais/INPE)

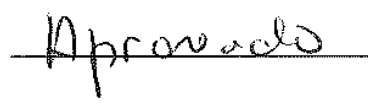

Prof. Dr. Edson Denis Leonel

Aprovado (Universidade Estadual Paulista "Júlio de Mesquita Filho"/UN"ESP-Rio Claro)

Prof. Dr. José Cláudio Geromel

aprovadio

(Universidade Estadual de Campinas/UNICAMP)

Coordenador do Programa de Pós-Graduação em Engenharia Elétrica:

Prof. Associado Luis Fernando Costa Alberto

Presidente da Comissão de Pós-Graduação:

Prof. Associado Paulo César Lima Segantine 
Dedicatória

Dedico à minha mãe Maria da Graça e às minhas tias Vitória Viana (in memoriam) e Noélia Viana. 


\section{Agradecimentos}

\section{À Deus.}

Agradeço a todas as pessoas que me incentivaram e me apoiaram ao longo da realização deste trabalho.

A minha família, em especial à minha mãe Maria da Graça, às minhas tias Vitória Viana (in memoriam) e Noélia Viana, às minhas irmãs, por todo amor, compreensão e incentivo, que me possibilitaram chegar até aqui.

Ao Professor Doutor Luís Fernando Costa Alberto pela orientação e ensinamentos transmitidos.

Ao Departamento de Pós-Graduação de Engenharia Elétrica da EESCUSP pela estrutura e a todos os funcionários e professores da pós-graduação que, direta ou indiretamente, contribuíram para a elaboração deste trabalho.

Aos amigos do LACO, pelo companheirismo.

À todos os meus amigos que, com incentivo ou amizade, contribuíram indiretamente para a realização deste trabalho.

Ao Instituto Federal da Bahia, pelo apoio financeiro para a realização desta pesquisa. 


\section{Resumo}

\section{Gouveia Jr., Josaphat R. R. Bifurcações da Região de Estabilidade}

Induzidas por Bifurcações Locais do Tipo Hopf. Escola de Engenharia de São Carlos, Universidade de São Paulo, São Carlos, 2015.

Pontos de equilíbrio assintoticamente estáveis de sistemas dinâmicos não lineares geralmente não são globalmente estáveis. Na maioria dos casos, há um subconjunto de condições iniciais, chamada região de estabilidade (ou área de atração), cujas trajetórias tendem ao ponto de equilíbrio quando o tempo tende ao infinito. Devido à importância das regiões de estabilidade em aplicações, e motivado principalmente pelo problema de análise de estabilidade transitória em sistemas elétricos de potência, uma caracterização completa da fronteira da região de estabilidade foi desenvolvida. Esta caracterização foi desenvolvida sob a suposição de que o sistema dinâmico é bem conhecido e que os parâmetros de seu modelo são constantes. Na prática, variações de parâmetros ocorrem e bifurcações desta podem ocorrer. Nesta tese, desenvolveremos uma caracterização completa da fronteira da região de estabilidade de sistemas dinâmicos autônomos não lineares admitindo a existência de pontos de equilíbrio não hiperbólicos do tipo Hopf na fronteira da região de estabilidade. Sob certas condições de transversalidade, apresentaremos uma caracterização completa da fronteira da região de estabilidade admitindo tanto a presença de pontos de equilíbrio não hiperbólicos do tipo Hopf como também a existência de órbitas periódicas na fronteira. Ofereceremos também uma caracterização da fronteira da região de estabilidade fraca do ponto de equilíbrio não hiperbólico Hopf supercrítico do tipo zero e uma caracterização topológica da sua região de atração. Além disso, exibiremos resultados relativos ao comportamento da região de estabilidade de um ponto de equilíbrio assintoticamente estável e da sua fronteira na vizinhança do valor crítico de bifurcação do tipo Hopf. 
Palavras-chave: Sistemas Dinâmicos, Sistemas Não Lineares, Região de Estabilidade, Região de Atração, Fronteira da Região de Estabilidade, Pontos de Equilíbrio Hopf, Bifurcação Hopf Supercrítica, Bifurcação Hopf Subcrítica, Região de Quase-Estabilidade, Conjuntos Minimais. 


\section{Abstract}

Gouveia Jr., Josaphat R. R. Bifurcations of the Stability Region Induced by Type-Hopf Local Bifurcations. Escola de Engenharia de São Carlos, Universidade de São Paulo, São Carlo, 2015.

Asymptotically stable equilibrium points of nonlinear dynamical systems are generally not globally stable. In most cases, there is a subset of initial conditions, called stability region (or attraction area), in which trajectories tend to the equilibrium point when time approaches infinity. Due to the importance of stability regions in applications, and mainly motivated by the problem of transient stability analysis in electric power systems, a complete characterization of the boundary of the stability region was developed. This characterization was developed under the assumption that the dynamic system is well known and the parameters of its model are constant. In practice, parameter variations happen and bifurcations may occur. In this thesis, we will develop a complete characterization of the boundary of the stability region of autonomous nonlinear dynamical systems admitting the existence of non-hyperbolic equilibrium points of the type Hopf on the boundary of the stability region. Under certain transversality conditions, we present a complete characterization of the boundary of the stability region admitting the presence of both non-hyperbolic equilibrium points of the type Hopf and periodic orbits on the boundary. Also a complete characterization of the boundary of the region of weak stability of a supercritical Hopf non-hyperbolic equilibrium point of the type zero and a topological characterization of its region of attraction is developed. Furthermore, the behavior of the stability region of an asymptotically stable equilibrium point and its boundary in the neighborhood of a critical value of bifurcation of the type Hopf is studied.

Keywords: Dynamic Systems, Nonlinear Systems, Stability Region, Region 
of Attraction, Boundary of the Stability Region, Hopf Equilibrium Points, Supercritical Hopf Bifurcation, Subcritical Hopf Bifurcation, Quasi-Stability Region, Minimal Sets. 


\section{Sumário}

Lista de Suposições sobre o Campo Vetorial xiii

Lista de Símbolos $\quad$ Xv

Lista de Figuras $\quad$ xvii

1 Introdução 1

1.1 Organização do Trabalho . . . . . . . . . . . . 4

2 Conceitos Preliminares $\quad 7$

2.1 Espaços Métricos . . . . . . . . . . . . . . . . . . . 7

2.1.1 Conjuntos Abertos . . . . . . . . . . . . 10

2.1.2 Conjuntos Fechados . . . . . . . . . . . . . . 12

2.1.3 Conjuntos Conexos . . . . . . . . . . . . . . . . . 14

2.1.4 Teorema da Função Implícita . . . . . . . . . . . . . . 15

2.2 Sistemas Dinâmicos . . . . . . . . . . . . . . . 16

2.2 .1 Teoria Geral . . . . . . . . . . . . . 16

2.2 .2 Trajetórias . . . . . . . . . . . . . 18

2.2 .3 Comportamento Assintótico . . . . . . . . . . . . . 21

2.2.4 Estabilidade Local . . . . . . . . . . . . . . . . 26

2.2.5 Pontos de Equilíbrio Hiperbólicos . . . . . . . . . . . . 29

2.2.6 Pontos de Equilíbrio Não Hiperbólicos . . . . . . . . . 37

2.2.7 $\lambda$-Lema e Ponto de Equilíbrio Hopf . . . . . . . . . . . 45

2.2.8 Transversalidade . . . . . . . . . . . . . . 48

3 Região de Estabilidade $\quad 51$

3.1 Conjuntos Atrativos . . . . . . . . . . . . . . . 51

3.2 Conjuntos Minimais . . . . . . . . . . . . . . 53

3.3 Região de Estabilidade . . . . . . . . . . . . . . . . . . . 54 
4 Caracterização da Fronteira da Região de Estabilidade $\quad 67$

4.1 Elementos Críticos na Fronteira da Região de Estabilidade . . 67

4.2 Ponto de Equilíbrio Sela-Nó do tipo zero na Fronteira da Região de Estabilidade . . . . . . . . . . . . . . . . . 75

5 Fronteira da Região de Estabilidade na Presença de um Ponto de Equilíbrio Hopf $\quad 79$

5.1 Lemas Auxiliares . . . . . . . . . . . . . . . . . . . . . 79

5.2 Ponto de equilíbrio Hopf Supercrítico em $\partial A\left(x^{s}\right) \ldots \ldots$

5.3 Ponto de equilíbrio Hopf Subcrítico em $\partial A\left(x^{s}\right) \quad \ldots \ldots$. . . 89

5.4 Exemplos . . . . . . . . . . . . . . . . . . 99

6 Fronteira da Região de Estabilidade na Presença de um Ponto de Equilíbrio Hopf com Elementos Críticos 101

6.1 Lemas Auxiliares e Condição de Transversalidade . . . . . . . 101

6.2 Ponto de equilíbrio Hopf Supercrítico em $\partial A\left(x^{s}\right) \ldots \ldots$

6.3 Ponto de equilíbrio Hopf Subcrítico em $\partial A\left(x^{s}\right) \ldots \ldots$

7 Fronteira e Região de Estabilidade Fraca de um Ponto de Equilíbrio Hopf Supercrítico do Tipo-Zero

7.1 Região de Estabilidade Fraca de um Ponto de equilíbrio Hopf Supercrítico do tipo zero . . . . . . . . . . . . . . . . . . . 119

7.2 Fronteira e Região de Quase-Estabilidade Fraca na Presença de um Ponto de Equilíbrio Hopf Supercrítico . . . . . . . . . 132

8 Bifurcação de Hopf

8.1 Teoria de Bifurcação . . . . . . . . . . . . . . . . . . . . . 141

8.2 Bifurcação de Hopf em Sistemas Bidimensionais . . . . . . . . 143

8.3 Bifurcação de Hopf $n$-dimensional . . . . . . . . . . . . 165

9 Bifurcação de Hopf na Fronteira da Região de Estabilidade179

9.1 Bifurcação de Hopf na Fronteira da Região de Estabilidade . 180

9.2 Região de Estabilidade Fraca de um Equilíbrio Hopf Supercrítico do tipo zero . . . . . . . . . . . . . . . 187

9.3 Exemplos . . . . . . . . . . . . . . . . . . . 190

10 Conclusões 203

10.1 Publicações Relacionadas à Tese . . . . . . . . . . . . . . 205 
A Algoritmo para o cálculo do primeiro coeficiente de Lyapunov para sistemas bidimensional

A.1 Cálculo do coeficiente de Lyapunov do sistema (7.1) . . . . 208

A.2 Cálculo do coeficiente de Lyapunov do sistema (7.3) . . . . 212

B Algoritmo para o cálculo do primeiro coeficiente de Lyapunov para sistemas $n$-dimensional

B.1 Cálculo do coeficiente de Lyapunov do sistema (5.2) . . . . 218

B.2 Cálculo do coeficiente de Lyapunov do sistema (7.2) . . . . 225

B.3 Cálculo do coeficiente de Lyapunov do sistema (7.4) . . . . . 232

B.4 Cálculo do coeficiente de Lyapunov do sistema (7.5) . . . . 238

Referências Bibliográficas 


\section{Lista de Suposições sobre o Campo Vetorial}

(A1) Todos os pontos de equilíbrios em $\partial A\left(x^{s}\right)$ são hiperbólicos;

(A2) As variedades estáveis e instáveis dos pontos de equilíbrio em $\partial A\left(x^{s}\right)$ satisfazem a condição de transversalidade;

(A3) Toda trajetória em $\partial A\left(x^{s}\right)$ se aproxima de um ponto de equilíbrio quando $t \rightarrow+\infty$;

(B1) Todos os elementos críticos de (2.1) em $\partial A\left(x^{s}\right)$ são hiperbólicos;

(B2) As variedades estável e instável dos elementos críticos de (2.1) na fronteira da área de atração satisfazem a condição de transversalidade;

(B3) Toda trajetória na fronteira da área de atração se aproxima de um dos elementos críticos do sistema (2.1) quando $t \rightarrow+\infty$;

(A1') Todos os pontos de equilíbrios em $\partial A\left(x^{s}\right)$ são hiperbólicos, exceto possivelmente o equilíbrio $x_{0}$ que é um ponto de equilíbrio sela-nó do tipo zero;

(A4) As variedades estáveis dos pontos de equilíbrio em $\partial A\left(x^{s}\right)$ e a variedade $W_{l o c}^{c^{+}}\left(x_{0}\right)$ satisfazem a condição de transversalidade;

(A1") Todos os pontos de equilíbrio em $\partial A\left(x^{s}\right)$ são hiperbólicos ou Hopf supercrítico;

(A2') As variedades estável, centro-estável e/ou central e a variedade instável dos pontos de equilíbrios em $\partial A\left(x^{s}\right)$ satisfazem a condição de transversalidade;

(A1"') Todos os pontos de equilíbrio em $\partial A\left(x^{s}\right)$ são pontos de equilíbrio hiperbólicos ou pontos de equilíbrio do tipo Hopf subcrítico; 
(A2") As variedades estáveis e as variedades centrais, centro-instáveis e/ou instáveis dos pontos de equilíbrio em $\partial A\left(x^{s}\right)$ satisfazem a condição de transversalidade;

(B1') Todos os elementos críticos em $\partial A\left(x^{s}\right)$ são elementos críticos hiperbólicos ou pontos de equilíbrio Hopf supercríticos;

(B2') As variedades estáveis, centro-estáveis e/ou centrais e as variedades instáveis dos elementos críticos em $\partial A\left(x^{s}\right)$ satisfazem segundo a condição de transversalidade definida no capítulo 6;

(B1") Todos os elementos críticos em $\partial A\left(x^{s}\right)$ são elementos críticos hiperbólicos ou pontos de equilíbrio Hopf subcrítico;

(B2") As variedades estáveis e as variedades centrais, centro-instáveis e/ou instáveis dos elementos críticos em $\partial A\left(x^{s}\right)$ satisfazem a condição de transversalidade definida no capítulo 6 ;

(S1) Todos os elementos críticos em $\partial A(p)$ são hiperbólicos;

(S2) A variedade estável e a variedade instável dos elementos críticos em $\partial A(p)$ satisfazem a condição de transversalidade;

(S3) Toda trajetória em $\partial A(p)$ se aproxima de um elemento crítico quando $t \rightarrow+\infty$; 


\section{Lista de Símbolos}

\begin{tabular}{ll}
$\Re(z)$ & Parte real do número complexo $z$ \\
$\Im(z)$ & Parte imaginária do número complexo $z$ \\
$\bar{z}$ & Conjugado do número complexo $z$ \\
$v^{T}$ & Transposta do vetor $v$ \\
$\|v\|$ & Norma do vetor $v$ \\
$d(x, y)$ & distância de $x$ a $y$ \\
$d(x, A)$ & distância de $x$ ao conjunto $A$ \\
$\langle x, y\rangle$ & Produto interno de $x$ com $y$ \\
$B(x ; \varepsilon)$ & Bola aberta de centro $x$ e raio $\varepsilon$ \\
$B[x ; \varepsilon]$ & Bola fechada de centro $x$ e raio $\varepsilon$ \\
$S[x ; \varepsilon]$ & Esfera de centro $x$ e raio $\varepsilon$ \\
$i n t X$ & Interior do conjunto $X$ \\
$\partial X$ & Fronteira do conjunto $X$ \\
$\bar{X}$ & Fecho do conjunto $X$ \\
$X \backslash Y$ & Conjunto dos pontos pertencentes a $X$ e não pertencentes a $Y$ \\
$A^{T}$ & Transposta da matriz $A$ \\
$\gamma(x)$ & Trajetória ou Órbita de $\varphi(t, x), t \in \mathbb{R}$ passando por $x$ \\
$\gamma^{+}(x)$ & Semitrajetória positiva de $\varphi(t, x), t \in \mathbb{R}^{+}$passando por $x$ \\
$\gamma^{-}(x)$ & Semitrajetória negativa de $\varphi(t, x), t \in \mathbb{R}^{-}$passando por $x$ \\
$\omega\left(x_{0}\right)$ & Conjunto $\omega$-limite de $x_{0}$ \\
$\alpha\left(x_{0}\right)$ & Conjunto $\alpha$-limite de $x_{0}$ \\
$\Gamma^{+}(x)\left(\Gamma^{-}(x)\right)$ & Primeiro prolongamento positivo (negativo) de $x$ \\
$\Lambda^{+}(x)\left(\Lambda^{-}(x)\right)$ & Primeiro prolongamento positivo (negativo) do conjunto $\omega(\alpha)$-limite de $x$ \\
$A_{\omega}(M)$ & Região de atração fraca do conjunto $M$ \\
$A(M)$ & Região de atração do conjunto $M$ \\
$A(M)$ & Região de atração uniforme do conjunto $M$ \\
$A^{\mathcal{Q}}(M)$ & \\
& \\
\hline &
\end{tabular}




\section{Lista de Figuras}

2.1 Trajetória do sistema dinâmico autonômo (2.1). . . . . . . . . 19

2.2 Exemplo de um conjunto $\omega$-limite desconexo e ilimitado. . . . 24

2.3 Trajetórias do sistema $\dot{x}=-x, \dot{y}=y \ldots \ldots \ldots \ldots$

2.4 Solução Estável do sistema dinâmico autonômo (2.1). . . . 26

2.5 Solução Assintoticamente estável do sistema dinâmico au-

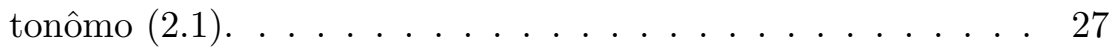

2.6 Ponto de equilíbrio estável do sistema dinâmico autonômo (2.1). 27

2.7 Ponto de equilíbrio atrativo do sistema dinâmico autonômo

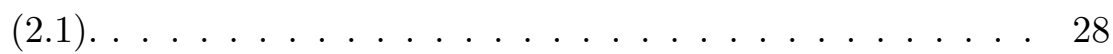

2.8 Ponto de equilíbrio assintoticamente estável do sistema dinâmico autonômo $(2.1) \ldots \ldots \ldots \ldots$

2.9 Variedades Estável $W^{s}(\bar{x})$ e Instável $W^{u}(\bar{x}) \ldots \ldots \ldots$. . . . . 31

2.10 A geometria da Aplicação de Poincaré para uma órbita pe-

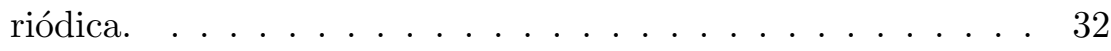

2.11 Variedades Estável $W^{s}(\phi)$ e Instável $W^{u}(\phi) . \quad \ldots . . .36$

2.12 Variedades Estável $W^{s}(\bar{x})$, Instável $W^{u}(\bar{x})$ e Central $W^{c}(\bar{x})$. 38

2.13 Variedades $W_{l o c}^{c^{+}}(p), W_{l o c}^{c^{-}}(p)$ e $W_{l o c}^{s}(p)$ para um ponto de equilíbrio sela-nó tipo-zero $p$ do sistema $(2.1)$ em $\mathbb{R}^{3}$. As variedades $W_{l o c}^{c^{+}}(p)$ e $W_{l o c}^{s}(p)$ são únicas. Três possíveis escolhas para $W_{l o c}^{c^{-}}(p)$ são mostradas nesta figura. . . . . . . . . . . . 39

2.14 Variedades $W_{l o c}^{c}(p)$ e $W_{l o c}^{s}(p)$ para um ponto de equilíbrio Hopf supercrítico do tipo-zero $p$ do sistema (2.1) em $\mathbb{R}^{3}$. $W_{l o c}^{c}(p)$ não é única. Três possíveis escolhas para $W_{l o c}^{c}(p)$ são mostradas nesta figura. . . . . . . . . . . . . . . . 42

2.15 Variedades $W_{l o c}^{c}(p)$ e $W_{l o c}^{u}(p)$ para um ponto de equilíbrio Hopf supercrítico do tipo-1 $p$ do sistema (2.1) em $\mathbb{R}^{3}$. Neste caso, $W_{l o c}^{c}(p)$ é único. . . . . . . . . . . . . . . . 43 
2.16 Variedades $W_{l o c}^{c}(p)$ e $W_{l o c}^{s}(p)$ para um ponto de equilíbrio Hopf subcrítico do tipo-1 $p$ do sistema $2.1 \mathrm{em} \mathbb{R}^{3}$. Neste caso, $W_{l o c}^{c}(p)$ é única. . . . . . . . . . . . . . . . . . . 44

2.17 Variedades $W_{l o c}^{c}(p)$ e $W_{l o c}^{u}(p)$ para um ponto de equilíbrio Hopf subcrítico do tipo-zero $p$ do sistema (2.1) em $\mathbb{R}^{3}$. $W_{l o c}^{c}(p)$ não é única. Três escolhas possíveis para $W_{l o c}^{c}(p)$ são mostradas nesta figura. . . . . . . . . . . . . . . .

2.18 Ilustração do Lema 2.2.23 e do Corolário 2.2.24 para um ponto de equilíbrio Hopf supercrítico $p$ do tipo- $k$, com $1 \leq k \leq n-3$, $\operatorname{com} q \in W_{l o c}^{u}(p) \cap D^{c s}$, onde $D^{c s}$ é um disco de dimensão $n-k$ transversal a $W_{l o c}^{u}(p)$ em $q . \ldots \ldots \ldots \ldots$

2.19 Ilustração do Lema 2.2.23 e do Corolário 2.2.24 para um ponto de equilíbrio Hopf supercrítico $p$ do tipo- $k$, com $1 \leq k \leq n-3$, com $q \in W_{l o c}^{c s}(p) \cap D^{u}$, onde $D^{u}$ um disco de dimensão $k$, transversal a $W_{l o c}^{c s}(p)$ em $q . \ldots \ldots \ldots \ldots$. . . . . . .

2.20 Ilustração do Lema 2.2.25 e do Corolário 2.2.26 para um ponto de equilíbrio Hopf subcrítico $p$ do tipo- $k$, com $1 \leq k \leq n-3$, $q \in W_{l o c}^{s}(p) \cap D^{c u}$, onde $D^{c u}$ é um disco de dimensão $k+2$, transversal a $W_{l o c}^{s}(p)$ em $q . \ldots \ldots \ldots \ldots$. . . . . . .

2.21 Ilustração do Lema 2.2.25 e do Corolário 2.2.26 para um ponto de equilíbrio Hopf subcrítico $p$ do tipo- $k$, com $1 \leq k \leq n-3$, $q \in W_{l o c}^{c u}(p) \cap D^{s}$, onde $D^{s}$ é um disco de dimensão $n-k-2$ transversal a $W_{l o c}^{c u}(p)$ em $q . \quad \ldots \ldots \ldots$. . . . . . 48

3.1 Atrator Fraco. . . . . . . . . . . . . . . . . 60

3.2 Trajetórias do sistema $\dot{r}=r(1-r), \dot{\theta}=\sin ^{2}\left(\frac{\theta}{2}\right)$. Fonte: [ver BS02, p. 59] . . . . . . . . . . . . . . . 6 . . . . . 61

3.3 Atrator Global. Fonte: [ver BS02, p. 59] . . . . . . . . . . 61

4.1 Retrato de fase do sistema (4.1). A fronteira da região de estabilidade de $x^{s}=(-10)$ é formada pela variedade estável $W^{s}\left(x_{1}\right)$ do ponto de equilíbrio hiperbólico do tipo-1 $x_{1}=$ $(2,3)$, a curva vermelha. . . . . . . . . . . . . . 
4.2 Retrato de fase do sistema (4.2). A fronteira da região de estabilidade de $x^{s_{1}}=(11)$ é formada pela união da variedade estável $W^{s}\left(x_{1}\right)$ do ponto de equilíbrio hiperbólico do tipo-1 $x_{1}=(-1,1)$, a curva verde, com a variedade estável $W^{s}\left(x_{2}\right)$ do ponto de equilíbrio hiperbólico do tipo-1 $x_{2}=(2,2)$, a curva vermelha. A fronteira da região de estabilidade de $x^{s_{2}}=$ $(-22)$ é formada pela variedade estável $W^{s}\left(x_{1}\right)$ do ponto de equilíbrio hiperbólico do tipo-1 $x_{1}=(-1,1)$, a curva verde. . 73

4.3 Retrato de fase do sistema (4.3). A fronteira da região de estabilidade $\partial A\left(x^{s_{1}}\right)$ é formada pela variedade estável $W^{s}\left(x_{1}\right)$ do ponto de equilíbrio hiperbólico do tipo-1 $x_{1}=(1,0)$, a curva em vermelho. A fronteira da região de estabilidade $\partial A\left(x^{s_{2}}\right)$ está contida na união da variedade estável $W^{s}\left(x_{1}\right)$ do ponto de equilíbrio hiperbólico do tipo-1 $x_{1}=(1,0)$ com a variedade estável $W^{s}\left(x_{2}\right)$ do ponto de equilíbrio hiperbólico do tipo-1 $x_{2}=(3,0)$, a curva em verde. . . . . . . . . . . .

4.4 Retrato de fase do sistema (4.4). A fronteira da região de estabilidade $\partial A\left(x^{s}\right)$ é formada pela união da variedade estável $W^{s}\left(x_{1}\right)$ do ponto de equilíbrio hiperbólico do tipo-1 $x_{1}=$ $(1,0)$, a curva verde, com a variedade estável $W^{s}\left(x_{0}\right)$ do ponto de equilíbrio sela-nó do tipo-zero $x_{0}=(0,-1)$, a curva vermelha. 77

5.1 Retrato de fase do sistema (5.2). A fronteira da região de estabilidade de $x^{s}=(0,0,3)$ é formada pela união da variedade estável $W^{s}(x)$ do ponto de equilíbrio hiperbólico do tipo-1 $x_{1}=(0,0,8)$ com a variedade central $W^{c}(p)$ do ponto de equilíbrio Hopf supercrítico do tipo-1 $p=(0,0,0) \ldots \ldots 100$

7.1 Retrato de fase do sistema (7.1). A fronteira da região de estabilidade fraca $\partial A(p)$ está contida na união da variedade estável $W^{s}\left(x_{1}\right)$ do ponto de equilíbrio hiperbólico do tipo-1 $x_{1}=(0,0)$, curva vermelha, com a variedade estável $W^{s}\left(x_{2}\right)$ do ponto de equilíbrio hiperbólico do tipo- $1 x_{2}=(6,0)$, curva preta. . . . . . . . . . . . . . 127 
7.2 Retrato de fase do sistema (7.2). A fronteira da região de estabilidade fraca $\partial A\left(x^{p}\right)$ está contida na união da variedade estável $W^{s}\left(x_{1}\right)$ do ponto de equilíbrio hiperbólico do tipo$1 x_{1}=(8 / 3,0,0)$, da variedade estável $W^{s}\left(x_{2}\right)$ do ponto de equilíbrio hiperbólico do tipo-1 $x_{2}=(2 / 3,0,3)$ com a variedade estável $W^{s}\left(x_{3}\right)$ do ponto de equilíbrio hiperbólico do tipo-1 $x_{3}=(1 / 3,7 / 3,0) \ldots \ldots \ldots \ldots 128$

7.3 Retrato de fase do sistema (7.3). A fronteira da região de estabilidade $\partial A\left(x^{s}\right)$ é formada pela variedade estável $W^{s}\left(x_{1}\right)$ do ponto de equilíbrio hiperbólico do tipo-1 $x_{1}=(0,0) \ldots$

7.4 Retrato de fase do sistema (7.4). A fronteira da região de estabilidade $\partial A(p)$ é formada pela união da variedade estável $W^{s}\left(x_{1}\right)$ do ponto de equilíbrio hiperbólico do tipo-1 $x_{1}=$ $(7.46,7.46,4)$, curva em vermelho, com a variedade estável $W^{s}\left(x_{2}\right)$ do ponto de equilíbrio hiperbólico do tipo-1 $x_{2}=$ $(-7.46,-7.46,4)$, curva em azul. . . . . . . . . . 131

7.5 Retrato de fase do sistema (7.5). A fronteira da região de estabilidade $\partial A(p)$ é formada pela variedade estável $W^{s}\left(x_{1}\right)$ do ponto de equilíbrio hiperbólico do tipo-1 $x_{1}=(1,0,0) \ldots 132$

7.6 Retrato de fase do sistema (7.6). A fronteira da região de estabilidade do ponto de equilíbrio assintoticamente estável $x^{s}=(0,0)$ é formada pela variedade estável $W^{s}\left(x_{2}\right)$, curva em azul, com a variedade estável $W^{s}\left(x_{1}\right)$, curva em vermelho, e com os pontos de equilíbrios hiperbólicos $x_{3}$ e $x_{4}$. A fronteira da região de quase-estabilidade $x^{s}=(0,0)$ é a união da variedade estável $W^{s}\left(x_{2}\right)$ e do ponto de equilíbrio hiperbólico do tipo-2 $x_{4}=(0,3) \ldots \ldots \ldots \ldots \ldots \ldots$

7.7 A fronteira da região de estabilidade fraca $A(p)$ é a união da curva que delimita a região de estabilidade fraca $A(p)$ com a variedade estável $W^{s}(\phi)$. A fronteira da região de quaseestabilidade é a curva que delimita a região de quase-estabilidade fraca $A_{\sigma}(p)$, área sombreada, em $(b) \ldots \ldots \ldots$. . . . . 134 
8.1 Bifurcação de Hopf Supercrítica. (a) Diagrama de Bifurcação de Hopf Supercrítica para o espaço de fase $r \times \mu$, onde $r(\mu)$ é uma família a um parâmetro de ciclos limite do sistema dinâmico (8.8). Para $\mu<0$, a origem $r=0$ é um ponto de equilíbrio assintoticamente estável e para $\mu>0$, a origem $r=0$ é um ponto de equilíbrio instável e o ciclo limite $r=\sqrt{\mu}$ é estável. (b) Diagrama de Bifurcação de Hopf Supercrítica para o espaço de estado versus parâmetro. Para $\mu<0$, toda trajetória tende para a origem quando $t$ cresce indefinidamente. Após a bifurcação, trajetórias que iniciam próximas do ponto de equilíbrio instável são atraídos pelo ciclo limite estável e as oscilações são limitadas. . . . . . . . . . . . . . . . . . . . 146

8.2 Bifurcação de Hopf Subcrítica. (a) Diagrama de Bifurcação de Hopf Subcrítica para o espaço de fase $r \times \mu$, onde $r(\mu)$ é uma família a um parâmetro de ciclos limites do sistema dinâmico (8.9). Para $\mu<0$, a origem $r=0$ é um ponto de equilíbrio assintoticamente estável e o ciclo limite $r=\sqrt{\mu}$ é instável e para $\mu>0$, a origem $r=0$ é um ponto de equilíbrio instável. (b) Diagrama de Bifurcação de Hopf Subcrítica para o espaço de estado versus parâmetro. Para $\mu<0$, a amplitude do ciclo limite instável diminui quando o parâmetro se aproxima do valor de bifurcação e desaparece quando o parâmetro cruza o eixo imaginário. As trajetórias, após o valor de bifurcação, têm oscilações com amplitudes crescentes. . . . . . . . . . . . 148

8.3 Aplicação de Poincaré para a Bifurcação de Hopf do sistema 8.13. . . . . . . . . . . . . . . . . . . 149

8.4 Ponto Fixo para a Aplicação de Poincaré do sistema (8.13). 152

8.5 Construção do homeomorfismo nas vizinhanças da bifurcação de Hopf. . . . . . . . . . . . . . . . . . . . . . . . . . 154

8.6 Variedade Central $W_{\mu}^{c}$ dependente do parâmetro $\mu$. . . . . 172 
9.1 Retrato de fase do sistema (9.1) para $\mu_{0}=0$. A fronteira da região de estabilidade de $x_{\mu_{0}}^{s}=(0,0,3)$ é formada pela união da variedade estável $W_{\mu_{0}}^{s}\left(x_{1}\right)$ do ponto de equilíbrio hiperbólico do tipo-1 $x_{1}=(0,0,8)$ com a variedade central $W_{\mu_{0}}^{c}\left(x_{\mu_{0}}\right)$ do ponto de equilíbrio Hopf supercrítico do tipo-1 $x_{\mu_{0}}=(0,0,0) \ldots \ldots \ldots \ldots \ldots \ldots \ldots \ldots \ldots \ldots \ldots \ldots \ldots \ldots$

9.2 Retrato de fase do sistema (9.1) para $\mu=-0.5$. A fronteira da região de estabilidade de $x_{\mu}^{s}=(0,0,3)$ é formada pela união da variedade estável $W_{\mu}^{s}\left(x_{1}\right)$ do ponto de equilíbrio hiperbólico do tipo-1 $x_{1}=(0,0,8)$ com a variedade estável $W_{\mu}^{s}\left(x_{\mu}^{H}\right)$ do ponto de equilíbrio hiperbólico do tipo- $1 x^{H}=(0,0,0) . \quad .192$

9.3 Retrato de fase do sistema (9.1) para $\mu=0.5$. A fronteira da região de estabilidade de $x_{\mu}^{s}=(0,0,3)$ é formada pela união da variedade estável $W_{\mu}^{s}\left(x_{1}\right)$ do ponto de equilíbrio hiperbólico do tipo-1 $x_{1}=(0,0,8)$ com a variedade estável $W_{\mu}^{s}\left(\phi_{\mu}^{H}\right)$ da órbita periódica hiperbólica do tipo-1 $\phi_{\mu}^{H}$. . . . . . . . 192

9.4 Retrato de fase do sistema (9.2) para $\mu_{0}=\frac{1}{6}$. A fronteira da região de estabilidade $\partial A_{\mu_{0}}\left(x_{\mu_{0}}\right)$ está contida na união da variedade estável $W_{\mu_{0}}^{s}\left(x_{1}\right)$ do ponto de equilíbrio hiperbólico do tipo-1 $x_{1}=(1,0)$, a curva em vermelho na Figura 9.4, com a variedade estável $W_{\mu_{0}}^{s}\left(x_{2}\right)$ do ponto de equilíbrio hiperbólico do tipo-1 $x_{2}=(6,0)$, a curva em preto na Figura 9.4 . . . 193

9.5 Retrato de fase do sistema (9.2) para $\mu=\frac{4}{10}$. A fronteira da região de estabilidade $\partial A_{\mu}\left(x_{\mu}^{s}\right)$ está contida na união da variedade estável $W_{\mu}^{s}\left(x_{1}\right)$ do ponto de equilíbrio hiperbólico do tipo-1 $x_{1}=(0,0)$, a curva em vermelho na Figura 9.5, com a variedade estável $W_{\mu}^{s}\left(x_{2}\right)$ do ponto de equilíbrio hiperbólico do tipo-1 $x_{2}=\left(\frac{5}{2}, 0\right)$, a curva em azul na Figura 9.5 . . . . 194 
9.6 Retrato de fase do sistema (9.2) para $\mu=\frac{8}{100}$. A fronteira da região de estabilidade $\partial A_{\mu}\left(\phi_{\mu}^{H}\right)$ é formada pela união da variedade estável $W_{\mu}^{s}\left(x_{1}\right)$ do ponto de equilíbrio hiperbólico do tipo-1 $x_{1}=(0,0)$, a curva em vermelho na Figura 9.6, com a variedade estável $W_{\mu}^{s}\left(x_{2}\right)$ do ponto de equilíbrio hiperbólico do tipo-1 $x_{2}=\left(\frac{25}{2}, 0\right)$, a curva em marrom na Figura 9.6, e com a variedade estável $W_{\mu}^{s}\left(x_{\mu}^{H}\right)$ do ponto de equilíbrio hiperbólico do tipo-2 $x_{\mu}^{H}=\left(2, \frac{42}{25}\right)$, a curva em verde na Figura

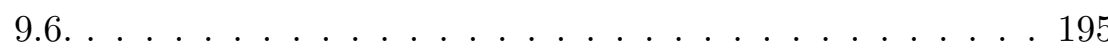

9.7 Retrato de fase do sistema (9.3) para $\mu_{0}=-\frac{4}{27}$. A fronteira da região de estabilidade $\partial A\left(x_{\mu_{0}}\right)$ é formada pela variedade estável $W_{\mu_{0}}^{s}\left(x_{1}\right)$ do ponto de equilíbrio hiperbólico do tipo-1 $x_{1}=(0,0)$, a curva em azul. . . . . . . . . . 19

9.8 Retrato de fase do sistema (9.3) para $\mu=-\frac{3}{10}$. A fronteira da região de estabilidade $\partial A\left(x_{\mu}^{H}\right)$ é formada pela variedade estável $W_{\mu}^{s}\left(x_{1}\right)$ do ponto de equilíbrio hiperbólico do tipo-1 $x_{1}=(1,0)$, a curva em vermelho. . . . . . . . . . 197

9.9 Retrato de fase do sistema (9.3) para $\mu=-\frac{1}{10}$. A fronteira da região de estabilidade $\partial A\left(\Omega_{\mu}^{H}\right)$ é formada pela união da variedade estável $W_{\mu}^{s}\left(x_{1}\right)$ do ponto de equilíbrio hiperbólico do tipo-1 $x_{1}=(1,0)$, a curva em vermelho, com a variedade estável $W_{\mu}^{s}\left(x_{\mu}^{H}\right)$ do ponto de equilíbrio hiperbólico do tipo-1 $x_{\mu}^{H}=\left(\frac{2}{3}, 0\right) \ldots \ldots \ldots \ldots \ldots \ldots \ldots \ldots \ldots \ldots \ldots \ldots \ldots \ldots \ldots$

9.10 Retrato de fase do sistema (9.4) para $\mu_{0}=0$. A fronteira da região de estabilidade fraca $\partial A\left(x_{\mu_{0}}^{H}\right)$ está contida na união da variedade estável $W_{\mu_{0}}^{s}\left(x_{1}\right)$ do ponto de equilíbrio hiperbólico do tipo-1 $x_{1}=(8 / 3,0,0)$, da variedade estável $W_{\mu_{0}}^{s}\left(x_{2}\right)$ do ponto de equilíbrio hiperbólico do tipo-1 $x_{2}=(2 / 3,0,3)$ com a variedade estável $W_{\mu_{0}}^{s}\left(x_{3}\right)$ do ponto de equilíbrio hiperbólico do tipo-1 $x_{3}=(1 / 3,7 / 3,0) . \ldots \ldots \ldots 199 \ldots$ 
9.11 Retrato de fase do sistema (9.4) para $\mu=-0.2$. A fronteira da região de estabilidade $\partial A_{\mu}\left(x_{\mu}^{H}\right)$ está contida na união da variedade estável $W_{\mu}^{s}\left(x_{1}\right)$ do ponto de equilíbrio hiperbólico do tipo-1 $x_{1}=(2.5625,0,0)$, da variedade estável $W_{\mu}^{s}\left(x_{2}\right)$ do ponto de equilíbrio hiperbólico do tipo-1 $x_{2}=$ $(0.8958,0,2.6667)$, da variedade estável $W_{\mu}^{s}\left(x_{3}\right)$ do ponto de equilíbrio hiperbólico do tipo-1 $x_{3}=(0,2.6667,0)$ e com a variedade estável $W_{\mu}^{s}\left(x_{4}\right)$ do ponto de equilíbrio hiperbólico do tipo-2 $x_{4}=(0,0,2.6667) \ldots \ldots \ldots \ldots$. . . . . . 200

9.12 Retrato de fase do sistema (9.4) para $\mu_{0}=0.02$. A fronteira da região de estabilidade $\partial A_{\mu}\left(\phi_{\mu}^{H}\right)$ está contida na união da variedade estável $W_{\mu}^{s}\left(x_{1}\right)$ do ponto de equilíbrio hiperbólico do tipo-1 $x_{1}=(2.6778,0,0)$, da variedade estável $W_{\mu}^{s}\left(x_{2}\right)$ do ponto de equilíbrio hiperbólico do tipo-1 $x_{2}=$ $(0.6370,0,3.0408)$, da variedade estável $W_{\mu}^{s}\left(x_{3}\right)$ do ponto de equilíbrio hiperbólico do tipo- $1 x_{3}=(0.3635,2.2989,0)$ com a variedade estável $W_{\mu}^{s}\left(x_{\mu}^{H}\right)$ do ponto de equilíbrio hiperbólico do tipo-2 $x_{\mu}^{H}=(1,1,1) \ldots \ldots \ldots \ldots . \ldots \ldots$ 


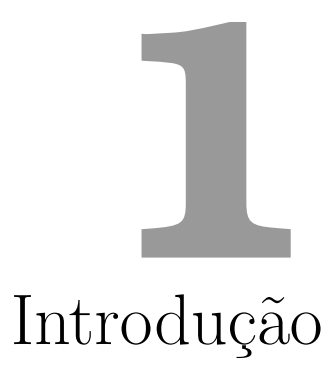

Pontos de equilíbrio assintoticamente estáveis de sistemas dinâmicos autônomos não lineares não são, em geral, globalmente estáveis. Na maioria dos casos, existe um subconjunto de condições iniciais, chamado de região de estabilidade, cujas trajetórias, iniciando dentro deste conjunto, tendem para o ponto de equilíbrio assintoticamente estável quando o tempo tende ao infinito.

Na literatura, esse conjunto de condições é também chamado de área de atração ou região de atração ou bacia de atração. Se $x_{0}$ é um ponto de equilíbrio assintoticamente estável, a região de estabilidade de $x_{0}$ é o conjunto:

$$
A\left(x_{0}\right)=\left\{x \in \mathbb{R}^{n}: \varphi(t, x) \rightarrow x_{0} \text { quando } t \rightarrow+\infty\right\} .
$$

O problema de determinar a região de estabilidade de um ponto de equilíbrio assintoticamente estável para um sistema dinâmico autônomo não linear é relevante em diversas aplicações no campo da engenharia, incluindo problemas de estabilidade em sistemas elétricos de potência [CWV87, SGAB09, VWC85, CCC95], técnicas de otimização global via sistemas dinâmicos [LC00] e em outras áreas tais como ecologia [May01] e economia $\left[\mathrm{AH}^{+} 71\right]$. Entretanto, determinar ou estimar a região de estabilidade de um sistema dinâmico é um problema difícil e métodos sistemáticos para este fim só existem para classes específicas de sistemas. Uma extensa pesquisa de métodos e técnicas para estimar regiões de estabilidade de sistemas dinâmicos autônomos pode ser encontrada em [GTV85]. O desenvolvimento de métodos para estimar regiões de estabilidade inicia com a consolidação da própria teoria de estabilidade. 
As primeiras noções de estabilidade remontam ao século $X V I I I$ onde os matemáticos Leonhard Paul Euler e Joseph-Louis Lagrange estabeleceram uma conexão entre os conceitos de estabilidade com os pontos de máximos e mínimos da função energia. Lyapunov apresentou condições suficientes para determinar a estabilidade de um ponto de equilíbrio mediante a existência de uma função escalar, chamada de Função de Lyapunov, com condições impostas sobre a função e sobre a sua derivada temporal. Logo percebeuse que a função de Lyapunov poderia, além de provar estabilidade fornecer estimativas da região de estabilidade. A grande dificuldade em se determinar uma estimativa da área de atração segundo a teoria de Lyapunov, é que o Teorema de Lyapunov não informa como obter a função de Lyapunov.

Em 1941, Joseph P. LaSalle apresentou o princípio de invariância de LaSalle que fornece estimativas da região de estabilidade em termos dos conjuntos de nível das funções de Lyapunov.

$\mathrm{Na}$ literatura existem vários métodos para estimar as regiões de estabilidade de sistemas não lineares, sendo que alguns deles usam função de Lyapunov e outros não. Porém, os métodos existentes, quase em sua totalidade, proporcionam estimativas muito conservadoras da região, ou seja, obtêm subconjuntos contidos na região de estabilidade.

Os métodos que utilizam funções de Lyapunov para obter estimativas da região de estabilidade de pontos de equilíbrio assintoticamente estáveis dependem da existência desta função. Entretanto, não existe nenhum procedimento sistemático para se obter funções de Lyapunov para sistemas dinâmicos não lineares.

O método de Zubov [YV67, DSR71] apresenta uma maneira de encontrar a região de estabilidade através de uma função de Lyapunov, porém para obtermos a função de Lyapunov é necessário resolver iterativamente um conjunto de equações diferenciais parciais. Na maioria dos casos, a resolução analítica destas equações é impossível e quando possível a convergência do método não é uniforme.

Nos anos 80, houve um enorme avanço na teoria da região de estabilidade com resultados acerca da caracterização global das regiões de estabilidade de sistemas dinâmicos autonômos não lineares [CHW88]. Motivado pelo problema de análise de estabilidade transitória em sistemas elétricos de potências, Chiang em [CHW88] apresentou uma caracterização completa da fronteira da região de estabilidade em termos das variedades estáveis dos pontos de equilíbrios instáveis e dos ciclos limites instáveis presentes na fronteira.

A partir desta caracterização, algoritmos numéricos para estimar a região de estabilidade foram desenvolvidos para classes importantes de sistemas dinâmicos com aplicações na área de sistemas elétricos de potência.

Chiang em [CWV87] propôs o método CUEP que oferece uma estimativa da região de estabilidade a partir do cálculo do ponto de equilíbrio instável de controle, que é um ponto de equilíbrio instável na fronteira da região 
de estabilidade [CT89]. O método BCU é um algoritmo numérico que foi desenvolvido para calcular o CUEP para uma classe particular de sistemas de segunda ordem [CWV94].

A modelagem de fenômenos dinâmicos, sejam eles sociais, econômicos, biológicos ou de engenharia, é realizado por conjuntos de equações diferenciais que usualmente dependem de um ou mais parâmetros.

Um estudo qualitativo do sistema dinâmico pode ser feito através da análise do comportamento das soluções ao perturbarmos os parâmetros num determinado conjunto e verificar se essas perturbações levam a comportamentos dinâmicos diferentes do original.

Ao variarmos um ou mais parâmetros do sistema de equações diferenciais, mudanças topológicas podem ocorrer no comportamento das soluções desse sistema. Por exemplo, o ponto de equilíbrio de um sistema poderá perder estabilidade em determinados valores dos parâmetros do sistema e até mesmo, ao perder estabilidade, ocasionar o surgimento de órbitas periódicas isoladas, ou ciclos limites, estáveis. Estas mudanças são chamadas de bifurcações e os valores dos parâmetros em que as mudanças ocorrem são chamados valores de bifurcação. Especificamente, a Teoria das Bifurcações permite-nos estudar como o sistema se comportará e em que momento perderá estabilidade ao variar um ou mais parâmetros do mesmo.

Devido a não linearidade do sistema de equações diferenciais que modela um sistema, ciclos limites podem surgir, como consequência à variação de parâmetros, fazendo com que o sistema entre em um modo oscilatório. Um dos mecanismos que originam estes comportamentos oscilatórios é a chamada Bifurcação de Hopf.

A caracterização completa da fronteira da região de estabilidade desenvolvida em [CHW88] admite que os elementos críticos na fronteira da região de estabilidade sejam hiperbólicos. Entretanto, apesar dessa suposição ser genérica, os sistemas dinâmicos estão sujeitos a variações de parâmetros que podem conduzir ao surgimento de pontos de equilíbrio não hiperbólicos na fronteira da região de estabilidade e portanto, a bifurcações na fronteira. Em sistemas elétricos de potência, por exemplo, o aparecimento de bifurcações sela-nó na fronteira da região de estabilidade como consequência da variação de carga foi relatado em [BMAG03]. Na análise da estabilidade de tensão em sistemas de energia, pontos de bifurcação de Hopf sobre a fronteira da região de estabilidade também foram encontrados, ver [Gao04].

Recentemente foi apresentado em [Ama10] um estudo do comportamento da região de estabilidade de sistemas dinâmicos autônomos não lineares e de sua fronteira, sujeitos a variações de parâmetros, quando da existência de uma bifurcação sela-nó do tipo zero na fronteira da região de estabilidade.

Em vista disto, o intuito deste trabalho é entender o comportamento da região de estabilidade de um ponto de equilíbrio assintoticamente estável e de sua fronteira quando ocorrem bifurcações de Hopf na fronteira da região de estabilidade. Nesta tese, faremos uma caracterização da região de estabi- 
lidade de um ponto de equilíbrio assintoticamente estável e da sua fronteira, mas admitindo a existência de uma Bifurcação de Hopf na fronteira da região de estabilidade.

Inicialmente, desenvolveremos uma caracterização da fronteira da região de estabilidade de um ponto de equilíbrio assintoticamente estável admitindo a existência de pontos de equilíbrio não hiperbólicos do tipo Hopf na fronteira da região de estabilidade. Exibiremos resultados que mostram que a fronteira da região de estabilidade é a união das variedades estáveis dos elementos críticos com as variedades central, centro-estável e/ou estável dos pontos de equilíbrio não hiperbólicos do tipo Hopf.

Apresentaremos a definição de região de estabilidade fraca de um ponto de equilíbrio não hiperbólico Hopf supercrítico do tipo zero e exploraremos a caracterização da fronteira da região de estabilidade como a união das variedades estáveis dos elementos críticos pertencentes à fronteira.

Posteriormente, enunciaremos a caracterização global da fronteira da região de estabilidade de um ponto de equilíbrio assintoticamente estável na vizinhança do valor crítico de uma bifurcação de Hopf do tipo- $k$, com $1 \leq k \leq n-2$. Finalmente, caracterizaremos a fronteira da região de estabilidade de um ponto de equilíbrio assintoticamente estável na vizinhança de um valor crítico de bifurcação de Hopf supercrítica do tipo zero.

Diversos exemplos em diversas áreas de aplicação são utilizados para ilustrar os resultados desta tese.

\subsection{Organização do Trabalho}

O presente trabalho será estruturado da seguinte forma.

No Capítulo 2, apresentar-se-ão alguns resultados sobre a Topologia dos Espaços Métricos e também da Teoria de Sistemas Dinâmicos. Além disso, apresentaremos o conceito de ponto de equilíbrio não hiperbólico do tipo Hopf, objeto de estudo deste trabalho, e enunciaremos uma versão do $\lambda$ lema para pontos de equilíbrio Hopf.

No Capítulo 3, apresentaremos um resumo da teoria existente de conjuntos atrativos e de conjuntos minimais e forneceremos uma caracterização topológica da região de estabilidade para esses conjuntos.

O capítulo 4 contém uma breve revisão da teoria de caracterização da fronteira da região de estabilidade de um ponto de equilíbrio assintoticamente estável proposta em [CHW88]. Também enunciaremos a caracterização da fronteira da região de estabilidade, admitindo a existência de um ponto de equilíbrio não hiperbólico sela-nó do tipo zero, desenvolvida em [Ama10].

No capítulo 5, exporemos condições suficientes e necessárias para que um ponto de equilíbrio não hiperbólico do tipo Hopf pertença à fronteira da região de estabilidade de um ponto de equilíbrio assintoticamente estável. 
Em seguida, caracterizaremos a fronteira da região de estabilidade como a união das variedades estáveis dos pontos de equilíbrio hiperbólicos com as variedades centrais, centro-estáveis ou estáveis dos pontos de equilíbrio não hiperbólicos do tipo Hopf.

No capítulo 6, exibiremos uma definição de transversalidade com uma condição sobre as dimensões das variedades dos equilíbrios para podermos acomodar na fronteira da região de estabilidade a presença de órbitas periódicas. Posteriormente, generalizaremos os resultados obtidos no capítulo anterior admitindo a existência de órbitas periódicas hiperbólicas na fronteira da região de estabilidade.

No capítulo 7, definiremos o conceito de região de estabilidade fraca de um ponto de equilíbrio não hiperbólico Hopf supercrítico do tipo zero e algumas de suas propriedades. Condições necessárias e suficientes para que elementos críticos hiperbólicos pertençam à fronteira da região de estabilidade fraca serão apresentadas e uma caracterização da fronteira como a união das variedades estáveis dos elementos críticos pertencentes à fronteira será desenvolvida. Finalmente, definiremos também o conceito de região de quase-estabilidade de um ponto de equilíbrio Hopf supercrítico do tipo zero e exploraremos a caracterização de sua fronteira.

No Capítulo 8, apresentaremos a bifurcacão de um sistema bidimensional dependente de um parâmetro na vizinhança do valor crítico de bifurcação do tipo Hopf. Em seguida, utilizaremos o Método da Projeção para analisarmos a Bifurcação de Hopf para um sistema $n$-dimensional.

No capítulo 9, mostraremos resultados relativos ao comportamento da região de estabilidade de um ponto de equilíbrio assintoticamente estável na vizinhança de um valor crítico de bifurcação de Hopf subcrítico do tipo- $k$, com $1 \leq k \leq n-2$, e a caracterização da fronteira nessa vizinhança em termos das variedades estáveis dos elementos críticos pertencentes à fronteira. Depois, exploraremos resultados relativos ao comportamento da região de estabilidade de um ponto de equilíbrio assintoticamente estável na vizinhança de um valor crítico de bifurcação de Hopf supercrítico do tipo- $k$, com $1 \leq k \leq n-2$, e a caracterização da fronteira nessa vizinhança em termos das variedades estáveis dos elementos críticos pertencentes à fronteira. Em seguida estudaremos o comportamento da região de estabilidade de um ponto de equilíbrio assintoticamente estável na vizinhança de um valor crítico de bifurcação de Hopf supercrítico do tipo zero e a caracterização de sua fronteira nessa vizinhança em termos das variedades estáveis dos elementos críticos pertencentes à fronteira.

No capítulo 10, algumas conclusões referentes ao presente trabalho serão discutidas e sugestões para trabalhos futuros serão apresentadas. Posteriormente apresentaremos as referências bibliográficas utilizadas no presente trabalho. E por fim, exibiremos os Apêndices A e B que complementam o trabalho, com os cálculos computacionais realizados para encontrar os pontos de equilíbrio Hopf, feitas com o auxílio do software MAPLE. 


\section{2 \\ Conceitos Preliminares}

Ao longo deste capítulo apresentaremos diversos conceitos e resultados preliminares que serão necessários ao desenvolvimento deste trabalho. Começaremos com uma breve introdução sobre a Topologia dos Espaços Métricos e em seguida apresentaremos alguns resultados da Teoria de Sistemas Dinâmicos. Este capítulo servirá de referência para os capítulos posteriores e tem por objetivo tornar o texto o mais autocontido possível.

\subsection{Espaços Métricos}

Nesta seção faremos uma revisão sobre conceitos referentes a um determinado tipo de conjunto onde podemos abstrair e generalizar a noção intuitiva de "distância" de modo a ser aplicada a outros tipos de conjuntos que não os familiares conjuntos euclidianos. Em outras palavras, revisaremos as noções de Espaços Métricos. As referências que utilizamos nesta seção são [Lim06], [Lim03] e [Lim81].

Uma métrica num conjunto $M$ é uma função $d: M \times M \rightarrow \mathbb{R}$, que associa a cada par ordenado de elementos $x, y \in M$ um número real $d(x, y)$, chamado distância de $x$ a $y$, de tal modo que satisfaça as seguintes propriedades, para quaisquer $x, y, z \in M$,

D1. $d(x, x)=0$;

D2. $d(x, y)>0$ se $x \neq y$; [Positividade]

D3. $d(x, y) \leq d(x, z)+d(z, y)$; [Desigualdade Triangular]

D4. $d(x, y)=d(y, x)$; [Comutatividade] 
Observe que as condições anteriormente impostas à métrica advêm das propriedades básicas da distância tradicional no conjunto $\mathbb{R}^{n}$.

Dado um conjunto $M$ e uma métrica $d$ em $M$, dizemos que o par $(M, d)$ é um Espaço Métrico ou simplesmente, que $M$ é um espaço métrico, deixando subentendido a métrica $d$.

O espaço euclidiano $\mathbb{R}^{n}$ é um exemplo de espaço métrico, onde $n$ é um número inteiro positivo qualquer, com a métrica euclidiana

$$
d(x, y)=\sqrt{\sum_{i=1}^{n}\left(x_{i}-y_{i}\right)^{2}},
$$

onde $x=\left(x_{1}, x_{2}, \cdots, x_{n}\right), y=\left(y_{1}, y_{2}, \cdots, y_{n}\right) \in \mathbb{R}^{n}$.

Consideremos o espaço vetorial real $E$. Uma norma no espaço $E$ é uma função real $\|\cdot\|: E \rightarrow \mathbb{R}$, que associa a cada vetor $x \in E$ o número real $\|x\|$, chamado a norma de $x$, satisfazendo as seguintes propriedades:

N1. $\|x\|>0$ se $x \neq 0$ e $\|x\|=0 \Leftrightarrow x=0$; [Positividade]

N2. $\|x+y\| \leq\|x\|+\|y\| ;$ [Desigualdade Triangular]

N3. $\|\alpha x\|=|\alpha| \cdot\|x\| ;$ [Homogeneidade]

onde $x, y \in E$ e $\alpha \in \mathbb{R}$.

Definimos o par $(E,\|\cdot\|)$ como Espaço Vetorial Normado, onde E é um espaço vetorial e $\|\cdot\|$ é uma norma em $E$. Por exemplo, a função

$$
\|x\|=\sqrt{\sum_{i=1}^{n} x_{i}^{2}}
$$

é uma norma no espaço vetorial $\mathbb{R}^{n}$, que chamaremos de norma euclidiana e, portanto, $\mathbb{R}^{n}$ é um espaço vetorial normado. Há uma infinidade de normas que se podem considerar no espaço euclidiano $\mathbb{R}^{n}$. Dentre elas, temos a norma do máximo dada por:

$$
\|x\|=\max \left\{\left|x_{1}\right|, \ldots,\left|x_{n}\right|\right\}
$$

e a norma da soma:

$$
\|x\|=\sum_{i=1}^{n}\left|x_{i}\right| .
$$

Vale ressaltar que essas normas junto com a norma euclidiana são equivalentes e, portanto, geram a mesma topologia, ver [Lim03] para maiores detalhes.

Outro exemplo, essencialmente idêntico ao espaço normado $\mathbb{R}^{n}$, é o espaço vetorial $\mathbb{C}^{n}$, onde $n$ é um inteiro positivo qualquer, dotado da norma 
$\|z\|=\sqrt{z \bar{z}}$, onde $z=\left(z_{1}, z_{2}, \ldots, z_{n}\right) \in \mathbb{C}^{n}$ e a barra sobre o número complexo $z$ denota seu conjugado $\bar{z}$.

Se $E$ é um espaço vetorial normado, a partir da norma podemos definir uma métrica em $E$ através da seguinte expressão, para quaisquer $x, y \in E$,

$$
d(x, y)=\|x-y\| .
$$

A métrica assim definida é dita ser a métrica proveniente da norma $\|\cdot\|$. Sendo assim, se $E$ é um espaço vetorial normado, então $E$ também é um espaço métrico com a métrica induzida pela norma definida acima. Por exemplo, o espaço $\mathbb{C}^{n}$ dotado da norma definida acima é um espaço métrico.

Um produto interno num espaço vetorial real $E$ é uma função $\langle\cdot, \cdot\rangle: E \times$ $E \longrightarrow \mathbb{R}$, que associa a cada par de vetores $x, y \in E$ um número real denotado por $\langle x, y\rangle$, chamado o produto interno de $x$ com $y$, de modo que sejam satisfeitas as seguintes condições, para quaisquer $x, y, z \in E \mathrm{e} \alpha \in \mathbb{R}$,

P1. $\langle x, y\rangle=\langle y, x\rangle ;$ [Simetria $]$

P2. $\langle x+z, y\rangle=\langle x, y\rangle+\langle z, y\rangle$; [Distributividade $]$

P3. $\langle\alpha x, y\rangle=\alpha \cdot\langle x, y\rangle=\langle x, \alpha y\rangle$; [Homogeneidade]

P4. $x \neq 0 \Rightarrow\langle x, x\rangle>0$ e $x=0 \Leftrightarrow\langle x, x\rangle=0$. [Positividade]

As três primeiras propriedades implicam que o produto interno no espaço vetorial $E$ é linear tanto na primeira variável quanto na segunda variável.

A partir do produto interno, podemos definir a norma de um vetor $x \in E$ segundo $\|x\|=\sqrt{\langle x, x\rangle}$ e que satisfaz as propriedades da definição de norma. O exemplo mais natural de espaço vetorial com produto interno é o Espaço Euclidiano $\mathbb{R}^{n}$ com o produto interno definido por

$$
\langle x, y\rangle=\sum_{i=1}^{n} x_{i} y_{i}
$$

onde $x, y \in \mathbb{R}^{n}$.

Podemos definir também um produto interno no espaço complexo $\mathbb{C}^{n}$, sabendo que o mesmo não é bilinear. De fato, se o produto interno no espaço complexo $\mathbb{C}^{n}$ fosse bilinear, então $\langle i x, i x\rangle=i^{2}\langle x, x\rangle=-\langle x, x\rangle$ e, assim sendo, não poderia ser positivo. Um produto interno no espaço complexo $\mathbb{C}^{n}$ é uma função $\langle\cdot, \cdot\rangle: \mathbb{C}^{n} \times \mathbb{C}^{n} \longrightarrow \mathbb{C}$ que associa a cada par de vetores $x, y \in \mathbb{C}^{n}$ um número complexo $\langle x, y\rangle$ e que goza das seguintes propriedades, para quaisquer $x, y, z \in \mathbb{C}^{n}$ e $\beta \in \mathbb{C}$,

H1. $\langle x, y\rangle=\overline{\langle y, x\rangle}$;

H2. $\langle x, y+z\rangle=\langle x, y\rangle+\langle x, z\rangle$; 
H3. $\langle x, \beta y\rangle=\beta \cdot\langle x, y\rangle$;

H4. $x \neq 0 \Rightarrow\langle x, x\rangle>0$ e $x=0 \Leftrightarrow\langle x, x\rangle=0$.

Das propriedades $\mathbf{H 1}$ e H2, temos que $\langle x+y, z\rangle=\langle x, z\rangle+\langle y, z\rangle$. As propriedades $\mathbf{H 1}$ e $\mathbf{H 3}$ implicam que $\langle\beta x, y\rangle=\bar{\beta} \cdot\langle x, y\rangle$. Diante disso, o produto interno no espaço complexo é anti-linear na primeira variável e linear na segunda.

No espaço complexo $\mathbb{C}^{n}$ definiremos o produto interno canônico, para $x, y \in \mathbb{C}^{n}$, como

$$
\langle x, y\rangle=\sum_{i=1}^{n} \bar{x}_{i} y_{i} .
$$

Ao longo do trabalho, salvo menção explícita em contrário, consideraremos sempre os espaços $\mathbb{C}^{n}$ e $\mathbb{R}^{n}$ providos do produto interno canônico e da distância que dela se origina.

\subsubsection{Conjuntos Abertos}

A definição de conjuntos abertos requer o uso de bolas de raio $\varepsilon$. Começaremos definindo o conceito de bola por ser a construção topológica mais básica. Consideremos $M$ um espaço métrico com a métrica $d$.

A bola aberta de centro num ponto $a \in M$ e raio $\varepsilon>0$ é o conjunto, denotado por $B(a ; \varepsilon)$, dos pontos $x \in M$ cuja distância ao ponto $a$ é menor do que $\varepsilon$, ou seja,

$$
B(a ; \epsilon)=\{x \in M ; d(x, a)<\varepsilon\} .
$$

A bola fechada de centro num ponto $a \in M$ e raio $\varepsilon>0$ é o conjunto, denotado por $B[a ; \varepsilon]$, dos pontos $x \in M$ cuja distância ao ponto $a$ é menor do que ou igual a $\varepsilon$, ou seja,

$$
B[a ; \epsilon]=\{x \in M ; d(x, a) \leq \varepsilon\} .
$$

A esfera de centro num ponto $a \in M$ e raio $\varepsilon>0$ é o conjunto, denotado por $S(a ; \varepsilon)$, dos pontos $x \in M$ cuja distância ao ponto $a$ é igual a $\varepsilon$, ou seja,

$$
S[a ; \epsilon]=\{x \in M ; d(x, a)=\varepsilon\} .
$$

Seja $X$ um subconjunto do espaço métrico $M$. Dizemos que o ponto $a \in X$ é um ponto interior a $X$ quando o ponto $a$ é centro de alguma bola aberta contida em $X$. Ou seja, quando existe $\varepsilon>0$ tal que para todo ponto $x \in M$ e $d(x, a)<\varepsilon$ implica em $x \in X$. Chama-se o interior de $X$ em $M$ ao conjunto, denotado por int $X$, formado pelos pontos interiores a $X$. Num espaço métrico, dizemos que o conjunto $X$ é uma vizinhança do ponto $a$ se $a \in$ int $X$. 
Uma condição necessária e suficiente para que o ponto $a$ não seja interior a $X$ é que toda bola aberta de centro em $a$ contenha algum ponto que não pertença a $X$, isto é, para todo $\varepsilon>0$ temos que $B(a ; \varepsilon) \cap(M \backslash X) \neq \emptyset$.

Dizemos que um subconjunto $X$ de um espaço métrico $M$ é aberto quando todos os seus pontos são interiores, isto é, se para cada $x \in X$ existe uma bola aberta de centro em $x$ e de raio $\varepsilon>0$ tal que $B(x ; \varepsilon) \subset X$. $\mathrm{E}$, portanto, $X$ é aberto se, e somente se, $i n t X=X$.

Intuitivamente da definição de aberto, podemos entender que a partir de cada ponto de $X$ podemos sempre nos movimentar a uma pequena distância em qualquer direção e ainda assim permanecer no conjunto.

Um exemplo trivial de conjunto aberto no espaço $\mathbb{R}^{n}$ é a bola aberta $B(a ; \varepsilon) \subset \mathbb{R}^{n}$, onde $a \in \mathbb{R}^{n}$. Outro exemplo de conjunto aberto que podemos considerar é o subconjunto aberto int $X$, para qualquer que seja o subconjunto $X \subset \mathbb{R}^{n}$.

Sendo assim, dados um conjunto $X$ e um ponto $a \in M$, ha três possibilidades que se excluem mutuamente: ou $a \in \operatorname{int} X$, ou $a \in \operatorname{int}(M \backslash X)$ ou então toda bola aberta de centro $a$ contém pontos de $X$ e pontos do complementar de $X$. Neste caso, dizemos que $a$ é um ponto de fronteira de $X$. A fronteira de $X$ em $M$ é o conjunto, denotado por $\partial X$, formado pelos pontos $a \in M$ tais que toda bola aberta de centro $a$ contém pelos menos um ponto de $X$ e um ponto do complementar de $X$. Logo, todo subconjunto $X \subset M$ determina a decomposição do espaço $M$ como reunião de três subconjuntos dois a dois disjuntos

$$
M=\operatorname{int} X \cup \partial X \cup \operatorname{int}(M \backslash X) .
$$

O próximo resultado fornece o comportamento de conjuntos abertos sujeitos às operações de união e interseção de conjuntos.

Teorema 2.1.1. [Lim03] Os conjuntos abertos do espaço métrico M gozam das seguintes propriedades:

(i) O conjunto vazio $\emptyset$ e o espaço $M$ são abertos;

(ii) A interseção $A=A_{1} \cap \cdots \cap A_{k}$ de um número finito de conjuntos abertos $A_{1}, \ldots, A_{k}$ é um conjunto aberto;

(iii) A reunião $A=\cup_{\lambda \in L} A_{\lambda}$ de uma família qualquer $\left(A_{\lambda}\right)_{\lambda \in L}$ de conjuntos abertos $A_{\lambda}$ é um conjunto aberto.

Consideremos o subconjunto $X \subset M$ munido da métrica induzida por $M$. Um conjunto $A$ em $X$ diz-se aberto em $X$ quando, para cada $a \in A$ existe $\varepsilon>0$ tal que $B(a ; \varepsilon) \cap X \subset A$. Ou seja, para cada $a \in A$ existe $\varepsilon>0$ tal que os pontos $x$, pertencentes a $X$, que cumprem a condição $d(x, a)<\varepsilon$ pertencem ao conjunto A. Por exemplo, se $0<\varepsilon<b-a$, o intervalo $[a, a+\varepsilon)$ é aberto no espaço $[a, b]$, pois $[a, a+\varepsilon)=(a-\varepsilon, a+\varepsilon) \cap[a, b]$, mas não é aberto em $\mathbb{R}$. 


\subsubsection{Conjuntos Fechados}

Apresentaremos agora o conceito de conjunto fechado que desempenha também um papel fundamental na geometria e análise de espaços euclidianos.

Diremos que um ponto $a \in M$ é ponto aderente a um conjunto $X \subset M$ quando, para todo $\varepsilon>0$, a bola aberta $B(a ; \varepsilon)$ contém algum ponto de $X$. Ou equivalentemente, uma condição necessária e suficiente para que o ponto $a$ seja aderente ao conjunto $X$ é que seja limite de uma sequência de pontos desse conjunto. Definamos o conjunto dos pontos aderentes a $X$ como fecho de $X$ e o denotaremos por $\bar{X}$.

Observemos que ao afirmar que $a$ não é um ponto aderente ao conjunto $X$ é o mesmo que dizer que $a$ é ponto interior do conjunto $M \backslash X$. Diante disso, utilizaremos o conceito de conjunto aberto para redefinir as condições para que um ponto seja aderente ou não aderente a um conjunto dado. Então:

a. Tem-se $a \in \bar{X}$ se, e somente se, todo aberto que contém $a$ intersecta o conjunto $X, \mathrm{e}$

b. Tem-se $b \notin \bar{X}$ se, e somente se, existe um aberto contendo $b$ disjunto de $X$.

Notemos que $\bar{\emptyset}=\emptyset, \bar{M}=M$ e $X \subset \bar{X}$ para todo $X \subset M$.

Um subconjunto $X$ do espaço métrico $M$ é fechado se contém todos seus pontos aderentes, isto é, quando $X=\bar{X}$. Por exemplo, a bola fechada $B[a ; \varepsilon]$ e a esfera $S[a ; \varepsilon]$ são exemplos triviais de subconjuntos fechados em $\mathbb{R}^{n}$.

Outro modo de definirmos conjunto fechado, possivelmente mais claro, é fazendo uso da noção de sequências convergentes. Sendo assim, uma condição necessária e suficiente para que um subconjunto $X$ do espaço métrico $M$ seja fechado é que o limite de qualquer sequência convergente formada por pontos em $X$ também pertença a $X$.

Os próximos resultados estabelecem algumas propriedades referentes aos conjuntos fechados.

Teorema 2.1.2. [Lim03] Um subconjunto é fechado em $M$ se, e somente se, seu complementar é aberto em $M$.

Proposição 2.1.3. [Lim03] O fecho de um subconjunto $X \subset M$ é um conjunto fechado.

Teorema 2.1.4. [Lim03] Os conjuntos fechados do espaco métrico M satisfazem as seguintes propriedades:

(i) O conjunto vazio $\emptyset$ e o espaco $M$ são fechados;

(ii) A união $F=F_{1} \cup \cdots \cup F_{k}$ de um número finito de conjuntos fechados $F_{1}, \ldots, F_{k}$ é um conjunto fechado; 
(iii) A interseção $F=\cap_{\lambda \in L} F_{\lambda}$ de uma família qualquer $\left(F_{\lambda}\right)_{\lambda \in L}$ de conjuntos fechados $F_{\lambda}$ é um conjunto fechado.

A Proposição 2.1.3 e o Teorema 2.1.4 garantem que a fronteira de todo conjunto $X$ em $M$ é um conjunto fechado. De fato, da definição de fronteira podemos concluir que todo ponto de fronteira de $X$ tem que ser aderente a $X$ e a $M \backslash X$. Isto é, $\partial X=\bar{X} \cap \overline{M \backslash X}$. Logo, $\partial X$ é a interseção finita de dois conjuntos fechados e, portanto, também é um conjunto fechado.

Vale ressaltar que o conjunto formado pela união infinita de conjuntos fechados pode não ser fechado. Por exemplo, consideremos os conjuntos fechados $F_{n}=\left[\frac{-n}{n+1}, \frac{n}{n+1}\right]$ para $n \geq 1$, temos que $\cup_{n \geq 1} F_{n}=(-1,1)$ é um intervalo aberto.

Teorema 2.1.5. [Mun75] Dados dois conjuntos fechados disjuntos $A, B \subset$ $\mathbb{R}^{n}$, então existem abertos disjuntos contendo $A$ e $B$.

Demonstração. Sejam $A, B$ subconjuntos fechados disjuntos de $\mathbb{R}^{n}$. Consequentemente, pelo Teorema 2.1.2, $\mathbb{R}^{n} \backslash A$ e $\mathbb{R}^{n} \backslash B$ são subconjuntos abertos de $\mathbb{R}^{n}$. Sendo assim, para cada $a \in A$ podemos afirmar que $a \in \mathbb{R}^{n} \backslash B$. Como $\mathbb{R}^{n} \backslash B$ é aberto, existe $\varepsilon_{a}>0$ tal que $B\left(a ; \varepsilon_{a}\right) \subset \mathbb{R}^{n} \backslash B$, isto é, $B\left(a ; \varepsilon_{a}\right)$ não intercepta $B$. Analogamente, para cada $b \in B$, existe $\varepsilon_{b}>0$ tal que $B\left(b ; \varepsilon_{b}\right)$ não intercepta $A$. Consideremos os conjuntos

$$
U=\cup_{a \in A} B\left(a ; \frac{\varepsilon_{a}}{2}\right) \text { e } V=\cup_{b \in B} B\left(b ; \frac{\varepsilon_{b}}{2}\right) .
$$

Então $U$ e $V$ são subconjuntos abertos contendo $A$ e $B$, respectivamente. Além disso, podemos afirmar ainda que os conjuntos $U$ e $V$ são disjuntos. De fato, suponhamos, por absurdo, que $U \cap V \neq \emptyset$. Se $z \in U \cap V$, então $z \in B\left(a ; \frac{\varepsilon_{a}}{2}\right) \cap B\left(b ; \frac{\varepsilon_{b}}{2}\right)$, para algum $a \in A$ e para algum $b \in B$. Pela desigualdade triangular, podemos concluir que $d(a, b) \leq d(a, z)+d(z, b)<$ $\frac{\varepsilon_{a}}{2}+\frac{\varepsilon_{b}}{2}$. Se $\varepsilon_{a} \leq \varepsilon_{b}$, então $d(a, b)<\frac{\varepsilon_{a}}{2}+\frac{\varepsilon_{b}}{2} \leq \frac{\varepsilon_{b}}{2}+\frac{\varepsilon_{b}}{2}=\varepsilon_{b}$, ou seja, $a \in B\left(b ; \varepsilon_{b}\right)$. Se $\varepsilon_{b} \leq \varepsilon_{a}$, então $d(a, b)<\frac{\varepsilon_{a}}{2}+\frac{\varepsilon_{b}}{2} \leq \frac{\varepsilon_{a}}{2}+\frac{\varepsilon_{a}}{2}=\varepsilon_{a}$, ou seja, $b \in B\left(a ; \varepsilon_{b}\right)$. Porém, nenhuma destas situações podem ocorrer, pela construção das bolas. E, portanto, os conjuntos $U$ e $V$ são abertos disjuntos contendo os conjuntos fechados disjuntos $A$ e $B$.

O conceito de conjunto fechado, assim como o conceito de conjunto aberto, é relativo a um espaço ambiente. Por exemplo, o subconjunto $\left[\frac{1}{2}, 1\right)$ é fechado em $[0,1)$ mas não é fechado em $\mathbb{R}$.

Dado um conjunto $X \subset M$. Um subconjunto $F$ em $X$ diz-se fechado em $X$ quando se tem $F=X \cap G$ onde $G$ é um conjunto fechado em $M$. Se $X \subset M$ é fechado, então um subconjunto $F \subset X$ é fechado em $X$ se, e somente se, é fechado em $M$.

Dados $Y \subset X \subset M$. Definamos o fecho de $Y$ relativamente a $X$ como sendo o conjunto $\bar{Y} \cap X$, dos pontos aderentes a $Y$ que pertencem ao conjunto $X$. 
Dado $X \subset Y \subset M$, dizemos que $X$ é denso em $Y$ quando $Y \subset \bar{X}$, ou seja, quando toda bola aberta em $Y$ contém algum ponto de $X$.

\subsubsection{Conjuntos Conexos}

Uma cisão de um espaço métrico $M$ é uma decomposição, $M=A \cup B$, de $M$ como reunião de dois subconjuntos abertos disjuntos $A$ e $B$. Consequentemente das condições $M=A \cup B$ e $A \cap B=\emptyset$, temos que $A=M \backslash B$ e $B=M \backslash A$. Por conseguinte, numa cisão $M=A \cup B$, os conjuntos $A, B$ são abertos e fechados em $M$.

A cisão $M=A \cup B$ diz-se trivial quando um dos abertos, $A$ ou $B$, é vazio e, consequentemente, o outro é igual a $M$. Caso contrário, é dita não trivial. Todo espaço métrico $M$ admite pelos menos a cisão trivial $M=M \cup \emptyset$. Um exemplo de cisão não trivial é $\mathbb{Q}=A \cup B$ onde $A=\{x \in \mathbb{Q}: x<\alpha\}$ e $B=\{x \in \mathbb{Q}: x>\alpha\}$ com $\alpha$ um número irracional.

Um espaço métrico $M$ chama-se conexo quando a única cisão possível em $M$ é a trivial. Um subconjunto $X$ de um espaço métrico $M$ diz-se um conjunto conexo quando o subespaço $X \subset M$ é conexo. Quando $X$ admite uma cisão não trivial, dizemos que $X$ é desconexo. Sendo assim, o conjunto Q é um conjunto desconexo, pois conforme vimos acima admite uma cisão não trivial.

Uma condição necessária e suficiente para que os únicos subconjuntos ao mesmo tempo abertos e fechados do espaço métrico conexo $M$ sejam $\emptyset$ e $M$ é que, para todo subconjunto $X \subset M$ com fronteira vazia tenha-se $X=M$ ou $X=\emptyset$. Uma outra propriedade importante é que a união de uma família de conjuntos conexos com um ponto em comum é um conjunto conexo.

A definição de conjunto conexo, como sendo aquele que admite apenas a cisão trivial, sugere que um conjunto conexo é formado por um só pedaço. Podemos redefinir o conceito de conjunto conexo utilizando para isso a idéia de unir dois pontos quaisquer do subconjunto por uma curva contínua contida no próprio conjunto. Diante disso, veremos a noção de espaço conexo por caminhos.

Um caminho num espaço métrico $M$ é uma aplicação contínua $f: I \rightarrow$ $M$, definida num intervalo $I$. Um exemplo trivial de caminho é a aplicação constante $f:[0,1] \rightarrow M, f(t)=c$ para todo $t \in[0,1]$. Outro exemplo de caminho, dado um espaço vetorial normado $E$, é a aplicação $f:[0,1] \rightarrow E$, definida por $f(t)=(1-t) a+t b$, que é contínua e se chama o caminho retilíneo que liga $a$ a $b$. Denotaremos o caminho retilíneo que liga os pontos $a$ e $b$ por $[a ; b]$.

Um subconjunto $X$ de um espaço vetorial $E$ diz-se convexo quando todo caminho retilíneo que liga dois pontos quaisquer em $X$ está contido em $X$. Por exemplo, todo subespaço vetorial de $E \subset \mathbb{R}^{n}$ é convexo, assim como toda bola aberta ou fechada em $\mathbb{R}^{n}$ é convexa.

Diremos que os pontos $a, b \in M$ podem ser ligados por um caminho em 
$M$ quando existir um caminho $f: I \rightarrow M$ tal que $a, b \in f(I)$. Um espaço métrico $M$ chama-se conexo por caminhos quando dois pontos quaisquer $a, b \in M$ podem ser ligados por um caminho em $M$. Um conjunto $X \subset M$ diz-se conexo por caminhos quando o subespaço $X$ tem essa propriedade. Por exemplo, todo conjunto convexo num espaço vetorial normado é conexo por caminhos.

Teorema 2.1.6. [Lim03] Se o espaço métrico $M$ é conexo por caminhos, então $M$ é conexo.

Considere um espaço vetorial normado $E$, com $\operatorname{dim} E>1$. Como $E \backslash\{a\}$, para todo $a \in E$, é conexo por caminhos então, pelo Teorema 2.1.6, $E \backslash\{a\}$ é conexo.

Vale ressaltar que a recíproca do Teorema 2.1.6 não é verdadeira. Por exemplo, seja $X \subset \mathbb{R}^{2}$ o gráfico da função $f:[0,+\infty) \rightarrow \mathbb{R}$, dada por $f(x)=\cos \left(\frac{1}{x}\right)$ se $x>0$ e $f(0)=0$. Temos que $X$ é conexo mas não é conexo por caminhos.(Para a demonstração, ver [Lim03, pp. 103].)

\subsubsection{Teorema da Função Implícita}

Nesta seção exibiremos um resultado central da Análise, o Teorema da Função Implícita. Dado a equação $F(x, y)=0$, para cada valor de $x$ pode existir um ou mais valores de $y$ ou também nenhum que satisfaça a equação dada. Seja $I$ um intervalo tal que para cada $x \in I$ existe apenas um $y$ satisfazendo a equação $F(x, y)=0$, então dizemos que a equação $F(x, y)=0$ define implicitamente $y$ como uma função de $x$ em $I$.

Consideremos a função $F(x, y)=x^{2}+y^{2}$. Então a equação $F(x, y)=1$ descreve o círculo unitário centrado na origem no plano $x y$. Considere o ponto $\left(x_{0}, y_{0}\right)=(1,0)$ e tomemos vizinhanças pequenas próximas do ponto $x_{0}=1$ e $y_{0}=0$. Não existe a possibilidade de representar o círculo unitário como o gráfico de uma única função de uma variável $y=f(x)$, pois numa vizinhança de $x_{0}=1$ existem duas opções para a função $y$, ou seja, $y=$ $\pm \sqrt{1-x^{2}}$.

Se considerarmos um ponto $\left(x_{0}, y_{0}\right)$ do círculo unitário com $y_{0}>0 \mathrm{e}$ vizinhanças pequenas próximas do ponto $x_{0}$ e $y_{0}$. Existe uma única função de uma variável $y=\sqrt{1-x^{2}}$ definida numa vizinhança próxima de $x_{0}$, com $y_{0}=f\left(x_{0}\right)$, e descreve o semicírculo superior.

O objetivo do teorema de função implícita é estabelecer condições sob as quais uma equação $F(x, y)=0$ define implicitamente $y$ como função de $x$ (ou $x$ como função de $y$ ), mesmo em situações em que não pode escrever fórmulas explícitas.

Teorema 2.1.7. [Lim81][Teorema da Função Implícita] Sejam U um subconjunto aberto de $\mathbb{R}^{m} \times \mathbb{R}^{n}$ e $f: U \rightarrow \mathbb{R}^{n}$ uma função de classe $\mathcal{C}^{k}, k>1$. Considere um ponto $(x, y) \in U$, onde $x \in \mathbb{R}^{m}$ e $y \in \mathbb{R}^{n}$, com $f(x, y)=c$. Se 
a matriz $D_{y} f(x, y)$ de ordem $n \times n$ das derivadas parciais de $f$ com relação a y é invertivel, então existem conjuntos abertos $V_{m} \subset \mathbb{R}^{m} e V_{n} \subset \mathbb{R}^{n}$ com $(x, y) \in V_{m} \times V_{n} \subset U$ e uma única função $\chi: V_{m} \rightarrow V_{n}$ de classe $\mathcal{C}^{k}$ tal que $f(x, \chi(x))=c$ para todo $x \in V_{m}$. Além disso, $f(x, y) \neq c$ se $(x, y) \in V_{m} \times V_{n}$ e $y \neq \chi(x)$. A derivada da função $\chi$ é dada pela fórmula:

$$
D \chi(x)=-\left[D_{y} f(x, \chi(x))\right]^{-1} D_{x} f(x, \chi(x)) .
$$

Se as suposições do Teorema da Função Implícita são satisfeitas, existe localmente uma função definida implicitamente, porém se as condições não são satisfeitas, nada podemos concluir quanto a existência local da função definida implicitamente, podendo ocorrer ou não. Diante disso, as condições do Teorema da Função Implícita são suficientes, mas não necessárias, para garatirmos que existe localmente uma função definida implicitamente pela equação $f(x, y)=c$.

\subsection{Sistemas Dinâmicos}

Nesta seção, introduziremos alguns conceitos básicos relacionados a Teoria de Sistemas Dinâmicos. Em particular, estudaremos os sistemas dinâmicos descritos pela Equação Diferencial Ordinária (EDO) autonôma não linear

$$
\dot{x}=f(x),
$$

onde $f: \mathbb{R}^{n} \rightarrow \mathbb{R}^{n}$ é um campo vetorial de classe $\mathcal{C}^{1}$. Iniciaremos esta seção discutindo a existência e unicidade das soluções de equações diferenciais ordinárias autonômas não lineares e estudaremos também a continuidade das soluções com relação às condições iniciais. Em seguida, introduziremos o conceito de conjunto invariante e veremos alguns exemplos de conjuntos invariantes relacionados ao sistema dinâmico (2.1). Além disso, discutiremos a estabilidade local e também o comportamento assintótico das soluções do sistema dinâmico autonômo (2.1) e, em particular, os pontos de equilíbrio hiperbólicos e não hiperbólicos. A última parte deste capítulo está reservada para o estudo do conceito de transversalidade, do qual faremos uso com frequência ao longo do texto para a caracterização da fronteira da região de estabilidade. Para complementar ou obter maiores detalhes a respeito das definições e resultados expostos nesta seção, sugere-se consultar [Alb06], [Ama10], [Hal69], [HPS70], [GH83], [Per13], [Shu13], [Sma67], [SHD03], [Sot79], [Wig03] e [Vid02].

\subsubsection{Teoria Geral}

Um sistema dinâmico é uma descrição funcional das soluções de um modelo matemático que descreve um dado sistema em estudo. Em outras palavras, podemos definir um sistema dinâmico como uma função $\varphi(t, x)$, 
definida para todo $t \in \mathbb{R}$ e $x \in \Omega \subset \mathbb{R}^{n}$, que descreve como pontos $x \in \Omega$ se movem ao longo do tempo.

Dizemos que um sistema dinâmico é autonômo quando o campo vetorial não depende explicitamente da variável independente $t$. Por outro lado, se o campo vetorial depende da variável $t$, o sistema dinâmico é dito nãoautonômo. De maneira intuitiva, o comportamento de um sistema autonômo muda com o tempo, mas suas "propriedades fundamentais" permanecem as mesmas independente do tempo. Um sistema dinâmico é dito linear se o campo vetorial não contém termos de ordem maiores ou iguais a $2, O\left(\|x\|^{2}\right)$. Ou também, se a equação diferencial ordinária é uma combinação linear da variável dependente $x$ e de suas derivadas, com os coeficientes como funções da variável independente ou funções constantes. Caso contrário, dizemos que o sistema dinâmico é não-linear.

Uma função diferenciável $\varphi: I \rightarrow \Omega$ chama-se solução da equação diferencial ordinária autonôma não linear

$$
\dot{x}=f(x)
$$

no intervalo I se

a. A imagem do gráfico de $\varphi$ em $I$ está contida em $\Omega$ e

b. $\frac{d \varphi(t)}{d t}=f(\varphi(t))$ para todo $t \in I$,

onde $f: \Omega \rightarrow \mathbb{R}^{n}$ é um campo vetorial de classe $\mathcal{C}^{1}, \Omega \subset \mathbb{R}^{n}$ é um conjunto aberto e $I$ é um intervalo não degenerado da reta.

Observemos que no caso de $t$ ser um ponto extremo do intervalo $I$, a derivada acima é a derivada lateral respectiva.

Fixado um ponto $x_{0} \in \Omega$, chamado de valor inicial para a equação diferencial ordinária autonôma (2.2), o problema de encontrar a solução $x(t)$ da EDO (2.2) satisfazendo a condição inicial $x(0)=x_{0}$ é conhecido como Problema de Valor Inicial(PVI):

$$
\text { PVI }:=\left\{\begin{array}{l}
\dot{x}=f(x) \\
x(0)=x_{0}
\end{array} .\right.
$$

O teorema a seguir estabelece condições suficientes sobre a função diferenciável $f$ para garantir a existência e a unicidade das soluções da equação diferencial ordinária autonôma (2.2).

Teorema 2.2.1 (Teorema de Existência e Unicidade). [Sot79] Sejam $f: \Omega$ $\rightarrow \mathbb{R}^{n}$ um campo vetorial de classe $\mathcal{C}^{1}$ e $x_{0} \in \Omega$ uma condição inicial. Então existe um $\eta>0$ tal que o problema de valor inicial

$$
\dot{x}=f(x), x(0)=x_{0}
$$


tem uma única solução $\varphi\left(t, x_{0}\right)$ no intervalo $[-\eta, \eta]$.

Admitindo as hipóteses do Teorema de Existência e Unicidade, é possível prolongar as soluções locais para um intervalo maximal de tempo. Ou seja, para cada $x_{0} \in \Omega$ existe uma única solução maximal $x(t)=\varphi\left(t, x_{0}\right)$ de

$$
\dot{x}=f(x), x(0)=x_{0}
$$

definida num intervalo maximal de existência $\left(\kappa_{-}\left(x_{0}\right), \kappa_{+}\left(x_{0}\right)\right)$. Ou seja, $\varphi$ é solução maximal se não admite nenhum prolongamento que também é solução da equação.

O próximo resultado fornece uma condição suficiente para que as soluções sejam contínuas com relação às condições iniciais.

Teorema 2.2.2. [Sot79] Seja $f: \Omega \rightarrow \mathbb{R}^{n}$ um campo vetorial de classe $\mathcal{C}^{1}$. Considere $\varphi\left(t, x_{0}\right)$ a solução do PVI

$$
\dot{x}=f(x), x(0)=x_{0}
$$

definida no seu intervalo maximal de existência. Então

$$
D=\left\{\left(t, x_{0}\right): x_{0} \in \Omega, t \in\left(\kappa_{-}\left(x_{0}\right), \kappa_{+}\left(x_{0}\right)\right)\right\}
$$

é aberto em $\mathbb{R} \times \Omega$ e $\varphi$ é contínua em $D$.

Considerando o espaço de estados do sistema como o $\mathbb{R}^{n}$, a equação diferencial ordinária autonôma não linear (2.2) define um sistema dinâmico autonômo de tempo contínuo. As soluções $\varphi_{t}(x)=\varphi(t, x)$ do sistema dinâmico autonômo (2.2) satisfazem as seguintes propriedades

a. $\varphi_{0}(x)=x$ para todo $x \in \Omega$

b. $\varphi_{t+s}(x)=\varphi_{t}\left(\varphi_{s}(x)\right)$ para todo $x \in \Omega$ e todos $t, s \in \mathbb{R}$ tais que $t+s \in$ $\left(\kappa_{-}(x), \kappa_{+}(x)\right)$ e $t \in\left(\kappa_{-}\left(\varphi_{s}(x)\right), \kappa_{+}\left(\varphi_{s}(x)\right)\right)$

A Figura 2.1 retrata o item $\mathbf{b}$ acima, segundo o qual a família de aplicações $\left\{\varphi_{t}: t \in \mathbb{R}\right\}$ é um grupo aditivo sob a operação de composição.

\subsubsection{Trajetórias}

Para uma melhor compreensão de como o sistema dinâmico autonômo (2.1) está evoluindo, faremos uso de determinados objetos geométricos básicos associados a um sistema dinâmico. Esses objetos aos quais nos referimos são chamados de órbitas ou trajetórias definidas pela solução do sistema dinâmico (2.1).

A aplicação $t \rightarrow \varphi(t, x)$ de $\mathbb{R}$ em $\mathbb{R}^{n}$ define uma curva em $\mathbb{R}^{n}$ passando por $x$ a qual denomina-se trajetória ou órbita passando por $x$ e que 


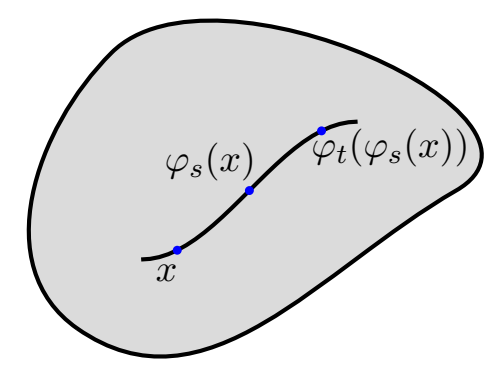

Figura 2.1: Trajetória do sistema dinâmico autonômo (2.1).

denotaremos pelo conjunto

$$
\gamma(x)=\left\{\varphi(t, x): t \in\left(\kappa_{-}\left(x_{0}\right), \kappa_{+}\left(x_{0}\right)\right)\right\} .
$$

Pelo Teorema de Existência e Unicidade, as trajetórias de um sistema dinâmico autonômo não se interceptam e, consequentemente, as órbitas $\gamma(x)$ são disjuntas duas a duas. Sendo assim, se $y=\varphi\left(t_{0}, x\right)$ para algum $t_{0}$, então as trajetórias $\gamma(y)$ e $\gamma(x)$ coincidem.

O conjunto $\gamma^{+}(x)=\left\{\varphi(t, x): t \in\left(0, \kappa_{+}\left(x_{0}\right)\right)\right\}$ chama-se semi-trajetória positiva passando pelo ponto $x$. Analogamente, o conjunto $\gamma^{-}(x)=\{\varphi(t, x)$ : $\left.t \in\left(\kappa_{-}\left(x_{0}\right), 0\right)\right\}$ chama-se semi-trajetória negativa passando pelo ponto $x$. Observemos que $\gamma(x)=\gamma^{+}(x) \cup \gamma^{-}(x)$.

Podemos também definir a trajetória de uma solução sobre um conjunto de condições iniciais. Sendo assim, dado $A$ um subconjunto de $\mathbb{R}^{n}$ e $t \in \mathbb{R}$, definamos o conjunto $\gamma(A)=\{\varphi(t, x): x \in A$ e $t \in \mathbb{R}\}=\cup_{x \in A} \gamma(x)$.

Definiremos agora dois tipos especiais de soluções do sistema dinâmico autonômo (2.1), os quais são relevantes para a caracterização da fronteira da região de estabilidade, que são eles os pontos de equilíbrio e as órbitas periódicas ou fechadas.

Dizemos que o ponto $\bar{x} \in \mathbb{R}^{n}$ é um ponto de equilíbrio do sistema dinâmico autonômo (2.1) se $f(\bar{x})=0$. Se $\bar{x}$ é um ponto de equilíbrio do sistema dinâmico autonômo (2.1), então a solução do sistema passando pelo ponto $\bar{x}$ em $t=0$ é a função $\varphi(t, \bar{x})=\bar{x}$, para todo $t \in \mathbb{R}$. Neste caso, $\gamma(\bar{x})=\{\bar{x}\}$.

Definamos uma trajetória do sistema dinâmico autonômo (2.1) como uma órbita periódica ou fechada se a trajetória não for um ponto de equilíbrio e para todo $x$ pertencente a trajetória, existe um tempo $T>0$ tal que $\varphi(T, x)=x$. Denotaremos a órbita periódica ou fechada por $\phi$ e o valor minímo $T>0$ que satisfaz a essa propriedade chamaremos de período da órbita $\phi$.

Segundo Poincaré, toda órbita fechada e isolada chamamos de ciclo limite. O termo isolado significa que não existe nenhuma outra trajetória 
fechada infinitesimalmente próxima a $\phi$. Sendo assim, todas trajetórias vizinhas a $\phi$ tendem ou a se aproximar ou a se afastar de $\phi$.

Os conjuntos definidos acima, ou seja, as órbitas, possuem uma característica em comum. Dada uma condição inicial pertencente a este conjunto, a solução passando por essa condição permanece no conjunto para todo tempo. Essa propriedade, a qual chamamos de invariância, é uma característica não somente das trajetórias como veremos, mas que é de fundamental importância para a análise de sistemas dinâmicos.

Um conjunto $A \subset \mathbb{R}^{n}$ é invariante com relação ao sistema (2.1) se para qualquer $x_{0} \in A$ temos $\varphi\left(t, x_{0}\right) \in A$ para todo $t \in \mathbb{R}$. Equivalentemente, $A$ é invariante se $\varphi(t, A) \subset A$ para todo $t \in \mathbb{R}$. Ou seja, toda solução iniciando no conjunto $A$ permanece em $A$ para todo o tempo $t$. Um exemplo trivial de conjunto invariante são as trajetórias passando por um ponto do sistema dinâmico autonômo (2.1).

Um conjunto $A \subset \mathbb{R}^{n}$ é positivamente invariante com relação ao sistema dinâmico autonômo (2.1) se, para qualquer $x_{0} \in A$ temos $\varphi\left(t, x_{0}\right) \in A$ para todo $t \geq 0$. Analogamente, um conjunto $A \subset \mathbb{R}^{n}$ é negativamente invariante com relação ao sistema dinâmico autonômo (2.1) se, para qualquer $x_{0} \in A$ temos $\varphi\left(t, x_{0}\right) \in A$ para todo $t \leq 0$.

Os resultados seguintes exibem algumas propriedades relacionados aos conjuntos invariantes.

Teorema 2.2.3. [BS02] Sejam $A, B \subset \mathbb{R}^{n}$ subconjuntos invariantes com relação ao sistema dinâmico autonômo (2.1). Então a interseção e a união dos conjuntos $A$ e $B$ são invariantes com relação ao sistema dinâmico autonômo (2.1).

Demonstração. Sejam $A, B$ invariantes com relação ao sistema dinâmico autonômo (2.1) e $x \in A \cup B$. Então $x \in A$ ou $x \in B$. Se $x \in A$ e $A$ é um conjunto invariante, então $\varphi(t, x) \in A$, para todo $t \in \mathbb{R}$. Por outro lado, se $x \in B$ e $B$ também é um conjunto invariante, então $\varphi(t, x) \in B$, para todo $t \in \mathbb{R}$. Segue-se que $\varphi(t, x) \in A \cup B$, para todo $t \in \mathbb{R}$ e, portanto, $A \cup B$ é um conjunto invariante. A demonstração é inteiramente análoga para mostrar que a interseção de conjuntos invariantes também é invariante.

O Teorema 2.2.3 continua válido se substituirmos a invariância pela invarância positiva ou negativa. E além disso, como consequência direta do Teorema 2.2.3, a união e a interseção finita de conjuntos invariantes também são invariantes.

Teorema 2.2.4. [BS02] $O$ conjunto $A \subset \mathbb{R}^{n}$ é invariante com relação ao sistema dinâmico autonômo (2.1) se, e somente se, $\mathbb{R}^{n} \backslash A$ também é invariante.

Demonstração. Seja $A \subset \mathbb{R}^{n}$ invariante em relação ao sistema dinâmico autonômo (2.1). Dado $x \in \mathbb{R}^{n} \backslash A$ e algum $t \in \mathbb{R}$. Suponhamos, por contradição, 
que $\varphi(t, x) \notin \mathbb{R}^{n} \backslash A$. Consequentemente, $\varphi(t, x) \in A$. E portanto, temos $x=\varphi(-t, \varphi(t, x)) \in A$, pois $A$ é invariante, o que é uma contradição pois $x \in \mathbb{R}^{n} \backslash A$. Logo, $\varphi(t, x) \in \mathbb{R}^{n} \backslash A$ e consequentemente, $\mathbb{R}^{n} \backslash A$ é invariante. A demonstração da recíproca é inteiramente análoga.

Observe que se considerarmos no Teorema 2.2.4 que o conjunto $A$ é positivamente invariante (ou negativamente invariante) com relação ao sistema dinâmico autonômo (2.1), então $\mathbb{R}^{n} \backslash A$ é negativamente invariante (ou positivamente invariante) e vice-versa.

Teorema 2.2.5. [BS02] Seja $A \subset \mathbb{R}^{n}$ um subconjunto invariante com relação ao sistema dinâmico autonômo (2.1). Então o fecho $\bar{A}$, a fronteira $\partial A$ e o interior int A também o são.

Demonstração. Seja $x \in \bar{A}$. Então existe uma sequência $\left\{x_{n}\right\}$ em $A$ tal que $x_{n} \rightarrow x$. Como $A$ é um conjunto invariante com relação ao sistema dinâmico autonômo (2.1), então $\varphi\left(t, x_{n}\right) \in A$, para cada $n$ e para todo $t \in \mathbb{R}$. Pela continuidade das soluções do sistema (2.1), temos $\varphi\left(t, x_{n}\right) \rightarrow \varphi(t, x)$ e, portanto, $\varphi(t, x) \in \bar{A}$. Logo, $\bar{A}$ é um conjunto invariante.

Se $A$ é um conjunto invariante com relação ao sistema dinâmico autonômo (2.1), então $\mathbb{R}^{n} \backslash A$ também é invariante pelo Teorema 2.2.4. Consequentemente, pela primeira parte do Teorema $2.2 .5, \bar{A}$ e $\overline{\mathbb{R}^{n} \backslash A}$ são conjuntos invariantes. Logo, $\partial A=\bar{A} \cap\left(\overline{\mathbb{R}^{n} \backslash A}\right)$ e $\operatorname{int} A=A \backslash\left(\overline{\mathbb{R}^{n} \backslash A}\right)$ são invariantes pelos Teoremas 2.2 .3 e 2.2.4, respectivamente.

\subsubsection{Comportamento Assintótico}

Para uma melhor análise do comportamento do sistema dinâmico autonômo (2.1) precisamos de informação adicional para determinarmos o comportamento assintótico das soluções do sistema quando o tempo tende para o infinito. Essa informação adicional ao qual nos referimos chamamos de conjuntos limites da trajetória.

Suponhamos que a solução $\varphi\left(t, x_{0}\right)$ exista para $0 \leq t<+\infty$. A semitrajetória positiva $\gamma\left(x_{0}\right)$ é definida como $\left\{\varphi\left(t, x_{0}\right): t \in(0,+\infty)\right\}$. Um ponto $p \in \mathbb{R}^{n}$ é um ponto $\omega$-limite da solução $\varphi\left(t, x_{0}\right)$ de $(2.1)$ se existir uma sequência $\left\{t_{j}\right\}$, com $t_{j} \rightarrow+\infty$ quando $j \rightarrow+\infty$ tal que $\varphi\left(t_{j}, x_{0}\right) \rightarrow p$ quando $j \rightarrow+\infty$. O conjunto de todos os pontos $\omega$-limite de $\varphi\left(t, x_{0}\right)$ é chamado conjunto $\omega$-limite da solução $\varphi\left(t, x_{0}\right)$, ou simplesmente $\omega$-limite de $x_{0}$, e é denotado por $\omega\left(x_{0}\right)$.

Suponhamos que a solução $\varphi\left(t, x_{0}\right)$ exista para $-\infty<t \leq 0$. A semitrajetória negativa $\gamma\left(x_{0}\right)$ é definida como $\left\{\varphi\left(t, x_{0}\right): t \in(-\infty, 0)\right\}$. Analogamente definimos o conjunto limite das soluções quando o tempo tende a $-\infty$. Um ponto $p \in \mathbb{R}^{n}$ é um ponto $\alpha$-limite da solução $\varphi\left(t, x_{0}\right)$ de $(2.1)$ se existir uma sequência $\left\{t_{j}\right\}$, com $t_{j} \rightarrow-\infty$ quando $j \rightarrow+\infty$ tal que $\varphi\left(t_{j}, x_{0}\right) \rightarrow p$ quando $j \rightarrow+\infty$. O conjunto de todos os pontos $\alpha$-limite de 
$\varphi\left(t, x_{0}\right)$ é chamado conjunto $\alpha$-limite da solução $\varphi\left(t, x_{0}\right)$, ou simplesmente $\alpha$-limite de $x_{0}$, e é denotado por $\alpha\left(x_{0}\right)$.

O próximo teorema nos fornece uma maneira alternativa de definir conjuntos limites de solução.

Teorema 2.2.6. $[L S \gamma 6] \omega\left(x_{0}\right)=\cap_{0<\tau<\infty} \overline{\varphi\left([\tau, \infty), x_{0}\right)}$.

Demonstração. Suponha que $p \in \omega\left(x_{0}\right)$, então pela definição de ponto $\omega$ limite, existe uma sequência $\left\{t_{j}\right\}$, com $t_{j} \rightarrow+\infty$ quando $j \rightarrow+\infty$ tal que $\varphi\left(t_{j}, x_{0}\right) \rightarrow p$ quando $j \rightarrow+\infty$. Logo, para qualquer $\tau \in[0,+\infty)$, existe um número inteiro $N>0$ tal que $t_{j} \in[\tau,+\infty)$ para todo $j \geq$ $N$. Assim, $\varphi\left(t_{j}, x_{0}\right) \in \varphi\left([\tau,+\infty), x_{0}\right)$ para todo $j \geq N$. Portanto, $p \in$ $\overline{\varphi\left([\tau, \infty), x_{0}\right)}$. Como $\tau$ pode ser escolhido arbitrariamente grande, tem-se que $p \in \cap_{0<\tau<\infty} \overline{\varphi\left([\tau, \infty), x_{0}\right)}$. Suponha agora que $p \in \cap_{0<\tau<\infty} \overline{\varphi\left([\tau, \infty), x_{0}\right)}$. Então dado $\tau>0$ arbitrário, $p \in \overline{\varphi\left([\tau, \infty), x_{0}\right)}$. Em particular, dado uma sequência $\left\{\tau_{j}\right\}$, com $\tau_{j} \rightarrow+\infty$ quando $j \rightarrow+\infty$, existe $t_{j} \in\left[\tau_{j},+\infty\right)$ tal que $\left\|\varphi\left(t_{j}, x_{0}\right)-p\right\| \leq \frac{1}{j}$. Obviamente, $t_{j} \rightarrow+\infty$ quando $j \rightarrow+\infty$ e, por construção, $\varphi\left(t_{j}, x_{0}\right) \rightarrow p$ quando $j \rightarrow+\infty$. Portanto, $p \in \omega\left(x_{0}\right)$.

Agora estabeleceremos algumas propriedades importantes dos conjuntos $\omega$-limite. Pelo resultado seguinte, observemos que os conjuntos $\omega$-limite das soluções são uma classe importante de conjuntos invariantes. Analogamente, valem as mesmas propriedades para os conjuntos $\alpha$-limite.

Teorema 2.2.7. [LS76] O conjunto w-limite de uma solução $\varphi\left(t, x_{0}\right)$ do sistema dinâmico autonômo (2.1) é fechado e invariante.

Demonstração. Como a interseção de conjuntos fechados é um conjunto fechado, obtém-se do Teorema 2.2.6 que $\omega\left(x_{0}\right)$ é um conjunto fechado. Para provar que $\omega\left(x_{0}\right)$ é um conjunto invariante, seja $p \in \omega\left(x_{0}\right)$. Vamos mostrar que $\varphi(t, p) \in \omega\left(x_{0}\right)$ para todo $t \in \mathbb{R}$. De acordo com a definição do conjunto $\omega$-limite, existe uma sequência $\left\{t_{j}\right\}$, com $t_{j} \rightarrow+\infty$ quando $j \rightarrow+\infty$ tal que $\varphi\left(t_{j}, x_{0}\right) \rightarrow p$ quando $j \rightarrow+\infty$. Seja $\varphi(t, p)$ a solução de (2.1) passando por $p$. Seja $\tau$ um número real arbitrário. Da continuidade das soluções com relação às condições iniciais, tem-se que dado $\varepsilon>0$ arbitrariamente pequeno, existe $\delta>0$ tal que $\|q-p\|<\delta$ implica em $\|\varphi(\tau, q)-\varphi(\tau, p)\|<\varepsilon$. Se escolhermos $\varepsilon_{m}=\frac{1}{m}$ onde $m=1,2, \ldots$, então para cada inteiro $m$, existe um número real $\delta_{m}^{m}>0$ e um inteiro $M>0$ (dependendo de $m$ ) tal que $\left\|\varphi\left(t_{M}, x_{0}\right)-p\right\|<\delta_{m}$ e, portanto, $\left\|\varphi\left(\tau, \varphi\left(t_{M}, x_{0}\right)\right)-\varphi(\tau, p)\right\| \leq \frac{1}{m}$. Pode-se, sem perda de generalidade, escolher a sequência $\left\{t_{M}\right\}$ estritamente crescente com $t_{M} \rightarrow+\infty$ quando $m \rightarrow+\infty$. Logo, tem-se que $\varphi\left(\tau, \varphi\left(t_{M}, x_{0}\right)\right)=$ $\varphi\left(\tau+t_{M}, x_{0}\right) \rightarrow \varphi(\tau, p)$ quando $m \rightarrow+\infty$. Portanto, $\varphi(t, p) \in \omega\left(x_{0}\right)$. A arbitrariedade da escolha de $p$ completa a demonstração mostrando que, para qualquer $p \in \omega\left(x_{0}\right), \varphi(t, p) \in \omega\left(x_{0}\right)$ para todo $t \in \mathbb{R}$. 
Se considerarmos a hipótese de que as trajetórias do sistema dinâmico autonômo (2.1) são limitadas, podemos obter algumas propriedades adicionais relacionadas ao conjunto $\omega$-limite. Precisaremos definir alguns conceitos antes de enunciarmos o resultado sobre o conjunto $\omega$-limite.

Dizemos que a solução $\varphi\left(t, x_{0}\right)$ do sistema dinâmico autonômo (2.1) é limitada se existe um número real positivo $M<\infty$ tal que

$$
\sup _{t \geq 0}\left\|\varphi\left(t, x_{0}\right)\right\| \leq M
$$

Suponhamos que exista uma métrica $d(x, y)$ definida em $M$. Definimos a distância entre um ponto $p$ e um conjunto $A$ a partir da distância usual entre dois pontos, ou seja, $d(p, A)=\inf _{x \in A}\|p-x\|$.

Teorema 2.2.8. [LS76] Se a solução $\varphi\left(t, x_{0}\right)$ de (2.1) é limitada, então o conjunto $\omega$-limite é não-vazio, conexo, fechado, limitado e invariante. Além disso, $d\left(\varphi\left(t, x_{0}\right), \omega\left(x_{0}\right)\right) \rightarrow 0$ quando $t \rightarrow+\infty$.

Demonstração. Já provamos no Teorema 2.2.7 que o conjunto $\omega\left(x_{0}\right)$ é fechado e invariante. O conjunto $\omega\left(x_{0}\right)$ é não vazio, pois toda sequência em um conjunto compacto possui subsequência convergente. A limitação da solução garante naturalmente a limitação do conjunto limite. Vamos provar que $d\left(\varphi\left(t, x_{0}\right), \omega\left(x_{0}\right)\right) \rightarrow 0$ quando $t \rightarrow+\infty$. Para isto, suponha, por contradição, que $\varphi\left(t, x_{0}\right)$ não se aproxima de $\omega\left(x_{0}\right)$ quando $t \rightarrow+\infty$. Então, dada uma sequência de tempos $\left\{T_{n}\right\}$ arbitrária, com $T_{n} \rightarrow+\infty$ quando $n \rightarrow+\infty$, existe $\varepsilon>0$ e $t_{n}>T_{n}$ tal que $d\left(\varphi\left(t_{n}, x_{0}\right), \omega\left(x_{0}\right)\right)>\varepsilon$ para todo $n=1,2,3, \ldots$ Como $\varphi\left(t_{n}, x_{0}\right)$ é uma sequência limitada em $\mathbb{R}^{n}$, então possui subsequência convergente, ou seja, existe $p \in \mathbb{R}^{n}$ e subsequência $\left\{t_{n_{j}}\right\}$ de $\left\{t_{n}\right\}$ tal que $\varphi\left(t_{n_{j}}, x_{0}\right) \rightarrow p$ quando $n_{j} \rightarrow+\infty$. Mas por definição, $p \in \omega\left(x_{0}\right)$, portanto chegamos a uma contradição provando o que se queria. Resta-nos provar que o conjunto $\omega\left(x_{0}\right)$ é conexo. Suponhamos, por absurdo, que $\omega\left(x_{0}\right)$ é desconexo, ou seja, $\omega\left(x_{0}\right)$ pode ser escrito como sendo a união de dois conjuntos disjuntos, fechados e não vazios. Sejam $A_{1}$ e $A_{2}$ estes conjuntos. Como eles são disjuntos, então pelo Teorema 2.1.5 existem dois conjuntos abertos e disjuntos $U_{1}$ e $U_{2}$ tal que $A_{1} \subset U_{1}$ e $A_{2} \subset U_{2}$. Como $d\left(\varphi\left(t, x_{0}\right), \omega\left(x_{0}\right)\right) \rightarrow 0$ quando $t \rightarrow+\infty$, então existe $T>0$ tal que $\varphi\left(t, x_{0}\right) \in U_{1} \cup U_{2}$ para todo $t>T$. Como a aplicação $t \rightarrow \varphi\left(t, x_{0}\right)$ é contínua e $U_{1}$ e $U_{2}$ são disjuntos, conclui-se que $\varphi\left(t, x_{0}\right)$ pertence apenas a um dos abertos para todo $t>T$. Suponha sem perda de generalidade que $\varphi\left(t, x_{0}\right) \in U_{1}$ para todo $t>T$. Então, necessariamente $A_{2}$ é um conjunto vazio. Isto nos leva a uma contradição e, portanto, o conjunto $\omega$-limite é conexo.

A limitação da solução é uma hipótese fundamental para demonstrarmos o Teorema 2.2.8. A Figura 2.2 mostra um exemplo de uma solução não 
limitada que possui um conjunto $\omega$-limite desconexo. Mas precisamente, o conjunto $\omega$-limite é constituído pela união de duas retas disjuntas. $\mathrm{O}$ conjunto $\omega$-limite é ilimitado e ainda, dado $T>0$ arbitrariamente grande e $\varepsilon>0$, existe $t>T$ tal que $d\left(\varphi\left(t, x_{0}\right), \omega\left(x_{0}\right)\right)>\varepsilon$, isto é, a solução não se aproxima do conjunto $\omega$-limite quando $t \rightarrow+\infty$.

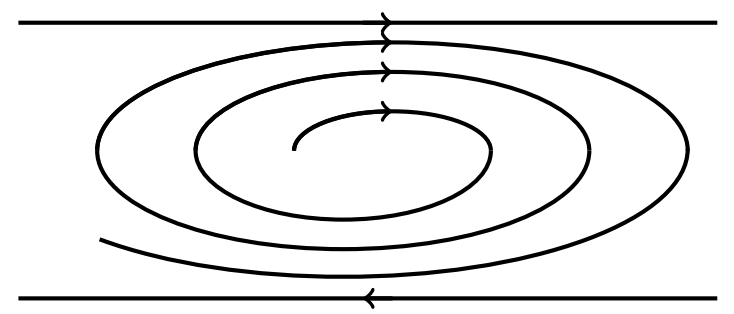

Figura 2.2: Exemplo de um conjunto $\omega$-limite desconexo e ilimitado.

Vejamos conjuntos que desempenharam um papel importante na teoria de estabilidade dos sistemas dinâmicos autonômos (2.1). Para cada $x \in \mathbb{R}^{n}$, definamos os conjuntos:

$\Gamma^{+}(x)=\left\{y \in \mathbb{R}^{n}\right.$ : existem sequências $\left\{x_{n}\right\}$ em $\mathbb{R}^{n}$ e $\left\{t_{n}\right\}$ em $\mathbb{R}^{+}$tal que $t_{n} \geq 0, x_{n} \rightarrow x$ e $\left.\varphi\left(t_{n}, x_{n}\right) \rightarrow y\right\}$

$\Gamma^{-}(x)=\left\{y \in \mathbb{R}^{n}:\right.$ existem sequências $\left\{x_{n}\right\}$ em $\mathbb{R}^{n}$ e $\left\{t_{n}\right\}$ em $\mathbb{R}^{-}$tal que $t_{n} \leq 0, x_{n} \rightarrow x$ e $\left.\varphi\left(t_{n}, x_{n}\right) \rightarrow y\right\}$

$\Lambda^{+}(x)=\left\{y \in \mathbb{R}^{n}:\right.$ existem sequências $\left\{x_{n}\right\}$ em $\mathbb{R}^{n}$ e $\left\{t_{n}\right\}$ em $\mathbb{R}^{+}$tal que $x_{n} \rightarrow x, t_{n} \rightarrow+\infty$ e $\left.\varphi\left(t_{n}, x_{n}\right) \rightarrow y\right\}$

$\Lambda^{-}(x)=\left\{y \in \mathbb{R}^{n}:\right.$ existem sequências $\left\{x_{n}\right\}$ em $\mathbb{R}^{n}$ e $\left\{t_{n}\right\}$ em $\mathbb{R}^{-}$tal que $x_{n} \rightarrow x, t_{n} \rightarrow-\infty$ e $\left.\varphi\left(t_{n}, x_{n}\right) \rightarrow y\right\}$.

Os conjuntos $\Gamma^{+}(x)$ e $\Gamma^{-}(x)$ são chamados primeiro prolongamento positivo e negativo de $x$, respectivamente. E os conjuntos $\Lambda^{+}(x)$ e $\Lambda^{-}(x)$ são chamados primeiro prolongamento positivo do conjunto $\omega$-limite de $x$ e primeiro prolongamento negativo do conjunto $\alpha$-limite de $x$, respectivamente. Observemos que para qualquer $x \in \mathbb{R}^{n}, \gamma^{+}(x) \subset \Gamma^{+}(x), \gamma^{-}(x) \subset \Gamma^{-}(x)$, $\omega(x) \subset \Lambda^{+}(x)$ e $\alpha(x) \subset \Lambda^{-}(x)$. Para um melhor entendimento dos conjuntos definidos acima, vejamos o seguinte exemplo devido a [BS02].

Exemplo 2.2.1. Consideremos o sistema

$$
\left\{\begin{array}{l}
\dot{x}=-x \\
\dot{y}=y
\end{array}\right.
$$

Note que a origem é o único ponto de equilíbrio do sistema (2.3) e suas trajetórias são mostradas na Figura 2.3. Seja $p=\left(x_{p}, y_{p}\right)$. Se p pertence ao eixo $x$, então $\Gamma^{+}(p)=\gamma^{+}(p) \cup\{(x, y): x=0\}, \Gamma^{-}(p)=\gamma^{-}(p), \Lambda^{+}(p)=$ $\{(x, y): x=0\}$. Se $x_{p} \neq 0$, então $\Lambda^{-}(p)=\emptyset$. Se $x_{p}=0$, então $\Gamma^{-}(p)=$ $\{(x, y): y=0\}$. Se $x_{p}, y_{p} \neq 0$, então $\Gamma^{+}(p)=\gamma^{+}(p), \Gamma^{-}(p)=\gamma^{-}(p)$, $\Lambda^{+}(p)=\Lambda^{-}(p)=\emptyset$. 


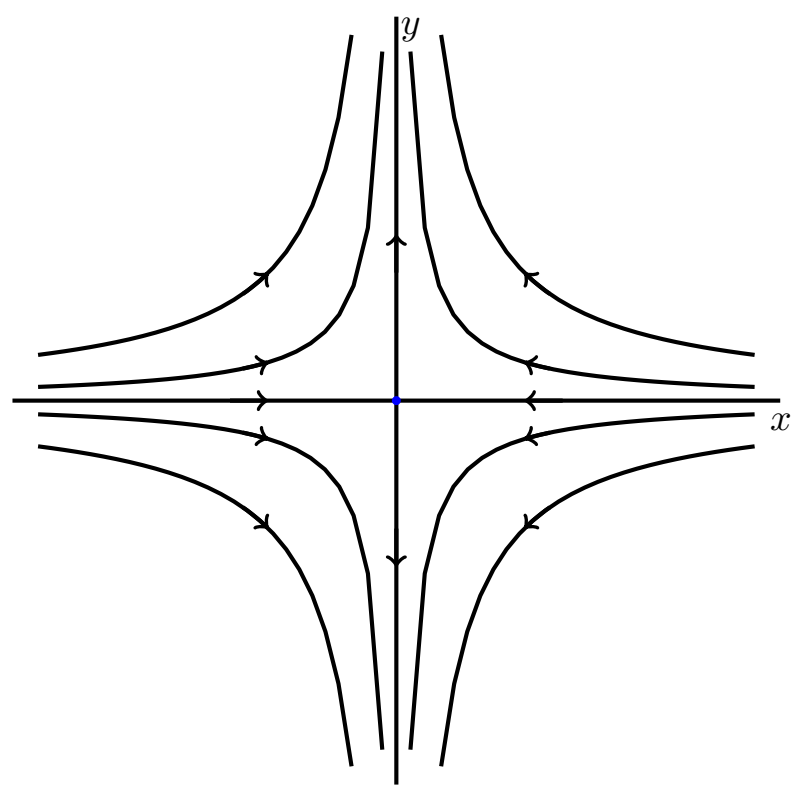

Figura 2.3: Trajetórias do sistema $\dot{x}=-x, \dot{y}=y$.

Da mesma forma como obtemos propriedades para os conjuntos $\omega, \alpha$-limite, os dois próximos resultados estabelecem algumas propriedades importantes desses conjuntos.

Teorema 2.2.9. [BS02] Para qualquer $x \in \mathbb{R}^{n}$,

i. $\Gamma^{+}(x)$ é positivamente invariante e fechado,

ii. $\Lambda^{+}(x)$ é invariante e fechado,

iii. $\Gamma^{+}(x)=\gamma^{+}(x) \cup \Lambda^{+}(x)$.

Analogamente, valem as mesmas propriedades do Lema 2.2.9 para os conjuntos $\Gamma^{-}(x)$ e $\Lambda^{-}(x)$.

Lema 2.2.10. [BS02] Seja $M \subset \mathbb{R}^{n}$ localmente compacto. Para qualquer $x \in M$, então $\omega(x) \neq \emptyset$ sempre que $\Lambda^{+}(x)$ é não vazio e compacto.

Se considerarmos a hipótese de que o conjunto $\Gamma^{+}(x)$ é compacto, podemos obter a propriedade de conexidade do conjunto.

Teorema 2.2.11. [BS02] Seja $M \subset \mathbb{R}^{n}$ localmente compacto. Para qualquer $x \in M$, se $\Gamma^{+}(x)$ é compacto, então $\Gamma^{+}(x)$ é conexo.

O Teorema 2.2.11 também é válido para os conjuntos $\Gamma^{-}(x), \Lambda^{+}(x)$ e $\Lambda^{-}(x)$. 


\subsubsection{Estabilidade Local}

Conceitos sobre estabilidade são importantes para se analisar o comportamento de um sistema dinâmico. Assim sendo, discutiremos a estabilidade das soluções do sistema dinâmico autonômo (2.1) e, em particular, a estabilidade dos pontos de equilíbrio e de órbitas fechadas que serão utilizados ao longo deste trabalho. Analisaremos se o comportamento destas soluções é compartilhada, quando $t \rightarrow+\infty$, pelas soluções vizinhas. Embora existam diversas definições de estabilidade, usaremos a definição de estabilidade no sentido de Lyapunov.

Seja $\varphi\left(t, x_{0}\right)$ uma solução de $(2.1)$ definida para $t \geq 0$ e passando por $x_{0}$. Dizemos que $\varphi\left(t, x_{0}\right)$ é estável se para todo $\varepsilon>0$ existir $\delta>0$ tal que se $\rho\left(t, y_{0}\right)$ também é solução de $(2.1)$ passando por $y_{0}$ e $\left\|\rho\left(0, y_{0}\right)-\varphi\left(0, x_{0}\right)\right\|<\delta$ então $\rho\left(t, y_{0}\right)$ está definida para todo $t \geq 0$ e $\left\|\rho\left(t, y_{0}\right)-\varphi\left(t, x_{0}\right)\right\|<\varepsilon$ para todo $t \geq 0$.

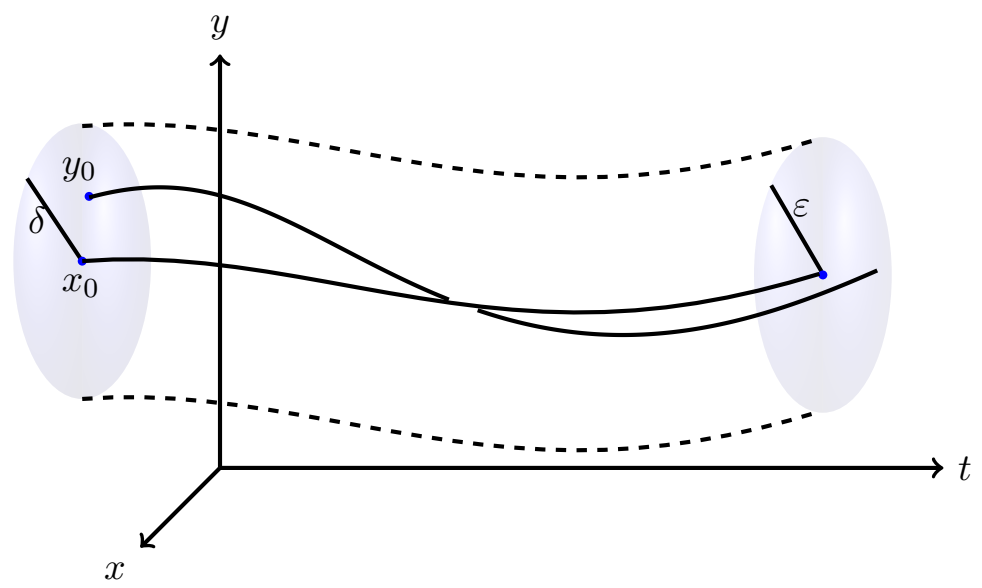

Figura 2.4: Solução Estável do sistema dinâmico autonômo (2.1).

Observemos que as trajetórias na Figura 2.4 que iniciam próximas de uma solução estável segundo Lyapunov devem permanecer nas proximidades dessa solução para todo $t \geq 0$, não necessariamente convergindo para a mesma.

Se na definição de solução estável, para todo $\varepsilon>0$ existir $\delta_{1}>0$ tal que $\left\|\rho\left(0, y_{0}\right)-\varphi\left(0, x_{0}\right)\right\|<\delta_{1}$ implica $\lim _{t \rightarrow+\infty}\left\|\rho\left(t, y_{0}\right)-\varphi\left(t, x_{0}\right)\right\|=0$, então dizemos que a solução $\varphi\left(t, x_{0}\right)$ é assintoticamente estável, veja Figura 2.5.

Agora podemos especializar as definições anteriores para o caso particular das soluções de (2.1), ou seja, os pontos de equilíbrio do sistema (2.1).

Um ponto de equilibrio $\bar{x}$ do sistema dinâmico autonômo (2.1) é estável se, para cada $\varepsilon>0$, existir um $\delta>0$ tal que, para toda condição inicial $x_{0}$ satisfazendo $\left\|x_{0}-\bar{x}\right\|<\delta$ tem-se $\left\|\varphi\left(t, x_{0}\right)-\bar{x}\right\|<\varepsilon$ para todo $t \geq 0$, veja Figura 2.6. 


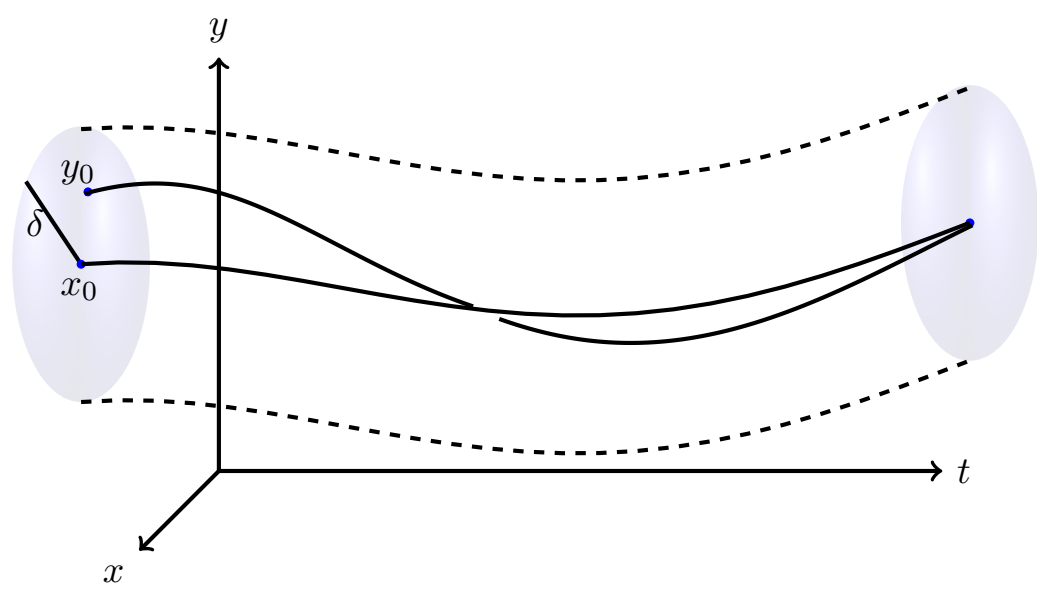

Figura 2.5: Solução Assintoticamente estável do sistema dinâmico autonômo (2.1).

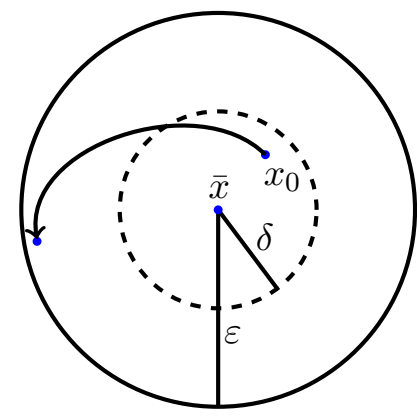

Figura 2.6: Ponto de equilíbrio estável do sistema dinâmico autonômo (2.1).

A Figura 2.6 ilustra a definição de ponto de equilíbrio estável. Note que as soluções que iniciam dentro da bola de raio $\delta$ não precisam se aproximar do equilíbrio $\bar{x}$ quando $t \rightarrow+\infty$, mas têm que permanecer dentro da bola de raio $\varepsilon$.

Um ponto de equilíbrio $\bar{x}$ do sistema dinâmico autonômo (2.1) é instável se ele não é estável.

Um ponto de equilíbrio $\bar{x}$ do sistema dinâmico autonômo (2.1) é atrativo se existir $\delta>0$ tal que $x_{0} \in B(\bar{x} ; \delta)$ implica que $\varphi\left(t, x_{0}\right) \rightarrow \bar{x}$ quando $t \rightarrow+\infty$.

A Figura 2.7 retrata a definição de ponto equilíbrio atrativo. Observemos que as soluções que iniciam dentro da bola de raio $\delta$ tendem para o equilíbrio $\bar{x}$ quando $t \rightarrow+\infty$. Além disso, as soluções que iniciam dentro da bola de raio $\delta$ podem se afastar do equilíbrio e até mesmo sair da bola de raio $\delta$, mas retornando e convergindo para o equilíbrio $\bar{x}$ quando $t \rightarrow+\infty$.

Note que estabilidade não implica em atratividade e atratividade também não implica em estabilidade. Porém, ao combinarmos os dois conceitos obtemos a noção de estabilidade assintótica. 


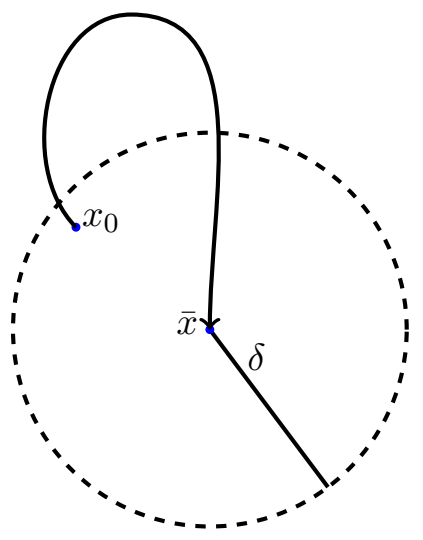

Figura 2.7: Ponto de equilíbrio atrativo do sistema dinâmico autonômo (2.1).

Um ponto de equilíbrio do sistema dinâmico autonômo (2.1) é assintoticamente estável se for estável e atrativo, veja Figura 2.8.

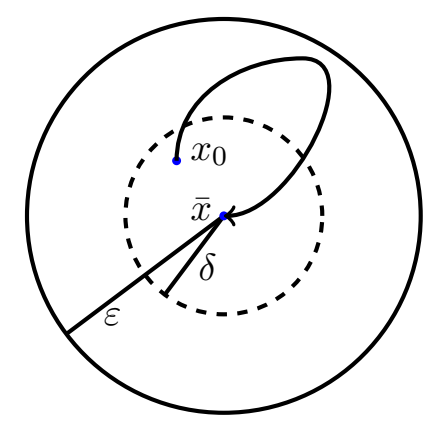

Figura 2.8: Ponto de equilíbrio assintoticamente estável do sistema dinâmico autonômo (2.1).

Na Figura 2.8, as soluções que iniciam dentro da bola de raio $\delta$, não se afastam do equilíbrio $\bar{x}$ e tendem para o equilíbrio $\bar{x}$ quando $t \rightarrow+\infty$.

$\mathrm{Na}$ análise qualitativa de alguns sistemas dinâmicos autônomos (2.1) desejamos não apenas que o ponto seja assintoticamente estável mas que tenha a propriedade de que todas as soluções tendam para este equilíbrio quando o tempo tende ao infinito. Para isto, definamos o conceito de estabilidade global assintótica.

Um ponto de equilíbrio $\bar{x}$ do sistema dinâmico autonômo (2.1) é globalmente assintoticamente estável se ele é estável e para todo $x_{0} \in \mathbb{R}^{n}$, $\varphi\left(t, x_{0}\right) \rightarrow \bar{x}$ quando $t \rightarrow+\infty$.

Observemos que se o sistema dinâmico autonômo (2.1) possui um ponto de equilíbrio globalmente estável, então para qualquer condição inicial que tomarmos no $\mathbb{R}^{n}$, a solução passando por esse ponto vai convergir para o equilíbrio globalmente estável quando $t \rightarrow+\infty$. Consequentemente, este ponto de equilíbrio será o único equilíbrio do sistema. 


\subsubsection{Pontos de Equilíbrio Hiperbólicos}

Ainda com o próposito de estudarmos o comportamento das soluções numa vizinhança do ponto de equilíbrio, veremos que existe uma classe de equilíbrios onde o sistema dinâmico autônomo não linear se comporta qualitativamente de forma equivalente ao sistema linear associado numa vizinhança do equilíbrio. Além disso, a fim de obter um conhecimento completo da dinâmica do sistema (2.1), estudaremos o comportamento de variedades invariantes dos pontos de equilíbrio e das órbitas fechadas.

Seja $\bar{x}$ um ponto de equilíbrio do sistema dinâmico autonômo não linear (2.1) e consideremos o sistema linearizado em torno do equilíbrio $\bar{x}$

$$
\dot{y}=J(\bar{x}) y
$$

onde $y=x-\bar{x}$ e $J(\bar{x})$ é a matriz Jacobiana do campo $f(x)$ calculada no ponto de equilíbrio $\bar{x}$.

Um ponto de equilíbrio $\bar{x}$ do sistema dinâmico autonômo (2.1) é hiperbólico se todos os autovalores da matriz Jacobiana do sistema linearizado associado (2.4) possuem parte real não nula. Se a parte real de algum autovalor for nulo, o ponto de equilíbrio será dito não hiperbólico ou degenerado.

Do Teorema da Função Inversa em [Lim81] podemos afirmar que os pontos de equilíbrio hiperbólicos são necessariamente pontos de equilíbrio isolados, isto é, existe uma vizinhança do equilíbrio que não contém outro ponto de equilíbrio.

Um ponto de equilíbrio $\bar{x}$ do sistema dinâmico (2.1) é do tipo- $k$ se a matriz Jacobiana $J(\bar{x})$ do sistema linearizado associado (2.4) possui $k$ autovalores com parte real positiva e $n-k$ autovalores com parte real negativa.

Consideremos $\bar{x}$ ponto de equilíbrio hiperbólico do sistema dinâmico autonômo (2.1). Seja $J(\bar{x})$ a matriz jacobiana do sistema linearizado associado ao sistema (2.1). Observemos que com a linearização, a origem é o ponto de equilíbrio do sistema linearizado (2.4). Suponhamos que a matriz Jacobiana $J(\bar{x})$ tenha $k$ autovalores com parte real positiva e $n-k$ autovalores com parte real negativa. Sejam $v_{1}, \ldots, v_{k}$ os autovetores generalizados associados aos autovalores com parte real positiva e $v_{k+1}, \ldots, v_{n}$ os autovetores generalizados associados com parte real negativa. Então o espaço $\mathbb{R}^{n}$ é decomposto como a soma direta de dois subespaços lineares definidos por

$$
E^{u}(\bar{x})=\operatorname{span}\left\{v_{1}, \ldots, v_{k}\right\} \text { e } E^{s}(\bar{x})=\operatorname{span}\left\{v_{k+1}, \ldots, v_{n}\right\} .
$$

Estes subespaços são invariantes em relação ao sistema dinâmico linearizado (2.4) e são conhecidos como subespaços Instável e Estável, respectivamente.

O subespaço instável $E^{u}(\bar{x})$ é o conjunto de todos pontos tais que as trajetórias passando por esse ponto permanecem no subespaço e se aproximam da origem quando o tempo tende a $-\infty$. O subespaço estável $E^{s}(\bar{x})$ é o conjunto de todos pontos tais que as trajetórias passando por esse ponto 
permanecem no subespaço e se aproximam da origem quando o tempo tende $\mathrm{a}+\infty$.

Para pontos de equilíbrio hiperbólicos, a dinâmica do sistema linearizado associado (2.4) é similar ao comportamento dinâmico numa vizinhança do ponto de equilíbrio do sistema não-linear (2.1). De fato, o resultado a seguir garante, numa vizinhança do ponto de equilíbrio hiperbólico do sistema dinâmico autonômo (2.1), a existência de variedades diferenciáveis invariantes tangentes aos subespaços estável e instável e com as propriedades assintóticas desses subespaços.

Intuitivamente podemos pensar em uma variedade invariante como sendo uma superfície com a propriedade de que toda trajetória passando por um ponto dessa superfície permanece na mesma para todo o tempo futuro ou passado. Sendo assim, essas variedades são nada mais do que conjuntos de trajetórias que tendem se aproximar ou se afastar do equilíbrio conforme o tempo tende para infinito. Essas variedades chamamos de Variedades Estável e Instável.

Teorema 2.2.12. [Per13] Seja $\bar{x}$ um ponto de equilíbrio hiperbólico do tipo$k$ do sistema dinâmico autonômo (2.1). Então existe uma variedade local $W_{\text {loc }}^{u}(\bar{x}), k$-dimensional de classe $\mathcal{C}^{1}$, tangente ao subespaço instável $E^{u}(\bar{x})$ em $\bar{x}$, tal que $\varphi_{t}\left(W_{\text {loc }}^{u}(\bar{x})\right) \subset W_{\text {loc }}^{u}(\bar{x})$ para todo $t \leq 0$ e para todo $x_{0} \in$ $W_{l o c}^{u}(\bar{x})$,

$$
\lim _{t \rightarrow-\infty} \varphi\left(t, x_{0}\right)=\bar{x}
$$

e existe uma variedade local $W_{\text {loc }}^{s}(\bar{x}),(n-k)$-dimensional de classe $\mathcal{C}^{1}$, tangente ao subespaço estável $E^{s}(\bar{x})$ em $\bar{x}$, tal que $\varphi_{t}\left(W_{l o c}^{s}(\bar{x})\right) \subset W_{l o c}^{s}(\bar{x})$ para todo $t \geq 0$ e para todo $x_{0} \in W_{l o c}^{s}(\bar{x})$,

$$
\lim _{t \rightarrow+\infty} \varphi\left(t, x_{0}\right)=\bar{x}
$$

O Teorema 2.2.12 garante a existência das variedades acima localmente, ou seja, apenas em uma vizinhança do ponto de equilíbrio $\bar{x}$. Definamos a seguir a noção de variedades globais estável ou instável ou simplesmente de variedades estável e instável.

As variedades estável e instável de um ponto de equilíbrio hiperbólico $\bar{x}$ do sistema dinâmico autonômo (2.1) são definidas a partir das variedades locais por

$$
W^{s}(\bar{x})=\bigcup_{t \leq 0} \varphi_{t}\left(W_{l o c}^{s}(\bar{x})\right) \text { e } W^{u}(\bar{x})=\bigcup_{t \geq 0} \varphi_{t}\left(W_{l o c}^{u}(\bar{x})\right)
$$

respectivamente.

Na Figura 2.9, ilustramos as variedades estável $W^{s}(\bar{x})$ e instável $W^{u}(\bar{x})$ do ponto de equilíbrio hiperbólico $\bar{x}$ do sistema dinâmico autonômo (2.1), com linhas sólidas, e seus espaços estável $E^{s}(\bar{x})$ e instável $E^{u}(\bar{x})$, com linhas 


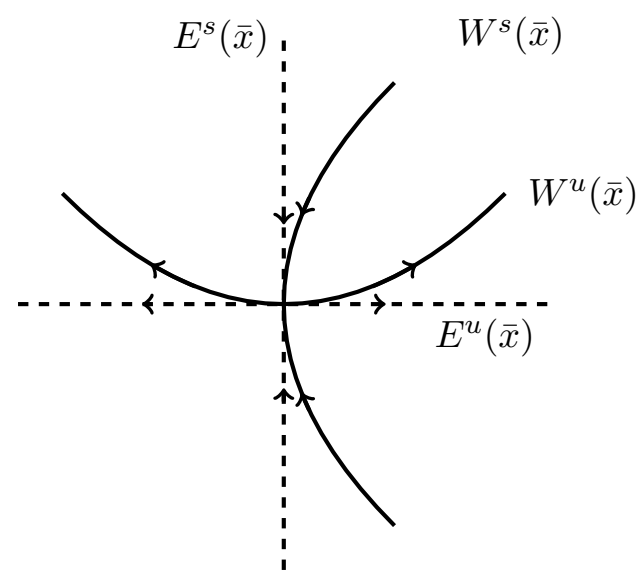

Figura 2.9: Variedades Estável $W^{s}(\bar{x})$ e Instável $W^{u}(\bar{x})$.

tracejadas.

As variedades estável $W^{s}(\bar{x})$ e instável $W^{u}(\bar{x})$ de um ponto de equilíbrio hiperbólico $\bar{x}$ são únicas e invariantes com relação ao sistema dinâmico autonômo (2.1). Podemos ainda reescrever essas variedades como

$$
W^{s}(\bar{x})=\left\{x \in \mathbb{R}^{n}: \varphi(t, x) \rightarrow \bar{x} \text { quando } t \rightarrow+\infty\right\}
$$

$$
W^{u}(\bar{x})=\left\{x \in \mathbb{R}^{n}: \varphi(t, x) \rightarrow \bar{x} \text { quando } t \rightarrow-\infty\right\}
$$

Depois de termos introduzido a noção de ponto equilíbrio hiperbólico e variedades invariantes destes equilíbrios, generalizemos a definição de hiperbolicidade e variedades invariantes para órbitas fechadas. Para determinar se uma uma órbita fechada é hiperbólica ou não faremos uso de uma ferramenta básica para o estudo de estabilidade de órbitas fechadas, a Aplicação de Poincaré. A estabilidade desta órbita será determinada pela estabilidade do ponto fixo correspondente à Aplicação de Poincaré.

Apresentaremos a idéia da Aplicação de Poincaré para determinar a estabilidade de uma órbita fechada. Consideremos o sistema dinâmico autonômo (2.1), com $f: \Omega \rightarrow \mathbb{R}^{n}$ um campo vetorial de classe $\mathcal{C}^{1}$ e $\Omega \subset \mathbb{R}^{n}$ um conjunto aberto. Sejam $\phi$ uma órbita fechada de período $T$ do sistema (2.1) passando pelo ponto $x_{0}$, isto é,

$$
\phi:=\left\{\varphi\left(t, x_{0}\right): 0 \leq t \leq T\right\}
$$

e $\Sigma$ o hiperplano ortogonal a $\phi$ em $x_{0}$ definido por:

$$
\Sigma=\left\{x \in \mathbb{R}^{n}:\left\langle\left(x-x_{0}\right), f\left(x_{0}\right)\right\rangle=0\right\} .
$$

Dado um ponto $x \in \Sigma$ na vizinhança $B\left(x_{0} ; \varepsilon\right)$ de $x_{0}$, a solução do sis- 
tema (2.1) passando por $x$ em $t=0, \varphi(t, x)$, interceptará o hiperplano $\Sigma$ novamente em algum ponto $P(x)$ próximo de $x_{0}$. Chamamos a aplicação que leva $x$ a $P(x)$ de Aplicação de Poincaré, veja Figura 2.10. O ponto $x_{0} \in \phi$ é um ponto fixo da Aplicação de Poincaré, ou seja, $P\left(x_{0}\right)=x_{0}$.

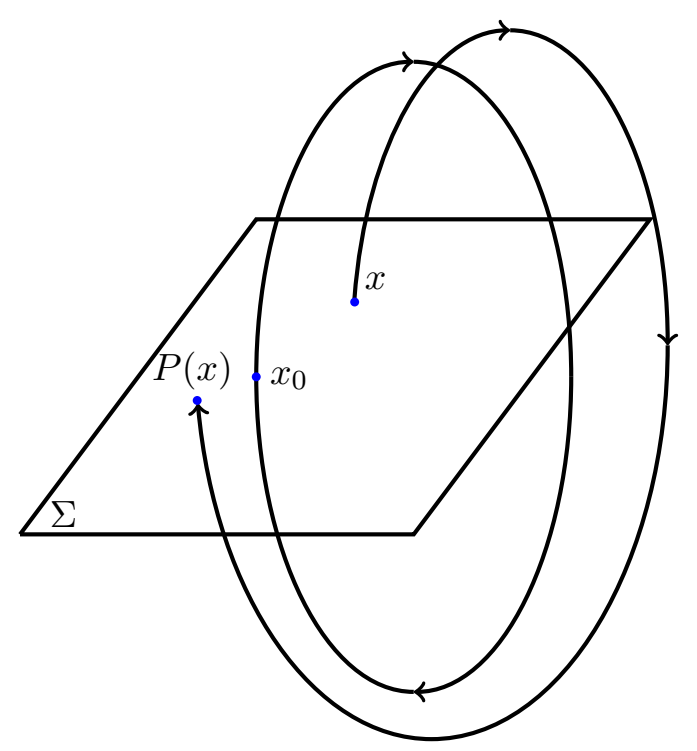

Figura 2.10: A geometria da Aplicação de Poincaré para uma órbita periódica.

O teorema que enunciaremos a seguir garante a existência e a continuidade da aplicação de Poincaré $P(x)$.

Teorema 2.2.13. [Per13] Seja $\Omega$ um subconjunto aberto do $\mathbb{R}^{n}$. Seja $\phi$ uma órbita fechada de período $T$ passando pelo ponto $x_{0}$ do sistema dinâmico autonômo (2.1). Suponhamos que o conjunto

$$
\phi=\left\{x \in \mathbb{R}^{n}: x=\varphi\left(t, x_{0}\right), 0 \leq t \leq T\right\},
$$

onde $\varphi_{T}\left(x_{0}\right)=x_{0}$, está contido em $\Omega$. Seja $\Sigma$ o hiperplano ortogonal a $\phi$ em $x_{0}$, isto é, seja

$$
\Sigma=\left\{x \in \mathbb{R}^{n}:\left\langle\left(x-x_{0}\right), f\left(x_{0}\right)\right\rangle=0\right\} .
$$

Então existe um $\delta>0$ e uma única função $\tau(x)$, definida e continuamente diferenciável para todo $x \in B\left(x_{0} ; \delta\right)$, tal que $\tau\left(x_{0}\right)=T e$

$$
\varphi(\tau(x), x) \in \Sigma
$$

para todo $x \in B\left(x_{0} ; \delta\right)$.

Demonstração. Definamos, com $x_{0} \in \phi \subset \Omega$, a função

$$
F(t, x)=\left\langle\left(\varphi(t, x)-x_{0}\right), f\left(x_{0}\right)\right\rangle .
$$


Segundo [Per13], temos que $F$ é de classe $\mathcal{C}^{1}(\mathbb{R} \times \Omega)$. E, devido a periodicidade de $\varphi\left(t, x_{0}\right)$, isto é, $\varphi\left(T, x_{0}\right)=x_{0}$, temos ainda que $F\left(T, x_{0}\right)=0$. Como $\varphi\left(t, x_{0}\right)$ é uma solução de $(2.1)$ e desde que $x_{0} \in \phi$ não seja um ponto de equilíbrio de (2.1), visto que neste caso o campo vetorial $f$ é nulo, temos

$$
\begin{aligned}
\frac{\partial F\left(T, x_{0}\right)}{\partial t} & =\left\langle\frac{\partial \varphi\left(T, x_{0}\right)}{\partial t}, f\left(x_{0}\right)\right\rangle \\
& =\left\langle f\left(x_{0}\right), f\left(x_{0}\right)\right\rangle \\
& =\left\|f\left(x_{0}\right)\right\|^{2} \neq 0 .
\end{aligned}
$$

Então, pelo Teorema 2.1.7, existe uma constante $\delta>0$ e uma única função $\tau(x)$ definida e continuamente diferenciável para todo $x \in B\left(x_{0} ; \delta\right)$ tal que

$$
\tau\left(x_{0}\right)=T \text { e } F(\tau(x), x)=0 .
$$

Portanto, para todo $x \in B\left(x_{0} ; \delta\right)$,

$$
\left\langle\varphi(\tau(x), x)-x_{0}, f\left(x_{0}\right)\right\rangle=0,
$$

ou seja, $\varphi(\tau(x), x) \in \Sigma$.

Sejam $\phi, \Sigma, \delta$ e $\tau(x)$ como definidos no Teorema 2.2.13. Então para $x \in B\left(x_{0} ; \delta\right) \cap \Sigma$, a função

$$
P(x)=\varphi_{\tau(x)}(x)
$$

é chamada Aplicação de Poincaré associada à órbita fechada $\phi$ em $x_{0}$.

Segue-se do Teorema 2.2.13 que a função $P$ é de classe $\mathcal{C}^{1}(V)$, onde $V=B\left(x_{0} ; \delta\right) \cap \Sigma$. Observemos também que se considerarmos o sistema (2.1) com $t \rightarrow-t$, podemos mostrar que a aplicação de Poincaré $P$ tem uma inversa $P^{-1}$ de classe $\mathcal{C}^{1}$ e, portanto, $P$ é um difeomorfismo.

O ponto $x \in \Sigma$ tal que $P(x)=x$ é chamado de ponto fixo da Aplicação de Poincaré. Os pontos fixos da Aplicação de Poincaré correspondem a órbitas periódicas do sistema (2.1).

Para determinarmos a hiperbolicidade da órbita fechada, linearizaremos o sistema (2.1) sobre $\phi$, obtendo-se assim o sistema linear não autonômo

$$
\dot{x}=A(t) x,
$$

onde $A(t)=D f\left(\varphi_{t}\left(x_{0}\right)\right)$ e $x_{0} \in \phi$. A matriz $A(t)$ de ordem $n$ é uma função contínua, periódica de periódo $T$ e definida para todo $t \in \mathbb{R}$. A matriz fundamental para o sistema (2.5) é uma matriz $\Phi(t)$ não singular de ordem $n$ que satisfaz a equação diferencial matricial

$$
\dot{\Phi}(t)=A(t) \Phi(t)
$$


para todo $t \in \mathbb{R}$. Vale ressaltar que as colunas de $\Phi(t)$ consistem de $n$ soluções linearmente independentes do sistema (2.5).

O próximo resultado, conhecido como Teorema de Floquet, fornece uma maneira de obtermos a matriz fundamental do sistema linear não autonômo $(2.5)$.

Teorema 2.2.14 (Teorema de Floquet). [Per13] Seja A(t) uma matriz contínua e periódica de período $T$. Então, para todo $t \in \mathbb{R}$, qualquer matriz fundamental do sistema (2.5) pode ser escrita na forma

$$
\Phi(t)=Q(t) e^{B t},
$$

onde $Q(t)$ é uma matriz não singular, diferenciável e periódica de periódo $T$ e $B$ é uma matriz constante de ordem n. Além disso, se $\Phi(0)=I$, onde $I$ é a matriz identidade, então $Q(0)=I$.

Qualquer matriz não singular $C$, tal que $\Phi(t+T)=\Phi(t) C$, onde $\Phi(t)$ é uma matriz fundamental do sistema (2.5), é chamada matriz monodromia de (2.5). Segue-se do Teorema de Floquet que $C=e^{B T}$. De fato,

$$
\begin{aligned}
\Phi(t+T) & =Q(t+T) e^{B(t+T)} \\
& =Q(t) e^{B t} e^{B T} \\
& =\Phi(t) e^{B T}
\end{aligned}
$$

Logo, $C=e^{B T}$ é a matriz de monodromia relativa a matriz fundamental $\Phi(t)$ do sistema (2.5).

Segundo o Teorema de Floquet a solução de $\dot{x}=A(t) x$ pode ser escrita como

$$
x(t)=Q(t) e^{B t} x_{0} .
$$

Como consequência do Teorema de Floquet, ao invés de estudarmos o sistema linear não autonômo (2.5), com uma mudança de variável podemos transformar esse sistema em um sistema linear autonômo de coeficientes constantes.

Teorema 2.2.15. [Per13] Seja $A(t)$ uma matriz contínua e periódica de periódo T. Então o sistema linear não autonômo (2.5), sob a mudança linear de coordenadas

$$
y=Q^{-1}(t) x
$$

reduz-se a um sistema linear autonômo

$$
\dot{y}=B y .
$$

Dados os autovalores $\xi_{j}, j=1, \ldots, n$ da matriz $B$, então $e^{\xi_{j} T}, j=$ $1, \ldots, n$ são os autovalores da matriz $e^{B T}$. Os autovalores $\xi_{j}, j=1, \ldots, n$ de 
$B$ são chamados de expoentes característicos de $\phi$. Os autovalores $e^{\xi_{j} T}, j=$ $1, \ldots, n$ de $e^{B T}$ são chamados de multiplicadores característicos de $\phi$. Os multiplicadores característicos são os autovalores da matriz de monodromia $e^{B T}$ de (2.5).

O resultado a seguir mostra que um dos expoentes característicos de $\phi$ é nulo, ou seja, um dos multiplicadores característicos é 1. Além disso, estabelece uma relação entre os multiplicadores característicos e os autovalores da Aplicação de Poincaré.

Teorema 2.2.16. [Per13] Suponhamos que $f: \Omega \rightarrow \mathbb{R}^{n}$ seja um campo vetorial de classe $\mathcal{C}^{1}$, onde $\Omega \subset \mathbb{R}^{n}$ é um subconjunto aberto e que $\phi:=$ $\left\{\varphi_{t}\left(x_{0}\right): 0 \leq t \leq T\right\}$ seja uma órbita fechada de (2.1) contida em $\Omega$. Para $\delta>0$ suficientemente pequeno e $x \in \Sigma \cap B\left(x_{0} ; \delta\right)$, seja $P(x)$ a Aplicação de Poincaré para $\phi$ em $x_{0}$ onde $\Sigma$ é o hiperplano ortogonal a $\phi$ em $x_{0}$ e de dimensão $n-1$. Se $\xi_{1}, \ldots, \xi_{n}$ são os expoentes característicos de $\phi$, então um deles, tomemos $\xi_{n}$, é nulo e os multiplicadores característicos $e^{\xi_{j} T}, j=$ $1, \ldots, n-1$, são os autovalores de $D P\left(x_{0}\right)$.

Dizemos que a órbita fechada $\phi$ do sistema dinâmico autonômo (2.1) é hiperbólica, se para qualquer $x_{0} \in \phi$, a matriz Jacobiana da Aplicação de Poincaré associada à órbita fechada $\phi$ em $x_{0}$ possui todos os multiplicadores característicos com módulo diferente de 1 , com exceção apenas de um multiplicador característico que têm módulo igual a 1.

Uma órbita fechada $\phi$ do sistema dinâmico (2.1) é do tipo- $k$ se a matriz Jacobiana da Aplicação de Poincaré associada à órbita $\phi$ possui $k$ multiplicadores característicos com módulo maior que 1 , com $0 \leq k \leq n-1$, e $n-k-1$ multiplicadores característicos com módulo menor que 1 .

Generalizando o Teorema 2.2.12 para órbitas periódicas, exibiremos a seguir um teorema que garante a existência de variedades locais invariantes para órbita periódicas. Para maiores detalhes da demonstração deste teorema ver [Per13].

Teorema 2.2.17. [Per13] Seja $\varphi(t, x)$ fluxo do sistema (2.1). Sejam $\phi=$ $\left\{\varphi\left(t, x_{0}\right): 0 \leq t \leq T\right\}$ uma órbita fechada do sistema dinâmico autonômo (2.1) de período $T$ e $x_{0} \in \phi$. Se $k$ multiplicadores característicos da aplicação de Poincaré associada a $\phi$ tem módulo maior que 1 , onde $0 \leq k \leq n-1$, e $n-k-1$ multiplicadores característicos tem módulo menor que 1 , então existe um $\delta>0$ tal que a variedade estável de $\phi$ dada por

$$
\begin{aligned}
W_{l o c}^{s}(\phi)= & \left\{x \in B(\phi, \delta) ; d\left(\varphi_{t}(x), \phi\right) \rightarrow 0 \text { quando } t \rightarrow+\infty \text { e } \varphi(t, x) \in\right. \\
& B(\phi, \delta) \text { quando } t \geq 0\}
\end{aligned}
$$

é uma variedade diferenciável $(n-k)$-dimensional que é positivamente in- 
variante e a variedade instável de $\phi$ dada por

$$
\begin{aligned}
W_{l o c}^{u}(\phi)= & \left\{x \in B(\phi, \delta) ; d\left(\varphi_{t}(x), \phi\right) \rightarrow 0 \text { quando } t \rightarrow-\infty e \varphi(t, x) \in\right. \\
& B(\phi, \delta) \text { quando } t \leq 0\}
\end{aligned}
$$

é uma variedade diferenciável $(k+1)$-dimensional que é negativamente invariante, onde $B(\phi, \delta)=\left\{x \in \mathbb{R}^{n} ; d(\phi, x)<\delta\right\}$. Além disso, as variedades estável e instável de $\phi$ se intersectam transversalmente em $\phi$.

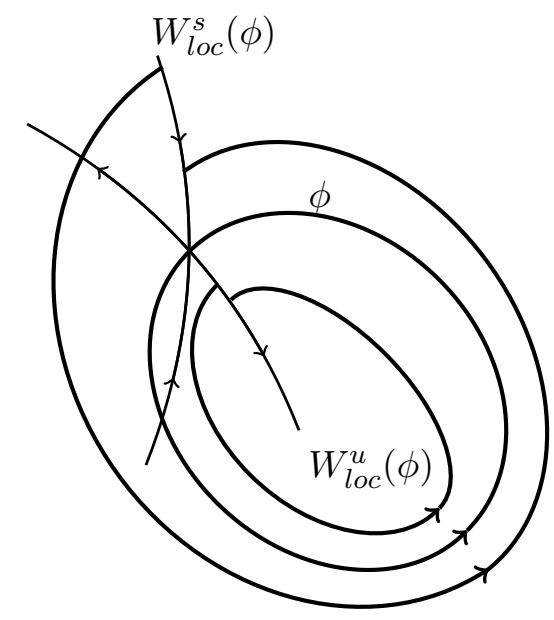

Figura 2.11: Variedades Estável $W^{s}(\phi)$ e Instável $W^{u}(\phi)$.

Na Figura 2.11, ilustramos as variedades estável $W_{l o c}^{s}(\phi)$ e instável $W_{l o c}^{u}(\phi)$ da órbita periódica do sistema dinâmico autonômo (2.1).

As variedades locais estável e instável $W_{l o c}^{s}(\phi)$ e $W_{l o c}^{u}(\phi)$, respectivamente, podem ser usadas para definir as variedades globais estável e instável de $\phi$. Analogamente, como definimos as variedades globais estável e instável de pontos de equilíbrios, definamos também a noção de variedades globais estável e instável, ou simplesmente de variedades estável e instável de órbitas fechadas.

As variedades estável e instável da órbita fechada $\phi$ do sistema dinâmico autonômo (2.1) são definidas por

$$
W^{s}(\phi)=\bigcup_{t \leq 0} \varphi_{t}\left(W_{l o c}^{s}(\phi)\right) \text { e } W^{u}(\phi)=\bigcup_{t \geq 0} \varphi_{t}\left(W_{l o c}^{u}(\phi)\right),
$$

respectivamente. 


\subsubsection{Pontos de Equilíbrio Não Hiperbólicos}

Na seção 2.2.5, estudamos o comportamento assintótico na vizinhança de um ponto de equilíbrio hiperbólico e determinamos suas variedades invariantes. Façamos o mesmo estudo para o caso em que o ponto de equilíbrio $\bar{x}$ do sistema dinâmico autonômo (2.1) seja não hiperbólico. Seja $J(\bar{x})$ a matriz jacobiana do sistema linearizado associado ao sistema (2.1) com a origem como ponto de equilíbrio do sistema linearizado (2.4). Suponhamos que a matriz Jacobiana $J(\bar{x})$ tenha $k_{u}$ autovalores com parte real positiva, $k_{s}$ autovalores com parte real negativa e $k_{c}$ autovalores com parte real nula, com $k_{u}+k_{s}+k_{c}=n$. Sejam $v_{1}, \ldots, v_{k_{u}}$ os autovetores generalizados associados aos autovalores com parte real positiva, $v_{k_{u}+1}, \ldots, v_{k_{u}+k_{s}}$ os autovetores generalizados associados aos autovalores com parte real negativa e $v_{k_{u}+k_{s}+1}, \ldots, v_{n}$ os autovetores generalizados associados aos autovalores com parte real nula. Então o espaço $\mathbb{R}^{n}$ é decomposto como a soma direta de três subespaços lineares definidos por

$$
\begin{gathered}
E^{u}(\bar{x})=\operatorname{span}\left\{v_{1}, \ldots, v_{k_{u}}\right\}, E^{s}(\bar{x})=\operatorname{span}\left\{v_{k_{u}+1}, \ldots, v_{k_{u}+k_{s}}\right\} \\
\text { e } E^{c}(\bar{x})=\operatorname{span}\left\{v_{k_{u}+k_{s}+1}, \ldots, v_{n}\right\} .
\end{gathered}
$$

Estes subespaços são invariantes em relação ao sistema dinâmico autonômo (2.4) e são conhecidos como subespaços Instável, Estável e Central, respectivamente. As soluções do sistema dinâmico linearizado (2.4) com condições iniciais em algum dos subespaços $E^{u}(\bar{x}), E^{s}(\bar{x})$ ou $E^{c}(\bar{x})$ devem permanecer em particular nestes subespaços para todo tempo $t$.

O Teorema 2.2.12 afirma que se um ponto de equilíbrio $\bar{x}$ é hiperbólico, então as variedades estável $W^{s}(\bar{x})$ e instável $W^{u}(\bar{x})$ tangenciam os espaços estável $E^{s}(\bar{x})$ e instável $E^{u}(\bar{x})$ no ponto de equilíbrio hiperbólico $\bar{x}$. O Teorema da Variedade Central estende esse resultado para o caso em que o ponto de equilíbrio $\bar{x}$ é não hiperbólico. Para maiores detalhes da demonstração deste teorema ver [HPS70].

Teorema 2.2.18. [HPS70] Seja $\bar{x}$ um ponto de equilíbrio não hiperbólico de (2.1). Então existem variedades locais invariantes $W_{l o c}^{s}(\bar{x}), W_{l o c}^{u}(\bar{x}), W_{l o c}^{c}(\bar{x})$, $W_{\text {loc }}^{\text {cs }}(\bar{x})$ e $W_{\text {loc }}^{\text {cu }}(\bar{x})$ de classe $\mathcal{C}^{1}$, tangentes a $E^{s}(\bar{x}), E^{u}(\bar{x}), E^{c}(\bar{x}), E^{c}(\bar{x}) \oplus$ $E^{s}(\bar{x})$ e $E^{c}(\bar{x}) \oplus E^{u}(\bar{x})$ em $\bar{x}$, respectivamente.

As variedades $W_{l o c}^{s}(x), W_{l o c}^{u}(x), W_{l o c}^{c}(x), W_{l o c}^{c s}(x)$ e $W_{l o c}^{c u}(x)$ são chamadas de variedades locais estável, instável, central, centro estável e centro instável, respectivamente. Soluções iniciando em qualquer que seja o ponto da variedade local estável $W_{l o c}^{s}(\bar{x})$ tendem para $\bar{x}$ quando $t$ tende a mais infinito. Analogamente, as soluções iniciando em $W_{l o c}^{u}(\bar{x})$ tendem para $\bar{x}$ quando $t$ tende a menos infinito. As variedades local estável e instável são únicas, mas a variedade local centro estável, central e centro instável não necessariamente têm que ser. 


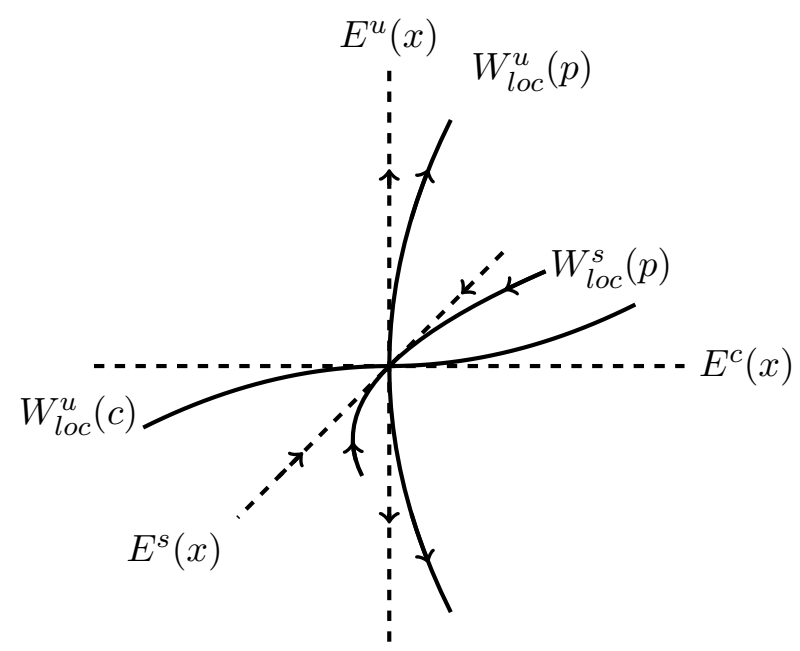

Figura 2.12: Variedades Estável $W^{s}(\bar{x})$, Instável $W^{u}(\bar{x})$ e Central $W^{c}(\bar{x})$.

Na Figura 2.12, ilustramos as variedades estável, instável e central, $W_{l o c}^{s}(\bar{x})$, $W_{l o c}^{u}(\bar{x})$ e $W^{c}(\bar{x})$, respectivamente, do ponto de equilíbrio não hiperbólico do sistema dinâmico autonômo (2.1).

Vejamos dois tipos de pontos de equilíbrio não hiperbólicos, o ponto de equilíbrio Sela-Nó e o ponto de equilíbrio Hopf. Apresentaremos o comportamento dinâmico desses equilíbrios e também o comportamento assintótico das soluções nas variedades invariantes.

Um ponto de equilíbrio não hiperbólico $p \in \mathbb{R}^{n}$ de (2.1) é chamado um ponto de equilíbrio sela-nó se as seguintes condições são satisfeitas:

a. $D_{x} f(p)$ tem um único autovalor simples igual a 0 com $v$ sendo um autovetor à direita e $w$ à esquerda, isto é, $D_{x} f(p) v=0$ e $\left(D_{x} f(p)\right)^{T} w=0$;

b. $w^{T}\left[D_{x}^{2} f(p) v\right] v \neq 0$.

A condição a caracteriza a singularidade no Jacobiano, enquanto a condição b caracteriza o tipo de bifurcação.

Pontos de equilíbrio sela-nó podem ser classificados em tipos de acordo com o número de autovalores de $D_{x} f(p)$ com parte real positiva.

Um ponto de equilíbrio sela-nó $p$ de (2.1) é chamado um ponto de equilíbrio sela-nó do tipo- $k$ se $D_{x} f(p)$ tem $k$, com $0 \leq k \leq n-1$, autovalores com parte real positiva e $n-k-1$ com parte real negativa.

Dado $p$ um ponto de equilíbrio sela-nó de (2.1), o Teorema 2.2.18 garante a existência de variedades locais invariantes de $p$ que são as variedades locais estável, instável, central, centro-estável e/ou centro-instável.

Vejamos algumas propriedades dessas variedades para o caso do ponto de equilíbrio sela-nó do tipo-zero de (2.1), para maiores detalhes ver [Sot79],: 


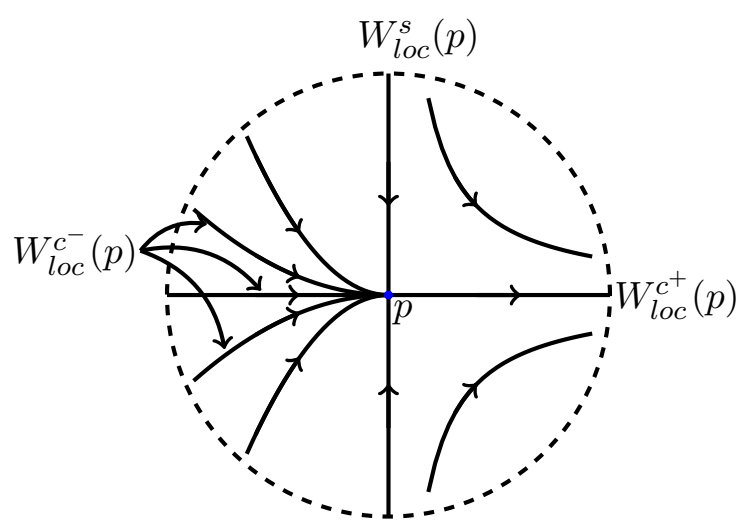

Figura 2.13: Variedades $W_{l o c}^{c^{+}}(p), W_{l o c}^{c^{-}}(p)$ e $W_{l o c}^{s}(p)$ para um ponto de equilíbrio sela-nó tipo-zero $p$ do sistema (2.1) em $\mathbb{R}^{3}$. As variedades $W_{l o c}^{c^{+}}(p)$ e $W_{l o c}^{s}(p)$ são únicas. Três possíveis escolhas para $W_{l o c}^{c^{-}}(p)$ são mostradas nesta figura.

(1) A variedade local central unidimensional $W_{l o c}^{c}(p)$ de $p$ pode ser decomposta em três subvariedades invariantes:

$$
W_{l o c}^{c}(p)=W_{l o c}^{c^{-}}(p) \cup\{p\} \cup W_{l o c}^{c^{+}}(p) .
$$

Se $q \in W_{l o c}^{c^{-}}(p)$ então $\varphi(t, q) \longrightarrow p$ quando $t \longrightarrow+\infty$. Se $q \in W_{l o c}^{c^{+}}(p)$ então $\varphi(t, q) \longrightarrow p$ quando $t \longrightarrow-\infty$. Além disso, $W_{l o c}^{c^{+}}(p)$ é única enquanto existem infinitas escolhas possíveis para $W_{l o c}^{c^{-}}(p)$.

(2) A variedade local estável $(n-1)$-dimensional $W_{l o c}^{s}(p)$ de $p$ existe, é única, e se $q \in W_{l o c}^{s}(p)$ então $\varphi(t, q) \longrightarrow p$ quando $t \longrightarrow+\infty$.

(3) Existe uma vizinhança $N$ de $p$ onde o retrato de fase do sistema (2.1) em $N$ é topologicamente equivalente ao retrato de fase da Figura 2.13

As variedades globais estável e instável de um ponto de equilíbrio hiperbólico são definidas estendendo-se as variedades locais estável e instável através do fluxo como foi mostrado na Seção 2.2.5. Geralmente, esta técnica para definirmos variedades globais não pode ser aplicada para pontos de equilíbrios não hiperbólicos. No caso particular de um ponto de equilíbrio sela-nó do tipo-zero, podemos ainda definir a variedade global estável $W^{s}(p)$ e a variedade global central $W^{c}(p)$ estendendo-se as variedades locais $W_{l o c}^{s}(p)$ e $W_{l o c}^{c}(p)$ através do fluxo como segue:

$$
\begin{gathered}
W^{s}(p):=\bigcup_{t \leq 0} \varphi\left(t, W_{l o c}^{s}(p)\right) \quad W^{c}(p):=W^{c^{-}}(p) \cup\{p\} \cup W^{c^{+}}(p) \\
W^{c^{+}}(p):=\bigcup_{t \geq 0} \varphi\left(t, W_{l o c}^{c^{+}}(p)\right) \quad W^{c^{-}}(p):=\bigcup_{t \leq 0} \varphi\left(t, W_{l o c}^{c^{-}}(p)\right) .
\end{gathered}
$$


A extensão definida acima é possível devido à invariância e ao comportamento assintótico das trajetórias nas variedades locais estável e central discutidas nos itens (1) e (2).

Exploraremos agora o comportamento dinâmico do outro ponto de equilíbrio não hiperbólico o qual estamos interessados, o ponto de equilíbrio Hopf. Analisaremos como as soluções se comportam numa vizinhança próxima desse ponto de equilíbrio. Dado o sistema dinâmico autonômo não linear (2.1), podemos realizar sempre uma mudança de coordenadas no sistema (2.1), deslocando o ponto de equilíbrio para origem. Deste modo, sem perda de generalidade, o sistema (2.1) pode ser reescrito como

$$
\dot{x}=A x+F(x), x \in \mathbb{R}^{n},
$$

onde $F, F(x)=O\left(\|x\|^{2}\right)$, é uma função suave que tem expansão de Taylor em $x$ iniciando com pelo menos os termos quadráticos. Podemos escrever a função $F(x)$ como

$$
F(x)=\frac{1}{2} B(x, x)+\frac{1}{6} C(x, x, x)+O\left(\|x\|^{4}\right)
$$

onde $B(x, y)$ e $C(x, y, z)$ são funções vetoriais multilineares simétricas de $x, y, z \in \mathbb{R}^{n}$ tal que

$$
B_{i}(x, y)=\left.\sum_{j, k=1}^{n} \frac{\partial^{2} F_{i}(\xi)}{\partial \xi_{j} \partial \xi_{k}}\right|_{\xi=0} x_{j} y_{k}, i=1, \ldots, n
$$

e

$$
C_{i}(x, y, z)=\left.\sum_{j, k, l=1}^{n} \frac{\partial^{3} F_{i}(\xi)}{\partial \xi_{j} \partial \xi_{k} \partial \xi_{l}}\right|_{\xi=0} x_{j} y_{k} z_{l}, i=1, \ldots, n .
$$

Um ponto de equilíbrio não-hiperbólico $p \in \mathbb{R}^{n}$ do sistema (2.1) é chamado um ponto de equilíbrio Hopf se as seguintes condições são satisfeitas:

a. $D_{x} f(p)$ tem um par simples de autovalores imaginários puros, $\pm i \omega$, e nenhum outro autovalor com parte real nula;

b. $l_{1} \neq 0$ onde $l_{1}$ é o Primeiro Coeficiente de Lyapunov, que pode ser calculado pela fórmula:

$$
\begin{aligned}
l_{1}= & \frac{1}{2 \omega} \Re\left[\langle u, C(v, v, \bar{v})\rangle-2\left\langle u, B\left(v, A^{-1} B(v, \bar{v})\right)\right\rangle\right. \\
& \left.+\left\langle u, B\left(\bar{v},(2 i \omega I-A)^{-1} B(v, v)\right)\right\rangle\right]
\end{aligned}
$$

onde $\Re: \mathbb{C} \rightarrow \mathbb{R}$ é a função que toma a parte real de um número complexo, $v$ é o autovetor complexo associado ao autovalor imaginário $i \omega, u$ é o autovetor adjunto complexo da matriz transposta $A$ associado ao seu autovalor $-i \omega$ 
e satisfazendo a condição de normalização $\langle u, v\rangle=1$. Nos Apêndices A e B foi disponibilizado um algoritmo para calcular o primeiro coeficiente de Lyapunov para um sistema dinâmico qualquer.

Se a segunda condição da definição de um ponto de equilíbrio Hopf não for satisfeita, podemos ainda ter um ponto de equilíbrio Hopf degenerado. Se o sistema não tem termos não lineares, por exemplo, temos um ponto de equilíbrio Hopf degenerado. Caso contrário, se o sistema tem termos não-lineares e ainda $l_{1}=0$, é preciso verificar se o segundo coeficiente de Lyapunov não se anula, veja [Kuz13] para maiores detalhes.

Pontos de equilíbrio Hopf podem ser classificados de acordo com o sinal do primeiro coeficiente de Lyapunov. Um ponto de equilíbrio Hopf $p \in \mathbb{R}^{n}$ de (2.1) é chamado um ponto de equilíbrio Hopf supercrítico se o primeiro coeficiente de Lyapunov $l_{1}<0$. Um ponto de equilíbrio Hopf $p \in \mathbb{R}^{n}$ de (2.1) é chamado um ponto de equilíbrio Hopf subcrítico se o primeiro coeficiente de Lyapunov $l_{1}>0$.

Coeficientes de Lyapunov estão relacionados com o comportamento assintótico do sistema restrito à variedade central. Pontos de equilíbrio Hopf supercríticos atraem órbitas na variedade central, enquanto pontos de equilíbrio Hopf subcríticos as repelem.

Pontos de equilíbrio Hopf podem ser classificados em tipos de acordo com o número de autovalores de $D_{x} f(p)$ com parte real positiva. Um ponto de equilíbrio Hopf $p$ de (2.1) é chamado um ponto de equilíbrio Hopf do tipo-k se $D_{x} f(p)$ tem $k(k \leq n-2)$ autovalores com parte real positiva e $n-k-2$ com parte real negativa.

Analogamente, os pontos de equilíbrio Hopf supercrítico e subcrítico também podem ser classificados em tipos de acordo com o número de autovalores de $D_{x} f(p)$ com parte real positiva.

Dado $p$ um ponto de equilíbrio Hopf de (2.1), o Teorema 2.2.18 garante a existência de variedades locais invariantes de $p$ que são as variedades locais estável, instável, central, centro-estável e/ou centro-instável. Vejamos algumas propriedades dessas variedades. Para maiores detalhes ver [Kuz13, Sot79].

Se $p$ é um ponto de equilíbrio Hopf supercrítico, então:

(1) $p$ é um ponto de equilíbrio Hopf supercrítico do tipo-zero de (2.1):

(a) A variedade local estável $(n-2)$-dimensional $W_{l o c}^{s}(p)$ de $p$ existe, é única, e se $q \in W_{l o c}^{s}(p)$ então $\varphi(t, q) \longrightarrow p$ quando $t \longrightarrow+\infty$.

(b) A variedade local central bidimensional $W_{l o c}^{c}(p)$ de $p$ existe, não é única, e se $q \in W_{l o c}^{c}(p)$ então $\varphi(t, q) \longrightarrow p$ quando $t \longrightarrow+\infty$.

(2) $p$ é um ponto de equilíbrio Hopf do supercrítico tipo- $k$ de (2.1), com $1 \leq k \leq n-3$ :

(a) A variedade local instável $k$-dimensional $W_{l o c}^{u}(p)$ de $p$ existe, é única, e se $q \in W_{l o c}^{u}(p)$ então $\varphi(t, q) \longrightarrow p$ quando $t \longrightarrow-\infty$. 
(b) A variedade local estável $(n-k-2)$-dimensional $W_{l o c}^{s}(p)$ de $p$ existe, é única, e se $q \in W_{l o c}^{s}(p)$ então $\varphi(t, q) \longrightarrow p$ quando $t \longrightarrow$ $+\infty$.

(c) A variedade local centro-estável $(n-k)$-dimensional $W_{l o c}^{c s}(p)$ de $p$ existe, é única, e se $q \in W_{l o c}^{c s}(p)$ então $\varphi(t, q) \longrightarrow p$ quando $t \longrightarrow+\infty$.

(3) $p$ é um ponto de equilíbrio Hopf supercrítico do tipo- $(n-2)$ de (2.1):

(a) A variedade local instável $(n-2)$-dimensional $W_{l o c}^{u}(p)$ de $p$ existe, é única, e se $q \in W_{l o c}^{u}(p)$ então $\varphi(t, q) \longrightarrow p$ quando $t \longrightarrow-\infty$.

(b) A variedade local central bidimensional $W_{l o c}^{c}(p)$ de $p$ existe, é única, e se $q \in W_{l o c}^{c}(p)$ então $\varphi(t, q) \longrightarrow p$ quando $t \longrightarrow+\infty$.

A Figura 2.14 ilustra as variedades invariantes do ponto de equilíbrio Hopf supercrítico do tipo-zero em $\mathbb{R}^{3}$ e a Figura 2.15 ilustra essas mesmas variedades invariantes para um ponto de equilíbrio Hopf supercrítico do tipo-1 em $\mathbb{R}^{3}$.

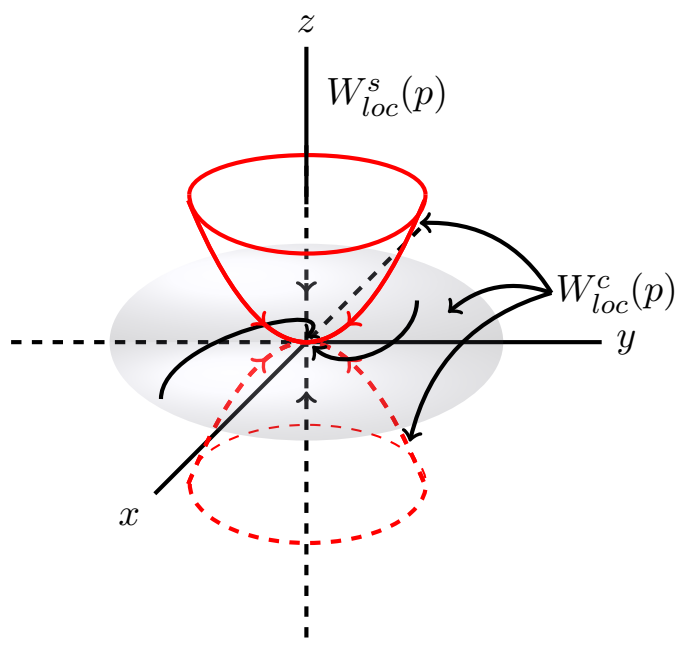

Figura 2.14: Variedades $W_{l o c}^{c}(p)$ e $W_{\text {loc }}^{s}(p)$ para um ponto de equilibrio Hopf supercrítico do tipo-zero $p$ do sistema (2.1) em $\mathbb{R}^{3}$. $W_{l o c}^{c}(p)$ não é única. Três possiveis escolhas para $W_{l o c}^{c}(p)$ são mostradas nesta figura.

Se $p$ é um ponto de equilíbrio Hopf subcrítico, então:

(1') $p$ é um ponto de equilíbrio Hopf subcrítico do tipo-zero de (2.1):

(a) A variedade local estável $(n-2)$-dimensional $W_{l o c}^{s}(p)$ de $p$ existe, é única, e se $q \in W_{l o c}^{s}(p)$ então $\varphi(t, q) \longrightarrow p$ quando $t \longrightarrow+\infty$.

(b) A variedade local central bidimensional $W_{l o c}^{c}(p)$ de $p$ existe, é única, e se $q \in W_{l o c}^{c}(p)$ então $\varphi(t, q) \longrightarrow p$ quando $t \longrightarrow-\infty$. 


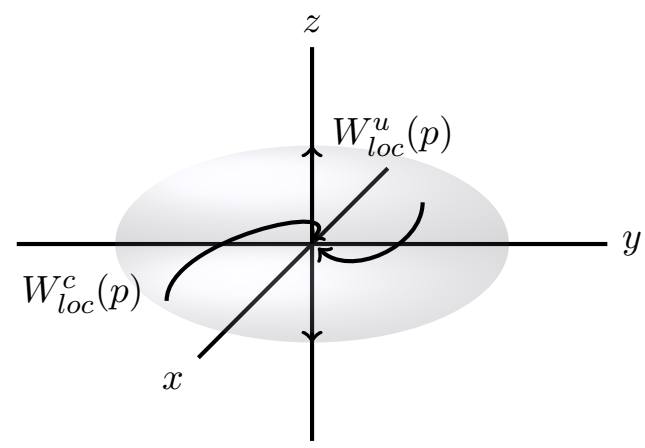

Figura 2.15: Variedades $W_{l o c}^{c}(p)$ e $W_{l o c}^{u}(p)$ para um ponto de equilíbrio Hopf supercrítico do tipo-1 $p$ do sistema (2.1) em $\mathbb{R}^{3}$. Neste caso, $W_{\text {loc }}^{c}(p)$ é único.

(2') $p$ é um ponto de equilíbrio Hopf subcrítico do tipo- $k$ de (2.1), com $1 \leq k \leq n-3$ :

(a) A variedade local instável $k$-dimensional $W_{l o c}^{u}(p)$ de $p$ existe, é única, e se $q \in W_{l o c}^{u}(p)$ então $\varphi(t, q) \longrightarrow p$ quando $t \longrightarrow-\infty$.

(b) A variedade local estável $(n-k-2)$-dimensional $W_{l o c}^{s}(p)$ de $p$ existe, é única, e se $q \in W_{l o c}^{s}(p)$ então $\varphi(t, q) \longrightarrow p$ quando $t \longrightarrow$ $+\infty$.

(c) A variedade local centro-instável $(k+2)$-dimensional $W_{l o c}^{c u}(p)$ de $p$ existe, é única, e se $q \in W_{l o c}^{c u}(p)$ então $\varphi(t, q) \longrightarrow p$ quando $t \longrightarrow-\infty$.

(3') $p$ é um ponto de equilíbrio Hopf subcrítico do tipo- $(n-2)$ de $(2.1)$ :

(a) A variedade local instável $(n-2)$-dimensional $W_{l o c}^{u}(p)$ de $p$ existe, é única, e se $q \in W_{l o c}^{u}(p)$ então $\varphi(t, q) \longrightarrow p$ quando $t \longrightarrow-\infty$.

(b) A variedade local central bidimensional $W_{l o c}^{c}(p)$ de $p$ existe, não é única, e se $q \in W_{l o c}^{c}(p)$ então $\varphi(t, q) \longrightarrow p$ quando $t \longrightarrow-\infty$.

A Figura 2.16 ilustra as variedades invariantes para um ponto de equilíbrio Hopf subcrítico do tipo-1 em $\mathbb{R}^{3}$ e a Figura 2.17 ilustra essas mesmas variedades invariantes para um ponto de equilíbrio Hopf subcrítico do tipozero em $\mathbb{R}^{3}$.

As variedades globais estável e instável de um ponto de equilíbrio hiperbólico são definidas estendendo-se as variedades locais estável e instável através do fluxo como foi mostrado na Seção 2.2.5. Geralmente, esta técnica para definir variedades globais não pode ser aplicada em geral para pontos de equilíbrio não-hiperbólicos. Entretanto, no caso particular de um ponto de equilíbrio de Hopf supercrítico, podemos ainda definir as variedades globais $W^{s}(p), W^{u}(p), W^{c}(p)$ e $W^{c s}(p)$ estendendo as variedades locais $W_{l o c}^{s}(p)$, $W_{l o c}^{u}(p), W_{l o c}^{c}(p)$ e $W_{l o c}^{c s}(p)$ através do fluxo como segue: 


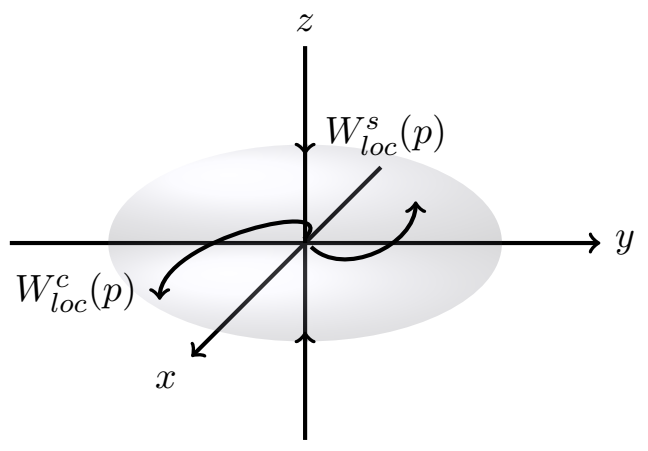

Figura 2.16: Variedades $W_{l o c}^{c}(p)$ e $W_{l o c}^{s}(p)$ para um ponto de equilíbrio Hopf subcrítico do tipo-1 $p$ do sistema $2.1 \mathrm{em} \mathbb{R}^{3}$. Neste caso, $W_{l o c}^{c}(p)$ é única.

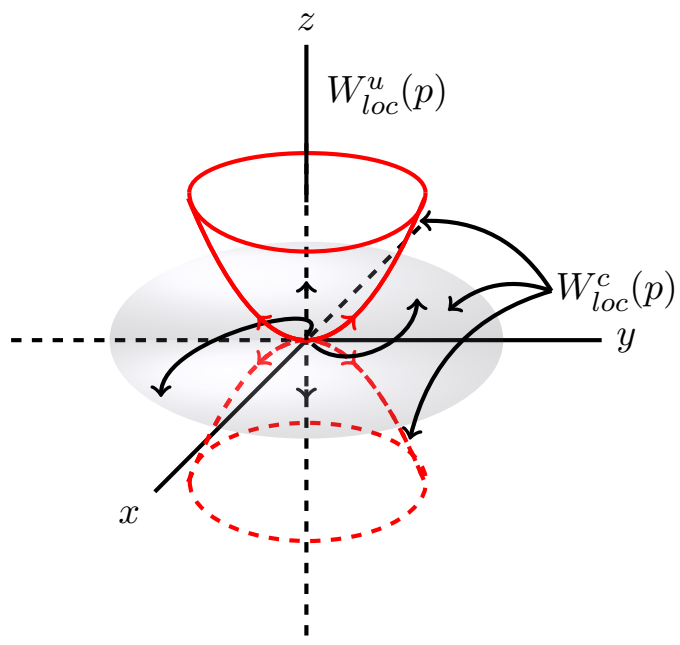

Figura 2.17: Variedades $W_{l o c}^{c}(p)$ e $W_{l o c}^{u}(p)$ para um ponto de equilibrio Hopf subcrítico do tipo-zero $p$ do sistema (2.1) em $\mathbb{R}^{3}$. $W_{l o c}^{c}(p)$ não é única. Três escolhas possiveis para $W_{l o c}^{c}(p)$ são mostradas nesta figura.

$$
\begin{aligned}
W^{s}(p) & :=\bigcup_{t \leq 0} \varphi\left(t, W_{l o c}^{s}(p)\right) & W^{u}(p) & :=\bigcup_{t \geq 0} \varphi\left(t, W_{l o c}^{u}(p)\right) \\
W^{c}(p) & :=\bigcup_{t \leq 0} \varphi\left(t, W_{l o c}^{c}(p)\right) & W^{c s}(p) & :=\bigcup_{t \leq 0} \varphi\left(t, W_{l o c}^{c s}(p)\right) .
\end{aligned}
$$

Se $p$ é um ponto de equilíbrio Hopf subcrítico, podemos definir as variedades globais $W^{s}(p), W^{u}(p), W^{c}(p)$ e $W^{c u}(p)$ estendendo as variedades locais $W_{l o c}^{s}(p), W_{l o c}^{u}(p), W_{l o c}^{c}(p)$ e $W_{l o c}^{c u}(p)$ através do fluxo como segue:

$$
W^{s}(p):=\bigcup_{t \leq 0} \varphi\left(t, W_{l o c}^{s}(p)\right) \quad W^{u}(p):=\bigcup_{t \geq 0} \varphi\left(t, W_{l o c}^{u}(p)\right)
$$




$$
W^{c}(p):=\bigcup_{t \geq 0} \varphi\left(t, W_{l o c}^{c}(p)\right) \quad W^{c u}(p):=\bigcup_{t \geq 0} \varphi\left(t, W_{l o c}^{c u}(p)\right) .
$$

As extensões definidas acima são possíveis devido à invariância e ao comportamento assintótico das trajetórias nas variedades locais estável, instável, central, centro-estável e centro-instável discutidas nos itens (1), (2), (3) e $\left(1^{\prime}\right),\left(2^{\prime}\right),\left(3^{\prime}\right)$.

\subsection{7 $\lambda$-Lema e Ponto de Equilíbrio Hopf}

Nesta seção, estudaremos o $\lambda$-Lema para pontos de equilíbrio hiperbólicos e versões deste lema para pontos de equilíbrio não hiperbólicos do tipo Hopf. A versão do $\lambda$-Lema para pontos de equilíbrio hiperbólicos afirma que dado uma seção transversal à variedade instável (ou estável), as iteradas do fluxo para um tempo passado (ou tempo futuro) dessa curva, com $t$ suficientemente grande, se aproxima da variedade estável (ou instável). Para maiores detalhes, ver [Pal69].

Sejam $\bar{x}$ um ponto de equilíbrio hiperbólico de (2.1) e $B^{u}$ uma vizinhança de $\bar{x}$ em $W_{l o c}^{u}(\bar{x})$ cuja fronteira $\partial B^{u}$ é transversal ao campo $f$ em $W_{l o c}^{u}(\bar{x})$. A fronteira $\partial B^{u}$ é denominada um domínio fundamental de $W_{l o c}^{u}(\bar{x})$. O domínio fundamental $\partial B^{u}$ possui a seguinte propriedade

$$
W_{l o c}^{u}(\bar{x}) \backslash\{\bar{x}\}=\bigcup_{t \in \mathbb{R}} \varphi\left(t, \partial B^{u}\right) .
$$

Qualquer vizinhança $N^{u}$ de $\partial B^{u}$ disjunta de $W_{l o c}^{s}(\bar{x})$ é denominada uma vizinhança fundamental de $W_{l o c}^{u}(\bar{x})$. Analogamente, define-se domínio fundamental e vizinhança fundamental para $W_{l o c}^{s}(\bar{x})$. A seguir, enunciamos a versão do $\lambda$-lema para ponto de equilíbrio hiperbólico $\bar{x}$.

Lema 2.2.19. ( $\lambda$-lema)[Pal69] Seja $\bar{x}$ um ponto de equilíbrio hiperbólico de (2.1). Considere $q \in W_{l o c}^{u}(\bar{x})$ e $D^{s}$ um disco de dimensão $r=\operatorname{dim} E^{s}(\bar{x})$, transversal a $W_{l o c}^{u}(\bar{x})$ em $q$. Então existe uma vizinhança $U$ de $\bar{x}$ em $\mathbb{R}^{n}$ tal que, dado $\varepsilon>0$, existe $t_{-}<0$ tal que $\varphi\left(t, D^{s}\right)$ contém um disco $\varepsilon$ $\mathcal{C}^{1}$-próximo a $U \cap W_{l o c}^{s}(\bar{x})$ para todo $t<t_{-}$.

Corolário 2.2.20. [Pal69] Seja $\bar{x}$ um ponto de equilíbrio hiperbólico de (2.1) e $N^{u}$ uma vizinhança fundamental de $W_{\text {loc }}^{u}(\bar{x})$. Então existe uma vizinhança $U$ de $\bar{x}$ tal que $\cup_{t \leq 0} \varphi\left(t, N^{u}\right) \supset U \backslash W_{l o c}^{s}(\bar{x})$.

O próximo resultado é uma versão do $\lambda$-Lema para órbitas periódicas que será usado na caracterização da fronteira da região de estabilidade.

Lema 2.2.21. [Pal69] Sejam $\phi$ uma órbita fechada hiperbólica do tipo-k do sistema (2.1). Seja $D$ um disco de dimensão $k$ em $W^{u}(\phi) \cap \Sigma$, onde $\Sigma$ é uma seção transversal em $p \in \phi$. Seja $N$ um disco de dimensão $k$ tendo um ponto de interseção transversal em $W^{s}(\phi)$. Então $D$ está contido no fecho do conjunto $\cap_{t \geq 0} \varphi(t, N)$. 
Corolário 2.2.22. [Pal69] Sejam $\phi$ uma órbita fechada hiperbólica de (2.1) e $N^{u}$ uma vizinhança fundamental de $W_{\text {loc }}^{u}(\phi)$. Então existe uma vizinhança $U$ de $\phi$ tal que $\cup_{t \leq 0} \varphi\left(t, N^{u}\right) \supset U \backslash W_{l o c}^{s}(\phi)$.

Como consequência da versão do $\lambda$-Lema e seu corolário para pontos de equilíbrio não hiperbólicos, ver [Pal69], apresentaremos a versão do $\lambda$ Lema para pontos de equilíbrio Hopf Supercrítico e Subcrítico. Além disso, define-se também domínio fundamental e vizinhança fundamental para as variedades estável, instável, central, centro-estável e/ou centro instável de um ponto de equilíbrio Hopf.

Lema 2.2.23. (Versão do $\lambda$-lema para ponto de equilíbrio Hopf Supercrítico do tipo $k$, com $1 \leq k \leq n-3)$ [Pal69] Seja $p$ um ponto de equilíbrio Hopf supercrítico do tipo- $k$, com $1 \leq k \leq n-3$ de (2.1). Considere $q \in W_{\text {loc }}^{u}(p)$ (ou $\left.q \in W_{\text {loc }}^{c s}(p)\right)$ e $D^{c s}$ (ou $D^{u}$ ) um disco de dimensão $n-k$ (ou $k$ ), transversal a $W_{\text {loc }}^{u}(p)$ (ou $\left.W_{\text {loc }}^{c s}(p)\right)$ em $q$, respectivamente. Então existe uma vizinhança $U$ de $p$ em $\mathbb{R}^{n}$ tal que, dado $\varepsilon>0$, existe $t_{-}<0$ (ou $t_{+}>0$ ) tal que $\varphi\left(t, D^{c s}\right.$ ) $\left(\right.$ ou $\varphi\left(t, D^{u}\right)$ ) contém um disco $\varepsilon \mathcal{C}^{1}$-próximo a $U \cap W_{\text {loc }}^{c s}(x)$ (ou $U \cap W_{l o c}^{u}(x)$ ) para todo $t<t_{-}$(ou $t>t_{+}$), respectivamente.

As Figuras 2.18 e 2.19 ilustram geometricamente o resultado do Lema 2.2.23. Como consequência do Lema 2.2.23, temos o seguinte o corolário.

Corolário 2.2.24. [Pal69] Seja p um ponto de equilíbrio Hopf supercrítico do tipo- $k$, com $1 \leq k \leq n-3$, de (2.1) e $N^{c s}$ (ou $N^{u}$ ) uma vizinhança fundamental de $W_{l o c}^{c s}(p)$ (ou $W_{l o c}^{u}(p)$ ). Então existe uma vizinhança $U$ de $p$ tal que $\cup_{t \geq 0} \varphi\left(t, N^{c s}\right) \supset U \backslash W_{l o c}^{u}(x)\left(\right.$ ou $\left.\cup_{t \leq 0} \varphi\left(t, N^{u}\right) \supset U \backslash W_{l o c}^{c s}(x)\right)$, respectivamente.

O Lema 2.2.25 e o corolário a seguir são versões dos Lema 2.2.23 e do Corolário 2.2.24 para pontos de equilíbrio Hopf subcríticos.

Lema 2.2.25. (Versão do $\lambda$-lema para ponto de equilíbrio Hopf Subcrítico do tipo $k, 0 \leq k \leq n-3$ )[Pal69] Seja $p$ um ponto de equilibrio Hopf subcrítico do tipo- $k$, com $0 \leq k \leq n-3$ de (2.1).Se $k=0$, considere $q \in W_{\text {loc }}^{c}(p)$ (ou $\left.q \in W_{l o c}^{s}(p)\right)$ e $D^{s}$ (ou $D^{c}$ ) um disco de dimensão $n-2$ (ou 2), transversal a $W_{\text {loc }}^{c}(p)$ (ou $\left.W_{\text {loc }}^{s}(p)\right)$ em $q$. Então existe uma vizinhança $U$ de $p$ em $\mathbb{R}^{n}$ tal que, dado $\varepsilon>0$, existe $t_{-}<0$ (ou $\left.t_{+}>0\right)$ tal que $\varphi\left(t, D^{s}\right)\left(\right.$ ou $\varphi\left(t, D^{c}\right)$ ) contém um disco $\varepsilon \mathcal{C}^{1}$-próximo a $U \cap W_{\text {loc }}^{s}(x)$ (ou $\left.U \cap W_{\text {loc }}^{c}(x)\right)$ para todo $t<t_{-}$(ou $t>t_{+}$), respectivamente. Se $k \neq 0$, considere $q \in W_{\text {loc }}^{\text {cu }}(p)$ (ou $\left.q \in W_{\text {loc }}^{s}(p)\right)$ e $D^{s}$ (ou $\left.D^{c u}\right)$ um disco de dimensão $n-k-2($ ou $k+2)$, transversal a $W_{\text {loc }}^{c u}(p)$ (ou $\left.W_{\text {loc }}^{\text {s }}(p)\right)$ em $q$. Então existe uma vizinhança $U$ de $p$ em $\mathbb{R}^{n}$ tal que, dado $\varepsilon>0$, existe $t_{-}<0$ (ou $t_{+}>0$ ) tal que $\varphi\left(t, D^{s}\right)$ (ou $\varphi\left(t, D^{c u}\right)$ ) contém um disco $\varepsilon \mathcal{C}^{1}$-próximo a $U \cap W_{\text {loc }}^{s}(x)$ (ou $\left.U \cap W_{\text {loc }}^{c u}(x)\right)$ para todo $t<t_{-}$(ou $t>t_{+}$), respectivamente. 


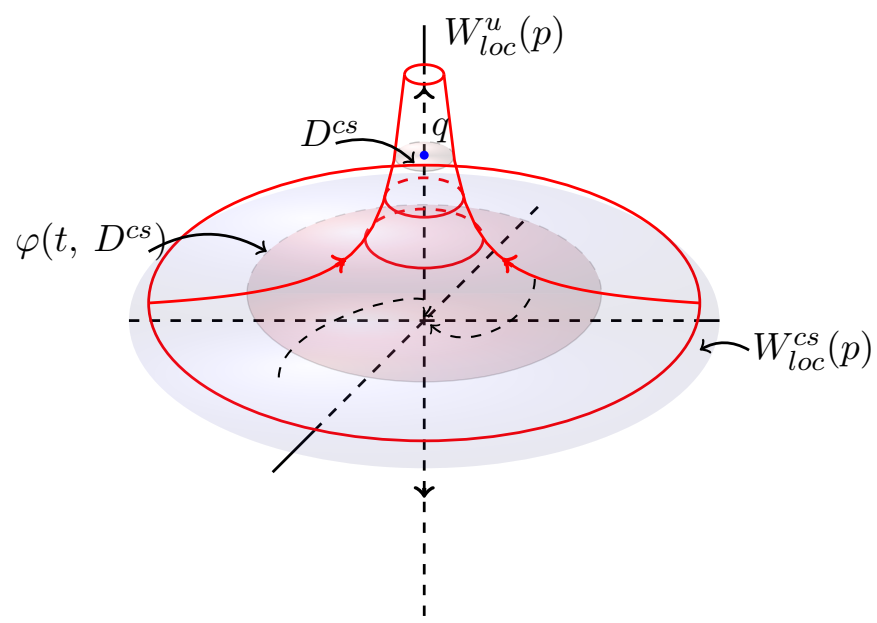

Figura 2.18: Ilustração do Lema 2.2.23 e do Corolário 2.2.24 para um ponto de

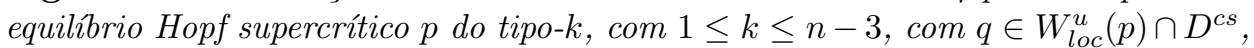
onde $D^{\text {cs }}$ é um disco de dimensão $n-k$ transversal a $W_{l o c}^{u}(p)$ em $q$.

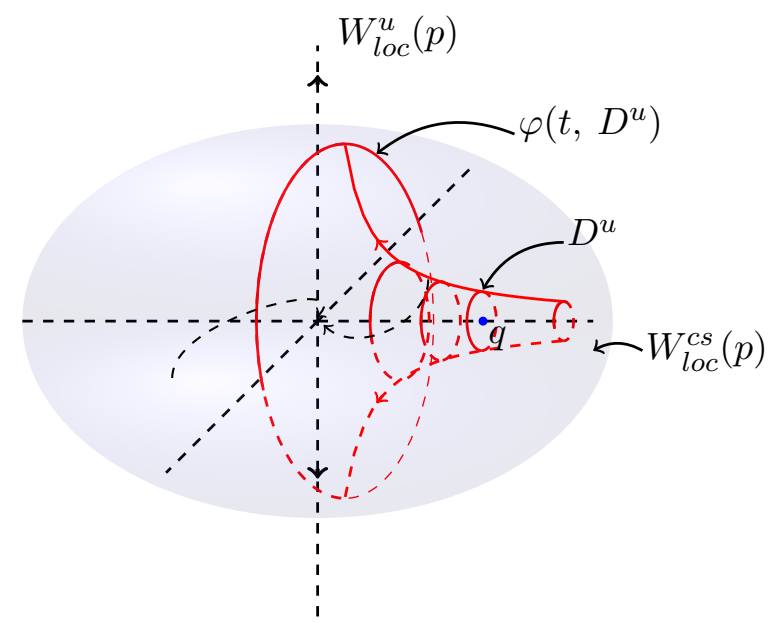

Figura 2.19: Ilustração do Lema 2.2.23 e do Corolário 2.2.24 para um ponto de equilíbrio Hopf supercrítico $p$ do tipo- $k$, com $1 \leq k \leq n-3$, com $q \in W_{l o c}^{c s}(p) \cap D^{u}$, onde $D^{u}$ um disco de dimensão $k$, transversal a $W_{\text {loc }}^{c s}(p)$ em $q$.

Corolário 2.2.26. [Pal69] Seja p um ponto de equilíbrio Hopf subcrítico do tipo-k, com $0 \leq k \leq n-3$, de (2.1). Se $k=0$ e $N^{s}$ (ou $N^{c}$ ) uma vizinhança fundamental de $W_{\text {loc }}^{s}(p)$ (ou $W_{l o c}^{c}(p)$ ), então existe uma vizinhança $U$ de $p$ tal que $\cup_{t \geq 0} \varphi\left(t, N^{s}\right) \supset U \backslash W_{l o c}^{c}(x)\left(o u \cup_{t \leq 0} \varphi\left(t, N^{c}\right) \supset U \backslash W_{l o c}^{s}(x)\right)$, respectivamente. Se $k \neq 0$ e $N^{s}$ (ou $N^{c u}$ ) uma vizinhança fundamental de $W_{\text {loc }}^{s}(p)$ (ou $W_{l o c}^{c u}(p)$ ), então existe uma vizinhança $U$ de $p$ tal que $\cup_{t \geq 0} \varphi\left(t, N^{s}\right) \supset$ $U \backslash W_{l o c}^{c u}(x)\left(\right.$ ou $\left.\cup_{t \leq 0} \varphi\left(t, N^{c u}\right) \supset U \backslash W_{l o c}^{s}(x)\right)$, respectivamente.

As Figuras 2.20 e 2.21 ilustram geometricamente as idéias do Lema 2.2.25 
e do Corolário 2.2.26.

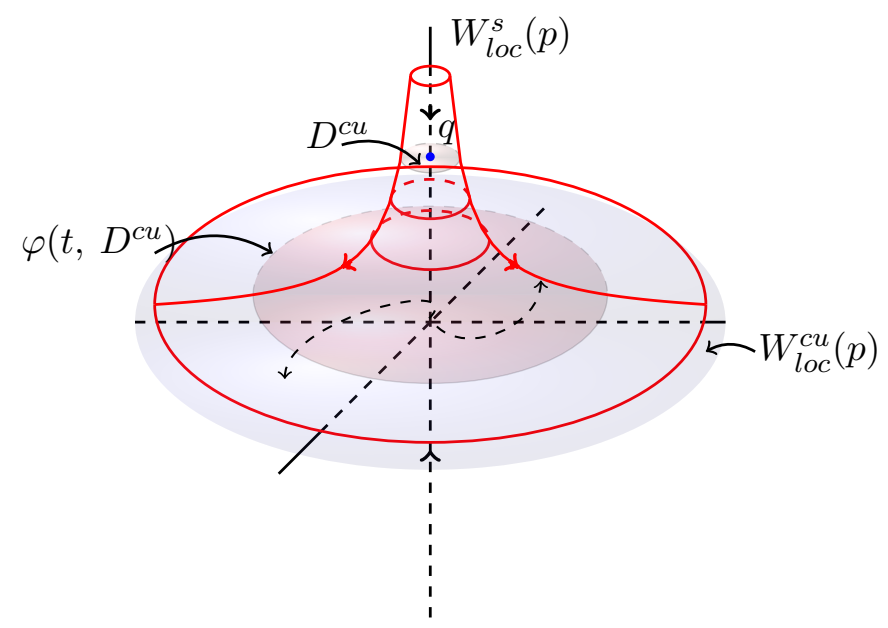

Figura 2.20: Ilustração do Lema 2.2.25 e do Corolário 2.2.26 para um ponto de equilíbrio Hopf subcrítico $p$ do tipo- $k$, com $1 \leq k \leq n-3, q \in W_{l o c}^{s}(p) \cap D^{c u}$, onde $D^{c u}$ é um disco de dimensão $k+2$, transversal a $W_{\text {loc }}^{s}(p)$ em $q$.

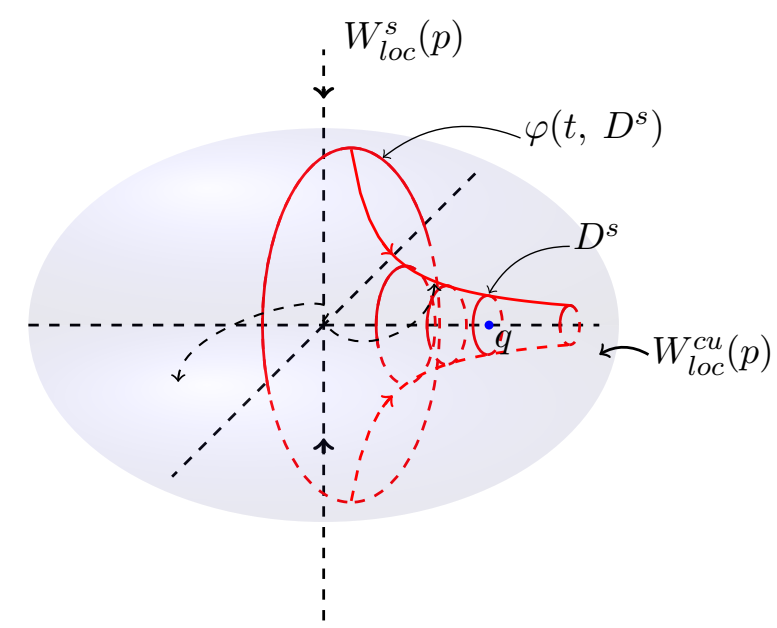

Figura 2.21: Ilustração do Lema 2.2.25 e do Corolário 2.2.26 para um ponto de equilibrio Hopf subcrítico $p$ do tipo- $k$, com $1 \leq k \leq n-3, q \in W_{\text {loc }}^{c u}(p) \cap D^{s}$, onde $D^{s}$ é um disco de dimensão $n-k-2$ transversal a $W_{l o c}^{c u}(p)$ em $q$.

\subsubsection{Transversalidade}

O conceito de transversalidade será de fundamental importância para a caracterização da fronteira da região de estabilidade que será desenvolvida nos próximos capítulos. Para tal caracterização, precisaremos saber se as variedades estável e instável dos equilíbrios se intersectam de maneira 
transversal. Se elas se intersectam transversalmente, então esta interseção persiste caso o sistema sofra pequenas perturbações. Caso contrário, a interseção pode ser "destruída" por alguma perturbação aplicada ao sistema.

Intuitivamente a transversalidade é um conceito geométrico que lida com a interseção de variedades ou superfícies e vem desempenhando um papel importante no desenvolvimento da teoria de sistemas dinâmicos.

Duas variedades $M$ e $N$ em $\mathbb{R}^{n}$ de classe $\mathcal{C}^{r}(r \geq 1)$ são transversais em $q \in \mathbb{R}^{n}$ se $q \notin M \cap N$; ou se $q \in M \cap N$, então $T_{q}(M)+T_{q}(N)=\mathbb{R}^{n}$, onde $T_{q}(M)$ e $T_{q}(N)$ denotam o espaço tangente de $M$ e $N$, respectivamente, no ponto $q$. Dizemos que $M$ e $N$ satisfazem a condição de transversalidade se elas são transversais em cada ponto $q \in M \cap N$.

Para ilustramos a definição anterior, por exemplo, no $\mathbb{R}^{3}$, duas retas nunca são transversais e uma reta e um plano só são transversais se a reta não é paralela ao plano e nem está contida nele. Enquanto no $\mathbb{R}^{2}$, duas retas só são transversais se não são paralelas ou coincidentes.

O próximo resultado estabelece uma conexão entre a dimensão das variedades e a interseção transversal das mesmas.

Lema 2.2.27. [Wig03] Se duas variedades $M$ e $N$ em $\mathbb{R}^{n}$ de classe $\mathcal{C}^{r}(r \geq$ 1) têm interseção não vazia, ou seja, $M \cap N \neq \emptyset$, e satisfazem a condição de transversalidade então

$$
\operatorname{dim}(M \cap N)=\operatorname{dim} M+\operatorname{dim} N-n .
$$

As interseções transversais têm a propriedade importante de serem "robustas" no sentido de que persistem se perturbarmos um pouco as variedades concorrentes, ver [Wig03]. 


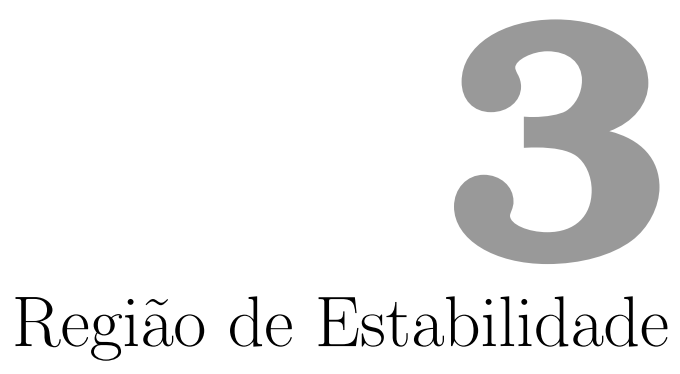

Neste capítulo, teremos como principal objetivo caracterizar topologicamente a região de estabilidade. Na literatura, região de estabilidade também é comumente chamada de domínio de atração, área de atração, região de atração ou bacia de atração. Entendemos por região de estabilidade de um conjunto atrativo ao subconjunto do espaço de estados do sistema dinâmico cujas trajetórias que iniciam nesse subconjunto tendem para o conjunto atrativo quando o tempo tende a infinito. Iniciaremos a seção introduzindo o conceito de conjunto atrativo e veremos algumas propriedades desses conjuntos. Depois enunciaremos a noção de conjunto minimal atrativo abordando algumas de suas propriedades topológicas. E finalmente, definiremos a região de estabilidade do conjunto minimal atrativo seguida de uma caracterização topológica da sua região de estabilidade e da fronteira da região de estabilidade.

\subsection{Conjuntos Atrativos}

No capítulo anterior, definimos o conceito de ponto de equilíbrio atrativo. Observemos que o ponto de equilíbrio é um conjunto fechado e invariante. Então pela definição de equilíbrio atrativo, vista na Seção 2.2.4 do Capítulo 2, afirmamos que o equilíbrio é atrativo se toda solução iniciando numa vizinhança desse equilíbrio tende para o equilíbrio quando o tempo tende para infinito. Generalizemos a definição de atratividade de ponto de equilíbrio para um conjunto fechado e invariante, obtendo assim o conceito de conjunto atrativo.

Um conjunto $H$ fechado, invariante com relação ao sistema dinâmico 
autonômo (2.1) é um conjunto atrativo se existir uma vizinhança $U$ de $H$ tal que, para toda condição inicial $x_{0} \in U, \varphi\left(t, x_{0}\right) \rightarrow H$ quando $t \rightarrow+\infty$.

Observemos que na definição acima de conjunto atrativo, a condição de que $\varphi\left(t, x_{0}\right) \rightarrow H$ quando $t \rightarrow+\infty$ pode ser reescrita como $d\left(\varphi\left(t, x_{0}\right), H\right) \rightarrow$ 0 quando $t \rightarrow+\infty$, isto é, à medida que o tempo tende para o infinito a distância entre a trajetória e o conjunto atrativo $H$ tende a zero. Definamos a vizinhança $U$ acima como vizinhança atrativa de $H$. Um exemplo trivial de conjunto atrativo é o ponto de equilíbrio atrativo.

O próximo resultado mostra que a união de conjuntos atrativos é ainda um conjunto atrativo.

Teorema 3.1.1. [Ama10] Sejam $H_{1}, H_{2} \subset \mathbb{R}^{n}$ conjuntos atrativos. Então $H=H_{1} \cup H_{2}$ é um conjunto atrativo.

Demonstração. Como $\mathrm{H}_{1}$ e $\mathrm{H}_{2}$ são conjuntos atrativos, em particular são fechados e invariantes. Como a união finita de conjuntos fechados e invariantes é um conjunto fechado e invariante, então $H=H_{1} \cup H_{2}$ tambem é fechado e invariante. Como $H_{1}$ é um conjunto atrativo, então existe uma vizinhança $U_{1}$ de $H_{1}$ tal que $x_{0} \in U_{1}$ implica $d\left(\varphi\left(t, x_{0}\right), H_{1}\right) \rightarrow 0$ quando $t \rightarrow+\infty$, ou seja, dado $\varepsilon>0$, existe $t_{1} \in \mathbb{R}$ tal que $d\left(\varphi\left(t, x_{0}\right), H_{1}\right)<\varepsilon$ para todo $t>t_{1}$. Analogamente, também existe uma vizinhança $U_{2}$ de $H_{2}$ tal que $x_{0} \in U_{2}$ implica $d\left(\varphi\left(t, x_{0}\right), H_{2}\right) \rightarrow 0$ quando $t \rightarrow+\infty$, ou seja, para todo $\varepsilon>0$, existe $t_{2} \in \mathbb{R}$ tal que $d\left(\varphi\left(t, x_{0}\right), H_{2}\right)<\varepsilon$ para todo $t>t_{2}$. Considere $U=U_{1} \cup U_{2}$ uma vizinhança de $H=H_{1} \cup H_{2}$. Mostraremos agora que $U=U_{1} \cup U_{2}$ é uma vizinhança atrativa de $H=H_{1} \cup H_{2}$. Para isso, seja $x_{0} \in U$. Sem perda de generalidade podemos supor que $x_{0} \in U_{1}$. Como $d\left(\varphi\left(t, x_{0}\right), H_{1} \cup H_{2}\right) \leq d\left(\varphi\left(t, x_{0}\right), H_{1}\right)$, então para todo $\varepsilon>0$, existe $t_{1} \in \mathbb{R}$ tal que $d\left(\varphi\left(t, x_{0}\right), H_{1} \cup H_{2}\right) \leq d\left(\varphi\left(t, x_{0}\right), H_{1}\right)<\varepsilon$ para todo $t>t_{1}$, ou seja, $d\left(\varphi\left(t, x_{0}\right), H_{1} \cup H_{2}\right) \rightarrow 0$ quando $t \rightarrow+\infty$. Portanto, como $x_{0} \in U$ foi tomado genérico, podemos afirmar que $U=U_{1} \cup U_{2}$ é uma vizinhança atrativa de $H=H_{1} \cup H_{2}$, o que finaliza a demonstração do teorema.

Generalizando o teorema anterior para uma coleção finita de conjuntos atrativos obtemos o seguinte resultado.

Corolário 3.1.2. [Ama10] Sejam $H_{1}, H_{2}, \ldots, H_{n} \subset \mathbb{R}^{n}$ conjuntos atrativos. Então o conjunto $H=H_{1} \cup H_{2} \cup \ldots \cup H_{n}$ é atrativo.

Vale salientar que a recíproca do Teorema 3.1.1 não é válida, isto é, se a união de conjuntos fechados e invariantes for atrativa, pode ser que pelo menos um dos conjuntos não seja atrativo. Considere, por exemplo, o sistema de equações diferenciais

$$
\left\{\begin{array}{l}
\dot{x}=y+x\left(1-x^{2}-y^{2}\right) \\
\dot{y}=-x+y\left(1-x^{2}-y^{2}\right)
\end{array}, \text { com }(x, y) \in \mathbb{R}^{2} .\right.
$$


O sistema acima possui um ciclo limite estável e um ponto de equilíbrio instável que é a origem. O conjunto formado, pela união do ciclo limite com a origem é um conjunto atrativo, porém a origem não é um conjunto atrativo, ver [Ama10].

\subsection{Conjuntos Minimais}

$\mathrm{Na}$ análise de sistemas dinâmicos, algumas vezes é importante estudar determinados tipos de conjuntos que não contêm subconjuntos com as mesmas propriedades. Tais conjuntos chamamos de conjuntos minimais.

Um subconjunto $M \subset \mathbb{R}^{n}$ é dito ser conjunto minimal se é não vazio, fechado e invariante e, além disso, nenhum subconjunto próprio de $M$ possui essas mesmas propriedades. O ponto de equilíbrio e a órbita periódica são exemplos de conjuntos minimais.

Um conjunto atrativo $H$ é minimal se nenhum subconjunto não vazio de $H$ têm as mesmas propriedades de ser fechado e invariante.

O próximo resultado fornece uma caracterização de conjuntos minimais através de suas trajetórias. Em particular, estabelece que um conjunto atrativo minimal é igual ao fecho de uma única órbita em $\gamma$.

Teorema 3.2.1. [BS02] Um subconjunto não vazio $M \subset \mathbb{R}^{n}$ é minimal se, e somente se, $\overline{\gamma(x)}=M$ para cada $x \in M$.

Demonstração. Seja $M$ minimal. Então, para cada $x \in M$, afirmamos que $\overline{\gamma(x)} \subset M$. De fato, como $M$ é invariante, temos que $\gamma(x) \subset M$. E como $M$ é fechado e, portanto, $\bar{M}=M$, temos que $\overline{\gamma(x)} \subset M$. Como $\overline{\gamma(x)}$ é um subconjunto não vazio, fechado e invariante, então pela definição de minimalidade temos que $\overline{\gamma(x)}=M$. Agora assumiremos que $\overline{\gamma(x)}=M$ para cada $x \in M$. Se $M$ não é minimal então existe um subconjunto $P \varsubsetneqq M$ não vazio, fechado e invariante. Escolhamos $x \in P$, então $\overline{\gamma(x)} \subset P$ e, consequentemente, $\overline{\gamma(x)} \varsubsetneqq M$, o que é uma contradição pois $\overline{\gamma(x)}=M$. Logo, $M$ é minimal.

O teorema abaixo estabelece uma propriedade de que todo conjunto minimal com interior não vazio é um conjunto aberto.

Teorema 3.2.2. [BS02] Se o subconjunto $M \subset \mathbb{R}^{n}$ é minimal e int $(M) \neq \emptyset$, então $M=\operatorname{int}(M)$.

Demonstração. Seja $y \in M$ e $x \in \operatorname{int}(M)$. Consequentemente, pelo Teorema 3.2.1, $x \in \overline{\gamma(y)}$. Então existe $t \in R$ tal que $x=\varphi_{t}(y) \in \operatorname{int}(M)$. Pela invariância do conjunto int $(M)$, temos que $y=\varphi_{-t}\left(\varphi_{t}(y)\right) \in \operatorname{int}(M)$, e, portanto, $M \subset \operatorname{int}(M)$. Sabemos que $\operatorname{int}(M)$ é um subconjunto aberto de $M$, então $\operatorname{int}(M) \subset M$. Logo, $M=\operatorname{int}(M)$. 
Ainda com o objetivo de caracterizar conjuntos minimais, o resultado a seguir permite reescrever um subconjunto compacto minimal não vazio através do seu conjunto $\omega$-limite.

Teorema 3.2.3. [BS02] Seja $M \subset \mathbb{R}^{n}$ um conjunto não vazio compacto. Então $M$ é minimal, se e somente se, $\omega(x)=M$ para cada $x \in M$.

Demonstração. Inicialmente, suponhamos que $M=\omega(x)$, para cada $x \in M$, porém $M$ não é um conjunto minimal. Então existe um conjunto não vazio, fechado e invariante $Y \subset M$. Como $Y$ é um conjunto fechado e invariante, então $\omega(x) \subset Y$ para todo $x \in Y$, o que nos leva a uma contradição pois $Y \subset M=\omega(x)$. Logo, $M$ é minimal.

Suponhamos agora que $M$ é minimal mas existe algum $x \in M$ tal que $\omega(x) \neq M$. Como $M$ é compacto, então $\omega(x)$ é compacto e invariante. Consequentemente, $M$ possui um subconjunto não vazio, fechado e invariante $\omega(x)$, o que é uma contradição visto que $M$ é minimal. Portanto, para cada $x \in M$ temos que $M=\omega(x)$.

O interesse por conjuntos minimais deve-se ao próximo resultado que estabelece uma condição necessária e suficiente para que um conjunto compacto não vazio invariante contenha um subconjunto compacto minimal.

Teorema 3.2.4. [BS02] Cada conjunto não vazio, compacto e invariante contém um subconjunto compacto minimal.

Demonstração. Dado conjunto compacto não vazio invariante, podemos obter uma família de subconjuntos não vazios, fechados e invariantes. Observe que um subconjunto fechado de um conjunto compacto é compacto e consequentemente, todo elemento desta família é compacto. Além disso, esta família é parcialmente ordenada pela inclusão de conjuntos. Dada uma cadeia desta família de subconjuntos não vazios, fechados e invariantes, a interseção dos elementos dessa cadeia é um subconjunto não vazio, fechado e invariante e pertence a cadeia. Logo, pelo Lema de Zorn, ver [Kel75], existe um subconjunto compacto invariante não vazio que não contém subconjuntos próprios que também são não vazios, fechados e invariantes. Portanto, tal subconjunto é não vazio, compacto e invariante e consequentemente, minimal.

\subsection{Região de Estabilidade}

Em sistemas dinâmicos não lineares é muito raro um ponto de equilíbrio assintoticamente estável ser globalmente assintoticamente estável. Diante disso, conhecer a região cujas trajetórias que iniciam em algum ponto dela se aproximam de um ponto de equilíbrio assintoticamente estável quando o tempo tende ao infinito permite uma análise do comportamento dinâmico do sistema. Sendo assim, definiremos o conceito de região de estabilidade. 
Nesta seção, revisaremos algumas das propriedades topológicas da região de estabilidade e de sua fronteira que serão de extrema importância para a caracterização da fronteira da região de estabilidade na presença de um ponto de equilíbrio não-hiperbólico do tipo Hopf. Por exemplo, exploraremos as propriedades de que a região de estabilidade é um conjunto aberto e invariante e que a sua fronteira também é invariante. As referências que utilizamos nesta seção são [BS02], [CHW88] e [Ama10].

Seja $M \subset \mathbb{R}^{n}$ um conjunto não vazio, compacto e invariante em relação ao sistema (2.1). Definimos os conjuntos

$$
\begin{gathered}
A_{\omega}(M)=\left\{x \in \mathbb{R}^{n}: \omega(x) \cap M \neq \emptyset\right\}, A(M)=\left\{x \in \mathbb{R}^{n}: \omega(x) \neq \emptyset \text { e } \omega(x) \subset M\right\} \\
\text { е } A_{u}(M)=\left\{x \in \mathbb{R}^{n}: \Lambda^{+}(x) \neq \emptyset \text { е } \Lambda^{+}(x) \subset M\right\}
\end{gathered}
$$

sendo respectivamente chamados de Região de Atração Fraca, Região de Atração e Região de Atração Uniforme do subconjunto $M$. Além disso, qualquer ponto x pertencente ao conjunto $A_{\omega}(M), A(M)$ ou $A_{u}(M)$ pode, respectivamente, ser chamado de fracamente atraído, atraído ou uniformemente atraído por $M$.

A proposição abaixo fornece condições para determinar quando um ponto é fracamente atraído, atraído ou uniformemente atraído por um conjunto.

Proposição 3.3.1. [BS02] Dado um subconjunto $M \subset \mathbb{R}^{n}$ não vazio, compacto e invariante em relação ao sistema (2.1), um ponto $x \in \mathbb{R}^{n}$ é

(i) fracamente atraído por $M$ se, e somente se, existe uma sequência $\left\{t_{n}\right\}$ em $\mathbb{R}$ com $_{n} \rightarrow+\infty$ e d $\left(\varphi_{t_{n}}(x), M\right) \rightarrow 0$,

(ii) atraído por $M$ se, e somente se, $d\left(\varphi_{t}(x), M\right) \rightarrow 0$ quando $t \rightarrow+\infty$,

(iii) uniformemente atraído por $M$ se, e somente se, para cada vizinhança $V$ de $M$ existem uma vizinhança $U$ de $x$ e um $T>0 \operatorname{com} \varphi_{t}(U) \subset V$ para $t \geq T$.

Demonstração. (i) Seja $x \in A_{\omega}(M)$. Então pela definição do conjunto $A_{\omega}(M)$, temos que $\omega(x) \cap M \neq \emptyset$. Seja $y \in \omega(x) \cap M$. Como $y \in \omega(x)$, então existe uma sequência $\left\{t_{n}\right\}$ em $\mathbb{R} \operatorname{com} t_{n} \rightarrow+\infty$ e $\varphi_{t_{n}}(x) \rightarrow y$ quando $n \rightarrow+\infty$. Ou ainda, existe uma sequência $\left\{t_{n}\right\}$ em $\mathbb{R}$ com $t_{n} \rightarrow+\infty$ e $d\left(\varphi_{t_{n}}(x), y\right) \rightarrow 0$ quando $n \rightarrow+\infty$. Como $d\left(\varphi_{t_{n}}(x), M\right)=\inf _{z \in M} d\left(\varphi_{t_{n}}(x), z\right)$ e $d\left(\varphi_{t_{n}}(x), z\right) \geq$ 0 , para todo $z \in M$, então $d\left(\varphi_{t_{n}}(x), M\right) \rightarrow 0$. Logo, existe uma sequência $\left\{t_{n}\right\}$ em $\mathbb{R}, t_{n} \rightarrow+\infty$ e $d\left(\varphi_{t_{n}}(x), M\right) \rightarrow 0$.

Reciprocamente, dado $x \in \mathbb{R}^{n}$ suponhamos que exista uma sequência $\left\{t_{n}\right\}$ em $\mathbb{R}$ com $t_{n} \rightarrow+\infty$ e $d\left(\varphi_{t_{n}}(x), M\right) \rightarrow 0$. Então para dado $\varepsilon>0$, existe um $k>0$ tal que $d\left(\varphi_{t_{n}}(x), M\right) \leq \varepsilon$ para todo $t_{n} \geq k$. Como $M$ é compacto e considerando a aplicação $y \mapsto d\left(\varphi_{t_{n}}(x), y\right)$, para todo $y \in M$, então pelo Teorema de Weierstrass, ver [Lim03, p. 215], temos que existe $y_{0} \in M$ tal que $d\left(\varphi_{t_{n}}(x), M\right)=d\left(\varphi_{t_{n}}(x), y_{0}\right)$. Então $d\left(\varphi_{t_{n}}(x), y_{0}\right) \leq \varepsilon$ para 
todo $t_{n} \geq k$. Logo, $y_{0} \in \omega(x)$ e consequentemente, $\omega(x) \cap M \neq \emptyset$. Portanto, $x \in A_{\omega}(M)$.

(ii) Seja $x \in A(M)$. Então temos que $\omega(x) \neq \emptyset$ e $\omega(x) \subset M$. Como a solução $\varphi(t, x)$ é contínua no conjunto compacto $M$, então $\varphi(t, x)$ é limitada em $M$. Então, pelo Teorema 2.2.8, $d\left(\varphi\left(t, x_{0}\right), \omega\left(x_{0}\right)\right) \rightarrow 0$ quando $t \rightarrow+\infty$. Consequentemente, $d\left(\varphi_{t}(x), M\right) \rightarrow 0$ quando $t \rightarrow+\infty$, pois $\omega(x) \subset M$.

Reciprocamente, dado $x \in \mathbb{R}^{n}$, suponhamos que $d\left(\varphi_{t}(x), M\right) \rightarrow 0$ quando $t \rightarrow+\infty$. Então para todo $\varepsilon>0$, existe um inteiro positivo $K$ tal que $d\left(\varphi_{t}(x), M\right) \leq \varepsilon$ para todo $t \geq K$. Tomando $\varepsilon_{1}=1$, existe um inteiro $t_{1}>K$ tal que $d\left(\varphi_{t_{1}}(x), M\right) \leq 1$. Seja $\varepsilon_{2}=\max \left\{d\left(\varphi_{t_{1}}(x), M\right), \frac{1}{2}\right\}$, existe um inteiro $t_{2}>t_{1}$ tal que $d\left(\varphi_{t_{2}}(x), M\right) \leq \varepsilon_{2}$. Tomando $\varepsilon_{3}=\max \left\{d\left(\varphi_{t_{2}}(x), M\right), \frac{1}{3}\right\}$, existe um inteiro $t_{3}>t_{2}$ tal que $d\left(\varphi_{t_{3}}(x), M\right) \leq \varepsilon_{3}$. E assim sucessivamente, obtemos uma sequência $\left\{t_{n}\right\}$ em $\mathbb{R}$, com $t_{n} \rightarrow+\infty$ e $d\left(\varphi_{t_{n}}(x), M\right) \rightarrow 0$. Consequentemente, existe $y \in M$ tal que $d\left(\varphi_{t_{n}}(x), y\right) \rightarrow 0$. Logo, $y \in \omega(x)$ e portanto, $\omega(x) \neq \emptyset$. Seja $z \in \omega(x)$. Então existe uma sequência $\left\{t_{n}\right\}$ em $\mathbb{R}$ com $t_{n} \rightarrow+\infty$ e $\varphi_{t_{n}}(x) \rightarrow z$ quando $n \rightarrow+\infty$. Ou ainda, para todo $\varepsilon>0$, existem inteiros $n_{0}>0$ e $\tau_{0}>0$ tal que $d\left(\varphi_{t_{n}}(x), z\right) \leq \frac{\varepsilon}{2}$ e $t_{n} \geq \tau_{0}$ para todo $n \geq n_{0}$. Além disso, existem inteiros $n_{1}>0$ e $\tau_{1}>0$ tal que $d\left(\varphi_{t_{n}}(x), M\right) \leq \frac{\varepsilon}{2}$ e $t_{n} \geq \tau_{1}$ para todo $n \geq n_{1}$. Sejam $\bar{n}=\max \left\{n_{0}, n_{1}\right\}$ e $\bar{\tau}=\max \left\{\tau_{0}, \tau_{1}\right\}$. Então

$$
\begin{aligned}
d(z, M) & \leq d\left(z, \varphi_{t_{n}}(x)\right)+d\left(\varphi_{t_{n}}(x), M\right) \\
& <\frac{\varepsilon}{2}+\frac{\varepsilon}{2} \\
& =\varepsilon .
\end{aligned}
$$

Como $\varepsilon$ pode ser escolhido arbitrariamente pequeno, então $d(z, M)=0$. Como $M$ é compacto, então existe $a \in M$ tal que $d(z, a)=0$. Consequentemente, $z=a \in M$ e portanto, $\omega(x) \subset M$. Logo, $x \in A(M)$.

(iii) Seja $x \in A_{u}(M)$. Pela definição do conjunto $A_{u}(M)$, temos que $\Lambda^{+}(x) \neq \emptyset$ e $\Lambda^{+}(x) \subset M$. Segue do Lema 2.2.10 que $\omega(x) \neq \emptyset$ e $\omega(x) \subset$ $M$. Suponhamos, por contradição, que exista uma vizinhança $V=\{z \in$ $\left.\mathbb{R}^{n}: d(z, M)<\varepsilon\right\}$ de $M$ de raio $\varepsilon>0$ tal que para toda vizinhança $U$ de $x$ e $T>0$ existe um $t>T$ tal que $\varphi_{t}(U) \not \subset V$. Pelo item (ii), temos que $d\left(\varphi_{t}(x), M\right) \rightarrow 0$ quando $t \rightarrow+\infty$. Ou seja, existe um $t_{0} \geq$ 0 tal que $\varphi_{t}(x) \in V$ para $t>t_{0}$. Assim, existem sequências $\left\{t_{n}\right\},\left\{\tau_{n}\right\}$ em $\mathbb{R}, t_{n}<\tau_{n}, t_{n} \rightarrow+\infty$ e uma sequência $\left\{x_{n}\right\}$ em $\mathbb{R}^{n}, x_{n} \rightarrow x$, tal que $\varphi_{t_{n}}\left(x_{n}\right) \in V$, mas $\varphi_{\tau_{n}}\left(x_{n}\right) \in H(M ; \varepsilon)=\left\{z \in \mathbb{R}^{n}: d(z, M)=\varepsilon\right\}$. Como $H(M ; \varepsilon)$ é compacto, pois $\mathbb{R}^{n}$ é localmente compacto, concluímos que existe uma sequência $\left\{x_{n}\right\}, x_{n} \rightarrow x$, e uma sequência $\left\{\tau_{n}\right\}, \tau_{n} \rightarrow+\infty$, tal que $\varphi_{\tau_{n}}\left(x_{n}\right) \rightarrow y \in H(M ; \varepsilon)$. Então $y \in \Lambda^{+}(x)$ mas $y \notin M$, o que é uma contradição pois $\Lambda^{+}(x) \subset M$. Logo, para cada vizinhança $V$ de $M$ existem uma vizinhança $U$ de $x$ e um $T>0$ com $\varphi_{t}(U) \subset V$ para $t \geq T$. Reciprocamente, suponhamos que para cada vizinhança $V$ de $M$ existem 
uma vizinhança $U$ de $x$ e um $T>0$ com $\varphi_{t}(U) \subset V$ para $t \geq T$. Então temos que $\overline{\varphi_{t}(U)} \subset \bar{V}$. Daí, temos que $\varphi_{t}(x) \in \bar{V}$ para todo $t \geq T$. Uma vez que possamos tomar $\bar{V}$ compacto, temos que $\omega(x) \neq \emptyset$. Consequentemente, $\Lambda^{+}(x) \neq \emptyset$. Observemos que $\Lambda^{+}(x) \subset \overline{\varphi_{[t,+\infty)}(U)}$ para cada vizinhança $U$ de $x$ e cada $t \in \mathbb{R}^{+}$. Por isso, $\Lambda^{+}(x) \subset \bar{V}$ para cada vizinhança $V$ de $M$. Deste modo, $\Lambda^{+}(x) \subset \cap\{\bar{V}: V$ é uma vizinhança de $M\}=M$. Portanto, $x \in A_{u}(M)$.

Como consequência do item (ii) da Proposição 3.3.1, se $M$ for um conjunto atrativo, a definição da região de atração devido a [BS02] coincide com a definição de região de atração definida em [Ama10].

No próximo teorema exibiremos as relações de inclusões entre as regiões de estabilidades $A_{\omega}(M), A(M), A_{u}(M)$ e além disso, veremos a invariância desses conjuntos. Mas primeiramente vejamos o seguinte lema.

Lema 3.3.2. [BS02] Seja $M \subset \mathbb{R}^{n}$ um espaço métrico e $x \in M$. Então

(i) $\omega(x)=\varphi_{t}(\omega(x))=\omega\left(\varphi_{t}(x)\right)$ para cada $t \in \mathbb{R}$,

(ii) $\Lambda^{+}(x)=\varphi_{t}\left(\Lambda^{+}(x)\right)=\Lambda^{+}\left(\varphi_{t}(x)\right)$ para cada $t \in \mathbb{R}$.

Demonstração. (i) Inicialmente vamos mostrar a primeira igualdade, consideremos $y \in \omega(x)$. Então existe uma sequência $\left\{t_{n}\right\}$ em $\mathbb{R}$ com $t_{n} \rightarrow+\infty$ e $\varphi_{t_{n}}(x) \rightarrow y$. Consideremos a sequência $\left\{t_{n}-t\right\}$ para um dado $t \in \mathbb{R}$. Obviamente que $t_{n}-t \rightarrow+\infty$ e pela continuidade das soluções do sistema (2.1), $\varphi_{t_{n}-t}(x)=\varphi_{-t} \circ \varphi_{t_{n}}(x) \rightarrow \varphi_{-t}(y)$. Logo, $\varphi_{-t}(y) \in \omega(x)$ ou ainda $y \in \varphi_{t}(\omega(x))$ para todo $t \in \mathbb{R}$. Logo, $\omega(x) \subset \varphi_{t}(\omega(x))$ para todo $t \in \mathbb{R}$.

Seja $y \in \varphi_{t}(\omega(x))$ para todo $t \in \mathbb{R}$. Seja $z \in \omega(x)$ tal que $y=\varphi_{t}(z)$. Como $z \in \omega(x)$, existe uma sequência $\left\{t_{n}\right\}$ em $\mathbb{R}$ com $t_{n} \rightarrow+\infty$ e $\varphi_{t_{n}}(x) \rightarrow z$. Consideremos a sequência $\left\{t_{n}+t\right\}$ em $\mathbb{R}$. Obviamente que $t_{n}+t \rightarrow+\infty$ e $\varphi_{t_{n}+t}(x)=\varphi_{t} \circ \varphi_{t_{n}}(x) \rightarrow \varphi_{t}(z)=y$. Logo, $y \in \omega(x)$. Logo, $\varphi_{t}(\omega(x)) \subset \omega(x)$ para todo $t \in \mathbb{R}$. Deste modo, $\omega(x)=\varphi_{t}(\omega(x))$ para cada $t \in \mathbb{R}$.

Mostraremos agora a segunda igualdade, isto é, $\varphi_{t}(\omega(x))=\omega\left(\varphi_{t}(x)\right)$ para cada $t \in \mathbb{R}$. Seja $y \in \varphi_{t}(\omega(x))$. Seja $z \in \omega(x)$ tal que $y=\varphi_{t}(z)$. Como $z \in \omega(x)$, existe uma sequência $\left\{t_{n}\right\}$ em $\mathbb{R} \operatorname{com} t_{n} \rightarrow+\infty$ e $\varphi_{t_{n}}(x) \rightarrow$ $z$. Então pela continuidade das soluções do sistema dinâmico (2.1) temos $\varphi_{t_{n}} \circ \varphi_{t}(x)=\varphi_{t} \circ \varphi_{t_{n}}(x) \rightarrow \varphi_{t}(z)=y$. Logo, $y \in \omega\left(\varphi_{t}(x)\right)$. Logo, $\varphi_{t}(\omega(x)) \subset$ $\omega\left(\varphi_{t}(x)\right)$ para todo $t \in \mathbb{R}$.

Seja $y \in \omega\left(\varphi_{t}(x)\right)$. Então existe uma sequência $\left\{t_{n}\right\}$ em $\mathbb{R}$ com $t_{n} \rightarrow$ $+\infty$ e $\varphi_{t_{n}}\left(\varphi_{t}(x)\right) \rightarrow y$. Consideremos a sequência $\left\{t_{n}-t\right\}$. Obviamente que $t_{n}-t \rightarrow+\infty$. Pela continuidade das soluções temos que $\varphi_{-t}\left(\varphi_{t_{n}}\left(\varphi_{t}(x)\right)\right) \rightarrow$ $\varphi_{-t}(y)$. Por outro lado, tem-se que $\varphi_{-t}\left(\varphi_{t_{n}}\left(\varphi_{t}(x)\right)\right)=\varphi_{-t}\left(\varphi_{t}\left(\varphi_{t_{n}}(x)\right)\right)=$ $\varphi_{-t+t}\left(\varphi_{t_{n}}(x)\right)=\varphi_{t_{n}}(x)$. Logo, $\varphi_{t_{n}}(x) \rightarrow \varphi_{-t}(y)$. Sendo assim, $\varphi_{-t}(y) \in$ $\omega(x)$ ou ainda $y \in \varphi_{t}(\omega(x))$ para todo $t \in \mathbb{R}$. Logo, $\omega\left(\varphi_{t}(x)\right) \subset \varphi_{t}(\omega(x))$ para todo $t \in \mathbb{R}$. Consequentemente, $\varphi_{t}(\omega(x))=\omega\left(\varphi_{t}(x)\right)$ para cada $t \in \mathbb{R}$. 
(ii) A demonstração para o conjunto $\Lambda^{+}(x)$ é inteiramente análoga ao item (i).

Os resultados a seguir estabelecem algumas propriedades topológicas da região de estabilidade e de sua fronteira importantes para a caracterização da fronteira da região de estabilidade, como por exemplo, a invariância da fronteira e da região de estabilidade como também a propriedade de que a região de estabilidade é um conjunto aberto.

Teorema 3.3.3. [BS02] Dado $M \subset \mathbb{R}^{n}$ um subconjunto não vazio compacto e invariante de (2.1),

(i) $A_{\omega}(M) \supset A(M) \supset A_{u}(M)$,

(ii) os conjuntos $A_{\omega}(M), A(M)$ e $A_{u}(M)$ são invariantes.

Demonstração. (i) Seja $x \in A_{u}(M)$. Pela definição do conjunto $A_{u}(M)$, temos que $\Lambda^{+}(x) \neq \emptyset$ e $\Lambda^{+}(x) \subset M$. Então $\omega(x) \neq \emptyset$ e $\omega(x) \subset M$, segue do Lema 2.2.10. Logo, $x \in A(M)$.

Seja $x \in A(M)$. Então $\omega(x) \neq \emptyset$ e $\omega(x) \subset M$. Sendo assim, temos que $\omega(x) \cap M=\omega(x) \neq \emptyset$. Logo, $x \in A_{\omega}(M)$.

(ii) A invariância dos conjuntos $A_{\omega}(M), A(M)$ e $A_{u}(M)$ segue do Lema 3.3.2.

Como uma consequência do Teorema 3.3 .3 e também pelo fato de que o fecho de todo conjunto invariante é invariante obtemos a seguinte propriedade.

Corolário 3.3.4. [Ama10] Seja $M \subset \mathbb{R}^{n}$ um subconjunto não vazio compacto e invariante de (2.1). Então $\overline{A(M)}$ é um conjunto invariante.

O Corolário 3.3.4 continua válido se substituirmos $A(M)$ por $A_{\omega}(M)$ ou $A_{u}(M)$.

Teorema 3.3.5. [Ama10] Sejam $H, K \subset \mathbb{R}^{n}$ compactos invariantes de (2.1) tais que $K \subset H$. Então $A(K) \subset A(H)$.

Demonstração. Seja $x_{0} \in A(K)$. Então $d\left(\varphi\left(t, x_{0}\right), K\right) \rightarrow 0$ quando $t \rightarrow+\infty$, ou seja, dado $\varepsilon>0$ existe $T>0$ tal que $d\left(\varphi\left(t, x_{0}\right), K\right)<\varepsilon$ para todo $t>T$. Como $d\left(\varphi\left(t, x_{0}\right), H\right) \leq d\left(\varphi\left(t, x_{0}\right), K\right)$, temos que $d\left(\varphi\left(t, x_{0}\right), H\right) \leq$ $d\left(\varphi\left(t, x_{0}\right), K\right)<\varepsilon$ para todo $t>T$, isto é, $d\left(\varphi\left(t, x_{0}\right), H\right) \rightarrow 0$ quando $t \rightarrow+\infty$ e consequentemente, $x_{0} \in A(H)$.

Teorema 3.3.6. [Ama10] Sejam $H, K \subset \mathbb{R}^{n}$ subconjuntos não vazios, compactos e invariantes de (2.1). Então $A(H \cup K)=A(H) \cup A(K)$. 
Demonstração. Mostraremos primeiramente que $A(H \cup K) \subset A(H) \cup A(K)$. De fato, se $x_{0} \in A(H \cup K)$ então $d\left(\varphi\left(t, x_{0}\right), H \cup K\right) \rightarrow 0$ quando $t \rightarrow+\infty$. Então pelo Teorema 2.1.5 existem dois conjuntos abertos e disjuntos $U$ e $V$ tais que $H \backslash K \subset U$ e $K \subset V$. Podemos afirmar que existe $T>0$ tal que $\varphi\left(T, x_{0}\right) \in U \cup V$, pois $d\left(\varphi\left(t, x_{0}\right), H \cup K\right) \rightarrow 0$ quando $t \rightarrow+\infty$. Logo, $p=\varphi\left(T, x_{0}\right) \in U$ ou $p=\varphi\left(T, x_{0}\right) \in V$. Sem perda de generalidade podemos supor que $p=\varphi\left(T, x_{0}\right) \in U$, ou seja, $d(\varphi(s, p), H) \rightarrow 0$ quando $s \rightarrow+\infty$. Tomando $s=t-T$ temos que $s \rightarrow+\infty$ quando $t \rightarrow+\infty$. Portanto, usando a propriedade que $\varphi\left(t_{1}+t_{2}, x\right)=\varphi\left(t_{1}, \varphi\left(t_{2}, x\right)\right)$ para todo $x \in \mathbb{R}^{n}$ e $t_{1}, t_{2} \in \mathbb{R}$ temos que, $d\left(\varphi\left(t, x_{0}\right), H\right)=d\left(\varphi\left(s+T, x_{0}\right), H\right)=d\left(\varphi\left(s, \varphi\left(T, x_{0}\right)\right), H\right)=$ $d(\varphi(s, p), H) \rightarrow 0$ quando $t \rightarrow+\infty$, ou seja, $x_{0} \in A(H)$ e consequentemente $A(H \cup K) \subset A(H) \cup A(K)$. A inclusão $A(H) \cup A(K) \subset A(H \cup K)$ segue do Teorema 3.3.5.

Como consequência imediata do Teorema 3.3.6 e por técnica de indução, mostramos que a região de atração de uma união finita de conjuntos compactos invariantes é a união finita das regiões de atração dos conjuntos compactos invariantes.

Teorema 3.3.7. [Ama10] Sejam $H$ e $K$ compactos invariantes de (2.1) tal que $H \cap K=\emptyset$. Então $A(H) \cap A(K)=\emptyset$.

Demonstração. Como $H$ e $K$ são conjuntos disjuntos e fechados, então pelo Teorema 2.1.5 existem abertos disjuntos $U$ e $V$ contendo $H$ e $K$, respectivamente. Seja $x_{0} \in A(H)$. Então $d\left(\varphi\left(t, x_{0}\right), H\right) \rightarrow 0$ quando $t \rightarrow+\infty$. Como $H \subset U$, existe $T \in \mathbb{R}$ tal que $\varphi\left(t, x_{0}\right) \in U$ para todo $t>T$. Sabendo que $U \cap V=\emptyset$, podemos afirmar que $\varphi\left(t, x_{0}\right) \notin V$ para todo $t>T$, ou seja, $\varphi\left(t, x_{0}\right)$ não se aproxima de $K$ quando $t \rightarrow+\infty$ e consequentemente $x_{0} \in A(K)$, o que mostra que $A(H) \cap A(K)=\emptyset$.

Apresentaremos a seguir algumas definições de atrator e estabilidade para um subconjunto do $\mathbb{R}^{n}$.

Seja $M \subset \mathbb{R}^{n}$ um subconjunto não vazio compacto e invariante em relação ao sistema (2.1). Dizemos que $M$ é um atrator fraco, atrator ou atrator uniforme se $A_{\omega}(M), A(M)$ ou $A_{u}(M)$ é uma vizinhança de $M$, respectivamente. O conjunto $M$ é um atrator minimal, se nenhum subconjunto $X \subset M$ é atrator.

O conjunto $M$ é estável se cada vizinhança $U$ de $M$ contém uma vizinhança positivamente invariante $V$ de $M$. Diz-se que $M$ é assintoticamente estável se $M$ é estável e também um atrator. Dizemos que $M$ é instável se não é estável.

Vale salientar que um atrator fraco pode ser chamado de atrator fraco global sempre que $A_{\omega}(M)=\mathbb{R}^{n}$. Analogamente, para conjuntos atratores ou atratores uniformes, o termo global é usado para indicar que a região de estabilidade correspondente é todo o espaço. 
Vejamos alguns exemplos, retirados de [BS02], que ilustram as definições acima.

Exemplo 3.3.1. Consideremos o sistema diferencial em $\mathbb{R}^{2}$

$$
\left\{\begin{array}{l}
\dot{x}=y+x\left(1-x^{2}-y^{2}\right) \\
\dot{y}=-x+y\left(1-x^{2}-y^{2}\right)
\end{array}, \operatorname{com}(x, y) \in \mathbb{R}^{2}\right.
$$

O sistema possui um ciclo limite, de raio 1, estável e um ponto de equilíbrio instável que é a origem. Consideremos o conjunto $M=\{x\}$, com $x$ pertencente ao ciclo limite. Então $M$ é apenas um atrator fraco, veja Figura 3.1. Da Figura 3.1, considerando $M$ apenas o ciclo limite unitário, então $M$

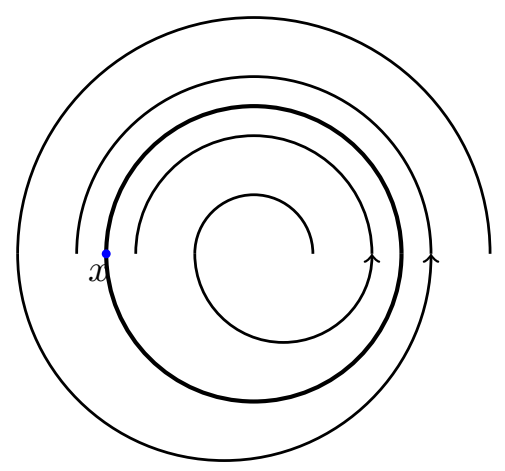

Figura 3.1: Atrator Fraco.

será assintoticamente estável e também atrator uniforme. Se considerarmos $M$ como sendo o ciclo limite unitário e contendo qualquer ponto diferente da origem e não pertecente ao ciclo, então $M$ ainda continua sendo atrator uniforme, porém não estável.

Exemplo 3.3.2. Consideremos o sistema de equações diferenciais, em coordenadas polares,

$$
\left\{\begin{array}{l}
\dot{r}=r(1-r), \\
\dot{\theta}=\sin ^{2}\left(\frac{\theta}{2}\right) .
\end{array}\right.
$$

A Figura 3.2 mostra o retrato de fase de um sistema com dois equilibrios $p_{1}=(0,0)$ e $p_{2}=(1,0)$. Observemos que qualquer que seja $p$, com $p \neq p_{1}$, temos $\omega(p)=\left\{p_{2}\right\}$ e, portanto, $\left\{p_{2}\right\}$ é um atrator. Observemos também que $\left\{p_{2}\right\}$ não é atrator uniforme, pois para qualquer $p=(\alpha, 0), \alpha>0$, temos que $\Lambda^{+}(p)$ é o círculo unitário.

Exemplo 3.3.3. A Figura 3.3 descreve o retrato de fase de um sistema possuindo um atrator global, no caso a origem, no plano, que não é assintoticamente estável.

Teorema 3.3.8. Se $M \subset \mathbb{R}^{n}$ é assintoticamente estável, então $M$ é conexo. 


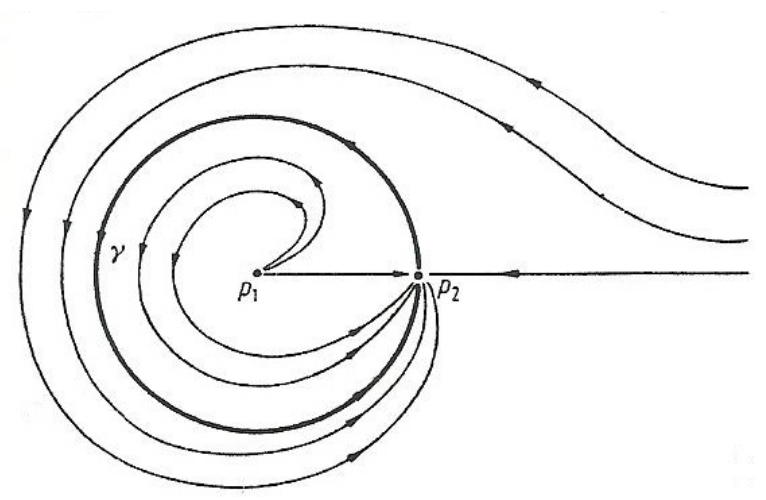

Figura 3.2: Trajetórias do sistema $\dot{r}=r(1-r), \dot{\theta}=\sin ^{2}\left(\frac{\theta}{2}\right)$. Fonte: [ver BS02, p. 59]

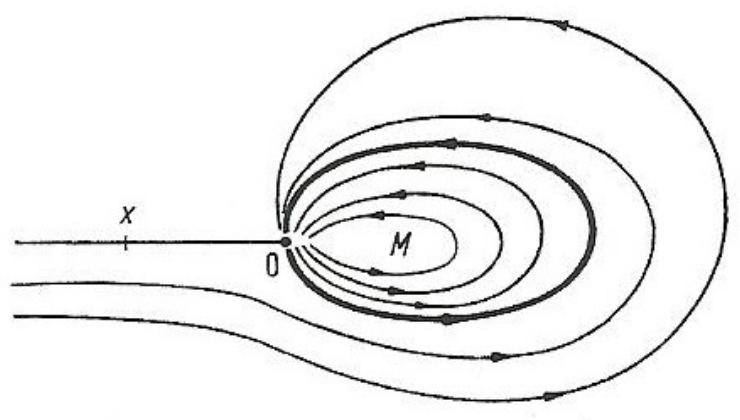

Figura 3.3: Atrator Global. Fonte: [ver BS02, p. 59]

Demonstração. Suponhamos que $M$ seja desconexo. Então $M$ pode ser escrito como sendo a união de dois conjuntos disjuntos, fechados e não vazios. Sejam $A$ e $B$ estes conjuntos. Como eles são disjuntos, então pelo Teorema 2.1.5 existem dois conjuntos abertos e disjuntos $U_{1}$ e $U_{2}$ tal que $A \subset U_{1}$ e $B \subset U_{2}$. Seja $x \in M$. Como $M$ é assintoticamente estável e portanto, estável e atrator então, pelo item (ii) da Proposição 3.3.1, $d(\varphi(t, x), M) \rightarrow 0$ quando $t \rightarrow+\infty$. Então existe $T>0$ tal que $\varphi(t, x) \in U_{1} \cup U_{2}$ para todo $t>T$. Como a aplicacao $t \rightarrow \varphi(t, x)$ é contínua, $U_{1}$ e $U_{2}$ são conjuntos abertos disjuntos e pela invariância de $M$, conclui-se que $\varphi(t, x)$ pertence apenas a um dos abertos para todo $t>T$. Suponha sem perda de generalidade que $\varphi(t, x) \in U_{1}$ para todo $t>T$. Então, necessariamente $B$ é um conjunto vazio. Isto nos leva a uma contradição e, portanto, o conjunto $M$ é conexo.

Com o propósito de caracterizarmos a fronteira da região de estabilidade, vejamos algumas propriedades relacionadas à fronteira da região que serão de suma importância para os capítulos posteriores. 
Teorema 3.3.9. [Ama10] Seja $H$ um conjunto compacto invariante de (2.1). Então a fronteira $\partial A(H)$ da região de estabilidade é um conjunto fechado e invariante.

Demonstração. Por definição, a fronteira de qualquer conjunto é um conjunto fechado. Logo, $\partial A(H)$ é um conjunto fechado. Como a região de estabilidade é um conjunto invariante, então, pelo Teorema 2.2.5, temos que $\partial A(H)$ é um conjunto invariante.

O Teorema 3.3.9 continua válido se substituirmos $A(H)$ por $A_{\omega}(H)$ ou $A_{u}(H)$.

O próximo teorema apresenta uma propriedade fundamental da região de estabilidade.

Teorema 3.3.10. [BS02] Se $M$ é um atrator fraco, atrator ou atrator uniforme, então os conjuntos $A_{\omega}(M), A(M)$ ou $A_{u}(M)$, respectivamente, são abertos.

Demonstração. Seja $M$ um atrator uniforme. Então, pela definição de atrator uniforme e pelo Teorema 3.3.3, $A_{u}(M)$ é uma vizinhança invariante de $M$. Consequentemente, $\partial A_{u}(M)$ também é invariante e disjunto de $M$ e além disso, é fechado. Para mostrarmos que $A_{u}(M)$ é um conjunto aberto, basta provarmos que todo ponto de $A_{u}(M)$ é ponto interior, ou seja, que $A_{u}(M)$ não contém nenhum ponto pertencente a $\partial A_{u}(M)$. Seja $x \in A_{u}(M)$. Pela definição do conjunto $A_{u}(M)$, temos que $\Lambda^{+}(x) \neq \emptyset$ e $\Lambda^{+}(x) \subset M$, para todo $x \in A_{u}(M)$. Consequentemente, pelo Lema 2.2.10, $\omega(x) \neq \emptyset$ e $\omega(x) \subset M$, a inclusão se deve ao fato de que $\omega(x) \subset \Lambda^{+}(x)$. Segue-se $\omega(x) \cap M \neq \emptyset$. Entretanto, se $x \in \partial A_{u}(M)$, então $\omega(x) \subset \partial A_{u}(M)$. De fato, seja $y \in \omega(x)$. Então existe sequência $\left\{t_{n}\right\}$, com $t_{n} \rightarrow+\infty$, tal que $\varphi_{t_{n}}(x) \rightarrow y$. Como $\partial A_{u}(M)$ é invariante, então $\varphi_{t_{n}}(x) \in \partial A_{u}(M)$, para todo $t_{n}$. Além disso, como $\partial A_{u}(M)$ é fechado, então $y \in \partial A_{u}(M)$. Como $\partial A_{u}(M) \cap M=\emptyset$, concluímos que $\partial A_{u}(M) \cap A_{u}(M)=\emptyset$. Logo, $A_{u}(M)$ é aberto.

A prova de que os conjuntos $A_{\omega}(M)$ e $A(M)$ também são abertos é inteiramente análoga a demonstração acima.

Definimos que para um conjunto ser assintoticamente estável além da propriedade de ser um conjunto estável, tem também que ser um atrator segundo [BS02]. Veremos nos próximos resultados que a combinação do conjunto ser estável com o fato de ser um atrator fraco ou ser atrator uniforme não proporciona novos conceitos.

Lema 3.3.11. [BS02] Seja $x \in \mathbb{R}^{n}$ e $y \in \omega(x)$. Então $\Lambda^{+}(x) \subset \Lambda^{+}(y)$.

Demonstração. Dado $y \in \omega(x)$ e seja $z \in \Lambda^{+}(x)$. Como $y \in \omega(x)$, então existe sequência $\left\{\tau_{n}\right\}, \tau_{n} \rightarrow+\infty, \varphi_{\tau_{n}}(x) \rightarrow y$. E como $z \in \Lambda^{+}(x)$, então existem sequências $\left\{t_{n}\right\}$ e $\left\{x_{n}\right\}$, com $x_{n} \rightarrow x, t_{n} \rightarrow+\infty$ e $\varphi_{t_{n}}\left(x_{n}\right) \rightarrow z$. 
Podemos, se necessário, escolher subsequências $\left\{t_{n}\right\}$ e $\left\{\tau_{n}\right\}$ tal que $t_{n}$ $\tau_{n}>n$ para cada $n$. Considere para cada $k$ fixado, com $k=1,2, \ldots$, a sequência $\left\{\varphi_{\tau_{k}}\left(x_{n}\right)\right\}$. Pela continuidade das soluções do sistema dinâmico autonômo (2.1), $\varphi_{\tau_{k}}\left(x_{n}\right) \rightarrow \varphi_{\tau_{k}}(x)$, para cada $k=1,2, \ldots$ quando $n \rightarrow+\infty$. Podemos, portanto, assumir sem perda de generalidade que para cada $k$ fixado, $d\left(\varphi_{\tau_{k}}\left(x_{n}\right), \varphi_{\tau_{k}}(x)\right) \leq \frac{1}{k}$ para $n \geq k$. Observemos que

$$
\begin{aligned}
d\left(y, \varphi_{\tau_{n}}\left(x_{n}\right)\right) & \leq d\left(y, \varphi_{\tau_{n}}(x)\right)+d\left(\varphi_{\tau_{n}}(x), \varphi_{\tau_{n}}\left(x_{n}\right)\right) \\
& \leq d\left(y, \varphi_{\tau_{n}}(x)\right)+\frac{1}{k} .
\end{aligned}
$$

Logo, $\varphi_{\tau_{n}}\left(x_{n}\right) \rightarrow y$, pois $\varphi_{\tau_{n}}(x) \rightarrow y$. Observemos também que $\varphi_{t_{n}}\left(x_{n}\right)=$ $\varphi_{\tau_{n}}\left(\varphi_{t_{n}-\tau_{n}}\left(x_{n}\right)\right)$. Tomemos $y_{n}=\varphi_{t_{n}-\tau_{n}}\left(x_{n}\right)$. Como $t_{n}-\tau_{n}>n$, para cada $n$, então $y_{n} \rightarrow y$. Como $\varphi_{t_{n}}\left(x_{n}\right) \rightarrow z$ então $\varphi_{\tau_{n}}\left(y_{n}\right)=\varphi_{t_{n}}\left(x_{n}\right) \rightarrow z$. Consequentemente, $z \in \Lambda^{+}(y)$. Logo, $\Lambda^{+}(x) \subset \Lambda^{+}(y)$.

Lema 3.3.12. [BS02] Dado o subconjunto $M \subset \mathbb{R}^{n}$ não vazio compacto $e$ invariante em relação ao sistema (2.1) e seja $x \in A_{\omega}(M)$, então

$$
\Lambda^{+}(x) \subset \Lambda^{+}(M) \subset \Gamma^{+}(M) .
$$

Demonstração. Como $x \in A_{\omega}(M)$, então $\omega(x) \cap M \neq \emptyset$. Tomemos $y \in$ $\omega(x) \cap M$. Então pelo Lema 3.3.11 temos que $\Lambda^{+}(x) \subset \Lambda^{+}(y)$. Para cada $y \in$ $\omega(x) \cap M$, tem-se $\Lambda^{+}(x) \subset \Lambda^{+}(y)$. Sendo assim, temos $\Lambda^{+}(x) \subset \Lambda^{+}(M)=$ $\cup_{y \in M} \Lambda^{+}(y)$. Pelo item (iii) do Teorema 2.2.9 temos que $\Lambda^{+}(y) \subset \Gamma^{+}(y)$ para todo $y \in M$. Logo, $\Lambda^{+}(M) \subset \Gamma^{+}(M)$.

O próximo teorema fornece uma importante caracterização da estabilidade de um conjunto $M$.

Teorema 3.3.13. [BS02] Um subconjunto $M \subset \mathbb{R}^{n}$ não vazio compacto $e$ invariante em relação ao sistema (2.1) é estável se, e somente se, $\Gamma^{+}(M)=$ $M$.

Demonstração. Seja $\Gamma^{+}(M)=M$. Suponhamos, por contradição, que $M$ não seja estável. Então existe $\varepsilon>0$, uma sequência $\left\{x_{n}\right\}$ e uma sequência $\left\{t_{n}\right\}$, com $t_{n} \geq 0$ tal que $d\left(x_{n}, M\right) \rightarrow 0$ e $d\left(\varphi_{t_{n}}\left(x_{n}\right), M\right) \geq \varepsilon$. Podemos assumir, sem perda de generalidade, que $\varepsilon>0$ pode ser escolhido tão pequeno tal que $B[M ; \varepsilon]$, onde $B[M ; \varepsilon]=\left\{y \in \mathbb{R}^{n}: d(y, M) \leq \varepsilon\right\}$, e consequentemente, $H(M, \varepsilon)$, onde $H(M ; \varepsilon)=\left\{y \in \mathbb{R}^{n}: d(y, M)=\varepsilon\right\}$, é compacto. Isto só é possível pois $\mathbb{R}^{n}$ é localmente compacto. Além disso, podemos assumir que $x_{n} \rightarrow x \in M$. Escolhamos uma sequência $\left\{\tau_{n}\right\}, 0 \leq \tau_{n} \leq t_{n}$, tal que $\varphi_{\tau_{n}}\left(x_{n}\right) \in H(M ; \varepsilon), n=1,2, \ldots$ Como $H(M ; \varepsilon)$ é compacto, podemos supor que $\varphi_{\tau_{n}}\left(x_{n}\right) \rightarrow y \in H(M, \varepsilon)$. Então obviamente $y \in \Gamma^{+}(x) \subset \Gamma^{+}(M)$, mas $y \notin M$, esta contradição mostra que $M$ é estável. Reciprocamente, suponhamos que $M$ seja estável. Dado uma vizinhança $V$ qualquer de $M$ existe 
uma vizinhança positivamente invariante $U$ de $M$ com $U \subset V$. Segue-se que para qualquer $x \in M, \Gamma^{+}(x) \subset \overline{\varphi_{\mathbb{R}^{+}}(W)}$ para qualquer vizinhança $W$ de $x$. Obtemos assim $\Gamma^{+}(x) \subset \bar{U}$ pois $U$ é positivamente invariante. Assim sendo, $\Gamma^{+}(x) \subset \bar{V}$ para qualquer vizinhança $V$ de $M$. Consequentemente, $\Gamma^{+}(x) \subset \cap\{\bar{V}: V$ é uma vizinhança de $M\}=M$ pois $M$ é compacto. Como $M \subset \Lambda^{+}(M)$, então $\Lambda^{+}(M)=M$.

Teorema 3.3.14. [BS02] Se $M$ é estável e é um atrator fraco, então $M$ é um atrator e consequentemente, assintoticamente estável.

Demonstração. Como $M$ é um atrator fraco, então $A_{\omega}(M)$ é uma vizinhança de $M$. Vamos mostrar que $A_{\omega}(M) \subset A(M)$. Seja $x \in A_{\omega}(M)$. Daí, tem-se que $\omega(x) \cap M \neq \emptyset$. Consequentemente, $\omega(x) \neq \emptyset$ e $\omega(x) \subset \Lambda^{+}(x) \subset \Gamma^{+}(M)$, onde a última inclusão segue pelo Lema 3.3.12. Como $M$ é estável, temos $\Gamma^{+}(M)=M$ e consequentemente, $\omega(x) \subset M$. Portanto, $M$ é um atrator.

Teorema 3.3.15. [BS02] Se $M$ é um subconjunto positivamente invariante e um atrator uniforme, então $M$ é estável e consequentemente, assintoticamente estável.

Demonstração. Pela definição de atrator uniforme, $M \subset A_{u}(M)$. Em particular,

$$
\begin{aligned}
\Gamma^{+}(M) & =\cup_{x \in M} \Gamma^{+}(x) \\
& =\cup_{x \in M}\left[\gamma^{+}(x) \cup \Lambda^{+}(x)\right] \\
& =\left[\cup_{x \in M} \gamma^{+}(x)\right] \cup\left[\cup_{x \in M} \Lambda^{+}(x)\right] \\
& =\varphi_{\mathbb{R}^{+}}(M) \cup \Lambda^{+}(M)=M,
\end{aligned}
$$

pois $M=\varphi_{\mathbb{R}^{+}}(M)$ e $\Lambda^{+}(M) \subset M$ visto que $M \subset A_{u}(M)$. Consequentemente pelo Teorema 3.3.13, $M$ é estável.

Teorema 3.3.16. [BS02] Se $M$ é assintoticamente estável então $M$ é um atrator uniforme.

Demonstração. Vamos mostrar que $A(M) \subset A_{u}(M)$. Seja $x \in A(M)$. Então $\omega(x) \neq \emptyset$ e $\omega(x) \subset M$. Seja $y \in \omega(x)$ e pelos Lemas 3.3.11 e 3.3.12 tem-se que $\Lambda^{+}(x) \subset \Lambda^{+}(y) \subset \Lambda^{+}(M) \subset \Gamma^{+}(M)$. Como $\Gamma^{+}(M)=M$ pois $M$ é estável, temos $\Lambda^{+}(M) \subset M$ para todo $x \in A(M)$ e, portanto, $x \in A_{u}(M)$.

Teorema 3.3.17. [BS02] Seja $p \in \mathbb{R}^{n}$ um ponto de equilíbrio assintoticamente estável. Então $A(p)$ é homeomorfa a $\mathbb{R}^{n}$.

Como consequência do Teorema 3.3.17, podemos concluir que $A(p) \backslash\{p\}$ é homeomorfa a $\mathbb{R}^{n} \backslash\{0\}$. 
Teorema 3.3.18. [BS02] Seja $M \subset \mathbb{R}^{n}$ um conjunto compacto, invariante em relação ao sistema (2.1) e também um atrator fraco. Seja $A_{\omega}(M)$ uma região de atração fraca de $M$ homeomorfa a $\mathbb{R}^{n}$. Então $M$ contém um ponto de equilíbrio.

Exemplo 3.3.4. Consideremos um sistema dinâmico no $\mathbb{R}^{n}$ com apenas dois pontos de equilibrios. Sejam $x, y$ os pontos de equilíbrios desse sistema. Pelo Teorema 3.3.17, podemos concluir que y não é assintoticamente estável com $A(\{y\})=\mathbb{R}^{n} \backslash\{x\}$, visto que $\mathbb{R}^{n} \backslash(\{x\} \cup\{y\})$ não é homeomorfa a $\mathbb{R}^{n} \backslash\{0\}$.

Teorema 3.3.19. [BS02] A região de atração de um conjunto $M$ compacto mininal e fracamente atrator não pode ser homeomorfa ao $\mathbb{R}^{n}$, ao menos que $M$ seja um ponto de equilíbrio. 


\section{4 \\ Caracterização da Fronteira da Região de Estabilidade}

Neste capítulo, faremos uma revisão da literatura sobre a teoria da região de estabilidade e de sua fronteira. Caracterizar regiões de estabilidade, ou regiões de atração, de sistemas dinâmicos não-lineares é um problema importante em muitas aplicações tanto na engenharia como nas ciências. A caracterização da região de estabilidade e também de sua fronteira tem sido estudada extensivamente nos últimos 30 anos, ver por exemplo [CHW88] e [Ama10]. Inicialmente, veremos quais são as condições necessárias e suficientes para que um ponto de equilíbrio hiperbólico pertença a fronteira da região de estabilidade e em seguida, caracterizaremos a fronteira da região de estabilidade como sendo a união das variedades estáveis dos pontos de equilíbrios hiperbólicos pertencentes à fronteira. Relaxando as condições de hiperbolicidade, exibiremos também condições necessárias e suficientes para que pontos de equilíbrio não hiperbólicos, e em particular pontos de equilíbrio sela-nó do tipo-zero, pertençam à fronteira da região de estabilidade. Uma caracterização dessa fronteira na presença de equilíbrio sela-nó do tipo-zero também será apresentada, ver [Ama10].

\subsection{Elementos Críticos na Fronteira da Região de Estabilidade}

Com o objetivo de caracterizarmos a fronteira da região de estabilidade de um ponto de equilíbrio assintoticamente estável, forneceremos condições para que um ponto de equilíbrio hiperbólico pertença à fronteira da região 
de estabilidade.

Teorema 4.1.1 (Caracterização de um ponto de equilíbrio na fronteira da região de estabilidade). [CHW88] Seja $x^{s}$ um ponto de equilíbrio assintoticamente estável do sistema dinâmico (2.1) e $A\left(x^{s}\right)$ sua correspondente região de estabilidade. Seja $\bar{x} \neq x^{s}$ um ponto de equilíbrio hiperbólico de (2.1). Então

(i) $\left(W^{u}(\bar{x}) \backslash\{\bar{x}\}\right) \cap \overline{A\left(x^{s}\right)} \neq \emptyset$ se, e somente se $\bar{x} \in \partial A\left(x^{s}\right)$.

(ii) Se $\bar{x}$ é um ponto de equilíbrio do tipo $k$, com $k<n$, então $\bar{x} \in \partial A\left(x^{s}\right)$ se, e somente se, $\left(W^{s}(\bar{x}) \backslash\{\bar{x}\}\right) \cap \partial A\left(x^{s}\right) \neq \emptyset$.

O Teorema 4.1 .1 oferece condições necessárias e suficientes para que um ponto de equilíbrio hiperbólico pertença à fronteira da região de estabilidade em termos de suas variedades estáveis e instáveis.

Generalizando o Teorema 4.1.1, o Teorema 4.1.2 oferece condições necessárias e suficientes para que uma órbita periódica pertença à fronteira da região de estabilidade.

Teorema 4.1.2 (Caracterização de uma órbita fechada na fronteira da região de estabilidade). [CHW88] Seja $x^{s}$ um ponto de equilíbrio assintoticamente estável do sistema dinâmico (2.1) e $A\left(x^{s}\right)$ sua correspondente região de estabilidade. Seja $\phi$ uma órbita fechada hiperbólica de (2.1). Então

(i) $\left(W^{u}(\phi) \backslash \phi\right) \cap \overline{A\left(x^{s}\right)} \neq \emptyset$ se, e somente se $\phi \subset \partial A\left(x^{s}\right)$.

(ii) Suponha $W^{s}(\phi) \backslash \phi \neq \emptyset$. Então $\phi \subset \partial A\left(x^{s}\right)$ se, e somente se, $\left(W^{s}(\phi) \backslash\right.$ $\phi) \cap \partial A\left(x^{s}\right) \neq \emptyset$.

Os resultados anteriores estabelecem condições suficientes e necessárias para que um ponto de equilíbrio hiperbólico instável ou uma órbita fechada hiperbólica pertençam à fronteira da região de estabilidade em função das suas variedades estável e instável. Impondo condições adicionais aos Teoremas 4.1.1 e 4.1.2 podemos refinar a caracterização de pontos de equilíbrio hiperbólicos ou órbitas periódicas hiperbólicas na fronteira da região de estabilidade.

O Teorema 4.1.1 indica que a condição $W^{u}(\bar{x}) \cap A\left(x^{s}\right) \neq \emptyset$ é uma condição suficiente para que $\bar{x}$ pertença à fronteira da região de estabilidade de $x^{s}$. A seguir estudaremos quando esta condição também é necessária.

Seja $x^{s}$ um ponto de equilíbrio assintoticamente estável do sistema dinâmico (2.1) e considere as seguintes condições sobre o sistema dinâmico (2.1):

(A1) Todos os pontos de equilíbrios em $\partial A\left(x^{s}\right)$ são hiperbólicos;

(A2) As variedades estáveis e instáveis dos pontos de equilíbrio em $\partial A\left(x^{s}\right)$ satisfazem a condição de transversalidade; 
(A3) Toda trajetória em $\partial A\left(x^{s}\right)$ se aproxima de um ponto de equilíbrio quando $t \rightarrow+\infty$.

As condições (A1) e (A2) são propriedades genéricas de sistemas dinâmicos na forma de (2.1). Isto significa que estas condições são satisfeitas para quase todo sistema dinâmico na classe de sistemas dinâmicos na forma de (2.1) e, na prática, não precisam ser verificadas. Porém, a condição (A3) não é uma propriedade genérica de sistemas dinâmicos. Entretanto, a existência de uma função energia é uma condição suficiente para garantir que a condição (A3) seja satisfeita, e, consequentemente, uma grande classe de sistemas dinâmicos satisfaz esta condição, ver [CHW88]. Uma função $V$ de classe $\mathcal{C}^{1}$ é uma função energia para o sistema (2.1) se satisfaz as seguintes propriedades:

(E1) $\dot{V}(x) \leq 0$;

(E2) Se $x_{0}$ não é um ponto de equilíbrio, então o conjunto $\left\{t \in \mathbb{R}: \dot{V}\left(\varphi\left(t, x_{0}\right)\right)\right.$ $=0\}$ tem medida de Lebesgue nula;

(E3) Se $V(\varphi(t, x))$ é limitada para $t \geq 0$ então a solução $\varphi(t, x)$ também é limitada para $t \geq 0$.

O próximo resultado oferece condições para que um ponto de equilíbrio hiperbólico pertença à fronteira da região de estabilidade admitindo as suposições (A1), (A2) e (A3).

Teorema 4.1.3 (Caracterização adicional de um Ponto de Equilíbrio na fronteira da região de estabilidade). [CHW88] Seja $x^{s}$ um ponto de equilíbrio assintoticamente estável de (2.1) e $A\left(x^{s}\right)$ sua correspondente região de estabilidade. Se as suposições (A1)-(A3) são satisfeitas, então:

(i) o ponto de equilíbrio $\bar{x} \in \partial A\left(x^{s}\right)$ se, e somente se, $W^{u}(\bar{x}) \cap A\left(x^{s}\right) \neq \emptyset$.

(ii) o ponto de equilíbrio $\bar{x} \in \partial A\left(x^{s}\right)$ se, e somente se, $W^{s}(\bar{x}) \subset \partial A\left(x^{s}\right)$.

A caracterização dos equilíbrios na fronteira da região de estabilidade oferecida pelo Teorema 4.1.3 pode ser numericamente verificada, bastando para isso verificar a existência de soluções iniciando em $W^{u}(\bar{x})$ que convergem para $x^{s}$. A verificação das condições do Teorema 4.1.2 não é simples do ponto de vista numérico.

Definimos elemento crítico do sistema dinâmico autonômo (2.1) como sendo ou uma órbita fechada ou um ponto de equilíbrio. Assim, como fizemos para ponto de equilíbrio hiperbólico, podemos refinar o Teorema 4.1.2, considerando as seguintes condições:

(B1) Todos os elementos críticos de (2.1) em $\partial A\left(x^{s}\right)$ são hiperbólicos. 
(B2) As variedades estável e instável dos elementos críticos de (2.1) na fronteira da área de atração satisfazem a condição de transversalidade.

(B3) Toda trajetória na fronteira da área de atração se aproxima de um dos elementos críticos do sistema (2.1) quando $t \rightarrow+\infty$.

Teorema 4.1.4 (Caracterização adicional de um Elemento Crítico na fronteira da região de estabilidade). [CHW88] Seja $x^{s}$ um ponto de equilíbrio assintoticamente estável de (2.1) e $A\left(x^{s}\right)$ sua correspondente região de estabilidade. Seja $r$ um elemento crítico. Se as suposições (B1)-(B3) são satisfeitas, então:

(i) o elemento crítico $r$ está em $\partial A\left(x^{s}\right)$ se, e somente se, $W^{u}(r) \cap A\left(x^{s}\right) \neq \emptyset$.

(ii) o elemento crítico $r$ está em $\partial A\left(x^{s}\right)$ se, e somente se, $W^{s}(r) \subset \partial A\left(x^{s}\right)$.

Os Teoremas 4.1.1 a 4.1.4 oferecem caracterizações locais da fronteira da região de estabilidade nas vizinhanças de elementos críticos.

Os dois teoremas a seguir exibem a caracterização global da fronteira da região de estabilidade de um ponto de equilíbrio assintoticamente estável, admitindo ou as condições (A1)-(A3) ou as condições (B1)-(B3), como sendo a união das variedades estáveis dos pontos de equilíbrio hiperbólicos ou elementos críticos hiperbólicos, respectivamente, que pertençam à fronteira da região de estabilidade.

Teorema 4.1.5 (Caracterização da fronteira da região de estabilidade). [CHW88] Seja $x^{s}$ um ponto de equilíbrio assintoticamente estável de (2.1) e $A\left(x^{s}\right)$ sua correspondente região de estabilidade. Se as suposições $(\boldsymbol{A} 1) e$ (A3) são satisfeitas, então

$$
\partial A\left(x^{s}\right) \subset \bigcup_{i} W^{s}\left(\bar{x}_{i}\right)
$$

onde $\bar{x}_{i}, i=1,2, \ldots$ são os pontos de equilíbrio em $\partial A\left(x^{s}\right)$. Se a condição (A2) também é satisfeita, então

$$
\partial A\left(x^{s}\right)=\bigcup_{i} W^{s}\left(\bar{x}_{i}\right)
$$

O Teorema 4.1.5 afirma, sob as condições (A1) e (A3) que a fronteira da região de estabilidade está contida na união das variedades estáveis dos pontos de equilíbrio hiperbólicos que pertencem à fronteira da região de estabilidade.

Se adicionalmente a condição (A2) é imposta então a fronteira da região de estabilidade é a união das variedades estáveis dos equilíbrios hiperbólicos que pertencem à fronteira da região de estabilidade. 
Substituindo as condições (A1)-(A3) pelas condições (B1)-(B3) obtémse o Teorema 4.1.6 que generaliza o Teorema 4.1.5 ao considerar a possibilidade de existirem órbitas periódicas fechadas na fronteira da região de estabilidade.

Teorema 4.1.6 (Caracterização da fronteira da região de estabilidade). [CHW88] Seja $x^{s}$ um ponto de equilíbrio assintoticamente estável de (2.1) e $A\left(x^{s}\right)$ sua correspondente região de estabilidade. Se as suposições (B1)-(B3) são satisfeitas, então:

$$
\partial A\left(x^{s}\right)=\bigcup_{i} W^{s}\left(\bar{x}_{i}\right) \bigcup_{j} W^{s}\left(\phi_{j}\right)
$$

onde $\bar{x}_{i}, i=1,2, \ldots$ são os pontos de equilíbrio $e \phi_{j}, j=1,2, \ldots$ são as órbitas fechadas em $\partial A\left(x^{s}\right)$.

Apresentaremos a seguir alguns exemplos que ilustram os Teoremas 4.1.3 à 4.1.6.

Exemplo 4.1.1. Considere o sistema de equações diferenciais extraído de [Mon06],

$$
\left\{\begin{array}{l}
\dot{x}=x^{2}-y-1 \\
\dot{y}=x-y+1
\end{array}\right.
$$

onde $(x, y) \in \mathbb{R}^{2}$.

O sistema (4.1) possui dois pontos de equilíbrios com o seguinte comportamento, um ponto de equilíbrio assintoticamente estável, $x^{s}=(-1,0)$, e um ponto de equilíbrio hiperbólico do tipo-1, $x_{1}=(2,3)$. Como todos os pontos são hiperbólicos então o sistema satisfaz a condição (A1), ver Figura 4.1. As suposições (A2) e (A3) são satisfeitas. A variedade instável $W^{u}\left(x_{1}\right)$ do ponto de equilíbrio hiperbólico do tipo-1 $x_{1}=(2,3)$ intercepta a região de estabilidade $A\left(x^{s}\right)$ e sua variedade estável $W^{s}\left(x_{1}\right)$ está contida na fronteira da região de estabilidade $\partial A\left(x^{s}\right)$, curva vermelha na Figura 4.1, de acordo com o Teorema 4.1.3. A fronteira da região de estabilidade $\partial A\left(x^{s}\right)$ é formada, de acordo com o Teorema 4.1.5, pela variedade estável $W^{s}\left(x_{1}\right)$ do ponto de equilíbrio hiperbólico do tipo-1 $x_{1}=(2,3)$, ver Figura 4.1.

Exemplo 4.1.2. Considere o sistema de equações diferenciais extraído de [Mon06],

$$
\left\{\begin{array}{l}
\dot{x}=y^{2}-3 y+2 \\
\dot{y}=x^{2}-y^{2}
\end{array}\right.
$$

onde $(x, y) \in \mathbb{R}^{2}$.

O sistema (4.2) possui quatro pontos de equilíbrios com o seguinte comportamento, dois pontos de equilíbrio assintoticamente estáveis, $x^{s_{1}}=(1,1)$ e $x^{s_{2}}=(-2,2)$, e dois pontos de equilíbrio hiperbólicos do tipo-1, $x_{1}=$ 


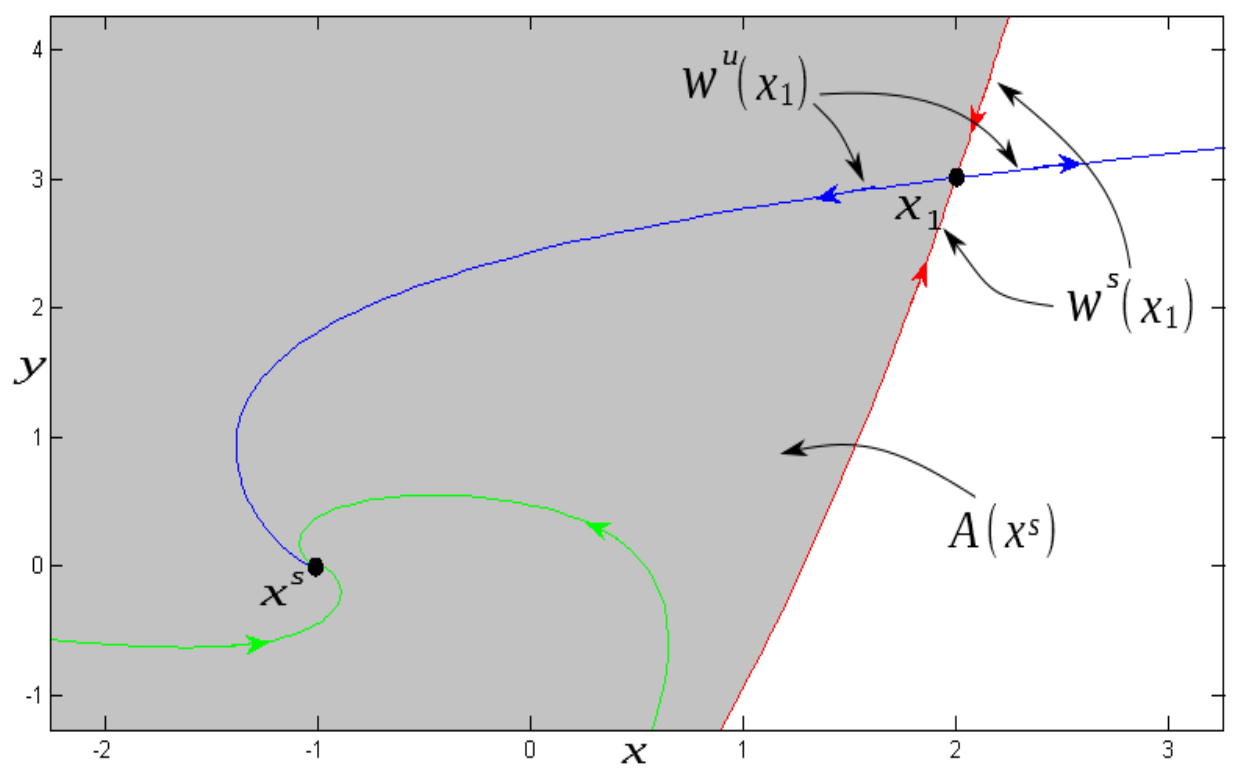

Figura 4.1: Retrato de fase do sistema (4.1). A fronteira da região de estabilidade de $x^{s}=(-10)$ é formada pela variedade estável $W^{s}\left(x_{1}\right)$ do ponto de equilíbrio hiperbólico do tipo-1 $x_{1}=(2,3)$, a curva vermelha.

$(-1,1)$ e $x_{2}=(2,2)$. Como todos os pontos são hiperbólicos então o sistema satisfaz a condição (A1), ver Figura 4.2. As suposições $(\boldsymbol{A} 2)$ e (A3) são satisfeitas. A variedade instável $W^{u}\left(x_{1}\right)$ do ponto de equilíbrio hiperbólico do tipo-1 $x_{1}=(-1,1)$ intercepta as regiões de estabilidade $A\left(x^{s_{1}}\right)$ e $A\left(x^{s_{2}}\right)$ e sua variedade estável $W^{s}\left(x_{1}\right)$ está contida na interseção das fronteiras das regiões de estabilidade $\partial A\left(x^{s_{1}}\right) \cap \partial A\left(x^{s_{2}}\right)$, de acordo com o Teorema 4.1.3, curva verde na Figura 4.2. A variedade instável $W^{u}\left(x_{2}\right)$ do ponto de equilíbrio hiperbólico do tipo-1 $x_{2}=(2,2)$ intercepta a região de estabilidade $A\left(x^{s_{1}}\right)$ e sua variedade estável $W^{s}\left(x_{2}\right)$ está contida na fronteira da região de estabilidade $\partial A\left(x^{s_{1}}\right)$, de acordo com o Teorema 4.1.3, curva vermelha na Figura 4.2. A fronteira da região de estabilidade $\partial A\left(x^{s_{1}}\right)$ é formada, de acordo com o Teorema 4.1.5, pela união da variedade estável $W^{s}\left(x_{1}\right)$ do ponto de equilíbrio hiperbólico do tipo-1 $x_{1}=(-1,1)$ com a variedade estável $W^{s}\left(x_{2}\right)$ do ponto de equilíbrio hiperbólico do tipo-1 $x_{2}=(2,2)$, ver Figura 4.2. E a fronteira da região de estabilidade $\partial A\left(x^{s_{2}}\right)$ é formada, de acordo com o Teorema 4.1.5, pela variedade estável $W^{s}\left(x_{2}\right)$ do ponto de equilíbrio hiperbólico do tipo-1 $x_{2}=(2,2)$, ver Figura 4.2.

Exemplo 4.1.3. Considere o sistema de equações diferenciais que modela 


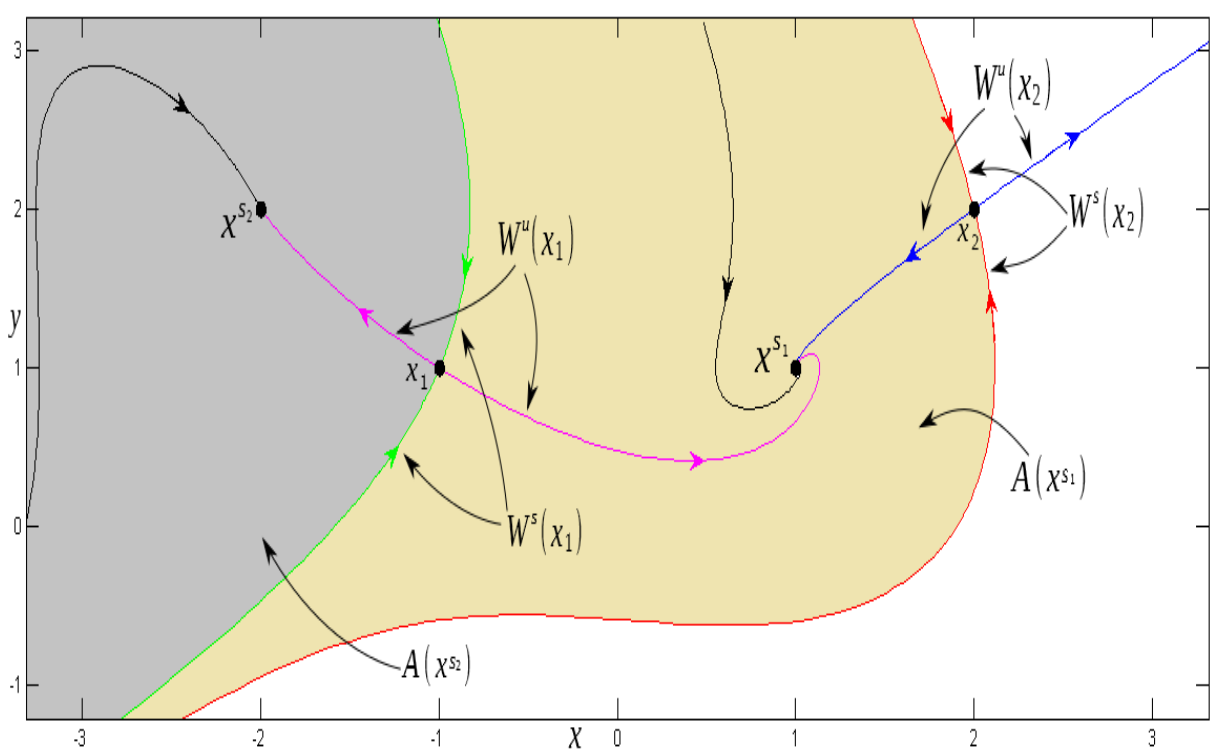

Figura 4.2: Retrato de fase do sistema (4.2). A fronteira da região de estabilidade de $x^{s_{1}}=\left(\begin{array}{ll}1 & 1\end{array}\right)$ é formada pela união da variedade estável $W^{s}\left(x_{1}\right)$ do ponto de equilíbrio hiperbólico do tipo-1 $x_{1}=(-1,1)$, a curva verde, com a variedade estável $W^{s}\left(x_{2}\right)$ do ponto de equilíbrio hiperbólico do tipo-1 $x_{2}=(2,2)$, a curva vermelha. A fronteira da região de estabilidade de $x^{s_{2}}=(-22)$ é formada pela variedade estável $W^{s}\left(x_{1}\right)$ do ponto de equilíbrio hiperbólico do tipo-1 $x_{1}=(-1,1)$, a curva verde.

a dinâmica de um sistema presa-predador extraído de [GTV85]

$$
\left\{\begin{array}{l}
\dot{x}=-3 x+4 x^{2}-0.5 x y-x^{3} \\
\dot{y}=-2.1 y+x y
\end{array}\right.
$$

onde $(x, y) \in \mathbb{R}^{2}$.

O sistema (4.3) possui quatro pontos de equilíbrios com o seguinte comportamento, dois pontos de equilíbrio assintoticamente estáveis, $x^{s_{1}}=(0,0)$ e $x^{s_{2}}=(2.10,1.98)$, e dois pontos de equilíbrio hiperbólicos do tipo-1, $x_{1}=(1,0)$ e $x_{2}=(3,0)$. Como todos os pontos são hiperbólicos então o sistema satisfaz a condição $(\boldsymbol{A} 1)$, ver Figura 4.3. A variedade instável $W^{u}\left(x_{1}\right)$ do ponto de equilíbrio hiperbólico do tipo-1 $x_{1}=(1,0)$ intercepta a região de estabilidade $A\left(x^{s_{1}}\right)$ e sua variedade estável $W^{s}\left(x_{1}\right)$ está contida na fronteira da região de estabilidade $\partial A\left(x^{s_{1}}\right)$, de acordo com o Teorema 4.1.3, curva vermelha na Figura 4.3. As suposiçôes (A2) e (A3) são satisfeitas. A fronteira da região de estabilidade $\partial A\left(x^{s_{1}}\right)$ é formada, de acordo com o Teorema 4.1.5, pela variedade estável $W^{s}\left(x_{1}\right)$ do ponto de equilíbrio hiperbólico do tipo-1 $x_{1}=(1,0)$, ver Figura 4.3.

A variedade instável $W^{u}\left(x_{2}\right)$ do ponto de equilíbrio hiperbólico do tipo- 
$1 x_{2}=(3,0)$ intercepta a região de estabilidade $A\left(x^{s_{2}}\right)$ e sua variedade estável $W^{s}\left(x_{2}\right)$ intercepta a fronteira da região de estabilidade $\partial A\left(x^{s_{2}}\right)$, de acordo com o Teorema 4.1.2, curva verde na Figura 4.3. A variedade instável $W^{u}\left(x_{1}\right)$ do ponto de equilíbrio hiperbólico do tipo-1 $x_{1}=(1,0)$ intercepta o fecho da região de estabilidade $\overline{A\left(x^{s_{2}}\right)}$ e sua variedade estável $W^{s}\left(x_{1}\right)$ intercepta a fronteira da região de estabilidade $\partial A\left(x^{s_{2}}\right)$, de acordo com o Teorema 4.1.1, curva vermelha na Figura 4.3. A suposição (A3) é satisfeita, mas a suposição (A2) não o é, pois a variedade instável $W^{u}\left(x_{1}\right)$ do ponto de equilíbrio $x_{1}$ não intercepta transversalmente a variedade estável $W^{s}\left(x_{2}\right)$ do ponto de equilíbrio $x_{2}$. Logo, a fronteira da regiãa de estabilidade $\partial A\left(x^{s_{2}}\right)$ está contida, de acordo com a primeira parte do Teorema 4.1.5, na união da variedade estável $W^{s}\left(x_{1}\right)$ do ponto de equilíbrio hiperbólico do tipo-1 $x_{1}=$ $(1,0)$ com a variedade estável $W^{s}\left(x_{2}\right)$ do ponto de equilíbrio hiperbólico do tipo-1 $x_{2}=(3,0)$, ver Figura 4.3.

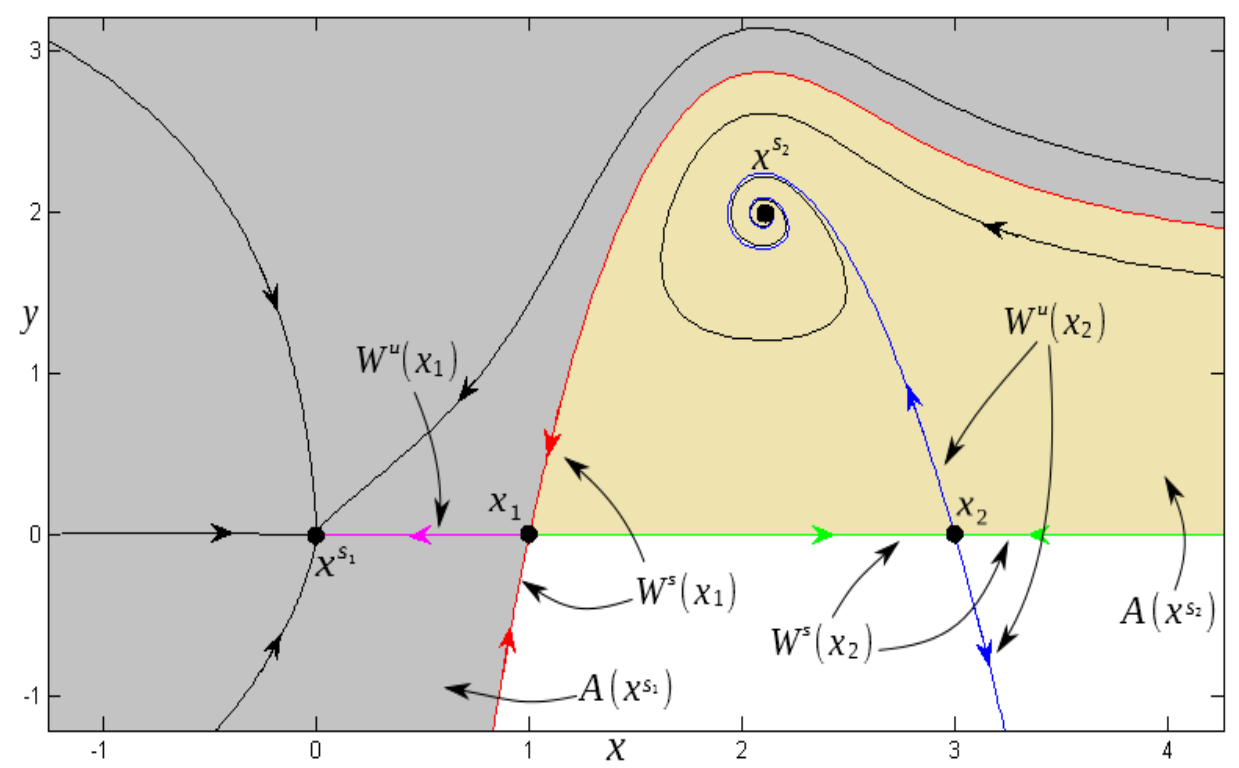

Figura 4.3: Retrato de fase do sistema (4.3). A fronteira da região de estabilidade $\partial A\left(x^{s_{1}}\right)$ é formada pela variedade estável $W^{s}\left(x_{1}\right)$ do ponto de equilíbrio hiperbólico do tipo-1 $x_{1}=(1,0)$, a curva em vermelho. A fronteira da região de estabilidade $\partial A\left(x^{s_{2}}\right)$ está contida na união da variedade estável $W^{s}\left(x_{1}\right)$ do ponto de equilíbrio hiperbólico do tipo-1 $x_{1}=(1,0)$ com a variedade estável $W^{s}\left(x_{2}\right)$ do ponto de equilibrio hiperbólico do tipo-1 $x_{2}=(3,0)$, a curva em verde. 


\subsection{Ponto de Equilíbrio Sela-Nó do tipo zero na Fronteira da Região de Estabilidade}

Nesta seção, apresentaremos a caracterização da fronteira da região de estabilidade de um ponto de equilíbrio assintoticamente estável admitindo a existência de um ponto de equilíbrio não hiperbólico do tipo sela-nó na fronteira da região de estabilidade.

O teorema a seguir apresenta uma caracterização de equilíbrio não hiperbólicos do tipo sela-nó do tipo-zero na fronteira da região de estabilidade.

Teorema 4.2.1 (Ponto de equilíbrio sela-nó do tipo zero na fronteira da região de estabilidade). [Ama10] Seja $x^{s}$ um ponto de equilíbrio assintoticamente estável do sistema dinâmico (2.1) e $A\left(x^{s}\right)$ sua correspondente região de estabilidade. Se $x_{0}$ é um ponto de equilíbrio sela-nó do tipo zero de (2.1), então

$$
x_{0} \in \partial A\left(x^{s}\right) \Longleftrightarrow W_{l o c}^{c^{+}}\left(x_{0}\right) \cap \overline{A\left(x^{s}\right)} \neq \emptyset .
$$

Relaxando a condição (A1), isto é, supondo a existência de pontos de equilíbrio não hiperbólicos na fronteira e com algumas suposições adicionais, uma caracterização mais refinada de um ponto de equilíbrio sela-nó do tipo zero na fronteira da região de estabilidade é obtida. Seja $x^{s}$ um ponto de equilíbrio assintoticamente estável do sistema dinâmico (2.1). Consideremos as seguintes condições sobre o sistema dinâmico:

(A1') Todos os pontos de equilíbrios em $\partial A\left(x^{s}\right)$ são hiperbólicos, exceto possivelmente o equilíbrio $x_{0}$ que é um ponto de equilíbrio sela-nó do tipo zero;

(A4) As variedades estáveis dos pontos de equilíbrio em $\partial A\left(x^{s}\right)$ e a variedade $W_{l o c}^{c^{+}}\left(x_{0}\right)$ satisfazem a condição de transversalidade.

Admitindo as suposições (A1'), (A2), (A3) e (A4), o próximo teorema oferece condições para que um ponto de equilíbrio sela-nó e um ponto de equilíbrio hiperbólico pertençam à fronteira da região de estabilidade.

Teorema 4.2.2 (Caracterizações adicionais de um ponto de equilíbrio sela-nó na fronteira da região de estabilidade). [Ama10] Sejam $x^{s}$ um ponto de equilíbrio assintoticamente estável de (2.1) e $A\left(x^{s}\right)$ sua correspondente região de estabilidade. Se $x_{0}$ é um ponto de equilíbrio sela-nó do tipo zero e $\bar{x}$ é um ponto de equilíbrio hiperbólico de (2.1) e as suposições (A1'), (A2), (A3) e (A4) são satisfeitas, então:

(i)

$$
\begin{gathered}
x_{0} \in \partial A\left(x^{s}\right) \text { se, e somente se, } W^{c^{+}}\left(x_{0}\right) \cap A\left(x^{s}\right) \neq \emptyset . \\
\bar{x} \in \partial A\left(x^{s}\right) \text { se, e somente se, } W^{u}(\bar{x}) \cap A\left(x^{s}\right) \neq \emptyset .
\end{gathered}
$$


(ii)

$$
\begin{gathered}
x_{0} \in \partial A\left(x^{s}\right) \text { se, e somente se, } W^{s}\left(x_{0}\right) \subset \partial A\left(x^{s}\right) . \\
\bar{x} \in \partial A\left(x^{s}\right) \text { se, e somente se, } W^{s}(\bar{x}) \subset \partial A\left(x^{s}\right) .
\end{gathered}
$$

O Teorema 4.2.2 oferece uma maneira de verificar numericamente se um ponto de equilíbrio sela-nó pertence à fronteira da região de estabilidade. Para isto, basta tomar um ponto em $W^{c^{+}}\left(x_{0}\right)$ e verificar se a trajetória do sistema converge para $x^{s}$ quando $t \rightarrow+\infty$.

O Teorema 4.2.2 mostra que a caracterização de um ponto de equilíbrio hiperbólico na fronteira da região de estabilidade não muda com a existência de um ponto de equilíbrio sela-nó na fronteira, ou seja, o ponto de equilíbrio hiperbólico pertence à fronteira da região de estabilidade se e só se sua variedade instável intercepta a região de estabilidade, vide Teorema $3.7 \mathrm{em}$ [CHW88].

O teorema a seguir fornece uma caracterização da fronteira da região de estabilidade de um ponto de equilíbrio assintoticamente estável na presença de um ponto de equilíbrio sela-nó do tipo zero em $\partial A\left(x^{s}\right)$.

Teorema 4.2.3 (Caracterização da fronteira da região de estabilidade). [Ama10] Seja $x^{s}$ um ponto de equilíbrio assintoticamente estável de (2.1) e $A\left(x^{s}\right)$ sua correspondente região de estabilidade. Se as suposições (A1'), (A2), (A3) e (A4) são satisfeitas, então:

$$
\partial A\left(x^{s}\right)=\bigcup_{i} W^{s}\left(\bar{x}_{i}\right) \bigcup_{j} W^{s}\left(x_{0}\right)
$$

onde $\bar{x}_{i}, i=1,2, \ldots$ são os pontos de equilíbrio hiperbólicos em $\partial A\left(x^{s}\right)$ e $x_{0}$ é um ponto de equilíbrio sela-nó do tipo zero em $\partial A\left(x^{s}\right)$.

Exibiremos a seguir um exemplo que ilustra os Teoremas 4.2.1, 4.2.2 e 4.2.3, ver [AAB10].

Exemplo 4.2.1. Considere o sistema de equações diferenciais extraído de [Ama10]

$$
\left\{\begin{array}{l}
\dot{x}=x^{2}+y^{2}-1 \\
\dot{y}=x^{2}-y-1
\end{array}\right.
$$

$\operatorname{com}(x, y) \in \mathbb{R}^{2}$.

O sistema (4.4) possui três pontos de equilíbrio com o seguinte comportamento, um ponto de equilíbrio sela-nó do tipo-zero, $x_{0}=(0,-1)$, um ponto de equilíbrio hiperbólico assintoticamente estável, $x^{s}=(-1,0)$, e um ponto de equilíbrio hiperbólico do tipo- $1, x_{1}=(1,0)$. O ponto de equilíbrio sela-nó do tipo-zero e o ponto de equilíbrio hiperbólico do tipo-1 pertencem à fronteira da região de estabilidade de $\partial A\left(x^{s}\right)$, ver Figura 4.4. As suposições (A1'), (A2), (A3) e (A4) são satisfeitas. A variedade central $W^{c^{+}}\left(x_{0}\right)$ do ponto de equilíbrio sela-nó do tipo-zero $x_{0}=(0,-1)$ intercepta a região de 
estabilidade $A\left(x^{s}\right)$ e sua variedade estável $W^{s}\left(x_{0}\right)$ está contida na fronteira da região de estabilidade $\partial A\left(x^{s}\right)$, de acordo com o Teorema 4.2.2, curva vermelha na Figura 4.4. Observemos também que a variedade instável $W^{u}\left(x_{1}\right)$ do ponto de equilíbrio hiperbólico do tipo-1 intercepta a região de estabilidade $A\left(x^{s}\right)$ e sua variedade estável $W^{s}\left(x_{1}\right)$ está contida na fronteira da região de estabilidade $\partial A\left(x^{s}\right)$, de acordo com o Teorema 4.2.2, curva verde na Figura 4.4. A fronteira da região de estabilidade $\partial A\left(x^{s}\right)$ é formada, de acordo com o Teorema 4.2.3, pela união da variedade estável $W^{s}\left(x_{1}\right)$ do ponto de equilíbrio hiperbólico do tipo-1, $x_{1}=(1,0)$ com a variedade estável $W^{s}\left(x_{0}\right)$ do ponto de equilíbrio sela-nó do tipo-zero, $x_{0}=(0,-1)$, ver Figura 4.4.

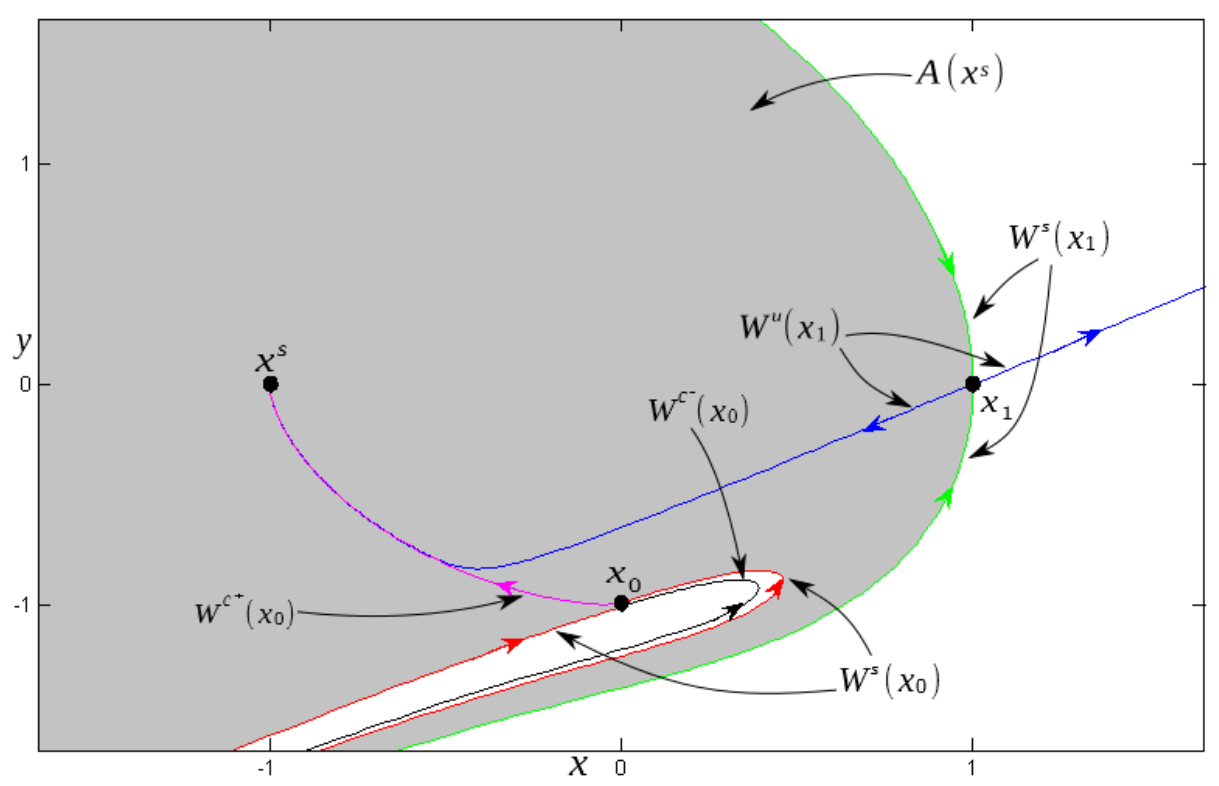

Figura 4.4: Retrato de fase do sistema (4.4). A fronteira da região de estabilidade $\partial A\left(x^{s}\right)$ é formada pela união da variedade estável $W^{s}\left(x_{1}\right)$ do ponto de equilíbrio hiperbólico do tipo-1 $x_{1}=(1,0)$, a curva verde, com a variedade estável $W^{s}\left(x_{0}\right)$ do ponto de equilíbrio sela-nó do tipo-zero $x_{0}=(0,-1)$, a curva vermelha. 


\section{5 \\ Fronteira da Região de Estabilidade na Presença de um Ponto de Equilíbrio Hopf}

No capítulo anterior, sob as suposições (A1), (A2) e (A3), caracterizamos a fronteira da região de estabilidade de um ponto de equilíbrio assintoticamente estável como sendo a união das variedades estáveis dos pontos de equilíbrios pertencentes à fronteira da região da estabilidade. Ainda no mesmo capítulo, relaxando a suposição (A1), e supondo a existência de um ponto de equilíbrio não hiperbólico do tipo sela-nó na fronteira da região de estabilidade, caracterizamos a fronteira da região de estabilidade como sendo a união das variedades estáveis dos equilíbrios hiperbólicos com a variedade estável do equilíbrio não hiperbólico sela-nó pertencentes à fronteira. Neste capítulo, suporemos a existência de um ponto de equilíbrio Hopf na fronteira da região de estabilidade e desenvolveremos uma caracterização da fronteira da região de estabilidade em termos das variedades estável, central e/ou centro-estável dos equilíbrios pertencentes à fronteira.

\subsection{Lemas Auxiliares}

Nesta seção, exibiremos alguns lemas auxiliares que serão importantes nas demonstrações dos resultados deste capítulo. Os seguintes lemas serão úteis para desenvolver uma caracterização da fronteira da região de estabilidade. Eles estabelecem uma relação entre as dimensões das variedades instáveis dos pontos de equilíbrio hiperbólicos e dos pontos de equilíbrio Hopf.

Lema 5.1.1. Sejam $\widetilde{x}$ um ponto de equilíbrio Hopf supercrítico do tipo-k 
ou um ponto de equilíbrio hiperbólico do tipo- $k$, com $k \geq 1$, e $x$ um ponto de equilíbrio hiperbólico do sistema (2.1). Se $W^{u}(\widetilde{x})$ e $W^{s}(x)$ satisfazem a condição de transversalidade e $W^{u}(\widetilde{x}) \cap W^{s}(x) \neq \emptyset$, então $\operatorname{dim} W^{u}(x)<$ $\operatorname{dim} W^{u}(\widetilde{x})$.

Demonstração. Seja $\widetilde{x}$ um ponto de equilíbrio Hopf supercrítico do tipo- $k$, com $k \geq 1$. Como $W^{u}(\widetilde{x})$ e $W^{s}(x)$ satisfazem a condição de transversalidade, então temos

$$
\operatorname{dim} W^{u}(\widetilde{x})+\operatorname{dim} W^{s}(x)=n+\operatorname{dim}\left(W^{u}(\widetilde{x}) \cap W^{s}(x)\right) .
$$

Se $x$ é um ponto de equilíbrio hiperbólico, temos

$$
\operatorname{dim} W^{u}(x)+\operatorname{dim} W^{s}(x)=n .
$$

Seja $y \in W^{u}(\widetilde{x}) \cap W^{s}(x)$. Como $W^{u}(\widetilde{x})$ e $W^{s}(x)$ são variedades invariantes, então cada órbita que passa pelo ponto $y$ está inteiramente contida em $W^{u}(\widetilde{x}) \cap W^{s}(x)$ e, portanto, temos $\operatorname{dim}\left(W^{u}(\widetilde{x}) \cap W^{s}(x)\right) \geq 1$. Consequentemente,

$$
\operatorname{dim} W^{u}(\widetilde{x})+\operatorname{dim} W^{s}(x) \geq n+1,
$$

$\mathrm{ou}$

$$
\operatorname{dim} W^{u}(\widetilde{x}) \geq n+1-\operatorname{dim} W^{s}(x) .
$$

Substituindo a equação (5.1) na desigualdade anterior, obtemos

$$
\operatorname{dim} W^{u}(\widetilde{x}) \geq \operatorname{dim} W^{u}(x)+1
$$

e, assim,

$$
\operatorname{dim} W^{u}(\widetilde{x})>\operatorname{dim} W^{u}(x) .
$$

A demonstração é análoga ao caso anterior se $\widetilde{x}$ é um ponto de equilíbrio hiperbólico do tipo- $k$, com $k \geq 1$.

Os Lemas 5.1.2 e 5.1.3 podem ser provados com a mesma técnica de demonstração do Lema 5.1.1 e portanto elas serão omitidas.

Lema 5.1.2. Seja $\widetilde{x}$ um ponto de equilíbrio Hopf subcrítico do tipo-0 e $x$ um ponto de equilíbrio hiperbólico do sistema (2.1). Se $W^{c}(\widetilde{x})$ e $W^{s}(x)$ satisfazem a condição de transversalidade e $W^{c}(\widetilde{x}) \cap W^{s}(x) \neq \emptyset$, então $\operatorname{dim} W^{u}(x)<\operatorname{dim} W^{c}(\widetilde{x})$.

Lema 5.1.3. Seja $\widetilde{x}$ um ponto de equilíbrio Hopf subcrítico do tipo-k, com $k \geq 1$, e $x$ um ponto de equilíbrio hiperbólico do sistema (2.1). Se $W^{c u}(\widetilde{x})$ $e W^{s}(x)$ satisfazem a condição de transversalidade e $W^{c u}(\widetilde{x}) \cap W^{s}(x) \neq \emptyset$, então $\operatorname{dim} W^{u}(x)<\operatorname{dim} W^{c u}(\widetilde{x})$.

Lema 5.1.4. Sejam $\widetilde{x}$ um ponto de equilíbrio hiperbólico do tipo-k ou um ponto de equilíbrio Hopf supercrítico do tipo-k, com $k \geq 1$, e p um ponto de 
equilíbrio Hopf supercrítico do tipo- $k^{\prime}$, com $1 \leq k^{\prime} \leq n-3$, do sistema (2.1). Se $W^{u}(\widetilde{x})$ e $W^{c s}(p)$ satisfazem a condição de transversalidade e $W^{u}(\widetilde{x}) \cap$ $W^{c s}(p) \neq \emptyset$, então $\operatorname{dim} W^{u}(p)<\operatorname{dim} W^{u}(\widetilde{x})$.

Demonstração. Seja $\widetilde{x}$ um ponto de equilíbrio Hopf supercrítico do tipo- $k$, com $k \geq 1$. Como $W^{u}(\widetilde{x})$ e $W^{c s}(p)$ satisfazem a condição de transversalidade, então temos

$$
\operatorname{dim} W^{u}(\widetilde{x})+\operatorname{dim} W^{c s}(p)=n+\operatorname{dim}\left(W^{u}(\widetilde{x}) \bigcap W^{c s}(p)\right) .
$$

Seja $y \in W^{u}(\widetilde{x}) \cap W^{c s}(p)$. Como $W^{u}(\widetilde{x})$ e $W^{c s}(p)$ são variedades invariantes, então cada órbita que passa pelo ponto $y$ está inteiramente contida em $W^{u}(\widetilde{x}) \cap W^{c s}(p)$ e, portanto, temos $\operatorname{dim}\left(W^{u}(\widetilde{x}) \cap W^{c s}(p)\right) \geq 1$. Consequentemente,

$$
\operatorname{dim} W^{u}(\widetilde{x})+\operatorname{dim} W^{c s}(p) \geq n+1,
$$

$\mathrm{Ou}$

$$
\operatorname{dim} W^{u}(\widetilde{x}) \geq n+1-\operatorname{dim} W^{c s}(p) .
$$

Como $p$ é um ponto de equilíbrio Hopf supercrítico do tipo- $k^{\prime}$, com $1 \leq k^{\prime} \leq$ $n-3$, temos que

$$
\operatorname{dim} W^{u}(p)+\operatorname{dim} W^{c s}(p)=n .
$$

Portanto, a desigualdade anterior nos fornece

$$
\operatorname{dim} W^{u}(\widetilde{x}) \geq \operatorname{dim} W^{u}(p)+1,
$$

e assim

$$
\operatorname{dim} W^{u}(\widetilde{x})>\operatorname{dim} W^{u}(p) .
$$

A demonstração é análoga ao caso anterior se $\widetilde{x}$ é um ponto de equilíbrio hiperbólico do tipo- $k$, com $k \geq 1$.

Lema 5.1.5. Sejam $\widetilde{x}$ um ponto de equilíbrio hiperbólico do tipo-k, com $k \geq 1$, (ou um ponto de equilíbrio Hopf subcrítico do tipo-0) e p um ponto de equilibrio Hopf subcrítico do tipo- $k^{\prime}$, com $0 \leq k^{\prime} \leq n-3$, do sistema (2.1). Se $W^{u}(\widetilde{x})$ (ou $W^{c}(\widetilde{x})$ ) e $W^{s}(p)$ satisfazem a condição de transversalidade e $W^{u}(\widetilde{x}) \cap W^{s}(p) \neq \emptyset\left(\right.$ ou $\left.W^{c}(\widetilde{x}) \cap W^{s}(p) \neq \emptyset\right)$, então $\operatorname{dim} W^{c u}(p)<$ $\operatorname{dim} W^{u}(\widetilde{x})$ (ou $\operatorname{dim} W^{c}(p)<\operatorname{dim} W^{c}(\widetilde{x})$ se p é do tipo-0 ou $\operatorname{dim} W^{c u}(p)<$ $\operatorname{dim} W^{c}(\widetilde{x})$ se $p$ é do tipo-k $k^{\prime}$, com $\left.k^{\prime} \geq 1\right)$, respectivamente.

Lema 5.1.6. Sejam $\widetilde{x}$ um ponto de equilíbrio Hopf subcrítico do tipo-k, com $k \geq 1$, e $p$ um ponto de equilíbrio Hopf subcrítico do tipo- $k^{\prime}$, com $0 \leq$ $k^{\prime} \leq n-3$, do sistema (2.1). Se $W^{c u}(\widetilde{x})$ e $W^{s}(p)$ satisfazem a condição de transversalidade e $W^{c u}(\widetilde{x}) \cap W^{s}(p) \neq \emptyset$, então $\operatorname{dim} W^{c}(p)<\operatorname{dim} W^{c u}(\widetilde{x})$ se $p$ é do tipo-0 ou $\operatorname{dim} W^{c u}(p)<\operatorname{dim} W^{c u}(\widetilde{x})$ se p é do tipo- $k^{\prime}$, com $k^{\prime} \geq 1$. 
Lema 5.1.7. Sejam $\widetilde{x}$ um ponto de equilíbrio Hopf supercrítico do tipo-k ou um ponto de equilibrio hiperbólico do tipo- $k$, com $k \geq 1$, e $p$ um ponto de equilíbrio Hopf supercrítico do tipo- $(n-2)$ do sistema (2.1). Se $W^{u}(\widetilde{x})$ $e W^{c}(p)$ satisfazem a condição de transversalidade e $W^{u}(\widetilde{x}) \cap W^{c}(p) \neq \emptyset$, então $\operatorname{dim} W^{u}(p)<\operatorname{dim} W^{u}(\widetilde{x})$.

A demonstração dos Lemas 5.1.5, 5.1.6 e 5.1.7 é inteiramente análoga à demonstração dos Lemas 5.1.1 e 5.1.4 e, portanto, serão omitidas.

O seguinte lema estabelece uma condição suficiente para garantir que as variedades instável e centro-estável (ou central) de um ponto de equilíbrio Hopf supercrítico tenham interseção nula.

Lema 5.1.8. Seja $p$ um ponto de equilíbrio Hopf supercrítico do sistema (2.1). Se p é do tipo- $k$, com $1 \leq k \leq n-3$, e $W^{u}(p)$ e $W^{c s}(p)$ satisfazem a condição de transversalidade, então $W^{u}(p) \cap W^{c s}(p)=\emptyset$. Se p é do tipo$(n-2)$ e $W^{u}(p)$ e $W^{c}(p)$ satisfazem a condição de transversalidade, então $W^{u}(p) \cap W^{c}(p)=\emptyset$.

Demonstração. Seja $p$ um ponto de equilíbrio Hopf supercrítico do tipo$k$, com $1 \leq k \leq n-3$. Como $W^{u}(p)$ e $W^{c s}(p)$ satisfazem a condição de transversalidade, então temos

$$
\operatorname{dim}\left(W^{u}(p) \cap W^{c s}(p)\right)=n-\operatorname{dim} W^{u}(p)-\operatorname{dim} W^{c s}(p) .
$$

Como $\operatorname{dim} W^{u}(p)=k$ e $\operatorname{dim} W^{c s}(p)=n-k$, então a igualdade anterior nos fornece

$$
\operatorname{dim}\left(W^{u}(p) \cap W^{c s}(p)\right)=n-k-(n-k)=0,
$$

isto é, $\operatorname{dim}\left(W^{u}(p) \cap W^{c s}(p)\right)=0$. Por outro lado, suponhamos que $y \in$ $W^{u}(p) \cap W^{c s}(p)$, então cada órbita que passa pelo ponto $y$ está inteiramente contida em $W^{u}(p) \cap W^{c s}(p)$ e, portanto, temos $\operatorname{dim}\left(W^{u}(p) \cap W^{c s}(p)\right) \geq 1$, o que é um absurdo. Portanto, a interseção $W^{u}(p) \cap W^{c s}(p)$ é vazia. Para $p$ do tipo- $(n-2)$, a demonstração é completamente análoga ao caso anterior.

O Lema 5.1.9 é uma versão do Lema 5.1 .8 para pontos de equilíbrio Hopf subcríticos. Ele estabelece, sob condições de transversalidade que as variedades estável e centro-instável (ou central) de um ponto de equilíbrio Hopf subcrítico tenham interseção nula.

Lema 5.1.9. Seja p um ponto de equilíbrio Hopf subcrítico do sistema (2.1). Se p é do tipo- $k$, com $1 \leq k \leq n-3$, e $W^{c u}(p)$ e $W^{s}(p)$ satisfazem a condição de transversalidade, então $W^{c u}(p) \cap W^{s}(p)=\emptyset$. Se p é do tipo-0 e $W^{s}(p)$ e $W^{c}(p)$ satisfazem a condição de transversalidade, então $W^{s}(p) \cap W^{c}(p)=\emptyset$. 


\subsection{Ponto de equilíbrio Hopf Supercrítico em $\partial A\left(x^{s}\right)$}

Nesta seção, mostraremos uma caracterização completa da fronteira da região de estabilidade na presença de um ponto de equilíbrio Hopf supercrítico.

A caracterização da fronteira da região de estabilidade na presença de um ponto de equilíbrio Hopf supercrítico será realizada em dois passos. Primeiramente, estudaremos a caracterização local da fronteira da região de estabilidade por meio do estudo e caracterização dos pontos de equilíbrio que pertencem à fronteira da região de estabilidade, e depois exibiremos a caracterização global.

Os próximos teoremas fornecem condições necessárias e suficientes para garantir que um ponto de equilíbrio Hopf supercrítico esteja na fronteira da região de estabilidade em termos de suas variedades estável, instável e/ou centro-estável.

Teorema 5.2.1. (Ponto de equilíbrio Hopf supercrítico na fronteira de estabilidade) Sejam $x^{s}$ um ponto de equilíbrio assintoticamente estável do sistema autonômo (2.1) e $A\left(x^{s}\right)$ sua região de estabilidade. Se p é um ponto de equilíbrio Hopf supercrítico do sistema (2.1), então

(i) se $p$ é um ponto de equilíbrio Hopf supercrítico tipo-0 de (2.1), então $p \notin \partial A\left(x^{s}\right)$

(ii) se $p$ é um ponto de equilíbrio Hopf supercrítico tipo-k de (2.1), com $1 \leq k \leq n-3$, então:

$$
\begin{gathered}
p \in \partial A\left(x^{s}\right) \Longleftrightarrow\left(W_{l o c}^{u}(p) \backslash\{p\}\right) \cap \overline{A\left(x^{s}\right)} \neq \emptyset \\
p \in \partial A\left(x^{s}\right) \Longleftrightarrow W_{l o c}^{c s}(p) \cap \partial A\left(x^{s}\right) \neq \emptyset
\end{gathered}
$$

(iii) se p é um ponto de equilíbrio Hopf supercrítico tipo- $(n-2)$ de (2.1), então:

$$
\begin{gathered}
p \in \partial A\left(x^{s}\right) \Longleftrightarrow\left(W_{l o c}^{u}(p) \backslash\{p\}\right) \cap \overline{A\left(x^{s}\right)} \neq \emptyset \\
p \in \partial A\left(x^{s}\right) \Longleftrightarrow W_{l o c}^{c}(p) \cap \partial A\left(x^{s}\right) \neq \emptyset
\end{gathered}
$$

Demonstração. (i) Se $p$ é um ponto de equilíbrio Hopf supercrítico tipo-0, então existe uma vizinhança $U$ de $p$ tal que $\varphi(t, q) \rightarrow p$ quando $t \rightarrow+\infty$ para todo $q \in U$. Suponhamos, por contradição, que $p \in \partial A\left(x^{s}\right)$, então, para qualquer vizinhança $U$ de $p$, existe $\bar{q} \in A\left(x^{s}\right) \cap U$ tal que $\varphi(t, \bar{q}) \rightarrow x^{s}$ quando $t \rightarrow+\infty$, o que é um absurdo. Portanto, $p \notin \partial A\left(x^{s}\right)$.

(ii) $(\Longleftarrow)$ Suponhamos que $\left(W_{l o c}^{u}(p) \backslash\{p\}\right) \cap \overline{A\left(x^{s}\right)} \neq \emptyset$. Então existe $q \in\left(W_{\text {loc }}^{u}(p) \backslash\{p\}\right) \cap \overline{A\left(x^{s}\right)}$. Observe que $\varphi(t, q) \longrightarrow p$ quando $t \longrightarrow-\infty$. Por outro lado, o conjunto $\overline{A\left(x^{s}\right)}$ é invariante e deste modo, $\varphi(t, q) \in \overline{A\left(x^{s}\right)}$ para 
todo $t \leq 0$. Consequentemente, $p \in \overline{A\left(x^{s}\right)}$, visto que $\overline{A\left(x^{s}\right)}$ é um conjunto fechado. Como $p \notin A\left(x^{s}\right)$, temos que $p \in \mathbb{R}^{n} \backslash A\left(x^{s}\right)$. Portanto, $p \in \partial A\left(x^{s}\right)$. Agora, suponhamos que $W_{l o c}^{c s}(p) \cap \partial A\left(x^{s}\right) \neq \emptyset$. Portanto, existe $q \in W_{l o c}^{c s}(p) \cap$ $\partial A\left(x^{s}\right)$. Note que $\varphi(t, q) \longrightarrow p$ quando $t \longrightarrow+\infty$. Como o conjunto $\partial A\left(x^{s}\right)$ é invariante e $q \in \partial A\left(x^{s}\right)$, temos $\varphi(t, q) \in \partial A\left(x^{s}\right)$ para todo $t \geq 0$. Como $\partial A\left(x^{s}\right)$ é um conjunto fechado, então $p \in \partial A\left(x^{s}\right)$.

$(\Longrightarrow)$ Suponhamos que $p \in \partial A\left(x^{s}\right)$. Seja $N^{u}$ um domínio fundamental de $W^{u}(p)$, isto é, $\bigcup_{t \in \mathbb{R}} \varphi\left(t, N^{u}\right)=W^{u}(p) \backslash\{p\}$. Seja $N_{\varepsilon}^{u}$ uma vizinhança fundamental de raio $\varepsilon$ de $N^{u}$, isto é, $N_{\varepsilon}^{u}=\left\{x \in \mathbb{R}^{n}: d\left(x, N^{u}\right)<\varepsilon\right\}$. Pelo Corolário 2.2.24, existe uma vizinhança $U$ de $p$ tal que $\bigcup_{t<0} \varphi\left(t, N_{\varepsilon}^{u}\right) \supset$ $U \backslash W_{l o c}^{c s}(p)$. Como $p \in \partial A\left(x^{s}\right)$, então $U \cap A\left(x^{s}\right) \neq \emptyset$. Por outro lado, $W_{l o c}^{c s}(p) \cap$ $A\left(x^{s}\right)=\emptyset$. Logo, $\left\{U \backslash W_{l o c}^{c s}(p)\right\} \cap A\left(x^{s}\right) \neq \emptyset$. Consequentemente, existe um ponto $q \in N_{\varepsilon}^{u}$ e um tempo $t_{q}$ tal que $\varphi\left(t_{q}, q\right) \in A\left(x^{s}\right)$. Como $A\left(x^{s}\right)$ é um conjunto invariante, então $q \in A\left(x^{s}\right)$. Como $\varepsilon$ pode ser escolhido arbitrariamente pequeno, podemos encontrar uma sequência de pontos $\left\{q_{i}\right\}$ com $q_{i} \in A\left(x^{s}\right)$ para todo $i=1,2, \ldots$ tal que $d\left(q_{i}, N^{u}\right) \rightarrow 0$ quando $i \rightarrow+\infty$. Por construção, a sequência $\left\{q_{i}\right\}$ é limitada e, portanto, admite uma subsequência convergente. Seja $\left\{q_{i_{k}}\right\}$ uma subsequência convergente, isto é, $q_{i_{k}} \rightarrow \bar{q}$ quando $i_{k} \rightarrow+\infty$. Observe que $d\left(q_{i_{k}}, N^{u}\right) \rightarrow d\left(\bar{q}, N^{u}\right)$ quando $i_{k} \rightarrow+\infty$ e, portanto, $\bar{q} \in \overline{N^{u}} \subset W_{l o c}^{u}(p) \backslash\{p\}$. Logo, $\bar{q} \in\left(W_{l o c}^{u}(p) \backslash\right.$ $\{p\}) \cap \overline{A\left(x^{s}\right)}$. A prova de que $W_{l o c}^{c s}(p) \cap \partial A\left(x^{s}\right) \neq \emptyset$ se $p \in \partial A\left(x^{s}\right)$ é muito similar a prova anterior e, portanto, será omitida.

A demonstração do item (iii) é similar à demonstração de $(i i)$ e também será omitida.

Como uma consequência do Teorema 5.2.1, sabemos que $W_{l o c}^{u}(p) \cap A\left(x^{s}\right) \neq$ $\emptyset$ é uma condição suficiente para guarantir que um ponto de equilíbrio Hopf supercrítico $p$ encontre-se na fronteira da região de estabilidade. Será relevante desenvolver uma caracterização da fronteira da região de estabilidade verificando quando esta condição também é necessária.

Os itens (i) e (ii) do Teorema 5.2.1 podem ser refinados se impusermos algumas condições para o campo vetorial. Sejam $x^{s}$ um ponto de equilíbrio assintoticamente estável e $p$ um ponto de equilíbrio Hopf supercrítico do sistema (2.1) e considere as seguintes afirmações:

(A1") Todos os pontos de equilíbrio em $\partial A\left(x^{s}\right)$ são hiperbólicos ou Hopf supercrítico;

(A2') As variedades estável, centro-estável e/ou central e a variedade instável dos pontos de equilíbrios em $\partial A\left(x^{s}\right)$ satisfazem a condição de transversalidade.

A condição (A1") é mais fraca do que a condição (A1), uma vez que permite a presença de pontos de equilíbrio não-hiperbólicos na fronteira da região de estabilidade. Os próximos resultados fornecem condições necessárias e suficientes para garantir que os pontos de equilíbrio hiperbólicos e os pontos de equilíbrio Hopf supercríticos pertençam à fronteira da região 
de estabilidade. Inicialmente, mostraremos a caracterização de pontos de equilíbrio do tipo-1 na fronteira da região de estabilidade e, em seguida, a caracterização para pontos de equilíbrios de tipos superiores a 1 seguirá por argumentos de indução.

Teorema 5.2.2. (Pontos de equilíbrio tipo-1 na fronteira de estabilidade) Seja $A\left(x^{s}\right)$ a região de estabilidade de um ponto de equilíbrio assintoticamente estável $x^{s}$ de (2.1). Sejam $p$ um ponto de equilíbrio Hopf supercrítico do tipo-1 e $\bar{x}$ um ponto de equilíbrio hiperbólico do tipo-1 de (2.1). Se as suposições (A1"), (A2') e (A3) são satisfeitas, então:

(i)

$$
\begin{aligned}
& p \in \partial A\left(x^{s}\right) \Longleftrightarrow W^{u}(p) \cap A\left(x^{s}\right) \neq \emptyset \\
& \bar{x} \in \partial A\left(x^{s}\right) \Longleftrightarrow W^{u}(\bar{x}) \cap A\left(x^{s}\right) \neq \emptyset
\end{aligned}
$$

(ii)

$$
\begin{aligned}
& p \in \partial A\left(x^{s}\right) \Longleftrightarrow W^{c s}(p) \subset \partial A\left(x^{s}\right) \\
& \bar{x} \in \partial A\left(x^{s}\right) \Longleftrightarrow W^{s}(\bar{x}) \subset \partial A\left(x^{s}\right)
\end{aligned}
$$

Demonstração. (i) $(\Longleftarrow)$ Suponhamos que $W^{u}(p) \cap A\left(x^{s}\right) \neq \emptyset$. Como $A\left(x^{s}\right) \subset$ $\overline{A\left(x^{s}\right)}$, então $\left(W_{l o c}^{u}(p) \backslash\{p\}\right) \cap \overline{A\left(x^{s}\right)} \neq \emptyset$. Portanto, pelo item (ii) do Teorema 5.2.1, temos que $p \in \partial A\left(x^{s}\right)$.

Suponhamos agora que $W^{u}(\bar{x}) \cap A\left(x^{s}\right) \neq \emptyset$. Como $A\left(x^{s}\right) \subset \overline{A\left(x^{s}\right)}$, então $\left(W_{l o c}^{u}(\bar{x}) \backslash\{\bar{x}\}\right) \cap \overline{A\left(x^{s}\right)} \neq \emptyset$. Portanto, pelo Teorema 3.7 de [CHW88], temos que $\bar{x} \in \partial A\left(x^{s}\right)$.

$(\Longrightarrow)$ Suponhamos que $p \in \partial A\left(x^{s}\right)$. Pelo Teorema 5.2.1, podemos concluir que $\left(W_{l o c}^{u}(p) \backslash\{p\}\right) \cap \overline{A\left(x^{s}\right)} \neq \emptyset$. Consequentemente, $\left(W^{u}(p) \backslash\{p\}\right) \cap$ $\overline{A\left(x^{s}\right)} \neq \emptyset$, pois $W_{l o c}^{u}(p) \subset W^{u}(p)$. Vamos mostrar, sob as suposições (A1"), (A2') e (A3) que $\left(W^{u}(p) \backslash\{p\}\right) \cap \overline{A\left(x^{s}\right)} \neq \emptyset$ implica em $W^{u}(p) \cap A\left(x^{s}\right) \neq \emptyset$. Seja $q \in\left(W^{u}(p) \backslash\{p\}\right) \cap \overline{A\left(x^{s}\right)}$. Se $q \in A\left(x^{s}\right)$, então não existe nada a ser provado. Suponhamos que $q \in \partial A\left(x^{s}\right)$. Da condição (A3), existe um ponto de equilíbro $\widehat{p} \in \partial A\left(x^{s}\right)$ tal que $\varphi(t, q) \rightarrow \widehat{p}$ quando $t \rightarrow+\infty$. Pelo Lema 5.1.8, podemos concluir que $\widehat{p} \neq p$. Então $\widehat{p}$ é um ponto de equilíbrio hiperbólico ou um ponto de equilíbrio Hopf supercrítico. Pelos Lemas 5.1.1 ou 5.1.4 ou 5.1.7, concluímos que $\operatorname{dim} W^{u}(\widehat{p})<\operatorname{dim} W^{u}(p)$ e, portanto, $\operatorname{dim} W^{u}(\widehat{p})<1$. Segue-se $\operatorname{dim} W^{u}(\widehat{p})=0$ e, consequentemente, $\widehat{p}$ é um ponto de equilíbrio Hopf supercrítico tipo zero ou um ponto de equilíbrio hiperbólico tipo zero. Isto nos leva a uma contradição, pois pontos de equilíbrio do tipo zero não podem pertencer a $\partial A\left(x^{s}\right)$. Sendo assim, $q \in A\left(x^{s}\right)$ e, portanto, $W^{u}(p) \cap A\left(x^{s}\right) \neq \emptyset$.

Suponhamos que $\bar{x} \in \partial A\left(x^{s}\right)$. Do Teorema 3.7 de [CHW88], podemos concluir que $\left(W_{l o c}^{u}(\bar{x}) \backslash\{\bar{x}\}\right) \cap \overline{A\left(x^{s}\right)} \neq \emptyset$. Consequentemente, $\left(W^{u}(\bar{x}) \backslash\right.$ 
$\{\bar{x}\}) \cap \overline{A\left(x^{s}\right)} \neq \emptyset$, pois $W_{l o c}^{u}(\bar{x}) \subset W^{u}(\bar{x})$. Vamos mostrar, sob as supo-

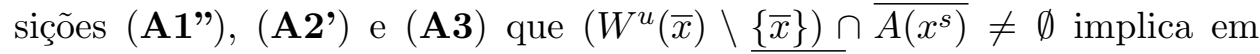
$W^{u}(\bar{x}) \cap A\left(x^{s}\right) \neq \emptyset$. Seja $q \in\left(W^{u}(\bar{x}) \backslash\{\bar{x}\}\right) \cap \overline{A\left(x^{s}\right)}$. Se $q \in A\left(x^{s}\right)$, não existe nada a ser provado. Suponhamos que $q \in \partial A\left(x^{s}\right)$. Da suposição (A3), existe um ponto de equilíbrio $\widehat{p} \in \partial A\left(x^{s}\right)$ tal que $\varphi(t, q) \rightarrow \widehat{p}$ quando $t \rightarrow+\infty$. Pela suposição (A1"), $\widehat{p}$ é um ponto de equilíbrio hiperbólico ou um ponto de equilíbrio Hopf supercrítico. Pelos Lemas 5.1.1 ou 5.1.4 ou 5.1.7, concluímos que $\operatorname{dim} W^{u}(\widehat{p})<\operatorname{dim} W^{u}(\bar{x})$ e, portanto, $\operatorname{dim} W^{u}(\widehat{p})<1$. Assim, $\operatorname{dim} W^{u}(\widehat{p})=0$ e, consenquentemente $\widehat{p}$ é um ponto de equilíbrio Hopf supercrítico tipo zero ou um ponto de equilíbrio hiperbólico tipo zero. Isto nos leva a uma contradição pois pontos de equilíbrio tipo zero não pertencem a $\partial A\left(x^{s}\right)$. Consequentemente, $q \in A\left(x^{s}\right)$ e, portanto, $W^{u}(\bar{x}) \cap A\left(x^{s}\right) \neq \emptyset$.

(ii) $(\Longleftarrow)$ Suponhamos que $W^{c s}(p) \subset \partial A\left(x^{s}\right)$. Como $p \in W^{c s}(p)$, então $p \in \partial A\left(x^{s}\right)$. Analogamente, suponhamos que $W^{s}(\bar{x}) \subset \partial A\left(x^{s}\right)$. Como $\bar{x} \in$ $W^{s}(\bar{x})$, então $\bar{x} \in \partial A\left(x^{s}\right)$.

$(\Longrightarrow)$ Suponhamos que $p \in \partial A\left(x^{s}\right)$. Pelo item (i) do Teorema 5.2.2, podemos concluir que $W^{u}(p) \cap A\left(x^{s}\right) \neq \emptyset$. Seja $y \in W^{u}(p) \cap A\left(x^{s}\right)$. Como $y \in W^{u}(p)$, então existe $t_{y}<0$ tal que $\varphi\left(t_{y}, y\right) \in W_{\text {loc }}^{u}(p)$. Seja $z=\varphi\left(t_{y}, y\right)$. Como $y \in A\left(x^{s}\right)$ e $A\left(x^{s}\right)$ é um conjunto invariante, então $z \in A\left(x^{s}\right)$. Seguese que $z \in W_{l o c}^{u}(p) \cap A\left(x^{s}\right)$. Seja $B(z, \varepsilon)$ uma bola aberta de raio $\varepsilon>0$ centrada em $z$, onde $\varepsilon$ é um número arbitrariamente pequeno. Seja $\widehat{q}$ um ponto arbitrário de $W^{c s}(p)$. Em particular, para algum $t_{\widehat{q}}>0$ temos $\widetilde{q}=$ $\varphi\left(t_{\widehat{q}}, \widehat{q}\right) \in W_{\text {loc }}^{c s}(p)$. Seja $S$ um disco no ponto $\widetilde{q} \operatorname{de} \operatorname{dim} S=1$ transversal a $W_{l o c}^{c s}(p)$. Pelo Lema 2.2.23, existe um ponto $w \in S$ e um tempo $t_{w}>0$ tal que $\varphi\left(t_{w}, w\right) \in B(z, \varepsilon)$. Como $A\left(x^{s}\right)$ é um conjunto invariante, então $w \in A\left(x^{s}\right)$. Como $\varepsilon$ e o disco $S$ podem ser escolhidos arbitrariamente pequenos, então existem pontos em $A\left(x^{s}\right)$ arbitrariamente próximos a $\widetilde{q}$. Consequentemente, $\widetilde{q} \in \overline{A\left(x^{s}\right)}$. Como $W_{l o c}^{c s}(p) \cap A\left(x^{s}\right)=\emptyset$, então $\widetilde{q} \in \partial A\left(x^{s}\right)$. Como $\partial A\left(x^{s}\right)$ é um conjunto invariante, $\widehat{q}=\varphi\left(-t_{\widehat{q}}, \widetilde{q}\right) \in \partial A\left(x^{s}\right)$. Como a escolha de $\widehat{q}$ em $W^{c s}(p)$ foi arbitrária, então podemos concluir que $W^{c s}(p) \subset \partial A\left(x^{s}\right)$.

A demonstração de que $W^{s}(\bar{x}) \subset \partial A\left(x^{s}\right)$ se $\bar{x} \in \partial A\left(x^{s}\right)$ é completamente análoga à primeira parte do item (i) do Teorema 5.2.2 e por isso será omitida.

O próximo teorema mostra a caracterização dos pontos de equilíbrio hiperbólico tipo- $k^{\prime}$, com $k^{\prime} \geq 2$ e Hopf supercrítico tipo- $k$, com $1<k \leq n-3$, na fronteira da região de estabilidade.

Teorema 5.2.3. (Pontos de equilíbrio tipo- $k$ na fronteira de estabilidade) Seja $A\left(x^{s}\right)$ a região de estabilidade de um ponto de equilíbrio assintoticamente estável $x^{s}$ do sistema autonômo (2.1). Sejam $p$ um ponto de equilíbrio Hopf supercrítico do tipo- $k$, com $1 \leq k \leq n-3$, e $\bar{x}$ um ponto de equilíbrio hiperbólico do tipo- $k^{\prime}$, com $1 \leq k^{\prime} \leq n$, do sistema (2.1). Se as suposições (A1"), (A2') e (A3) são satisfeitas, então: 
(i)

$$
\begin{aligned}
& p \in \partial A\left(x^{s}\right) \Longleftrightarrow W^{u}(p) \cap A\left(x^{s}\right) \neq \emptyset \\
& \bar{x} \in \partial A\left(x^{s}\right) \Longleftrightarrow W^{u}(\bar{x}) \cap A\left(x^{s}\right) \neq \emptyset
\end{aligned}
$$

(ii)

$$
\begin{aligned}
& p \in \partial A\left(x^{s}\right) \Longleftrightarrow W^{c s}(p) \subset \partial A\left(x^{s}\right) \\
& \bar{x} \in \partial A\left(x^{s}\right) \Longleftrightarrow W^{s}(\bar{x}) \subset \partial A\left(x^{s}\right)
\end{aligned}
$$

Demonstração. (i) $(\Longleftarrow)$ A demonstração é análoga à demonstração do Teorema 5.2 .2 e será omitida.

$(\Longrightarrow)$ Suponhamos que $x \in \partial A\left(x^{s}\right)$, onde $x$ é um ponto de equilíbrio hiperbólico ou um ponto de equilíbrio Hopf supercrítico. Demonstraremos o teorema usando indução finita na dimensão de $W^{u}(x)$. Se $\operatorname{dim} W^{u}(x)=1$, então, pelo Teorema 5.2.2, temos que $W^{u}(x) \cap A\left(x^{s}\right) \neq \emptyset$. Suponhamos que $W^{u}(x) \cap A\left(x^{s}\right) \neq \emptyset$ para todo os pontos de equilíbrio $x$ na fronteira $\partial A\left(x^{s}\right)$ com $\operatorname{dim} W^{u}(x) \leq k$. Agora, suponhamos que $\operatorname{dim} W^{u}(x)=k+1$. Pelo Teorema 5.2.1 e Teorema 3.7 de [CHW88], podemos concluir que $\left(W_{l o c}^{u}(x) \backslash\right.$ $\{x\}) \cap \overline{A\left(x^{s}\right)} \neq \emptyset$. Consequentemente, $\left(W^{u}(x) \backslash\{x\}\right) \cap \overline{A\left(x^{s}\right)} \neq \emptyset$, pois $W_{l o c}^{u}(x) \subset W^{u}(x)$. Vamos mostrar, sob as suposições (A1"), (A2') e (A3) que $\left(W^{u}(x) \backslash\{x\}\right) \cap \overline{A\left(x^{s}\right)} \neq \emptyset$ implica em $W^{u}(x) \cap A\left(x^{s}\right) \neq \emptyset$. Seja $q \in$ $\left(W^{u}(x) \backslash\{x\}\right) \cap \overline{A\left(x^{s}\right)}$. Se $q \in A\left(x^{s}\right)$, não existe nada a ser provado. Suponha que $q \in \partial A\left(x^{s}\right)$. Da condição (A3), existe um ponto de equilíbrio $\widehat{p} \in \partial A\left(x^{s}\right)$ tal que $\varphi(t, q) \rightarrow \widehat{p}$ quando $t \rightarrow+\infty$. De (A1"), conclui-se que $\widehat{p}$ é um ponto de equilíbrio hiperbólico ou um ponto de equilíbrio Hopf supercrítico. Pelos Lemas 5.1.1 ou 5.1.4 ou 5.1.7, se $x$ é um ponto de equilíbrio hiperbólico do tipo- $k$, com $1 \leq k \leq n$, ou um ponto de equilíbrio Hopf supercrítico do tipo- $k^{\prime}$, com $1 \leq k^{\prime} \leq n-2$, concluímos que $\operatorname{dim} W^{u}(\widehat{p})<\operatorname{dim} W^{u}(x)$. Seja $\widehat{p}$ um ponto de equilíbrio hiperbólico. Como $\operatorname{dim} W^{u}(\widehat{p})<\operatorname{dim} W^{u}(x)$, então concluímos que $\operatorname{dim} W^{u}(\widehat{p}) \leq k$. Portanto, pela hipótese de indução $W^{u}(\widehat{p}) \cap A\left(x^{s}\right) \neq \emptyset$. Sejam $y \in W^{u}(\widehat{p}) \cap A\left(x^{s}\right)$ e $B(y, \varepsilon)$ uma bola aberta de raio $\varepsilon>0$ centrada em $y$. Como $A\left(x^{s}\right)$ é um conjunto aberto, então $B(y, \varepsilon) \subset A\left(x^{s}\right)$ para $\varepsilon$ suficientemente pequeno. Seja $N^{u}$ uma vizinhança de $q$ em $W^{u}(x)$. A vizinhança $N^{u}$ contém uma seção transversal $D$ de $W^{s}(\widehat{p})$ no ponto $q$ com dimensão $\operatorname{dim} D \leq k$. Pelo Lema 2.2.19, existe um ponto $w \in D$ e um tempo $t_{w}>0$ tal que $\varphi\left(t_{w}, w\right) \in N^{u}$. Como $A\left(x^{s}\right)$ é um conjunto invariante, então $w \in A\left(x^{s}\right)$. Portanto, $w \in W^{u}(x) \cap A\left(x^{s}\right)$ e, consequentemente, $W^{u}(x) \cap A\left(x^{s}\right) \neq \emptyset$.

Se $\widehat{p}$ é um ponto de equilíbrio Hopf supercrítico e como $\operatorname{dim} W^{u}(\widehat{p})<$ $\operatorname{dim} W^{u}(x)$, então também concluímos que $\operatorname{dim} W^{u}(\widehat{p}) \leq k$. Portanto, pela hipótese de indução $W^{u}(\widehat{p}) \cap A\left(x^{s}\right) \neq \emptyset$. Seja $y \in W^{u}(\widehat{p}) \cap A\left(x^{s}\right)$ e $B(y, \varepsilon)$ uma bola aberta de raio $\varepsilon>0$ centrado em $y$. Como $A\left(x^{s}\right)$ é um conjunto aberto, então $B(y, \varepsilon) \subset A\left(x^{s}\right)$ para $\varepsilon$ suficientemente pequeno. Seja $N^{u}$ uma vizinhança de $q$ em $W^{u}(x)$. A vizinhança $N^{u}$ contém um seção transversal 
$D$ de $W^{c s}(\widehat{p})$, se $\widehat{p}$ é do tipo- $k$, com $1 \leq k \leq n-3$, no ponto $q$ com dimensão $\operatorname{dim} D \leq k$. Pelo Lema 2.2.23, existem um ponto $w \in D$ e um tempo $t_{w}>0$ tal que $\varphi\left(t_{w}, w\right) \in B(y ; \varepsilon)$. Como $A\left(x^{s}\right)$ é um conjunto invariante, então $w \in$ $A\left(x^{s}\right)$. Portanto, $w \in W^{u}(x) \cap A\left(x^{s}\right)$ e consequentemente, $W^{u}(x) \cap A\left(x^{s}\right) \neq \emptyset$.

(ii) A demonstração é análoga à demonstração do Teorema 5.2.2 e será omitida.

Para completar a caracterização dos pontos de equilíbrio na fronteira da região de estabilidade, resta mostrar a caracterização dos pontos de equilíbrio Hopf supercrítico na fronteira da região de estabilidade para tipo- $(n-2)$.

Teorema 5.2.4. (Ponto de equilíbrio Hopf supercrítico tipo- $(n-2)$ na fronteira de estabilidade): Seja $A\left(x^{s}\right)$ a região de estabilidade de um ponto de equilíbrio assintoticamente estável $x^{s}$ de (2.1). Seja $p$ um ponto de equilíbrio Hopf supercrítico do tipo-(n-2) de (2.1). Se as suposições (A1"), (A2') e (A3) são satisfeitas, então:

$$
\begin{gathered}
p \in \partial A\left(x^{s}\right) \Longleftrightarrow W^{u}(p) \cap A\left(x^{s}\right) \neq \emptyset \\
p \in \partial A\left(x^{s}\right) \Longleftrightarrow W^{c}(p) \subset \partial A\left(x^{s}\right)
\end{gathered}
$$

Demonstração. A demonstração é similar à demonstração do Teorema 5.2.2 e será omitida.

O próximo teorema oferece uma caracterização completa da fronteira da região de estabilidade quando existem pontos de equilíbrio Hopf supercríticos em $\partial A\left(x^{s}\right)$.

Teorema 5.2.5. (Caracterização da Fronteira de Estabilidade para Pontos de Equilíbrios): Seja $x^{s}$ um ponto de equilíbrio assintoticamente estável de (2.1) e $A\left(x^{s}\right)$ sua região de estabilidade. Se as suposições (A1") $e$ (A3) são satisfeitas, então

$$
\partial A\left(x^{s}\right) \subset \bigcup_{i} W^{s}\left(x_{i}\right) \bigcup_{j} W^{c s}\left(p_{j}\right) \bigcup_{l} W^{c}\left(q_{l}\right)
$$

onde $x_{i}$ são os pontos de equilíbrio hiperbólicos, $p_{j}$ os pontos de equilíbrio Hopf supercríticos do tipo- $k$, com $1 \leq k \leq n-3$, e $q_{l}$ os pontos de equilíbrio Hopf supercríticos do tipo- $(n-2)$ em $\partial A\left(x^{s}\right), i, j, l=1,2, \ldots$ Se a suposição (A2') é satisfeita, então

$$
\partial A\left(x^{s}\right)=\bigcup_{i} W^{s}\left(x_{i}\right) \bigcup_{j} W^{c s}\left(p_{j}\right) \bigcup_{l} W^{c}\left(q_{l}\right) .
$$

Demonstração. Seja $q \in \partial A\left(x^{s}\right)$. Pela hipótese (A3), podemos afirmar que existe um ponto de equilíbrio $x$ tal que $\varphi(t, q) \rightarrow x$ quando $t \rightarrow+\infty$. Pela hipótese (A1"), podemos afirmar que $x$ é um ponto de equilíbrio hiperbólico 
$x_{i}$ ou um ponto de equilíbrio Hopf supercrítico do tipo- $k$, com $1 \leq k \leq n-3$, $x=p_{j}$ ou um ponto de equilíbrio Hopf supercrítico do tipo- $(n-2) x=q_{l}$, ou seja, $x=x_{i}$ or $x=p_{j}$ or $x=q_{l}$ para algum $i, j, l$. Portanto, concluímos que $q \in \bigcup_{i} W^{s}\left(x_{i}\right) \bigcup_{j} W^{c s}\left(p_{j}\right) \bigcup_{l} W^{c}\left(q_{l}\right)$. Portanto,

$$
\partial A\left(x^{s}\right) \subset \bigcup_{i} W^{s}\left(x_{i}\right) \bigcup_{j} W^{c s}\left(p_{j}\right) \bigcup_{l} W^{c}\left(q_{l}\right) .
$$

Pelos Teoremas 5.2.2, 5.2.3 e 5.2.4, sabemos que $W^{s}\left(x_{i}\right) \subset \partial A\left(x^{s}\right), W^{c s}\left(p_{j}\right) \subset$ $\partial A\left(x^{s}\right)$ e $W^{c}\left(q_{l}\right) \subset \partial A\left(x^{s}\right)$. Logo,

$$
\bigcup_{i} W^{s}\left(x_{i}\right) \bigcup_{j} W^{c s}\left(p_{j}\right) \bigcup_{l} W^{c}\left(q_{l}\right) \subset \partial A\left(x^{s}\right)
$$

e, portanto,

$$
\partial A\left(x^{s}\right)=\bigcup_{i} W^{s}\left(x_{i}\right) \bigcup_{j} W^{c s}\left(p_{j}\right) \bigcup_{l} W^{c}\left(q_{l}\right) .
$$

\subsection{Ponto de equilíbrio Hopf Subcrítico em $\partial A\left(x^{s}\right)$}

Nesta seção, uma caracterização completa da fronteira da região de estabilidade na presença de pontos de equilíbrio Hopf Subcríticos na fronteira da região de estabilidade será desenvolvida.

A caracterização da fronteira da região de estabilidade na presença de um ponto de equilíbrio Hopf Subcrítico será desenvolvida em dois estágios. Primeiramente, estudaremos uma caracterização local da fronteira da região de estabilidade, estudando e caracterizando os pontos de equilíbrio que pertencem à fronteira da região de estabilidade, e em seguida, uma caracterização global será desenvolvida.

Os próximos teoremas oferecem condições necessárias e suficientes para garantir que um ponto de equilíbrio Hopf Subcrítico pertença à fronteira da região de estabilidade em termos das propriedades das suas variedades estável local, centro instável local e centro local.

Teorema 5.3.1. (Ponto de equilíbrio Hopf subcrítico na fronteira da região de estabilidade) Sejam $x^{s}$ um ponto de equilíbrio assintoticamente estável e $A\left(x^{s}\right)$ sua região de estabilidade. Seja $p$ um ponto de equilíbrio Hopf subcrítico de (2.1).

(i) Se p é um ponto de equilíbrio Hopf subcrítico tipo-0 de (2.1), então:

$$
\begin{gathered}
p \in \partial A\left(x^{s}\right) \Longleftrightarrow\left(W_{l o c}^{c}(p) \backslash\{p\}\right) \cap \overline{A\left(x^{s}\right)} \neq \emptyset \\
p \in \partial A\left(x^{s}\right) \Longleftrightarrow W_{l o c}^{s}(p) \cap \partial A\left(x^{s}\right) \neq \emptyset
\end{gathered}
$$


(ii) Se p é um ponto de equilíbrio Hopf subcrítico tipo-k de (2.1), com $1 \leq$ $k \leq n-3$, então:

$$
\begin{gathered}
p \in \partial A\left(x^{s}\right) \Longleftrightarrow\left(W_{l o c}^{c u}(p) \backslash\{p\}\right) \cap \overline{A\left(x^{s}\right)} \neq \emptyset \\
p \in \partial A\left(x^{s}\right) \Longleftrightarrow W_{l o c}^{s}(p) \cap \partial A\left(x^{s}\right) \neq \emptyset
\end{gathered}
$$

(iii) Se p é um ponto de equilíbrio Hopf subcrítico tipo-(n-2) de (2.1), então:

$$
p \in \partial A\left(x^{s}\right) \Longleftrightarrow\left(W_{l o c}^{c u}(p) \backslash\{p\}\right) \cap \overline{A\left(x^{s}\right)} \neq \emptyset
$$

Demonstração. (i) $(\Longleftarrow)$ Suponhamos que $\left(W_{l o c}^{c}(p) \backslash\{p\}\right) \cap \overline{A\left(x^{s}\right)} \neq \emptyset$. Então existe $q \in\left(W_{l o c}^{c}(p) \backslash\{p\}\right) \cap \overline{A\left(x^{s}\right)}$. Observe que $\varphi(t, q) \longrightarrow p$ quando $t \longrightarrow$ $-\infty$. Por outro lado, o conjunto $\overline{A\left(x^{s}\right)}$ é invariante e deste modo, $\varphi(t, q) \in$ $\overline{A\left(x^{s}\right)}$ para todo $t \leq 0$. Consequentemente, $p \in \overline{A\left(x^{s}\right)}$, visto que $\overline{A\left(x^{s}\right)}$ é um conjunto fechado. Como $p \notin A\left(x^{s}\right)$, temos que $p \in \mathbb{R}^{n} \backslash A\left(x^{s}\right)$. Portanto, $p \in \partial A\left(x^{s}\right)$. Agora suponhamos que $W_{\text {loc }}^{s}(p) \cap \partial A\left(x^{s}\right) \neq \emptyset$. Portanto, existe $q \in W_{l o c}^{s}(p) \cap \partial A\left(x^{s}\right)$. Note que $\varphi(t, q) \longrightarrow p$ quando $t \longrightarrow+\infty$. Como o conjunto $\partial A\left(x^{s}\right)$ é invariante e $q \in \partial A\left(x^{s}\right)$, então $\varphi(t, q) \in \partial A\left(x^{s}\right)$ para todo $t \geq 0$. Como $\partial A\left(x^{s}\right)$ é um conjunto fechado, portanto $p \in \partial A\left(x^{s}\right)$.

$(\Longrightarrow)$ Suponhamos que $p \in \partial A\left(x^{s}\right)$. Seja $N^{c}$ um domínio fundamental de $W^{c}(p)$, isto é, $\bigcup_{t \in \mathbb{R}} \varphi\left(t, N^{c}\right)=W^{c}(p) \backslash\{p\}$. Seja $N_{\varepsilon}^{c}$ uma vizinhança fundamental de raio $\varepsilon$ de $N^{c}$, isto é, $N_{\varepsilon}^{c}=\left\{x \in \mathbb{R}^{n}: d\left(x, N^{c}\right)<\varepsilon\right\}$. Pelo Corolário 2.2.26, existe uma vizinhança $U$ de $p$ tal que $\bigcup_{t \leq 0} \varphi\left(t, N_{\varepsilon}^{c}\right) \supset$ $U \backslash W_{l o c}^{s}(p)$. Como $p \in \partial A\left(x^{s}\right)$, então $U \cap A\left(x^{s}\right) \neq \emptyset$. Por outro lado, $W_{l o c}^{s}(p) \cap$ $A\left(x^{s}\right)=\emptyset$. Logo, $\left\{U \backslash W_{l o c}^{s}(p)\right\} \cap A\left(x^{s}\right) \neq \emptyset$. Consequentemente, existe um ponto $z \in N_{\varepsilon}^{c}$ e um tempo $\bar{t}$ tal que $\varphi(\bar{t}, z) \in A\left(x^{s}\right)$. Como $A\left(x^{s}\right)$ é um conjunto invariante, então $z \in A\left(x^{s}\right)$. Como $\varepsilon$ pode ser escolhido arbitrariamente pequeno, podemos encontrar uma sequência de pontos $\left\{z_{i}\right\}$ com $z_{i} \in A\left(x^{s}\right)$ para todo $i=1,2, \ldots$ tal que $d\left(z_{i}, N^{u}\right) \rightarrow 0$ quando $i \rightarrow+\infty$. Por construção, a sequência $\left\{z_{i}\right\}$ é limitada e, portanto, admite uma subsequência convergente. Seja $\left\{z_{i_{k}}\right\}$ uma subsequência convergente, isto é, $z_{i_{k}} \rightarrow \bar{z}$ quando $i_{k} \rightarrow+\infty$. Observe que $d\left(z_{i_{k}}, N^{c}\right) \rightarrow d\left(\bar{z}, N^{c}\right)$ quando $i_{k} \rightarrow+\infty$ e, portanto, $\bar{z} \in \overline{N^{c}} \subset W_{l o c}^{c}(q) \backslash\{q\}$. Logo, $\bar{z} \in\left(W_{l o c}^{c}(p) \backslash\right.$ $\{p\}) \cap \overline{A\left(x^{s}\right)}$. A demonstração de que $W_{l o c}^{s}(p) \cap \partial A\left(x^{s}\right) \neq \emptyset$ se $p \in \partial A\left(x^{s}\right)$ é muito similar à demonstração anterior e, portanto, será omitida.

As demonstrações dos itens (ii) e (iii) são similares à demonstração de (i) e serão omitidas.

O Teorema 5.3.1, além de ser relevante para o desenvolvimento de uma caracterização completa da fronteira da região de estabilidade na presença de pontos de equilíbrio Hopf Subcríticos na fronteira da região de estabilidade, fornece uma maneira de verificar se um ponto de equilíbrio Hopf Subcrítico pertence à fronteira da região de estabilidade. Para isto basta verificar se a sua variedade central e/ou centro-instável intersecta a região de estabilidade. 
Como uma consequência do Teorema 5.3.1, sabemos que $W_{l o c}^{c u}(p) \cap A\left(x^{s}\right) \neq$ $\emptyset$ é uma condição suficiente para guarantir que um ponto de equilíbrio Hopf subcrítico $p$ pertença à fronteira da região de estabilidade. Sob certas condições de transversalidade, verificaremos que esta condição é também necessária.

Os itens (i) e (ii) do Teorema 5.3.1 podem ser melhorados impondo-se algumas condições para o campo vetorial. Sejam $x^{s}$ um ponto de equilíbrio assintoticamente estável, $p$ um ponto de equilíbrio Hopf Subcrítico de (2.1), e consideremos as seguintes suposições:

(A1"') Todos os pontos de equilíbrio em $\partial A\left(x^{s}\right)$ são pontos de equilíbrio hiperbólicos ou pontos de equilíbrio do tipo Hopf subcrítico;

(A2") As variedades estável, instável, central e/ou centro instável dos pontos de equilíbrio em $\partial A\left(x^{s}\right)$ satisfazem a condição de transversalidade.

A condição (A1"') é mais fraca do que a condição (A1), uma vez que permite a presença de pontos de equilíbrio não hiperbólicos na fronteira da região de estabilidade.

Os próximos resultados fornecem condições necessárias e suficientes para garantir que os pontos de equilíbrio hiperbólicos e os pontos de equilíbrio Hopf Subcríticos pertençam à fronteira da região de estabilidade. Inicialmente apresentamos uma caracterização para pontos de equilíbrio hiperbólicos do tipo-1 e pontos de equilíbrio Hopf subcrítico tipo-zero na fronteira da região de estabilidade e então a caracterização para pontos de equilíbrio de tipos maiores seguirá por argumentos de indução.

Teorema 5.3.2. (Ponto de equilíbrio hiperbólico tipo-1 na fronteira de estabilidade) Seja $A\left(x^{s}\right)$ a região de estabilidade de um ponto de equilíbrio assintoticamente estável $x^{s}$ de (2.1). Seja $x^{\star}$ um ponto de equilíbrio hiperbólico do tipo-1 de (2.1). Se as suposições (A1"), (A2") e (A3) são satisfeitas, então:

(i)

$$
x^{\star} \in \partial A\left(x^{s}\right) \Longleftrightarrow W^{u}\left(x^{\star}\right) \cap A\left(x^{s}\right) \neq \emptyset
$$

$$
x^{\star} \in \partial A\left(x^{s}\right) \Longleftrightarrow W^{s}\left(x^{\star}\right) \subset \partial A\left(x^{s}\right)
$$

Demonstração. (i) $(\Longleftarrow)$ Suponhamos agora que $W^{u}\left(x^{\star}\right) \cap A\left(x^{s}\right) \neq \emptyset$. Como $A\left(x^{s}\right) \subset \overline{A\left(x^{s}\right)}$, então $\left(W_{l o c}^{u}\left(x^{\star}\right) \backslash\left\{x^{\star}\right\}\right) \cap \overline{A\left(x^{s}\right)} \neq \emptyset$. Portanto, pelo Teorema 3.7 de [CHW88], temos que $x^{\star} \in \partial A\left(x^{s}\right)$.

$(\Longrightarrow)$ Suponhamos que $x^{\star} \in \partial A\left(x^{s}\right)$. Do Teorema 3.7 de [CHW88], podemos concluir que $\left(W_{l o c}^{u}\left(x^{\star}\right) \backslash\left\{x^{\star}\right\}\right) \cap \overline{A\left(x^{s}\right)} \neq \emptyset$. Consequentemente, $\left(W^{u}\left(x^{\star}\right) \backslash\left\{x^{\star}\right\}\right) \cap \overline{A\left(x^{s}\right)} \neq \emptyset$, pois $W_{l o c}^{u}\left(x^{\star}\right) \subset W^{u}\left(x^{\star}\right)$. Vamos mostrar, sob as suposições (A1"'), (A2") e (A3) que $\left(W^{u}\left(x^{\star}\right) \backslash\left\{x^{\star}\right\}\right) \cap \overline{A\left(x^{s}\right)} \neq \emptyset$ implica em $W^{u}\left(x^{\star}\right) \cap A\left(x^{s}\right) \neq \emptyset$. Seja $q \in\left(W^{u}\left(x^{\star}\right) \backslash\left\{x^{\star}\right\}\right) \cap \overline{A\left(x^{s}\right)}$. Se $q \in A\left(x^{s}\right)$, 
não existe nada a ser provado. Suponhamos que $q \in \partial A\left(x^{s}\right)$. Da suposição (A3), existe um ponto de equilíbrio $\widehat{p} \in \partial A\left(x^{s}\right)$ tal que $\varphi(t, q) \rightarrow \widehat{p}$ quando $t \rightarrow-\infty$. Pela suposição (A1"'), $\widehat{p}$ é um ponto de equilíbrio hiperbólico ou um ponto de equilíbrio Hopf subcrítico. Pelo Lema 5.1.5, concluímos que $\operatorname{dim} W^{u}(\widehat{p})<\operatorname{dim} W^{u}\left(x^{\star}\right)$ e, portanto, $\operatorname{dim} W^{u}(\widehat{p})<1$. Assim, $\operatorname{dim} W^{u}(\widehat{p})=0$ e, consenquentemente $\widehat{p}$ é um ponto de equilíbrio hiperbólico tipo zero. Isto nos leva a uma contradição pois pontos de equilíbrio tipo zero não pertencem a $\partial A\left(x^{s}\right)$. Consequentemente, $q \in A\left(x^{s}\right)$ e, portanto, $W^{u}\left(x^{\star}\right) \cap A\left(x^{s}\right) \neq \emptyset$.

(ii) $(\Longleftarrow)$ Suponhamos que $W^{s}\left(x^{\star}\right) \subset \partial A\left(x^{s}\right)$. Como $p \in W^{s}(p)$, então $p \in \partial A\left(x^{s}\right)$.

$(\Longrightarrow)$ Suponhamos que $x^{\star} \in \partial A\left(x^{s}\right)$. Pela primeira parte do Teorema 5.3.2, podemos concluir que $W^{u}\left(x^{\star}\right) \cap A\left(x^{s}\right) \neq \emptyset$. Seja $y \in W^{u}\left(x^{\star}\right) \cap A\left(x^{s}\right)$. Como $y \in W^{u}\left(x^{\star}\right)$, então existe $T<0$ tal que $\varphi(T, y) \in W_{l o c}^{u}\left(x^{\star}\right)$. Seja $z=\varphi(T, y)$. Como $y \in A\left(x^{s}\right)$ e $A\left(x^{s}\right)$ é um conjunto invariante, então $z \in A\left(x^{s}\right)$. Segue-se que $z \in W_{l o c}^{u}\left(x^{\star}\right) \cap A\left(x^{s}\right)$. Seja $B(z, \varepsilon)$ uma bola aberta de raio $\varepsilon>0$ centrada em $z$ onde $\varepsilon$ é um número arbitrariamente pequeno. Seja $\widehat{q}$ um ponto arbitrário de $W^{s}\left(x^{\star}\right)$. Em particular, para algum $\bar{T}>0$ temos $\widetilde{q}=\varphi(\bar{T}, \widehat{q}) \in W_{l o c}^{s}\left(x^{\star}\right)$. Seja $S$ um disco em um ponto $\widetilde{q} \operatorname{de} \operatorname{dim} S=1$ transversal a $W_{l o c}^{s}\left(x^{\star}\right)$. Pelo Lema 2.2.25, existe um ponto $w \in S$ e um tempo $t_{w}>0$ tal que $\varphi\left(t_{w}, w\right) \in B(z, \varepsilon)$. Como $A\left(x^{s}\right)$ é um conjunto invariante, então $w \in A\left(x^{s}\right)$. Como $\varepsilon$ e o disco $S$ podem ser escolhidos arbitrariamente pequenos, então existem pontos em $A\left(x^{s}\right)$ arbitrariamente próximos a $\widetilde{q}$. Consequentemente, $\widetilde{q} \in \overline{A\left(x^{s}\right)}$. Como $W_{l o c}^{s}\left(x^{\star}\right) \cap A\left(x^{s}\right)=\emptyset$, então $\widetilde{q} \in \partial A\left(x^{s}\right)$. Como $\partial A\left(x^{s}\right)$ é um conjunto invariante, $\widehat{q}=\varphi(-\bar{T}, \widetilde{q}) \in \partial A\left(x^{s}\right)$. Como a escolha de $\widehat{q}$ em $W^{s}\left(x^{\star}\right)$ foi arbitrária, então podemos concluir que $W^{s}\left(x^{\star}\right) \subset \partial A\left(x^{s}\right)$.

Teorema 5.3.3. (Ponto de equilíbrio Hopf subcrítico tipo-0 na fronteira de estabilidade) Seja $A\left(x^{s}\right)$ a região de estabilidade de um ponto de equilíbrio assintoticamente estável $x^{s}$ de (2.1). Seja $p$ um ponto de equilíbrio Hopf subcrítico do tipo-0 de (2.1). Se as suposições (A1"'), (A2") e (A3) são satisfeitas, então:

(i)

$$
p \in \partial A\left(x^{s}\right) \Longleftrightarrow W^{c}(p) \cap A\left(x^{s}\right) \neq \emptyset
$$

(ii)

$$
p \in \partial A\left(x^{s}\right) \Longleftrightarrow W^{s}(p) \subset \partial A\left(x^{s}\right)
$$

Demonstração. (i) $(\Longleftarrow)$ Suponhamos que $W^{c}(p) \cap A\left(x^{s}\right) \neq \emptyset$. Como $A\left(x^{s}\right) \subset$ $\overline{A\left(x^{s}\right)}$, então $\left(W_{l o c}^{c}(p) \backslash\{p\}\right) \cap \overline{A\left(x^{s}\right)} \neq \emptyset$. Portanto, pelo item (i) do Teorema 5.3.1, temos que $p \in \partial A\left(x^{s}\right)$.

$(\Longrightarrow)$ Suponhamos que $p \in \partial A\left(x^{s}\right)$. Pelo Teorema 5.3.1, podemos concluir que $\left(W_{l o c}^{c}(p) \backslash\{p\}\right) \cap \overline{A\left(x^{s}\right)} \neq \emptyset$. Consequentemente, $\left(W^{c}(p) \backslash\{p\}\right) \cap$ 
$\overline{A\left(x^{s}\right)} \neq \emptyset$, pois $W_{l o c}^{c}(p) \subset W^{c}(p)$. Vamos mostrar, sob as suposições (A1"'),

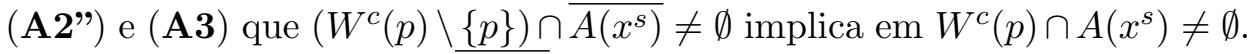
Seja $q \in\left(W^{c}(p) \backslash\{p\}\right) \cap \overline{A\left(x^{s}\right)}$. Se $q \in A\left(x^{s}\right)$, então não existe nada a ser provado. Suponhamos que $q \in \partial A\left(x^{S}\right)$. Da condição (A3), existe um ponto de equilíbro $\widehat{p} \in \partial A\left(x^{s}\right)$ tal que $\varphi(t, q) \rightarrow \widehat{p}$ quando $t \rightarrow-\infty$. Pelo Lema 5.1.9, podemos concluir que $\widehat{p} \neq p$. Então $\widehat{p}$ é um ponto de equilíbrio hiperbólico ou um ponto de equilíbrio Hopf subcrítico. Pelos Lemas 5.1.2 ou 5.1.5 ou 5.1.3 ou 5.1.6, concluímos que $\operatorname{dim} W^{c u}(\widehat{p})<\operatorname{dim} W^{c u}(p)$ ou $\operatorname{dim} W^{c}(\widehat{p})<\operatorname{dim} W^{c u}(p)$ se $\widehat{p}$ é um ponto de equilíbrio Hopf subcrítico ou $\operatorname{dim} W^{u}(\widehat{p})<\operatorname{dim} W^{c u}(p)$ se $\widehat{p}$ é um ponto de equilíbrio hiperbólico. Se $\widehat{p}$ é um ponto de equilíbrio Hopf subcrítico, então $\operatorname{dim} W^{c u}(\widehat{p})<2$ ou $\operatorname{dim} W^{c}(\widehat{p})<2$, o que é uma contradição pois a variedade central de um ponto de equilíbrio Hopf subcrítico tem pelo menos dimensão 2. Seja $\widehat{p}$ um ponto de equilíbrio hiperbólico e, portanto, $\operatorname{dim} W^{u}(\widehat{p})<2$. Segue-se que $\operatorname{dim} W^{u}(\widehat{p})=1$, pois pontos de equilíbrio hiperbólicos do tipo zero não podem pertencer à fronteira da região de estabilidade. Logo, pelo Teorema 5.3.2, $W^{u}(\widehat{p}) \cap A\left(x^{s}\right) \neq \emptyset$. Sejam $y \in W^{u}(\widehat{p}) \cap A\left(x^{s}\right)$ e $B(y, \varepsilon)$ uma bola aberta de raio $\varepsilon>0$ centrada em $y$. Como $A\left(x^{s}\right)$ é um conjunto aberto, então $B(y, \varepsilon) \subset A\left(x^{s}\right)$ para $\varepsilon$ suficientemente pequeno. Seja $N^{c}$ uma vizinhança de $q$ em $W^{c}(p)$. A vizinhança $N^{c}$ contém uma seção transversal $D$ de $W^{s}(\widehat{p})$ no ponto $q$ com dimensão $\operatorname{dim} D=2$. Pelo Lema 2.2.25, existe um ponto $w \in D$ e um tempo $t_{w}>0$ tal que $\varphi\left(t_{w}, w\right) \in N^{c}$. Como $A\left(x^{s}\right)$ é um conjunto invariante, então $w \in A\left(x^{s}\right)$. Portanto, $w \in W^{c}(p) \cap A\left(x^{s}\right)$ e, consequentemente, $W^{c}(p) \cap A\left(x^{s}\right) \neq \emptyset$.

(ii) $(\Longleftarrow)$ Suponhamos que $W^{s}(p) \subset \partial A\left(x^{s}\right)$. Como $p \in W^{s}(p)$, então $p \in \partial A\left(x^{s}\right)$.

$(\Longrightarrow)$ Suponhamos que $p \in \partial A\left(x^{s}\right)$. Pela primeira parte do Teorema 5.3.2, podemos concluir que $W^{c}(p) \cap A\left(x^{s}\right) \neq \emptyset$. Seja $y \in W^{c}(p) \cap A\left(x^{s}\right)$. Como $y \in W^{c}(p)$, então existe $T<0$ tal que $\varphi(T, y) \in W_{l o c}^{c}(p)$. Seja $z=\varphi(T, y)$. Como $y \in A\left(x^{s}\right)$ e $A\left(x^{s}\right)$ é um conjunto invariante, então $z \in A\left(x^{s}\right)$. Segue-se que $z \in W_{l o c}^{c}(p) \cap A\left(x^{s}\right)$. Seja $B(z, \varepsilon)$ uma bola aberta de raio $\varepsilon>0$ centrada em $z$ onde $\varepsilon$ é um número arbitrariamente pequeno. Seja $\widehat{q}$ um ponto arbitrário de $W^{s}(p)$. Em particular, para algum $\bar{T}>0$ temos $\widetilde{q}=\varphi(\bar{T}, \widehat{q}) \in W_{\text {loc }}^{s}(p)$. Seja $S$ um disco em um ponto $\widetilde{q} \operatorname{de} \operatorname{dim} S=2$ transversal a $W_{l o c}^{s}(p)$. Pelo Lema 2.2.25, existe um ponto $w \in S$ e um tempo $t_{w}>0$ tal que $\varphi\left(t_{w}, w\right) \in B(z, \varepsilon)$. Como $A\left(x^{s}\right)$ é um conjunto invariante, então $w \in A\left(x^{S}\right)$. Como $\varepsilon$ e o disco $S$ podem ser escolhidos arbitrariamente pequenos, então existem pontos em $A\left(x^{s}\right)$ arbitrariamente próximos a $\widetilde{q}$. Consequentemente, $\widetilde{q} \in \overline{A\left(x^{s}\right)}$. Como $W_{l o c}^{s}(p) \cap A\left(x^{s}\right)=\emptyset$, então $\widetilde{q} \in \partial A\left(x^{s}\right)$. Como $\partial A\left(x^{s}\right)$ é um conjunto invariante, $\widehat{q}=\varphi(-\bar{T}, \widetilde{q}) \in \partial A\left(x^{s}\right)$. Como a escolha de $\widehat{q}$ em $W^{s}(p)$ foi arbitrária, então podemos concluir que $W^{s}(p) \subset$ $\partial A\left(x^{s}\right)$.

Teorema 5.3.4. (Ponto de equilíbrio hiperbólico tipo-2 na fronteira 
de estabilidade) Seja $A\left(x^{s}\right)$ a região de estabilidade de um ponto de equilíbrio assintoticamente estável $x^{s}$ de (2.1). Seja $x^{\star}$ um ponto de equilíbrio hiperbólico do tipo-2 de (2.1). Se as suposições (A1"'), (A2") e (A3) são satisfeitas, então:

(i)

$$
x^{\star} \in \partial A\left(x^{s}\right) \Longleftrightarrow W^{u}\left(x^{\star}\right) \cap A\left(x^{s}\right) \neq \emptyset
$$

(ii)

$$
x^{\star} \in \partial A\left(x^{s}\right) \Longleftrightarrow W^{s}\left(x^{\star}\right) \subset \partial A\left(x^{s}\right)
$$

Demonstração. (i) $(\Longleftarrow)$ Suponhamos que $W^{u}\left(x^{\star}\right) \cap A\left(x^{s}\right) \neq \emptyset$. Como $A\left(x^{s}\right) \subset \overline{A\left(x^{s}\right)}$, então $\left(W_{l o c}^{u}\left(x^{\star}\right) \backslash\left\{x^{\star}\right\}\right) \cap \overline{A\left(x^{s}\right)} \neq \emptyset$. Portanto, pelo Teorema 3.7 de [CHW88], temos que $x^{\star} \in \partial A\left(x^{s}\right)$.

$(\Longrightarrow)$ Suponhamos que $x^{\star} \in \partial A\left(x^{s}\right)$. Do Teorema 3.7 de [CHW88], podemos concluir que $\left(W_{l o c}^{u}\left(x^{\star}\right) \backslash\left\{x^{\star}\right\}\right) \cap \overline{A\left(x^{s}\right)} \neq \emptyset$. Consequentemente, $\left(W^{u}\left(x^{\star}\right) \backslash\left\{x^{\star}\right\}\right) \cap \overline{A\left(x^{s}\right)} \neq \emptyset$, pois $W_{l o c}^{u}\left(x^{\star}\right) \subset W^{u}\left(x^{\star}\right)$. Vamos mostrar, sob as suposições (A1"'), (A2") e (A3) que $\left(W^{u}\left(x^{\star}\right) \backslash\left\{x^{\star}\right\}\right) \cap \overline{A\left(x^{s}\right)} \neq \emptyset$ implica em $W^{u}\left(x^{\star}\right) \cap A\left(x^{s}\right) \neq \emptyset$. Seja $q \in\left(W^{u}\left(x^{\star}\right) \backslash\left\{x^{\star}\right\}\right) \cap \overline{A\left(x^{s}\right)}$. Se $q \in A\left(x^{s}\right)$, não existe nada a ser provado. Suponhamos que $q \in \partial A\left(x^{s}\right)$. Da suposição (A3), existe um ponto de equilíbrio $\widehat{p} \in \partial A\left(x^{s}\right)$ tal que $\varphi(t, q) \rightarrow \widehat{p}$ quando $t \rightarrow+\infty$. Pela suposição (A1"'), $\widehat{p}$ é um ponto de equilíbrio hiperbólico ou um ponto de equilíbrio Hopf subcrítico. Pelos Lemas 5.1.2 ou 5.1.5 ou 5.1.3 ou 5.1.6, concluímos que $\operatorname{dim} W^{c u}(\widehat{p})<\operatorname{dim} W^{u}\left(x^{\star}\right)$ ou $\operatorname{dim} W^{c}(\widehat{p})<\operatorname{dim} W^{u}\left(x^{\star}\right)$ se $\widehat{p}$ é um ponto de equilíbrio Hopf subcrítico ou $\operatorname{dim} W^{u}(\widehat{p})<\operatorname{dim} W^{u}\left(x^{\star}\right)$ se $\widehat{p}$ é um ponto de equilíbrio hiperbólico. Se $\widehat{p}$ é um ponto de equilíbrio Hopf subcrítico, então $\operatorname{dim} W^{c u}(\widehat{p})<2$ ou $\operatorname{dim} W^{c}(\widehat{p})<2$, o que é uma contradição pois a variedade central de um ponto de equilíbrio Hopf subcrítico tem pelo menos dimensão 2. Seja $\widehat{p}$ um ponto de equilíbrio hiperbólico e, portanto, $\operatorname{dim} W^{u}(\widehat{p})<2$. Segue-se que $\operatorname{dim} W^{u}(\widehat{p})=1$, pois pontos de equilíbrios hiperbólicos do tipo zero não podem pertencer à fronteira da região de estabilidade. Logo, pelo Teorema 5.3.2, $W^{u}(\widehat{p}) \cap A\left(x^{s}\right) \neq \emptyset$. Sejam $y \in W^{u}(\widehat{p}) \cap A\left(x^{s}\right)$ e $B(y, \varepsilon)$ uma bola aberta de raio $\varepsilon>0$ centrada em $y$. Como $A\left(x^{s}\right)$ é um conjunto aberto, então $B(y, \varepsilon) \subset A\left(x^{s}\right)$ para $\varepsilon$ suficientemente pequeno. Seja $N^{c}$ uma vizinhança de $q$ em $W^{u}\left(x^{\star}\right)$. A vizinhança $N^{c}$ contém uma seção $D$ transversal a $W^{s}(\widehat{p})$ no ponto $q$ com dimensão $\operatorname{dim} D=1$. Pelo Lema 2.2.19, existe um ponto $w \in D$ e um tempo $t_{w}>0$ tal que $\varphi\left(t_{w}, w\right) \in N^{c}$. Como $A\left(x^{s}\right)$ é um conjunto invariante, então $w \in A\left(x^{s}\right)$. Portanto, $w \in W^{u}\left(x^{\star}\right) \cap A\left(x^{s}\right)$ e, consequentemente, $W^{u}\left(x^{\star}\right) \cap A\left(x^{s}\right) \neq \emptyset$.

(ii) $(\Longleftarrow)$ Suponhamos $W^{s}\left(x^{\star}\right) \subset \partial A\left(x^{s}\right)$. Como $p \in W^{s}(p)$, então $p \in$ $\partial A\left(x^{s}\right)$.

$(\Longrightarrow)$ Suponhamos que $x^{\star} \in \partial A\left(x^{s}\right)$. Pela primeira parte do Teorema 5.3.2, podemos concluir que $W^{u}\left(x^{\star}\right) \cap A\left(x^{s}\right) \neq \emptyset$. Seja $y \in W^{u}\left(x^{\star}\right) \cap A\left(x^{s}\right)$. 
Como $y \in W^{u}\left(x^{\star}\right)$, então existe $T<0$ tal que $\varphi(T, y) \in W_{l o c}^{u}\left(x^{\star}\right)$. Seja $z=\varphi(T, y)$. Como $y \in A\left(x^{s}\right)$ e $A\left(x^{s}\right)$ é um conjunto invariante, então $z \in A\left(x^{s}\right)$. Segue-se que $z \in W_{\text {loc }}^{u}\left(x^{\star}\right) \cap A\left(x^{s}\right)$. Seja $B(z, \varepsilon)$ uma bola aberta de raio $\varepsilon>0$ centrada em $z$ onde $\varepsilon$ é um número arbitrariamente pequeno. Seja $\widehat{q}$ um ponto arbitrário de $W^{s}\left(x^{\star}\right)$. Em particular, para algum $\bar{T}>0$ temos $\widetilde{q}=\varphi(\bar{T}, \widehat{q}) \in W_{l o c}^{s}\left(x^{\star}\right)$. Seja $S$ um disco em um ponto $\widetilde{q} \operatorname{de} \operatorname{dim} S=1$ transversal a $W_{l o c}^{s}\left(x^{\star}\right)$. Pelo Lema 2.2.19, existe um ponto $w \in S$ e um tempo $t_{w}>0$ tal que $\varphi\left(t_{w}, w\right) \in B(z, \varepsilon)$. Como $A\left(x^{s}\right)$ é um conjunto invariante, então $w \in A\left(x^{s}\right)$. Como $\varepsilon$ e o disco $S$ podem ser escolhidos arbitrariamente pequenos, então existem pontos em $A\left(x^{s}\right)$ arbitrariamente próximos a $\widetilde{q}$. Consequentemente, $\widetilde{q} \in \overline{A\left(x^{s}\right)}$. Como $W_{l o c}^{s}\left(x^{\star}\right) \cap A\left(x^{s}\right)=\emptyset$, então $\widetilde{q} \in \partial A\left(x^{s}\right)$. Como $\partial A\left(x^{s}\right)$ é um conjunto invariante, $\widehat{q}=\varphi(-\bar{T}, \widetilde{q}) \in \partial A\left(x^{s}\right)$. Como a escolha de $\widehat{q} \mathrm{em} W^{s}\left(x^{\star}\right)$ foi arbitrária, então podemos concluir que $W^{s}\left(x^{\star}\right) \subset \partial A\left(x^{s}\right)$.

Teorema 5.3.5. (Ponto de equilíbrio Hopf subcrítico tipo-1 na fronteira de estabilidade) Seja $A\left(x^{s}\right)$ a região de estabilidade de um ponto de equilíbrio assintoticamente estável $x^{s}$ de (2.1). Seja $p$ um ponto de equilíbrio Hopf subcrítico do tipo-1 de (2.1). Se as suposições (A1"'), (A2") e (A3) são satisfeitas, então:

(i)

$$
p \in \partial A\left(x^{s}\right) \Longleftrightarrow W^{c u}(p) \cap A\left(x^{s}\right) \neq \emptyset
$$

(ii)

$$
p \in \partial A\left(x^{s}\right) \Longleftrightarrow W^{s}(p) \subset \partial A\left(x^{s}\right)
$$

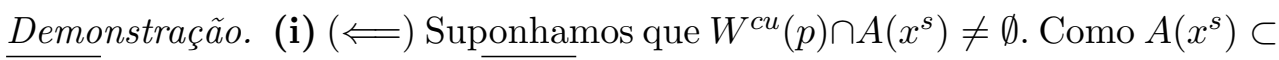
$\overline{A\left(x^{s}\right)}$, então $\left(W_{l o c}^{c u}(p) \backslash\{p\}\right) \cap \overline{A\left(x^{s}\right)} \neq \emptyset$. Portanto, pelo item (ii) do Teorema 5.3.2, temos que $p \in \partial A\left(x^{s}\right)$.

$(\Longrightarrow)$ Suponhamos que $p \in \partial A\left(x^{s}\right)$. Pelo Teorema 5.3.1, podemos concluir que $\left(W_{l o c}^{c u}(p) \backslash\{p\}\right) \cap \overline{A\left(x^{s}\right)} \neq \emptyset$. Consequentemente, $\left(W^{c u}(p) \backslash\{p\}\right) \cap$ $\overline{A\left(x^{s}\right)} \neq \emptyset$, pois $W_{l o c}^{c u}(p) \subset W^{c u}(p)$. Vamos mostrar, sob as suposições $\left(\mathbf{A} 1^{\prime \prime \prime}\right)$, (A2") e (A3) que $\left(W^{c u}(p) \backslash\{p\}\right) \cap \overline{A\left(x^{s}\right)} \neq \emptyset$ implica em $W^{c u}(p) \cap A\left(x^{s}\right) \neq \emptyset$. Seja $q \in\left(W^{c u}(p) \backslash\{p\}\right) \cap \overline{A\left(x^{s}\right)}$. Se $q \in A\left(x^{s}\right)$, então não existe nada a ser provado. Suponhamos que $q \in \partial A\left(x^{s}\right)$. Da condição (A3), existe um ponto de equilíbro $\widehat{p} \in \partial A\left(x^{s}\right)$ tal que $\varphi(t, q) \rightarrow \widehat{p}$ quando $t \rightarrow+\infty$. Pelo Lema 5.1.9, podemos concluir que $\widehat{p} \neq p$. Então $\widehat{p}$ é um ponto de equilíbrio hiperbólico ou um ponto de equilíbrio Hopf subcrítico. Pelos Lemas 5.1.2 ou 5.1.5 ou 5.1.3 ou 5.1.6, concluímos que $\operatorname{dim} W^{c u}(\widehat{p})<\operatorname{dim} W^{c u}(p)$ ou $\operatorname{dim} W^{c}(\widehat{p})<\operatorname{dim} W^{c u}(p)$ se $\widehat{p}$ é um ponto de equilíbrio Hopf subcrítico ou $\operatorname{dim} W^{u}(\widehat{p})<\operatorname{dim} W^{c u}(p)$ se $\widehat{p}$ é um ponto de equilíbrio hiperbólico. Se $\widehat{p}$ é um ponto de equilíbrio Hopf subcrítico, então $\operatorname{dim} W^{c u}(\widehat{p})<3$ ou $\operatorname{dim} W^{c}(\widehat{p})<3$. Segue-se que $\operatorname{dim} W^{c}(\widehat{p})=2$, pois a variedade central de um ponto de equilíbrio Hopf subcrítico tem pelo menos dimensão 2. Logo, 
pelo Teorema 5.3.3, $W^{c}(\widehat{p}) \cap A\left(x^{s}\right) \neq \emptyset$. Sejam $y \in W^{c}(\widehat{p}) \cap A\left(x^{s}\right)$ e $B(y, \varepsilon)$ uma bola aberta de raio $\varepsilon>0$ centrada em $y$. Como $A\left(x^{s}\right)$ é um conjunto aberto, então $B(y, \varepsilon) \subset A\left(x^{s}\right)$ para $\varepsilon$ suficientemente pequeno. Seja $N^{c}$ uma vizinhança de $q$ em $W^{c u}(p)$. A vizinhança $N^{c}$ contém uma seção transversal $D$ de $W^{s}(\widehat{p})$ no ponto $q$ com dimensão $\operatorname{dim} D=2$. Pelo Lema 2.2.25, existe um ponto $w \in D$ e um tempo $t_{w}>0$ tal que $\varphi\left(t_{w}, w\right) \in N^{c}$. Como $A\left(x^{s}\right)$ é um conjunto invariante, então $w \in A\left(x^{s}\right)$. Portanto, $w \in W^{c u}(p) \cap A\left(x^{s}\right)$ e, consequentemente, $W^{c u}(p) \cap A\left(x^{s}\right) \neq \emptyset$.

Se $\widehat{p}$ é um ponto de equilíbrio hiperbólico e, portanto, $\operatorname{dim} W^{u}(\widehat{p})<3$, segue-se que $\operatorname{dim} W^{u}(\widehat{p})=1$ ou $\operatorname{dim} W^{u}(\widehat{p})=2$, pois pontos de equilíbrios hiperbólicos do tipo zero não podem pertencer à fronteira da região de estabilidade. Logo, pelos Teorema 5.3.2 ou 5.3.4, $W^{u}(\widehat{p}) \cap A\left(x^{s}\right) \neq \emptyset$. Sejam $y \in W^{u}(\widehat{p}) \cap A\left(x^{s}\right)$ e $B(y, \varepsilon)$ uma bola aberta de raio $\varepsilon>0$ centrada em $y$. Como $A\left(x^{s}\right)$ é um conjunto aberto, então $B(y, \varepsilon) \subset A\left(x^{s}\right)$ para $\varepsilon$ suficientemente pequeno. Seja $N^{c}$ uma vizinhança de $q$ em $W^{c u}(p)$. A vizinhança $N^{c}$ contém uma seção transversal $D$ de $W^{s}(\widehat{p})$ no ponto $q$ com dimensão $\operatorname{dim} D=2$. Pelo Lema 2.2.19, existe um ponto $w \in D$ e um tempo $t_{w}>0$ tal que $\varphi\left(t_{w}, w\right) \in N^{c}$. Como $A\left(x^{s}\right)$ é um conjunto invariante, então $w \in A\left(x^{s}\right)$. Portanto, $w \in W^{c u}(p) \cap A\left(x^{s}\right)$ e, consequentemente, $W^{c u}(p) \cap A\left(x^{s}\right) \neq \emptyset$.

(ii) $(\Longleftarrow)$ Suponhamos que $W^{s}(p) \subset \partial A\left(x^{s}\right)$. Como $p \in W^{s}(p)$, então $p \in \partial A\left(x^{s}\right)$.

$(\Longrightarrow)$ Suponhamos que $p \in \partial A\left(x^{s}\right)$. Pela primeira parte do Teorema 5.3.5, podemos concluir que $W^{c u}(p) \cap A\left(x^{s}\right) \neq \emptyset$. Seja $y \in W^{c u}(p) \cap A\left(x^{s}\right)$. Como $y \in W^{c u}(p)$, então existe $T<0$ tal que $\varphi(T, y) \in W_{l o c}^{c u}(p)$. Seja $z=\varphi(T, y)$. Como $y \in A\left(x^{s}\right)$ e $A\left(x^{s}\right)$ é um conjunto invariante, então $z \in A\left(x^{s}\right)$. Segue-se que $z \in W_{l o c}^{c u}(p) \cap A\left(x^{s}\right)$. Seja $B(z, \varepsilon)$ uma bola aberta de raio $\varepsilon>0$ centrada em $z$ onde $\varepsilon$ é um número arbitrariamente pequeno. Seja $\widehat{q}$ um ponto arbitrário de $W^{s}(p)$. Em particular, para algum $\bar{T}>0$ temos $\widetilde{q}=\varphi(\bar{T}, \widehat{q}) \in W_{l o c}^{s}(p)$. Seja $S$ um disco em um ponto $\widetilde{q} \operatorname{de} \operatorname{dim} S=3$ transversal a $W_{l o c}^{s}(p)$. Pelo Lema 2.2.25, existe um ponto $w \in S$ e um tempo $t_{w}>0$ tal que $\varphi\left(t_{w}, w\right) \in B(z, \varepsilon)$. Como $A\left(x^{s}\right)$ é um conjunto invariante, então $w \in A\left(x^{s}\right)$. Como $\varepsilon$ e o disco $S$ podem ser escolhidos arbitrariamente pequenos, então existem pontos em $A\left(x^{s}\right)$ arbitrariamente próximos a $\widetilde{q}$. Consequentemente, $\widetilde{q} \in \overline{A\left(x^{s}\right)}$. Como $W_{l o c}^{s}(p) \cap A\left(x^{s}\right)=\emptyset$, então $\widetilde{q} \in \partial A\left(x^{s}\right)$. Como $\partial A\left(x^{s}\right)$ é um conjunto invariante, $\widehat{q}=\varphi(-\bar{T}, \widetilde{q}) \in \partial A\left(x^{s}\right)$. Como a escolha de $\widehat{q}$ em $W^{s}(p)$ foi arbitrária, então podemos concluir que $W^{s}(p) \subset$ $\partial A\left(x^{s}\right)$.

O próximo teorema mostra a caracterização dos pontos de equilíbrio hiperbólicos e Hopf subcríticos na fronteira da região de estabilidade para tipo- $k$, com $1 \leq k \leq n-3$.

Teorema 5.3.6. (Pontos de equilíbrio tipo- $k$ na fronteira de estabilidade): Seja $A\left(x^{s}\right)$ a região de estabilidade de um ponto de equilíbrio 
assintoticamente estável $x^{s}$ de (2.1) e suponha que as seguintes suposições (A1"), (A2") e (A3) sejam satisfeitas. Sejam p um ponto equilibrio Hopf subcrítico do tipo- $k$, com $1 \leq k \leq n-3$, e $x^{\star}$ um ponto de equilíbrio hiperbólico do tipo-k', com $k^{\prime} \leq n$, de (2.1). Então:

(i)

$$
\begin{gathered}
p \in \partial A\left(x^{s}\right) \Longleftrightarrow W^{c u}(p) \cap A\left(x^{s}\right) \neq \emptyset \\
x^{\star} \in \partial A\left(x^{s}\right) \Longleftrightarrow W^{u}\left(x^{\star}\right) \cap A\left(x^{s}\right) \neq \emptyset
\end{gathered}
$$

(ii)

$$
\begin{aligned}
p \in \partial A\left(x^{s}\right) & \Longleftrightarrow W^{s}(p) \subset \partial A\left(x^{s}\right) \\
x^{\star} \in \partial A\left(x^{s}\right) & \Longleftrightarrow W^{s}\left(x^{\star}\right) \subset \partial A\left(x^{s}\right)
\end{aligned}
$$

Demonstração. (i) ( ) A demonstração é análoga à demonstração dos teorema anteriores e será omitida.

$(\Longrightarrow)$ Demonstraremos o teorema usando indução finita na dimensão de $W^{c u}(x)$ ou $W^{u}(x)$ se $x \in \partial A\left(x^{s}\right)$ é um ponto de equilíbrio Hopf subcrítico ou um ponto de equilíbrio hiperbólico. Se $\operatorname{dim} W^{u}(x)=1$, então pelo Teorema 5.3.2 ou 5.3.5 sabemos que $W^{u}(x) \cap A\left(x^{s}\right) \neq \emptyset$ ou $W^{c u}(x) \cap A\left(x^{s}\right) \neq \emptyset$. Suponha que $W^{u}(x) \cap A\left(x^{s}\right) \neq \emptyset$ ou $W^{c u}(x) \cap A\left(x^{s}\right) \neq \emptyset$ para todos os pontos de equilíbrio $x$ na fronteira $\partial A\left(x^{s}\right) \operatorname{com} \operatorname{dim} W^{u}(x) \leq k$. Agora, suponhamos que $\operatorname{dim} W^{u}(x)=k+1$. Pelo Teorema 5.3.1 ou Teorema $3.7 \mathrm{de}$ [CHW88], podemos concluir que $\left(W_{l o c}^{i}(x) \backslash\{x\}\right) \cap \overline{A\left(x^{s}\right)} \neq \emptyset$, para $i=c u$ ou $i=u$ respectivamente. Consequentemente $\left(W^{i}(x) \backslash\{x\}\right) \cap \overline{A\left(x^{s}\right)} \neq \emptyset$, pois $W_{l o c}^{i}(x) \subset W^{i}(x)$. Vamos mostrar, sob as suposições (A1"'), (A2") e (A3) que $\left(W^{i}(x) \backslash\{x\}\right) \cap \overline{A\left(x^{s}\right)} \neq \emptyset$ implica em $W^{i}(x) \cap A\left(x^{s}\right) \neq \emptyset$. Seja $q \in\left(W^{i}(x) \backslash\{p\}\right) \cap \overline{A\left(x^{s}\right)}$. Se $q \in A\left(x^{s}\right)$, não existe nada a ser provado. Suponha que $q \in \partial A\left(x^{s}\right)$. Da condição (A3), existe um ponto de equilíbrio $\widehat{p} \in \partial A\left(x^{s}\right)$ tal que $\varphi(t, q) \rightarrow \widehat{p}$ quando $t \rightarrow+\infty$. De (A1"'), concluise que $\widehat{p}$ é um ponto de equilíbrio hiperbólico ou um ponto de equilíbrio Hopf subcrítico. Pelos Lemas 5.1.2 ou 5.1.5 ou 5.1.3 ou 5.1.6, se $x$ é um ponto de equilíbrio hiperbólico ou um ponto de equilíbrio Hopf subcrítico do tipo- $k$, com $1 \leq k \leq n-3$, ou um ponto de equilíbrio Hopf subcrítico do tipo- $(n-2)$ respectivamente, concluímos que $\operatorname{dim} W^{j}(\widehat{p})<\operatorname{dim} W^{i}(x)$, para $j=u$ se $\widehat{p}$ é um ponto de equilíbrio hiperbólico ou $j=c u$ se $\widehat{p}$ é um ponto de equilíbrio Hopf subcrítico. Seja $\widehat{p}$ um ponto de equilíbrio hiperbólico. Como $\operatorname{dim} W^{u}(\widehat{p})<\operatorname{dim} W^{i}(x)$, então concluímos que $\operatorname{dim} W^{u}(\widehat{p}) \leq k$. Portanto, pela hipótese de indução $W^{u}(\widehat{p}) \cap A\left(x^{s}\right) \neq \emptyset$. Sejam $y \in W^{u}(\widehat{p}) \cap A\left(x^{s}\right)$ e $B(y, \varepsilon)$ uma bola aberta de raio $\varepsilon>0$ centrada em $y$. Como $A\left(x^{s}\right)$ é um conjunto aberto, então $B(y, \varepsilon) \subset A\left(x^{s}\right)$ para $\varepsilon$ suficientemente pequeno. Seja $N^{i}$ uma vizinhança de $q$ em $W^{i}(x)$. A vizinhança $N^{i}$ contém uma seção transversal $D$ de $W^{s}(\widehat{p})$ no ponto $q$ com dimensão $\operatorname{dim} D \leq k$. Pelo Lema 2.2.19, existe um ponto $w \in D$ e um tempo $t_{w}>0$ tal que $\varphi\left(t_{w}, w\right) \in N^{i}$. 
Como $A\left(x^{s}\right)$ é um conjunto invariante, então $w \in A\left(x^{s}\right)$. Portanto, $w \in$ $W^{i}(x) \cap A\left(x^{s}\right)$ e, consequentemente, $W^{i}(x) \cap A\left(x^{s}\right) \neq \emptyset$.

Se $\widehat{p}$ é um ponto de equilíbrio Hopf subcrítico e como $\operatorname{dim} W^{c u}(\widehat{p})<$ $\operatorname{dim} W^{i}(x)$, então também concluímos que $\operatorname{dim} W^{c u}(\widehat{p}) \leq k$. Portanto, pela hipótese de indução $W^{c u}(\widehat{p}) \cap A\left(x^{s}\right) \neq \emptyset$. Sejam $y \in W^{c u}(\widehat{p}) \cap A\left(x^{s}\right)$ e $B(y, \varepsilon)$ uma bola aberta de raio $\varepsilon>0$ centrada em $y$. Como $A\left(x^{s}\right)$ é um conjunto aberto, então $B(y, \varepsilon) \subset A\left(x^{s}\right)$ para $\varepsilon$ suficientemente pequeno. Seja $N^{i}$ uma vizinhança de $q$ em $W^{i}(x)$. A vizinhança $N^{i}$ contém um seção transversal $D$ de $W^{s}(\widehat{p})$ no ponto $q$ com dimensão $\operatorname{dim} D \leq k$. Pelo Lema 2.2.25, existem um ponto $w \in D$ e um tempo $t_{w}>0$ tal que $\varphi\left(t_{w}, w\right) \in N^{i}$. Como $A\left(x^{s}\right)$ é um conjunto invariante, então $w \in A\left(x^{s}\right)$. Portanto, $w \in W^{i}(x) \cap A\left(x^{s}\right)$ e consequentemente, $W^{i}(x) \cap A\left(x^{s}\right) \neq \emptyset$.

(ii) A demonstração é análoga à demonstração dos Teoremas $5.3 .2 \mathrm{ou}$ 5.3.5 e será omitida.

Para completar a caracterização dos pontos de equilíbrio na fronteira da região de estabilidade, resta mostrar a caracterização dos pontos de equilíbrio Hopf subcrítico na fronteira da região de estabilidade para tipo- $(n-2)$.

Teorema 5.3.7. (Ponto de equilíbrio Hopf subcrítico tipo- $(n-2)$ na fronteira da região de estabilidade): Seja $A\left(x^{s}\right)$ a região de estabilidade de um ponto de equilíbrio assintoticamente estável $x^{s}$ de (2.1). Seja $p$ um ponto de equilíbrio Hopf subcrítico do tipo- $(n-2)$ de (2.1). Se as suposições (A1"'), (A2") e (A3) são satisfeitas, então:

$$
p \in \partial A\left(x^{s}\right) \Longleftrightarrow W^{c u}(p) \cap A\left(x^{s}\right) \neq \emptyset
$$

Demonstração. A demonstração é similar à demonstração do Teorema 5.3.5 e será omitida.

O próximo teorema oferece uma caracterização completa da fronteira da região de estabilidade quando existem pontos de equilíbrio Hopf subcríticos em $\partial A\left(x^{s}\right)$.

Teorema 5.3.8. (Caracterização da Fronteira de Estabilidade): Seja $x^{s}$ um ponto de equilíbrio assintoticamente estável de (2.1) e $A\left(x^{s}\right)$ sua região de estabilidade. Se as suposições (A1"') e (A3) são satisfeitas, então

$$
\partial A\left(x^{s}\right) \subset \bigcup_{i} W^{s}\left(x_{i}\right) \bigcup_{j} W^{s}\left(p_{j}\right)
$$

onde $x_{i}$ são os pontos de equilíbrio hiperbólicos e $p_{j}$ os pontos de equilíbrio Hopf subcríticos em $\partial A\left(x^{s}\right), i, j=1,2, \ldots$ Se a suposição (A2") é satisfeita, então

$$
\partial A\left(x^{s}\right)=\bigcup_{i} W^{s}\left(x_{i}\right) \bigcup_{j} W^{s}\left(p_{j}\right) .
$$


Demonstração. Seja $q \in \partial A\left(x^{s}\right)$. Pela hipótese (A3), podemos afirmar que existe um ponto de equilíbrio $x$ tal que $\varphi(t, q) \rightarrow x$ quando $t \rightarrow+\infty$. Pela hipótese (A1"'), podemos afirmar que $x$ é um ponto de equilíbrio hiperbólico $x_{i}$ ou um ponto de equilíbrio Hopf subcrítico $p_{j}$, ou seja, $x=x_{i}$ ou $x=p_{j}$ para algum $i, j$. Portanto, concluímos que $q \in \bigcup_{i} W^{s}\left(x_{i}\right) \bigcup_{j} W^{s}\left(p_{j}\right)$. Portanto, $\partial A\left(x^{s}\right) \subset \bigcup_{i} W^{s}\left(x_{i}\right) \bigcup_{j} W^{s}\left(p_{j}\right)$. Pelos Teoremas 5.3.3, 5.3.6 e 5.3.7, sabemos que $W^{s}\left(x_{i}\right) \subset \partial A\left(x^{s}\right)$ e $W^{s}\left(p_{j}\right) \subset \partial A\left(x^{s}\right)$. Logo, $\bigcup_{i} W^{s}\left(x_{i}\right) \bigcup_{j} W^{s}\left(p_{j}\right) \subset$ $\partial A\left(x^{s}\right)$ e, portanto,

$$
\partial A\left(x^{s}\right)=\bigcup_{i} W^{s}\left(x_{i}\right) \bigcup_{j} W^{s}\left(p_{j}\right)
$$

\subsection{Exemplos}

Exibiremos um exemplo que ilustra os teoremas discutidos nas seções 5.2 e 5.3 .

Exemplo 5.4.1. Considere o sistema dinâmico autônomo não-linear extraído de [GJAA13]

$$
\left\{\begin{array}{l}
\dot{x}=-x z-y-x\left(x^{2}+y^{2}\right) \\
\dot{y}=-y z+x-y\left(x^{2}+y^{2}\right) \\
\dot{z}=-0.1\left(z+0.5\left(x^{2}+y^{2}\right)\right)(z-3)(8-z)
\end{array}\right.
$$

onde $(x, y, z) \in \mathbb{R}^{3}$.

O sistema (5.2) tem três pontos de equilíbrio, são eles: um ponto de equilíbrio assintoticamente estável $x^{s}=(0,0,3)$, um ponto de equilíbrio hiperbólico do tipo-1 $x_{1}=(0,0,8)$ e um ponto de equilibrio Hopf supercrítico do tipo-1 $p=(0,0,0)$. Consequentemente, as suposições $\left(\boldsymbol{A} \mathbf{1}^{\prime \prime}\right)$ e $\left(\boldsymbol{A} \mathscr{2}^{\prime}\right)$ são satisfeitas.

A função $V(x, y, z)=\frac{x^{2}}{2}+\frac{y^{2}}{2}-\frac{z^{4}}{4}+\frac{11 z^{3}}{3}-12 z^{2}$ é um função energia para o sistema (5.2). De fato, $\dot{V}=-\left(x^{2}+y^{2}\right) z^{2}-\left(x^{2}+y^{2}\right)^{2}-z^{2}(z-$ $3)^{2}(8-z)^{2} \leq 0$ e a afirmação (E1) é satisfeita. A derivada de $V$ é igual a zero somente nos pontos de equilíbrio, consequentemente a afirmação (E2) também é satisfeita. E finalmente, se a solução $\varphi\left(t, x_{0}\right)$ é ilimitada para $t \geq 0$, então $V\left(\varphi\left(t, x_{0}\right)\right)$ é também ilimitada para $t \geq 0$. Como consequência, a afirmação (E3) é satisfeita e $V$ é uma função energia para o sistema (5.2).

A existência de uma função energia implica que a afirmação (A3) é satisfeita. Consequentemente, as afirmações dos Teoremas 5.2.2 e 5.2.4 são satisfeitas e a caracterização da fronteira da região de estabilidade desenvolvida no Teorema 5.2.5 também é satisfeita. A variedade instável do ponto de equilíbrio Hopf supercrítico do tipo-1 p intersecta a região de estabilidade de 
$x^{s}$, consequentemente, de acordo com o Teorema 5.2.4, p pertence à fronteira da região de estabilidade $\partial A\left(x^{s}\right)$ e a variedade central está contida na fronteira da região de estabilidade de $x^{s}$, veja Figura 5.1. A variedade instável do ponto de equilíbrio hiperbólico do tipo-1 $x_{1}$ também intersecta a região de estabilidade de $x^{s}$ e, portanto, $x_{1}$ também pertence à fronteira da região de estabilidade de $x^{s}$ e a variedade estável está contida na fronteira da região de estabilidade de $x^{s}$, de acordo com o Teorema 5.2.2, veja Figura 5.1.

A Figura 5.1 ilustra a fronteira da região de estabilidade do ponto de equilibrio assintoticamente estável $x^{s}=(0,0,3)$. A fronteira da região de estabilidade de $x^{s}=(0,0,3)$ é formada, de acordo com o Teorema 5.2.5, pela união da variedade estável do ponto de equilíbrio hiperbólico do tipo-1 $x_{1}=(0,0,8)$, a superfície sombreada mais alta passando por $x_{1}$ na Figura 5.1, com a variedade central do ponto de equilíbrio Hopf supercrítico do tipo$1 p=(0,0,0)$, a superfície sombreada mais baixa passando por $p$ na Figura 5.1

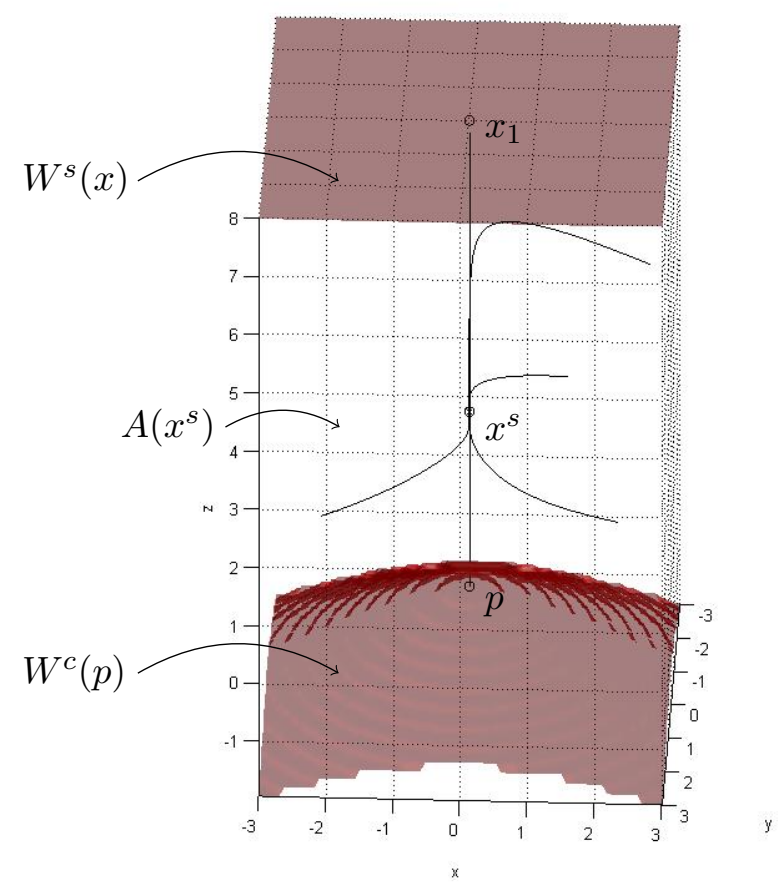

Figura 5.1: Retrato de fase do sistema (5.2). A fronteira da região de estabilidade de $x^{s}=(0,0,3)$ é formada pela união da variedade estável $W^{s}(x)$ do ponto de equilíbrio hiperbólico do tipo-1 $x_{1}=(0,0,8)$ com a variedade central $W^{c}(p)$ do ponto de equilíbrio Hopf supercrítico do tipo-1 $p=(0,0,0)$. 


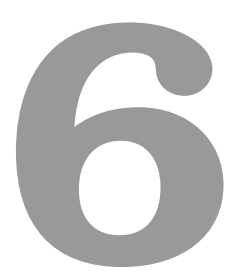

\section{Fronteira da Região de Estabilidade na Presença de um Ponto de Equilíbrio Hopf com Elementos Críticos}

No capítulo anterior, sob as suposições $\left(\mathbf{A} 1^{\prime \prime}\right),\left(\mathbf{A} 1^{\prime \prime \prime}\right),\left(\mathbf{A} 2^{\prime}\right),\left(\mathbf{A} 2^{\prime \prime}\right)$ e (A3), caracterizamos a fronteira da região de estabilidade de um ponto de equilíbrio asssintoticamente estável, na presença de um ponto de equilíbrio Hopf na fronteira, como sendo a união das variedades dos pontos de equilíbrio hiperbólicos com as variedades centro-estável, central e/ou estável dos pontos de equilíbrio Hopf pertencentes à fronteira da região da estabilidade. Neste capítulo, impondo uma condição sobre a transversalidade das variedades, faremos uma generalização da caracterização da fronteira da região de estabilidade desenvolvida no capítulo anterior, admitindo agora também a existência de órbitas periódicas na fronteira da região de estabilidade do ponto de equilíbrio assintoticamente estável.

\subsection{Lemas Auxiliares e Condição de Transversali- dade}

Sabemos que se $x$ é um ponto de equilíbrio hiperbólico do tipo- $k$, então existem as variedades invariantes $k$-dimensional instável $W_{l o c}^{u}(x)$ e $(n-k)$ dimensional estável $W_{l o c}^{s}(x)$ e, portanto,

$$
\operatorname{dim} W_{l o c}^{u}(x)+\operatorname{dim} W_{l o c}^{s}(x)=n .
$$

O resultado a seguir estabelece também uma equação envolvendo as di- 
Capítulo 6. Fronteira da Região de Estabilidade na Presença de um Ponto 102 de Equilíbrio Hopf com Elementos Críticos

mensões das variedades estável e instável de uma órbita periódica hiperbólica.

Lema 6.1.1. Se $\phi$ é uma órbita periódica hiperbólica, então

$$
\operatorname{dim} W_{l o c}^{u}(\phi)+\operatorname{dim} W_{l o c}^{s}(\phi)=n+1 .
$$

Demonstração. Como $\phi$ é uma órbita fechada hiperbólica então, pelo Teorema 2.2.17, existem as variedades invariantes $(k+1)$-dimensional instável $W_{l o c}^{u}(\phi)$ e $(n-k)$-dimensional estável $W_{l o c}^{s}(\phi)$. Além disso, como as variedades $W_{l o c}^{u}(\phi)$ e $W_{l o c}^{s}(\phi)$ se intersectam transversalmente em $\phi$, então

$$
\operatorname{dim} W_{l o c}^{u}(\phi)+\operatorname{dim} W_{l o c}^{s}(\phi)=n+1 .
$$

Para acomodar a presença de órbitas periódicas na fronteira da região de estabilidade, redefiniremos a condição de transversalidade enunciada no Capítulo 2.

Sejam $\Psi_{i}$ e $\Psi_{j}$ variedades de dimensões $n$ e $l$. Dizemos que as variedades $W^{u}\left(\Psi_{i}\right)$ e $W^{s}\left(\Psi_{j}\right)$ em $\mathbb{R}^{n}$ de classe $C^{r}(r \geq 1)$ são transversais, se $W^{u}\left(\Psi_{i}\right) \cap W^{s}\left(\Psi_{j}\right)=\emptyset$; ou se $x \in W^{u}\left(\Psi_{i}\right) \cap W^{s}\left(\Psi_{j}\right) \neq \emptyset$, então $\operatorname{dim}\left(W^{u}\left(\Psi_{i}\right) \cap W^{s}\left(\Psi_{j}\right)\right) \geq 1+\max \{n, l\}$ e $T_{x}\left(W^{u}\left(\Psi_{i}\right)\right)+T_{x}\left(W^{s}\left(\Psi_{j}\right)\right)=\mathbb{R}^{n}$, onde $T_{x}\left(W^{u}\left(\Psi_{i}\right)\right)$ e $T_{x}\left(W^{s}\left(\Psi_{j}\right)\right)$ denotam o espaço tangente de $W^{u}\left(\Psi_{i}\right)$ e $W^{s}\left(\Psi_{j}\right)$, respectivamente, no ponto $x$.

Observemos que a definição de transversalidade acima implica na definição de transversalidade enunciada na seção 2.2.8 do Capítulo 2. Por exemplo, se $\Psi_{i}$ e $\Psi_{j}$ são pontos de equilibrios hiperbólicos e que satisfazem a condição de transversalidade definida acima, então $W^{u}\left(\Psi_{i}\right) \cap W^{s}\left(\Psi_{j}\right)=\emptyset$ ou se $x \in W^{u}\left(\Psi_{i}\right) \cap W^{s}\left(\Psi_{j}\right) \neq \emptyset$, então $T_{x}\left(W^{u}\left(\Psi_{i}\right)\right)+T_{x}\left(W^{s}\left(\Psi_{j}\right)\right)=\mathbb{R}^{n}$ e $\operatorname{dim}\left(W^{u}\left(\Psi_{i}\right) \cap W^{s}\left(\Psi_{j}\right)\right) \geq 1$, pois $\operatorname{dim}\left(\Psi_{i}\right)=\operatorname{dim}\left(\Psi_{j}\right)=0$.

Com a nova definição de condição de transversalidade, generalizaremos os resultados da Seção 5.1 do Capítulo 5 com o propósito de desenvolver uma caracterização da fronteira da região de estabilidade supondo também a existência de órbitas pediódicas na fronteira da região de estabilidade.

Os Lemas 6.1.2 a 6.1.7 generalizam os Lemas 5.1.1 a 5.1.7 do capítulo anterior considerando a possibilidade do elemento crítico ser uma órbita periódica.

Lema 6.1.2. Sejam $\widetilde{x}$ um ponto de equilíbrio Hopf supercrítico do tipo-k ou um elemento crítico hiperbólico do tipo- $k$, com $k \geq 1$, e $x$ um elemento crítico hiperbólico do sistema (2.1). Se $W^{u}(\widetilde{x})$ e $W^{s}(x)$ satisfazem a condição de transversalidade e $W^{u}(\widetilde{x}) \cap W^{s}(x) \neq \emptyset$, então $\operatorname{dim} W^{u}(x)<\operatorname{dim} W^{u}(\widetilde{x})$.

Demonstração. Seja $\widetilde{x}$ um ponto de equilíbrio Hopf supercrítico do tipo- $k$, com $k \geq 1$. Como $W^{u}(\widetilde{x})$ e $W^{s}(x)$ satisfazem a condição de transversalidade, 
então temos

$$
\operatorname{dim} W^{u}(\widetilde{x})+\operatorname{dim} W^{s}(x)=n+\operatorname{dim}\left(W^{u}(\widetilde{x}) \cap W^{s}(x)\right) .
$$

Se $x$ é uma órbita periódica, temos

$$
\operatorname{dim} W^{u}(x)+\operatorname{dim} W^{s}(x)=n+1 .
$$

Como $W^{u}(\widetilde{x})$ e $W^{s}(x)$ satisfazem a condição de transversalidade e $W^{u}(\widetilde{x})$ $\bigcap W^{s}(x) \neq \emptyset$, então $\operatorname{dim}\left(W^{u}(\widetilde{x}) \cap W^{s}(x)\right) \geq 2$. Consequentemente,

$$
\operatorname{dim} W^{u}(\widetilde{x})+\operatorname{dim} W^{s}(x) \geq n+2,
$$

$\mathrm{ou}$

$$
\operatorname{dim} W^{u}(\widetilde{x}) \geq n+2-\operatorname{dim} W^{s}(x) .
$$

Substituindo a equação (6.1) na desigualdade anterior, obtemos

$$
\operatorname{dim} W^{u}(\widetilde{x}) \geq \operatorname{dim} W^{u}(x)+1
$$

e, assim,

$$
\operatorname{dim} W^{u}(\widetilde{x})>\operatorname{dim} W^{u}(x) .
$$

Para os demais casos a demonstração é análoga e portanto, será omitida.

As demonstrações dos Lemas 6.1.3 e 6.1.4 são inteiramente análogas à demonstração do Lema 6.1.2 e, portanto, serão omitidas.

Lema 6.1.3. Seja $\widetilde{x}$ um ponto de equilíbrio Hopf subcrítico do tipo-0 e $x$ um elemento crítico hiperbólico do sistema (2.1). Se $W^{c}(\widetilde{x})$ e $W^{s}(x)$ satisfazem a condição de transversalidade e $W^{c}(\widetilde{x}) \cap W^{s}(x) \neq \emptyset$, então $\operatorname{dim} W^{u}(x)<$ $\operatorname{dim} W^{c}(\widetilde{x})$.

Lema 6.1.4. Seja $\widetilde{x}$ um ponto de equilíbrio Hopf subcrítico do tipo-k, com $k \geq 1$, e $x$ um elemento crítico hiperbólico do sistema (2.1). Se $W^{c u}(\widetilde{x})$ e $W^{s}(x)$ satisfazem a condição de transversalidade e $W^{c u}(\widetilde{x}) \cap W^{s}(x) \neq \emptyset$, então $\operatorname{dim} W^{u}(x)<\operatorname{dim} W^{c u}(\widetilde{x})$.

Lema 6.1.5. Sejam $\widetilde{x}$ um elemento crítico hiperbólico do tipo-k ou um ponto de equilíbrio Hopf supercrítico do tipo- $k$, com $k \geq 1$, e $p$ um ponto de equilibrio Hopf supercrítico do tipo- $k^{\prime}$, com $1 \leq k^{\prime} \leq n-3$, do sistema (2.1). Se $W^{u}(\widetilde{x})$ e $W^{c s}(p)$ satisfazem a condição de transversalidade $e$ $W^{u}(\widetilde{x}) \cap W^{c s}(p) \neq \emptyset$, então $\operatorname{dim} W^{u}(p)<\operatorname{dim} W^{u}(\widetilde{x})$.

Demonstração. Seja $\widetilde{x}$ uma órbita periódica hiperbólica do tipo- $k, \operatorname{com} k \geq$ 1. Como $W^{u}(x)$ e $W^{c s}(p)$ satisfazem a condição de transversalidade, então 
Capítulo 6. Fronteira da Região de Estabilidade na Presença de um Ponto 104 de Equilíbrio Hopf com Elementos Críticos

temos

$$
\operatorname{dim} W^{u}(x)+\operatorname{dim} W^{c s}(p)=n+\operatorname{dim}\left(W^{u}(x) \bigcap W^{c s}(p)\right) .
$$

Como $W^{u}(\widetilde{x})$ e $W^{c s}(p)$ satisfazem a condição de transversalidade e $W^{u}(\widetilde{x})$ $\cap W^{c s}(p) \neq \emptyset$, então $\operatorname{dim}\left(W^{u}(\widetilde{x}) \cap W^{c s}(p)\right) \geq 2$. Consequentemente,

$$
\operatorname{dim} W^{u}(x)+\operatorname{dim} W^{c s}(p) \geq n+2,
$$

$\mathrm{ou}$

$$
\operatorname{dim} W^{u}(x) \geq n+2-\operatorname{dim} W^{c s}(p) .
$$

Como $p$ é um ponto de equilíbrio Hopf supercrítico do tipo- $k^{\prime}$, com $1 \leq k^{\prime} \leq$ $n-3$, temos que

$$
\operatorname{dim} W^{u}(p)+\operatorname{dim} W^{c s}(p)=n .
$$

Portanto, a desigualdade anterior nos fornece

$$
\operatorname{dim} W^{u}(x) \geq \operatorname{dim} W^{u}(p)+2,
$$

e assim

$$
\operatorname{dim} W^{u}(x)>\operatorname{dim} W^{u}(p) .
$$

A prova é análoga ao caso anterior para os demais casos.

As demonstrações dos Lemas 6.1 .6 e 6.1 .7 são inteiramente análogas às demonstrações dos Lemas 6.1.2 e 6.1.5 e, portanto, serão omitidas.

Lema 6.1.6. Sejam $\widetilde{x}$ um elemento crítico hiperbólico do tipo-k (ou um ponto de equilíbrio Hopf subcrítico do tipo-k), com $k \geq 0$, e p um ponto de equilíbrio Hopf subcrítico do tipo- $k^{\prime}$, com $0 \leq k^{\prime} \leq n-3$, do sistema (2.1). Se $W^{u}(\widetilde{x})$ (ou $\left.W^{c u}(\widetilde{x})\right)$ e $W^{s}(p)$ satisfazem a condição de transversalidade e $W^{u}(\widetilde{x}) \cap W^{s}(p) \neq \emptyset$ (ou $\left.W^{c u}(\widetilde{x}) \bigcap W^{s}(p) \neq \emptyset\right)$, então $\operatorname{dim} W^{c u}(p)<$ $\operatorname{dim} W^{u}(\widetilde{x})$ (ou $\operatorname{dim} W^{c u}(p)<\operatorname{dim} W^{c u}(\widetilde{x})$, respectivamente).

Lema 6.1.7. Sejam $\widetilde{x}$ ou um ponto de equilíbrio Hopf supercrítico do tipo$k$ ou um elemento crítico hiperbólico do tipo- $k$, com $k \geq 1$, e $p$ um ponto de equilíbrio Hopf supercrítico do tipo- $(n-2)$ do sistema (2.1). Se $W^{u}(\widetilde{x})$ e $W^{c}(p)$ satisfazem a condição de transversalidade e $W^{u}(\widetilde{x}) \cap W^{c}(p) \neq \emptyset$, então $\operatorname{dim} W^{u}(p)<\operatorname{dim} W^{u}(\widetilde{x})$.

\subsection{Ponto de equilíbrio Hopf Supercrítico em $\partial A\left(x^{s}\right)$}

Nesta seção, considerando a nova definição de condição de transversalidade, generalizaremos a caracterização da fronteira de estabilidade na presença de um ponto de equilíbrio Hopf supercrítico na fronteira da região de 
estabilidade admitindo também na fronteira a existência de órbitas periódicas.

A generalização do Teorema 5.2.2 pode ser melhorada se impusermos novas condições para o campo vetorial. Sejam $x^{s}$ um ponto de equilíbrio assintoticamente estável e $p$ um ponto de equilíbrio Hopf supercrítico do sistema (2.1) e considere as seguintes afirmações:

(B1') Todos os elementos críticos em $\partial A\left(x^{s}\right)$ são elementos críticos hiperbólicos ou pontos de equilíbrio Hopf supercríticos;

(B2') As variedades estáveis, centro-estáveis e/ou centrais e as variedades instáveis dos elementos críticos em $\partial A\left(x^{s}\right)$ satisfazem segundo a condição de transversalidade definida neste capítulo.

Assim como na condição $\left(\mathbf{A} 1^{\prime}\right)$, a condição $\left(\mathbf{B} 1^{\prime}\right)$ é mais fraca do que a condição (B1), uma vez que permite a presença de pontos de equilíbrio nãohiperbólicos na fronteira da região de estabilidade. Os próximos resultados fornecem condições necessárias e suficientes para garantir que os elementos críticos hiperbólicos e os pontos de equilíbrio Hopf supercríticos pertençam à fronteira da região de estabilidade. Inicialmente, mostraremos a caracterização dos elementos críticos do tipo-1 na fronteira da região de estabilidade e, em seguida, a caracterização para elementos criticos de tipos superiores a 1 seguirá por argumentos de indução.

Teorema 6.2.1. (Elementos críticos do tipo-1 na fronteira da região de estabilidade) Seja $A\left(x^{s}\right)$ a região de estabilidade de um ponto de equilíbrio assintoticamente estável $x^{s}$ do sistema autonômo (2.1). Sejam $p$ um ponto de equilbrio Hopf supercrítico do tipo-1 e $\psi$ um elemento crítico do tipo-1 do sistema (2.1). Se as suposições (B1'), (B2') e (B3) são satisfeitas, então:

(i)

$$
\begin{gathered}
p \in \partial A\left(x^{s}\right) \Longleftrightarrow W^{u}(p) \cap A\left(x^{s}\right) \neq \emptyset \\
\psi \text { está na fronteira } \partial A\left(x^{s}\right) \Longleftrightarrow W^{u}(\psi) \cap A\left(x^{s}\right) \neq \emptyset
\end{gathered}
$$

$$
\begin{gathered}
p \in \partial A\left(x^{s}\right) \Longleftrightarrow W^{c s}(p) \subset \partial A\left(x^{s}\right) \\
\psi \text { está na fronteira } \partial A\left(x^{s}\right) \Longleftrightarrow W^{s}(\psi) \subset \partial A\left(x^{s}\right)
\end{gathered}
$$

Demonstração. (i) $(\Longleftarrow)$ A demonstração de que $p \in \partial A\left(x^{s}\right)$ se $W^{u}(p) \cap$ $A\left(x^{s}\right) \neq \emptyset$ é análoga à demonstração do Teorema 5.2 .2 e será omitida.

Suponhamos que $W^{u}(\psi) \cap A\left(x^{s}\right) \neq \emptyset$. Como $A\left(x^{s}\right) \subset \overline{A\left(x^{s}\right)}$, então $W^{u}(\psi) \cap \overline{A\left(x^{s}\right)} \neq \emptyset$. Portanto, pelo Teorema 3.8 de [CHW88], temos que $\psi$ está em $\partial A\left(x^{s}\right)$.

$(\Longrightarrow)$ Suponhamos que $p \in \partial A\left(x^{s}\right)$. Pelo Teorema 5.2.1, podemos concluir que $\left(W_{l o c}^{u}(p) \backslash\{p\}\right) \cap \overline{A\left(x^{s}\right)} \neq \emptyset$. Consequentemente, $\left(W^{u}(p) \backslash\{p\}\right) \cap$ 
Capítulo 6. Fronteira da Região de Estabilidade na Presença de um Ponto 106 de Equilíbrio Hopf com Elementos Críticos

$\overline{A\left(x^{s}\right)} \neq \emptyset$, pois $W_{\text {loc }}^{u}(p) \subset W^{u}(p)$. Vamos mostrar, sob as suposições (B1'), (B2') e (B3) que $\left(W^{u}(p) \backslash\{p\}\right) \cap \overline{A\left(x^{s}\right)} \neq \emptyset$ implica em $W^{u}(p) \cap A\left(x^{s}\right) \neq \emptyset$. Seja $q \in\left(W^{u}(p) \backslash\{p\}\right) \cap \overline{A\left(x^{s}\right)}$. Se $q \in A\left(x^{s}\right)$, então não existe nada a ser provado. Suponhamos que $q \in \partial A\left(x^{s}\right)$. Da condição (B3), existe um elemento crítico $\widehat{\psi} \in \partial A\left(x^{s}\right)$ tal que $\varphi(t, q) \rightarrow \widehat{\psi}$ quando $t \rightarrow+\infty$. Da condição (B1'), $\widehat{\psi}$ é ou um elemento crítico hiperbólico ou um ponto de equilíbrio Hopf supercrítico. Pelos Lemas 6.1.2 ou 6.1.5 ou 6.1.7, concluímos que $\operatorname{dim} W^{u}(\widehat{\psi})<\operatorname{dim} W^{u}(p)$ e, portanto, $\operatorname{dim} W^{u}(\widehat{\psi})<1$. Então $\operatorname{dim} W^{u}(\widehat{\psi})=0$, isto é, $\widehat{\psi}$ é um ponto de equilíbrio Hopf supercrítico tipozero ou um ponto de equilíbrio hiperbólico tipo-zero. Isto nos leva a uma contradição, pois pontos de equilíbrio do tipo-zero não podem pertencer a $\partial A\left(x^{s}\right)$. Sendo assim, $q \in A\left(x^{s}\right)$ e, portanto, $W^{u}(p) \cap A\left(x^{s}\right) \neq \emptyset$.

Suponhamos que $\psi$ está na fronteira $\partial A\left(x^{s}\right)$. Do Teorema 3.8 em [CHW88], podemos concluir que $\left(W_{l o c}^{u}(\psi) \backslash\{\psi\}\right) \cap \overline{A\left(x^{s}\right)} \neq \emptyset$. Consequentemente, $\left(W^{u}(\psi) \backslash\{\psi\}\right) \cap \overline{A\left(x^{s}\right)} \neq \emptyset$, pois $W_{l o c}^{u}(\psi) \subset W^{u}(\psi)$. Vamos mostrar, sob as suposições (B1'), (B2') e (B3) que $\left(W^{u}(\psi) \backslash\{\psi\}\right) \cap \overline{A\left(x^{s}\right)} \neq \emptyset$ implica em $W^{u}(\psi) \cap A\left(x^{s}\right) \neq \emptyset$. Seja $q \in\left(W^{u}(\psi) \backslash\{\psi\}\right) \cap \overline{A\left(x^{s}\right)}$. Se $q \in A\left(x^{s}\right)$, não existe nada a ser provado. Suponhamos que $q \in \partial A\left(x^{s}\right)$. Da suposição (B3), existe um elemento crítico $\widehat{\psi} \in \partial A\left(x^{s}\right)$ tal que $\varphi(t, q) \rightarrow \widehat{\psi}$ quando $t \rightarrow+\infty$. Pela suposição $\left(\mathbf{B 1}^{\prime}\right), \widehat{\psi}$ é um elemento crítico hiperbólico ou um ponto de equilíbrio Hopf supercrítico. Pelos Lemas 6.1.2 ou 6.1.5 ou 6.1.7, concluímos que $\operatorname{dim} W^{u}(\widehat{\psi})<\operatorname{dim} W^{u}(\psi)$ e, portanto, $\operatorname{dim} W^{u}(\widehat{\psi})<1$. Então $\operatorname{dim} W^{u}(\widehat{\psi})=0$, isto é, $\widehat{\psi}$ é um ponto de equilíbrio Hopf supercrítico tipo-zero ou um ponto de equilíbrio hiperbólico tipo-zero. Isto nos leva a uma contradição, pois pontos de equilíbrios do tipo-zero não podem pertencer a $\partial A\left(x^{s}\right)$. Sendo assim, $q \in A\left(x^{s}\right)$ e, portanto, $W^{u}(\psi) \cap A\left(x^{s}\right) \neq \emptyset$.

(ii) A demonstração é análoga à demonstração do Teorema 5.2 .2 e por isso será omitida.

O próximo teorema mostra a caracterização dos pontos de equilíbrio hiperbólico e Hopf supercrítico na fronteira da região de estabilidade para tipo- $k$, com $1<k \leq n-3$.

Teorema 6.2.2. (Elementos críticos do tipo- $k$ na fronteira da região de estabilidade): Seja $A\left(x^{s}\right)$ a região de estabilidade de um ponto de equilíbrio assintoticamente estável $x^{s}$ de (2.1). Sejam $p$ um ponto equilíbrio Hopf supercrítico do tipo- $k$, com $1 \leq k \leq n-3$, e $\psi$ um elemento crítico hiperbólico do tipo- $k^{\prime}$, com $k^{\prime} \leq n$, de (2.1). Se as suposições (B1'), (B2') e (B3) são satisfeitas, então:

(i)

$$
\begin{gathered}
p \in \partial A\left(x^{s}\right) \Longleftrightarrow W^{u}(p) \cap A\left(x^{s}\right) \neq \emptyset \\
\psi \text { está na fronteira } \partial A\left(x^{s}\right) \Longleftrightarrow W^{u}(\psi) \cap A\left(x^{s}\right) \neq \emptyset
\end{gathered}
$$


(ii)

$$
\begin{gathered}
p \in \partial A\left(x^{s}\right) \Longleftrightarrow W^{c s}(p) \subset \partial A\left(x^{s}\right) \\
\psi \text { está na fronteira } \partial A\left(x^{s}\right) \Longleftrightarrow W^{s}(\psi) \subset \partial A\left(x^{s}\right)
\end{gathered}
$$

Demonstração. (i) $(\Longleftarrow)$ A prova é análoga à prova do Teorema 5.2.2 e será omitida.

$(\Longrightarrow)$ Suponha que $x$ esteja na fronteira $\partial A\left(x^{s}\right)$, onde $x$ é um elemento crítico hiperbólico ou um ponto de equilíbrio Hopf supercrítico. Pelo Teorema 5.2.1 ou Teorema $3.8 \mathrm{em}$ [CHW88], podemos concluir que $\left(W_{l o c}^{u}(x) \backslash\right.$ $\{x\}) \cap \overline{A\left(x^{s}\right)} \neq \emptyset$. Consequentemente, $\left(W^{u}(x) \backslash\{x\}\right) \cap \overline{A\left(x^{s}\right)} \neq \emptyset$, pois $W_{\text {loc }}^{u}(x) \subset W^{u}(x)$. Vamos mostrar, sob as suposições (B1'), (B2') e (B3) que $\left(W^{u}(x) \backslash\{x\}\right) \cap \overline{A\left(x^{s}\right)} \neq \emptyset$ implica em $W^{u}(x) \cap A\left(x^{s}\right) \neq \emptyset$. Seja $q \in\left(W^{u}(x) \backslash\{x\}\right) \cap \overline{A\left(x^{s}\right)}$. Se $q \in A\left(x^{s}\right)$, então não existe nada a ser provado. Suponhamos que $q \in \partial A\left(x^{s}\right)$. Da condição (B3), existe um elemento crítico $\widehat{\psi} \in \partial A\left(x^{s}\right)$ tal que $\varphi(t, q) \rightarrow \widehat{\psi}$ quando $t \rightarrow+\infty$. Da condição (B1'), $\widehat{\psi}$ é um elemento crítico hiperbólico ou um ponto de equilíbrio Hopf supercrítico. Pelos Lemas 6.1.2 ou 6.1.5 ou 6.1.7, concluímos que $\operatorname{dim} W^{u}(\widehat{\psi})<\operatorname{dim} W^{u}(x)$. Demonstraremos o teorema usando indução finita na dimensão de $W^{u}(x)$. Se $\operatorname{dim} W^{u}(x)=1$, então pelo Teorema 5.2.2 temos que $W^{u}(x) \cap A\left(x^{s}\right) \neq \emptyset$. Suponha que $\operatorname{dim} W^{u}(x)=2$. Segue-se que $\operatorname{dim} W^{u}(\widehat{\psi})<2$. Então $\operatorname{dim} W^{u}(\widehat{\psi}) \leq 1$, isto é, $\widehat{\psi}$ é um ponto de equilíbrio Hopf supercrítico tipo-1 ou um elemento crítico hiperbólico tipo-1. Se $\widehat{\psi}$ é um ponto de equilíbrio Hopf supercrítico tipo-1 ou um ponto de equilíbrio hiperbólico tipo-1, então pelo Teorema 5.2.2 temos que $W^{u}(\widehat{\psi}) \cap A\left(x^{s}\right) \neq \emptyset$. Seja $B(\widehat{\psi}, \varepsilon)$ uma bola aberta de raio $\varepsilon>0$ centrada em $\widehat{\psi}$. Observe que $B(\widehat{\psi}, \varepsilon) \cap A\left(x^{s}\right) \neq \emptyset$. Seja $B=B(\widehat{\psi}, \varepsilon) \cap A\left(x^{s}\right)$. Seja $N^{u}$ uma vizinhança de $q$ em $W^{u}(x)$. A vizinhança $N^{u}$ contém uma seção transversal $D$ de $W^{s}(\psi)$ no ponto $q$ com dimensão $\operatorname{dim} D=1$. Pelo Lema 2.2.21, existe um ponto $w \in D$ e um tempo $t_{w}>0$ tal que $\varphi\left(t_{w}, w\right) \in N^{u} \cap B$. Então $w \in A\left(x^{s}\right)$. Portanto, $w \in W^{u}(x) \cap A\left(x^{s}\right)$ e, consequentemente, $W^{u}(x) \cap A\left(x^{s}\right) \neq \emptyset$.

Suponha que $\widehat{\psi}$ seja uma órbita fechada com $\operatorname{dim} W^{u}(\widehat{\psi})=1$. Então $\widehat{\psi}$ é uma órbita fechada estável e portanto, não pertence a $\partial A\left(x^{s}\right)$.

Suponha que $W^{u}(x) \cap A\left(x^{s}\right) \neq \emptyset$ para todo os elementos críticos $x$ na fronteira $\partial A\left(x^{s}\right)$ com $\operatorname{dim} W^{u}(x) \leq k$. Suponha que $\operatorname{dim} W^{u}(x)=k+1$. Consequentemente, $\operatorname{dim} W^{u}(\widehat{\psi})<k+1$. Então $\operatorname{dim} W^{u}(\widehat{\psi}) \leq k$ onde $\widehat{\psi}$ é um ponto de equilíbrio Hopf supercrítico do tipo- $k$ ou um elemento crítico hiperbólico do tipo- $k$. Portanto, pela hipótese de indução $W^{u}(\widehat{\psi}) \cap A\left(x^{s}\right) \neq \emptyset$. Seja $y \in W^{u}(\widehat{\psi}) \cap A\left(x^{s}\right)$ e $B(y, \varepsilon)$ uma bola aberta de raio $\varepsilon>0$ centrada em $y$. Como $A\left(x^{s}\right)$ é um conjunto aberto, então $B(y, \varepsilon) \subset A\left(x^{s}\right)$ para $\varepsilon$ suficientemente pequeno. Seja $N^{u}$ uma vizinhança de $q$ em $W^{u}(x)$. A vizinhança $N^{u}$ contém uma seção transversal $D$ de $W^{s}(\widehat{\psi})$ no ponto $q$ com dimensão $\operatorname{dim} D \leq k$. Pelo Lema 2.2.21, existe um ponto $w \in D$ e um tempo $t_{w}>0$ tal que $\varphi\left(t_{w}, w\right) \in N^{u}$. Como $A\left(x^{s}\right)$ é um conjunto invariante, então 
Capítulo 6. Fronteira da Região de Estabilidade na Presença de um Ponto 108 de Equilibrio Hopf com Elementos Críticos

$w \in A\left(x^{s}\right)$. Portanto, $w \in W^{u}(x) \cap A\left(x^{s}\right)$ e, consequentemente, $W^{u}(x) \cap$ $A\left(x^{s}\right) \neq \emptyset$.

(ii) A prova é análoga à prova do Teorema 5.2.2 e será omitida.

O próximo teorema oferece uma caracterização completa da fronteira da região de estabilidade quando pontos de equilíbrio Hopf supercrítico encontram-se em $\partial A\left(x^{s}\right)$.

Teorema 6.2.3. (Caracterização da Fronteira de Estabilidade para Elementos Críticos) Seja $x^{s}$ um ponto de equilíbrio assintoticamente estável de (2.1) e $A\left(x^{s}\right)$ sua região de estabilidade. Se as suposições (B1') e (B3) são satisfeitas, então

$$
\partial A\left(x^{s}\right) \subset \bigcup_{i} W^{s}\left(x_{i}\right) \bigcup_{k} W^{s}\left(\phi_{k}\right) \bigcup_{j} W^{c s}\left(p_{j}\right) \bigcup_{l} W^{c}\left(q_{l}\right)
$$

onde $x_{i}$ são os pontos de equilíbrio hiperbólicos, $\phi_{k}$ são as órbitas fechadas hiperbólicas, $p_{j}$ os pontos de equilíbrio Hopf supercríticos do tipo-k, com $1 \leq k \leq n-3$, e $q_{l}$ os pontos de equilíbrio Hopf supercríticos do tipo- $(n-2)$ em $\partial A\left(x^{s}\right), i, k, j, l=1,2, \ldots$ Se a suposição (B2') é satisfeita, então

$$
\partial A\left(x^{s}\right)=\bigcup_{i} W^{s}\left(x_{i}\right) \bigcup_{k} W^{s}\left(\phi_{k}\right) \bigcup_{j} W^{c s}\left(p_{j}\right) \bigcup_{l} W^{c}\left(q_{l}\right) .
$$

Demonstração. Seja $q \in \partial A\left(x^{s}\right)$. Pela hipótese (B3), podemos afirmar que existe um elemento crítico $x$ tal que $\varphi(t, q) \rightarrow x$ quando $t \rightarrow+\infty$. Pela hipótese (B1'), podemos afirmar que $x$ é um ponto de equilíbrio hiperbólico $x_{i}$ ou uma órbita fechada hiperbólica $\phi_{k}$ ou um ponto de equilíbrio Hopf supercrítico $p_{j}$ do tipo- $k$, com with $1 \leq k \leq n-3$, ou um ponto de equilíbrio Hopf supercrítico $q_{l}$ do tipo- $(n-2)$, ou seja, $x=x_{i}$ ou $x=\phi_{k}$ ou $x=p_{j}$ ou $x=q_{l}$ para algum $i, j, k, l$. Portanto, concluímos que $q \in \bigcup_{i} W^{s}\left(x^{i}\right) \bigcup_{k} W^{s}\left(\phi^{k}\right) \bigcup_{j} W^{c s}\left(p_{j}\right) \bigcup_{l} W^{c}\left(q_{l}\right)$. Portanto, $\partial A\left(x^{s}\right) \subset$ $\bigcup_{i} W^{s}\left(x^{i}\right) \bigcup_{k} W^{s}\left(\phi^{k}\right) \bigcup_{j} W^{c s}\left(p_{j}\right) \bigcup_{l} W^{c}\left(q_{l}\right)$. Pelos Teoremas 6.2.1, 6.2.2 e 5.2.4, sabemos que $W^{s}\left(x_{i}\right) \subset \partial A\left(x^{s}\right), W^{s}\left(\phi_{k}\right) \subset \partial A\left(x^{s}\right), W^{c s}\left(p_{j}\right) \subset \partial A\left(x^{s}\right)$ e $W^{c}\left(q_{l}\right) \subset \partial A\left(x^{s}\right)$. Logo, $\bigcup_{i} W^{s}\left(x_{i}\right) \bigcup_{k} W^{s}\left(\phi_{k}\right) \bigcup_{j} W^{c s}\left(p_{j}\right) \bigcup_{l} W^{c}\left(q_{l}\right) \subset$ $\partial A\left(x^{s}\right)$ e, portanto,

$$
\partial A\left(x^{s}\right)=\bigcup_{i} W^{s}\left(x_{i}\right) \bigcup_{k} W^{s}\left(\phi_{k}\right) \bigcup_{j} W^{c s}\left(p_{j}\right) \bigcup_{l} W^{c}\left(q_{l}\right) .
$$

\subsection{Ponto de equilíbrio Hopf Subcrítico em $\partial A\left(x^{s}\right)$}

Assim como fizemos na seção 6.2, generalizando os resultados da caracterização da fronteira da região de estabilidade admitindo a existência de 
órbitas periódicas, faremos uma generalização da caracterização da fronteira da região de estabilidade na presença também de pontos de equilíbrio Hopf subcríticos na fronteira de estabilidade.

Para admitirmos a presença de órbitas periódicas na fronteira, as afirmações (A1"") e (A2") do Teorema 5.3.1 podem ser reformuladas. Sejam $x^{s}$ um ponto de equilíbrio assintoticamente estável, $q$ um ponto de equilíbrio Hopf subcrítico de (2.1) e considere as seguintes afirmações:

(B1") Todos os elementos críticos em $\partial A\left(x^{s}\right)$ são elementos críticos hiperbólicos ou pontos de equilíbrio Hopf subcrítico;

(B2") As variedades estáveis e as variedades centrais, centro-instáveis e/ou instáveis dos elementos críticos em $\partial A\left(x^{s}\right)$ satisfazem a condição de transversalidade.

A condição (B1") é mais fraca do que a condição (B1), uma vez que permite a presença de pontos de equilíbrio não-hiperbólicos na fronteira da região de estabilidade. Os próximos resultados fornecem condições necessárias e suficientes para garantir que os elementos críticos hiperbólicos e os pontos de equilíbrio Hopf subcríticos pertençam à fronteira da região de estabilidade. Inicialmente, mostraremos a caracterização dos elementos critícos hiperbólicos do tipo-1 e tipo-2 e dos pontos de equilíbrio Hopf subcríticos do tipo-0 e tipo-1 na fronteira da região de estabilidade e, em seguida, a caracterização de elementos critícos para tipos superiores seguirá por argumentos de indução.

Teorema 6.3.1. (redPonto de equilÃ brio tipo-1 na fronteira da região de estabilidade) Seja $A\left(x^{s}\right)$ a região de estabilidade de um ponto de equilíbrio assintoticamente estável $x^{s}$ de (2.1). Seja $x^{\star}$ um ponto de equilíbrio hiperbólico do tipo-1 de (2.1). Se as suposições $\left(\boldsymbol{B 1}^{\prime \prime}\right),\left(\boldsymbol{B 2}^{\prime \prime}\right)$ e (B3) são satisfeitas, então:

$$
x^{\star} \in \partial A\left(x^{s}\right) \Longleftrightarrow W^{u}\left(x^{\star}\right) \cap A\left(x^{s}\right) \neq \emptyset
$$

$$
x^{\star} \in \partial A\left(x^{s}\right) \Longleftrightarrow W^{s}\left(x^{\star}\right) \subset \partial A\left(x^{s}\right)
$$

Demonstração. (i) $(\Longleftarrow)$ Suponhamos que $W^{u}\left(x^{\star}\right) \cap A\left(x^{s}\right) \neq \emptyset$. Como $A\left(x^{s}\right) \subset \overline{A\left(x^{s}\right)}$, então $\left(W_{l o c}^{u}\left(x^{\star}\right) \backslash\left\{x^{\star}\right\}\right) \cap \overline{A\left(x^{s}\right)} \neq \emptyset$. Portanto, pelo Teorema 3.8 de [CHW88], temos que $x^{\star} \in \partial A\left(x^{s}\right)$.

$(\Longrightarrow)$ Suponhamos que $x^{\star} \in \partial A\left(x^{s}\right)$. Do Teorema 3.8 de [CHW88], podemos concluir que $\left(W_{l o c}^{u}\left(x^{\star}\right) \backslash\left\{x^{\star}\right\}\right) \cap \overline{A\left(x^{s}\right)} \neq \emptyset$. Consequentemente, $\left(W^{u}\left(x^{\star}\right) \backslash\left\{x^{\star}\right\}\right) \cap \overline{A\left(x^{s}\right)} \neq \emptyset$, pois $W_{l o c}^{u}\left(x^{\star}\right) \subset W^{u}\left(x^{\star}\right)$. Vamos mostrar, sob as suposições $\left(\mathbf{B 1}^{\prime \prime}\right),\left(\mathbf{B 2}^{\prime \prime}\right)$ e $(\mathbf{B 3})$ que $\left(W^{u}\left(x^{\star}\right) \backslash\left\{x^{\star}\right\}\right) \cap \overline{A\left(x^{s}\right)} \neq \emptyset$ implica em $W^{u}\left(x^{\star}\right) \cap A\left(x^{s}\right) \neq \emptyset$. Seja $q \in\left(W^{u}\left(x^{\star}\right) \backslash\left\{x^{\star}\right\}\right) \cap \overline{A\left(x^{s}\right)}$. Se $q \in A\left(x^{s}\right)$, não existe nada a ser provado. Suponhamos que $q \in \partial A\left(x^{s}\right)$. Da suposição (B3), existe um ponto de equilíbrio $\widehat{p} \in \partial A\left(x^{s}\right)$ tal que $\varphi(t, q) \rightarrow \widehat{p}$ 
Capítulo 6. Fronteira da Região de Estabilidade na Presença de um Ponto 110 de Equilíbrio Hopf com Elementos Críticos

quando $t \rightarrow+\infty$. Pela suposição $\left(\mathbf{B} 1^{\prime \prime}\right), \widehat{p}$ é um elemento crítico hiperbólico ou um ponto de equilíbrio Hopf subcrítico. Pelos Lemas 6.1.2 ou 6.1.3 ou 6.1.4 ou 6.1.6, concluímos que $\operatorname{dim} W^{u}(\widehat{p})<\operatorname{dim} W^{u}\left(x^{\star}\right)$ e, portanto, $\operatorname{dim} W^{u}(\widehat{p})<1$. Assim, $\operatorname{dim} W^{u}(\widehat{p})=0$ e, consenquentemente $\widehat{p}$ é um ponto de equilíbrio hiperbólico tipo zero. Isto nos leva a uma contradição pois pontos de equilíbrio tipo zero não pertencem a $\partial A\left(x^{s}\right)$. Consequentemente, $q \in A\left(x^{s}\right)$ e, portanto, $W^{u}\left(x^{\star}\right) \cap A\left(x^{s}\right) \neq \emptyset$.

(ii) $(\Longleftarrow)$ Suponhamos $W^{s}\left(x^{\star}\right) \subset \partial A\left(x^{s}\right)$. Como $x^{\star} \in W^{s}\left(x^{\star}\right)$, então $x^{\star} \in \partial A\left(x^{s}\right)$.

$(\Longrightarrow)$ Suponhamos que $x^{\star} \in \partial A\left(x^{s}\right)$. Pela primeira parte do Teorema 6.3.2, podemos concluir que $W^{u}\left(x^{\star}\right) \cap A\left(x^{s}\right) \neq \emptyset$. Seja $y \in W^{u}\left(x^{\star}\right) \cap A\left(x^{s}\right)$. Como $y \in W^{u}\left(x^{\star}\right)$, então existe $T<0$ tal que $\varphi(T, y) \in W_{l o c}^{u}\left(x^{\star}\right)$. Seja $z=\varphi(T, y)$. Como $y \in A\left(x^{s}\right)$ e $A\left(x^{s}\right)$ é um conjunto invariante, então $z \in A\left(x^{s}\right)$. Segue-se que $z \in W_{l o c}^{u}\left(x^{\star}\right) \cap A\left(x^{s}\right)$. Seja $B(z, \varepsilon)$ uma bola aberta de raio $\varepsilon>0$ centrada em $z$ onde $\varepsilon$ é um número arbitrariamente pequeno. Seja $\widehat{q}$ um ponto arbitrário de $W^{s}\left(x^{\star}\right)$. Em particular, para algum $\bar{T}>0$ temos $\widetilde{q}=\varphi(\bar{T}, \widehat{q}) \in W_{l o c}^{s}\left(x^{\star}\right)$. Seja $S$ um disco em um ponto $\widetilde{q}$ $\operatorname{de} \operatorname{dim} S=1$ transversal a $W_{l o c}^{s}\left(x^{\star}\right)$. Pelo Corolário 2.2 .20 ou 2.2.22, existe um ponto $w \in S$ e um tempo $t_{w}>0$ tal que $\varphi\left(t_{w}, w\right) \in B(z, \varepsilon)$. Como $A\left(x^{s}\right)$ é um conjunto invariante, então $w \in A\left(x^{s}\right)$. Como $\varepsilon$ e o disco $S$ podem ser escolhidos arbitrariamente pequenos, então existem pontos em $A\left(x^{s}\right)$ arbitrariamente próximos a $\widetilde{q}$. Consequentemente, $\widetilde{q} \in \overline{A\left(x^{s}\right)}$. Como $W_{l o c}^{s}\left(x^{\star}\right) \cap A\left(x^{s}\right)=\emptyset$, então $\widetilde{q} \in \partial A\left(x^{s}\right)$. Como $\partial A\left(x^{s}\right)$ é um conjunto invariante, $\widehat{q}=\varphi(-\bar{T}, \widetilde{q}) \in \partial A\left(x^{s}\right)$. Como a escolha de $\widehat{q} \mathrm{em} W^{s}\left(x^{\star}\right)$ foi arbitrária, então podemos concluir que $W^{s}\left(x^{\star}\right) \subset \partial A\left(x^{s}\right)$.

Teorema 6.3.2. (Ponto de equilíbrio Hopf subcrítico tipo-0 na fronteira da região de estabilidade) Seja $A\left(x^{s}\right)$ a região de estabilidade de um ponto de equilibrio assintoticamente estável $x^{s}$ de (2.1). Seja $p$ um ponto de equilíbrio Hopf subcrítico do tipo-0 de (2.1). Se as suposições (B1"), (B2") $e$ (B3) são satisfeitas, então:

(i)

$$
p \in \partial A\left(x^{s}\right) \Longleftrightarrow W^{c}(p) \cap A\left(x^{s}\right) \neq \emptyset
$$

$$
p \in \partial A\left(x^{s}\right) \Longleftrightarrow W^{s}(p) \subset \partial A\left(x^{s}\right)
$$

Demonstração. (i) $(\Longleftarrow)$ Suponhamos que $W^{c}(p) \cap A\left(x^{s}\right) \neq \emptyset$. Como $A\left(x^{s}\right) \subset$ $\overline{A\left(x^{s}\right)}$, então $\left(W_{l o c}^{c}(p) \backslash\{p\}\right) \cap \overline{A\left(x^{s}\right)} \neq \emptyset$. Portanto, pelo item (i) do Teorema 5.3.1, temos que $p \in \partial A\left(x^{s}\right)$.

$(\Longrightarrow)$ Suponhamos que $p \in \partial A\left(x^{s}\right)$. Pelo Teorema 5.3.1, podemos concluir que $\left(W_{l o c}^{c}(p) \backslash\{p\}\right) \cap \overline{A\left(x^{s}\right)} \neq \emptyset$. Consequentemente, $\left(W^{c}(p) \backslash\{p\}\right) \cap$ $\overline{A\left(x^{s}\right)} \neq \emptyset$, pois $W_{\text {loc }}^{c}(p) \subset W^{c}(p)$. Vamos mostrar, sob as suposições $\left(\mathbf{B} 1^{\prime \prime}\right)$, (B2 $\left.^{\prime \prime}\right)$ e $\left(\right.$ B3) que $\left(W^{c u}(p) \backslash\{p\}\right) \cap \overline{A\left(x^{s}\right)} \neq \emptyset$ implica em $W^{c}(p) \cap A\left(x^{s}\right) \neq \emptyset$. 
Seja $q \in\left(W^{c}(p) \backslash\{p\}\right) \cap \overline{A\left(x^{s}\right)}$. Se $q \in A\left(x^{s}\right)$, então não existe nada a ser provado. Suponhamos que $q \in \partial A\left(x^{s}\right)$. Da condição (B3), existe um ponto de equilíbro $\widehat{p} \in \partial A\left(x^{s}\right)$ tal que $\varphi(t, q) \rightarrow \widehat{p}$ quando $t \rightarrow+\infty$. Então $\widehat{p}$ é ou um elemento crítico hiperbólico ou um ponto de equilíbrio Hopf subcrítico. Pelos Lemas 6.1.2 ou 6.1.3 ou 6.1.4 ou 6.1.6, concluímos que $\operatorname{dim} W^{c u}(\widehat{p})<\operatorname{dim} W^{c u}(p)$ ou $\operatorname{dim} W^{c}(\widehat{p})<\operatorname{dim} W^{c u}(p)$ se $\widehat{p}$ é um ponto de equilíbrio Hopf subcrítico ou $\operatorname{dim} W^{u}(\widehat{p})<\operatorname{dim} W^{c u}(p)$ se $\widehat{p}$ é um elemento crítico hiperbólico. Se $\widehat{p}$ é um ponto de equilíbrio Hopf subcrítico, então $\operatorname{dim} W^{c u}(\widehat{p})<2$ ou $\operatorname{dim} W^{c}(\widehat{p})<2$, o que é uma contradição pois a variedade central de um ponto de equilibrio Hopf subcritico tem pelo menos dimensão 2. Seja $\widehat{p}$ um elemento crítico hiperbólico e, portanto, $\operatorname{dim} W^{u}(\widehat{p})<2$. Segue-se que $\operatorname{dim} W^{u}(\widehat{p})=1$ e $\widehat{p}$ é um ponto de equiíbrio hiperbólico, pois elementos críticos hiperbólicos do tipo zero não podem pertencer à fronteira da região de estabilidade. Logo, pelo Teorema 6.3.2, $W^{u}(\widehat{p}) \cap A\left(x^{s}\right) \neq \emptyset$. Sejam $y \in W^{u}(\widehat{p}) \cap A\left(x^{s}\right)$ e $B(y, \varepsilon)$ uma bola aberta de raio $\varepsilon>0$ centrada em $y$. Como $A\left(x^{s}\right)$ é um conjunto aberto, então $B(y, \varepsilon) \subset A\left(x^{s}\right)$ para $\varepsilon$ suficientemente pequeno. Seja $N^{c}$ uma vizinhança de $q$ em $W^{c}(p)$. A vizinhança $N^{c}$ contém uma seção transversal $D$ de $W^{s}(\widehat{p})$ no ponto $q$ com dimensão $\operatorname{dim} D=2$. Pelo Lema 2.2.25, existe um ponto $w \in D$ e um tempo $t_{w}>0$ tal que $\varphi\left(t_{w}, w\right) \in N^{c}$. Como $A\left(x^{s}\right)$ é um conjunto invariante, então $w \in A\left(x^{s}\right)$. Portanto, $w \in W^{c}(p) \cap A\left(x^{s}\right)$ e, consequentemente, $W^{c}(p) \cap A\left(x^{s}\right) \neq \emptyset$.

(ii) $(\Longleftarrow)$ Suponhamos que $W^{s}(p) \subset \partial A\left(x^{s}\right)$. Como $p \in W^{s}(p)$, então $p \in \partial A\left(x^{s}\right)$.

$(\Longrightarrow)$ Suponhamos que $p \in \partial A\left(x^{s}\right)$. Pela primeira parte do Teorema 6.3.2, podemos concluir que $W^{c}(p) \cap A\left(x^{s}\right) \neq \emptyset$. Seja $y \in W^{c}(p) \cap A\left(x^{s}\right)$. Como $y \in W^{c}(p)$, então existe $T<0$ tal que $\varphi(T, y) \in W_{l o c}^{c}(p)$. Seja $z=\varphi(T, y)$. Como $y \in A\left(x^{s}\right)$ e $A\left(x^{s}\right)$ é um conjunto invariante, então $z \in A\left(x^{s}\right)$. Segue-se que $z \in W_{l o c}^{c}(p) \cap A\left(x^{s}\right)$. Seja $B(z, \varepsilon)$ uma bola aberta de raio $\varepsilon>0$ centrada em $z$ onde $\varepsilon$ é um número arbitrariamente pequeno. Seja $\widehat{q}$ um ponto arbitrário de $W^{s}(p)$. Em particular, para algum $\bar{T}>0$ temos $\widetilde{q}=\varphi(\bar{T}, \widehat{q}) \in W_{l o c}^{s}(p)$. Seja $S$ um disco em um ponto $\widetilde{q} \operatorname{de} \operatorname{dim} S=1$ transversal a $W_{l o c}^{s}(p)$. Pelo Corolário 2.2.26, existe um ponto $w \in S$ e um tempo $t_{w}>0$ tal que $\varphi\left(t_{w}, w\right) \in B(z, \varepsilon)$. Como $A\left(x^{s}\right)$ é um conjunto invariante, então $w \in A\left(x^{s}\right)$. Como $\varepsilon$ e o disco $S$ podem ser escolhidos arbitrariamente pequenos, então existem pontos em $A\left(x^{s}\right)$ arbitrariamente próximos a $\widetilde{q}$. Consequentemente, $\widetilde{q} \in \overline{A\left(x^{s}\right)}$. Como $W_{l o c}^{s}(p) \cap A\left(x^{s}\right)=\emptyset$, então $\widetilde{q} \in \partial A\left(x^{s}\right)$. Como $\partial A\left(x^{s}\right)$ é um conjunto invariante, $\widehat{q}=\varphi(-\bar{T}, \widetilde{q}) \in \partial A\left(x^{s}\right)$. Como a escolha de $\widehat{q}$ em $W^{s}(p)$ foi arbitrária, então podemos concluir que $W^{s}(p) \subset \partial A\left(x^{s}\right)$.

Teorema 6.3.3. (Elemento crítico tipo-2 na fronteira da região de estabilidade) Seja $A\left(x^{s}\right)$ a região de estabilidade de um ponto de equilíbrio assintoticamente estável $x^{s}$ de (2.1). Seja $x^{\star}$ um elemento crítico hiperbólico 
Capítulo 6. Fronteira da Região de Estabilidade na Presença de um Ponto 112 de Equilíbrio Hopf com Elementos Críticos

do tipo-2 de (2.1). Se as suposições $\left(\boldsymbol{B 1}^{\prime \prime}\right),\left(\boldsymbol{B 2}^{\prime \prime}\right)$ e (B3) são satisfeitas, então:

(i)

$$
x^{\star} \in \partial A\left(x^{s}\right) \Longleftrightarrow W^{u}\left(x^{\star}\right) \cap A\left(x^{s}\right) \neq \emptyset
$$

(ii)

$$
x^{\star} \in \partial A\left(x^{s}\right) \Longleftrightarrow W^{s}\left(x^{\star}\right) \subset \partial A\left(x^{s}\right)
$$

Demonstração. (i) $(\Longleftarrow)$ Suponhamos que $W^{u}\left(x^{\star}\right) \cap A\left(x^{s}\right) \neq \emptyset$. Como $A\left(x^{s}\right) \subset \overline{A\left(x^{s}\right)}$, então $\left(W_{l o c}^{u}\left(x^{\star}\right) \backslash\left\{x^{\star}\right\}\right) \cap \overline{A\left(x^{s}\right)} \neq \emptyset$. Portanto, pelo Teorema 3.8 de [CHW88], temos que $x^{\star} \in \partial A\left(x^{s}\right)$.

$(\Longrightarrow)$ Suponhamos que $x^{\star} \in \partial A\left(x^{s}\right)$. Do Teorema 3.8 de [CHW88], podemos concluir que $\left(W_{l o c}^{u}\left(x^{\star}\right) \backslash\left\{x^{\star}\right\}\right) \cap \overline{A\left(x^{s}\right)} \neq \emptyset$. Consequentemente, $\left(W^{u}\left(x^{\star}\right) \backslash\left\{x^{\star}\right\}\right) \cap \overline{A\left(x^{s}\right)} \neq \emptyset$, pois $W_{l o c}^{u}\left(x^{\star}\right) \subset W^{u}\left(x^{\star}\right)$. Vamos mostrar, sob as suposições $\left(\mathbf{B} 1^{\prime \prime}\right),\left(\mathbf{B 2}^{\prime \prime}\right)$ e $(\mathbf{B 3})$ que $\left(W^{u}\left(x^{\star}\right) \backslash\left\{x^{\star}\right\}\right) \cap \overline{A\left(x^{s}\right)} \neq \emptyset$ implica em $W^{u}\left(x^{\star}\right) \cap A\left(x^{s}\right) \neq \emptyset$. Seja $q \in\left(W^{u}\left(x^{\star}\right) \backslash\left\{x^{\star}\right\}\right) \cap \overline{A\left(x^{s}\right)}$. Se $q \in A\left(x^{s}\right)$, não existe nada a ser provado. Suponhamos que $q \in \partial A\left(x^{s}\right)$. Da suposição (B3), existe um elemento crítico $\widehat{p} \in \partial A\left(x^{s}\right)$ tal que $\varphi(t, q) \rightarrow \widehat{p}$ quando $t \rightarrow+\infty$. Pela suposição (B1"), $\widehat{p}$ é um elemento crítico hiperbólico ou um ponto de equilíbrio Hopf subcrítico. Pelos Lemas 6.1.2 ou 6.1.3 ou 6.1.4 ou 6.1.6, concluímos que $\operatorname{dim} W^{c u}(\widehat{p})<\operatorname{dim} W^{u}\left(x^{\star}\right)$ ou $\operatorname{dim} W^{c}(\widehat{p})<\operatorname{dim} W^{u}\left(x^{\star}\right)$ se $\widehat{p}$ é um ponto de equilíbrio Hopf subcrítico ou $\operatorname{dim} W^{u}(\widehat{p})<\operatorname{dim} W^{u}\left(x^{\star}\right)$ se $\widehat{p}$ é um elemento crítico hiperbólico. Se $\widehat{p}$ é um ponto de equilíbrio Hopf subcrítico, então $\operatorname{dim} W^{c u}(\widehat{p})<2$ ou $\operatorname{dim} W^{c}(\widehat{p})<2$, o que é uma contradição pois a variedade central de um ponto de equilibrio Hopf subcrítico tem pelo menos dimensão 2. Seja $\widehat{p}$ um elemento crítico hiperbólico e, portanto, $\operatorname{dim} W^{u}(\widehat{p})<2$. Segue-se que $\operatorname{dim} W^{u}(\widehat{p})=1$ e $\widehat{p}$ é um ponto de equilíbrio hiperbólico, pois elementos críticos hiperbólicos do tipo zero não pode pertencer a fronteira da região de estabilidade. Logo, pelo Teorema 6.3.2, $W^{u}(\widehat{p}) \cap A\left(x^{s}\right) \neq \emptyset$. Seja $y \in W^{u}(\widehat{p}) \cap A\left(x^{s}\right)$ e $B(y, \varepsilon)$ uma bola aberta de raio $\varepsilon>0$ centrada em $y$. Como $A\left(x^{s}\right)$ é um conjunto aberto, então $B(y, \varepsilon) \subset A\left(x^{s}\right)$ para $\varepsilon$ suficientemente pequeno. Seja $N^{c}$ uma vizinhança de $q$ em $W^{u}\left(x^{\star}\right)$. A vizinhança $N^{c}$ contém uma seção transversal $D$ de $W^{s}(\widehat{p})$ no ponto $q$ com dimensão $\operatorname{dim} D=2$. Pelo Lema 2.2 .19 , existe um ponto $w \in D$ e um tempo $t_{w}>0$ tal que $\varphi\left(t_{w}, w\right) \in N^{c}$. Como $A\left(x^{s}\right)$ é um conjunto invariante, então $w \in A\left(x^{s}\right)$. Portanto, $w \in W^{u}\left(x^{\star}\right) \cap A\left(x^{s}\right)$ e, consequentemente, $W^{u}\left(x^{\star}\right) \cap A\left(x^{s}\right) \neq \emptyset$.

(ii) $(\Longleftarrow)$ Suponhamos $W^{s}\left(x^{\star}\right) \subset \partial A\left(x^{s}\right)$. Como $x^{\star} \in W^{s}\left(x^{\star}\right)$, então $x^{\star} \in \partial A\left(x^{s}\right)$.

$(\Longrightarrow)$ Suponhamos que $x^{\star} \in \partial A\left(x^{s}\right)$. Pela primeira parte do Teorema 6.3.3, podemos concluir que $W^{u}\left(x^{\star}\right) \cap A\left(x^{s}\right) \neq \emptyset$. Seja $y \in W^{u}\left(x^{\star}\right) \cap A\left(x^{s}\right)$. Como $y \in W^{u}\left(x^{\star}\right)$, então existe $T<0$ tal que $\varphi(T, y) \in W_{l o c}^{u}\left(x^{\star}\right)$. Seja $z=\varphi(T, y)$. Como $y \in A\left(x^{s}\right)$ e $A\left(x^{s}\right)$ é um conjunto invariante, então 
$z \in A\left(x^{s}\right)$. Segue-se que $z \in W_{\text {loc }}^{u}\left(x^{\star}\right) \cap A\left(x^{s}\right)$. Seja $B(z, \varepsilon)$ uma bola aberta de raio $\varepsilon>0$ centrada em $z$ onde $\varepsilon$ é um número arbitrariamente pequeno. Seja $\widehat{q}$ um ponto arbitrário de $W^{s}\left(x^{\star}\right)$. Em particular, para algum $\bar{T}>0$ temos $\widetilde{q}=\varphi(\bar{T}, \widehat{q}) \in W_{l o c}^{s}\left(x^{\star}\right)$. Seja $S$ um disco em um ponto $\widetilde{q}$ de $\operatorname{dim} S=1$ transversal a $W_{l o c}^{s}\left(x^{\star}\right)$. Pelo Corolário 2.2.20 ou 2.2.22, existe um ponto $w \in S$ e um tempo $t_{w}>0$ tal que $\varphi\left(t_{w}, w\right) \in B(z, \varepsilon)$. Como $A\left(x^{s}\right)$ é um conjunto invariante, então $w \in A\left(x^{s}\right)$. Como $\varepsilon$ e o disco $S$ podem ser escolhidos arbitrariamente pequenos, então existem pontos em $A\left(x^{s}\right)$ arbitrariamente próximos a $\widetilde{q}$. Consequentemente, $\widetilde{q} \in \overline{A\left(x^{s}\right)}$. Como $W_{l o c}^{s}\left(x^{\star}\right) \cap A\left(x^{s}\right)=\emptyset$, então $\widetilde{q} \in \partial A\left(x^{s}\right)$. Como $\partial A\left(x^{s}\right)$ é um conjunto invariante, $\widehat{q}=\varphi(-\bar{T}, \widetilde{q}) \in \partial A\left(x^{s}\right)$. Como a escolha de $\widehat{q} \mathrm{em} W^{s}\left(x^{\star}\right)$ foi arbitrária, então podemos concluir que $W^{s}\left(x^{\star}\right) \subset \partial A\left(x^{s}\right)$.

Teorema 6.3.4. (Ponto de equilíbrio Hopf subcrítico tipo-1 na fronteira da região de estabilidade) Seja $A\left(x^{s}\right)$ a região de estabilidade de um ponto de equilíbrio assintoticamente estável $x^{s}$ de (2.1). Seja $p$ um ponto de equilíbrio Hopf subcrítico do tipo-1 de (2.1). Se as suposições $\left(\boldsymbol{B} 1^{\prime \prime}\right),\left(\boldsymbol{B 2}^{\prime \prime}\right)$ e (B3) são satisfeitas, então:

(i)

$$
p \in \partial A\left(x^{s}\right) \Longleftrightarrow W^{c u}(p) \cap A\left(x^{s}\right) \neq \emptyset
$$

$$
p \in \partial A\left(x^{s}\right) \Longleftrightarrow W^{s}(p) \subset \partial A\left(x^{s}\right)
$$

Demonstração. i. $(\Longleftarrow)$ Suponhamos que $W^{c u}(p) \cap A\left(x^{s}\right) \neq \emptyset$. Como $A\left(x^{s}\right) \subset$ $\overline{A\left(x^{s}\right)}$, então $\left(W_{l o c}^{c u}(p) \backslash\{p\}\right) \cap \overline{A\left(x^{s}\right)} \neq \emptyset$. Portanto, pelo item (ii) do Teorema 5.3.1, temos que $p \in \partial A\left(x^{s}\right)$.

$(\Longrightarrow)$ Suponhamos que $p \in \partial A\left(x^{s}\right)$. Pelo item (ii) do Teorema 5.3.2, podemos concluir que $\left(W_{l o c}^{c u}(p) \backslash\{p\}\right) \cap \overline{A\left(x^{s}\right)} \neq \emptyset$. Consequentemente, $\left(W^{c u}(p) \backslash\right.$ $\{p\}) \cap \overline{A\left(x^{s}\right)} \neq \emptyset$, pois $W_{l o c}^{c u}(p) \subset W^{c u}(p)$. Vamos mostrar, sob as suposições $\left(\mathbf{B 1}^{\prime \prime}\right),\left(\mathbf{B 2}^{\prime \prime}\right)$ e $(\mathbf{B 3})$ que $\left(W^{c u}(p) \backslash\{p\}\right) \cap \overline{A\left(x^{s}\right)} \neq \emptyset$ implica em $W^{c u}(p) \cap A\left(x^{s}\right) \neq \emptyset$. Seja $q \in\left(W^{c u}(p) \backslash\{p\}\right) \cap \overline{A\left(x^{s}\right)}$. Se $q \in A\left(x^{s}\right)$, então não existe nada a ser provado. Suponhamos que $q \in \partial A\left(x^{s}\right)$. Da condição (B3), existe um ponto de equilíbro $\widehat{p} \in \partial A\left(x^{s}\right)$ tal que $\varphi(t, q) \rightarrow \widehat{p}$ quando $t \rightarrow+\infty$. Então $\widehat{p}$ é um elemento crítico hiperbólico ou um ponto de equilíbrio Hopf subcrítico. Pelos Lemas 6.1.2 ou 6.1.3 ou 6.1.4 ou 6.1.6, concluímos que $\operatorname{dim} W^{c u}(\widehat{p})<\operatorname{dim} W^{c u}(p)$ ou $\operatorname{dim} W^{c}(\widehat{p})<\operatorname{dim} W^{c u}(p)$ se $\widehat{p}$ é um ponto de equilíbrio Hopf subcrítico ou $\operatorname{dim} W^{u}(\widehat{p})<\operatorname{dim} W^{c u}(p)$ se $\widehat{p}$ é um elemento crítico hiperbólico. Se $\widehat{p}$ é um ponto de equilíbrio Hopf subcrítico, então $\operatorname{dim} W^{c u}(\widehat{p})<3$ ou $\operatorname{dim} W^{c}(\widehat{p})<3$. Segue-se que $\operatorname{dim} W^{c}(\widehat{p})<3$, pois a variedade central de um ponto de equilíbrio Hopf subcrítico tem pelo menos dimensão 2. Consequentemente, $\operatorname{dim} W^{c}(\widehat{p})=2$ e portanto, $\widehat{p}$ é um ponto de equilíbrio Hopf subcrítico tipo-0. Logo, pelo Teorema 6.3.2, $W^{c}(\widehat{p}) \cap A\left(x^{s}\right) \neq \emptyset$. Seja $y \in W^{c}(\widehat{p}) \cap A\left(x^{s}\right)$ e $B(y, \varepsilon)$ uma bola aberta 
Capítulo 6. Fronteira da Região de Estabilidade na Presença de um Ponto 114 de Equilíbrio Hopf com Elementos Críticos

de raio $\varepsilon>0$ centrada em $y$. Como $A\left(x^{s}\right)$ é um conjunto aberto, então $B(y, \varepsilon) \subset A\left(x^{s}\right)$ para $\varepsilon$ suficientemente pequeno. Seja $N^{c u}$ uma vizinhança de $q$ em $W^{c u}(p)$. A vizinhança $N^{c u}$ contém uma seção transversal $D$ de $W^{s}(\widehat{p})$ no ponto $q$ com dimensão $\operatorname{dim} D=2$. Pelo Lema 2.2.25, existe um ponto $w \in D$ e um tempo $t_{w}>0$ tal que $\varphi\left(t_{w}, w\right) \in N^{c}$. Como $A\left(x^{s}\right)$ é um conjunto invariante, então $w \in A\left(x^{s}\right)$. Portanto, $w \in W^{c u}(p) \cap A\left(x^{s}\right)$ e, consequentemente, $W^{c u}(p) \cap A\left(x^{s}\right) \neq \emptyset$.

Se $\widehat{p}$ é um elemento crítico hiperbólico e, portanto, $\operatorname{dim} W^{u}(\widehat{p})<3$. Segue-se que $\operatorname{dim} W^{u}(\widehat{p})=1$ ou $\operatorname{dim} W^{u}(\widehat{p})=2$, pois elementos críticos hiperbólicos do tipo zero não pode pertencer a fronteira da região de estabilidade. Logo, pelos Teorema 6.3.1 ou 6.3.3, $W^{u}(\widehat{p}) \cap A\left(x^{s}\right) \neq \emptyset$. Seja $y \in W^{u}(\widehat{p}) \cap A\left(x^{s}\right)$ e $B(y, \varepsilon)$ uma bola aberta de raio $\varepsilon>0$ centrada em $y$. Como $A\left(x^{s}\right)$ é um conjunto aberto, então $B(y, \varepsilon) \subset A\left(x^{s}\right)$ para $\varepsilon$ suficientemente pequeno. Seja $N^{u}$ uma vizinhança de $q$ em $W^{c u}(p)$. A vizinhança $N^{u}$ contém uma seção transversal $D$ de $W^{s}(\widehat{p})$ no ponto $q$ com dimensão $\operatorname{dim} D=1$ ou $\operatorname{dim} D=2$. Pelo Lema 2.2.19, existe um ponto $w \in D$ e um tempo $t_{w}>0$ tal que $\varphi\left(t_{w}, w\right) \in N^{u}$. Como $A\left(x^{s}\right)$ é um conjunto invariante, então $w \in A\left(x^{s}\right)$. Portanto, $w \in W^{u}(p) \cap A\left(x^{s}\right)$ e, consequentemente, $W^{u}(p) \cap A\left(x^{s}\right) \neq \emptyset$.

(ii) $(\Longleftarrow)$ Suponhamos que $W^{s}(p) \subset \partial A\left(x^{s}\right)$. Como $p \in W^{s}(p)$, então $p \in \partial A\left(x^{s}\right)$.

$(\Longrightarrow)$ Suponhamos que $p \in \partial A\left(x^{s}\right)$. Pela primeira parte do Teorema 6.3.4, podemos concluir que $W^{c u}(p) \cap A\left(x^{s}\right) \neq \emptyset$. Seja $y \in W^{c u}(p) \cap A\left(x^{s}\right)$. Como $y \in W^{c u}(p)$, então existe $T<0$ tal que $\varphi(T, y) \in W_{l o c}^{c u}(p)$. Seja $z=\varphi(T, y)$. Como $y \in A\left(x^{s}\right)$ e $A\left(x^{s}\right)$ é um conjunto invariante, então $z \in A\left(x^{s}\right)$. Segue-se que $z \in W_{l o c}^{c u}(p) \cap A\left(x^{s}\right)$. Seja $B(z, \varepsilon)$ uma bola aberta de raio $\varepsilon>0$ centrada em $z$ onde $\varepsilon$ é um número arbitrariamente pequeno. Seja $\widehat{q}$ um ponto arbitrário de $W^{s}(p)$. Em particular, para algum $\bar{T}>0$ temos $\widetilde{q}=\varphi(\bar{T}, \widehat{q}) \in W_{l o c}^{s}(p)$. Seja $S$ um disco em um ponto $\widetilde{q} \operatorname{de} \operatorname{dim} S=1$ transversal a $W_{l o c}^{s}(p)$. Pelo Corolário 2.2.26, existe um ponto $w \in S$ e um tempo $t_{w}>0$ tal que $\varphi\left(t_{w}, w\right) \in B(z, \varepsilon)$. Como $A\left(x^{s}\right)$ é um conjunto invariante, então $w \in A\left(x^{s}\right)$. Como $\varepsilon$ e o disco $S$ podem ser escolhidos arbitrariamente pequenos, então existem pontos em $A\left(x^{s}\right)$ arbitrariamente próximos a $\widetilde{q}$. Consequentemente, $\widetilde{q} \in \overline{A\left(x^{s}\right)}$. Como $W_{l o c}^{s}(p) \cap A\left(x^{s}\right)=\emptyset$, então $\widetilde{q} \in \partial A\left(x^{s}\right)$. Como $\partial A\left(x^{s}\right)$ é um conjunto invariante, $\widehat{q}=\varphi(-\bar{T}, \widetilde{q}) \in \partial A\left(x^{s}\right)$. Como a escolha de $\widehat{q}$ em $W^{s}(p)$ foi arbitrária, então podemos concluir que $W^{s}(p) \subset \partial A\left(x^{s}\right)$.

O próximo teorema mostra a caracterização dos elementos críticos hiperbólico e pontos de equilíbrio Hopf subcrítico na fronteira da região de estabilidade para tipo- $k$, com $1 \leq k \leq n-3$.

Teorema 6.3.5. (Elementos críticos tipo-k na fronteira da região de estabilidade): Seja $A\left(x^{s}\right)$ a região de estabilidade de um ponto de equilíbrio assintoticamente estável $x^{s}$ de (2.1) e suponha as seguintes suposições 
(B1"), (B2") e (B3) são satisfeitas. Sejam p um ponto equilíbrio Hopf subcrítico do tipo- $k$, com $1 \leq k \leq n-3$, e $\psi$ um elemento crítico do tipo- $k^{\prime}$, com $k^{\prime} \leq n$, de (2.1). Então:

i.

$$
\begin{aligned}
& p \in \partial A\left(x^{s}\right) \Longleftrightarrow W^{c u}(p) \cap A\left(x^{s}\right) \neq \emptyset \\
& \psi \in \partial A\left(x^{s}\right) \Longleftrightarrow W^{u}(\psi) \cap A\left(x^{s}\right) \neq \emptyset
\end{aligned}
$$

ii.

$$
\begin{aligned}
p \in \partial A\left(x^{s}\right) & \Longleftrightarrow W^{s}(p) \subset \partial A\left(x^{s}\right) \\
\psi \in \partial A\left(x^{s}\right) & \Longleftrightarrow W^{s}(\psi) \subset \partial A\left(x^{s}\right)
\end{aligned}
$$

Demonstração. i. $(\Longleftarrow)$ A prova é análoga a prova dos Teoremas 6.3 .1 a 6.3.4 e será omitida.

$(\Longrightarrow)$ Demonstraremos o teorema usando indução finita na dimensão de $W^{c u}(x)$ ou $W^{u}(x)$ se $x \in \partial A\left(x^{s}\right)$ é um ponto de equilíbrio Hopf subcritico ou um elemento crítico hiperbólico. Se $\operatorname{dim} W^{u}(x)=1$, então pelo Teorema 6.3.1 ou 6.3.3 sabemos que $W^{u}(x) \cap A\left(x^{s}\right) \neq \emptyset$. Suponha que $W^{i}(x) \cap A\left(x^{s}\right) \neq$ $\emptyset$, para $i=u$ ou $i=c u$ para todo os elementos críticos $x$ na fronteira $\partial A\left(x^{s}\right)$ com $\operatorname{dim} W^{u}(x) \leq k$. Agora, suponhamos que $\operatorname{dim} W^{i}(x)=k+1$. Pelo Teorema 5.3.1 ou Teorema 3.8 de [CHW88], podemos concluir que $\left(W_{l o c}^{i}(x) \backslash\right.$ $\{x\}) \cap \overline{A\left(x^{s}\right)} \neq \emptyset$, para $i=c u$ ou $i=u$ respectivamente. Consequentemente $\left(W^{i}(x) \backslash\{x\}\right) \cap \overline{A\left(x^{s}\right)} \neq \emptyset$, pois $W_{l o c}^{i}(x) \subset W^{i}(x)$. Vamos mostrar, sob as suposições $\left(\right.$ B1 $\left.^{\prime \prime}\right),\left(\right.$ B2 $\left.^{\prime \prime}\right)$ e $($ B3 $)$ que $\left(W^{i}(x) \backslash\{x\}\right) \cap \overline{A\left(x^{s}\right)} \neq \emptyset$ implica em $W^{i}(x) \cap A\left(x^{s}\right) \neq \emptyset$. Seja $q \in\left(W^{i}(x) \backslash\{p\}\right) \cap \overline{A\left(x^{s}\right)}$. Se $q \in A\left(x^{s}\right)$, não existe nada a ser provado. Suponha que $q \in \partial A\left(x^{s}\right)$. Da condição (B3), existe um ponto de equilíbrio $\widehat{p} \in \partial A\left(x^{s}\right)$ tal que $\varphi(t, q) \rightarrow \widehat{p}$ quando $t \rightarrow+\infty$. De $\left(\mathbf{B 1}^{\prime \prime}\right)$, então $\widehat{p}$ é um elemento crítico hiperbólico ou ponto de equilíbrio Hopf subcrítico. Pelos Lemas 6.1.2 ou 6.1.3 ou 6.1.4 ou 6.1.6, se $x$ é um elemento crítico hiperbólico ou um ponto de equilíbrio Hopf subcrítico do tipo- $k$, com $1 \leq k \leq n-3$, ou um ponto de equilíbrio Hopf subcrítico do tipo- $(n-2)$ respectivamente, concluimos que $\operatorname{dim} W^{j}(\widehat{p})<\operatorname{dim} W^{i}(x)$, para $j=u$ se $\widehat{p}$ é um elemento crítico hiperbólico ou $j=c u$ se $\widehat{p}$ é um ponto de equilíbrio Hopf subcrítico. Seja $\widehat{p}$ um elemento crítico hiperbólico. Como $\operatorname{dim} W^{u}(\widehat{p})<\operatorname{dim} W^{u}(x)$, então concluímos que $\operatorname{dim} W^{u}(\widehat{p}) \leq k$. Portanto, pela hipótese de indução $W^{u}(\widehat{p}) \cap A\left(x^{s}\right) \neq \emptyset$. Seja $y \in W^{u}(\widehat{p}) \cap A\left(x^{s}\right)$ e $B(y, \varepsilon)$ uma bola aberta de raio $\varepsilon>0$ centrada em $y$. Como $A\left(x^{s}\right)$ é um conjunto aberto, então $B(y, \varepsilon) \subset A\left(x^{s}\right)$ para $\varepsilon$ suficientemente pequeno. Seja $N^{u}$ uma vizinhança de $q$ em $W^{u}(x)$. A vizinhança $N^{u}$ contém uma seção transversal $D$ de $W^{s}(\widehat{p})$ no ponto $q$ com dimensão $\operatorname{dim} D \leq k$. Como uma consequência do $\lambda$-Lemma, existe um ponto $w \in D$ e um tempo $t_{w}>0$ tal que $\varphi\left(t_{w}, w\right) \in N^{u}$. Como $A\left(x^{s}\right)$ é um conjunto invariante, então $w \in A\left(x^{s}\right)$. Portanto, $w \in W^{u}(x) \cap A\left(x^{s}\right)$ e, consequentemente, $W^{u}(x) \cap A\left(x^{s}\right) \neq \emptyset$. 
Capítulo 6. Fronteira da Região de Estabilidade na Presença de um Ponto 116 de Equilíbrio Hopf com Elementos Críticos

Se $\widehat{p}$ é um ponto de equilíbrio Hopf subcrítico e como $\operatorname{dim} W^{c u}(\widehat{p})<$ $\operatorname{dim} W^{u}(x)$, então também concluimos que $\operatorname{dim} W^{c u}(\widehat{p}) \leq k$. Portanto, pela hipótese de indução $W^{c u}(\widehat{p}) \cap A\left(x^{s}\right) \neq \emptyset$. Seja $y \in W^{c u}(\widehat{p}) \cap A\left(x^{s}\right)$ e $B(y, \varepsilon)$ uma bola aberta de raio $\varepsilon>0$ centrada em $y$. Como $A\left(x^{s}\right)$ é um conjunto aberto, então $B(y, \varepsilon) \subset A\left(x^{s}\right)$ para $\varepsilon$ suficientemente pequeno. Seja $N^{u}$ uma vizinhança de $q$ em $W^{u}(x)$. A vizinhança $N^{u}$ contém um seção transversal $D$ de $W^{s}(\widehat{p})$ no ponto $q$ com dimensão $\operatorname{dim} D \leq k$. Como uma consequência da vers $\tilde{A} £$ o $\lambda$-Lemma para pontos de equilíbrios Hopf subcríticos, existem um ponto $w \in D$ e um tempo $t_{w}>0$ tal que $\varphi\left(t_{w}, w\right) \in N^{u}$. Como $A\left(x^{s}\right)$ é um conjunto invariante, então $w \in A\left(x^{s}\right)$. Portanto, $w \in W^{u}(x) \cap A\left(x^{s}\right) \mathrm{e}$ consequentemente, $W^{u}(x) \cap A\left(x^{s}\right) \neq \emptyset$.

ii. A prova é análoga a prova dos Teoremas 6.3 .1 a 6.3 .4 e será omitida.

Para completar a caracterização dos elementos críticos na fronteira da região de estabilidade, resta mostrar a caracterização dos pontos de equilíbrio Hopf subcrítico na fronteira da região de estabilidade para tipo- $(n-2)$.

Teorema 6.3.6. (Ponto de equilíbrio Hopf subcrítico tipo- $(n-2)$ na fronteira da região de estabilidade): Seja $A\left(x^{s}\right)$ a região de estabilidade de um ponto de equilibrio assintoticamente estável $x^{s}$ de (2.1). Seja $p$ um ponto de equilíbrio Hopf subcrítico do tipo-n -2 de (2.1). Se as suposições $\left(\boldsymbol{B 1 ^ { \prime \prime }}\right),\left(\boldsymbol{B}^{\prime \prime}\right)$ e (B3) são satisfeitas, então:

$$
p \in \partial A\left(x^{s}\right) \Longleftrightarrow W^{c u}(p) \cap A\left(x^{s}\right) \neq \emptyset
$$

Demonstração. A prova é similar a prova do Teorema 6.3 .5 e será omitida.

O próximo teorema oferece uma completa caracterização da fronteira de estabilidade quando pontos de equilíbrio Hopf subcríticos encontram-se em $\partial A\left(x^{s}\right)$.

Teorema 6.3.7. (Caracterização da Fronteira da Região de Estabilidade): Seja $x^{s}$ um ponto de equilíbrio assintoticamente estável de (2.1) e $A\left(x^{s}\right)$ sua região de estabilidade. Se as suposições (B1"), e (B3) são satisfeitas, então

$$
\partial A\left(x^{s}\right) \subset \bigcup_{i} W^{s}\left(\psi_{i}\right) \bigcup_{j} W^{s}\left(p_{j}\right)
$$

onde $\psi_{i}$ são os elementos críticos hiperbólico e $p_{j}$ os pontos de equilíbrio Hopf subcríticos em $\partial A\left(x^{s}\right), i, j=1,2, \ldots$ Se a suposição (B2") é satisfeita, então

$$
\partial A\left(x^{s}\right)=\bigcup_{i} W^{s}\left(\psi_{i}\right) \bigcup_{j} W^{s}\left(p_{j}\right) .
$$


Demonstração. Seja $q \in \partial A\left(x^{s}\right)$. Pela hipótese (B3), podemos afirmar que existe um ponto de equilíbrio $x$ tal que $\varphi(t, q) \rightarrow x$ quando $t \rightarrow+\infty$. Pela hipótese $\left(\mathbf{B} 1^{\prime \prime}\right)$, podemos afirmar que $x$ é um elemento crítico hiperbólico $\psi_{i}$ ou um ponto de equilíbrio Hopf subcrítico $x=p_{j}$, ou seja, $x=\psi_{i}$ or $x=p_{j}$ para algum $i, j$. Portanto, concluimos que $q \in \bigcup_{i} W^{s}\left(\psi_{i}\right) \bigcup_{j} W^{s}\left(p_{j}\right)$. Portanto,

$$
\partial A\left(x^{s}\right) \subset \bigcup_{i} W^{s}\left(\psi_{i}\right) \bigcup_{j} W^{c s}\left(p_{j}\right) \bigcup_{n} W^{c}\left(q_{l}\right) .
$$

Pelos Teoremas 6.3.1, 6.3.2, 6.3.3, 6.3.4 e 6.3.5, sabemos que $W^{s}\left(\psi_{i}\right) \subset$ $\partial A\left(x^{s}\right)$ e $W^{s}\left(p_{j}\right) \subset \partial A\left(x^{s}\right)$. Logo, $\bigcup_{i} W^{s}\left(\psi_{i}\right) \bigcup_{j} W^{s}\left(p_{j}\right) \subset \partial A\left(x^{s}\right)$ e, portanto,

$$
\partial A\left(x^{s}\right)=\bigcup_{i} W^{s}\left(\psi_{i}\right) \bigcup_{j} W^{s}\left(p_{j}\right)
$$


Capítulo 6. Fronteira da Região de Estabilidade na Presença de um Ponto 118 de Equilíbrio Hopf com Elementos Críticos 


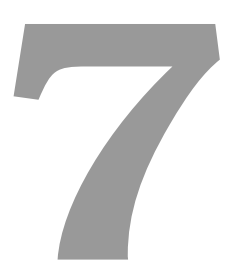

\section{Fronteira e Região de Estabilidade Fraca de um Ponto de Equilíbrio Hopf Supercrítico do Tipo-Zero}

Numa bifurcação de Hopf supercrítica do tipo-0, um ponto de equilíbrio hiperbólico assintoticamente estável perde a estabilidade e surge uma órbita periódica hiperbólica assintoticamente estável. Deseja-se estudar como as regiões de estabilidade do equilíbrio e da órbita periódica se relacionam. No capítulo 9, apresentaremos uma caracterização da fronteira da região de estabilidade na vizinhança de um parâmetro de bifurcação de Hopf supercrítico do tipo-0. Para tal caracterização, precisaremos definir o conceito de região de estabilidade fraca de um ponto de equilíbrio Hopf supercrítico do tipo-0 e exibiremos o comportamento de sua fronteira. Além disso, também definiremos a região de quase-estabilidade de um ponto de equilíbrio Hopf supercrítico do tipo-0 e faremos uma caracterização da sua fronteira de região de quase-estabilidade de um ponto de equilíbrio Hopf supercrítico do tipo-zero.

\subsection{Região de Estabilidade Fraca de um Ponto de equilíbrio Hopf Supercrítico do tipo zero}

Nesta seção, inicialmente, definiremos região de estabilidade fraca de um ponto de equilíbrio Hopf supercrítico do tipo-0 e estudaremos algumas de suas propriedades topológicas. Em seguida, exibiremos uma caracterização da região de estabilidade de um ponto de equilíbrio Hopf supercrítico do tipo- 
Capítulo 7. Fronteira e Região de Estabilidade Fraca de um Ponto de 120 Equilíbrio Hopf Supercrítico do Tipo-Zero

0. $\mathrm{O}$ adjetivo fraca é utilizado porque o ponto de equilíbrio Hopf supercrítico do tipo-0 é um ponto de equilíbrio atrativo mas que perde esta propriedade com a variação dos parâmetros.

Seja $p$ um ponto de equilíbrio Hopf supercrítico do tipo-zero do sistema (2.1). A região de estabilidade fraca de $p$ é o conjunto $A(p)$ dos pontos $q \in \mathbb{R}^{n}$ cujas trajetórias convergem para $p$ quando $t \rightarrow+\infty$, isto é,

$$
A(p)=\left\{q \in \mathbb{R}^{n}: \varphi(t, q) \rightarrow p \text { quando } t \rightarrow+\infty\right\} .
$$

Os próximos resultados estabelecem que a região de estabilidade fraca $A(p)$ é um conjunto aberto invariante.

Teorema 7.1.1. Seja p um ponto de equilíbrio Hopf supercrítico do tipo-zero do sistema (2.1). Então a região de estabilidade fraca $A(p)$ é um conjunto aberto em $\mathbb{R}^{n}$.

Demonstração. Como o ponto de equilíbrio Hopf supercrítico do tipo-zero $p$ é um ponto de equilíbrio atrativo, então existe $\varepsilon>0$ tal que $B(p, \varepsilon) \subset A(p)$. Seja $q \in A(p)$. Então $\varphi(t, q) \rightarrow p$ quando $t \rightarrow+\infty$, ou seja, existe um tempo $T>0$ tal que $d(\varphi(T, q), p)<\frac{\varepsilon}{2}$. Pela continuidade das soluções com relação às condições iniciais, existe $\delta>0$ tal que $\varphi(T, x) \in B\left(\varphi(T, q), \frac{\varepsilon}{2}\right)$ sempre que $x \in B(q, \delta)$. Temos $\varphi(t, x)=\varphi(t-T, \varphi(T, x))$. Então $s=t-T \rightarrow+\infty$ quando $t \rightarrow+\infty$ e consequentemente,

$$
\lim _{t \rightarrow+\infty} \varphi(t, x)=\lim _{s \rightarrow+\infty} \varphi(s, \varphi(T, x))=p .
$$

Segue-se que existe $\delta>0$ tal que para todo $x \in B(q, \delta)$ temos $x \in A(p)$. Logo, $A(p)$ é um conjunto aberto.

Teorema 7.1.2. Seja $p$ um ponto de equilíbrio Hopf supercrítico do tipozero do sistema (2.1). Então a região de estabilidade fraca $A(p)$, o seu fecho $\overline{A(p)}$ e a sua fronteira $\partial A(p)$ são conjuntos invariantes.

Demonstração. Seja $q \in A(p)$. Segue-se que $\varphi(t, q) \rightarrow p$ quando $t \rightarrow+\infty$. Seja $\bar{q}=\varphi(s, q)$ para algum $s \in \mathbb{R}$ arbitrário. Pela propriedade de fluxos, $\varphi(t, \bar{q})=\varphi(t, \varphi(s, q))=\varphi(t+s, q)$. Então, para cada $s$ fixo, temos que $t+s \rightarrow+\infty$ quando $t \rightarrow+\infty$ e consequentemente,

$$
\varphi(t, \bar{q})=\varphi(t+s, q) \rightarrow p .
$$

Portanto, $\bar{q} \in A(p)$. Como a escolha de $q$ em $A(p)$ foi arbitrária, concluímos que $A(p)$ é um conjunto invariante com relação ao sistema (2.1).

Pelos Teoremas 7.1 .1 e 2.2 .5 e pela primeira parte do Teorema 7.1.2, podemos concluir que $\overline{A(p)}$ e $\partial A(p)$ também são invariantes. 
Para a caracterização da fronteira da região de estabilidade de um ponto de equilíbrio Hopf supercrítico do tipo-zero, inicialmente, faremos uma caracterização local da fronteira da região de estabilidade por meio do estudo e caracterização dos pontos de equilíbrio que pertencem à fronteira da região de estabilidade, e depois exibiremos uma caracterização global.

Os próximos teoremas fornecem condições necessárias e suficientes para garantir que pontos de equilíbrio estejam na fronteira da região de estabilidade em termos de suas variedades estável e instável.

Teorema 7.1.3. (Ponto de equilíbrio hiperbólico na fronteira da região de estabilidade fraca) Sejam $p$ um ponto de equilíbrio Hopf supercrítico do tipo-zero do sistema (2.1) e $A(p)$ sua região de estabilidade fraca. Se $x$ é um ponto de equilíbrio hiperbólico do sistema (2.1), então

$$
\begin{gathered}
x \in \partial A(p) \Longleftrightarrow\left(W_{l o c}^{u}(x) \backslash\{x\}\right) \cap \overline{A(p)} \neq \emptyset \\
x \in \partial A(p) \Longleftrightarrow W_{l o c}^{s}(x) \cap \partial A(p) \neq \emptyset
\end{gathered}
$$

Demonstração. Suponhamos que $\left(W_{l o c}^{u}(x) \backslash\{x\}\right) \cap \overline{A(p)} \neq \emptyset$. Então existe $q \in$ $\left(W_{l o c}^{u}(x) \backslash\{x\}\right) \cap \overline{A(p)}$. Observe que $\varphi(t, q) \longrightarrow x$ quando $t \longrightarrow-\infty$. Por outro lado, o conjunto $\overline{A(p)}$ é invariante e deste modo, $\varphi(t, q) \in \overline{A(p)}$ para todo $t \leq$ 0 . Consequentemente, $x \in \overline{A(p)}$, visto que $\overline{A(p)}$ é um conjunto fechado. Como $x \notin A(p)$, temos que $x \in \mathbb{R}^{n} \backslash A(p)$. Portanto, $x \in \partial A(p)$. Reciprocamente, suponhamos que $x \in \partial A(p)$. Seja $N^{u}$ um domínio fundamental de $W^{u}(x)$, isto é, $\bigcup_{t \in \mathbb{R}} \varphi\left(t, N^{u}\right)=W^{u}(x) \backslash\{x\}$. Seja $N_{\varepsilon}^{u}$ uma vizinhança fundamental de raio $\varepsilon$ de $N^{u}$, isto é, $N_{\varepsilon}^{u}=\left\{y \in \mathbb{R}^{n}: d\left(y, N^{u}\right)<\varepsilon\right\}$. Pelo Corolário 2.2.20, existe uma vizinhança $U$ de $x$ tal que $\bigcup_{t \leq 0} \varphi\left(t, N_{\varepsilon}^{u}\right) \supset U \backslash W_{l o c}^{s}(x)$. Como $x \in \partial A(p)$, então $U \cap A(p) \neq \emptyset$. Por outro lado, $W_{l o c}^{s}(x) \cap A(p)=\emptyset$. Logo, $\left\{U \backslash W_{\text {loc }}^{s}(x)\right\} \cap A(p) \neq \emptyset$. Consequentemente, existe um ponto $q \in N_{\varepsilon}^{u}$ e um tempo $t_{q}$ tal que $\varphi\left(t_{q}, q\right) \in A(p)$. Como $A(p)$ é um conjunto invariante, então $q \in A(p)$. Como $\varepsilon$ pode ser escolhido arbitrariamente pequeno, podemos encontrar uma sequência de pontos $\left\{q_{i}\right\}$ com $q_{i} \in A(p)$ para todo $i=1,2, \ldots$ tal que $d\left(q_{i}, N^{u}\right) \rightarrow 0$ quando $i \rightarrow+\infty$. Por construção, a sequência $\left\{q_{i}\right\}$ é limitada e, portanto, admite uma subsequência convergente. Seja $\left\{q_{i_{k}}\right\}$ uma subsequência convergente, isto é, $q_{i_{k}} \rightarrow \bar{q}$ quando $i_{k} \rightarrow+\infty$. Observe que $d\left(q_{i_{k}}, N^{u}\right) \rightarrow d\left(\bar{q}, N^{u}\right)$ quando $i_{k} \rightarrow+\infty$ e, portanto, $\bar{q} \in \overline{N^{u}} \subset W_{l o c}^{u}(x) \backslash\{x\}$. Logo, $\bar{q} \in\left(W_{l o c}^{u}(x) \backslash\{p\}\right) \cap \overline{A(p)}$.

Suponhamos que $W_{l o c}^{s}(x) \cap \partial A(p) \neq \emptyset$. Portanto, existe $q \in W_{l o c}^{s}(x) \cap$ $\partial A(p)$. Note que $\varphi(t, q) \longrightarrow x$ quando $t \longrightarrow+\infty$. Como o conjunto $\partial A(p)$ é invariante e $q \in \partial A(p)$, temos $\varphi(t, q) \in \partial A(p)$ para todo $t \geq 0$. Como $\partial A(p)$ é um conjunto fechado, então $x \in \partial A(p)$. A prova de que $W_{l o c}^{s}(x) \cap \partial A(p) \neq \emptyset$ se $x \in \partial A(p)$ é muito similar à demonstração da primeira parte e, portanto, será omitida.

Generalizando o Teorema 7.1.3, enunciaremos o Teorema 7.1.4 que oferece condições necessárias e suficientes para que uma órbita periódica per- 
Capítulo 7. Fronteira e Região de Estabilidade Fraca de um Ponto de 122 Equilíbrio Hopf Supercrítico do Tipo-Zero

tença à fronteira da região de estabilidade fraca do ponto de equilíbrio Hopf supercrítico do tipo-zero.

Teorema 7.1.4. (Órbita periódica hiperbólica na fronteira da região de estabilidade fraca) Sejam $p$ um ponto de equilibrio Hopf supercrítico do tipo-zero do sistema (2.1) e A(p) sua região de estabilidade fraca. Se $\phi$ é uma órbita periódica hiperbólica do sistema (2.1), então

$$
\begin{gathered}
\phi \text { está em } \partial A(p) \Longleftrightarrow\left(W_{l o c}^{u}(\phi) \backslash\{\phi\}\right) \cap \overline{A(p)} \neq \emptyset \\
\phi \text { está em } \partial A(p) \Longleftrightarrow W_{l o c}^{s}(\phi) \cap \partial A(p) \neq \emptyset
\end{gathered}
$$

Demonstração. Suponhamos que $\left(W_{l o c}^{u}(\phi) \backslash\{\phi\}\right) \cap \overline{A(p)} \neq \emptyset$. Então existe $q \in\left(W_{l o c}^{u}(\phi) \backslash\{\phi\}\right) \cap \overline{A(p)}$. Observe que $\varphi(t, q) \longrightarrow \phi$ quando $t \longrightarrow-\infty$. Ou seja, para todo $\varepsilon>0$, existe $t_{0}<0$ tal que $d(\varphi(t, q), \phi) \leq \varepsilon$ para todo $t \leq t_{0}$. Por outro lado, o conjunto $\overline{A(p)}$ é invariante e deste modo, $\varphi(t, q) \in \overline{A(p)}$ para todo $t \leq 0$. Então $d(\overline{A(p)}, \phi) \leq d(\varphi(t, q), \phi) \leq \varepsilon$ para todo $t \leq t_{0}$. Portanto, $d(\overline{A(p)}, \phi)=0$ e consequentemente, $\phi \subset \overline{A(p)}$. Como $\phi \notin A(p)$, temos que $\phi \subset \mathbb{R}^{n} \backslash A(p)$. Portanto, $\phi \subset \partial A(p)$. Reciprocamente, suponhamos que $\phi \subset \partial A(p)$. Seja $N^{u}$ um domínio fundamental de $W^{u}(\phi)$, isto é, $\bigcup_{t \in \mathbb{R}} \varphi\left(t, N^{u}\right)=W^{u}(\phi) \backslash \phi$. Seja $N_{\varepsilon}^{u}$ uma vizinhança fundamental de raio $\varepsilon$ de $N^{u}$, isto é, $N_{\varepsilon}^{u}=\left\{y \in \mathbb{R}^{n}: d\left(y, N^{u}\right)<\varepsilon\right\}$. Pelo Corolário 2.2.22, existe uma vizinhança $U$ de $\phi$ tal que $\bigcup_{t \leq 0} \varphi\left(t, N_{\varepsilon}^{u}\right) \supset U \backslash W_{l o c}^{s}(\phi)$. Como $\phi \subset \partial A(p)$, então $U \cap A(p) \neq \emptyset$. Por outro lado, $W_{l o c}^{s}(\phi) \cap A(p)=\emptyset$. Logo, $\left\{U \backslash W_{l o c}^{s}(\phi)\right\} \cap A(p) \neq \emptyset$. Consequentemente, existe um ponto $q \in N_{\varepsilon}^{u}$ e um tempo $t_{q}$ tal que $\varphi\left(t_{q}, q\right) \in A(p)$. Como $A(p)$ é um conjunto invariante, então $q \in A(p)$. Como $\varepsilon$ pode ser escolhido arbitrariamente pequeno, podemos encontrar uma sequência de pontos $\left\{q_{i}\right\} \operatorname{com} q_{i} \in A(p)$ para todo $i=1,2, \ldots$ tal que $d\left(q_{i}, N^{u}\right) \rightarrow 0$ quando $i \rightarrow+\infty$. Por construção, a sequência $\left\{q_{i}\right\}$ é limitada e, portanto, admite uma subsequência convergente. Seja $\left\{q_{i_{k}}\right\}$ uma subsequência convergente, isto é, $q_{i_{k}} \rightarrow \bar{q}$ quando $i_{k} \rightarrow+\infty$. Observe que $d\left(q_{i_{k}}, N^{u}\right) \rightarrow d\left(\bar{q}, N^{u}\right)$ quando $i_{k} \rightarrow+\infty$ e, portanto, $\bar{q} \in \overline{N^{u}} \subset W_{l o c}^{u}(x) \backslash\{\phi\}$. Logo, $\bar{q} \in\left(W_{l o c}^{u}(\phi) \backslash\{p\}\right) \cap \overline{A(p)}$.

Suponhamos que $W_{l o c}^{s}(\phi) \cap \partial A(p) \neq \emptyset$. Portanto, existe $q \in W_{l o c}^{s}(\phi) \cap$ $\partial A(p)$. Note que $\varphi(t, q) \longrightarrow \phi$ quando $t \longrightarrow+\infty$. Ou seja, para todo $\varepsilon>0$, existe $t_{1}>0$ tal que $d(\varphi(t, q), \phi) \leq \varepsilon$ para todo $t \geq t_{1}$. Por outro lado, o conjunto $A(p)$ é invariante e deste modo, $\varphi(t, q) \in A(p)$ para todo $t \geq 0$. Então $d(A(p), \phi) \leq d(\varphi(t, q), \phi) \leq \varepsilon$ para todo $t \leq t_{0}$. Portanto, $d(A(p), \phi)=$ 0 e consequentemente, $\phi \subset A(p)$. A prova de que $W_{l o c}^{s}(\phi) \cap \partial A(p) \neq \emptyset$ se $\phi \subset \partial A(p)$ é muito similar à demonstração da primeira parte e, portanto, será omitida.

Desenvolveremos uma caracterização da fronteira da região de estabilidade fraca de um ponto de equilíbrio Hopf supercrítico do tipo-0. Para isto, admitiremos algumas condições sobre o campo vetorial. 
Sejam $p$ um ponto de equilíbrio Hopf supercrítico do tipo-0 do sistema (2.1) e considere as seguintes suposições:

(S1) Todos os elementos críticos em $\partial A(p)$ são hiperbólicos;

(S2) A variedade estável e a variedade instável dos elementos críticos em $\partial A(p)$ satisfazem a condição de transversalidade;

(S3) Toda trajetória em $\partial A(p)$ se aproxima de um elemento crítico quando $t \rightarrow+\infty$.

Os próximos resultados estabelecem condições necessárias e suficientes para garantir que os elementos críticos hiperbólicos pertençam à fronteira da região de estabilidade. Inicialmente, desenvolveremos uma caracterização de pontos de equilíbrio do tipo-1 na fronteira da região de estabilidade fraca e depois a caracterização para pontos de equilíbrios de tipos superiores a 1 seguirá por argumentos de indução.

Teorema 7.1.5. (Elementos críticos hiperbólicos do tipo-1 na fronteira da região de estabilidade fraca) Sejam $A(p)$ a região de estabilidade fraca de um ponto de equilíbrio Hopf supercrítico do tipo-zero $p$ e $\psi$ um elemento crítico hiperbólico do tipo-1 de (2.1). Se as suposições (S1), (S2) e (S3) são satisfeitas, então:

(i) o elemento crítico $\psi$ está em $\partial A(p)$ se, e somente se, $W^{u}(\psi) \cap A(p) \neq \emptyset$

(ii) o elemento crítico $\psi$ está em $x \in \partial A(p)$ se, e somente se, $W^{s}(\psi) \subset$ $\partial A(p)$

Demonstração. (i) $(\Longleftarrow)$ Suponhamos que $W^{u}(\psi) \cap A(p) \neq \emptyset$. Como $A(p) \subset$ $\overline{A(p)}$, então $\left(W_{l o c}^{u}(\psi) \backslash\{\psi\}\right) \cap \overline{A(p)} \neq \emptyset$. Portanto, pelos Teoremas $7.1 .3 \mathrm{e}$ 7.1.4, temos que $\psi$ está em $\partial A(p)$.

$(\Longrightarrow)$ Suponhamos que $\psi \in \partial A(p)$. Pelo Teorema 7.1.3 e 7.1.4, podemos concluir que $\left(W_{\text {loc }}^{u}(\psi) \backslash\{x\}\right) \cap \overline{A(p)} \neq \emptyset$. Consequentemente, $\left(W^{u}(\psi) \backslash\{\psi\}\right) \cap$ $\overline{A(p)} \neq \emptyset$, pois $W_{\text {loc }}^{u}(\psi) \subset W^{u}(x)$. Vamos mostrar, sob as suposições (S1), (S2) e (S3) que $\left(W^{u}(\psi) \backslash\{\psi\}\right) \cap \overline{A(p)} \neq \emptyset$ implica em $W^{u}(\psi) \cap A(p) \neq \emptyset$. Seja $q \in\left(W^{u}(\psi) \backslash\{x\}\right) \cap \overline{A(p)}$. Se $q \in A(p)$, então não existe nada a ser provado. Suponhamos que $q \in \partial A(p)$. Da condição (S3) e (S1), existe um elemento crítico hiperbólico $\widehat{\psi} \in \partial A(p)$ tal que $\varphi(t, q) \rightarrow \widehat{\psi}$ quando $t \rightarrow$ $+\infty$. Então $\widehat{\psi}$ é um elemento crítico hiperbólico e consequentemente, $q \in$ $W^{s}(\widehat{\psi}) \cap W^{u}(\psi)$. Pelo Lema 6.1.2, concluímos que $\operatorname{dim} W^{u}(\widehat{\psi})<\operatorname{dim} W^{u}(\psi)$ e, portanto, $\operatorname{dim} W^{u}(\widehat{\psi})<1$. Segue-se $\operatorname{dim} W^{u}(\widehat{\psi})=0$ e, consequentemente, $\widehat{\psi}$ é um ponto de equilíbrio hiperbólico do tipo zero. Isto nos leva a uma contradição, pois pontos de equilíbrio do tipo zero não podem pertencer a $\partial A(p)$. Sendo assim, $q \in A(p)$ e, portanto, $W^{u}(\psi) \cap A(p) \neq \emptyset$.

(ii) $(\Longleftarrow)$ Suponhamos que $W^{s}(\psi) \subset \partial A(p)$. Como $\psi \in W^{s}(x)$, então $\psi \in \partial A(p)$.

$(\Longrightarrow)$ Suponhamos que $\psi \in \partial A(p)$. Pelo item (i) do Teorema 7.1.5, podemos concluir que $W^{u}(\psi) \cap A(p) \neq \emptyset$. Seja $y \in W^{u}(\psi) \cap A(p)$. Como 
Capítulo 7. Fronteira e Região de Estabilidade Fraca de um Ponto de 124 Equilíbrio Hopf Supercrítico do Tipo-Zero

$y \in W^{u}(\psi)$, então existe $t_{y}<0$ tal que $\varphi\left(t_{y}, y\right) \in W_{l o c}^{u}(\psi)$. Seja $z=\varphi\left(t_{y}, y\right)$. Como $y \in A(p)$ e $A(p)$ é um conjunto invariante, então $z \in A(p)$. Seguese que $z \in W_{l o c}^{u}(\psi) \cap A(p)$. Seja $B(z, \varepsilon)$ uma bola aberta de raio $\varepsilon>0$ centrado em $z$, onde $\varepsilon$ é um número arbitrariamente pequeno. Seja $\widehat{q}$ um ponto arbitrário de $W^{s}(\psi)$. Em particular, para algum $t_{\widehat{q}}>0$ temos $\widetilde{q}=$ $\varphi\left(t_{\widehat{q}}, \widehat{q}\right) \in W_{l o c}^{s}(\psi)$. Seja $S$ um disco no ponto $\widetilde{q} \operatorname{de} \operatorname{dim} S=1$ transversal a $W_{l o c}^{s}(\psi)$. Pelos Lemas 2.2.19 ou 2.2.21, existe um ponto $w \in S$ e um tempo $t_{w}>0$ tal que $\varphi\left(t_{w}, w\right) \in B(z, \varepsilon)$. Como $A(p)$ é um conjunto invariante, então $w \in A(p)$. Como $\varepsilon$ e o disco $S$ podem ser escolhidos arbitrariamente pequenos, então existem pontos em $A(p)$ arbitrariamente próximos a $\widetilde{q}$. Consequentemente, $\widetilde{q} \in \overline{A(p)}$. Como $W_{l o c}^{s}(\psi) \cap A(p)=\emptyset$, então $\widetilde{q} \in \partial A(p)$. Como $\partial A(p)$ é um conjunto invariante, $\widehat{q}=\varphi\left(-t_{\widehat{q}}, \widetilde{q}\right) \in \partial A(p)$. Como a escolha de $\widehat{q}$ em $W^{s}(\psi)$ foi arbitrária, então podemos concluir que $W^{s}(\psi) \subset \partial A(p)$.

O próximo teorema mostra a caracterização dos elementos críticos hiperbólicos tipo- $k$, com $k \geq 1$, na fronteira da região de estabilidade.

Teorema 7.1.6. (Elementos críticos hiperbólicos tipo-k na fronteira da região de estabilidade fraca) Sejam $A(p)$ a região de estabilidade fraca de um ponto de equilíbrio Hopf supercrítico do tipo-zero $p$ e $\psi$ um ulemento crítico hiperbólico do tipo-k, com $1 \leq k \leq n$, do sistema (2.1). Se as suposições (S1), (S2) e (S3) são satisfeitas, então:

(i)

$$
\psi \in \partial A(p) \Longleftrightarrow W^{u}(\psi) \cap A(p) \neq \emptyset
$$

$$
\psi \in \partial A(p) \Longleftrightarrow W^{s}(\psi) \subset \partial A(p)
$$

Demonstração. i. $(\Longleftarrow)$ A demonstração é análoga à demonstração do Teorema 7.1.5 e será omitida.

$(\Longrightarrow)$ Suponhamos que $\psi \in \partial A(p)$. Demonstraremos o teorema usando indução finita na dimensão de $W^{u}(\psi)$. Se $\operatorname{dim} W^{u}(\psi)=1$, então pelo Teorema 7.1.5 temos que $W^{u}(\psi) \cap A(p) \neq \emptyset$. Suponhamos que $W^{u}(\psi) \cap A(p) \neq \emptyset$ para todo os pontos de equilíbrio $\psi$ na fronteira $\partial A(p) \operatorname{com} \operatorname{dim} W^{u}(\psi) \leq k$. Suponhamos que $\operatorname{dim} W^{u}(\psi)=k+1$. Pelo Teorema 7.1.3 e 7.1.4, podemos concluir que $\left(W_{l o c}^{u}(\psi) \backslash\{\psi\}\right) \cap \overline{A(p)} \neq \emptyset$. Consequentemente, $\left(W^{u}(\psi) \backslash\{\psi\}\right) \cap$ $\overline{A(p)} \neq \emptyset$, pois $W_{\text {loc }}^{u}(\psi) \subset W^{u}(\psi)$. Vamos mostrar, sob as suposições (S1), (S2) e (S3) que $\left(W^{u}(\psi) \backslash\{\psi\}\right) \cap \overline{A(p)} \neq \emptyset$ implica em $W^{u}(\psi) \cap A(p) \neq \emptyset$. Seja $q \in\left(W^{u}(\psi) \backslash\{\psi\}\right) \cap \overline{A(p)}$. Se $q \in A(p)$, não existe nada a ser provado. Suponha que $q \in \partial A(p)$. Da condição (S3), existe um elemento crítico $\widehat{\psi} \in \partial A(p)$ tal que $\varphi(t, q) \rightarrow \widehat{\psi}$ quando $t \rightarrow+\infty$. Então $\widehat{\psi}$ é um elemento crítico hiperbólico e consequentemente, $q \in W s(\widehat{\psi}) \cap W^{u}(\psi)$. Pelo Lema 5.1.1, concluímos que $\operatorname{dim} W^{u}(\widehat{\psi})<\operatorname{dim} W^{u}(\psi)$, ou seja, $\operatorname{dim} W^{u}(\widehat{\psi})<k+1$. Segue-se que 
$\operatorname{dim} W^{u}(\widehat{\psi}) \leq k$. Portanto, pela hipótese de indução $W^{u}(\widehat{\psi}) \cap A(p) \neq \emptyset$. Seja $y \in W^{u}(\widehat{\psi}) \cap A(p)$ e $B(y, \varepsilon)$ uma bola aberta de raio $\varepsilon>0$ centrada em $y$. Como $A(p)$ é um conjunto aberto, então $B(y, \varepsilon) \subset A(p)$ para $\varepsilon$ suficientemente pequeno. Seja $N^{u}$ uma vizinhança de $q$ em $W^{u}(\psi)$. A vizinhança $N^{u}$ contém uma seção transversal $D$ de $W^{s}(\widehat{\psi})$ no ponto $q$ com dimensão $\operatorname{dim} D \leq k$. Pelo Lema 2.2.19 e 2.2.21, existe um ponto $w \in D$ e um tempo $t_{w}>0$ tal que $\varphi\left(t_{w}, w\right) \in N^{u}$. Como $A(p)$ é um conjunto invariante, então $w \in A(p)$. Portanto, $w \in W^{u}(\psi) \cap A(p)$ e, consequentemente, $W^{u}(\psi) \cap A(p) \neq \emptyset$.

(ii) A demonstração é análoga à demonstração do Teorema 7.1.5 e será omitida.

O próximo teorema fornece uma completa caracterização da fronteira da região de estabilidade fraca quando elementos críticos hiperbólico pertencem à $\partial A(p)$.

Teorema 7.1.7. (Caracterização da Fronteira da Região de Estabilidade Fraca para Elementos críticos) Sejam p um ponto de equilíbrio Hopf supercrítico do tipo-zero de (2.1) e $A(p)$ sua região de estabilidade fraca. Se as suposições (S1) e (S3) são satisfeitas, então

$$
\partial A(p) \subset \bigcup_{i} W^{s}\left(\psi_{i}\right)
$$

onde $\psi_{i}, i=1,2, \ldots$ são os elementos críticos hiperbólicos em $\partial A\left(x^{s}\right)$. Se a suposição (S2) é satisfeita, então

$$
\partial A(p)=\bigcup_{i} W^{s}\left(\psi_{i}\right)
$$

Demonstração. Seja $q \in \partial A(p)$. Pela hipótese (S3), podemos afirmar que existe um elemento crítico $\psi$ tal que $\varphi(t, q) \rightarrow \psi$ quando $t \rightarrow+\infty$. Pela hipótese $(\mathbf{S 1})$, podemos afirmar que $\psi$ é um elemento crítico hiperbólico $\psi_{i}$ para algum $i$. Portanto, concluimos que $q \in \bigcup_{i} W^{s}\left(\psi_{i}\right)$. Portanto, $\partial A(p) \subset$ $\bigcup_{i} W^{s}\left(\psi_{i}\right)$. Pelos Teoremas 7.1.5 e 7.1.6, sabemos que $W^{s}\left(\psi_{i}\right) \subset \partial A(p)$. Logo, $\bigcup_{i} W^{s}\left(\psi_{i}\right) \subset \partial A(p)$ e, portanto,

$$
\partial A(p)=\bigcup_{i} W^{s}\left(\psi_{i}\right)
$$

Exibiremos alguns exemplos que ilustram os Teoremas 7.1.5, 7.1.6 e 7.1.7.

Exemplo 7.1.1. Considere o sistema Predador-Presa em $\mathbb{R}^{2}$ extraído de 
Capítulo 7. Fronteira e Região de Estabilidade Fraca de um Ponto de 126 Equilíbrio Hopf Supercrítico do Tipo-Zero

[Vér13]

$$
\left\{\begin{array}{l}
\dot{x}=x-\frac{x y}{1+0.5 x}-\frac{x^{2}}{6} \\
\dot{y}=-y\left(1-\frac{x}{1+0.5 x}\right)
\end{array}\right.
$$

onde $(x, y) \in \mathbb{R}^{2}$.

O sistema (7.1) possui três pontos de equilíbrio, são eles: dois pontos de equilíbrio hiperbólicos do tipo-1, $x_{1}=(0,0)$ e $x_{2}=(6,0)$, e um ponto de equilíbrio Hopf supercrítico do tipo-zero, $p=\left(2, \frac{4}{3}\right)$. Como os pontos $x_{1}=$ $(0,0)$ e $x_{2}=(6,0)$ são hiperbólicos então o sistema satisfaz a condição (S1), ver Figura 7.1. A suposição (S3) é satisfeita, mas a suposição (S2) não o é, pois a variedade instável $W^{u}\left(x_{1}\right)$ do ponto de equilíbrio $x_{1}$ não intersecta transversalmente a variedade estável $W^{s}\left(x_{2}\right)$ do ponto de equilíbrio $x_{2}$. A variedade estável $W^{s}\left(x_{1}\right)$ intercepta a fronteira da região de estabilidade fraca $\partial A(p)$, de acordo com o Teorema 7.1.3. A variedade instável do ponto de equilíbrio hiperbólico do tipo-1 $x_{2}=(6,0), W^{u}\left(x_{2}\right)$, intersecta a região de estabilidade fraca $A\left(x^{s}\right)$ e sua variedade estável $W^{s}\left(x_{2}\right)$ está contida na fronteira da região de estabilidade fraca $\partial A(p)$, de acordo com o Teorema 7.1.5.

A Figura 7.1 ilustra a fronteira da região de estabilidade do ponto de equilíbrio Hopf supercrítico do tipo-zero, $p=\left(2, \frac{4}{3}\right)$. A fronteira da região de estabilidade de $p=\left(2, \frac{4}{3}\right)$ está contida, de acordo com a primeira parte do Teorema 7.1.7, na união da variedade estável $W^{s}\left(x_{1}\right)$ do ponto de equilíbrio hiperbólico do tipo-1 $x_{1}=(0,0)$, curva em vermelho na Figura 7.1, com a variedade estável $W^{s}\left(x_{2}\right)$ do ponto de equilibrio hiperbólico do tipo-1 $x_{2}=(6,0)$, curva em preto na Figura 7.1.

Exemplo 7.1.2. Considere o sistema Predador-Presa em $\mathbb{R}^{3}$ extraído de [Vér13]

$$
\left\{\begin{array}{l}
\dot{x}=x(8-3 x-3 y-2 z) \\
\dot{y}=y(3-2 x-y) \\
\dot{z}=z(3-2 y-z)
\end{array},\right.
$$

onde $(x, y, z) \in \mathbb{R}^{3}$.

O sistema (7.2) possui oitos pontos de equilíbrio, são eles: três pontos de equilíbrio hiperbólicos do tipo- $1, x_{1}=(8 / 3,0,0), x_{2}=(2 / 3,0,3)$ e $x_{3}=$ $(1 / 3,7 / 3,0)$, dois pontos de equilíbrio hiperbólicos do tipo-2, $x_{4}=(0,0,3)$ e $x_{5}=(0,3,-3)$, um ponto de equilíbrio hiperbólico do tipo-3, $x_{6}=(0,0,0)$, um ponto de equilíbrio assintoticamente estável, $x_{s}=(0,3,0)$, e um ponto de equilíbrio Hopf supercrítico do tipo-zero, $p=(1,1,1)$. Como todos os pon- 


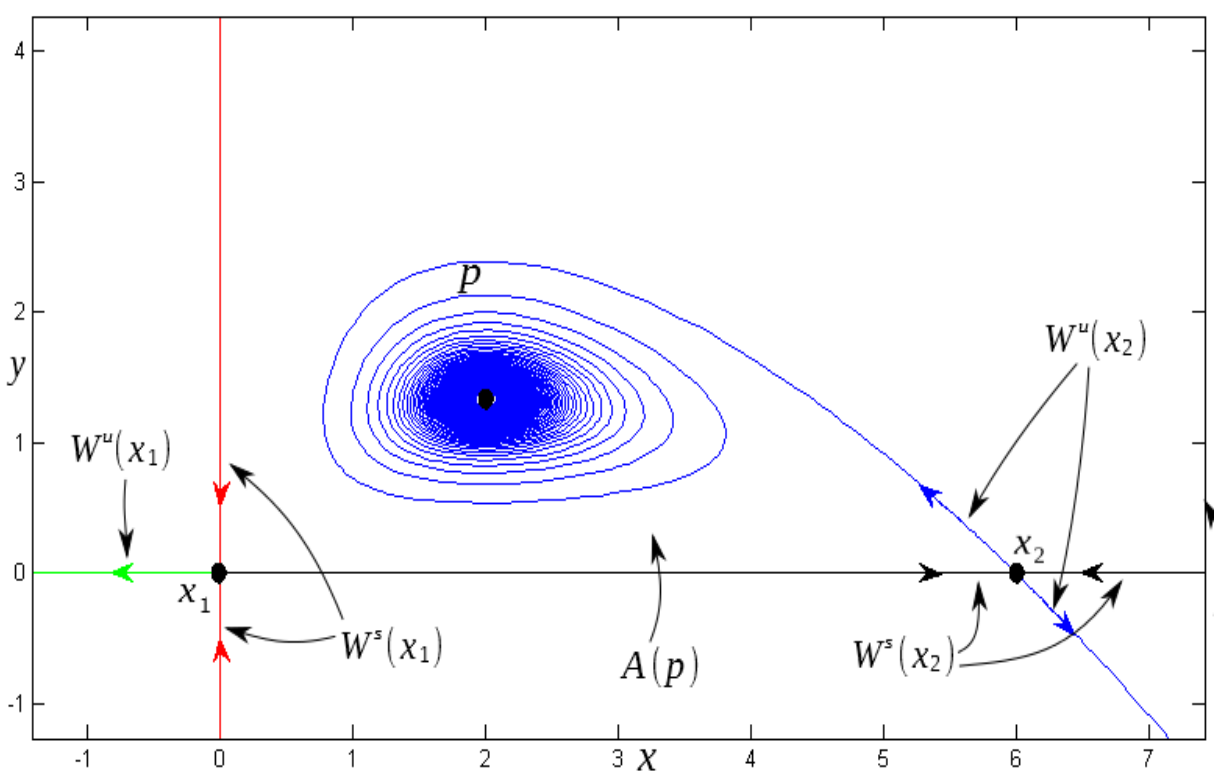

Figura 7.1: Retrato de fase do sistema (7.1). A fronteira da região de estabilidade fraca $\partial A(p)$ está contida na união da variedade estável $W^{s}\left(x_{1}\right)$ do ponto de equilíbrio hiperbólico do tipo-1 $x_{1}=(0,0)$, curva vermelha, com a variedade estável $W^{s}\left(x_{2}\right)$ do ponto de equilíbrio hiperbólico do tipo-1 $x_{2}=(6,0)$, curva preta.

tos são hiperbólicos com exceção de p então o sistema satisfaz a condição (S1), ver Figura 7.2. A suposição (S3) é satisfeita, mas a suposição (S2) não o é, pois a variedade instável $W^{u}\left(x_{1}\right)$ do ponto de equilíbrio $x_{1}$ não intercepta transversalmente a variedade estável $W^{s}\left(x_{2}\right)$ do ponto de equilíbrio $x_{2}$. A variedade estável $W^{s}\left(x_{1}\right)$ intercepta a fronteira da região de estabilidade fraca $\partial A\left(x^{p}\right)$, de acordo com o Teorema 7.1.3. A variedade instável do ponto de equilíbrio hiperbólico do tipo-1 $x_{2}, W^{u}\left(x_{2}\right)$, intercepta a região de estabilidade $A\left(x^{s}\right)$ e sua variedade estável $W^{s}\left(x_{2}\right)$ está contida na fronteira da região de estabilidade fraca $\partial A\left(x^{p}\right)$, de acordo com o Teorema 7.1.5. $A$ variedade estável $W^{s}\left(x_{3}\right)$ intercepta a fronteira da região de estabilidade fraca $\partial A\left(x^{p}\right)$, de acordo com o Teorema 7.1.3.

A Figura 7.2 ilustra a fronteira da região de estabilidade do ponto de equilíbrio Hopf supercrítico do tipo-zero, $p=(1,1,1)$. A fronteira da região de estabilidade de $p$ está contida, de acordo com a primeira parte do Teorema 7.1.7, na união da variedade estável $W^{s}\left(x_{1}\right)$ do ponto de equilíbrio hiperbólico do tipo-1 $x_{1}=(8 / 3,0,0)$, curva em vermelho na Figura 7.2, com a variedade estável $W^{s}\left(x_{2}\right)$ do ponto de equilibrio hiperbólico do tipo-1 $x_{2}=(2 / 3,0,3)$, curva em ciano na Figura 7.2, e com a variedade estável $W^{s}\left(x_{3}\right)$ do ponto de equilíbrio hiperbólico do tipo-1 $x_{3}=(1 / 3,7 / 3,0)$, curva em magenta na Figura 7.2. 
Capitulo 7. Fronteira e Região de Estabilidade Fraca de um Ponto de
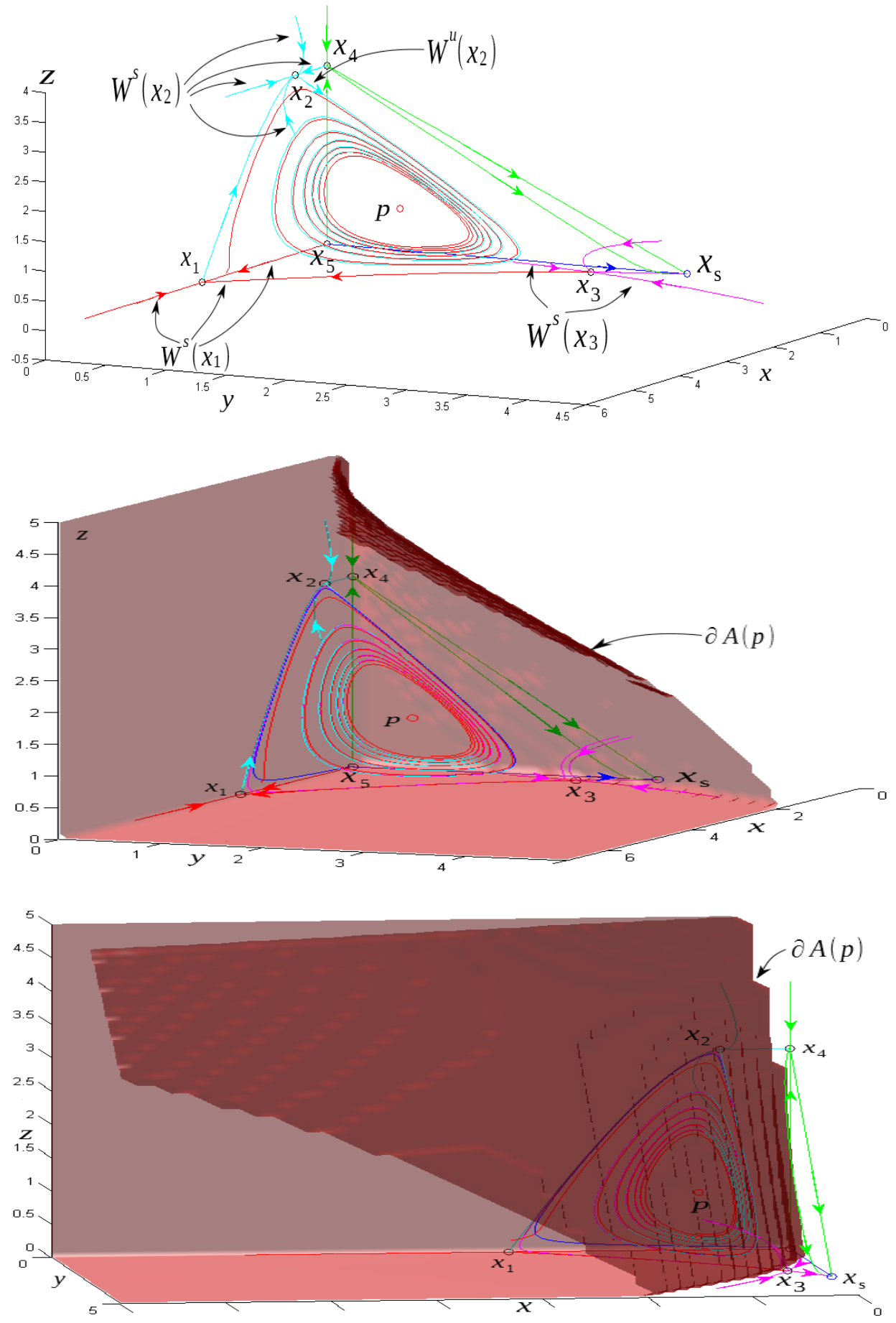

Figura 7.2: Retrato de fase do sistema (7.2). A fronteira da região de estabilidade fraca $\partial A\left(x^{p}\right)$ está contida na união da variedade estável $W^{s}\left(x_{1}\right)$ do ponto de equilíbrio hiperbólico do tipo-1 $x_{1}=(8 / 3,0,0)$, da variedade estável $W^{s}\left(x_{2}\right)$ do ponto de equilíbrio hiperbólico do tipo-1 $x_{2}=(2 / 3,0,3)$ com a variedade estável $W^{s}\left(x_{3}\right)$ do ponto de equilíbrio hiperbólico do tipo-1 $x_{3}=(1 / 3,7 / 3,0)$. 
Exemplo 7.1.3. Considere o sistema extraído de [Mon06]

$$
\left\{\begin{array}{l}
\dot{x}=2 y \\
\dot{y}=2 x-3 x^{2}-y\left(x^{3}-x^{2}+y^{2}+\frac{4}{27}\right),
\end{array}\right.
$$

onde $(x, y) \in \mathbb{R}^{2}$.

O sistema (7.3) possui dois pontos de equilíbrio, são eles: um ponto de equilíbrio hiperbólico do tipo-1, $x_{1}=(0,0)$, e um ponto de equilíbrio Hopf supercrítico do tipo-zero, $x_{s}=\left(\frac{2}{3}, 0\right)$. Como o ponto $x_{1}$ é hiperbólico então o sistema satisfaz a condição (S1), ver Figura 7.3. As suposições (S3) e (S2) são satisfeitas. A variedade instável do ponto de equilíbrio hiperbólico do tipo-1 $x_{1}=(0,0), W^{u}\left(x_{1}\right)$, intersecta a região de estabilidade $A\left(x^{s}\right)$ e sua variedade estável $W^{s}\left(x_{1}\right)$ está contida na fronteira da região de estabilidade $\partial A\left(x^{s}\right)$, de acordo com o Teorema 7.1.5, curva vermelha na Figura 7.3.

A Figura 7.3 ilustra a fronteira da região de estabilidade do ponto de equilíbrio Hopf supercrítico do tipo-zero, $x_{s}=\left(\frac{2}{3}, 0\right)$. A fronteira da região de estabilidade de $x^{s}=\left(\frac{2}{3}, 0\right)$ é formada, de acordo com a primeira parte do Teorema 7.1.7, pela variedade estável $W^{s}\left(x_{1}\right)$ do ponto de equilíbrio hiperbólico do tipo-1 $x_{1}=(0,0)$, curva em vermelho na Figura 7.3.

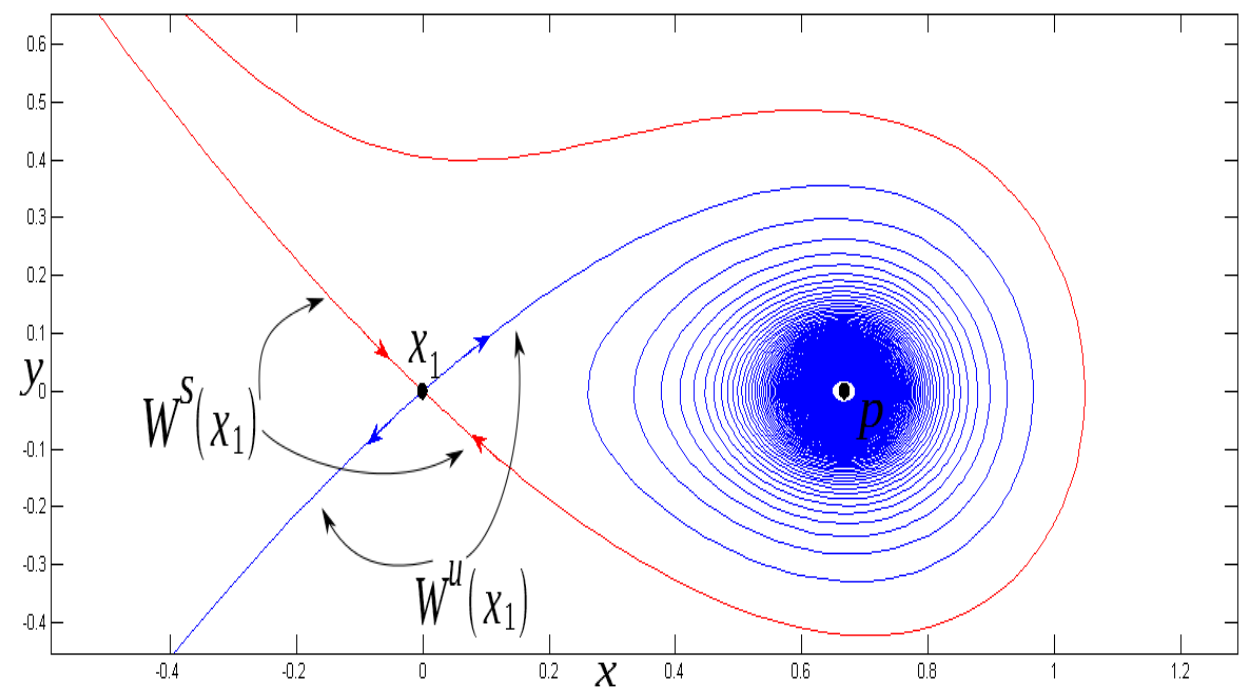

Figura 7.3: Retrato de fase do sistema (7.3). A fronteira da região de estabilidade $\partial A\left(x^{s}\right)$ é formada pela variedade estável $W^{s}\left(x_{1}\right)$ do ponto de equilíbrio hiperbólico do tipo-1 $x_{1}=(0,0)$. 
Capítulo 7. Fronteira e Região de Estabilidade Fraca de um Ponto de 130 Equilíbrio Hopf Supercrítico do Tipo-Zero

Exemplo 7.1.4. Considere o sistema extraído de [Mon06]

$$
\left\{\begin{array}{l}
\dot{x}=x-y \\
\dot{y}=5 x-y-x z \\
\dot{z}=x y-13.93 z
\end{array},\right.
$$

onde $(x, y, z) \in \mathbb{R}^{3}$.

O sistema (7.4) possui três pontos de equilíbrio, são eles: dois pontos de equilíbrio hiperbólicos do tipo-1, $x_{1}=(7.46,7.46,4)$ e $x_{2}=(-7.46,-7.46,4)$, e um ponto de equilíbrio Hopf supercrítico do tipo-zero, $p=(0,0,0)$. Os pontos de equilíbrio $x_{1}=(7.46,7.46,4)$ e $x_{2}=(-7.46,-7.46,4)$ pertencem à fronteira da região de estabilidade fraca $\partial A(p)$. As suposições $(S 1),(S 2) e$ (S3) são satisfeitas. A variedade instável do ponto de equilíbrio hiperbólico do tipo-1 $x_{1}=(7.46,7.46,4), W^{u}\left(x_{1}\right)$, intersecta a região de estabilidade fraca $A\left(x^{1}\right)$ e sua variedade estável $W^{s}\left(x_{1}\right)$ está contida na fronteira da região de estabilidade fraca $A(p)$, de acordo com o Teorema 7.1.5. A variedade instável do ponto de equilíbrio hiperbólico do tipo-1 $x_{2}=(-7.46,-7.46,4), W^{u}\left(x_{2}\right)$, intersecta a região de estabilidade fraca $A(p)$ e sua variedade estável $W^{s}\left(x_{2}\right)$ está contida na fronteira da região de estabilidade fraca $A\left(x^{1}\right)$, de acordo com o Teorema 7.1.5.

A Figura 7.5 ilustra a fronteira da região de estabilidade do ponto de equilíbrio Hopf supercrítico do tipo-zero, $p=(0,0,0)$. A fronteira da região de estabilidade $\partial A\left(x^{1}\right)$ é formada, de acordo com o Teorema 7.1.6, pela união da variedade estável do ponto de equilíbrio hiperbólico do tipo-1 $x_{1}=$ $(7.46,7.46,4), W^{s}\left(x_{1}\right)$, curva em vermelho na Figura 7.3, com a variedade estável do ponto de equilíbrio hiperbólico do tipo-1 $x_{2}=(-7.46,-7.46,4)$, $W^{s}\left(x_{2}\right)$, curva em azul na Figura 7.3.

Exemplo 7.1.5. Considere o sistema extraído de [Mon06] que descreve um sistema de controle de retorno do tipo Lur'e

$$
\left\{\begin{array}{l}
\dot{x}=y \\
\dot{y}=z \\
\dot{z}=-0.5 z-2 y-x+x^{2}
\end{array},\right.
$$

onde $(x, y, z) \in \mathbb{R}^{3}$.

O sistema (7.5) possui dois pontos de equilíbrio, são eles: um ponto de equilíbrio hiperbólico do tipo-1, $x_{1}=(1,0,0)$, e um ponto de equilíbrio Hopf supercrítico do tipo-zero, $p=(0,0,0)$. As suposições $(S 1),(S 2)$ e $(S 3)$ são satisfeitas. A variedade instável do ponto de equilíbrio hiperbólico do tipo-1 $x_{1}=(1,0,0), W^{u}\left(x_{1}\right)$, intersecta a região de estabilidade fraca $A\left(x^{1}\right)$ e sua variedade estável $W^{s}\left(x_{1}\right)$ está contida na fronteira das região de estabilidade fracas $A\left(x^{1}\right)$, de acordo com o Teorema 7.1.5. 

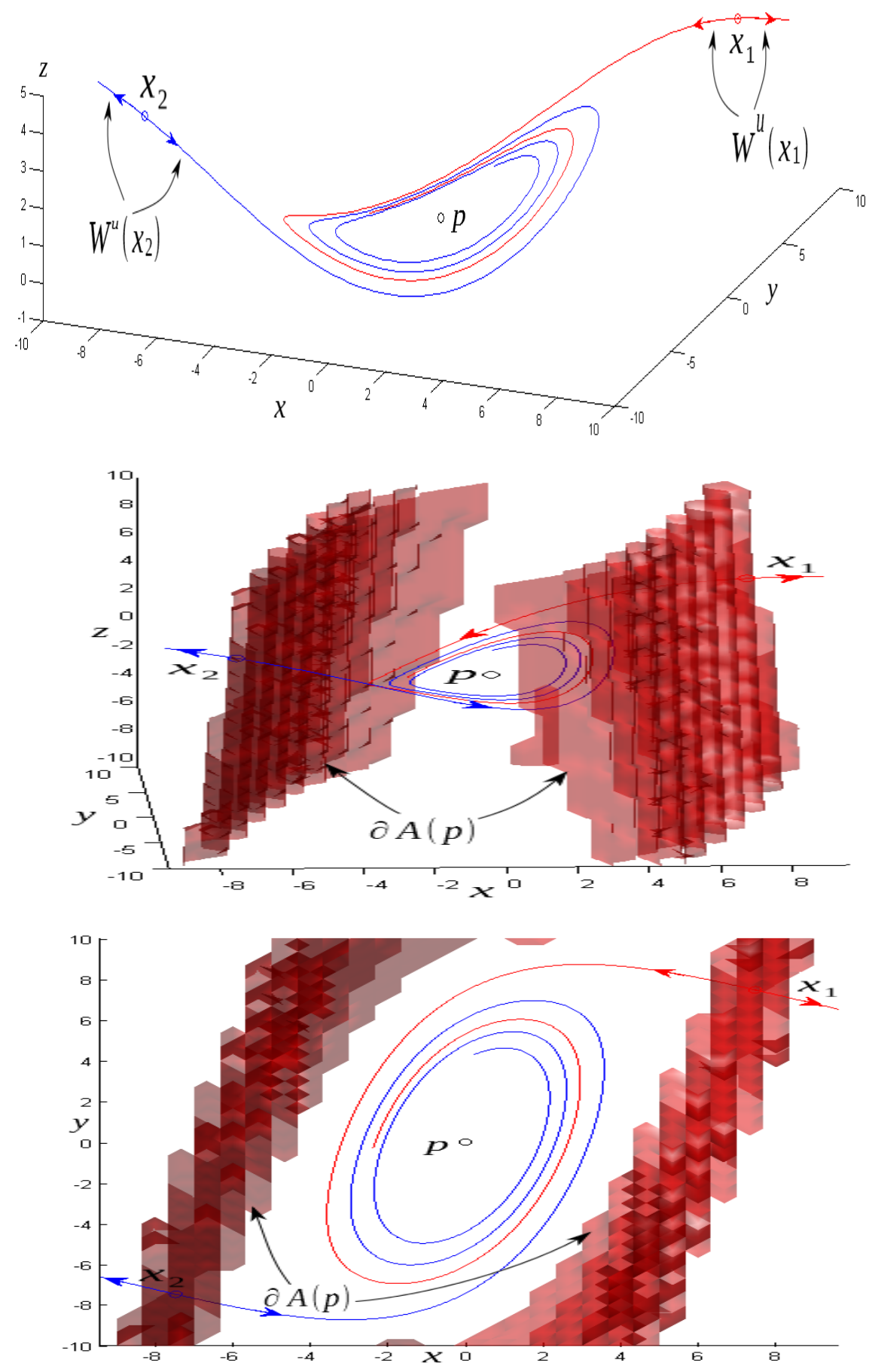

Figura 7.4: Retrato de fase do sistema (7.4). A fronteira da região de estabilidade $\partial A(p)$ é formada pela união da variedade estável $W^{s}\left(x_{1}\right)$ do ponto de equilibrio hiperbólico do tipo-1 $x_{1}=(7.46,7.46,4)$, curva em vermelho, com a variedade estável $W^{s}\left(x_{2}\right)$ do ponto de equilíbrio hiperbólico do tipo-1 $x_{2}=(-7.46,-7.46,4)$, curva em azul. 
Capítulo 7. Fronteira e Região de Estabilidade Fraca de um Ponto de 132 Equilíbrio Hopf Supercrítico do Tipo-Zero

A Figura 7.5 ilustra a fronteira da região de estabilidade do ponto de equilíbrio Hopf supercrítico do tipo-zero, $p=(0,0,0)$. A fronteira da região de estabilidade de $p=(0,0,0)$ é formada, de acordo com a primeira parte do Teorema 7.1.6, pela variedade estável $W^{s}\left(x_{1}\right)$ do ponto de equilíbrio hiperbólico do tipo-1 $x_{1}=(1,0,0)$, curva em vermelho na Figura 7.5 .

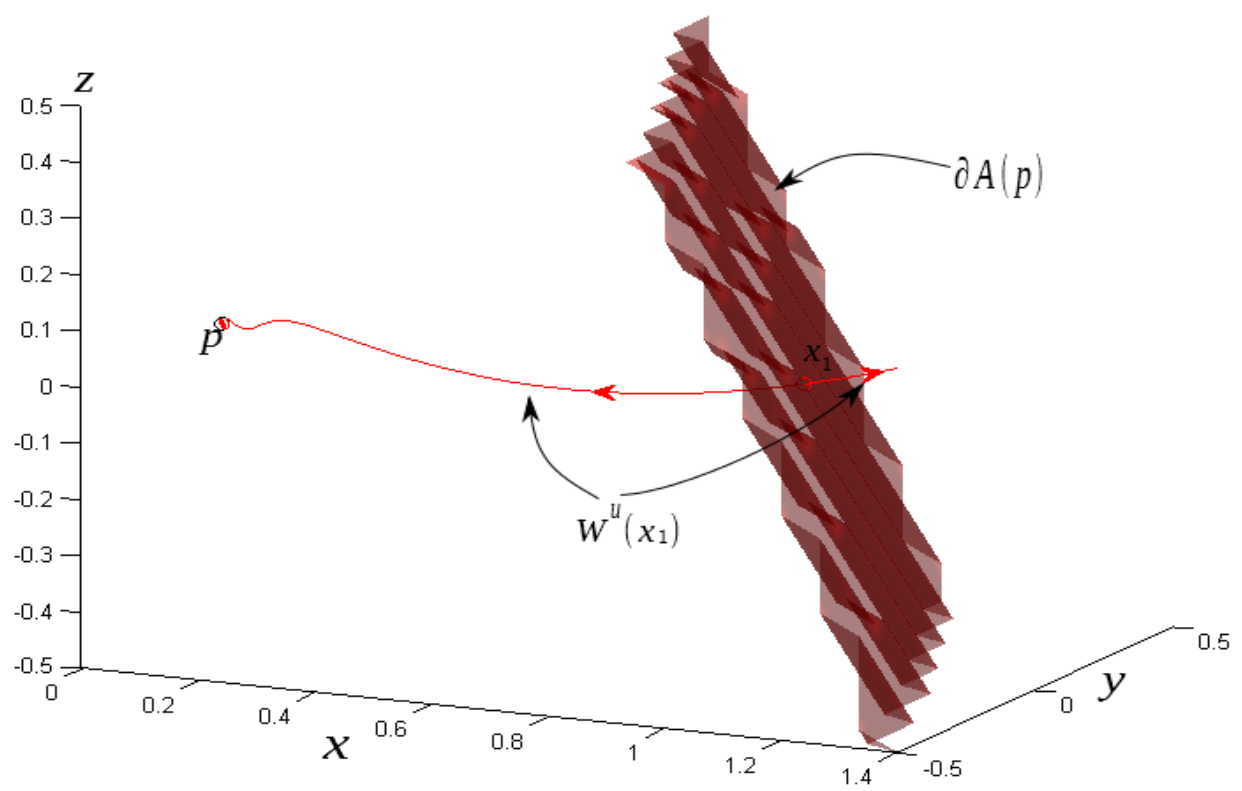

Figura 7.5: Retrato de fase do sistema (7.5). A fronteira da região de estabilidade $\partial A(p)$ é formada pela variedade estável $W^{s}\left(x_{1}\right)$ do ponto de equilíbrio hiperbólico do tipo-1 $x_{1}=(1,0,0)$.

\subsection{Fronteira e Região de Quase-Estabilidade Fraca na Presença de um Ponto de Equilíbrio Hopf Supercrítico}

Nesta seção, começaremos definindo a noção de região de quase-estabilidade fraca de um ponto de equilíbrio Hopf supercrítico do tipo-0 e exploraremos algumas de suas propriedades topológicas. E finalmente, exibiremos uma caracterização da fronteira de região de quase-estabilidade fraca de um ponto de equilíbrio Hopf supercrítico do tipo-0 como a união das variedades estáveis de todos os elementos críticos pertencentes à fronteira de quase-estabilidade fraca.

Antes de enunciarmos a definição de região de quase-estabilidade fraca de um ponto de equilíbrio Hopf supercrítico do tipo-0, ilustraremos a definição de região de quase-estabilidade de um ponto de equilíbrio hiperbólico assintoticamente estável através de um exemplo extraído de [CFA96]. Considere 
o sistema de equações diferenciais

$$
\left\{\begin{array}{l}
\dot{x}=\left(\left(\sqrt{(} x^{2}+y^{2}\right)-3\right)\left(x^{2}+y^{2}+(y-2) \sqrt{\left.\left.\left(x^{2}+y^{2}\right)-2 y+1.5\right)+y\right) x}\right. \\
\dot{y}=\left(\left(\sqrt{(} x^{2}+y^{2}\right)-3\right)\left(x^{2}+y^{2}+(y-2) \sqrt{\left.\left.\left(x^{2}+y^{2}\right)-2 y+1.5\right) y\right)-x^{2}}\right.
\end{array}\right.
$$

onde $(x, y) \in \mathbb{R}^{2}$.

O sistema (7.6) possui cincos pontos de equilíbrio, são eles: dois pontos de equilíbrio hiperbólicos do tipo- $1, x_{1}=(0,1.5)$ e $x_{2}=(0,-3)$, dois pontos de equilíbrio hiperbólicos do tipo- $2, x_{3}=(0,0.5)$ e $x_{4}=(0,3)$, e um ponto de equilíbrio assintoticamente estável, $x_{s}=(0,0)$. A Figura 7.6, ilustra a fronteira da região de estabilidade $\partial A\left(x^{s}\right)$ do ponto de equilíbrio assintoticamente estável, $x_{s}=(0,0)$. A fronteira da região de estabilidade de $x_{s}=(0,0)$ é formada pela união da variedade estável $W^{s}\left(x_{1}\right)$ com a variedade estável $W^{s}\left(x_{2}\right)$ e com os pontos de equilíbrios hiperbólicos $x_{3}$ e $x_{4}$. Como $W^{s}\left(x_{1}\right)$ encontra-se no interior do fecho da região de estabilidade $\overline{A\left(x^{s}\right)}$, ver Figura 7.6, pequenas perturbações nas trajetórias que se encontram na variedade estável $W^{s}\left(x_{1}\right)$ podem vir a convergir para o ponto de equilíbrio assintoticamente estável $x_{s}=(0,0)$. Diante disso, a região limitada pela variedade estável $W^{s}\left(x_{2}\right)$ do ponto de equilíbrio hiperbólico do tipo-1 $x_{2}=(0,-3)$ chamamos de região de quase-estabilidade de $x_{s}=(0,0)$ e a fronteira da região de quase-estabilidade é a união da variedade estável $W^{s}\left(x_{2}\right)$ com o ponto de equilíbrio hiperbólico do tipo-2 $x_{4}=(0,3)$. Observe que as variedades instáveis dos pontos de equilíbrio hiperbólicos do tipo-1 intersectam a região de estabilidade $A\left(x^{s}\right)$. Porém, a variedade instável $W^{u}\left(x_{1}\right)$ está contida em $\overline{A\left(x^{s}\right)}$, enquanto a variedade instável $W^{u}\left(x_{2}\right)$ intersecta tanto $\overline{A\left(x^{s}\right)}$ quanto $\left(\overline{A\left(x^{s}\right)}\right)^{c}$.

Considere $p$ um ponto de equilíbrio Hopf supercrítico do tipo-0 e $A(p)$ e $A_{\sigma}(p)$ suas regiões de estabilidade fraca e de quase-estabilidade fraca, respectivamente. Um elemento crítico $\phi$ está na fronteira de quase-estabilidade fraca $\partial A_{\sigma}(p)$ se, e somente se, as seguintes condições são satisfeitas

a. $\phi \in \partial A(p)$;

b. $W^{u}(\phi) \cap(\overline{A(p)})^{c} \neq \emptyset$.

Na Figura 7.7 (a), observe que as variedades instável e estável do elemento crítico $\phi$ estão contidas no interior da região de estabilidade fraca $A(p)$. A fronteira da região de estabilidade fraca $A(p)$ é formada pela união da curva que delimita a região de estabilidade fraca $A(p)$, área sombreada, com a variedade estável $W^{s}(\phi)$. Na Figura 7.7(b), a região de quasi-estabilidade do elemento crítico $\phi$ é a área sombreada e a fronteira da região de quaseestabilidade é a curva que delimita a região de quase-estabilidade.

Os dois próximos resultados estabelecem uma condição necessária para um elemento crítico hiperbólico $\phi$ pertencer a $\partial A_{\sigma}(p)$. 
Capítulo 7. Fronteira e Região de Estabilidade Fraca de um Ponto de

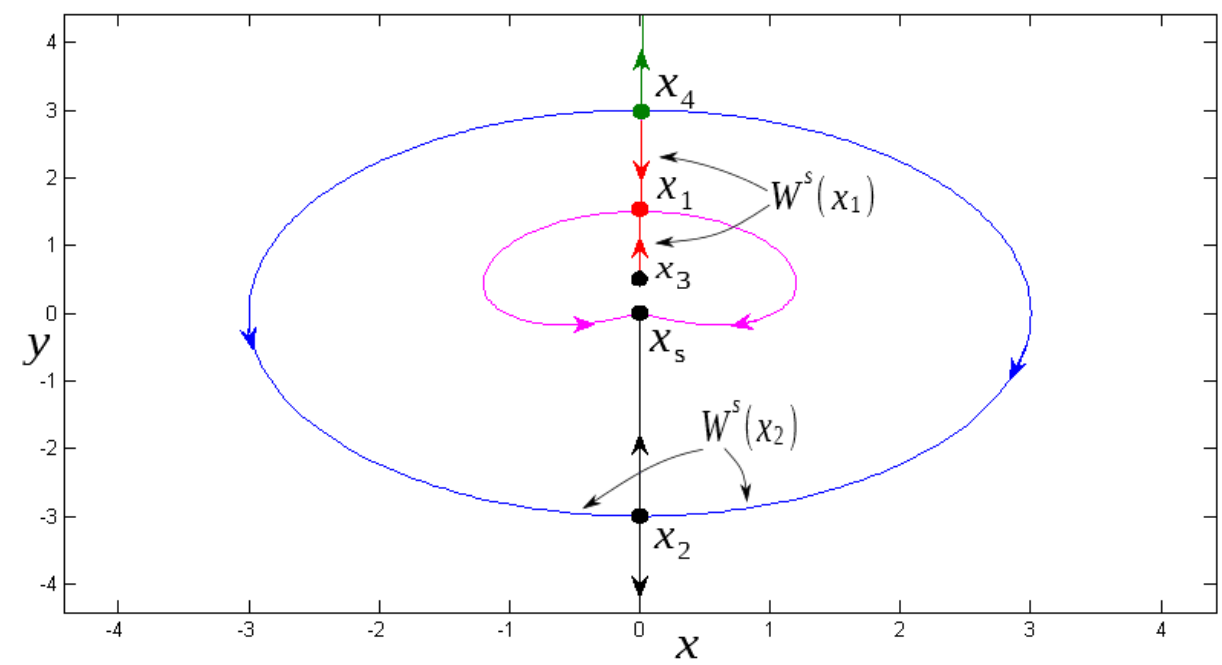

Figura 7.6: Retrato de fase do sistema (7.6). A fronteira da região de estabilidade do ponto de equilíbrio assintoticamente estável $x^{s}=(0,0)$ é formada pela variedade estável $W^{s}\left(x_{2}\right)$, curva em azul, com a variedade estável $W^{s}\left(x_{1}\right)$, curva em vermelho, e com os pontos de equilíbrios hiperbólicos $x_{3}$ e $x_{4}$. A fronteira da região de quaseestabilidade $x^{s}=(0,0)$ é a união da variedade estável $W^{s}\left(x_{2}\right)$ e do ponto de equilíbrio hiperbólico do tipo-2 $x_{4}=(0,3)$.

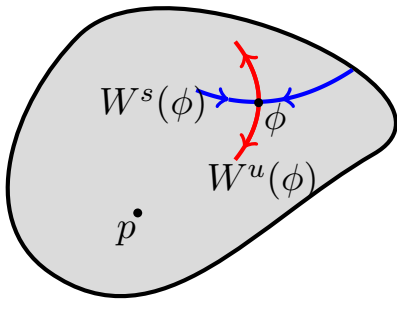

(a)

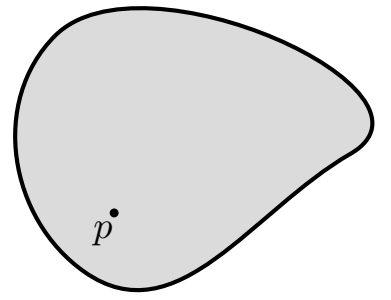

(b)

Figura 7.7: A fronteira da região de estabilidade fraca $A(p)$ é a união da curva que delimita a região de estabilidade fraca $A(p)$ com a variedade estável $W^{s}(\phi)$. A fronteira da região de quase-estabilidade é a curva que delimita a região de quaseestabilidade fraca $A_{\sigma}(p)$, área sombreada, em $(b)$. 
7.2. Fronteira e Região de Quase-Estabilidade Fraca na Presença de um Ponto de Equilibrio Hopf Supercrítico

Proposição 7.2.1. Seja $\phi$ um elemento crítico hiperbólico. Se $\phi \in \partial A_{\sigma}(p)$ então $\left.W^{s}(\phi) \subset \overline{(\overline{A(p)})^{c}}\right)$.

Demonstração. Suponhamos que $\phi \in \partial A_{\sigma}$. Pela definição da fronteira de região de quase-estabilidade $\partial A_{\sigma}(p)$ temos que $\phi \in \partial A$ e $W^{u}(\phi) \cap(\overline{A(p)})^{c} \neq \emptyset$. Seja $D$ um disco em $W^{u}(\phi) \cap(\overline{A(p)})^{c} \operatorname{com} \operatorname{dim} D=k+1$, onde $k$ é o número de multiplicadores característicos da aplicação de Poincaré associada a $\phi$ com módulo maior que 1 e $0 \leq k \leq n-1$. Seja $y \in W^{s}(\phi)$ escolhido arbitrariamente. Para qualquer $\varepsilon>0$, seja $S$ um disco transversal a $W^{s}(\phi)$ em $y, \operatorname{com} \operatorname{dim} S=k+1$, e contido numa vizinhança $N$ de $y$ de raio $\varepsilon$. Pelo Lema 2.2.21, existe um $t>0$ tal que $\varphi(t, S)$ está próxima de $D$ tal que $\varphi(t, N)$ contém um ponto $q \in(\overline{A(p)})^{c}$. Segue-se que $\varphi(-t, q) \in N$. Como $(\overline{A(p)})^{c}$ é um conjunto invariante, então $\varphi(-t, q) \in(\overline{A(p)})^{c}$ e consequentemente, $N \cap(\overline{A(p)})^{c} \neq \emptyset$. Como $\varepsilon$ pode ser escolhido arbitrariamente pequeno, obtemos $y \in \overline{\overline{A(p)}^{c}}$. Logo, $W^{s}(\phi) \subset \overline{\overline{A(p)}^{c}}$.

Proposição 7.2.2. Seja $\phi$ um elemento crítico hiperbólico. Se $\phi \in \partial A_{\sigma}(p)$ então $\phi \in \partial \overline{A(p)}$.

Demonstração. Como $\phi \in \partial A_{\sigma}(p)$, então da definição de fronteira da região de quase-estabilidade temos que $\phi \in \partial A(p)$ e $W^{u}(\phi) \cap(\overline{A(p)})^{c} \neq \emptyset$. Seja $y \in W^{u}(\phi) \cap(\overline{A(p)})^{c}$. Como $y \in W^{u}(\phi)$ então $\lim _{t \rightarrow-\infty} \varphi(t, y)=\phi$. Como $y \in(\overline{A(p)})^{c}$ e $(\overline{A(p)})^{c}$ é um conjunto invariante então $\varphi(t, y) \in(\overline{A(p)})^{c}$ para todo $t \in R^{-}$e consequentemente, $\phi \in \overline{\left(\overline{A(p)^{c}}\right)}$. Como $\phi \in \partial A(p)$ então $\phi \in \overline{A(p)}$. Então $\phi \in \overline{\left.(\overline{A(p)})^{c}\right)} \cap \overline{A(p)}$ e portanto, $\phi \in \overline{A(p)}$.

Nos dois próximos resultados exibiremos uma caracterização dos elementos críticos pertencentes à fronteira do fecho da região de estabilidade de um ponto de equilíbrio Hopf supercrítico do tipo-zero.

Proposição 7.2.3. Seja $\phi$ um elemento crítico hiperbólico, então

(i) se $\phi \in \partial \overline{A(p)}$ ent $\tilde{a} o\left\{W^{u}(\phi)-\phi\right\} \cap \overline{\overline{A(p)}^{c}} \neq \emptyset$;

(ii) $s e\left\{W^{u}(\phi)-\phi\right\} \cap \overline{\overline{A(p)}} \bar{c}^{c} \neq \emptyset e\left\{W^{u}(\phi)-\phi\right\} \cap \overline{A(p)} \neq \emptyset$, então $\phi \in \partial A_{\sigma}(p)$.

Demonstração. (i) Suponhamos que $\phi \in \partial \overline{A(p)}$. Seja $N^{u}$ um domínio fundamental de $W^{u}(\phi)$, isto é, $\bigcup_{t \in \mathbb{R}} \varphi\left(t, N^{u}\right)=W^{u}(\phi) \backslash \phi$. Seja $N_{\varepsilon}^{u}$ uma vizinhança fundamental de raio $\varepsilon$ de $N^{u}$, isto é, $N_{\varepsilon}^{u}=\left\{x \in \mathbb{R}^{n}: d\left(x, N^{u}\right)<\varepsilon\right\}$. Pelo Corolário 2.2.22, existe uma vizinhança $U$ de $\phi$ tal que $\bigcup_{t \leq 0} \varphi\left(t, N_{\varepsilon}^{u}\right) \supset$ $U \backslash W_{l o c}^{s}(\phi)$.

Como $\phi \in \partial \overline{A(p)}$ e portanto, $\phi \in \partial(\overline{A(p)})^{c}$, então $U \cap(\overline{A(p)})^{c} \neq \emptyset$. Como $\phi \in \partial \overline{A(p)}$ e $\partial \overline{A(p)} \subset \partial A(p)$ então $W_{l o c}^{s}(\phi) \cap(\overline{A(p)})^{c}=\emptyset$. Portanto, $\left\{U \backslash W_{l o c}^{s}(\phi)\right\} \cap(\overline{A(p)})^{c} \neq \emptyset$ e consequentemente, temos que $\bigcup_{t \leq 0} \varphi\left(t, N_{\varepsilon}^{u}\right) \cap$ $(\overline{A(p)})^{c} \neq \emptyset$. Segue-se que $N_{\varepsilon}^{u} \cap \varphi\left(t,(\overline{A(p)})^{c}\right) \neq \emptyset$ para algum $t$. 
Capítulo 7. Fronteira e Região de Estabilidade Fraca de um Ponto de 136 Equilíbrio Hopf Supercrítico do Tipo-Zero

Como $(\overline{A(p)})^{c}$ é um conjunto invariante, então $N_{\varepsilon}^{u} \cap(\overline{A(p)})^{c} \neq \emptyset$. Como $\varepsilon$ foi escolhido arbitrariamente e $N^{u}$ é compacto, então $N^{u}$ contém um ponto de $\overline{\overline{A(p)}}^{c}$. Logo, $\left\{W^{u}(\phi)-\phi\right\} \cap \overline{\overline{A(p)}}^{c} \neq \emptyset$.

(ii) Suponhamos que $\left.\left\{W^{u}(\phi)-\phi\right\} \cap \overline{\overline{A(p)}}\right)^{c} \neq \emptyset$ e $\left\{W^{u}(\phi)-\phi\right\} \cap \overline{A(p)} \neq \emptyset$. Seja $y \in\left\{W^{u}(\phi)-\phi\right\} \cap \overline{\overline{A(p)}}^{c}$. Então $\lim _{t \rightarrow-\infty} \varphi(t, y)=\phi$ e portanto, $\phi \in \overline{\overline{A(p)}}^{c}$. Analogamente, temos que $\phi \in \overline{A(p)}$. Portanto, $\phi \in \partial \overline{A(p)}$.

Proposição 7.2.4. (Caracterização de Elementos Críticos em $\partial \overline{A(p)}$ ) Sejam $p$ um ponto de equilíbrio Hopf supercrítico do tipo-zero do sistema (2.1) e $A(p)$ a sua região de estabilidade fraca. Se as suposições (S1), (S2) e (S3) são satisfeitas e $\phi$ é um elemento crítico hiperbólico, então $\phi \in \partial \overline{A(p)}$ implica que $\left\{W^{u}(\phi)-\phi\right\} \cap \overline{A(p)}^{c} \neq \emptyset$.

Demonstração. Seja $x$ um ponto de equilíbrio hiperbólico com $\operatorname{dim} W^{u}(x) \geq$ 1. Demonstraremos o teorema usando indução finita na dimensão de $W^{u}(x)$. Como $x \in \partial \overline{A(p)}$ então, pela Proposição 7.2.3, $\left\{W^{u}(x)-\{x\}\right\} \cap \overline{\overline{A(p)}}^{c} \neq \emptyset$. Seja $y \in\left\{W^{u}(x)-\{x\}\right\} \cap \overline{\overline{A(p)}}^{c}$. Se $y \in \overline{A(p)}^{c}$, então a prova está completa. Se $y \in \partial \overline{A(p)}^{c}$ e consequentemente, $y \in \partial \overline{A(p)}$, então existe um ponto de equilíbrio $z \in \overline{A(p)}$ e $y \in W^{s}(z)-\{z\}$. Pela condição $(\mathbf{S 1})$, temos que $\operatorname{dim} W^{u}(z) \geq 1$. Pela condição $(\mathbf{S 2}), W^{u}(x)$ intersecta $W^{s}(z)$ transversalmente em $y$. Segue-se do Lema 6.1.2 que $\operatorname{dim} W^{u}(z)<\operatorname{dim} W^{u}(x)$.

Se $\operatorname{dim} W^{u}(x)=1$, então $\operatorname{dim} W^{u}(z)<1$ e portanto, $\operatorname{dim} W^{u}(z)=0$, o que é uma contradição pois pontos de equilíbrios do tipo-zero não pode pertencer a fronteira da região de estabilidade. Logo, $\left\{W^{u}(x)-x\right\} \cap \overline{A(p)}^{c} \neq \emptyset$. Suponhamos que $\left\{W^{u}(x)-x\right\} \cap \overline{A(p)}^{c} \neq \emptyset$ para todo os pontos de equilíbrio $x$ na fronteira $\partial \overline{A(p)} \operatorname{com} \operatorname{dim} W^{u}(x) \leq k$. Suponhamos que $\operatorname{dim} W^{u}(x)=$ $k+1$. Então $\operatorname{dim} W^{u}(z)<k+1$. Segue-se que $\operatorname{dim} W^{u}(z) \leq k+1$ e consequentemente, por hipótese de indução $\left\{W^{u}(z)-z\right\} \cap \overline{A(p)}^{c} \neq \emptyset$. Sejam $q \in\left\{W^{u}(z)-z\right\} \cap \overline{A(p)}^{c}$ e $B(q, \varepsilon)$ uma bola aberta de raio $\varepsilon>0$ centrada em $q$. Seja $N^{u}$ uma vizinhança de $y$ em $W^{u}(x)$. A vizinhança $N^{u}$ contém uma seção transversal $D$ de $W^{s}(z)$ no ponto $y \operatorname{com} \operatorname{dimensão~} \operatorname{dim} D \leq k$. Pelo Lema 2.2.19, existe um ponto $w \in D$ e um tempo $t_{w}>0$ tal que $\varphi\left(t_{w}, w\right) \in N^{u}$. Como $\overline{A(p)}^{c}$ é um conjunto invariante, então $w \in \overline{A(p)}^{c}$. Portanto, $w \in W^{u}(x) \cap \overline{A(p)}^{c}$ e, consequentemente, $W^{u}(x) \cap \overline{A(p)}^{c} \neq \emptyset$.

A demonstração é análoga à demonstração para ponto de equilíbrio hiperbólico se $\phi$ é uma órbita periódica hiperbólica.

O teorema a seguir estabelece uma relação entre as fronteiras da região de quase-estabilidade e o fecho da região de estabilidade do ponto de equilíbrio Hopf supercrítico do tipo-zero. Em outras palavras, todo elemento crítico pertencente à fronteira da região de quase-estabilidade do ponto de equilíbrio Hopf supercrítico do tipo-zero, consequentemente pertence à fronteira do 
7.2. Fronteira e Região de Quase-Estabilidade Fraca na Presença de um Ponto de Equilibrio Hopf Supercrítico

fecho da região de estabilidade do ponto de equilíbrio Hopf supercrítico do tipo-zero e vice-versa.

Teorema 7.2.5. Sejam $p$ um ponto de equilíbrio Hopf supercrítico do tipozero do sistema (2.1) e $A(p)$ a sua região de estabilidade fraca. Se as suposições $(\boldsymbol{S} 1),(\boldsymbol{S 2})$ e (S3) são satisfeitas e $\phi$ é um elemento crítico, então $\phi \in \partial A_{\sigma}(p)$ se, e somente se, $\phi \in \partial \overline{A(p)}$.

Demonstração. Se $\phi \in \partial A_{\sigma}(p)$, então da Proposição 7.2.2 temos que $\phi \in$ $\partial \overline{A(p)}$. Reciprocamente, se $\phi \in \partial \overline{A(p)}$, então $\phi \in \partial A(p)$, pois $\partial \overline{A(p)} \subset$ $\partial A(p)$. Além disso, da Proposição 7.2.5, temos também que $\left\{W^{u}(\phi)-\phi\right\} \cap$ $\overline{A(p)} \neq \emptyset$. Logo, da definição de fronteira da região de quase-estabilidade, $\phi \in \partial A_{\sigma}(p)$.

Para definirmos a região de quase-estabilidade do ponto de equilíbrio Hopf supercrítico do tipo-zero, exibiremos uma propriedade topológica relacionada com a região de estabilidade fraca do ponto de equilíbrio Hopf supercrítico do tipo-zero.

Proposição 7.2.6. Sejam $p$ um ponto de equilíbrio Hopf supercrítico do tipo-zero do sistema (2.1) e $A(p)$ a sua região de estabilidade fraca. Então

$$
\partial \overline{\operatorname{int} \overline{A(p)}} \subset \partial \operatorname{int} \overline{A(p)} \subset \partial \overline{A(p)} \subset \partial A(p) .
$$

Demonstração. Inicialmente mostraremos a última inclusão. Seja $y \in \partial \overline{A(p)}$. Segue-se que $y \in \overline{A(p)} \cap \overline{\overline{A(p)}}^{c}$. Se $y \in \overline{\overline{A(p)}^{c}}$, então existe uma sequência $\left\{x_{n}\right\}$ em $\overline{A(p)^{c}}, \forall n \geq 1$, e $x_{n} \rightarrow y$. Como $\left\{x_{n}\right\} \in \overline{A(p)}^{c}$, então $\left\{x_{n}\right\} \notin \overline{A(p)}$ e portanto, $x_{n} \notin A(p)$, pois $A(p) \subset \overline{A(p)}$. Consequentemente, $x_{n} \in A(p)^{c}$. Como $x_{n} \in A(p)^{c}$ e $x_{n} \rightarrow y$ então $y \in \overline{A(p)^{c}}$. Logo, $y \in \partial A(p)$ e com isso concluímos que $\partial \overline{A(p)} \subset \partial A(p)$. Daí temos que $\operatorname{\partial int\overline {A(p)}} \subset \partial \operatorname{int} \overline{A(p)}$.

Seja $y \in \operatorname{dint} \overline{A(p)}$. Segue-se que $y \in \overline{\operatorname{int} \overline{A(p)}} \cap \overline{(i n t \overline{A(p)})^{c}}$. Se $y \in \overline{\text { int } \overline{A(p)}}$, então existe uma sequência $\left\{x_{n}\right\}$ em int $\overline{A(p)}, \forall n \geq 1$, e $x_{n} \rightarrow y$. Como $\left\{x_{n}\right\} \in \operatorname{int} \overline{A(p)}$, então $\left\{x_{n}\right\} \in \overline{A(p)}$. Como $x_{n} \in \overline{A(p)}$ e $x_{n} \rightarrow y$ então $y \in \overline{A(p)}$. Como $y \in \overline{(i n t \overline{A(p)})^{c}}$, então existe uma sequência $\left\{x_{n}\right\}$ em $(\operatorname{int} \overline{A(p)})^{c}, \forall n \geq 1$, e $x_{n} \rightarrow y$. Como $\left\{x_{n}\right\} \in(\operatorname{int} \overline{A(p)})^{c}$, então $\left\{x_{n}\right\} \notin$ int $\overline{A(p)}$. Daí temos que $x_{n} \notin \overline{A(p)}$ ou $x_{n} \in \partial \overline{A(p)}$. Se $x_{n} \in \partial \overline{A(p)}$ e como $\partial \overline{A(p)}$ é um conjunto fechado, então $y \in \partial \overline{A(p)}$. Se $x_{n} \notin \overline{A(p)}$ então $x_{n} \in \overline{A(p)}^{c}$. Daí, temos $y \in \overline{\overline{A(p)}}^{c}$. Logo, $y \in \partial \overline{A(p)}$ e com isso concluímos que $\operatorname{\partial int} \overline{A(p)} \subset \partial \overline{A(p)}$.

Pelo Teorema 7.2.5 e pela Proposição 7.2.6, podemos definir a fronteira e a região de quase-estabilidade do ponto de equilíbrio Hopf supercrítico do tipo-zero. Definamos a fronteira da região de quase-estabilidade do ponto de equilíbrio Hopf supercrítico do tipo-zero $\partial A_{\sigma}(p)$ como sendo $\partial \overline{A(p)}$ e a região de quase-estabilidade $A_{\sigma}(p)$ o conjunto aberto int $\overline{A(p)}$. 
Capítulo 7. Fronteira e Região de Estabilidade Fraca de um Ponto de 138 Equilíbrio Hopf Supercrítico do Tipo-Zero

A proposição a seguir mostra que a definição da fronteira e da região de quase-estabilidade do ponto de equilíbrio Hopf supercrítico do tipo-zero está bem definida.

Proposição 7.2.7. Sejam $p$ um ponto de equilíbrio Hopf supercrítico do tipo-zero do sistema (2.1) e $A(p)$ a sua região de estabilidade. Então dint $\bar{A}=$ $\partial A_{\sigma}$.

Demonstração. Pela Proposição 7.2.6 temos que $\partial i n t \overline{A(p)} \subset \partial \overline{A(p)}$. Se $y \in$ $\partial \overline{A(p)}$, então $y \in \overline{A(p)} \cap \overline{\overline{A(p)}})^{c}$. Como $y \in \overline{A(p)}$, então existe uma sequência $\left\{y_{n}\right\}$ em $A(p)$ e $y_{n} \rightarrow y$. Como $A(p)$ é um conjunto aberto, então existe um $\varepsilon_{n}>0$ tal que $B\left(y_{n}, \varepsilon_{n}\right) \subset A(p) \subset \overline{A(p)}$, para todo $n>0$. Seguese que $y_{n} \in \operatorname{int} \overline{A(p)}$, para todo $n>0$, e portanto, $y \in \overline{i n t \overline{A(p)}}$. Como $y \in \overline{\overline{A(p)}}^{c}$, então existe uma sequência $\left\{z_{n}\right\}$ em $\overline{A(p)}^{c}$ e $z_{n} \rightarrow y$. Daí tem-se que $z_{n} \notin \overline{A(p)}$, para todo $n>0$, e consequentemente, $z_{n} \notin i n t \overline{A(p)}$, para todo $n>0$. Então $z_{n} \in \operatorname{int} \overline{A(p)}^{c}$, para todo $n>0$, e $y \in \overline{i n t} \overline{A(p)}^{c}$. Logo, $y \in \operatorname{dint} \overline{A(p)}$.

Exibiremos, sob as afirmações (S1), (S2) e (S3), condições para que um elemento crítico pertença à fronteira de região de quase-estabilidade do ponto de equilpibrio Hopf supercrítico do tipo-zero e depois, caracterizaremos a fronteira de região de quase-estabilidade.

Teorema 7.2.8. Sejam $A_{\sigma}(p)$ e $A(p)$, respectivamente, a região de quaseestabilidade e a região de estabilidade do ponto de equilíbrio Hopf supercrítico do tipo-zero $p$ do sistema (2.1). Seja $\psi$ um elemento crítico hiperbólico do sistema (2.1). Se as suposições (S1), (S2) e (S3) são satisfeitas, então

(i)

$$
\psi \in \partial A_{\sigma}(p) \Longleftrightarrow W^{u}(\psi) \cap A(p) \neq \emptyset e W^{u}(\psi) \cap(\overline{A(p)})^{c} \neq \emptyset
$$

$$
\psi \in \partial A_{\sigma}(p) \Longleftrightarrow W^{s}(\psi) \subset \partial A_{\sigma}(p)
$$

Demonstração. (i.) Suponha $\psi \in \partial A_{\sigma}(p)$. Então, da definição de fronteira de região de quase-estabilidade fraca, $\psi \in \partial A(p)$ e $W^{u}(\psi) \cap(\overline{A(p)})^{c} \neq \emptyset$. Como $\psi \in \partial A(p)$ então, pelo Teorema 7.1.5, $W^{u}(\psi) \cap A(p) \neq \emptyset$.

Suponha $W^{u}(\psi) \cap A(p) \neq \emptyset$ e $W^{u}(\psi) \cap(\overline{A(p)})^{c} \neq \emptyset$. Se $W^{u}(\psi) \cap A(p) \neq \emptyset$, então $\psi \in \partial A(p)$. Como $\psi \in \partial A(p)$ e $W^{u}(\psi) \cap(\overline{A(p)})^{c} \neq \emptyset$ então pela definição de fronteira de região de quase-estabilidade fraca, $\psi \in \partial A_{\sigma}(p)$.

(ii) Suponha que $\psi \in \partial A_{\sigma}(p)$. Então, da Proposição 7.2.1, temos $W^{s}(\psi) \subset$ $\overline{(\overline{A(p)})^{c}}$. Por outro lado, pelo Teorema 7.1.5, $W^{s}(\psi) \subset \partial A(p)$. Consequentemente, $\left.W^{s}(\psi) \nsubseteq \overline{A(p)}\right)^{c}$. Daí, e do fato que $\overline{(\overline{A(p)})^{c}}=(\overline{A(p)})^{c} \cup \partial(\overline{A(p)})^{c}=$ $(\overline{A(p)})^{c} \cup \partial \overline{A(p)}$, então $W^{s}(\psi) \subset \partial \overline{A(p)}$.

Suponha $W^{s}(\psi) \subset \partial A_{\sigma}(p)$. Como $x \in W^{s}(\psi)$ então $\psi \in \partial A_{\sigma}(p)$. 
7.2. Fronteira e Região de Quase-Estabilidade Fraca na Presença de um Ponto de Equilibrio Hopf Supercrítico

Teorema 7.2.9. (Caracterização da Fronteira da região de quaseEstabilidade fraca para Pontos de Equilíbrios): Sejam $A_{\sigma}(p)$ e $A(p)$, respectivamente, a região de quase-estabilidade fraca e a região de estabilidade fraca do ponto de equilibrio Hopf supercrítico do tipo-zero $p$ do sistema (2.1). Seja $\phi$ um elemento crítico hiperbólico do sistema (2.1). Se as suposições (S1), (S2) e (S3) são satisfeitas, então

$$
\partial A_{\sigma}(p)=\bigcup_{i} W^{s}\left(x^{i}\right)
$$

onde $x^{i}, i=1,2, \ldots$ são os elementos críticos hiperbólicos na fronteira da região de quase-estabilidade $\partial A_{\sigma}(p)$.

Demonstração. Seja $q \in \partial A_{\sigma}(p)$. Pela hipótese (S3), podemos afirmar que existe um elemento crítico hiperbólico $x$ tal que $\varphi(t, q) \rightarrow x$ quando $t \rightarrow+\infty$. Pela hipótese (S1), $x$ é um elemento crítico hiperbólico $x^{i}$ para algum $i$. Portanto, concluímos que $q \in \bigcup_{i} W^{s}\left(x^{i}\right)$. Portanto, $\partial A_{\sigma}(p) \subset \bigcup_{i} W^{s}\left(x^{i}\right)$. Pelo Teorema 7.2.8, $\bigcup_{i} W^{s}\left(x^{i}\right) \subset \partial A\left(x^{s}\right)$ e, portanto,

$$
\partial A_{\sigma}(p)=\bigcup_{i} W^{s}\left(x^{i}\right) .
$$

Ainda com o próposito de obtermos uma caracterização completa da região de quase-estabilidade do ponto de equilíbrio Hopf supercrítico do tipozero, os próximos resultados caracterizam os elementos críticos que pertencem à região de quase-estabilidade em termos de suas variedades instável e estável.

Teorema 7.2.10. Sejam $A_{\sigma}(p)$ e $A(p)$, respectivamente, a região de quaseestabilidade e a região de estabilidade do ponto de equilíbrio Hopf supercrítico do tipo-zero $p$ do sistema (2.1). Seja $\phi$ um elemento crítico hiperbólico do sistema (2.1). Então $\phi \in A_{\sigma}(p)$ se, e somente se, $\left\{W^{u}(\phi)-\phi\right\} \subset A(p)$.

Demonstração. Suponhamos $\phi \in A_{\sigma}(p)$, isto é, $\phi \in i n t \overline{A(p)}$. Sejam $B(\varepsilon, \phi)$ uma vizinhança de raio $\varepsilon>0$ e $G$ um domínio fundamental de $\phi$ tal que $G \subset B(\varepsilon, \phi) \subset A_{\sigma}(p)$, pois $A_{\sigma}(p)$ é um conjunto aberto. Como $A_{\sigma}(p)$ é um conjunto invariante e $\left\{W^{u}(\phi)-\phi\right\}=\cup_{t \in \mathbb{R}} \varphi(t, G)$, então $W^{u}(\phi) \subset$ int $\overline{A(p)}$. Como $\partial A(p)$ é um conjunto invariante e disjunto de $A(p)$, então $\left\{W^{u}(\phi)-\phi\right\} \cap \partial A(p)=\emptyset$. Logo, $W^{u}(\phi)-\phi \subset A(p)$.

Reciprocamente, suponha que $\left\{W^{u}(\phi)-\phi\right\} \subset A(p)$. Como $A(p) \subset$ $\operatorname{int} \overline{A(p)}$ então $\left\{W^{u}(\phi)-\phi\right\} \subset \operatorname{int} \overline{A(p)}$. Como $\overline{\overline{A(p)}}^{c}=\overline{A(p)}^{c} \cup \partial \overline{A(p)}^{c}=$ $\overline{A(p)}^{c} \cup \partial \overline{A(p)}$ e $\overline{A(p)}=\operatorname{int} \overline{A(p)} \cup \partial \overline{A(p)}$, então $\left\{W^{u}(\phi)-\phi\right\} \cap \partial \overline{A(p)}=\emptyset$ e $\left\{W^{u}(\phi)-\phi\right\} \cap \partial \overline{A(p)}^{c}=\emptyset$. Segue-se que $\left\{W^{u}(\phi)-\phi\right\} \cap \partial \overline{\overline{A(p)}}^{c}=\emptyset$. 
Capítulo 7. Fronteira e Região de Estabilidade Fraca de um Ponto de 140 Equilíbrio Hopf Supercrítico do Tipo-Zero

Consequentemente, pelo item (i) da Proposição 7.2.3, $\phi \notin \partial \overline{A(p)}$ e portanto, concluímos que $\phi \in \operatorname{int} \overline{A(p)}$.

Corolário 7.2.11. Sejam $A_{\sigma}(p)$ e $A(p)$, respectivamente, a região de quaseestabilidade e a região de estabilidade do ponto de equilíbrio Hopf supercrítico do tipo-zero $p$ do sistema (2.1). Seja $\phi$ um elemento crítico hiperbólico do sistema (2.1). Então $\phi \in A_{\sigma}(p)$ se, e somente se, $W^{s}(\phi) \subset \partial A(p) \cap A_{\sigma}(p)$.

Demonstração. Suponhamos $\phi \in A_{\sigma}$. Então da definição de região de quaseestabilidade $\phi \in \operatorname{int} \overline{A(p)}$. Consequentemente, $\phi \in \overline{A(p)}$ e como $\phi \notin A(p)$, então temos que $\phi \in \partial A(p)$. Pelo Teorema 7.2.10, $W^{u}(\phi) \cap A(p) \neq \emptyset$. Seja $A(p)=W^{c s}(p)$. Então temos que $W^{u}(\phi) \cap W^{c s}(p) \neq \emptyset$. Observemos que a interseção $W^{u}(\phi) \cap W^{c s}(p)$ é transversal pois $W^{c s}(p)$ tem dimensão $n$. Sejam $y \in W^{u}(\phi) \cap W^{c s}(p)$ e $D$ um disco fechado em $W^{c s}(p)$ contendo $y$. Como a interseção é transversal, então pelo Lema $W^{s}(\phi) \overline{\cup_{t \leq 0}(t, D)} \subset \overline{W^{c s}(p)}=\overline{A(p)}$. Como os conjuntos $W^{s}(\phi)$ e $A(p)$ são disjuntos, então $W^{s}(\phi) \subset \partial A(p)$. Como $\partial A(p) \cap i n t \overline{A(p)}$ é um conjunto invariante e $\phi \in i n t \overline{A(p)}$, então $W^{s}(\phi) \subset \partial A(p) \cap i n t \overline{A(p)}$.

O próximo teorema apresenta uma caracterização do conjunto $A_{\sigma}(p) \backslash$ $A(p)$, isto é, o conjunto $\partial A(p) \cap A_{\sigma}(p)$, sob as afirmações (S1) e (S3), como a união das variedades estáveis dos elementos críticos pertencentes a região de quase-estabilidade do ponto de equilíbrio Hopf supercrítico do tipo-zero.

Teorema 7.2.12. Sejam $A_{\sigma}(p)$ e $A(p)$, respectivamente, a região de quaseestabilidade e a região de estabilidade do ponto de equilíbrio Hopf supercrítico do tipo-zero $p$ do sistema (2.1). Sejam $\phi_{i}, i=1,2, \ldots$ elementos crítico hiperbólicos do sistema (2.1). Se as suposições $(\boldsymbol{S 1})$ e (S3) são satisfeitas, então

$$
\partial A(p) \cap A_{\sigma}(p)=\cup_{\phi \in A_{\sigma}(p)} W^{s}(\phi) .
$$

Demonstração. Observe que

$$
\begin{aligned}
\partial A(p) \cap A_{\sigma}(p) & =\partial A(p) \cap i n t \overline{A(p)} \\
& =\partial A(p) \cap(\overline{A(p)} \backslash \partial \overline{A(p)}) \\
& =\partial A(p) \backslash \partial \overline{A(p)} .
\end{aligned}
$$

Se $\phi_{i} \in A_{\sigma}(p)$, então pelo Corolário 7.2.11 $\cup_{\phi_{i} \in A_{\sigma}(p)} W^{s}\left(\phi_{i}\right) \subset \partial A(p) \cap A_{\sigma}(p)$. Da invariância do conjunto $\partial A(p) \cap A_{\sigma}(p)$ e da suposição (S3), $\partial A(p) \cap$ $A_{\sigma}(p) \subset \cup_{\phi_{i} \in A_{\sigma}(p)} W^{s}\left(\phi_{i}\right)$. Logo,

$$
\partial A(p) \cap A_{\sigma}(p)=\cup_{\phi \in A_{\sigma}(p)} W^{s}(\phi) .
$$




\section{8 Bifurcação de Hopf}

Neste capítulo apresentaremos um estudo da Bifurcação de Hopf para um sistema dinâmico autonômo dependente de um parâmetro. Bifurcação de Hopf é uma bifurcação local no qual o ponto de equilíbrio não hiperbólico do sistema dinâmico perde ou ganha estabilidade quando coalesce com uma órbita periódica. Isto ocorre quando um par de autovalores complexos da matriz jacobiana sobre o ponto de equilíbrio não hiperbólico cruza o eixo imaginário. Inicialmente, faremos uma breve revisão sobre conceitos da Teoria de Bifurcação, ou seja, veremos que uma bifurcação num sistema dinâmico surge com uma mudança qualitativa no retrato de fase do sistema dinâmico devido à variação de um ou mais parâmetros. Existem vários tipos de bifurcações que ocorrem em sistemas dinâmicos não lineares dependentes de um parâmetro $\mu \in \mathbb{R}^{m}$. Em particular, trataremos da Bifurcação de Hopf em sistemas bidimensionais e posteriormente, estudaremos a bifurcação de Hopf para sistemas $n$-dimensionais. Para tanto, utilizaremos o método da projeção para reduzir o caso $n$-dimensional ao caso bidimensional. Para maiores detalhes sobre a Teoria da Bifurcação, o leitor deve consultar [GH83], [Hal69], [Kuz13], [Per13] e [Wig03].

\subsection{Teoria de Bifurcação}

Em geral, sistemas físicos muitas das vezes são modelados por sistemas dinâmicos autonômos não lineares dependentes de um ou mais parâmetros. Ao variarmos estes parâmetros, mudanças podem surgir no comportamento das soluções para determinados valores de parâmetros. Ao variarmos um conjunto de parâmetros, o fluxo do sistema dinâmico resultante pode ser 
ou não topologicamente equivalente ao fluxo do sistema dinâmico antes da perturbação. Antes de definirmos estabilidade estrutural de um sistema dinâmico, definamos o conceito de equivalência topológica.

Dois sistemas

$$
\begin{aligned}
& \dot{x}=f_{1}\left(x, \mu_{1}\right), x \in \mathbb{R}^{n}, \mu_{1} \in \mathbb{R}^{m}, \\
& \dot{y}=f_{2}\left(y, \mu_{2}\right), y \in \mathbb{R}^{n}, \mu_{2} \in \mathbb{R}^{m},
\end{aligned}
$$

são ditos localmente topologicamente equivalentes em torno da origem se existir uma aplicação $\left(x, \mu_{1}\right) \rightarrow\left(h_{\mu_{1}}(x), g\left(\mu_{1}\right)\right)$, definida em uma vizinhança $U_{0} \times V_{0}$ de $(x, \zeta)=(0,0)$, contida em $\mathbb{R}^{n} \times \mathbb{R}^{m}$, satisfazendo

a. $g: \mathbb{R}^{m} \rightarrow \mathbb{R}^{m}$ é um homeomorfismo definido em $V_{0}$;

b. $h_{\mu_{1}}: \mathbb{R}^{n} \rightarrow \mathbb{R}^{n}$ é um homeomorfismo para cada $\mu_{1}$, definido na vizinhança $U_{0}$ de $x=0, h_{0}(0)=0$, e levando órbitas de (8.1) contidas em $U_{0}$ em órbitas de (8.2) em $h_{\mu_{1}}\left(U_{0}\right)$, preservando a direção do tempo.

Uma característica importante de equivalência topológica é que o homeomorfismo leva fluxos do sistema (8.1) em fluxos em (8.2). Isto é, a definição de equivalência topológica acima implica que para qualquer $\left(x, \mu_{1}\right)$ e $t_{1}$, existe um $t_{2}$ tal que

$$
h_{\mu_{1}}\left(\varphi_{1}\left(t_{1}, x, \mu_{1}\right)\right)=\varphi_{2}\left(t_{2}, h_{\mu_{1}}(x), g\left(\mu_{1}\right)\right)
$$

onde $\varphi_{1}$ e $\varphi_{2}$ são fluxos dos sistemas (8.1) e (8.2), respectivamente.

Consideremos o sistema dinâmico autonômo dependente de um parâmetro

$$
\dot{x}=f(x, \mu), x \in \mathbb{R}^{n}, \mu \in \mathbb{R}
$$

onde $f: \mathbb{R}^{n} \times \mathbb{R} \rightarrow \mathbb{R}^{n}$ é um campo vetorial de classe $\mathcal{C}^{1}$.

A seguir, apresenta-se uma definição particular de perturbação de um campo vetorial.

Dado $\mu_{0} \in \mathbb{R}$, dizemos que o campo vetorial $f$ é estruturalmente estável na vizinhança de $\mu_{0}$ se o campo $f(x, \mu)$ é topologicamente equivalente a $f\left(x, \mu_{0}\right)$ para todo $\mu \in\left[-\varepsilon+\mu_{0}, \varepsilon+\mu_{0}\right]$. Em outras palavras, um campo é estruturalmente estável, se sob pequenas perturbações o retrato de fase desse novo sistema têm o mesmo comportamento dinâmico do que o retrato fase do sistema original.

Por exemplo, o sistema $\dot{x}=x$ é estruturalmente estável para qualquer pequena perturbação, mas o sistema $\dot{x}=x^{3}$ não é estruturalmente estável. Se perturbamos o sistema $\dot{x}=x^{3}$, o novo sistema $\dot{x}=x^{3}-\varepsilon$, com $\varepsilon>0$ tem dois equilíbrios $-\sqrt[3]{\varepsilon}$ e $\sqrt[3]{\varepsilon}$ e possui um equilíbrio a mais que o sistema original.

Dado um sistema dinâmico autonômo não linear, se o retrato de fase do sistema se altera com o surgimento ou desaparecimento de um ponto 
de equilíbrio ou até mesmo de uma órbita periódica após pequenas perturbações, dizemos que o sistema é estruturalmente instável. Então mudanças qualitativas no retrato de fase do sistema dinâmico podem ocorrer quando o parâmetro $\mu$ varia numa vizinhança de um determinado valor de parâmetro $\mu_{0}$, estas mudanças chamamos de bifurcação.

A Teoria de Bifurcação trata das mudanças que podem surgir no comportamento qualitativo das soluções do sistema (8.3) quando o parâmetro $\lambda$ é variado. Sendo assim, em um sistema dinâmico não linear, ao variarmos um ou mais parâmetros, a estabilidade dos pontos de equilíbrio poderá mudar com o surgimento de uma órbita periódica ou de um novo ponto de equilíbrio. O valor do parâmetro para o qual estas mudanças ocorrem chamamos de valor de bifurcação e o parâmetro de parâmetro de bifurcação.

Veremos que uma condição necessária para ocorrer uma bifurcação local em um ponto de equilíbrio é que o equilíbrio seja não hiperbólico. Observaremos também que uma bifurcação ocorre quando conjuntos com estabilidades diferentes colidem ao perturbamos o sistema.

\subsection{Bifurcação de Hopf em Sistemas Bidimensio- nais}

Ao longo deste trabalho, utilizaremos o termo suave para nos referirmos às funções onde a classe de diferenciabilidade é suficientemente grande. Isto é, o campo vetorial $f$ suave implicará em um campo vetorial $f$ de classe $\mathcal{C}^{n}$, com $n$ suficientemente grande. Quando acharmos necessário explicitaremos a classe de diferenciabilidade do campo.

A notação $f(x)=O\left(\|x\|^{n}\right)$ representará uma função suave cuja expansão de Taylor em $x$ inicia com os termos de ordem $n$ ou superiores.

Considere o seguinte sistema dinâmico dependente do parâmetro $\mu \in \mathbb{R}$,

$$
\left(\begin{array}{c}
\dot{x}_{1} \\
\dot{x}_{2}
\end{array}\right)=\left(\begin{array}{cc}
\mu & -1 \\
1 & \mu
\end{array}\right)\left(\begin{array}{l}
x_{1} \\
x_{2}
\end{array}\right) \pm\left(x_{1}^{2}+x_{2}^{2}\right)\left(\begin{array}{l}
x_{1} \\
x_{2}
\end{array}\right)
$$

ou ainda

$$
\left\{\begin{array}{l}
\dot{x}_{1}=\mu x_{1}-x_{2} \pm\left(x_{1}^{2}+x_{2}^{2}\right) x_{1} \\
\dot{x}_{2}=x_{1}+\mu x_{2} \pm\left(x_{1}^{2}+x_{2}^{2}\right) x_{2}
\end{array}\right.
$$

Observemos que o ponto $\left(x_{1}, x_{2}\right)=(0,0)$ é um ponto de equilíbrio do sistema (8.4) ou (8.5), para qualquer que seja $\mu \in \mathbb{R}$. De fato, multiplicando a primeira e a segunda equação do sistema (8.5) por $-x_{2}$ e $x_{1}$, respectivamente, e somando os resultados, obtemos

$$
x_{1}^{2}+x_{2}^{2}=0 \Leftrightarrow x_{1}=x_{2}=0 .
$$


A matriz Jacobiana do sistema (8.5) é dada por

$$
D f(x, \mu)=\left(\begin{array}{cc}
\mu \pm\left(3 x_{1}^{2}+x_{2}^{2}\right) & -1 \pm 2 x_{1} x_{2} \\
1 \pm 2 x_{1} x_{2} & \mu \pm\left(x_{1}^{2}+3 x_{2}^{2}\right)
\end{array}\right)
$$

e calculada no ponto de equilíbrio:

$$
\begin{aligned}
A & =D f(0, \mu) \\
& =\left(\begin{array}{cc}
\mu & -1 \\
1 & \mu
\end{array}\right)
\end{aligned}
$$

$A$ possui os seguintes autovalores $\lambda_{1,2}=\mu \pm i$.

Considere a variável complexa $z=x_{1}+i x_{2}, \operatorname{com} x_{1}, x_{2} \in \mathbb{R}$. Derivando $z$ e usando as equações do sistema (8.5), tem-se o sistema:

$$
\begin{aligned}
\dot{z} & =\dot{x}_{1}+i \dot{x}_{2} \\
& =\left[\mu x_{1}-x_{2} \pm\left(x_{1}^{2}+x_{2}^{2}\right) x_{1}\right]+i\left[x_{1}+\mu x_{2} \pm\left(x_{1}^{2}+x_{2}^{2}\right) x_{2}\right] \\
& =\mu\left(x_{1}+i x_{2}\right)+i\left(x_{1}+i x_{2}\right) \pm\left(x_{1}^{2}+x_{2}^{2}\right)\left(x_{1}+i x_{2}\right) \\
& =\mu z+i z \pm|z|^{2} z \\
& =(\mu+i) z \pm|z|^{2} z .
\end{aligned}
$$

que é a forma complexa de (8.4). Podemos ainda reescrever o sistema (8.6) na sua forma polar, para isso usaremos a representação polar $z=\rho e^{i \theta}$, obtendo assim

$$
\dot{z}=\dot{\rho} e^{i \theta}+\rho e^{i \theta} i \dot{\theta} .
$$

Substituindo o valor de $\dot{z}$ da equação acima e $z=\rho e^{i \theta}$ na equação (8.6), temos

$$
\dot{\rho} e^{i \theta}+i \rho e^{i \theta} \dot{\theta}=\rho e^{i \theta}\left(\mu+i \pm \rho^{2}\right)
$$

$\mathrm{ou}$

$$
\dot{\rho}+i \rho \dot{\theta}=\rho\left(\mu+i \pm \rho^{2}\right) .
$$

Consequentemente, da igualdade acima temos a seguinte representação do sistema (8.4) na sua forma polar

$$
\left\{\begin{array}{l}
\dot{\rho}=\rho\left(\mu \pm \rho^{2}\right) \\
\dot{\theta}=1
\end{array}\right.
$$

Observemos que para qualquer valor de $\mu$, a componente angular é sempre crescente, pois $\dot{\theta}=1>0$. Observemos também que da primeira equação do sistema (8.7), $\rho=0$ é um ponto de equilíbrio para qualquer valor de $\mu$. À depender do sinal do termo cúbico do sistema (8.7), obtemos outro ponto de equilíbrio para determinado valores do parâmetro $\mu$.

Inicialmente, analisaremos o retrato de fase para o sistema (8.7) com o 
sinal negativo do termo cúbico, ou seja, o sistema

$$
\left\{\begin{array}{l}
\dot{\rho}=\rho\left(\mu-\rho^{2}\right) \\
\dot{\theta}=1
\end{array}\right.
$$

Se $\mu>0, \rho(\mu)=\sqrt{\mu}$ é um ponto de equilíbrio do sistema (8.8). Este equilíbrio representa uma órbita periódica circular de raio $\sqrt{\mu}$ com velocidade constante no espaço dos $x_{s}$. A origem, que também é um ponto de equilíbrio, fica isolada por um ciclo limite, que é único e atrativo. De fato, como $\dot{\rho}>0$ em $0<\rho<\sqrt{\mu}$ e $\dot{\rho}<0$ em $\rho>\sqrt{\mu}$, então a componente radial é crescente para $0<\rho<\sqrt{\mu}$ e decrescente para $\rho>\sqrt{\mu}$. Assim, todas as órbitas internas ou externas a este ciclo, com exceção da origem, tendem ao ciclo limite exponencialmente quando $t \rightarrow+\infty$, isto é, o ciclo limite $\rho=\sqrt{\mu}$ é estável, veja Figura 8.1.

Se $\mu<0$, temos que $\dot{\rho}=\rho\left(\mu-\rho^{2}\right)<0$, isto é, a componente radial é decrescente. Consequentemente, toda trajetória tende exponencialmente para a origem quando $t \rightarrow+\infty$ e neste caso, a origem é um foco atrator.

Se $\mu=0$, temos $\dot{\rho}=-\rho^{3}<0$. Quando $\mu=0$, o equilíbrio deixa de ser hiperbólico, pois a parte real dos autovalores se anulam. A função $V\left(x_{1}, x_{2}\right)=\frac{x_{1}^{2}}{2}+\frac{x_{2}^{2}}{2}$ é uma candidata à função de Lyapunov para o sistema (8.5) para $\mu=0$ com o sinal do termo cúbico negativo. Temos $\dot{V}\left(x_{1}, x_{2}\right)=-\left(x_{1}^{4}+x_{2}^{4}\right)-2 x_{1}^{2} x_{2}^{2} \leq 0$, para todo $\left(x_{1}, x_{2}\right) \neq(0,0)$ e a função $V$ só é igual a zero na origem. Além disso, a função de Lyapunov $V$ é sempre positiva. E finalmente, se a solução $\varphi(t, x)$ é ilimitada para $t \geq 0$, então $V(\varphi(t, x))$ é também ilimitado para $t \geq 0$. Consequentemente, pelo Teorema 3.1 de [KG96], a origem é um foco atrator "fraco" e assim, toda trajetória tende para origem quando $t \rightarrow+\infty$, porém o equilíbrio não atrai exponencialmente, veja Figura 8.1.

Quando um sistema dinâmico autonômo possui um ponto de equilíbrio assintoticamente estável que perde estabilidade, tornando-se instável e surge um ciclo limite estável contendo esse equilíbrio no seu interior ao variarmos o parâmetro $\mu$, dizemos que o sistema sofreu uma Bifurcação de AndronovHopf Supercrítica ou simplesmente, Bifurcação de Hopf Supercrítica.

Pela Figura 8.1, em uma Bifurcação de Hopf Supercrítica, à esquerda do valor crítico de bifurcação de Hopf, o ciclo limite tem amplitude zero e o ponto de equilíbrio hiperbólico é estável. O ciclo limite surge quando o parâmetro cruza o eixo imaginário. Neste caso, a amplitude do ciclo limite cresce junto com o parâmetro e é estável, enquanto o ponto de equilíbrio hiperbólico, para esses valores de parâmetros, é instável. A curva $r(\mu)$ representa uma família a um parâmetro de ciclos limite do sistema dinâmico. O retrato de fase para este sistema é mostrado na Figura 8.1 junto com seu diagrama de bifurcação. Os pontos de equilíbrio assintoticamente estáveis e as órbitas fechadas estáveis são representados com traço contínuo e os equilíbrios e as órbitas fechadas instáveis por tracejado. 


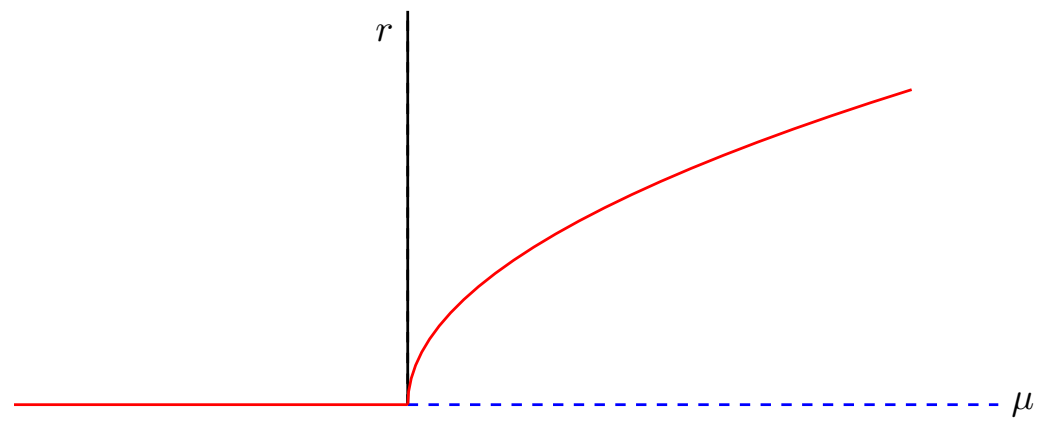

(a)

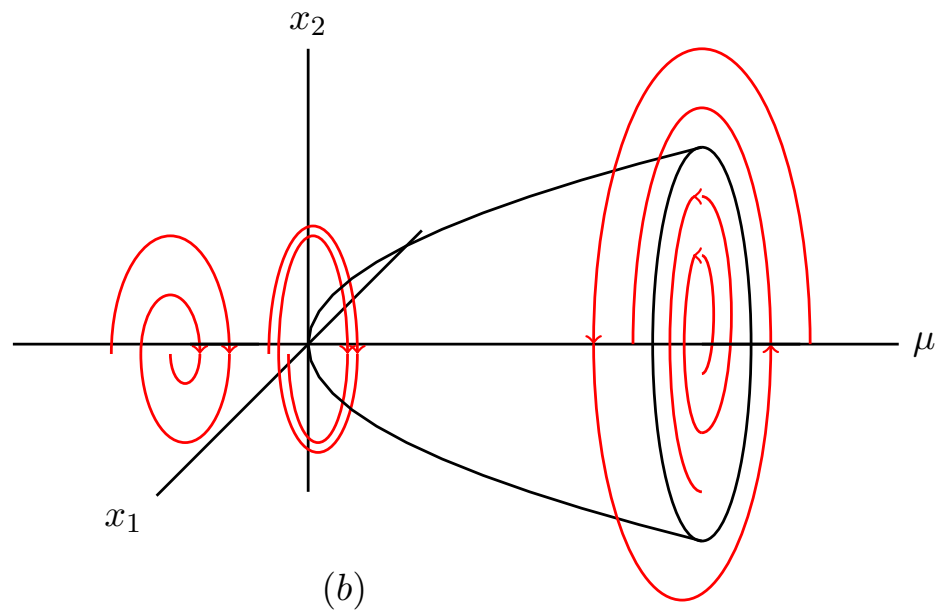

Figura 8.1: Bifurcação de Hopf Supercrítica. (a) Diagrama de Bifurcação de Hopf Supercrítica para o espaço de fase $r \times \mu$, onde $r(\mu)$ é uma familia a um parâmetro de ciclos limite do sistema dinâmico (8.8). Para $\mu<0$, a origem $r=0$ é um ponto de equilíbrio assintoticamente estável e para $\mu>0$, a origem $r=0$ é um ponto de equilíbrio instável e o ciclo limite $r=\sqrt{\mu}$ é estável. (b) Diagrama de Bifurcação de Hopf Supercrítica para o espaço de estado versus parâmetro. Para $\mu<0$, toda trajetória tende para a origem quando t cresce indefinidamente. Após a bifurcação, trajetórias que iniciam próximas do ponto de equilíbrio instável são atraídos pelo ciclo limite estável e as oscilações são limitadas.

A análise do retrato de fase do sistema (8.7) com o sinal positivo no termo cúbico, isto é,

$$
\left\{\begin{array}{l}
\dot{\rho}=\rho\left(\mu+\rho^{2}\right) \\
\dot{\theta}=1 .
\end{array}\right.
$$

é análoga à análise do sistema (8.8). Ocorrerá uma bifurcação de Hopf para $\mu=0$, porém ao contrário de (8.8), o ciclo limite surgirá para $\mu<0$ e será instável.

Se $\mu>0$, então $\dot{\rho}=\rho\left(\mu+\rho^{2}\right)>0$ e deste modo, a componente radial 
é crescente. Assim, toda trajetória afasta-se da origem exponencialmente quando $t \rightarrow+\infty$. Neste caso, a origem é um foco repulsor.

Se $\mu<0$, a origem será um foco atrator e teremos um equilíbrio $\rho(\mu)=$ $\sqrt{-\mu}$ que é um ciclo limite instável centrado na origem de raio $\rho(\mu)=\sqrt{-\mu}$. Assim, todas as órbitas iniciando externa ou internamente ao ciclo, com exceção da origem, tendem a este ciclo quando $t \rightarrow-\infty$, veja Figura 8.2.

Se $\mu=0$, teremos que $\dot{\rho}=\rho^{3}>0$. Observemos que quando $\mu=0$ o sistema deixa de ser hiperbólico, pois a parte real dos autovalores se anula. A função $V\left(x_{1}, x_{2}\right)=\frac{x_{1}^{2}}{2}+\frac{x_{2}^{2}}{2}$ é uma candidata a função de Lyapunov para o sistema (8.5) para $\mu=0$ com sinal do termo cúbico positivo. Temos $\dot{V}\left(x_{1}, x_{2}\right)=x_{1}^{4}+x_{2}^{4}+2 x_{1}^{2} x_{2}^{2} \geq 0$, para todo $\left(x_{1}, x_{2}\right) \neq(0,0)$ e a função $V$ é igual a zero somente na origem. Além disso, a função de Lyapunov $V$ é sempre positiva. Consequentemente, pelo Teorema 3.3 de [KG96], a origem é um foco repulsor "fraco" e assim, toda trajetória afasta-se da origem mas não exponencialmente quando $t \rightarrow+\infty$, veja Figura 8.2.

A bifurcação definida acima chamamos de Bifurcação de Andronov-Hopf Subcrítica ou simplesmente, Bifurcação de Hopf Subcrítica. Nesta bifurcação, o sistema dinâmico autonômo possui um ponto de equilíbrio assintoticamente estável isolado por um ciclo limite instável que perde a sua estabilidade ao coalescer com o ciclo limite.

Na Figura 8.2, em uma Bifurcação de Hopf Subcrítica, à esquerda do valor crítico de bifurcação de Hopf, existe um ciclo limite instável, em torno de um ponto de equilíbrio hiperbólico estável, que vai encolhendo e coalesce quando se funde com o ponto de equilíbrio na bifurcação. À direita do valor de bifurcação, o ciclo limite desaparece e o ponto de equilíbrio hiperbólico passa ser instável.

Os sistemas (8.4), ou equivalentemente (8.6) e (8.7) serão denominadas formas normais das Bifurcações de Hopf. De um modo geral, entendemos por forma normal de um objeto matemático a uma forma simplificada do objeto obtida por uma mudança de coordenadas que preserva as características essenciais do mesmo.

Exploraremos condições para que um sistema bidimensional seja localmente topologicamente equivalente à forma normal da bifurcação de Hopf. Este resultado será apresentado no Teorema 8.2.9.

Considere o sistema

$$
\left(\begin{array}{c}
\dot{x}_{1} \\
\dot{x}_{2}
\end{array}\right)=\left(\begin{array}{cc}
\mu & -1 \\
1 & \mu
\end{array}\right)\left(\begin{array}{c}
x_{1} \\
x_{2}
\end{array}\right)-\left(x_{1}^{2}+x_{2}^{2}\right)\left(\begin{array}{l}
x_{1} \\
x_{2}
\end{array}\right)
$$

que representa a forma normal da Bifurcação de Hopf, cujo sinal dos termos cúbicos é negativo e, consequentemente, apresenta um ciclo limite estável para cada $\mu>0$. Para o sistema (8.4), cujo sinal do termo cúbico é positivo, os resultados a seguir são análogos. 


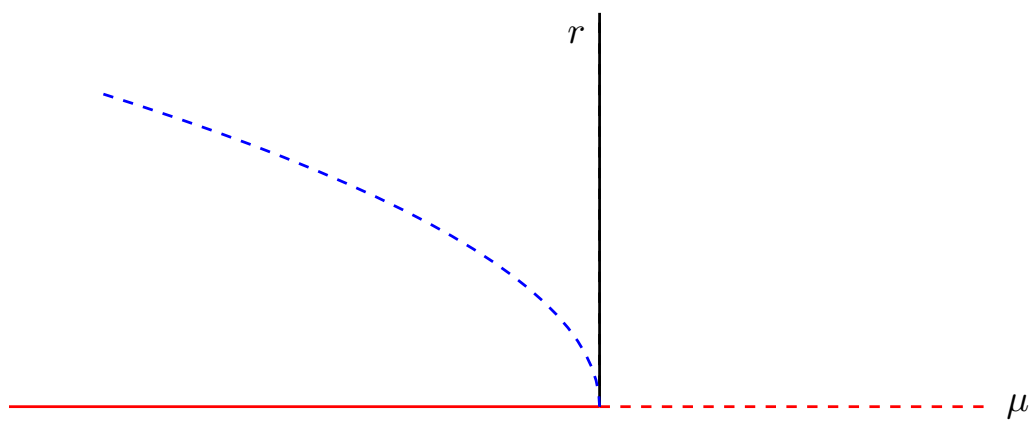

(a)

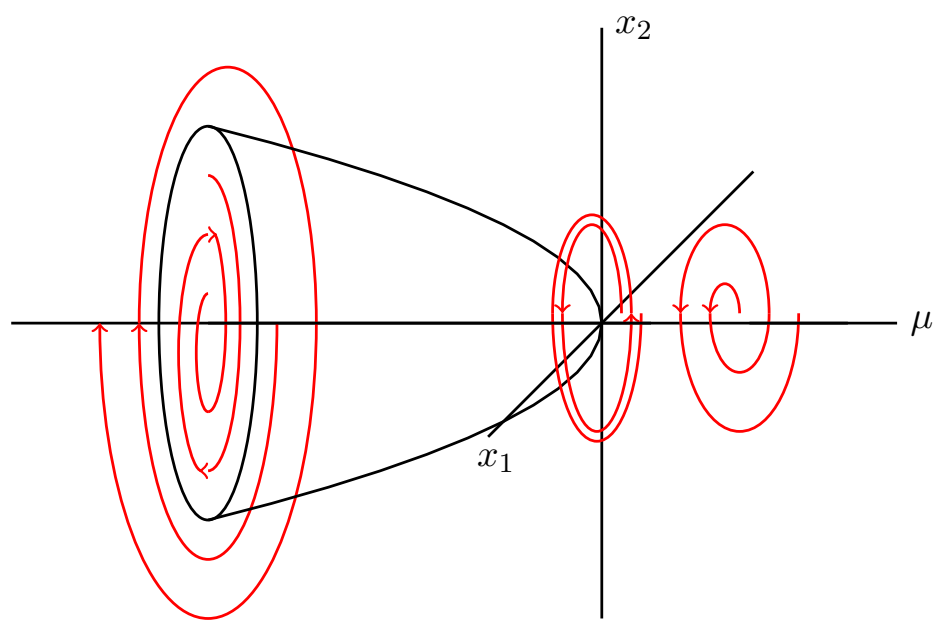

(b)

Figura 8.2: Bifurcação de Hopf Subcrítica. (a) Diagrama de Bifurcação de Hopf Subcrítica para o espaço de fase $r \times \mu$, onde $r(\mu)$ é uma familia a um parâmetro de ciclos limites do sistema dinâmico (8.9). Para $\mu<0$, a origem $r=0$ é um ponto de equilíbrio assintoticamente estável e o ciclo limite $r=\sqrt{\mu}$ é instável e para $\mu>0$, a origem $r=0$ é um ponto de equilíbrio instável. (b) Diagrama de Bifurcação de Hopf Subcrítica para o espaço de estado versus parâmetro. Para $\mu<0$, a amplitude do ciclo limite instável diminui quando o parâmetro se aproxima do valor de bifurcação e desaparece quando o parâmetro cruza o eixo imaginário. As trajetórias, após o valor de bifurcação, têm oscilações com amplitudes crescentes.

Lema 8.2.1. [Kuz13] Considere o sistema dependendo do parâmetro $\mu \in \mathbb{R}$,

$$
\left(\begin{array}{c}
\dot{x}_{1} \\
\dot{x}_{2}
\end{array}\right)=\left(\begin{array}{cc}
\mu & -1 \\
1 & \mu
\end{array}\right)\left(\begin{array}{l}
x_{1} \\
x_{2}
\end{array}\right)-\left(x_{1}^{2}+x_{2}^{2}\right)\left(\begin{array}{l}
x_{1} \\
x_{2}
\end{array}\right)+O\left(\|x\|^{4}\right)
$$

onde $x=\left(x_{1}, x_{2}\right)^{T} \in \mathbb{R}^{2}, \mu \in \mathbb{R}$ e $O\left(\|x\|^{4}\right)$ representa os termos de ordem maiores ou iguais a 4 e que dependem suavemente de $\mu$. O sistema (8.11) é localmente topologicamente equivalente ao sistema (8.10) em torno 
da origem.

Demonstração. Inicialmente, provaremos a existência e unicidade do ciclo limite do sistema (8.11) e depois exibiremos um homeomorfismo que leva órbitas do sistema (8.11) no sistema (8.10). Reescrevendo o sistema (8.11) em coordenadas polares $(\rho, \theta)$, obtemos

$$
\left\{\begin{array}{l}
\dot{\rho}=\rho\left(\mu-\rho^{2}\right)+\Phi(\rho, \theta), \\
\dot{\theta}=1+\Psi(\rho, \theta),
\end{array}\right.
$$

onde $\Phi=O\left(|\rho|^{4}\right)$ e $\Psi=O\left(|\rho|^{3}\right)$ dependem suavemente de $\mu$. Omitiremos esta dependência para não carregar na notação.

Para provar a existência de um ciclo limite para o sistema (8.12), basta mostrar a existência de um ponto fixo para a Aplicação de Poincaré associada ao sistema.

Para qualquer trajetória do sistema (8.12) com $\rho$ e $\mu$ próximos da origem, temos que $\dot{\theta}>0$. Então podemos eliminar a variável $t$, isto é, escrever $\rho$ como uma função de $\theta$. Assim, uma órbita do sistema (8.12) partindo de $(\rho, \theta)=\left(\rho_{0}, 0\right)$ tem a seguinte representação, veja Figura 8.3, com $\rho=$ $\rho\left(\theta, \rho_{0}\right), \rho_{0}=\rho\left(0, \rho_{0}\right)$ :

$$
\begin{aligned}
\frac{d \rho}{d \theta} & =\frac{\rho\left(\mu-\rho^{2}\right)+\Phi(\rho, \theta)}{1+\Psi(\rho, \theta)} \\
& =\rho\left(\mu-\rho^{2}\right)+R(\rho, \theta),
\end{aligned}
$$

onde $R=O\left(|\rho|^{4}\right)$. Observe que com essa reparametrização do tempo, o

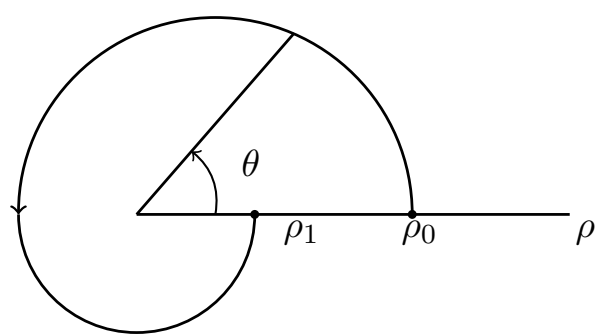

Figura 8.3: Aplicação de Poincaré para a Bifurcação de Hopf do sistema 8.13.

tempo de retorno para o semi-eixo $\theta=0$ é o mesmo para todas as órbitas que partem desse eixo com $\rho_{0}>0$. Como $\rho(\theta, 0) \equiv 0$, então

$$
\frac{d \rho}{d \theta}(\theta, 0)=\frac{d^{2} \rho}{d \rho d \theta}(\theta, 0)=\frac{d^{2} \rho}{d^{2} \theta}(\theta, 0)=\cdots=0
$$

e, portanto, podemos escrever a expansão de Taylor para $\rho\left(\theta, \rho_{0}\right)$ da seguinte 
forma

$$
\rho\left(\theta, \rho_{0}\right)=\left.\frac{d \rho}{d \rho_{0}}\right|_{\rho_{0}=0} \rho_{0}+\left.\frac{1}{2 !} \frac{d^{2} \rho}{d \rho_{0}^{2}}\right|_{\rho_{0}=0} \rho_{0}^{2}+\left.\frac{1}{3 !} \frac{d^{3} \rho}{d \rho_{0}^{3}}\right|_{\rho_{0}=0} \rho_{0}^{3}+O\left(\left|\rho_{0}\right|^{4}\right),
$$

ou também

$$
\rho\left(\theta, \rho_{0}\right)=u_{1}(\theta) \rho_{0}+u_{2}(\theta) \rho_{0}^{2}+u_{3}(\theta) \rho_{0}^{3}+O\left(\left|\rho_{0}\right|^{4}\right),
$$

onde

$$
u_{1}=\left.\frac{d \rho}{d \rho_{0}}\right|_{\rho_{0}=0}, u_{2}=\left.\frac{1}{2 !} \frac{d^{2} \rho}{d \rho_{0}^{2}}\right|_{\rho_{0}=0}, u_{3}(\theta)=\left.\frac{1}{3 !} \frac{d^{3} \rho}{d \rho_{0}^{3}}\right|_{\rho_{0}=0}, \cdots
$$

Derivando a expressão (8.14) em relação a $\theta$, obtemos

$$
\frac{d \rho}{d \theta}=\frac{d u_{1}(\theta)}{d \theta} \rho_{0}+\frac{d u_{2}(\theta)}{d \theta} \rho_{0}^{2}+\frac{d u_{3}(\theta)}{d \theta} \rho_{0}^{3}+\cdots .
$$

Substituindo (8.15) e (8.14) em (8.13), obtemos

$$
\begin{aligned}
\frac{d \rho}{d \theta}= & \frac{d}{d \theta}\left(u_{1}(\theta) \rho_{0}+u_{2}(\theta) \rho_{0}^{2}+u_{3}(\theta) \rho_{0}^{3}+\cdots\right) \\
= & \left(u_{1}(\theta) \rho_{0}+u_{2}(\theta) \rho_{0}^{2}+u_{3}(\theta) \rho_{0}^{3}+\cdots\right)\left(\mu-\left(u_{1}(\theta) \rho_{0}+u_{2}(\theta) \rho_{0}^{2}+\cdots\right)^{2}\right) \\
& +R(\rho, \theta) \\
= & u_{1}(\theta) \mu \rho_{0}+u_{2}(\theta) \mu \rho_{0}^{2}+\left(u_{3}(\theta) \mu-u_{1}^{3}\right) \rho_{0}^{3}+\cdots+R(\rho, \theta) .
\end{aligned}
$$

Da igualdade acima, resultam as seguintes equações diferenciais

$$
\begin{gathered}
\frac{d u_{1}}{d \theta}=u_{1}(\theta) \mu, \\
\frac{d u_{2}}{d \theta}=u_{2}(\theta) \mu, \\
\frac{d u_{3}}{d \theta}=u_{3}(\theta) \mu-u_{1}^{3} .
\end{gathered}
$$

Para $\theta=0, \rho=\rho_{0}$, obtém-se da equação (8.14) as condições iniciais $u_{1}(0)=1, u_{2}(0)=u_{3}(0)=0$. Resolvendo as equações diferenciais com suas respectivas condições iniciais, temos

$$
\begin{gathered}
u_{1}(\theta)=e^{\mu \theta}, \\
u_{2}(\theta) \equiv 0, \\
u_{3}(\theta)=e^{\mu \theta} \frac{1-e^{2 \mu \theta}}{2 \mu} .
\end{gathered}
$$

Essas expressões independem da função $R(\rho, \theta)$. Notemos que podemos 
reescrever o valor da expressão

$$
u_{3}(2 \pi)=e^{2 \mu \pi} \frac{1-e^{4 \mu \pi}}{2 \mu}
$$

como

$$
\begin{aligned}
& u_{3}(2 \pi)=e^{2 \mu \pi} \frac{1-e^{4 \mu \pi}}{2 \mu} \\
& =\frac{e^{2 \mu \pi}}{2 \mu}\left(1-\left[1+2(2 \pi) \mu+\frac{2^{2}(2 \pi)^{2} \mu^{2}}{2 !}+\cdots\right]\right) \\
& =\frac{e^{2 \mu \pi}}{2 \mu}\left(-2(2 \pi) \mu-\frac{2^{2}(2 \pi)^{2} \mu^{2}}{2 !}-\cdots\right) \\
& =\frac{-e^{2 \mu \pi}}{2 \mu}\left(2(2 \pi) \mu\left[1+\frac{2(2 \pi) \mu}{2 !}+\frac{2^{2}(2 \pi)^{2} \mu^{2}}{3 !}+\cdots\right]\right) \\
& =-e^{2 \mu \pi}\left(2 \pi\left[1+\frac{2(2 \pi) \mu}{2 !}+\frac{2^{2}(2 \pi)^{2} \mu^{2}}{3 !}+\cdots\right]\right) \\
& =-e^{2 \mu \pi}\left(2 \pi+\frac{2(2 \pi)^{2} \mu}{2 !}+\frac{2^{2}(2 \pi)^{3} \mu^{2}}{3 !}+\cdots\right) \\
& =-e^{2 \mu \pi}(2 \pi+O(\mu)) \text {, onde } O(\mu)=\frac{2(2 \pi)^{2} \mu}{2 !}+\frac{2^{2}(2 \pi)^{3} \mu^{2}}{3 !}+\cdots \text {. }
\end{aligned}
$$

De onde podemos concluir que a Aplicação de Poincaré

$$
\rho_{0} \mapsto \rho_{1}=\rho\left(2 \pi, \rho_{0}\right)
$$

tem a forma

$$
\rho_{1}=e^{2 \mu \pi} \rho_{0}-e^{2 \mu \pi}[2 \pi+O(\mu)] \rho_{0}^{3}+O\left(\rho_{0}^{4}\right)
$$

para todo $R=O\left(\rho^{4}\right)$. Para $\rho_{0}$ e $\mu$ suficientemente pequenos, existe uma vizinhança da origem onde a Aplicação de Poincaré tem somente o ponto fixo trivial para pequenos valores de $\mu<0$ e um ponto fixo extra $\rho_{0}^{\star}=\sqrt{\mu}+\cdots$ para pequenos valores de $\mu>0$, veja Figura 8.4. Para verificar essa última afirmação, podemos reescrever a função (8.16) como

$$
\rho_{1}=\rho_{0} \widetilde{S}\left(\mu, \rho_{0}\right)
$$

onde

$$
\widetilde{S}\left(\mu, \rho_{0}\right)=e^{2 \mu \pi}\left(1-[2 \pi+O(\mu)] \rho_{0}^{2}+O\left(\rho_{0}^{3}\right)\right) .
$$




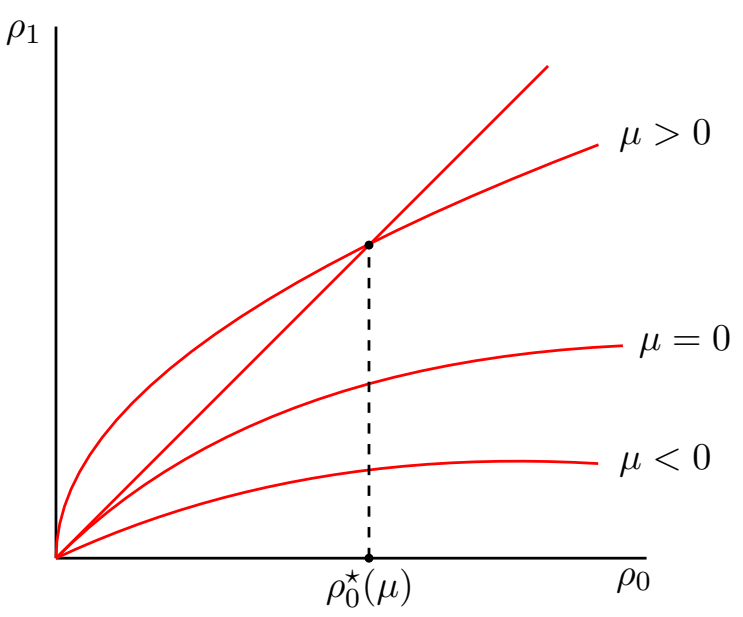

Figura 8.4: Ponto Fixo para a Aplicação de Poincaré do sistema (8.13).

Obtemos assim a equação dos pontos fixos, para $\rho_{0}>0$, dada por

$$
\begin{aligned}
\widetilde{S}\left(\mu, \rho_{0}\right) & =1 \\
e^{2 \mu \pi}\left(1-[2 \pi+O(\mu)] \rho_{0}^{2}+O\left(\rho_{0}^{3}\right)\right) & =1 \\
1-[2 \pi+O(\mu)] \rho_{0}^{2}+e^{-2 \mu \pi} O\left(\rho_{0}^{3}\right) & =e^{-2 \mu \pi} \\
1-[2 \pi+O(\mu)] \rho_{0}^{2}+e^{-2 \mu \pi} O\left(\rho_{0}^{3}\right)-e^{-2 \mu \pi} & =0 .
\end{aligned}
$$

Seja

$$
S\left(\mu, \rho_{0}\right)=1-[2 \pi+O(\mu)] \rho_{0}^{2}+e^{-2 \mu \pi} O\left(\rho_{0}^{3}\right)-e^{-2 \mu \pi} .
$$

Derivando a expressão acima em relação a $\mu$, obtemos

$$
S_{\mu}\left(\mu, \rho_{0}\right)=O_{\mu}(\mu) \rho_{0}^{2}-2 \pi e^{-2 \mu \pi} O\left(\rho_{0}^{3}\right)+2 \pi e^{-2 \mu \pi} .
$$

Como, para $\left(\mu, \rho_{0}\right)=(0,0), S(0,0)=0$ e $S_{\mu}(0,0)=2 \pi$, então pelo Teorema 2.1.7 podemos escrever $\mu$ como função de $\rho_{0}$ numa vizinhança de $\rho_{0}=0$. Escrevendo a expansão de Taylor de $\mu$ em torno de $\rho_{0}=0$, temos

$$
\mu\left(\rho_{0}\right)=\mu(0)+\mu^{\prime}(0) \rho_{0}+\frac{1}{2 !} \mu^{\prime \prime}(0) \rho_{0}^{2}+\cdots .
$$

Sabemos que $\mu(0)=0$. Calculando a derivada de $\mu$ em relação a $\rho_{0}$, tem-se que

$$
\begin{aligned}
\mu^{\prime}\left(\rho_{0}\right) & =\frac{-S_{\rho_{0}}\left(\rho_{0}, \mu\left(\rho_{0}\right)\right)}{S_{\mu}\left(\rho_{0}, \mu\left(\rho_{0}\right)\right)} \\
& =\frac{2(2 \pi+O(\mu)) \rho_{0}+e^{-2 \pi \mu} O\left(\rho_{0}^{2}\right)}{(\ldots) \rho_{0}^{2}-2 \pi e^{-2 \pi \mu} O\left(\rho_{0}^{3}\right)+2 \pi e^{-2 \pi \mu}}
\end{aligned}
$$


e, em $\rho_{0}=0$, temos que $\mu^{\prime}(0)=0$. Calculando a segunda derivada de $\mu$ em relação a $\rho$, temos

$$
\mu^{\prime \prime}\left(\rho_{0}\right)=\frac{-S_{\rho_{0} \rho_{0}}-\left(S_{\rho_{0} \mu}+S_{\mu \rho_{0}}\right) \mu^{\prime}\left(\rho_{0}\right)-S_{\mu \mu}\left(\mu^{\prime}\left(\rho_{0}\right)\right)^{2}}{S_{\mu}}
$$

$\mathrm{e}$, em $\rho_{0}=0$,

$$
\begin{gathered}
\mu^{\prime \prime}\left(\rho_{0}\right)=\frac{-S_{\rho_{0} \rho_{0}}}{S_{\mu}} \\
\mu^{\prime \prime}\left(\rho_{0}\right)=\frac{2[2 \pi+O(\mu)]+2 e^{-2 \mu \pi} O\left(\rho_{0}\right)}{(\ldots) \rho_{0}^{2}-2 \pi e^{-2 \pi \mu} O\left(\rho_{0}^{3}\right)+2 \pi e^{-2 \pi \mu}}, \mu^{\prime \prime}\left(\rho_{0}\right)=2 .
\end{gathered}
$$

Assim sendo, a expansão de Taylor de $\mu\left(\rho_{0}\right)$ em torno de $\rho_{0}=0$ é dada por

$$
\mu\left(\rho_{0}\right)=\rho_{0}^{2}+\cdots,
$$

que é uma função injetora no domínio $\rho_{0} \geq 0$.

Para provarmos a estabilidade de $\rho_{0}^{\star}$ basta mostrarmos que

$$
\frac{d \rho_{1}}{d \rho_{0}}\left(\rho_{0}^{\star}\right)<1 .
$$

De fato, para determinarmos a estabilidade dos pontos fixos da função (8.16), derivaremos (8.17) em relação a $\rho_{0}$. Então

$$
\frac{d \rho_{1}}{d \rho_{0}}=\widetilde{S}\left(\mu, \rho_{0}\right)+\rho_{0} \widetilde{S}_{\rho_{0}}\left(\mu, \rho_{0}\right) .
$$

Como $\widetilde{S}\left(\mu, \rho_{0}\right)=1$ para $\rho_{0}=\rho_{0}^{\star}, \mu=\mu\left(\rho_{0}^{\star}\right)$, então para mostrarmos a desigualdade acima basta provarmos que $\rho_{0} \widetilde{S}_{\rho_{0}}\left(\mu\left(\rho_{0}^{\star}\right), \rho_{0}^{\star}\right)$ é negativo. Calculando $\rho_{0} \widetilde{S}_{\rho_{0}}\left(\mu\left(\rho_{0}^{\star}\right), \rho_{0}^{\star}\right)$, obtemos

$$
\rho_{0} \widetilde{S}_{\rho_{0}}\left(\mu, \rho_{0}\right)=\rho_{0}^{2}\left[-2 e^{2 \pi \mu}[2 \pi+O(\mu)]+O\left(\rho_{0}\right)\right],
$$

que, para pequenos valores de $\rho_{0}^{\star}>0, \mu\left(\rho_{0}^{\star}\right)>0$, satisfaz o desejado.

Levando em consideração que o ponto fixo positivo da função corresponde a um ciclo limite do sistema, podemos concluir que o sistema (8.10), ou (8.11), com quaisquer termos $O\left(|\rho|^{4}\right)$, tem um único ciclo limite (e estável) bifurcando da origem quando $\mu>0$ como no sistema (8.4). Portanto, os termos de ordem superior não afetam o surgimento do ciclo limite numa vizinhança de $\left(x_{1}, x_{2}\right)=(0,0)$ com $|\mu|$ suficientemente pequeno.

Construiremos agora o homeomorfismo necessário para provar a equivalência topológica dos retratos de fase. Fixemos $\mu>0$ pequeno. Ambos os sistemas (8.10) e (8.11) tem um ciclo limite em alguma vizinhança da origem. Assumiremos uma reparametrização de tempo no sistema (8.11) de modo que o tempo de retorno seja constante $2 \pi$. Além disso, aplicaremos 
um escalonameno linear nas coordenadas do sistema (8.11) de modo que o ponto de intersecção do ciclo e o semi-eixo horizontal seja $x_{1}=\sqrt{\mu}$.

Defina a função $x \mapsto \widetilde{x}$ do seguinte modo, considere o ponto $x=\left(x_{1}, x_{2}\right)$ e encontre valores $\left(\rho_{0}, \tau_{0}\right)$, onde $\tau_{0}$ é o tempo mínimo que uma órbita do sistema (8.10) leva para alcançar o ponto $x$ partindo do semi-eixo horizontal com $\rho=\rho_{0}$. Com um ponto deste eixo $\rho=\rho_{0}$, construíremos uma órbita do sistema (8.11) no intervalo [0, $\left.\tau_{0}\right]$ partindo desse ponto. Denotemos o ponto resultante por $\widetilde{x}=\left(\widetilde{x}_{1}, \widetilde{x}_{2}\right)$, veja Figura 8.5. Assumindo $\widetilde{x}=0$ para $x=0$. A continuidade do homeomorfismo e de sua inversa segue da continuidade das soluções do sistema de equações diferenciais ordinárias.
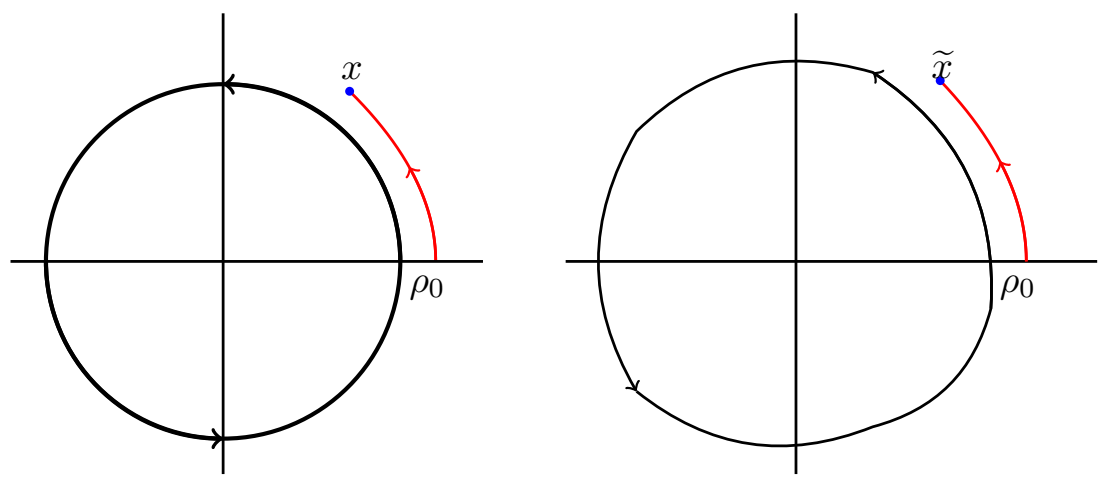

Figura 8.5: Construção do homeomorfismo nas vizinhanças da bifurcação de Hopf.

A função construída assim é um homeomorfismo que, para $\mu>0$, leva órbitas do sistema (8.10), em alguma vizinhança da origem, em órbitas de (8.11), preservando a direção do tempo. $\mathrm{O}$ caso $\mu<0$ pode ser considerado da mesma forma com uma nova mudança de coordenadas.

Considere o sistema

$$
\dot{x}=f(x, \mu), x=\left(x_{1}, x_{2}\right)^{T} \in \mathbb{R}^{2}, \mu \in \mathbb{R},
$$

com $f$ suave, e para $\mu=0$ temos o ponto de equilíbrio $x_{0}$ com autovalores $\lambda_{1,2}= \pm i \omega_{0}, \omega_{0}>0$. Pelo Teorema 2.1.7, como $\lambda=0$ não é um autovalor da matriz Jacobiana, o sistema tem um único equilíbrio $x_{0}(\mu)$ em alguma vizinhança da origem para todo $|\mu|$ suficientemente pequeno. De fato, como

$$
\operatorname{det} f_{x}\left(x_{0}, 0\right)=\lambda_{1}(0) \lambda_{2}(0)=\omega_{0}^{2} \neq 0
$$

então o Teorema 2.1.7 garante a unicidade e a suavidade local do equilíbrio $x_{0}(\mu)$, para $|\mu|$ pequeno. Podemos então, através de uma mudança de coordenadas, deslocar este equilíbrio para a origem. Portanto, vamos assumir, sem perda de generalidade, que $x_{0}=0$ é o ponto de equilíbrio do sistema para $|\mu|$ suficientemente pequeno. Então, o sistema (8.18) pode ser escrito 
como

$$
\dot{x}=A(\mu) x+F(x, \mu)
$$

onde $F$ é uma função suave com componentes $F_{1,2}$, tendo expansão de Taylor em $x$ iniciando com os termos quadráticos, $F=O\left(\|x\|^{2}\right)$. A matriz Jacobiana $A(\mu)=D f_{x}\left(0, \mu_{0}\right)$ possui dois autovalores $\lambda_{1,2}=\sigma(\mu) \pm i \omega(\mu)$, com a condição para a bifurcação de Hopf $\sigma(0)=0, \omega(0)=\omega_{0}>0$.

Lema 8.2.2. [Kuz13] O sistema (8.19) pode ser escrito, para $\|\mu\|$ suficientemente pequeno, na forma

$$
\dot{z}=\lambda(\mu) z+g(z, \bar{z}, \mu)
$$

onde $g=O\left(\|z\|^{2}\right)$ é uma função suave de $(z, \bar{z}, \mu)$, dada por

$$
g(z, \bar{z}, \mu)=\langle p(\mu), F(z q(\mu)+\overline{z q}(\mu), \mu)\rangle
$$

onde $F=O\left(\|x\|^{2}\right)$.

Demonstração. Seja $q(\mu) \in \mathbb{C}^{2}$ o autovetor de $A(\mu)$ correspondente ao autovalor $\lambda(\mu)$,

$$
A(\mu) q(\mu)=\lambda(\mu) q(\mu)
$$

e seja $p(\mu) \in \mathbb{C}^{2}$ o autovetor da matriz transposta $A^{T}(\mu)$ correspondente ao autovalor $\bar{\lambda}(\mu)$,

$$
A^{T}(\mu) p(\mu)=\bar{\lambda}(\mu) p(\mu)
$$

Observemos que sempre é possível normalizar o autovetor $p(\mu)$ com respeito ao autovetor $q(\mu)$, visto que múltiplos escalares dos autovetores $p(\mu)$ e $q(\mu)$ também são autovetores e portanto,

$$
\langle p(\mu), q(\mu)\rangle=1
$$

Dado $x \in \mathbb{R}^{2}$, para todo $\mu$ pequeno, existe uma representação única de $x$ em função de $q(\mu)$ e $\bar{q}(\mu)$,

$$
x=z q(\mu)+\overline{z q}(\mu),
$$

para algum complexo z. De fato, podemos determinar $z$ a partir da equação

$$
z=\langle p(\mu), x\rangle
$$

Observemos que

$$
\begin{aligned}
\langle p(\mu), x\rangle & =\langle p(\mu), z q(\mu)+\overline{z q}(\mu)\rangle \\
& =z\langle p(\mu), q(\mu)\rangle+\bar{z}\langle p(\mu), \bar{q}(\mu)\rangle \\
& =z+\bar{z}\langle p(\mu), \bar{q}(\mu)\rangle, \text { pois }\langle p(\mu), q(\mu)\rangle=1 .
\end{aligned}
$$


Falta provarmos que $\langle p(\mu), \bar{q}(\mu)\rangle=0$. Então

$$
\begin{aligned}
\langle p(\mu), \bar{q}(\mu)\rangle & =\left\langle p(\mu), \frac{1}{\bar{\lambda}(\mu)} A(\mu) \bar{q}(\mu)\right\rangle \\
& =\frac{1}{\bar{\lambda}(\mu)}\langle p(\mu), A(\mu) \bar{q}(\mu)\rangle \\
& =\frac{1}{\bar{\lambda}(\mu)}\left\langle A^{T}(\mu) p(\mu), \bar{q}(\mu)\right\rangle \\
& =\frac{1}{\bar{\lambda}(\mu)}\langle\bar{\lambda}(\mu) p(\mu), \bar{q}(\mu)\rangle \\
& =\frac{\lambda(\mu)}{\bar{\lambda}(\mu)}\langle p(\mu), \bar{q}(\mu)\rangle .
\end{aligned}
$$

Daí, temos que

$$
\left(1-\frac{\lambda(\mu)}{\bar{\lambda}(\mu)}\right)\langle p(\mu), \bar{q}(\mu)\rangle=0 .
$$

Como $\lambda(\mu) \neq \bar{\lambda}(\mu)$, para $|\mu|$ suficientemente pequeno, podemos concluir que

$$
\langle p(\mu), \bar{q}(\mu)\rangle=0 .
$$

Da equação (8.21) temos que a variável complexa $z$ satisfaz a equação

$$
\begin{aligned}
\dot{z} & =\langle p(\mu), \dot{x}\rangle \\
& =\langle p(\mu), A(\mu) x+F(x, \mu)\rangle \\
& =\langle p(\mu), A(\mu)(z q(\mu)+\overline{z q}(\mu))+F(z q(\mu)+\overline{z q}(\mu), \mu)\rangle \\
& =\langle p(\mu), A(\mu)(z q(\mu)+\overline{z q}(\mu))+F(z q(\mu)+\overline{z q}(\mu), \mu)> \\
& =\lambda(\mu) z\langle p(\mu), q(\mu)>+\bar{\lambda}(\mu) \bar{z}\langle p(\mu), \bar{q}(\mu)\rangle+\langle p(\mu), F(z q(\mu)+\overline{z q}(\mu), \mu)\rangle \\
& =\lambda(\mu) z+g(z, \bar{z}, \mu),
\end{aligned}
$$

onde $g(z, \bar{z}, \mu)=\langle p(\mu), F(z q(\mu)+\overline{z q}(\mu), \mu)\rangle$, obtendo assim a forma da equação (8.20), como queríamos.

Escrevendo a função $g$ em série de Taylor em relação as variáveis complexas $z$ e $\bar{z}$, temos

$$
g(z, \bar{z}, \mu)=\sum_{k+l \geq 2} \frac{1}{k ! l !} g_{k l}(\mu) z^{k} \bar{z}^{l}
$$

onde

$$
g_{k l}(\mu)=\left.\frac{\partial^{k+l}}{\partial z^{k} \partial \bar{z}^{l}}\langle p(\mu), F(z q(\mu)+\overline{z q}(\mu), \mu)\rangle\right|_{z=0}
$$

para $k+l \geq 2, k, l=0,1, \ldots$ 
Suponha que, para $\mu=0$, a função $F(x, \mu)$ em (8.19) seja representada por

$$
F(x, 0)=\frac{1}{2} B(x, x)+\frac{1}{6} C(x, x, x)+O\left(\|x\|^{4}\right),
$$

onde $B(x, y)$ e $C(x, y, z)$ são funções multilineares simétricas de $x, y, z \in \mathbb{R}^{2}$. Em coordenadas, temos

$$
B_{i}(x, y)=\left.\sum_{j, k=1}^{2} \frac{\partial^{2} F_{i}(\xi, 0)}{\partial \xi_{j} \partial \xi_{k}}\right|_{\xi=0} x_{j} y_{k}, i=1,2
$$

e

$$
C_{i}(x, y, z)=\left.\sum_{j, k, l=1}^{2} \frac{\partial^{3} F_{i}(\xi, 0)}{\partial \xi_{j} \partial \xi_{k} \partial \xi_{l}}\right|_{\xi=0} x_{j} y_{k} z_{l}, i=1,2 .
$$

Daí, tem-se

$$
B(z q+\overline{z q}, z q+\overline{z q})=z^{2} B(q, q)+2 z \bar{z} B(q, \bar{q})+\bar{z}^{2} B(\bar{q}, \bar{q}),
$$

onde $q=q(0)$. Sendo assim, os coeficentes de Taylor $g_{k l}$, com $k+l=2$, dos termos quadráticos em $g(z, \bar{z}, 0)$ podem ser expressos pelas fórmulas

$$
g_{20}=\langle p, B(q, q)\rangle, g_{11}=\langle p, B(q, \bar{q})\rangle, g_{02}=\langle p, B(\bar{q}, \bar{q})\rangle,
$$

onde $p=p(0)$.

Analogamente, cálculo similar com a função multilinear $C(x, y, z)$ fornece

$$
\begin{aligned}
& g_{30}=\langle p, C(q, q, q)\rangle, g_{21}=\langle p, C(q, q, \bar{q})\rangle, \\
& g_{12}=\langle p, C(q, \bar{q}, \bar{q})\rangle, g_{03}=\langle p, C(\bar{q}, \bar{q}, \bar{q})\rangle .
\end{aligned}
$$

Grosso modo o metódo de determinar a forma normal de um sistema consiste em estabelecer mudanças de coordenadas de tal maneira que o sistema dinâmico toma uma forma mais simples e assim sendo, a sua manipulação torna-se mais simples.

Lema 8.2.3. [Kuz13] A equação

$$
\dot{z}=\lambda z+\frac{g_{20}}{2} z^{2}+g_{11} z \bar{z}+\frac{g_{02}}{2} \bar{z}^{2}+O\left(|z|^{3}\right),
$$

onde $\lambda=\lambda(\mu)=\sigma(\mu)+i \omega(\mu), \gamma(0)=0, \omega(0)=\omega_{0}>0$ e $g_{i j}=g_{i j}(\mu)$, pode ser transformada, pela mudança de coordenadas complexa

$$
z=w+\frac{h_{20}}{2} w^{2}+h_{11} w \bar{w}+\frac{h_{02}}{2} \bar{w}^{2},
$$


para $|\mu|$ suficientemente pequeno, na equação sem termos quadráticos

$$
\dot{w}=\lambda w+O\left(|w|^{3}\right) .
$$

Demonstração. A mudança de variável inversa de (8.26) é dada pela expressão

$$
w=z-\frac{h_{20}}{2} z^{2}-h_{11} z \bar{z}-\frac{h_{02}}{2} \bar{z}^{2}+O\left(|z|^{3}\right) .
$$

Derivando a equação acima, obtém-se

$$
\begin{aligned}
\dot{w}= & \dot{z}-h_{20} z \dot{z}-h_{11}(\dot{z} \bar{z}+z \dot{\bar{z}})-h_{02} \dot{\bar{z} \dot{z}}+\cdots \\
= & \lambda z+\left(\frac{g_{20}}{2}-\lambda h_{20}\right) z^{2}+\left(g_{11}-\lambda h_{11}-\bar{\lambda} h_{11}\right) z \bar{z}+\left(\frac{g_{02}}{2}-\bar{\lambda} h_{02}\right) \bar{z}^{2}+\cdots \\
= & \lambda w+\left(\lambda \frac{h_{20}}{2}+\frac{g_{20}}{2}-\lambda h_{20}\right) w^{2}+\left(\lambda h_{11}+g_{11}-\lambda h_{11}-\bar{\lambda} h_{11}\right) w \bar{w}+ \\
& +\left(\lambda \frac{h_{02}}{2}+\frac{g_{02}}{2}-\bar{\lambda} h_{02}\right) \bar{w}^{2}+O\left(|w|^{3}\right) \\
= & \lambda w+\frac{1}{2}\left(g_{20}-\lambda h_{20}\right) w^{2}+\left(g_{11}-\bar{\lambda} h_{11}\right) w \bar{w}+\frac{1}{2}\left(g_{02}-(2 \bar{\lambda}-\lambda) h_{02}\right) \bar{w}^{2}+ \\
& +O\left(|w|^{3}\right)
\end{aligned}
$$

Escolhem-se

$$
h_{20}=\frac{g_{20}}{\lambda}, h_{11}=\frac{g_{11}}{\bar{\lambda}} \text { e } h_{02}=\frac{g_{02}}{2 \bar{\lambda}-\lambda}
$$

de tal forma a eliminimar os termos quadráticos da equação (8.25). Observemos que essas substituições são sempres possíveis, pois para $|\mu|$ suficientemente pequeno, os denominadores $\lambda(0)=i \omega_{0}$ com $\omega_{0}>0$ nunca se anulam.

Lema 8.2.4. [Kuz13] A equação

$$
\dot{z}=\lambda z+\frac{g_{30}}{6} z^{3}+\frac{g_{21}}{2} z^{2} \bar{z}+\frac{g_{12}}{2} z \bar{z}^{2}+\frac{g_{03}}{6} \bar{z}^{3}+O\left(|z|^{4}\right),
$$

onde $\lambda=\lambda(\mu)=\sigma(\mu)+i \omega(\mu), \gamma(0)=0, \omega(0)=\omega_{0}>0$ e $g_{i j}=g_{i j}(\mu)$, pode ser transformada, pela mudança de coordenadas complexa

$$
z=w+\frac{h_{30}}{6} w^{3}+\frac{h_{21}}{2} w^{2} \bar{w}+\frac{h_{12}}{2} w \bar{w}^{2}+\frac{h_{03}}{6} \bar{w}^{3},
$$

para $|\mu|$ suficientemente pequeno, na equação com apenas um termo cúbico

$$
\dot{w}=\lambda w+c_{1} w^{2} \bar{w}+O\left(|w|^{4}\right)
$$


onde $c_{1}=c_{1}(\mu)$.

Demonstração. A transformação inversa de (8.28) é

$$
w=z-\frac{h_{30}}{6} z^{3}-\frac{h_{21}}{2} z^{2} \bar{z}-\frac{h_{12}}{2} z \bar{z}^{2}-\frac{h_{03}}{6} \bar{z}^{3}+O\left(|z|^{4}\right) .
$$

Derivando a equação acima têm-se que

$$
\begin{aligned}
\dot{w}= & \dot{z}-\frac{h_{30}}{2} z^{2} \dot{z}-\frac{h_{21}}{2}\left(2 z \bar{z} \dot{z}+z^{2} \dot{\bar{z}}\right)-\frac{h_{12}}{2}\left(\bar{z}^{2} \dot{z}+2 z \bar{z} \dot{z}\right)-\frac{h_{03}}{2} \bar{z}^{2} \dot{\bar{z}}+\cdots \\
= & \lambda z+\left(\frac{g_{30}}{6}-\lambda \frac{h_{30}}{2}\right) z^{3}+\left(\frac{g_{21}}{2}-\lambda h_{21}-\bar{\lambda} \frac{h_{21}}{2}\right) z^{2} \bar{z}+ \\
& +\left(\frac{g_{12}}{2}-\lambda \frac{h_{12}}{2}-\bar{\lambda} h_{12}\right) z \bar{z}^{2}+\left(\frac{g_{03}}{6}-\bar{\lambda} \frac{h_{03}}{2}\right) \bar{z}^{3}+\cdot \\
=\quad & \lambda w+\left(\lambda \frac{h_{30}}{6}+\frac{g_{30}}{6}-\lambda \frac{h_{30}}{2}\right) w^{3}+\left(\lambda \frac{h_{21}}{2}+\frac{g_{21}}{2}-\lambda h_{21}-\bar{\lambda} \frac{h_{21}}{2}\right) w^{2} \bar{w} \\
& +\left(\lambda \frac{h_{12}}{2}+\frac{g_{12}}{2}-\lambda \frac{h_{12}}{2}-\bar{\lambda} h_{12}\right) w \bar{w}^{2}+\left(\lambda \frac{h_{03}}{6}+\frac{g_{03}}{6}-\bar{\lambda} \frac{h_{03}}{2}\right) \bar{w}^{3}+ \\
+ & O\left(|w|^{4}\right) \\
= & \lambda w+\frac{1}{6}\left(g_{30}-2 \lambda h_{30}\right) w^{3}+\frac{1}{2}\left(g_{21}-(\lambda+\bar{\lambda}) h_{21}\right) w^{2} \bar{w}+\frac{1}{2}\left(g_{12}-2 \bar{\lambda} h_{12}\right) w \bar{w}^{2} \\
+ & \frac{1}{6}\left(g_{03}+(\lambda-3 \bar{\lambda}) h_{03}\right) \bar{w}^{3}+O\left(|w|^{4}\right)
\end{aligned}
$$

Fazendo, portanto,

$$
h_{30}=\frac{g_{30}}{2 \lambda}, h_{12}=\frac{g_{12}}{2 \bar{\lambda}} \text { e } h_{03}=\frac{g_{03}}{3 \bar{\lambda}-\lambda},
$$

eliminam-se todos os termos cúbicos, com exceção do termo $w^{2} \bar{w}$. Assim sendo, as substituições são válidas, pois os denominadores envolvidos são diferentes de zero para todo $|\mu|$ suficientemente pequeno.

Para eliminar o termo $w^{2} \bar{w}$, teríamos que escolher $h_{21}=\frac{g_{21}}{\lambda+\bar{\lambda}}$, o que não é possível, visto que para $\mu=0$ o denominador se anula, pois $\lambda(0)+\bar{\lambda}(0)=$ $i \omega_{0}-i \omega_{0}=0$. Para obtermos uma transformação que dependa suavemente de $\mu$, escolhemos $h_{21}=0$, resultando em

$$
c_{1}=\frac{g_{21}}{2} .
$$

Chamamos o termo $w^{2} \bar{w}$ de termo ressonante. Observe que o seu coeficiente é o mesmo coeficiente do termo cúbico $z^{2} \bar{z}$ na equação (8.27). 
No próximo resultado, combinaremos os Lemas (8.2.3) e (8.2.4) para eliminar os termos quadráticos e cúbicos do sistema simultaneamente.

Lema 8.2.5. [Kuz13] A equação

$$
\dot{z}=\lambda z+\sum_{2 \leq k+l \leq 3} \frac{1}{k ! l !} g_{k l} z^{k} \bar{z}^{l}+O\left(|z|^{4}\right),
$$

onde $\lambda=\lambda(\mu)=\sigma(\mu)+i \omega(\mu), \gamma(0)=0, \omega(0)=\omega_{0}>0$ e $g_{i j}=g_{i j}(\mu)$, pode ser transformada, pela mudança de coordenadas complexa, suavemente dependendo do parâmetro,

$$
z=w+\frac{h_{20}}{2} w^{2}+h_{11} w \bar{w}+\frac{h_{02}}{2} \bar{w}^{2}+\frac{h_{30}}{6} w^{3}+\frac{h_{12}}{2} w \bar{w}^{2}+\frac{h_{03}}{6} \bar{w}^{3},
$$

para $|\mu|$ suficientemente pequeno, na equação com apenas o termo cúbico ressonante

$$
\dot{w}=\lambda w+c_{1} w^{2} \bar{w}+O\left(|w|^{4}\right)
$$

onde $c_{1}=c_{1}(\mu)$.

Demonstração. Obviamente a superposição das transformações definidas nos Lemas (8.2.3) e (8.2.4) nos levam a este resultado. Inicialmente, usaremos a transformação

$$
z=w+\frac{h_{20}}{2} w^{2}+h_{11} w \bar{w}+\frac{h_{02}}{2} \bar{w}^{2}
$$

com

$$
h_{20}=\frac{g_{20}}{\lambda}, h_{11}=\frac{g_{11}}{\bar{\lambda}}, h_{02}=\frac{g_{02}}{2 \bar{\lambda}-\lambda},
$$

definidas no Lema 8.2.3, anulando assim todos os termos quadráticos, mas também alterando os coeficientes dos termos cúbicos. Assim sendo, o coeficiente do termo cúbico $w^{2} \bar{w}$ passará a ser $\frac{1}{2} \widetilde{g}_{21}$, ao invés de $\frac{1}{2} g_{21}$. Agora, aplicaremos a transformação do Lema 8.2.4

$$
z=w+\frac{h_{30}}{6} w^{3}+\frac{h_{21}}{2} w^{2} \bar{w}+\frac{h_{12}}{2} w \bar{w}^{2}+\frac{h_{03}}{6} \bar{w}^{3}
$$

com

$$
h_{30}=\frac{g_{30}}{2 \lambda}, h_{12}=\frac{g_{12}}{2 \bar{\lambda}}, h_{03}=\frac{g_{03}}{3 \bar{\lambda}-\lambda},
$$

eliminando todos os termos cúbicos menos o termo ressonante, sendo que o coeficiente desse termo será $\frac{1}{2} \widetilde{g}_{21}$. Como os termos de ordem 4 e superiores constantes na superposição afetam somente os termos $O\left(|w|^{4}\right)$ da equação (8.29), então eles podem ser truncados.

O resultado a seguir fornecerá um modo de obtermos o valor do coeficiente do termo ressonante no valor de bifurcação de Hopf. 
Lema 8.2.6. [Kuz13] O coeficiente $c_{1}(\mu)$ da equação (8.30), para $\mu=0$, é dado por

$$
c_{1}(0)=\frac{i}{2 \omega_{0}}\left(g_{20} g_{11}-2\left|g_{11}\right|^{2}-\frac{1}{3}\left|g_{02}\right|^{2}\right)+\frac{g_{21}}{2} .
$$

Demonstração. Derivando a equação (8.31), obtemos

$$
\dot{z}=\dot{w}+h_{20} w \dot{w}+h_{11}(\bar{w} \dot{w}+w \dot{\bar{w}})+h_{02} \dot{w} \dot{w} .
$$

Substituindo $\dot{w}$ e seu complexo conjugado da equação (8.30) e rearrumando os seus termos, temos

$$
\dot{z}=\lambda w+\lambda h_{20} w^{2}+(\lambda+\bar{\lambda}) h_{11} w \bar{w}+\bar{\lambda} h_{02} \bar{w}^{2}+c_{1} w^{2} \bar{w}+\cdots .
$$

Por outro lado, na equação (8.29), tem-se

$$
\dot{z}=\lambda z+\frac{1}{2} g_{20} z^{2}+g_{11} z \bar{z}+\frac{1}{2} g_{02} \bar{z}^{2}+\frac{1}{6} g_{30} z^{3}+\frac{1}{2} g_{21} z^{2} \bar{z}+\frac{1}{2} g_{12} z \bar{z}^{2}+\frac{1}{6} g_{03} \bar{z}^{3}+\cdots .
$$

Se substituirmos $z$ e $\bar{z}$, dados pela equação (8.31), e novamente rearrumarmos os termos, obtemos

$$
\begin{aligned}
\dot{z}= & \lambda w+\frac{1}{2}\left(\lambda h_{20}+g_{20}\right) w^{2}+\left(\lambda h_{11}+g_{11}\right) w \bar{w}+\frac{1}{2}\left(\lambda h_{02}+g_{02}\right) \bar{w}^{2}+ \\
& +\left(g_{20} h_{11}+g_{11}\left(\frac{h_{20}}{2}+\bar{h}_{11}\right)+g_{02} \frac{\bar{h}_{02}}{2}+\frac{g_{21}}{2}\right) w^{2} \bar{w}+\cdots .
\end{aligned}
$$

Comparando os coeficientes do termo $w^{2} \bar{w}$ das duas equações obtidas para $\dot{z}$ e utilizando os valores encontrados para $h_{20}, h_{11}$ e $h_{02}$,

$$
h_{20}=\frac{g_{20}}{\lambda}, h_{11}=\frac{g_{11}}{\bar{\lambda}}, h_{02}=\frac{g_{02}}{2 \bar{\lambda}-\lambda}
$$

temos

$$
\begin{aligned}
c_{1} & =g_{20} h_{11}+g_{11}\left(\frac{h_{20}}{2}+\bar{h}_{11}\right)+g_{02} \frac{\bar{h}_{02}}{2}+\frac{g_{21}}{2} \\
& =\frac{g_{20} g_{11}}{\bar{\lambda}}+g_{11}\left(\frac{g_{20}}{2 \lambda}+\frac{\bar{g}_{11}}{\lambda}\right)+\frac{\left|g_{02}\right|^{2}}{2(2 \lambda-\bar{\lambda})}+\frac{g_{21}}{2} \\
& =g_{20} g_{11}\left(\frac{1}{\bar{\lambda}}+\frac{1}{2 \lambda}\right)+\frac{\left|g_{11}\right|^{2}}{\lambda}+\frac{\left|g_{02}\right|^{2}}{2(2 \lambda-\bar{\lambda})}+\frac{g_{21}}{2} \\
& =g_{20} g_{11}\left(\frac{2 \lambda+\bar{\lambda}}{2|\lambda|^{2}}\right)+\frac{\left|g_{11}\right|^{2}}{\lambda}+\frac{\left|g_{02}\right|^{2}}{2(2 \lambda-\bar{\lambda})}+\frac{g_{21}}{2}
\end{aligned}
$$

Observe que $c_{1}$ depende suavemente de $\mu$, pois $\lambda$ e $g_{i j}$ são funções suaves do parâmetro $\mu$. Para o valor de bifurcação de Hopf $\mu=0$, o valor do coeficiente 
do termo cúbico se reduz a

$$
\begin{aligned}
c_{1}(0) & =g_{20} g_{11}\left(\frac{2 i \omega_{0}-i \omega_{0}}{2 \omega_{0}^{2}}\right)+\frac{\left|g_{11}\right|^{2}}{i \omega_{0}}+\frac{\left|g_{02}\right|^{2}}{2\left(2 i \omega_{0}+i \omega_{0}\right)}+\frac{g_{21}}{2} \\
& =\frac{g_{20} g_{11} i}{2 \omega_{0}}+\frac{\left|g_{11}\right|^{2} i}{\omega_{0}}-\frac{\left|g_{02}\right|^{2} i}{6 \omega_{0}}+\frac{g_{21}}{2} \\
& =\frac{i}{2 \omega_{0}}\left(g_{20} g_{11}-2\left|g_{11}\right|^{2}-\frac{1}{3}\left|g_{02}\right|^{2}\right)+\frac{g_{21}}{2} .
\end{aligned}
$$

Lema 8.2.7. [Kuz13] Considere a equação

$$
\frac{d w}{d t}=(\sigma(\mu)+i w(\mu)) w+c_{1}(\mu) w|w|^{2}+O\left(|w|^{4}\right)
$$

onde $\sigma(0)=0$ e $\omega(0)=\omega_{0}>0$. Suponha $\sigma^{\prime}(0) \neq 0$ e $\Re\left(c_{1}(0)\right) \neq 0$. Então, a equação (8.34) será transformada, por mudança de coordenadas, na equação

$$
\frac{d u}{d \theta}=(\beta+i) u+s u|u|^{2}+O\left(|u|^{4}\right)
$$

onde u é a nova coordenada complexa, $\theta$ e $\beta$ são, respectivamente, os novos tempo e parâmetro e $s=\operatorname{sinal}\left(\Re\left(c_{1}(0)\right)\right)= \pm 1$.

Demonstração. Introduzindo a nova coordenada de tempo $\tau=\omega(\mu) t$, que preserva a direção, pois $\omega(\mu)>0$, para todo $\mu$ suficientemente pequeno, então

$$
\frac{d w}{d \tau}=\frac{d w}{d t} \frac{d t}{d \tau} \frac{d w}{d \tau}=\frac{1}{\omega(\mu)} \frac{d w}{d t} .
$$

Substituindo o valor de $\frac{d w}{d t}$, dado pela equação (8.34), obtemos

$$
\begin{array}{r}
\frac{d w}{d \tau}=\frac{1}{\omega(\mu)}\left((\sigma(\mu)+i \omega(\mu)) w+c_{1}(\mu) w|w|^{2}+O\left(|w|^{4}\right)\right) \\
\frac{d w}{d \tau}=(\beta+i) w+d_{1}(\beta) w|w|^{2}+O\left(|w|^{4}\right)
\end{array}
$$

onde

$$
\beta=\beta(\mu)=\frac{\sigma(\mu)}{\omega(\mu)}, d_{1}(\beta)=\frac{c_{1}(\mu(\beta))}{\omega(\mu(\beta))} .
$$

Podemos considerar $\beta$ como um novo parâmetro, pois

$$
\beta(0)=0
$$


e a derivada de $\beta(\mu)$ em relação a $\mu$ é dada por

$$
\beta^{\prime}(\mu)=\frac{\sigma^{\prime}(\mu) \omega(\mu)-\sigma(\mu) \omega^{\prime}(\mu)}{\omega^{2}(\mu)} \Rightarrow \beta^{\prime}(0)=\frac{\sigma^{\prime}(0)}{\omega_{0}} \neq 0
$$

e, portanto, o Teorema da Função Inversa em [Lim81] nos garante a existência local e suave de $\mu$ como função de $\beta$.

Vamos agora reparametrizar o tempo ao longo das órbitas com a nova mudança de tempo $\theta=\theta(\tau, \beta)$, onde

$$
d \theta=\left(1+e_{1}(\beta)|w|^{2}\right) d \tau,
$$

com $e_{1}(\beta)=\Im\left(d_{1}(\beta)\right)$. Esta mudança é próxima da identidade numa pequena vizinhança da origem. Usando esse valor de tempo definido, obtemos

$$
\frac{d w}{d \theta}=(\beta+i) w+l_{1}(\beta) w|w|^{2}+O\left(|w|^{4}\right)
$$

onde $l_{1}(\beta)=\Re\left(d_{1}(\beta)\right)-\beta e_{1}(\beta)$ é real e

$$
l_{1}(0)=\frac{\Re c_{1}(0)}{\omega(0)} .
$$

De fato,

$$
\begin{gathered}
\frac{d w}{d \theta}=\frac{\frac{d w}{d \tau}}{\frac{d \theta}{d \tau}} \\
\frac{\frac{d w}{d \tau}}{1+e_{1}(\beta)|w|^{2}}=(\beta+i) w+l_{1}(\beta) w|w|^{2}+\cdots \\
\frac{d w}{d \tau}=\left(1+e_{1}(\beta)|w|^{2}\right)\left[(\beta+i) w+l_{1}(\beta) w|w|^{2}+\cdots\right] \\
=(\beta+i) w+\left[l_{1}(\beta)+e_{1}(\beta)(\beta+i)\right] w|w|^{2}+\cdots \\
=(\beta+i) w+\left[\Re\left(d_{1}(\beta)\right)-\beta e_{1}(\beta)+\beta e_{1}(\beta)+\right. \\
=(\beta+i) w+\left[\Re\left(d_{1}(\beta)\right)+i \Im\left(d_{1}(\beta)\right)\right] w|w|^{2}+\cdots \\
=(\beta+i) w+d_{1}(\beta) w|w|^{2}+\cdots
\end{gathered}
$$

Finalmente, introduzindo a nova variável complexa $u$,

$$
w=\frac{u}{\sqrt{\left|l_{1}(\beta)\right|}}
$$


o que é sempre possível, pois $\Re\left(c_{1}(0)\right) \neq 0$, conclui-se que $l_{1}(0) \neq 0$. Então temos

$$
\begin{aligned}
\frac{1}{\sqrt{\left|l_{1}(\beta)\right|}} \frac{d u}{d \theta} & =(\beta+i) \frac{u}{\sqrt{\left|l_{1}(\beta)\right|}}+l_{1}(\beta) \frac{u}{\sqrt{\left|l_{1}(\beta)\right|}}\left|\frac{u}{\sqrt{\left|l_{1}(\beta)\right|}}\right|^{2}+\cdots \\
\Rightarrow \frac{d u}{d \theta} & =(\beta+i) u+\frac{l_{1}(\beta)}{\left|l_{1}(\beta)\right|} u|u|^{2}+O\left(|u|^{4}\right) \\
& =(\beta+i) u+s u|u|^{2}+O\left(|u|^{4}\right),
\end{aligned}
$$

onde $s=\operatorname{sinal}\left(l_{1}(0)\right)$.

A função $l_{1}(\beta)$ é chamada de Primeiro Coeficiente de Lyapunov. $\mathrm{O}$ valor do Primeiro Coeficiente de Lyapunov, para $\beta=0$, pode ser calculado pela fórmula

$$
l_{1}(0)=\frac{1}{2 \omega_{0}^{2}} \Re\left(i g_{20} g_{11}+\omega_{0} g_{21}\right) .
$$

Observe que precisamos apenas das segundas e terceiras derivadas parciais no ponto de bifurcação para calcularmos o Primeiro Coeficiente de Lyapunov $l_{1}(0)$. O valor de $l_{1}(0)$ dependerá da normalização dos autovetores $q$ e $p$, contudo seu sinal é invariante pela normalização dos autovetores.

Observemos também que a equação (8.35) com sinal $s=-1$ escrita em coordenadas cartesianas coincidirá com o sistema (8.11) do Lema 8.2.1.

Teorema 8.2.9 (Teorema da Bifurcação de Hopf). [Kuz13] Considere o sistema bidimensional

$$
\dot{x}=f(x, \mu)
$$

onde $f$ é suave, $x \in \mathbb{R}^{2}$ e $\mu \in \mathbb{R}$. Suponha que para todo $|\mu|$ suficientemente pequeno a origem seja um equilíbrio com autovalores

$$
\lambda_{1,2}=\sigma(\mu) \pm i \omega(\mu)
$$

onde $\sigma(0)=0, \omega(0)=\omega_{0}>0$. Se as seguintes condições são satisfeitas:

(i) $l_{1}(0) \neq 0$ (condição de não degenerescência);

(ii) $\sigma^{\prime}(0) \neq 0$ (condição de transversalidade).

Então o sistema (8.38) é localmente topologicamente equivalente, em torno do ponto de equilibrio $x_{0}=0$, a uma das seguintes formas normais

$$
\left(\begin{array}{l}
\dot{y}_{1} \\
\dot{y}_{2}
\end{array}\right)=\left(\begin{array}{cc}
\zeta & -1 \\
1 & \zeta
\end{array}\right)\left(\begin{array}{l}
y_{1} \\
y_{2}
\end{array}\right) \pm\left(y_{1}^{2}+y_{2}^{2}\right)\left(\begin{array}{l}
y_{1} \\
y_{2}
\end{array}\right)
$$

Demonstração. Utilizando os Lemas 8.2 .2 a 8.2.7, transformamos o sistema (8.38) no sistema (8.35) do Lema 8.2.7. Consequentemente, pelo Lema 8.2.1 concluimos o resultado. 
A suposição (ii) é a condição de transversalidade, que garante o cruzamento transversal do eixo imaginário pelos autovalores da matriz jacobiana. Numa bifurcação estacionária, do tipo sela-nó, o cruzamento do eixo imaginário pelos autovalores é feito no eixo real.

Coeficientes de Lyapunov indicam o nível de degeneração do campo vetorial. Se o primeiro coeficiente de Lyapunov é diferente de zero, então o campo vetorial possui uma degeneração de ordem cúbica mostrando que termos cúbicos são os que determinam o tipo de comportamento dinâmico local na vizinhança do ponto de equilíbrio não hiperbólico na variedade central, ver [Kuz13] para mais detalhes.

\subsection{Bifurcação de Hopf $n$-dimensional}

Nesta seção, estudaremos a Bifurcação de Hopf em um sistema $n$-dimensional, para tal usaremos o Método da Projeção a fim de restringirmos o sistema ao caso bidimensional da Bifurcação de Hopf estudado na seção anterior. Este método consiste em transformar o sistema

$$
\dot{x}=f(x, \mu), x \in \mathbb{R}^{n}, \mu \in \mathbb{R}^{m},
$$

escrevendo-o em uma base formada pelos seus autovetores. Em seguida, utilizaremos apenas os autovetores correspondentes aos autovalores críticos, ou seja, ao par de autovalores com parte real nula, e projetaremos o sistema a sua Variedade Central invariante tangente ao espaço associado a esses autovetores e com isso restringindo ao caso bidimensional.

Diante disso, faremos agora um breve resumo de alguns resultados de Álgebra Linear necessários para um bom entendimento do método proposto, ver [Kuz13] e [Pon], para estudar a Bifurcação de Hopf no caso ndimensional.

Seja $A$ uma matriz quadrada de ordem $n$ com $\lambda$ um autovalor de multiplicidade $m$, com $v_{1}, v_{2}, \ldots, v_{l}, 1 \leq l \leq m$, autovetores linearmente independentes correspondentes a $\lambda$. Para cada autovetor $v_{j}, 1 \leq j \leq l$, existe uma escolha maximal de vetores $w_{1}^{j}, w_{2}^{j}, \ldots, w_{k}^{j}$, onde $k=k(j) \in \mathbb{N}$, satisfazendo

$$
\begin{aligned}
A w_{1}^{j} & =\lambda w_{1}^{j} \\
A w_{2}^{j} & =\lambda w_{2}^{j}+w_{1}^{j} \\
& \cdots \\
A w_{k}^{j} & =\lambda w_{k}^{j}+w_{k-1}^{j}
\end{aligned}
$$

Observemos que não há nenhum problema em escolher o vetor $w_{1}=w_{1}^{j}$ como sendo o próprio autovetor $v_{j}$. Os vetores $w_{k}^{j}, k \geq 2$, são denominados autovetores generalizados da matriz $A$ correspondentes ao autovalor $\lambda$. O conjunto formado pelos autovetores generalizados, correspondentes ao auto- 
valor $\lambda$, é linearmente independente e o subespaço

$$
E=\left\{x \in \mathbb{C}^{n}: x=a_{1} w_{1}^{j}+a_{2} w_{2}^{j}+\cdots+a_{k} w_{k}^{j}, a_{i} \in \mathbb{C}\right\}
$$

é invariante segundo a matriz $A$.

Segundo as formas canônicas de Jordan, o espaço vetorial $\mathbb{C}^{n}$ pode ser decomposto em subespaços $A$-invariantes correspondentes aos autovalores de $A$ e esses subespaços são gerados pelos seus respectivos autovetores e autovetores generalizados. Chamaremos esses subespaços de autoespaços generalizados de $A$. Para uma matriz $A$ real, esses subespaços $A$-invariantes do $\mathbb{R}^{n}$ são gerados pelos autovetores e autovetores generalizados de $A$, correspondentes aos autovalores reais e as partes real e imaginária dos autovalores complexos, veja [Kuz13] e [Pon].

Consideremos o sistema, com um equilíbrio não hiperbólico em $x_{0}=0$,

$$
\dot{x}=A x+F(x), x \in R^{n},
$$

onde $F(x)=O\left(\|x\|^{2}\right)$ é uma função suave e $A$, a parte linear do sistema, com um par simples de autovalores complexos no eixo imaginário $\lambda_{1,2}=$ $\pm i \omega_{0}, \omega_{0}>0$ e não adimintindo outros autovalores sobre o eixo imaginário, isto é, parte real nula.

Seja $q \in \mathbb{C}^{n}$ um autovetor correspondente a $\lambda_{1}$. Então

$$
A q=i \omega_{0} q, A \bar{q}=-i \omega_{0} \bar{q}
$$

Consideremos também o autovetor autoadjunto $p \in \mathbb{C}^{n}$ satisfazendo as propriedades

$$
A^{T} p=-i \omega_{0} p, A^{T} \bar{p}=i \omega_{0} \bar{p}
$$

e a propriedade de normalização $\langle p, q\rangle=1$, onde $\langle p, q\rangle=\sum_{i=1}^{n} \bar{p}_{i} q_{i}$ é o produto escalar padrão em $\mathbb{C}^{n}$.

Seja $T^{c}$ o autoespaço bidimensional real generalizado, correspondente aos autovalores $\lambda_{1,2}= \pm i \omega_{0}$, gerado por $\left.\Re(q), \Im(q)\right\}$. Seja $T^{s u}$ o autoespaço $(n-2)$-dimensional real generalizado correspondente a todos autovalores de $A$, com exceção de $\lambda_{1,2}= \pm i \omega_{0}$.

Como $\mathbb{R}^{n}=T^{s u} \oplus T^{c}$, podemos decompor $x \in \mathbb{R}^{n}$ como

$$
x=z q+\overline{z q}+y,
$$

onde $z \in \mathbb{C}, z q+\overline{z q} \in T^{c}$ e $y \in T^{s u}$. Diante disso, utilizaremos o próximo resultado para explicitarmos os valores $y$ e $z$ com relação à variável $x$.

Lema 8.3.1. [Kuz13] Seja $y \in \mathbb{R}^{n}$. Então, $y \in T^{s u}$ se, e somente se, $\langle p, y\rangle=$ $0, p \in \mathbb{C}^{n}$ complexo.

Demonstração. Seja $y \in T^{s u}$. Sejam $\nu_{1}, \nu_{2}, \ldots, \nu_{l}$ os autovalores reais de $A$ e $\eta_{1}, \bar{\eta}_{1}, \eta_{2}, \bar{\eta}_{2}, \ldots, \eta_{k}, \bar{\eta}_{k}$ os autovalores complexos de $A$, diferentes de $\lambda_{1}$ 
e $\lambda_{2}$.

Sejam $T_{\nu_{i}}$ o autoespaço generalizado correspondente ao autovalor $\nu_{i} \mathrm{e}$ $T_{\eta_{j}, \bar{\eta}_{j}}$ o autoespaço real generalizado correspondente aos autovalores $\eta_{j}, \bar{\eta}_{j}$.

Então, tem-se que

$$
T^{s u}=T_{\nu_{1}} \oplus T_{\nu_{2}} \oplus \cdots \oplus T_{\nu_{l}} \oplus T_{\eta_{1}, \bar{\eta}_{1}} \oplus T_{\eta_{2}, \bar{\eta}_{2}} \oplus \cdots \oplus T_{\eta_{k}, \bar{\eta}_{k}} .
$$

Como $T_{\nu_{i}}$ são espaços generalizados, é fato que para cada $i$ existe um $N_{\nu_{i}} \in \mathbb{N}$ tal que se $y \in T_{\nu_{i}}$, então $\left(A-\nu_{i} I_{n}\right)^{N_{\nu_{i}}} y=0$. Portanto,

$$
\begin{aligned}
0 & =\left\langle p,\left(A-\nu_{i} I_{n}\right)^{N_{\nu_{i}}} y\right\rangle=\left\langle\left[\left(A-\nu_{i} I_{n}\right)^{N_{\nu_{i}}}\right]^{T} p, y\right\rangle \\
& =\left\langle\left(A^{T}-\nu_{i} I_{n}\right)^{N_{\nu_{i}}} p, y\right\rangle=\left\langle\left(\bar{\lambda}-\nu_{i}\right)^{N_{\nu_{i}}} p, y\right\rangle \\
& =\left(\lambda-\nu_{i}\right)^{N_{\nu_{i}}}\langle p, y\rangle .
\end{aligned}
$$

Como $\lambda \neq \nu_{i}$, temos que

$$
\langle p, y\rangle=0 .
$$

Analogamente, como $T_{\eta_{j}, \bar{\eta}_{j}}$ são espaços generalizados, para cada $j$ existe um $N_{\eta_{j}} \in \mathbb{N}$ tal que se $y \in T_{\eta_{j}}, \bar{\eta}_{j}$, então $\left(A-\eta_{j} I_{n}\right)^{N_{\eta_{j}}}\left(A-\bar{\eta}_{j} I_{n}\right)^{N_{\eta_{j}}} y=0$. Portanto,

$$
\begin{aligned}
0 & =\left\langle p,\left(A-\eta_{j} I_{n}\right)^{N_{\eta_{j}}}\left(A-\overline{\eta_{j}} I_{n}\right)^{N_{\eta_{j}}} y\right\rangle=\left\langle\left[\left(A-\eta_{j} I_{n}\right)^{N_{\eta_{j}}}\right]^{T} p,\left(A-\overline{\eta_{j}} I_{n}\right)^{N_{\eta_{j}}} y\right\rangle \\
& =\left\langle\left(A^{T}-\bar{\eta}_{j} I_{n}\right)^{N_{\eta_{j}}} p,\left(A-\overline{\eta_{j}} I_{n}\right)^{N_{\eta_{j}}} y\right\rangle=\left\langle\left(\bar{\lambda}-\bar{\eta}_{j}\right)^{N_{\eta_{j}}} p,\left(A-\overline{\eta_{j}} I_{n}\right)^{N_{\eta_{j}}} y\right\rangle \\
& =\left(\lambda-\eta_{j}\right)^{N_{\eta_{j}}}\left\langle\left[\left(A-\overline{\eta_{j}} I_{n}\right)^{N_{\eta_{j}}}\right]^{T} p, y\right\rangle=\left(\lambda-\eta_{j}\right)^{N_{\eta_{j}}}\left\langle\left(A^{T}-\eta_{j} I_{n}\right)^{N_{\eta_{j}}} p, y\right\rangle \\
& =\left(\lambda-\eta_{j}\right)^{N_{\eta_{j}}}\left\langle\left(\bar{\lambda}-\eta_{j}\right)^{N_{\eta_{j}}} p, y\right\rangle=\left(\lambda-\eta_{j}\right)^{N_{\eta_{j}}}\left(\lambda-\overline{\eta_{j}}\right)^{N_{\eta_{j}}}\langle p, y\rangle .
\end{aligned}
$$

Como $\lambda \neq \eta_{j}$ e $\lambda \neq \bar{\eta}_{j}$, temos que

$$
\langle p, y\rangle=0
$$

Portanto, para qualquer $y \in T^{s u}$, então podemos escrever $y$ como

$$
y=\sum_{i=1}^{l} y_{\nu_{i}}+\sum_{j=1}^{k} y_{\eta_{j}},
$$

$\operatorname{com} y_{\nu_{i}} \in T_{\nu_{i}}$, para $i=1,2, \ldots, l$, e $y_{\eta_{j}} \in T_{\eta_{j} \bar{\eta}_{j}}$, para $j=1,2, \ldots, k$. Então 
concluímos que

$$
\begin{aligned}
\langle p, y\rangle & =\left\langle p, \sum_{i=1}^{l} y_{\nu_{i}}+\sum_{j=1}^{k} y_{\eta_{j}}\right\rangle \\
& =\sum_{i=1}^{l}\left\langle p, y_{\nu_{i}}\right\rangle+\sum_{j=1}^{k}\left\langle p, y_{\eta_{j}}\right\rangle \\
& =0 .
\end{aligned}
$$

Reciprocamente, suponhamos $y \in \mathbb{R}^{n}$ tal que $<p, y>=0$, com $p \in \mathbb{C}^{n}$. Como $\mathbb{R}^{n}=T^{c} \oplus T^{s u}$, então

$$
y=y_{s u}+y_{c}
$$

com $y_{s u} \in T^{s u}$ e $y_{c} \in T^{c}$. Como $T^{c}$ é gerado por $q$, $\bar{q}$, mas $y_{c} \in \mathbb{R}^{n}$, então

$$
y_{c}=\alpha q+\overline{\alpha q},
$$

com $\alpha \in \mathbb{C}$ e, portanto,

$$
y=y_{s u}+\alpha q+\overline{\alpha q} .
$$

Mostraremos que $y_{c}=0$, para isso precisamos provar que $\alpha=0$. Temos

$$
\begin{aligned}
0 & =\langle p, y\rangle=\left\langle p, y_{s u}+y_{c}\right\rangle \\
& =\left\langle p, y_{s u}\right\rangle+\left\langle p, y_{c}\right\rangle
\end{aligned}
$$

Como $\left\langle p, y_{s u}\right\rangle=0$, pois $y_{s u} \in T^{s u}$, então $\left\langle p, y_{c}\right\rangle=0$ e, portanto,

$$
\begin{aligned}
0 & =\langle p, \alpha q+\overline{\alpha q}\rangle \\
& =\alpha\langle p, q\rangle+\bar{\alpha}\langle p, \bar{q}\rangle .
\end{aligned}
$$

Como $\langle p, q\rangle=1$ e $\langle p, \bar{q}\rangle=0$, então $\alpha=0$.

Pelo Lema 8.3.1, podemos explicitar as variáveis $z$ e $y$ em relação a $x$. Sendo assim, dado $x=z q+\overline{z q}+y \in \mathbb{R}^{n}$, com $z q+\overline{z q} \in T^{c}$ e $y \in T^{s u}$, então

$$
\begin{aligned}
\langle p, x\rangle & =\langle p, z q+\overline{z q}+y\rangle \\
& =z\langle p, q\rangle+\bar{z}\langle p, \bar{q}\rangle+\langle p, y\rangle .
\end{aligned}
$$

Como $y \in T^{s u}$, então pelo Lema 8.3.1 temos que $\langle p, y\rangle=0$. Consequentemente,

$$
\begin{aligned}
\langle p, x\rangle & =z\langle p, q\rangle+\bar{z}\langle p, \bar{q}\rangle \\
& =z
\end{aligned}
$$


pois $\langle p, q\rangle=1$ e $\langle p, \bar{q}\rangle=0$. Concluímos então

$$
\left\{\begin{array}{l}
z=\langle p, x\rangle \\
y=x-\langle p, x\rangle q-\langle\bar{p}, x\rangle \bar{q}
\end{array} .\right.
$$

Reescrevendo o sistema (8.40) utilizando as coordenadas de (8.41), temos

$$
\begin{aligned}
\dot{z} & =\langle p, \dot{x}\rangle \\
& =\langle p, A x+F(x)\rangle \\
& =\langle p, A(z q+\overline{z q}+y)+F(z q+\overline{z q}+y)\rangle \\
& =\langle p, z A q\rangle+\langle p, \bar{z} A \bar{q}\rangle+\langle p, A y\rangle+\langle p, F(z q+\overline{z q}+y)\rangle \\
& =\left\langle p, i \omega_{0} z q\right\rangle+\left\langle p,-i \omega_{0} \overline{z q}\right\rangle+\langle p, A y\rangle+\langle p, F(z q+\overline{z q}+y)\rangle \\
& =i \omega_{0} z\langle p, q\rangle-i \omega_{0} \bar{z}\langle p, \bar{q}\rangle+\langle p, A y\rangle+\langle p, F(z q+\overline{z q}+y)\rangle \\
& =i \omega_{0} z+\langle p, F(z q+\overline{z q}+y)\rangle
\end{aligned}
$$

pois $\langle p, q\rangle=1,\langle p, \bar{q}\rangle=0$. E além disso, como $T^{s u}$ é $A$-invariante e, consequentemente, $A y \in T^{s u}$, para todo $y \in T^{s u}$, então $\langle p, A y\rangle=0$. Portanto,

$$
\dot{z}=i \omega_{0} z+\langle p, F(z q+\overline{z q}+y)\rangle .
$$

Da segunda equação do sistema (8.41), temos que

$$
\begin{aligned}
\dot{y}= & \dot{x}-\langle p, \dot{x}\rangle q-\langle\bar{p}, \dot{x}\rangle \bar{q} \\
= & A x+F(x)-\langle p, A x+F(x)\rangle q-\langle\bar{p}, A x+F(x)\rangle \bar{q} \\
= & A(z q+\overline{z q}+y)+F(z q+\overline{z q}+y)-\langle p, A(z q+\overline{z q}+y)+F(z q+\overline{z q}+y)\rangle q- \\
& -\langle\bar{p}, A(z q+\overline{z q}+y)+F(z q+\overline{z q}+y)\rangle \bar{q} \\
= & z A q+\bar{z} A \bar{q}+A y+F(z q+\overline{z q}+y)- \\
& -(\langle p, z A q\rangle+\langle p, \bar{z} A \bar{q}\rangle+\langle p, A y\rangle+\langle p, F(z q+\overline{z q}+y)\rangle) q- \\
& -(\langle\bar{p}, z A q\rangle+\langle\bar{p}, \bar{z} A \bar{q}\rangle+\langle\bar{p}, A y\rangle+\langle\bar{p}, F(z q+\overline{z q}+y)\rangle) \bar{q} \\
& \\
= & i \omega_{0} z q-i \omega_{0} \overline{z q}+A y+F(z q+\overline{z q}+y)- \\
& -\left(i \omega_{0} z\langle p, q\rangle-i \omega_{0} \bar{z}\langle p, \bar{q}\rangle+\langle p, A y\rangle+\langle p, F(z q+\overline{z q}+y)\rangle\right) q- \\
& -\left(i \omega_{0} z\langle\bar{p}, q\rangle-i \omega_{0} \bar{z}\langle\bar{p}, \bar{q}\rangle+\langle\bar{p}, A y\rangle+\langle\bar{p}, F(z q+\overline{z q}+y)\rangle\right) \bar{q} \\
= & i \omega_{0} z q-i \omega_{0} \overline{z q}+A y+F(z q+\overline{z q}+y)-i \omega_{0} z q-\langle p, F(z q+\overline{z q}+y)\rangle q+i \omega_{0} \overline{z q}- \\
& -\langle\bar{p}, F(z q+\overline{z q}+y)\rangle \bar{q} \\
= & A y+F(z q+\overline{z q}+y)-\langle p, F(z q+\overline{z q}+y)\rangle q-\langle\bar{p}, F(z q+\overline{z q}+y)\rangle \bar{q},
\end{aligned}
$$

pois $\langle p, A y\rangle,\langle\bar{p}, A y\rangle,\langle p, \bar{q}\rangle$ e $\langle\bar{p}, q\rangle$ são todos nulos. 
Portanto,

$$
\left\{\begin{array}{l}
\dot{z}=i \omega_{0} z+\langle p, F(z q+\overline{z q}+y)\rangle \\
\dot{y}=A y+F(z q+\overline{z q}+y)-\langle p, F(z q+\overline{z q}+y)\rangle q-\langle\bar{p}, F(z q+\overline{z q}+y)\rangle \bar{q}
\end{array}\right.
$$

Usando a expansão de Taylor, podemos reescrever o sistema (8.42) em termos das variáveis $z, \bar{z}$ e $y$, então

$$
\left\{\begin{array}{l}
\dot{z}=i \omega_{0} z+\frac{1}{2} G_{20} z^{2}+G_{11} z \bar{z}+\frac{1}{2} G_{02} \bar{z}^{2}+\frac{1}{2} G_{21} z^{2} \bar{z}+\left\langle G_{10}, y\right\rangle z+\left\langle G_{01}, y\right\rangle \bar{z}+\cdots \\
\dot{y}=A y+\frac{1}{2} H_{20} z^{2}+H_{11} z \bar{z}+\frac{1}{2} H_{02} \bar{z}^{2}+\cdots
\end{array}\right.
$$

onde $G_{20}, G_{11}, G_{02}, G_{21} \in \mathbb{C}, G_{10}, G_{01}, H_{i j} \in \mathbb{C}^{n}$ e podem ser calculados por

$$
\begin{gathered}
G_{i j}=\left.\frac{\partial^{i+j}}{\partial z^{i} \partial \bar{z}^{j}}\langle p, F(z q+\overline{z q})\rangle\right|_{z=0}, i+j \geq 2, \\
\bar{G}_{10, i}=\left.\frac{\partial^{2}}{\partial y^{i} \partial z}\langle p, F(z q+\overline{z q}+y)\rangle\right|_{z=0, y=0}, i=1, \ldots, n, \\
\bar{G}_{01, i}=\left.\frac{\partial^{2}}{\partial y^{i} \partial \bar{z}}\langle p, F(z q+\overline{z q}+y)\rangle\right|_{z=0, y=0}, i=1, \ldots, n, \\
H_{i j}=\left.\frac{\partial^{i+j}}{\partial z^{i} \partial \bar{z}^{j}} F(z q+\overline{z q})\right|_{z=0}-G_{i j} q-\bar{G}_{j i} \bar{q}, i+j=2
\end{gathered}
$$

$\mathrm{e}\langle G, y\rangle=\sum_{i=1}^{n} \bar{G}_{i} y_{i}$.

Com o objetivo de analisar o sistema (8.43), restringiremos o sistema a sua Variedade Central invariante tangente ao autoespaço generalizado $T^{c}$ e analisaremos a dinâmica do sistema para todos $\mu$ na vizinhança da origem. Vejamos a dependência da Variedade Central em relação ao parâmetro $\mu$.

Considere o sistema de equações diferenciais dependente de parâmetro na forma

$$
\dot{x}=f(x, \mu),
$$

onde $x \in \mathbb{R}^{n}, \mu \in \Omega \subset \mathbb{R}^{m}$ e $f: \mathbb{R}^{n} \rightarrow \mathbb{R}^{n}$ é um campo vetorial de classe $\mathcal{C}^{1}$. Seja $\left(x_{0}, \mu_{0}\right)$ um ponto de bifurcação do sistema (8.44). Consequentemente, a matriz jacobiana do sistema (8.44) tem $n_{0}$ autovalores sobre o eixo imaginário, com $n_{0} \geq 1$. Em $\mu=\mu_{0}$, o ponto de equilíbrio $x_{0}$ é um ponto de equilíbrio não hiperbólico e portanto, admite uma variedade central. Com o propósito de estudarmos o comportamento assintótico do sistema (8.44) numa vizinhança de $\mu=\mu_{0}$, visto que para valores de $\mu \neq 0$ o Teorema da Variedade Central não pode ser aplicado, consideremos o seguinte sistema

$$
\left\{\begin{array}{l}
\dot{x}=f(x, \mu) \\
\dot{\mu}=0
\end{array}\right.
$$


onde $(x, \mu) \in \mathbb{R}^{n+m}$. Observemos que o ponto $\left(x_{0}, \mu_{0}\right)$ é um ponto de equilíbrio não hiperbólico do novo sistema 8.45 e sua matriz jacobiana no ponto de equilíbrio é dado por

$$
A=\left(\begin{array}{cc}
A & \frac{\partial f}{\partial \lambda}\left(x_{0}, \mu_{0}\right) \\
0 & 0
\end{array}\right)
$$

com $n_{0}+m$ autovalores sobre o eixo imaginário. Utilizando uma mudança linear de variáveis, podemos reescrever o sistema 8.45 como

$$
\left\{\begin{array}{l}
\dot{u}=B u+m(u, v, \mu) \\
\dot{v}=C v+D \mu+n(u, v, \mu) \\
\dot{\mu}=0
\end{array}\right.
$$

onde $B$ é uma matriz de ordem $\left(n-n_{0}\right) \times\left(n-n_{0}\right)$ com autovalores com parte real não nula, $C$ é uma matriz de ordem $n_{0} \times n_{0}$ com autovalores com parte real nula e $D$ é uma matriz de ordem $\left(n_{0}+m\right) \times\left(n_{0}+m\right)$. Então pelo Teorema 2.2.18, existe uma variedade central local invariante $u=h(v, \mu)$ e sua dinâmica é dada pela equação diferencial $n_{0}$-dimensional reduzida a variedade central

$$
\dot{v}=C v+D \mu+g_{2}(h(v, \mu), v, \mu) .
$$

O Teorema 2.2.18 garante a existência de uma variedade central invariante $W^{c} \subset \mathbb{R} \times \mathbb{R}^{n}$ tangente ao autoespaço generalizado $T^{c}$ com $n_{0}+1$ autovalores com parte real nula e $\operatorname{dim} W^{c}=n_{0}+1$. Como $\dot{\mu}=0$, os hiperplanos $\sigma_{\mu_{0}}=\left\{(\mu, x): \mu=\mu_{0}\right\}$ são invariantes em relação ao sistema (8.45). Então concluímos que a variedade central é uma folheação de variedades invariantes $n_{0}$-dimensional $W_{\mu}^{c}=W^{c} \cap \sigma_{\mu}$, veja Figura 8.6.

A Figura 8.6 ilustra a dependência da variedade central do sistema (8.44) em função do parâmetro $\mu$.

Observe que $W_{\mu_{0}}^{c}$ é a variedade central do sistema (8.41) quando $\mu_{0}=0$ e pode ser localmente representada como um gráfico de uma função suave

$$
W_{0}^{c}(0)=\{(v, \bar{v}, u): u=V(v, \bar{v})\} .
$$

Como $V: T^{c} \rightarrow T^{s u}$ e devido a propriedade de tangência da variedade $W_{0}^{c}(0)$, temos $V=O\left(|v|^{2}\right)$.

Lema 8.3.2. [Kuz13] O sistema (8.43), restrito a variedade central $W_{\text {loc }}^{c}(0)$, pode ser escrito como

$$
\begin{aligned}
\dot{z}= & i \omega_{0} z+\frac{1}{2} G_{20} z^{2}+G_{11} z \bar{z}+\frac{1}{2} G_{02} \bar{z}^{2}+\frac{1}{2}\left(G_{21}-\right. \\
& \left.2\left\langle G_{10}, A^{-1} H_{11}\right\rangle+\left\langle G_{01},\left(2 i \omega_{0} I-A\right)^{-1} H_{20}\right\rangle\right) z^{2} \bar{z}+\cdots,
\end{aligned}
$$




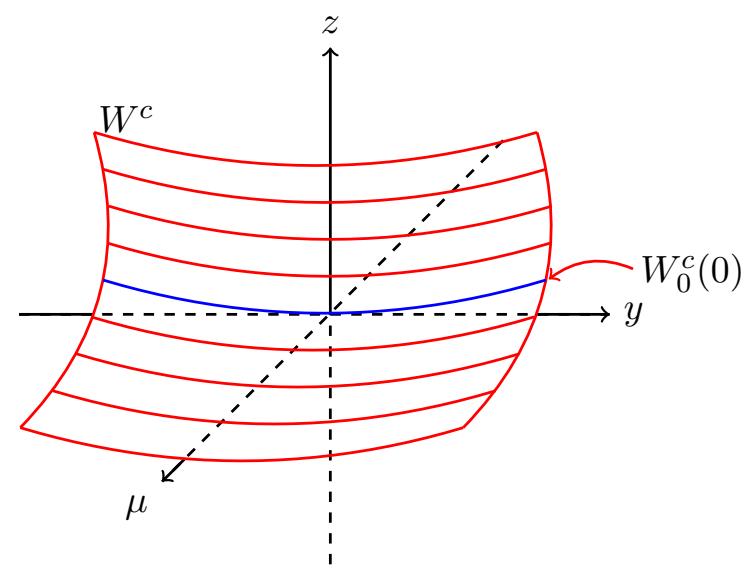

Figura 8.6: Variedade Central $W_{\mu}^{c}$ dependente do parâmetro $\mu$.

onde I é a matriz identidade.

Demonstração. Consideremos a seguinte representação para a variedade central $W_{l o c}^{c}(0)$ dada por

$$
y=V(z, \bar{z})=\frac{1}{2} w_{20} z^{2}+w_{11} z \bar{z}+\frac{1}{2} w_{02} \bar{z}^{2}+O\left(|z|^{3}\right),
$$

onde $\left\langle p, w_{i j}\right\rangle=0$. Derivando a equação (8.48), obtemos

$$
\dot{y}=w_{20} z \dot{z}+w_{11}(\bar{z} \dot{z}+z \dot{\bar{z}})+w_{02} \dot{z \bar{z}}+\cdots .
$$

Substituindo $\dot{z}$ e e seu conjugado da equação $\dot{z}$ em (8.43) na equação acima, temos

$$
\begin{aligned}
\dot{y}= & w_{20} z\left(i \omega_{0} z+\frac{1}{2} G_{20} z^{2}+\cdots\right)+w_{11}\left(i \omega_{0} z+\frac{1}{2} G_{20} z^{2}+\cdots\right) \bar{z}+ \\
& +w_{11}\left(-i \omega_{0} \bar{z}+\frac{1}{2} \bar{G}_{20} \bar{z}^{2}+\cdots\right) z+w_{02} \bar{z}\left(-i \omega_{0} \bar{z}+\frac{1}{2} \bar{G}_{20} z^{2}+\cdots\right)+\cdots \\
= & i \omega_{0} w_{20} z^{2}+i \omega_{0} w_{11} z \bar{z}-i \omega_{0} w_{11} z \bar{z}-i \omega_{0} w_{02} \bar{z}^{2}+\cdots \\
= & i \omega_{0} w_{20} z^{2}-i \omega_{0} w_{02} \bar{z}^{2}+\cdots
\end{aligned}
$$

Por outro lado, substituindo $y$ da equação (8.48) na segunda equação do sistema (8.43), obtemos

$$
\begin{aligned}
\dot{y} & =A\left(\frac{1}{2} w_{20} z^{2}+w_{11} z \bar{z}+\frac{1}{2} w_{02} \bar{z}^{2}+\cdots\right)+\frac{1}{2} H_{20} z^{2}+H_{11} z \bar{z}+\frac{1}{2} H_{02} \bar{z}^{2}+\cdots \\
& =\frac{1}{2}\left(A w_{20}+H_{20}\right) z^{2}+\left(A w_{11}+H_{11}\right) z \bar{z}+\frac{1}{2}\left(A w_{02}+H_{02}\right) \bar{z}^{2}+\cdots
\end{aligned}
$$

Comparando os coeficientes dos termos quadráticos destas duas últimas 
equações de $\dot{y}$, tem-se que

$$
\left\{\begin{array}{l}
H_{20}=\left(2 i \omega_{0} I-A\right) w_{20} \\
H_{11}=-A w_{11} \\
H_{02}=\left(-2 i \omega_{0} I-A\right) w_{02}
\end{array} .\right.
$$

As equações acima tem solução única visto que as matrizes $-A, 2 i \omega_{0} I-A$ e $-2 i \omega_{0} I-A$ são inversíveis, pois $0, \pm 2 i \omega_{0}$ não são autovalores da matriz $A$. Portanto, podemos explicitar os vetores $w_{i j} \in \mathbb{C}^{n}$ das equações acima,

$$
\left\{\begin{array}{l}
w_{20}=\left(2 i \omega_{0} I-A\right)^{-1} H_{20} \\
w_{11}=-A^{-1} H_{11} \\
w_{02}=\left(-2 i \omega_{0} I-A\right)^{-1} H_{02}
\end{array} .\right.
$$

Finalmente, vejamos o termo ressonante da primeira equação do sistema (8.43) restringindo-o à variedade central. Diante disso, observemos que

$$
\begin{aligned}
\langle G, y\rangle & =\left\langle G, \frac{1}{2} w_{20} z^{2}+w_{11} z \bar{z}+\frac{1}{2} w_{02} \bar{z}^{2}+\cdots\right\rangle \\
& =\frac{1}{2}\left\langle G, w_{20}\right\rangle z^{2}+\left\langle G, w_{11}\right\rangle z \bar{z}+\frac{1}{2}\left\langle G, w_{02}\right\rangle \bar{z}^{2}+\cdots
\end{aligned}
$$

Observemos que nas parcelas $\left\langle G_{10}, y\right\rangle z$ e $\left\langle G_{10}, y\right\rangle \bar{z}$ da equação de $\dot{z}$ em (8.43) aparecem o termo ressonante $z^{2} \bar{z}$ cujos coeficientes são

$$
\begin{aligned}
\left\langle G_{10}, y\right\rangle z= & \frac{1}{2}\left\langle G_{10}, w_{20}\right\rangle z^{3}+\left\langle G_{10}, w_{11}\right\rangle z^{2} \bar{z}+\frac{1}{2}\left\langle G_{10}, w_{02}\right\rangle z \bar{z}^{2}+\cdots \\
= & \frac{1}{2}\left\langle G_{10},\left(2 i \omega_{0} I-A\right)^{-1} H_{20}\right\rangle z^{3}+\left\langle G_{10},-A^{-1} H_{11}\right\rangle z^{2} \bar{z}+ \\
& +\frac{1}{2}\left\langle G_{10},\left(-2 i \omega_{0} I-A\right)^{-1} H_{02}\right\rangle z \bar{z}^{2}+\cdots \\
= & \frac{1}{2}\left\langle G_{10},\left(2 i \omega_{0} I-A\right)^{-1} H_{20}\right\rangle z^{3}-\left\langle G_{10}, A^{-1} H_{11}\right\rangle z^{2} \bar{z}+ \\
& +\frac{1}{2}\left\langle G_{10},\left(-2 i \omega_{0} I-A\right)^{-1} H_{02}\right\rangle z \bar{z}^{2}+\cdots
\end{aligned}
$$

$\mathrm{e}$

$$
\begin{aligned}
\left\langle G_{01}, y\right\rangle \bar{z}= & \frac{1}{2}\left\langle G_{01}, w_{20}\right\rangle z^{2} \bar{z}+\left\langle G_{01}, w_{11}\right\rangle z \bar{z}^{2}+\frac{1}{2}\left\langle G_{01}, w_{02}\right\rangle \bar{z}^{3}+\cdots \\
= & \frac{1}{2}\left\langle G_{01},\left(2 i \omega_{0} I-A\right)^{-1} H_{20}\right\rangle z^{2} \bar{z}+\left\langle G_{01},-A^{-1} H_{11}\right\rangle z \bar{z}^{2}+ \\
& +\frac{1}{2}\left\langle G_{01},\left(-2 i \omega_{0} I-A\right)^{-1} H_{02}\right\rangle \bar{z}^{3}+\cdots
\end{aligned}
$$


Consequentemente,

$$
\begin{aligned}
\dot{z}= & i \omega_{0} z+\frac{1}{2} G_{20} z^{2}+G_{11} z \bar{z}+\frac{1}{2} G_{02} \bar{z}^{2}+\frac{1}{2} G_{21} z^{2} \bar{z}+\left\langle G_{10}, y\right\rangle z+\left\langle G_{01}, y\right\rangle \bar{z}+\cdots \\
= & i \omega_{0} z+\frac{1}{2} G_{20} z^{2}+G_{11} z \bar{z}+\frac{1}{2} G_{02} \bar{z}^{2}+\frac{1}{2} G_{21} z^{2} \bar{z}-\left\langle G_{10}, A^{-1} H_{11}\right\rangle z^{2} \bar{z}+ \\
& +\frac{1}{2}\left\langle G_{01},\left(2 i \omega_{0} I-A\right)^{-1} H_{20}\right\rangle z^{2} \bar{z}+\cdots \\
= & i \omega_{0} z+\frac{1}{2} G_{20} z^{2}+G_{11} z \bar{z}+\frac{1}{2} G_{02} \bar{z}^{2}+\frac{1}{2}\left(G_{21}-2\left\langle G_{10}, A^{-1} H_{11}\right\rangle+\right. \\
& \left.+\left\langle G_{01},\left(2 i \omega_{0} I-A\right)^{-1} H_{20}\right\rangle\right) z^{2} \bar{z}+\cdots .
\end{aligned}
$$

Lema 8.3.3 (Condição de Não Degenerescência). [Kuz13] O Primeiro Coeficiente de Lyapunov para o sistema (8.40) é dado pela fórmula

$$
\begin{aligned}
l_{1}(0)= & \frac{1}{2 \omega_{0}} \operatorname{Re}\left[\langle p, C(q, q, \bar{q})\rangle-2\left\langle p, B\left(q, A^{-1} B(q, q)\right)\right\rangle+\right. \\
& \left.+\left\langle p, B\left(\bar{q},\left(2 i \omega_{0} I-A\right)^{-1} B(q, q)\right)\right\rangle\right]
\end{aligned}
$$

Demonstração. A expansão de Taylor da função $F(x)$ do sistema (8.40) é dada pela seguinte fórmula

$$
F(x)=\frac{1}{2} B(x, x)+\frac{1}{6} C(x, x, x)+O\left(\|x\|^{4}\right),
$$

onde $B(x, y)$ e $C(x, y, z)$ são funções multilineares simétricas de $x, y, z \in \mathbb{R}^{n}$,

$$
B_{i}(x, y)=\left.\sum_{j, k=1}^{n} \frac{\partial^{2} F_{i}(\xi, 0)}{\partial \xi_{j} \partial \xi_{k}}\right|_{\xi=0} x_{j} y_{k}
$$

e

$$
C_{i}(x, y, z)=\left.\sum_{j, k, l=1}^{n} \frac{\partial^{3} F_{i}(\xi, 0)}{\partial \xi_{j} \partial \xi_{k} \partial \xi_{l}}\right|_{\xi=0} x_{j} y_{k} z_{l},
$$

$i=1,2, \ldots, n$. Dado $x=z q+\overline{z q}+y \in \mathbb{R}^{n}, \operatorname{com} z q+\overline{z q} \in T^{c}$ e $y \in T^{s u}$, então

$$
\begin{aligned}
B(z q+\overline{z q}+y, z q+\overline{z q}+y)= & z^{2} B(q, q)+2 z \bar{z} B(q, \bar{q})+\bar{z}^{2} B(\bar{q}, \bar{q})+2 z B(q, y)+ \\
& 2 \bar{z} B(\bar{q}, y)+B(y, y)
\end{aligned}
$$

Segue-se daí, que os coeficentes dos termos quadráticos do sistema (8.43) 
podem ser expressos como

$$
\left\{\begin{array}{l}
\left\langle G_{10}, y\right\rangle=\langle p, B(q, y)\rangle,\left\langle G_{01}, y\right\rangle=\langle p, B(\bar{q}, y)\rangle \\
G_{20}=\langle p, B(q, q)\rangle, G_{11}=\langle p, B(q, \bar{q})\rangle, G_{02}=\langle p, B(\bar{q}, \bar{q})\rangle .
\end{array}\right.
$$

$$
\left\{\begin{array}{l}
H_{20}=B(q, q)-\langle p, B(q, q)\rangle q-\langle\bar{p}, B(q, q)\rangle \bar{q} \\
H_{11}=B(q, \bar{q})-\langle p, B(q, \bar{q})\rangle q-\langle\bar{p}, B(q, \bar{q})\rangle \bar{q} \\
H_{02}=B(\bar{q}, \bar{q})-\langle p, B(\bar{q}, \bar{q})\rangle q-\langle\bar{p}, B(\bar{q}, \bar{q})\rangle \bar{q}
\end{array}\right.
$$

Analogamente, o coeficente do termo cúbico ressonante do sistema (8.43) pode ser expresso como

$$
G_{21}=\langle p, C(q, q, \bar{q})\rangle
$$

Substituindo (8.50), (8.51) e (8.52) na equação restrita à variedade central (8.47) e, tendo em conta as seguintes identidades

$$
\begin{gathered}
A^{-1} q=\frac{1}{i \omega_{0}} q,\left(2 i \omega_{0} I-A\right)^{-1} q=\frac{1}{i \omega_{0}} q, \\
A^{-1} \bar{q}=-\frac{1}{i \omega_{0}} \bar{q},\left(2 i \omega_{0} I-A\right)^{-1} \bar{q}=\frac{1}{3 i \omega_{0}} \bar{q}
\end{gathered}
$$

obtemos a seguinte equação

$$
\dot{z}=i \omega_{0} z+\frac{1}{2} g_{20} z^{2}+g_{11} z \bar{z}+\frac{1}{2} g_{02} \bar{z}^{2}+\frac{1}{2} g_{21} z^{2} \bar{z}+\ldots,
$$

onde

$$
\begin{aligned}
g_{20}= & \langle p, B(q, q)\rangle, g_{11}=\langle p, B(q, \bar{q})\rangle, g_{02}=\langle p, B(\bar{q}, \bar{q})\rangle, \\
g_{21}= & \langle p, C(q, q, \bar{q})\rangle-2\left\langle p, B\left(q, A^{-1} B(q, \bar{q})\right)\right\rangle+ \\
& \left\langle p, B\left(\bar{q},\left(2 i \omega_{0} I-A\right)^{-1} B(q, q)\right)\right\rangle+\frac{1}{i \omega_{0}}\langle p, B(q, q)\rangle\langle p, B(q, \bar{q})\rangle \\
& -\frac{2}{i \omega_{0}}|\langle p, B(q, \bar{q})\rangle|^{2}-\frac{1}{3 i \omega_{0}}|\langle p, B(\bar{q}, \bar{q})\rangle|^{2} .
\end{aligned}
$$

Sendo assim, a aplicação da fórmula do primeiro coeficiente de Lyapunov para o sistema bidimensional

$$
l_{1}(0)=\frac{1}{2 \omega_{0}^{2}} \Re\left(i g_{20} g_{11}+\omega_{0} g_{21}\right)
$$

fornece a seguinte expressão invariante para o primeiro coeficiente de Lya- 
punov para um sistema $n$-dimensional

$$
\begin{aligned}
l_{1}(0)= & \frac{1}{2 \omega_{0}} \operatorname{Re}\left[\langle p, C(q, q, \bar{q})\rangle-2\left\langle p, B\left(q, A^{-1} B(q, q)\right)\right\rangle+\right. \\
& \left.\left\langle p, B\left(\bar{q},\left(2 i \omega_{0} I-A\right)^{-1} B(q, q)\right)\right\rangle\right] .
\end{aligned}
$$

O próximo resultado estabelece condição suficiente para que o sistema restrito à variedade central satisfaça a condição de transversalidade de Hopf.

Lema 8.3.4 (Condição de Transversalidade de Hopf). [Kuz13] Consideremos o sistema (8.40), com matriz jacobiana $A(\mu)$ dependendo suavemente do parâmetro $\mu \in \mathbb{R}$ e além disso, com um par de autovalores complexos $\lambda_{1,2}=\sigma(\mu) \pm i \omega(\mu), \sigma(0)=0, \omega(0)>0$. Então

$$
\sigma^{\prime}(0)=\operatorname{Re}\left\langle p, A^{\prime}(0) q\right\rangle
$$

onde $p, q \in \mathbb{C}^{n}$ satisfazem

$$
A(0) q=i \omega_{0} q, A^{T}(0) p=-i \omega_{0} p,\langle p, q\rangle=1 .
$$

Demonstração. Derivando ambos os membros da equação $A(\mu) q(\mu)=\lambda(\mu) q(\mu)$ com relação a $\mu$, visto que $A$ depende suavemente de $\mu$, obtemos

$$
A^{\prime}(\mu) q(\mu)+A(\mu) q^{\prime}(\mu)=\lambda^{\prime}(\mu) q(\mu)+\lambda(\mu) q^{\prime}(\mu) .
$$

Aplicando o produto escalar por $p$ em ambos os membros da equação acima, temos

$$
\begin{aligned}
\left\langle p, A^{\prime}(\mu) q(\mu)+A(\mu) q^{\prime}(\mu)\right\rangle & =\left\langle p, \lambda^{\prime}(\mu) q(\mu)+\lambda(\mu) q^{\prime}(\mu)\right\rangle \\
\left\langle p, A^{\prime}(\mu) q(\mu)\right\rangle+\left\langle p, A(\mu) q^{\prime}(\mu)\right\rangle & =\left\langle p, \lambda^{\prime}(\mu) q(\mu)\right\rangle+\left\langle p, \lambda(\mu) q^{\prime}(\mu)\right\rangle \\
\left\langle p, A^{\prime}(\mu) q(\mu)\right\rangle+\left\langle A^{T}(\mu) p, q^{\prime}(\mu)\right\rangle & =\lambda^{\prime}(\mu)\langle p, q(\mu)\rangle+\lambda(\mu)\left\langle p, q^{\prime}(\mu)\right\rangle .
\end{aligned}
$$

Para $\mu=0, A^{T}(0) p=-i \omega_{0} p$, então

$$
\begin{aligned}
\left\langle p, A^{\prime}(0) q\right\rangle+\left\langle-i \omega_{0} p, q^{\prime}(0)\right\rangle & =\lambda^{\prime}(0)\langle p, q\rangle+\lambda(0)\left\langle p, q^{\prime}(0)\right\rangle \\
\left\langle p, A^{\prime}(0) q\right\rangle+i \omega_{0}\left\langle p, q^{\prime}(0)\right\rangle & =\lambda^{\prime}(0)+i \omega_{0}\left\langle p, q^{\prime}(0)\right\rangle \\
\left\langle p, A^{\prime}(0) q\right\rangle & =\lambda^{\prime}(0)
\end{aligned}
$$

pois $\langle p, q\rangle=1$. Logo,

$$
\lambda^{\prime}(0)=\left\langle p, A^{\prime}(0) q\right\rangle
$$

e, portanto,

$$
\sigma^{\prime}(0)=\operatorname{Re}\left\langle p, A^{\prime}(0) q\right\rangle
$$


Diante do que foi exposto, apresentaremos o Teorema da Bifurcação de Hopf para o caso $n$-dimensional. E a sua demonstração segue dos resultados discutidos nesta seção e da seção anterior.

Teorema 8.3.5 (Teorema da Bifurcação de Hopf). [Kuz13, Per13] Considere o sistema

$$
\dot{x}=f(x, \mu), x \in \mathbb{R}^{n}, \mu \in \mathbb{R},
$$

com $f$ suave, tendo para todo $\left|\mu-\mu_{0}\right|$ suficientemente pequeno, o equilíbrio $x_{\mu_{0}}$ com autovalores complexos

$$
\lambda_{1,2}(\mu)=\sigma(\mu) \pm i \omega(\mu)
$$

onde $\gamma\left(\mu_{0}\right)=0, \omega\left(\mu_{0}\right)=\omega>0$, e os demais autovalores com partes reais diferentes de zero. Se as seguintes condições são satisfeitas

(a) $l_{1}\left(\mu_{0}\right) \neq 0$ (Condição de não degenerescência)

(b) $\sigma^{\prime}\left(\mu_{0}\right) \neq 0$ (Condição de Transversalidade).

Então, introduzindo uma variável complexa, aplicada a transformações de coordenadas suaves e inversiveis que dependem suavemente do parâmetro $\mu$, o sistema pode ser reduzido à sequinte forma complexa restrita à variedade central,

$$
\dot{z}=(\beta+i) z+s z|z|^{2}+O\left(|z|^{4}\right)
$$

com $s=\operatorname{sinal}\left(l_{1}\left(\mu_{0}\right)\right)=\operatorname{sinal}\left(\Re\left(c_{1}\left(\mu_{0}\right)\right)\right)$. Além disso, existe uma vizinhança $U$ de $x_{\mu_{0}}$ e $\epsilon>0$ tal que:

(i) se $l_{1}\left(\mu_{0}\right)<0$, então existe um ponto de equilíbrio hiperbólico $x_{\mu}^{H}$ do tipo- $k$, com $1 \leq k \leq n-2$, em $U$ para todo $\mu \in\left(\mu_{0}-\epsilon, \mu_{0}\right)$ e uma órbita fechada hiperbólica $\Omega_{\mu}^{H}$ do tipo- $k$, com $1 \leq k \leq n-2$, e um ponto de equilíbrio hiperbólico $x_{\mu}^{H}$ do tipo- $k+2$, com $1 \leq k \leq n-2$, em $U$ para todo $\mu \in\left(\mu_{0}, \mu_{0}+\epsilon\right)$. Além disso, a variedade instável do ponto de equilíbrio hiperbólico $x_{\mu}^{H}$ do tipo- $(k+2)$ intercepta a variedade estável da órbita periódica hiperbólica $\Omega_{\mu}^{H}$ do tipo-k ao longo de uma variedade bidimensional.

(ii) se $l_{1}\left(\mu_{0}\right)>0$, então existe uma órbita fechada hiperbólica $\Omega_{\mu}^{H}$ do tipo$(k+1)$, com $1 \leq k \leq n-2$, e um ponto de equilíbrio hiperbólico $x_{\mu}^{H}$ do tipo- $k$, com $1 \leq k \leq n-2$, em $U$ para todo $\mu \in\left(\mu_{0}-\epsilon, \mu_{0}\right)$ e um ponto de equilíbrio hiperbólico $x_{\mu}^{H}$ do tipo- $(k+2)$, com $1 \leq k \leq n-2$, em $U$ para todo $\mu \in\left(\mu_{0}, \mu_{0}+\epsilon\right)$. Além disso, a variedade estável do ponto de equilíbrio hiperbólico $x_{\mu}^{H}$ do tipo-k intercepta a variedade instável da órbita periódica hiperbólica $\Omega_{\mu}^{H}$ do tipo- $(k+1)$ ao longo de uma variedade bidimensional para todo $\mu \in\left(\mu_{0}-\epsilon, \mu_{0}\right)$. 


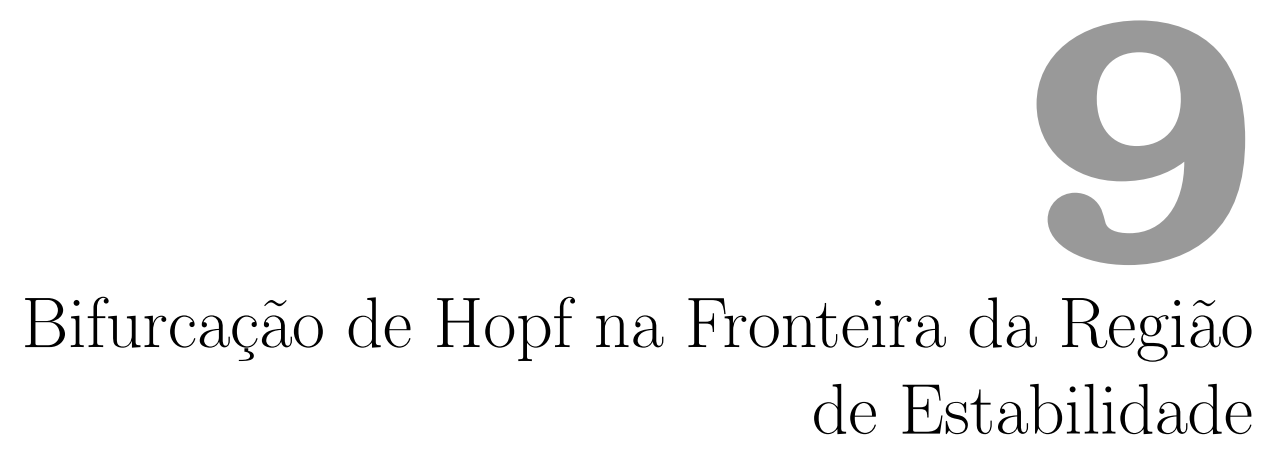

No capítulo 5 caracterizamos a fronteira da região de estabilidade de um ponto de equilíbrio assintoticamente estável na presença de equilibrios não hiperbólicos do tipo Hopf. No capítulo seguinte, para acomodarmos órbitas periódicas na fronteira da região de estabilidade, impusemos uma condição sobre a transversalidade das variedades e caracterizamos a fronteira da região de estabilidade admitindo a existência de elementos críticos e pontos de equilíbrio Hopf. No capítulo 7 fizemos uma caracterização completa da fronteira da região de estabilidade fraca de um ponto de equilíbrio não hiperbólico Hopf Supercrítico do tipo-zero admitindo apenas a existência de elementos críticos na fronteira. Neste capítulo, inicialmente, exploraremos o comportamento da fronteira da região de estabilidade a variações de parâmetros na vizinhança de um ponto de equilíbrio Hopf do tipo- $k$ com $k \geq 1 \mathrm{e}$ exibiremos uma caracterização global da fronteira da região de estabilidade próximo a um parâmetro de bifurcação Hopf do tipo- $k$ com $k \geq 1$. Posteriormente, exploraremos também o comportamento da fronteira da região de estabilidade de um ponto de equilíbrio Hopf Supercrítico do tipo-zero a variações de parâmetros e exibiremos uma caracterização global da fronteira da região de estabilidade próximo ao parâmetro de bifurcação Hopf supercrítico do tipo-zero. Também veremos que o ponto de equilíbrio instável resultante da bifurcação de Hopf supercrítica do tipo-zero pertence à fronteira de quase-estabilidade da órbita fechada estável. Finalmente, apresentaremos alguns exemplos que ilustram os resultados discutidos nas seções anteriores. 


\subsection{Bifurcação de Hopf na Fronteira da Região de Estabilidade}

Com o objetivo de estudarmos o comportamento da fronteira da região de estabilidade na presença de um ponto de equilíbrio Hopf do tipo- $k$ com $k \geq 1$ e obtermos a caracterização da sua fronteira na vizinhança do ponto de equilíbrio Hopf, vejamos o próximo resultado que garante a persistência dos pontos de equilíbrio hiperbólicos na fronteira da região de estabilidade perturbada ao variarmos o parâmetro na vizinhança de $\bar{\mu}$.

Teorema 9.1.1. [CC95][Persistência dos pontos de equilíbrio hiperbólicos na fronteira da região de estabilidade] Seja $x_{\bar{\mu}}$ um ponto de equilíbrio hiperbólico assintoticamente estável de (8.3), para $\mu=\bar{\mu}$ e $A_{\bar{\mu}}\left(x_{\bar{\mu}}^{s}\right)$ sua região de estabilidade. Sejam $x_{\bar{\mu}}^{i}, i=1,2, \ldots, k$ os pontos de equilibrio hiperbólicos em $\partial A_{\bar{\mu}}\left(x_{\bar{\mu}}^{s}\right)$ e admita que as suposições $(\boldsymbol{A} 1)$, (A2) e (A3) sejam satisfeitas para $\mu=\bar{\mu}$. Então, existe um $\varepsilon>0$ tal que, os pontos de equilíbrio perturbados $x_{\mu}^{i}, i=1,2, \ldots, k$ também pertencem à fronteira da região de estabilidade perturbada de $\partial A_{\bar{\mu}}\left(x_{\bar{\mu}}^{s}\right)$ para todo $\mu \in(\bar{\mu}-\varepsilon, \bar{\mu}+\varepsilon)$.

O Teorema 9.1.1, sob as hipóteses (A1), (A2) e (A3), garante a persistência dos equilíbrios hiperbólicos na fronteira da região de estabilidade ao perturbarmos o sistema (8.3). Para estudarmos o comportamento da fronteira da região de estabilidade na presença de um ponto de equilíbrio Hopf, relaxaremos a hipótese (A1), ou seja, a hipótese de que todos os pontos de equilíbrio na fronteira são hiperbólicos. O Teorema 9.1.3 garante a persistência dos elementos críticos na fronteira admitindo as hipóteses (B1'), (B2') e (B3) (ou (B1"), (B2") e (B3)) porém vejamos um lema auxiliar que nos será útil para a demonstração do teorema.

Lema 9.1.2. [Shu13] Sejam $x_{\mu_{\star}}$ um elemento crítico e $y_{\mu_{\star}}$ um ponto de equilibrio do sistema (8.3) para $\mu=\mu_{\star}$. Se $W_{\mu_{\star}}^{u}\left(x_{\mu_{\star}}\right) \cap W_{\mu_{\star}}^{s}\left(y_{\mu_{\star}}\right) \neq \emptyset$, e $W_{\mu_{\star}}^{u}\left(x_{\mu_{\star}}\right)$ e $W_{\mu_{\star}}^{s}\left(y_{\mu_{\star}}\right) \neq \emptyset$ satisfazem a condição de transversalidade, então existe $\varepsilon$ tal que $W_{\mu}^{u}\left(x_{\mu}\right) \cap W_{\mu}^{s}\left(y_{\mu}\right) \neq \emptyset$ para todo $\mu \in\left(\mu_{\star}-\varepsilon, \mu_{\star}+\varepsilon\right)$ onde $x_{\mu}$ é o elemento crítico perturbado e $y_{\mu}$ é o ponto de equilíbrio perturbado.

O Lema 9.1.2 afirma que a condição de interseção das variedades dos elementos críticos ser não vazia persiste se a interseção é transversal.

Teorema 9.1.3. (Persistência de Elementos Críticos Hiperbólicos na fronteira da região de estabilidade) Seja $x_{\mu_{0}}$ um ponto de equilíbrio Hopf na fronteira da região de estabilidade $\partial A_{\mu_{0}}\left(x_{\mu_{0}}^{s}\right)$ de um ponto de equilíbrio hiperbólico assintoticamente estável $x_{\mu_{0}}^{s}$ de (8.3) para $\mu=\mu_{0}$. Admita que as suposições (B1'), (B2')(ou (B1"), (B2")) e (B3) sejam satisfeitas para $\mu=\mu_{0}$. Se $r_{\mu_{0}}^{i}, i=1, \ldots, k$ são os elementos críticos hiperbólicos em $\partial A_{\mu_{0}}\left(x_{\mu_{0}}^{s}\right)$, então existe $\epsilon>0$ tal que o elemento crítico perturbado $r_{\mu}^{i} \in \partial A_{\mu}\left(x_{\mu}^{s}\right)$, para todo $i=1, \ldots, k$ e $\mu \in\left(\mu_{0}-\epsilon, \mu_{0}+\epsilon\right)$. 
Demonstração. O Teorema 2.1.7 garante a persistência dos pontos de equilíbrio hiperbólicos $x_{\mu_{0}}^{i}, i=1, \ldots, k$, e do ponto de equilíbrio hiperbólico assintoticamente estável $x_{\mu_{0}}^{s}$, sob pequenas variações do parâmetro $\mu$. Seja $\phi_{\mu_{0}}^{i}$ uma órbita periódica hiperbólica do sistema. Seja $P$ a aplicação de Poincaré para $\phi$. Pelo Teorema 2.2.13 temos que $P \in C(U)$, onde $U=B\left(x_{0} ; \delta\right) \cap \Sigma$, $\Sigma$ é o hiperplano ortogonal a $\phi_{\mu_{0}}^{i}$ em $x_{0} \in \phi_{\mu_{0}}^{i}$ e $x_{0}$ é um ponto fixo hiperbólico de $P$. Seja $g(x, \mu)=P_{\mu}(x)-x_{0}$. Observe que $g\left(x_{0}, \mu_{0}\right)=0$ e $D_{x} g\left(x_{0}, \mu_{0}\right)=D P_{\mu_{0}}\left(x_{0}\right)-I$ é invertível, pois $\phi_{\mu_{0}}^{i}$ é uma órbita hiperbólica e portanto, os autovalores de $D P_{\mu_{0}}\left(x_{0}\right)$ tem módulo diferente de 1 . Então pelo Teorema 2.1.7, existe uma única solução $x_{\mu}^{i}$ da equação $g(x, \mu)=0$ para todo $\mu$ próximo de $\mu_{0}$. Como $x_{\mu}^{i}$ é ponto fixo hiperbólico de $P$, então existe uma órbita periódica perturbada $\phi_{\mu}^{i}$ passando por $x_{\mu}^{i}$ e, consequentemente, $\phi_{\mu}^{i}$ é hiperbólica. Então existem vizinhanças $N_{i}$ e $U$ de $r_{\mu_{0}}^{i}$ e $x_{\mu_{0}}^{s}$, respectivamente, e $\varepsilon_{1}>0$ tal que existe um único elemento crítico hiperbólico $r_{\mu}^{i} \in N_{i}$ e um único ponto de equilíbrio hiperbólico assintoticamente estável $x_{\mu}^{s} \in U$ para todo $i=1, \ldots, k$ e $\mu \in\left(\mu_{0}-\varepsilon_{1}, \mu_{0}+\varepsilon_{1}\right)$. Como $r_{\mu_{0}}^{i}$ está em $\partial A_{\mu_{0}}\left(x_{\mu_{0}}^{s}\right)$ e (B1'), (B2') (ou (B1"), (B2")) e (B3) são satisfeitas para $\mu=\mu_{0}$, o Teorema 6.3 .5 garante que $W_{\mu_{0}}^{u}\left(r_{\mu_{0}}^{i}\right) \cap A_{\mu_{0}}\left(x_{\mu_{0}}^{s}\right) \neq \emptyset$ para todo $i=1, \ldots, k$. Além disto, esta interseção é transversal. Por outro lado, como $A_{\mu_{0}}\left(x_{\mu_{0}}^{s}\right)=W_{\mu_{0}}^{s}\left(x_{\mu_{0}}^{s}\right)$, temos que $W_{\mu_{0}}^{u}\left(r_{\mu_{0}}^{i}\right) \cap W_{\mu_{0}}^{s}\left(x_{\mu_{0}}^{s}\right) \neq \emptyset$ para todo $i=1, \ldots, k$. Logo, pelo Lema 9.1.2 podemos afirmar que existe $\varepsilon_{2}>0$ tal que $W_{\mu}^{u}\left(r_{\mu}^{i}\right) \cap W_{\mu}^{s}\left(x_{\mu}^{s}\right) \neq \emptyset$ para todo $i=1, \ldots, k$ e $\mu \in\left(\mu_{0}-\varepsilon_{2}, \mu_{0}+\varepsilon_{2}\right)$, ou seja, $W_{\mu}^{u}\left(r_{\mu}^{i}\right) \cap A_{\mu}\left(x_{\mu}^{s}\right) \neq \emptyset$. Portanto, o Teorema 6.3.5 garante que $r_{\mu}^{i} \in \partial A_{\mu}\left(x_{\mu}^{s}\right)$ para todo $i=1, \ldots, k$ e $\mu \in\left(\mu_{0}-\varepsilon, \mu_{0}+\varepsilon\right) \operatorname{com} \varepsilon=\min \left\{\varepsilon_{1}, \varepsilon_{2}\right\}$.

Nos teoremas a seguir exploraremos o comportamento da fronteira da região de estabilidade do ponto de equilíbrio assintoticamente estável numa vizinhança pequena do parâmetro $\mu$ próxima ao parâmetro $\mu_{0}$ de bifurcação Hopf subcrítico do tipo- $k$, com $k \geq 1$. Admitiremos como hipótese, para o valor do parâmetro de bifurcação Hopf subcrítico $\mu_{0}$, apenas a existência de elementos críticos hiperbólicos do sistema (8.3) em $\mu=\mu_{0}$, com exceção do ponto de equilíbrio não hiperbólico Hopf subcrítico do tipo- $k$, com $k \geq 1$, $x_{\mu_{0}}$. Além disso, numa vizinhança pequena do parâmetro $\mu_{0}$, também admitiremos apenas a existência de elementos críticos que são os elementos críticos perturbados do sistema original (8.3) em $\mu=\mu_{0}$. Inicialmente, estabeleceremos o comportamento da fronteira da região de estabilidade na vizinhança de um ponto de equilíbrio Hopf Subcrítico do tipo- $k$ com $k \geq 1$ e, em seguida, apresentaremos uma caracterização global da fronteira nessa vizinhança.

Teorema 9.1.4. (Comportamento da fronteira da região de estabilidade na vizinhança de um ponto de equilíbrio Hopf Subcrítico do tipo- $k$ com $k \geq 1)$ Seja $\left(\mu_{0}, x_{\mu_{0}}\right)$ um ponto de bifurcação Hopf subcrítico do tipo- $k, k \geq 1$, de (8.3) para $\mu=\mu_{0}$. Suponha que o ponto de equilíbrio Hopf subcrítico do tipo- $k x_{\mu_{0}}$ pertença à fronteira da região de estabilidade 
$\partial A_{\mu_{0}}\left(x_{\mu_{0}}^{s}\right)$ de um ponto de equilíbrio hiperbólico assintoticamente estável $x_{\mu_{0}}^{s}$ de (8.3) para $\mu=\mu_{0}$. Admita que as suposições (B1), (B2) e (B3) sejam satisfeitas para todo $\mu$ pertencente a um intervalo aberto $I$ contendo $\mu=\mu_{0}$, exceto em $\mu_{0}$ onde as condições (B1") e (B2") são satisfeitas. Além disso, assuma que $x_{\mu_{0}}$ seja o único ponto de equilíbrio não hiperbólico em $\mu=\mu_{0}$. Suponha também, que para todo $\mu \in I$, todos os elementos críticos do sistema perturbado $\dot{x}=f(x, \mu)$ são elementos críticos perturbados originados do sistema $\dot{x}=f\left(x, \mu_{0}\right)$. Então existe uma vizinhança $U$ de $x_{\mu_{0}}$ e $\epsilon_{1} \geq \epsilon>0$ tal que:

(i) Existe uma órbita fechada hiperbólica $\Omega_{\mu}^{H}$ do tipo- $(k+1)$, com $1 \leq k \leq$ $n-2$, e um ponto de equilíbrio hiperbólico $x_{\mu}^{H}$ do tipo- $k$, com $1 \leq k \leq$ $n-2$, em $U$ para todo $\mu \in\left(\mu_{0}-\epsilon_{1}, \mu_{0}\right)$ e um ponto de equilibrio hiperbólico $x_{\mu}^{H}$ do tipo- $(k+2)$, com $1 \leq k \leq n-2$, em $U$ para todo $\mu \in\left(\mu_{0}, \mu_{0}+\epsilon_{1}\right)$.

(ii) Para $\mu \in\left(\mu_{0}-\epsilon, \mu_{0}\right)$ temos que

$$
\Omega_{\mu}^{H} \in \partial A_{\mu}\left(x_{\mu}^{s}\right) \quad e \quad x_{\mu}^{H} \in \partial A_{\mu}\left(x_{\mu}^{s}\right) .
$$

(iii) Para $\mu \in\left(\mu_{0}, \mu_{0}+\epsilon\right)$ temos que

$$
x_{\mu}^{H} \in \partial A_{\mu}\left(x_{\mu}^{s}\right) .
$$

Demonstração. (i) A existência da vizinhança $U$, do escalar $\epsilon_{1}$ e dos elementos críticos segue do Teorema 8.3.5.

(ii) Mostraremos a existência de $\epsilon>0$ tal que $\Omega_{\mu}^{H} \in \partial A_{\mu}\left(x_{\mu}^{s}\right)$ e $x_{\mu}^{H} \in$ $\partial A_{\mu}\left(x_{\mu}^{s}\right)$ para todo $\mu \in\left(\mu_{0}-\epsilon, \mu_{0}\right)$. Como $x_{\mu_{0}} \in \partial A_{\mu_{0}}\left(x_{\mu_{0}}^{s}\right)$ e as suposições (B1"), (B2") e (B3) são satisfeitas para $\mu=\mu_{0}$, então de acordo com o Teorema 6.3.5 $W_{\mu_{0}}^{c u}\left(x_{\mu_{0}}\right) \cap A_{\mu_{0}}\left(x_{\mu_{0}}^{s}\right) \neq \emptyset$ e esta interseção é transversal. Como $A_{\mu_{0}}\left(x_{\mu_{0}}^{s}\right)=W_{\mu_{0}}^{s}\left(x_{\mu_{0}}^{s}\right)$, temos que $W_{\mu_{0}}^{c u}\left(x_{\mu_{0}}\right) \cap W_{\mu_{0}}^{s}\left(x_{\mu_{0}}^{s}\right) \neq$ $\emptyset$. Como $W_{\mu}^{u}\left(x_{\mu}^{H}\right)$ e $W_{\mu}^{s}\left(x_{\mu}^{s}\right)$ dependem continuamente de $\mu$, ver [Sot73], então podemos afirmar que existe $\epsilon_{2}>0$ tal que $W_{\mu}^{u}\left(x_{\mu}^{H}\right) \cap W_{\mu}^{s}\left(x_{\mu}^{s}\right) \neq \emptyset$ para todo $\mu \in\left(\mu_{0}-\epsilon_{2}, \mu_{0}\right)$, ou seja, $W_{\mu}^{u}\left(x_{\mu}^{H}\right) \cap A_{\mu}\left(x_{\mu}^{s}\right) \neq \emptyset$ para todo $\mu \in\left(\mu_{0}-\epsilon_{2}, \mu_{0}\right)$. Logo, pelo Teorema 6.3.5, $x_{\mu}^{H} \in \partial A_{\mu}\left(x_{\mu}^{s}\right)$ para todo $\mu \in\left(\mu_{0}-\epsilon, \mu_{0}\right)$, onde $\epsilon=\min \left\{\epsilon_{1}, \epsilon_{2}\right\}$. Pelo Teorema 8.3.5, a variedade estável do ponto de equilíbrio $x_{\mu}^{H}$ hiperbólico do tipo- $k$ intercepta a variedade instável da órbita periódica $\Omega_{\mu}^{H}$ hiperbólica do tipo- $(k+1)$ ao longo de uma variedade bidimensional, ou seja, $W_{\mu}^{s}\left(x_{\mu}^{H}\right) \cap W_{\mu}^{u}\left(\Omega_{\mu}^{H}\right) \neq \emptyset$. Como $x_{\mu}^{H} \in \partial A_{\mu}\left(x_{\mu}^{s}\right)$ para todo $\mu \in\left(\mu_{0}-\epsilon, \mu_{0}\right)$, então pelo Teorema 6.3.5 $W_{\mu}^{s}\left(x_{\mu}^{H}\right) \subset \partial A_{\mu}\left(x_{\mu}^{s}\right)$ e portanto, $W_{\mu}^{u}\left(\Omega_{\mu}^{H}\right) \cap \overline{A_{\mu}\left(x_{\mu}^{s}\right)} \neq \emptyset$ para todo $\mu \in\left(\mu_{0}-\epsilon, \mu_{0}\right)$. Consequentemente, de acordo com o Corolário 3.4 de [CHW88] $\Omega_{\mu}^{H} \in A_{\mu}\left(x_{\mu}^{s}\right)$ para todo $\mu \in\left(\mu_{0}-\epsilon, \mu_{0}\right)$. 
(iii) Seja $U$ a vizinhança de $x_{\mu_{0}}$ e $\epsilon_{1}>0$ como definidos no item (i). Como $W_{\mu}^{u}\left(x_{\mu}^{H}\right)$ e $W_{\mu}^{s}\left(x_{\mu}^{s}\right)$ dependem continuamente de $\mu$, ver [Sot73], então podemos afirmar que existe $\epsilon_{2}>0$ tal que $W_{\mu}^{u}\left(x_{\mu}^{H}\right) \cap W_{\mu}^{s}\left(x_{\mu}^{s}\right) \neq \emptyset$ para todo $\mu \in\left(\mu_{0}, \mu_{0}+\epsilon_{2}\right)$, ou seja, $W_{\mu}^{u}\left(x_{\mu}^{H}\right) \cap A_{\mu}\left(x_{\mu}^{s}\right) \neq \emptyset$ para todo $\mu \in\left(\mu_{0}, \mu_{0}+\epsilon_{2}\right)$. Logo, pelo Teorema $6.3 .5, x_{\mu}^{H} \in \partial A_{\mu}\left(x_{\mu}^{s}\right)$ para todo $\mu \in\left(\mu_{0}, \mu_{0}+\epsilon\right)$, onde $\epsilon=\min \left\{\epsilon_{1}, \epsilon_{2}\right\}$.

Observe do Teorema 9.1.4 que para $\mu \in\left(\mu_{0}-\epsilon, \mu_{0}\right)$, a órbita fechada hiperbólica $\Omega_{\mu}^{H}$ do tipo- $(k+1)$, com $1 \leq k \leq n-2$, e o ponto de equilíbrio hiperbólico $x_{\mu}^{H}$ do tipo- $k$, com $1 \leq k \leq n-2$, na vizinhança em $U$ pertencem à fronteira da região de estabilidade de $x_{\mu}^{s}$. À medida que o parâmetro $\mu$ cresce, a amplitude da órbita fechada descresce e vai se aproximando do ponto de equilíbrio hiperbólico do tipo- $k$, com $1 \leq k \leq n-2$ em $U$. Em $\mu=\mu_{0}$, a órbita periódica coalesce com o ponto de equilíbrio hiperbólico em $U$, ocasionando o surgimento do ponto de equilíbrio Hopf subcrítico do tipo- $k$, com $k \geq 1$. O ponto de equilíbrio Hopf subcrítico está na fronteira da região de estabilidade de $x_{\mu}^{s}$. Para valores de $\mu>\mu_{0}$, temos um ponto de equilíbrio hiperbólico $x_{\mu}^{H}$ em $U$ e que pertence à fronteira da região de estabilidade. O Teorema 9.1.4 estabelece que tanto a região de estabilidade quanto a fronteira da região de estabilidade sofrem mudanças quando o parâmetro varia no intervalo $\left(\mu_{0}-\epsilon, \mu_{0}+\epsilon\right)$.

Combinando os Teoremas 6.3.5, 9.1.3 e 9.1.4, obtemos o próximo resultado que estabelece a caracterização da fronteira da região de estabilidade numa vizinhança pequena do valor de parâmetro de bifurcação Hopf subcrítico do tipo- $k$, com $k \geq 1$.

Corolário 9.1.5. (Caracterização da fronteira da região de estabilidade na vizinhança de um ponto de equilíbrio Hopf Subcrítico do tipo-k com $k \geq 1)$ Seja $\left(\mu_{0}, x_{\mu_{0}}\right)$ um ponto de bifurcação Hopf subcrítico do tipo- $k, k \geq 1$, de (8.3) para $\mu=\mu_{0}$. Suponha que o ponto de equilíbrio Hopf subcrítico do tipo- $k x_{\mu_{0}}$ pertença à fronteira da região de estabilidade $\partial A_{\mu_{0}}\left(x_{\mu_{0}}^{s}\right)$ de um ponto de equilíbrio hiperbólico assintoticamente estável $x_{\mu_{0}}^{s}$ de (8.3) para $\mu=\mu_{0}$. Admita que as suposições (B1), (B2) e (B3) sejam satisfeitas para todo $\mu$ pertencente a um intervalo aberto $I$ contendo $\mu=\mu_{0}$, exceto em $\mu_{0}$ onde as condições (B1") e (B2") são satisfeitas. Além disso, assuma que $x_{\mu_{0}}$ seja o único ponto de equilíbrio não hiperbólico em $\mu=\mu_{0}$. Suponha também, que para todo $\mu \in I$, todos os elementos críticos do sistema perturbado $\dot{x}=f(x, \mu)$ sejam elementos críticos perturbados originados do sistema $\dot{x}=f\left(x, \mu_{0}\right)$. Se $r_{\mu_{0}}^{i}$ são os elementos críticos em $\partial A_{\mu_{0}}\left(x_{\mu_{0}}^{s}\right)$, $i=1, \ldots, k$, então: 
(i) Para $\mu=\mu_{0}$ temos

$$
\partial A_{\mu_{0}}\left(x_{\mu_{0}}^{s}\right)=\bigcup_{i} W_{\mu_{0}}^{s}\left(r_{\mu_{0}}^{i}\right) \bigcup_{j} W_{\mu_{0}}^{s}\left(x_{\mu_{0}}\right)
$$

(ii) Existe $\epsilon>0$ tal que, para todo $\mu \in\left(\mu_{0}-\epsilon, \mu_{0}\right)$,

$$
\partial A_{\mu}\left(x_{\mu}^{s}\right)=\bigcup_{i} W_{\mu}^{s}\left(r_{\mu}^{i}\right) \bigcup W_{\mu}^{s}\left(x_{\mu}^{H}\right) \bigcup W_{\mu}^{s}\left(\Omega_{\mu}^{H}\right)
$$

onde $r_{\mu}^{i}, i=1,2, \ldots, k$ são os elementos críticos hiperbólicos perturbados em $\partial A_{\mu}\left(x_{\mu}^{s}\right)$ e $x_{\mu}^{H}$ e $\Omega_{\mu}^{H}$ são o ponto de equilíbrio hiperbólico do tipo-k, com $1 \leq k \leq n-2$, e a órbita periódica do tipo- $(k+1)$, com $1 \leq k \leq n-2$, respectivamente, originados da bifurcação Hopf subcrítica do tipo- $k, k \geq 1$.

(iii) Existe $\epsilon>0$ tal que, para todo $\mu \in\left(\mu_{0}, \mu_{0}+\epsilon\right)$,

$$
\partial A_{\mu}\left(x_{\mu}^{s}\right)=\bigcup_{i} W_{\mu}^{s}\left(r_{\mu}^{i}\right) \bigcup W_{\mu}^{s}\left(x_{\mu}^{H}\right)
$$

onde $r_{\mu}^{i}, i=1,2, \ldots, k$ são os elementos críticos hiperbólicos perturbados em $\partial A_{\mu}\left(x_{\mu}^{s}\right)$ e $x_{\mu}^{H}$ é o ponto de equilíbrio hiperbólico do tipo- $(k+2)$, com $1 \leq k \leq n-2$, originado da bifurcação Hopf subcrítica do tipo-k, $k \geq 1$.

Nos dois próximos teoremas apresentaremos o comportamento da fronteira da região de estabilidade do ponto de equilíbrio assintoticamente estável numa vizinhança pequena do parâmetro $\mu$ próximo ao parâmetro $\mu_{0}$ de bifurcação Hopf supercrítica do tipo- $k$, com $k \geq 1$. Admitiremos como hipótese, para o valor do parâmetro de bifurcação Hopf supercrítico $\mu_{0}$, apenas a existência de elementos críticos hiperbólicos do sistema (8.3) em $\mu=\mu_{0}$, com exceção do ponto de equilíbrio não hiperbólico Hopf supercrítico $x_{\mu_{0}}$ do tipo- $k$, com $k \geq 1$. Além disso, numa vizinhança pequena do parâmetro $\mu_{0}$, também admitiremos apenas a existência de elementos críticos que são os pontos de equilíbrio perturbados do sistema original (8.3) em $\mu=\mu_{0}$. Procedendo da mesma forma quando da ocorrência da bifurcação Hopf subcrítica, estabeleceremos o comportamento da fronteira da região de estabilidade na vizinhança de um ponto de equilíbrio Hopf Supercrítico do tipo- $k$ com $k \geq 1$ e em seguida, apresentaremos uma caracterização global da fronteira nessa vizinhança.

Teorema 9.1.6. (Comportamento da fronteira da região de estabilidade na vizinhança de um ponto de equilíbrio Hopf Supercrítico do tipo- $k$ com $k \geq 1)$ Seja $\left(\mu_{0}, x_{\mu_{0}}\right)$ um ponto de bifurcação Hopf supercrítico do tipo-k, $k \geq 1$, de (8.3) para $\mu=\mu_{0}$. Suponha que o ponto de equilíbrio Hopf supercrítico do tipo-k $x_{\mu_{0}}$ pertença à fronteira da região de estabilidade $\partial A_{\mu_{0}}\left(x_{\mu_{0}}^{s}\right)$ de um ponto de equilíbrio hiperbólico assintoticamente estável $x_{\mu_{0}}^{s}$ 
de (8.3) para $\mu=\mu_{0}$. Admita que as suposições (B1), (B2) e (B3) sejam satisfeitas para todo $\mu$ pertencente a um intervalo aberto $I$ contendo $\mu=\mu_{0}$, exceto em $\mu_{0}$ onde as condições (B1') e (B2') são satisfeitas. Além disso, assuma que $x_{\mu_{0}}$ seja o único ponto de equilibrio não hiperbólico em $\mu=\mu_{0}$. Suponha também, que para todo $\mu \in I$, todos os elementos críticos do sistema perturbado $\dot{x}=f(x, \mu)$ sejam elementos críticos perturbados originados do sistema $\dot{x}=f\left(x, \mu_{0}\right)$. Então existe uma vizinhança de $x_{\mu_{0}}$ e $\epsilon_{1} \geq \epsilon>0$ tal que:

(i) Existe um ponto de equilíbrio hiperbólico $x_{\mu}^{H}$ do tipo- $k$, com $1 \leq k \leq$ $n-2$, em $U$ para todo $\mu \in\left(\mu_{0}-\epsilon_{1}, \mu_{0}\right)$ e uma órbita fechada hiperbólica $\Omega_{\mu}^{H}$ do tipo-k, com $1 \leq k \leq n-2$, e um ponto de equilíbrio hiperbólico $x_{\mu}^{H}$ do tipo- $k+2$, com $1 \leq k \leq n-2$, em $U$ para todo $\mu \in\left(\mu_{0}, \mu_{0}+\epsilon_{1}\right)$.

(ii) Para $\mu \in\left(\mu_{0}, \mu_{0}+\epsilon\right)$ temos que

$$
\Omega_{\mu}^{H} \in \partial A_{\mu}\left(x_{\mu}^{s}\right) \quad e \quad x_{\mu}^{H} \in \partial A_{\mu}\left(x_{\mu}^{s}\right) .
$$

(iii) Para $\mu \in\left(\mu_{0}-\epsilon, \mu_{0}\right)$ temos que

$$
x_{\mu}^{H} \in \partial A_{\mu}\left(x_{\mu}^{s}\right) .
$$

Demonstração. (i) A existência da vizinhança $U$, do escalar $\epsilon_{1}$ e dos elementos críticos segue do Teorema 8.3.5.

(ii) Mostraremos a existência de $\epsilon>0$ tal que $\Omega_{\mu}^{H} \in \partial A_{\mu}\left(x_{\mu}^{s}\right)$ e $x_{\mu}^{H} \in$ $\partial A_{\mu}\left(x_{\mu}^{s}\right)$ para todo $\mu \in\left(\mu_{0}, \mu_{0}+\epsilon\right)$. Como $x_{\mu_{0}} \in \partial A_{\mu_{0}}\left(x_{\mu_{0}}^{s}\right)$ e as suposições (B1'), (B2') e (B3) são satisfeitas para $\mu=\mu_{0}$, então de acordo com o Teorema 6.2.2 $W_{\mu_{0}}^{u}\left(x_{\mu_{0}}\right) \cap A_{\mu_{0}}\left(x_{\mu_{0}}^{s}\right) \neq \emptyset$ e esta interseção é transversal. Como $A_{\mu_{0}}\left(x_{\mu_{0}}^{s}\right)=W_{\mu_{0}}^{s}\left(x_{\mu_{0}}^{s}\right)$, temos que $W_{\mu_{0}}^{u}\left(x_{\mu_{0}}\right) \cap$ $W_{\mu_{0}}^{s}\left(x_{\mu_{0}}^{s}\right) \neq \emptyset$. Como $W_{\mu}^{u}\left(\Omega_{\mu}^{H}\right)$ e $W_{\mu}^{s}\left(x_{\mu}^{s}\right)$ dependem continuamente de $\mu$, ver [Sot73], então podemos afirmar que existe $\epsilon_{2}>0$ tal que $W_{\mu}^{u}\left(\Omega_{\mu}^{H}\right) \cap W_{\mu}^{s}\left(x_{\mu}^{s}\right) \neq \emptyset$ para todo $\mu \in\left(\mu_{0}, \mu_{0}+\epsilon_{2}\right)$, ou seja, $W_{\mu}^{u}\left(\Omega_{\mu}^{H}\right) \cap$ $A_{\mu}\left(x_{\mu}^{s}\right) \neq \emptyset$ para todo $\mu \in\left(\mu_{0}, \mu_{0}+\epsilon_{2}\right)$. Logo, pelo Teorema 6.2.2, $\Omega_{\mu}^{H} \subset \partial A_{\mu}\left(x_{\mu}^{s}\right)$ para todo $\mu \in\left(\mu_{0}, \mu_{0}+\epsilon\right)$, onde $\epsilon=\min \left\{\epsilon_{1}, \epsilon_{2}\right\}$. Pelo Teorema 8.3.5, a variedade instável do ponto de equilíbrio hiperbólico $x_{\mu}^{H}$ do tipo- $(k+2)$ intercepta a variedade estável da órbita periódica hiperbólica $\Omega_{\mu}^{H}$ do tipo- $k$ ao longo de uma variedade bidimensional, ou seja, $W_{\mu}^{u}\left(x_{\mu}^{H}\right) \cap W_{\mu}^{s}\left(\Omega_{\mu}^{H}\right) \neq \emptyset$. Como $\Omega_{\mu}^{H} \in \partial A_{\mu}\left(x_{\mu}^{s}\right)$ para todo $\mu \in\left(\mu_{0}, \mu_{0}+\epsilon\right)$, então pelo Teorema 6.2.2 $W_{\mu}^{s}\left(\Omega_{\mu}^{H}\right) \subset \partial A_{\mu}\left(x_{\mu}^{s}\right)$ e portanto, $W_{\mu}^{u}\left(x_{\mu}^{H}\right) \cap \overline{A_{\mu}\left(x_{\mu}^{s}\right)} \neq \emptyset$ para todo $\mu \in\left(\mu_{0}, \mu_{0}+\epsilon\right)$. Consequentemente, de acordo com o Teorema 3.3 de [CHW88] $x_{\mu}^{H} \in A_{\mu}\left(x_{\mu}^{s}\right)$ para todo $\mu \in\left(\mu_{0}, \mu_{0}+\epsilon\right)$. 
(iii) Seja $U$ a vizinhança de $x_{\mu_{0}}$ e $\epsilon_{1}>0$ como definidos no item (i). Como $W_{\mu}^{u}\left(x_{\mu}^{H}\right)$ e $W_{\mu}^{s}\left(x_{\mu}^{s}\right)$ dependem continuamente de $\mu$, ver [Sot73], então podemos afirmar que existe $\epsilon_{2}>0$ tal que $W_{\mu}^{u}\left(x_{\mu}^{H}\right) \cap W_{\mu}^{s}\left(x_{\mu}^{s}\right) \neq \emptyset$ para todo $\mu \in\left(\mu_{0}-\epsilon_{2}, \mu_{0}\right)$, ou seja, $W_{\mu}^{u}\left(x_{\mu}^{H}\right) \cap A_{\mu}\left(x_{\mu}^{s}\right) \neq \emptyset$ para todo $\mu \in\left(\mu_{0}-\epsilon_{2}, \mu_{0}\right)$. Logo, pelo Teorema 6.2.2, $x_{\mu}^{H} \in \partial A_{\mu}\left(x_{\mu}^{s}\right)$ para todo $\mu \in\left(\mu_{0}-\epsilon, \mu_{0}\right)$, onde $\epsilon=\min \left\{\epsilon_{1}, \epsilon_{2}\right\}$.

Observe do Teorema 9.1.6 que para $\mu \in\left(\mu_{0}-\epsilon, \mu_{0}\right)$, o ponto de equilíbrio hiperbólico $x_{\mu}^{H}$ do tipo- $k$, com $1 \leq k \leq n-2$, na vizinhança em $U$ pertence à fronteira da região de estabilidade de $x_{\mu}^{s}$. Em $\mu=\mu_{0}$, o ponto de equilíbrio perde a hiperbolicidade em $U$, ocasionando o surgimento do ponto de equilíbrio Hopf supercrítico do tipo- $k$, com $k \geq 1$. O ponto de equilíbrio Hopf supercrítico está na fronteira da região de estabilidade de $x_{\mu}^{s}$. Para valores de $\mu>\mu_{0}$, o ponto de equilíbrio hiperbólico $x_{\mu}^{H}$ do tipo- $k+2$, com $1 \leq k \leq n-2$ em $U$ perde estabilidade e surge uma órbita fechada hiperbólica $\Omega_{\mu}^{H}$ do tipo- $(k+1)$, com $1 \leq k \leq n-2$, em $U$ na fronteira da região de estabilidade de $x_{\mu}^{s}$. O Teorema 9.1.6 estabelece que tanto a região de estabilidade quanto a fronteira da região de estabilidade sofrem mudanças quando o parâmetro varia no intervalo $\left(\mu_{0}-\epsilon, \mu_{0}+\epsilon\right)$.

Combinando os Teoremas 6.2.2, 9.1.3 e 9.1.6, obtemos o próximo resultado que estabelece a caracterização da fronteira da região de estabilidade numa vizinhança pequena do valor de parâmetro de bifurcação Hopf supercrítico do tipo- $k$, com $k \geq 1$.

Corolário 9.1.7. (Caracterização da fronteira da região de estabilidade na vizinhança de um ponto de equilíbrio Hopf Supercrítico do tipo-k com $k \geq 1)$ Seja $\left(\mu_{0}, x_{\mu_{0}}\right)$ um ponto de bifurcação Hopf supercrítico do tipo- $k, k \geq 1$, de (8.3) para $\mu=\mu_{0}$. Suponha que o ponto de equilíbrio Hopf supercrítico do tipo- $k x_{\mu_{0}}$ pertença à fronteira da região de estabilidade $\partial A_{\mu_{0}}\left(x_{\mu_{0}}^{s}\right)$ de um ponto de equilíbrio hiperbólico assintoticamente estável $x_{\mu_{0}}^{s}$ de (8.3) para $\mu=\mu_{0}$. Admita que as suposições (B1), (B2) e (B3) sejam satisfeitas para todo $\mu$ pertencente a um intervalo aberto $I$ contendo $\mu=\mu_{0}$, exceto em $\mu_{0}$ onde as condições (B1') e (B2') são satisfeitas. Além disso, assuma que $x_{\mu_{0}}$ seja o único ponto de equilíbrio não hiperbólico em $\mu=\mu_{0}$. Suponha também, que para todo $\mu \in I$, todos os elementos críticos do sistema perturbado $\dot{x}=f(x, \mu)$ sejam elementos críticos perturbados originados do sistema $\dot{x}=f\left(x, \mu_{0}\right)$. Se $r_{\mu_{0}}^{i}$ são os elementos críticos em $\partial A_{\mu_{0}}\left(x_{\mu_{0}}^{s}\right)$, $i=1, \ldots, k$, então:

(i) Para $\mu=\mu_{0}$ temos

$$
\partial A_{\mu_{0}}\left(x_{\mu_{0}}^{s}\right)=\bigcup_{i} W_{\mu_{0}}^{s}\left(r_{\mu_{0}}^{i}\right) \bigcup_{j} W_{\mu_{0}}^{c}\left(x_{\mu_{0}}\right)
$$


(ii) Existe $\epsilon>0$ tal que, para todo $\mu \in\left(\mu_{0}-\epsilon, \mu_{0}\right)$,

$$
\partial A_{\mu}\left(x_{\mu}^{s}\right)=\bigcup_{i} W_{\mu}^{s}\left(r_{\mu}^{i}\right) \bigcup W_{\mu}^{s}\left(x_{\mu}^{H}\right)
$$

onde $r_{\mu}^{i}, i=1,2, \ldots, k$ são os elementos críticos hiperbólicos perturbados em $\partial A_{\mu}\left(x_{\mu}^{s}\right)$ e $x_{\mu}^{H}$ é o ponto de equilíbrio hiperbólico do tipo-k, com $1 \leq k \leq n-2$, originado da bifurcação Hopf supercrítico do tipo- $k, k \geq 1$.

(iii) Existe $\epsilon>0$ tal que, para todo $\mu \in\left(\mu_{0}, \mu_{0}+\epsilon\right)$,

$$
\partial A_{\mu}\left(x_{\mu}^{s}\right)=\bigcup_{i} W_{\mu}^{s}\left(r_{\mu}^{i}\right) \bigcup W_{\mu}^{s}\left(x_{\mu}^{H}\right) \bigcup W_{\mu}^{s}\left(\Omega_{\mu}^{H}\right)
$$

onde $r_{\mu}^{i}, i=1,2, \ldots, k$ são os elementos críticos hiperbólicos perturbados em $\partial A_{\mu}\left(x_{\mu}^{s}\right)$ e $x_{\mu}^{H}$ e $\Omega_{\mu}^{H}$ são o ponto de equilíbrio hiperbólico do tipo- $(k+2)$, com $1 \leq k \leq n-2$, e a órbita periódica do tipo- $k$, com $1 \leq k \leq n-2$, respectivamente, originados da bifurcação Hopf supercrítica do tipo- $k, k \geq 1$.

\subsection{Região de Estabilidade Fraca de um Equilíbrio Hopf Supercrítico do tipo zero}

Exibiremos agora o comportamento da fronteira da região de estabilidade de um ponto de equilíbrio Hopf supercrítico do tipo-zero na vizinhança do valor de bifurcação e depois, obteremos a caracterização da sua fronteira na vizinhança da bifurcação de Hopf supercrítica do tipo zero. Analogamente, como procedemos para a caracterização da fronteira da região de estabilidade na presença de um ponto de equilíbrio Hopf subcrítico, exploraremos o comportamento da fronteira da região de estabilidade do ponto de equilíbrio Hopf supercrítico do tipo-zero na vizinhança desse equilíbrio não hiperbólico. E posteriormente, apresentaremos a caracterização global da fronteira da região de estabilidade na vizinhança do parâmetro de Bifurcação Hopf supercrítico do tipo-zero.

Teorema 9.2.1. (Comportamento da fronteira da região de estabilidade de um ponto de equilíbrio Hopf Supercrítico do tipo-zero numa vizinhança desse equilíbrio) Seja $\left(\mu_{0}, x_{\mu_{0}}\right)$ um ponto de bifurcação Hopf supercrítico do tipo-zero de (8.3) para $\mu=\mu_{0}$. Seja $A_{\mu_{0}}\left(x_{\mu_{0}}\right)$ a região de estabilidade fraca do ponto de equilíbrio Hopf supercrítico do tipozero de (8.3) para $\mu=\mu_{0}$. Admita que as suposições (B1), (B2) e (B3) sejam satisfeitas para todo $\mu$ pertencente a um intervalo aberto I contendo $\mu=\mu_{0}$, exceto em $\mu_{0}$ onde as condições (S1), (S2) e (S3) são satisfeitas. Suponha também, que para todo $\mu \in I$, todos os elementos críticos do sistema perturbado $\dot{x}=f(x, \mu)$ sejam elementos críticos perturbados origi- 
nados do sistema $\dot{x}=f\left(x, \mu_{0}\right)$. Seja $\psi_{\mu_{0}}^{*}$ um elemento crítico hiperbólico tal que $\psi_{\mu_{0}}^{*} \in \partial A_{\mu_{0}}\left(x_{\mu_{0}}\right)$ onde $\partial A_{\mu_{0}}\left(x_{\mu_{0}}\right)$ é a fronteira da região de estabilidade fraca. Então existe $\epsilon>0$ tal que

(i) Existem uma órbita fechada $\Omega_{\mu}^{H}$ hiperbólica de tipo-zero e um ponto de equilíbrio hiperbólico do tipo-2, $x_{\mu}^{H}$ para todo $\mu \in\left(\mu_{0}, \mu_{0}+\epsilon_{1}\right)$ e um ponto de equilíbrio hiperbólico do tipo-zero $x_{\mu}^{H}$ para todo $\mu \in\left(\mu_{0}-\right.$ $\left.\epsilon_{1}, \mu_{0}\right)$.

(ii) Para $\mu \in\left(\mu_{0}-\epsilon, \mu_{0}\right)$ temos que

$$
\psi_{\mu}^{*} \in \partial A_{\mu}\left(x_{\mu}^{H}\right)
$$

onde $x_{\mu}^{H}$ é o equilibrio assintoticamente estável originado da bifurcação de Hopf supercrítica do tipo-zero.

(iii) Para $\mu \in\left(\mu_{0}, \mu_{0}+\epsilon\right)$ temos que

$$
\psi_{\mu}^{*} \in \partial A_{\mu}\left(\Omega_{\mu}^{H}\right)
$$

onde $\Omega_{\mu}^{H}$ é o ciclo limite estável originado da bifurcação de Hopf supercrítica do tipo-zero e $x_{\mu}^{H} \in \partial A_{\mu}\left(\Omega_{\mu}^{H}\right) \cap A_{\mu}^{\mathcal{Q}}\left(\Omega_{\mu}^{H}\right)$, onde $A_{\mu}^{\mathcal{Q}}\left(\Omega_{\mu}^{H}\right)$ é a região de quase-estabilidade do ciclo limite estável $\Omega_{\mu}^{H}$.

Demonstração. (i) A existência de $\epsilon_{1}$ e dos elementos críticos segue do Teorema 8.3.5.

(ii) Mostraremos a existência de $\epsilon>0$ tal que $\psi_{\mu}^{*} \in \partial A_{\mu}\left(x_{\mu}^{H}\right)$ para todo $\mu \in\left(\mu_{0}-\epsilon, \mu_{0}\right)$. Seja $\psi_{\mu_{0}}^{*} \in \partial A_{\mu_{0}}\left(x_{\mu_{0}}\right)$. Pelo Teorema 7.1.6 concluí$\operatorname{mos}$ que $W_{\mu_{0}}^{u}\left(\psi_{\mu_{0}}^{*}\right) \cap A_{\mu_{0}}\left(x_{\mu_{0}}\right) \neq \emptyset$. Seja $q \in W_{\mu_{0}}^{u}\left(\psi_{\mu_{0}}^{*}\right) \cap A_{\mu_{0}}\left(x_{\mu_{0}}\right)$. Como $A_{\mu_{0}}\left(x_{\mu_{0}}\right)$ é um conjunto aberto, então existe $r>0$ tal que $B(q, r) \subset A_{\mu_{0}}\left(x_{\mu_{0}}\right)$. Dado $\epsilon_{1} \geq \epsilon_{2}>0$ suficientemente pequeno, podemos escolher $r$ também suficientemente pequeno de tal maneira que $B(q, r) \subset A_{\mu}\left(x_{\mu}^{H}\right)$ para todo $\mu \in\left(\mu_{0}-\epsilon_{2}, \mu_{0}\right)$, ver [Sot73], onde $x_{\mu}^{H}$ é o ponto de equilíbrio hiperbólico originado da bifurcação de Hopf supercrítica. Como $W_{\mu_{0}}^{u}\left(\psi_{\mu_{0}}^{*}\right)$ intertecepta transversalmente $B(q, r)$ e além disso, interseções transversais persistem sob pequenas perturbações, então existe $\epsilon_{3}$ tal que $W_{\mu}^{u}\left(\psi_{\mu}^{*}\right) \cap B(q, r) \neq \emptyset$ para todo $\mu \in\left(\mu_{0}-\epsilon_{3}, \mu_{0}+\epsilon_{3}\right)$. Tomemos $\epsilon=\min \left\{\epsilon_{1}, \epsilon_{2}, \epsilon_{3}\right\}$, temos $W_{\mu}^{u}\left(\psi_{\mu}^{*}\right) \cap B(q, r) \neq \emptyset$ e $B(q, r) \subset A_{\mu}\left(x_{\mu}^{H}\right)$, para todo $\mu \in\left(\mu_{0}-\epsilon, \mu_{0}\right)$. Consequentemente, $W_{\mu}^{u}\left(\psi_{\mu}^{*}\right) \cap A_{\mu}\left(x_{\mu}^{H}\right) \neq \emptyset$, para todo $\mu \in\left(\mu_{0}-\epsilon, \mu_{0}\right)$, e portanto, pelo Teorema 7.1.6, $\psi_{\mu}^{*} \in \partial A_{\mu}\left(x_{\mu}^{H}\right)$ para todo $\mu \in\left(\mu_{0}-\epsilon, \mu_{0}\right)$.

(iii) Sejam $r, \epsilon_{1}, \epsilon_{2}, \epsilon_{3}$ como definidos no item (ii) tal que $B(q, r) \subset A_{\mu}\left(\Omega_{\mu}^{H}\right)$ para todo $\mu \in\left(\mu_{0}, \mu_{0}+\epsilon_{2}\right)$, ver [Sot73], onde $\Omega_{\mu}^{H}$ é a órbita periódica hiperbólica originada da bifurcação de Hopf supercrítica, e $W_{\mu}^{u}\left(\psi_{\mu}^{*}\right) \cap$ 
$B(q, r) \neq \emptyset$ para todo $\mu \in\left(\mu_{0}-\epsilon_{3}, \mu_{0}+\epsilon_{3}\right)$. Assim como procedemos no item anterior, tomemos $\epsilon=\min \left\{\epsilon_{1}, \epsilon_{2}, \epsilon_{3}\right\}$, então $W_{\mu}^{u}\left(\psi_{\mu}^{*}\right) \cap B(q, r) \neq \emptyset$ e $B(q, r) \subset A_{\mu}\left(\Omega_{\mu}^{H}\right)$, para todo $\mu \in\left(\mu_{0}, \mu_{0}+\epsilon\right)$. Consequentemente, $W_{\mu}^{u}\left(\psi_{\mu}^{*}\right) \cap A_{\mu}\left(\Omega_{\mu}^{H}\right) \neq \emptyset$, para todo $\mu \in\left(\mu_{0}, \mu_{0}+\epsilon\right)$, e portanto, pelo Teorema 7.1.6, $\psi_{\mu}^{*} \in \partial A_{\mu}\left(\Omega_{\mu}^{H}\right)$ para todo $\mu \in\left(\mu_{0}, \mu_{0}+\epsilon\right)$.

Tomando o mesmo $r$ e $\epsilon$ definido anteriormente, temos $W_{\mu}^{u}\left(x_{\mu}^{H}\right) \cap$ $B(q, r) \neq \emptyset$ para todo $\mu \in\left(\mu_{0}, \mu_{0}+\epsilon\right)$. Consequentemente, $W_{\mu}^{u}\left(x_{\mu}^{H}\right) \cap$ $A_{\mu}\left(\Omega_{\mu}^{H}\right) \neq \emptyset$, para todo $\mu \in\left(\mu_{0}, \mu_{0}+\epsilon\right)$, e portanto, pelo Teorema 7.1.6 $x_{\mu}^{H} \in \partial A_{\mu}\left(\Omega_{\mu}^{H}\right)$ para todo $\mu \in\left(\mu_{0}, \mu_{0}+\epsilon\right)$.

Consequentemente, trajetórias iniciando na vizinhança de $x_{\mu}^{H}$ tendem para o ciclo limite estável quando $t \rightarrow+\infty$. Logo, $W_{\mu}^{u}\left(x_{\mu}^{H}\right) \subset A_{\mu}\left(\Omega_{\mu}^{H}\right)$ para todo $\mu \in\left(\mu_{0}, \mu_{0}+\epsilon\right)$. Portanto, pelo Teorema 5.1 em [CFA96] tem-se que $x_{\mu}^{H} \in A_{\mu}^{\mathcal{Q}}\left(\Omega_{\mu}^{H}\right)$

Analogamente, com os Teoremas 7.1.5, 9.1.3 e 9.2.1, obtemos a caracterização da fronteira da região de estabilidade de um ponto de bifurcação Hopf supercrítico do tipo-zero numa vizinhança pequena do valor de parâmetro de bifurcação Hopf supercrítico do tipo-zero.

Corolário 9.2.2. (Caracterização da fronteira da região de estabilidade de um ponto de bifurcação Hopf Supercrítico do tipo-zero numa vizinhança desse equilíbrio) Seja $\left(\mu_{0}, x_{\mu_{0}}\right)$ um ponto de bifurcação Hopf supercrítico do tipo-zero de (8.3) para $\mu=\mu_{0}$. Seja $A_{\mu_{0}}\left(x_{\mu_{0}}\right)$ a região de estabilidade fraca do ponto bifurcação Hopf supercrítico do tipozero de (8.3) para $\mu=\mu_{0}$. Admita que as suposiçôes (B1), (B2) e (B3) sejam satisfeitas para todo $\mu$ pertencente a um intervalo aberto $I$ contendo $\mu=\mu_{0}$, exceto em $\mu_{0}$ onde as condições (S1), (S2) e (S3) são satisfeitas. Suponha também, que para todo $\mu \in I$, todos os pontos de equilibrios do sistema perturbado $\dot{x}=f(x, \mu)$ são pontos de equilíbrios perturbados originados do sistema $\dot{x}=f\left(x, \mu_{0}\right)$. Se $r_{\mu_{0}}^{i}$ são os elementos críticos hiperbólico na fronteira da região de estabilidade fraca $\partial A_{\mu_{0}}\left(x_{\mu_{0}}\right)$. Então

(i) Para $\mu=\mu_{0}$ temos

$$
\partial A_{\mu_{0}}\left(x_{\mu_{0}}\right)=\bigcup_{i} W_{\mu_{0}}^{s}\left(r_{\mu_{0}}^{i}\right)
$$

(ii) Existe $\epsilon>0$ tal que, para todo $\mu \in\left(\mu_{0}-\epsilon, \mu_{0}\right)$,

$$
\partial A_{\mu}\left(x_{\mu}^{H}\right)=\bigcup_{i} W_{\mu}^{s}\left(r_{\mu}^{i}\right)
$$

onde $r_{\mu}^{i}, i=1,2, \ldots, k$ são os elementos críticos hiperbólicos perturbados 
em $\partial A_{\mu}\left(x_{\mu}^{H}\right)$ e $x_{\mu}^{H}$ é o ponto de equilíbrio hiperbólico assintoticamente estável originado da bifurcação Hopf supercrítica do tipo-zero.

(iii) Existe $\epsilon>0$ tal que, para todo $\mu \in\left(\mu_{0}, \mu_{0}+\epsilon\right)$,

$$
\begin{gathered}
\partial A_{\mu}\left(\Omega_{\mu}^{H}\right)=\bigcup_{i} W_{\mu}^{s}\left(r_{\mu}^{i}\right) \bigcup_{i} W_{\mu}^{s}\left(x_{\mu}^{H}\right) \\
\partial A_{\mu}^{\mathcal{Q}}\left(\Omega_{\mu}^{H}\right)=\bigcup_{i} W_{\mu}^{s}\left(r_{\mu}^{i}\right)
\end{gathered}
$$

onde $r_{\mu}^{i}, i=1,2, \ldots, k$ são os elementos críticos hiperbólicos perturbados em $\partial A_{\mu}\left(x_{\mu}^{s}\right)$ e $\Omega_{\mu}^{H}$ e $x_{\mu}^{H}$ são o ciclo limite estável e o ponto de equilíbrio hiperbólico do tipo- $(k+2)$, com $0 \leq k \leq n-2$, originados da bifurcação Hopf supercrítica do tipo-zero e $\partial A_{\mu}^{\mathcal{Q}}\left(\Omega_{\mu}^{H}\right)$ é a fronteira da região de quaseestabilidade de $\Omega_{\mu}^{H}$.

\subsection{Exemplos}

Exibiremos alguns exemplos que ilustram os Teoremas discutidos nas seções 9.1 e 9.2 .

Exemplo 9.3.1. Considere o sistema dinâmico autônomo não-linear extraído de [GJAA13]

$$
\left\{\begin{array}{l}
\dot{x}=(-z+\mu) x-y-x\left(x^{2}+y^{2}\right) \\
\dot{y}=(-z+\mu) y+x-y\left(x^{2}+y^{2}\right) \\
\dot{z}=-0.1\left(z+0.5\left(x^{2}+y^{2}\right)\right)(z-3)(8-z)
\end{array}\right.
$$

onde $(x, y, z) \in \mathbb{R}^{3}$ e $\mu \in \mathbb{R}$.

Para $\mu_{0}=0$, vimos no Exemplo 5.4.1 que a fronteira da região de estabilidade de $x_{\mu_{0}}^{s}=(0,0,3)$ é formada pela união da variedade estável do ponto de equilíbrio hiperbólico do tipo-1 $x_{1}=(0,0,8)$ com a variedade central do ponto de equilíbrio Hopf supercrítico do tipo-1 $x_{\mu_{0}}^{H}=(0,0,0)$, ver Figura 9.1.

Para $\mu=-0.5$, o sistema (9.1) possui três pontos de equilíbrio, são eles: dois pontos de equilíbrio hiperbólicos do tipo- $1, x_{\mu}^{H}=(0,0,0)$ e $x_{1}=(0,0,8)$, e um ponto de equilíbrio assintoticamente estável, $x_{\mu}^{s}=(0,0,3)$. O ponto de equilíbrio $x_{\mu}^{H}$ é originado do ponto de equilíbrio Hopf supercrítico do tipo1 em uma bifurcação Hopf supercrítica do tipo-1. Os pontos de equilíbrio hiperbólicos $x_{\mu}^{H}=(0,0,0)$ e $x_{1}=(0,0,8)$ pertencem à fronteira da região de estabilidade $\partial A_{\mu}\left(x_{\mu}^{s}\right)$, de acordo com o Teorema 9.1.6, ver Figura 9.2.

Para $\mu=0.5$, o sistema (9.1) possui quatro elementos críticos, são eles: um ponto de equilíbrio hiperbólico do tipo-3, $x_{\mu}^{H}=(0,0,0)$, um ponto de 
equilíbrio hiperbólico do tipo-1, $x_{1}=(0,0,8)$, um ponto de equilíbrio assintoticamente estável, $x_{\mu}^{s}=(0,0,3)$, e uma órbita periódica hiperbólico do tipo-1 $\phi_{\mu}^{H}$. Os elementos críticos $x_{\mu}^{H}$ e $\phi_{\mu}^{H}$ foram originados do ponto de equilíbrio Hopf supercrítico do tipo-zero em uma bifurcação Hopf supercrítica do tipo-zero. Os pontos de equilíbrio hiperbólicos $x_{1}$ e $\phi_{\mu}^{H}$ pertencem à fronteira da região de estabilidade $\partial A_{\mu}\left(x_{\mu}^{s}\right)$, de acordo com o Teorema 9.1.6, ver Figura 9.3.

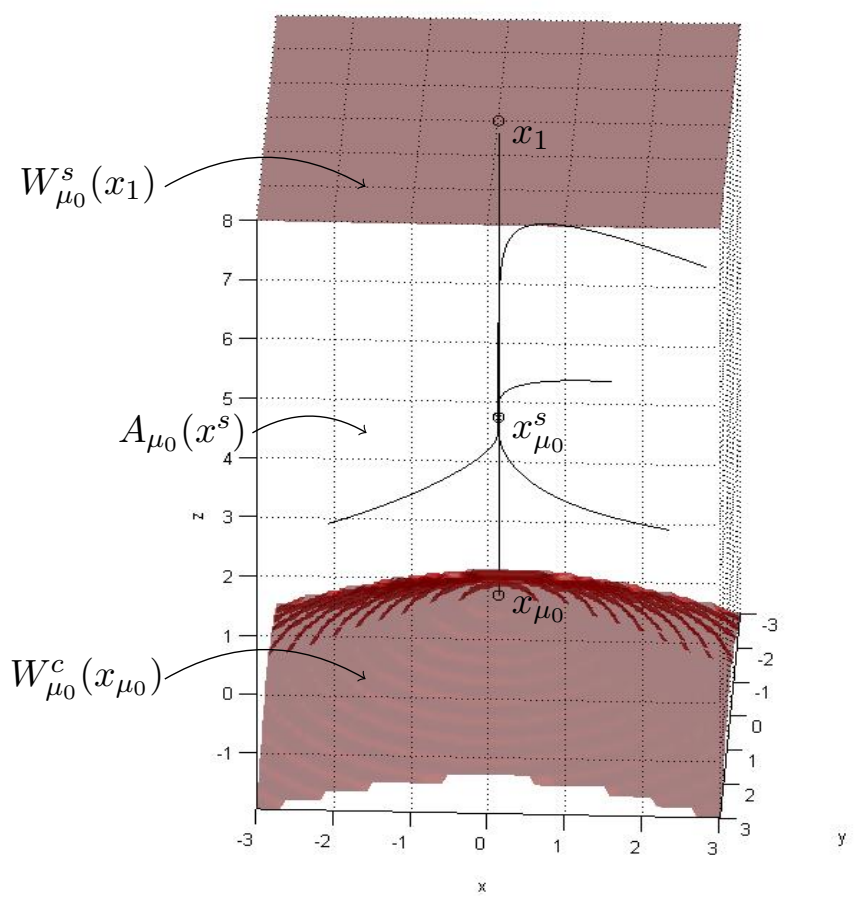

Figura 9.1: Retrato de fase do sistema (9.1) para $\mu_{0}=0$. A fronteira da região de estabilidade de $x_{\mu_{0}}^{s}=(0,0,3)$ é formada pela união da variedade estável $W_{\mu_{0}}^{s}\left(x_{1}\right)$ do ponto de equilíbrio hiperbólico do tipo- $1 x_{1}=(0,0,8)$ com a variedade central $W_{\mu_{0}}^{c}\left(x_{\mu_{0}}\right)$ do ponto de equilibrio Hopf supercrítico do tipo-1 $x_{\mu_{0}}=(0,0,0)$. 


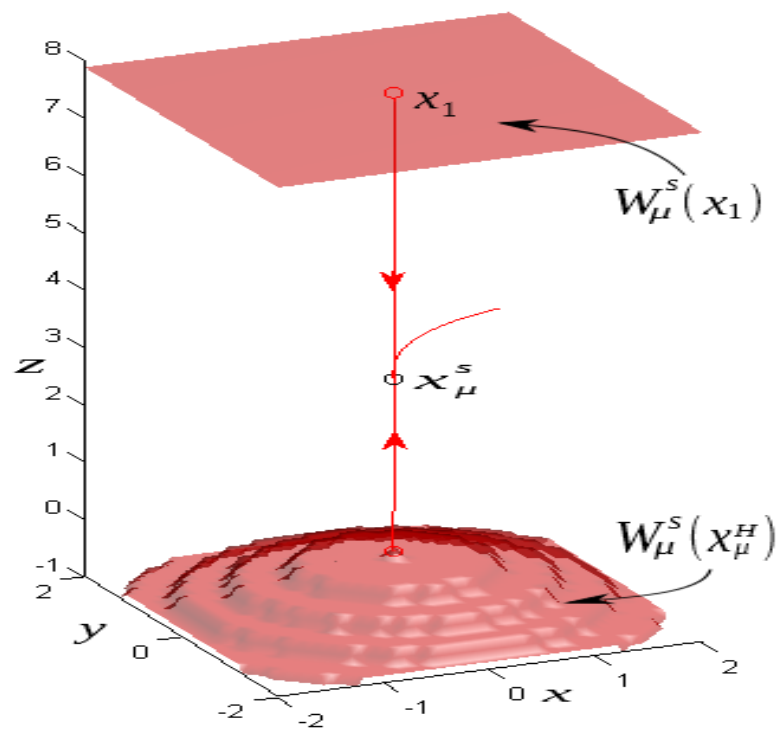

Figura 9.2: Retrato de fase do sistema (9.1) para $\mu=-0.5$. A fronteira da região de estabilidade de $x_{\mu}^{s}=(0,0,3)$ é formada pela união da variedade estável $W_{\mu}^{s}\left(x_{1}\right)$ do ponto de equilíbrio hiperbólico do tipo-1 $x_{1}=(0,0,8)$ com a variedade estável $W_{\mu}^{s}\left(x_{\mu}^{H}\right)$ do ponto de equilíbrio hiperbólico do tipo-1 $x^{H}=(0,0,0)$.

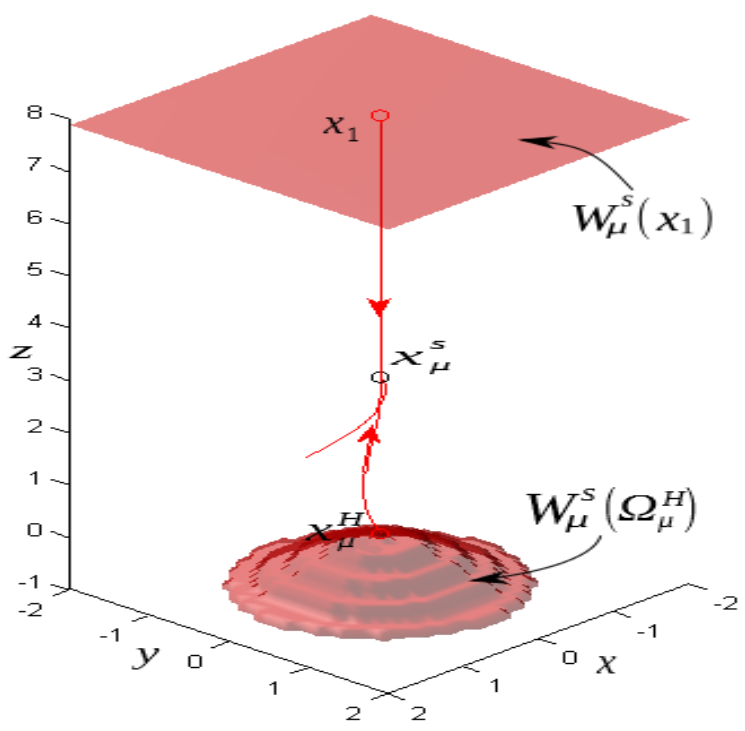

Figura 9.3: Retrato de fase do sistema (9.1) para $\mu=0.5$. A fronteira da região de estabilidade de $x_{\mu}^{s}=(0,0,3)$ é formada pela união da variedade estável $W_{\mu}^{s}\left(x_{1}\right)$ do ponto de equilíbrio hiperbólico do tipo-1 $x_{1}=(0,0,8)$ com a variedade estável $W_{\mu}^{s}\left(\phi_{\mu}^{H}\right)$ da órbita periódica hiperbólica do tipo-1 $\phi_{\mu}^{H}$. 
Exemplo 9.3.2. Considere o sistema Predador-Presa em $\mathbb{R}^{2}$ extraído de [Vér13]

$$
\left\{\begin{array}{l}
\dot{x}=x-\frac{x y}{1+0.5 x}-\mu x^{2} \\
\dot{y}=-y\left(1-\frac{x}{1+0.5 x}\right)
\end{array},\right.
$$

onde $(x, y) \in \mathbb{R}^{2}$ e $\mu \in \mathbb{R}$.

Para $\mu_{0}=\frac{1}{6}$, vimos no Exemplo 7.1.1 que a fronteira da região de estabilidade fraca $\partial A_{\mu_{0}}\left(x_{\mu_{0}}\right)$ do ponto de equilíbrio Hopf supercrítico do tipo-0 $x_{\mu_{0}}=\left(2, \frac{4}{3}\right)$ está contida na união da variedade estável $W_{\mu_{0}}^{s}\left(x_{1}\right)$ do ponto de equilíbrio hiperbólico do tipo-1 $x_{1}=(0,0)$, a curva em vermelho na Figura 9.4, com a variedade estável $W_{\mu_{0}}^{s}\left(x_{2}\right)$ do ponto de equilíbrio hiperbólico do tipo-1 $x_{2}=(6,0)$, a curva em preto na Figura 9.4.

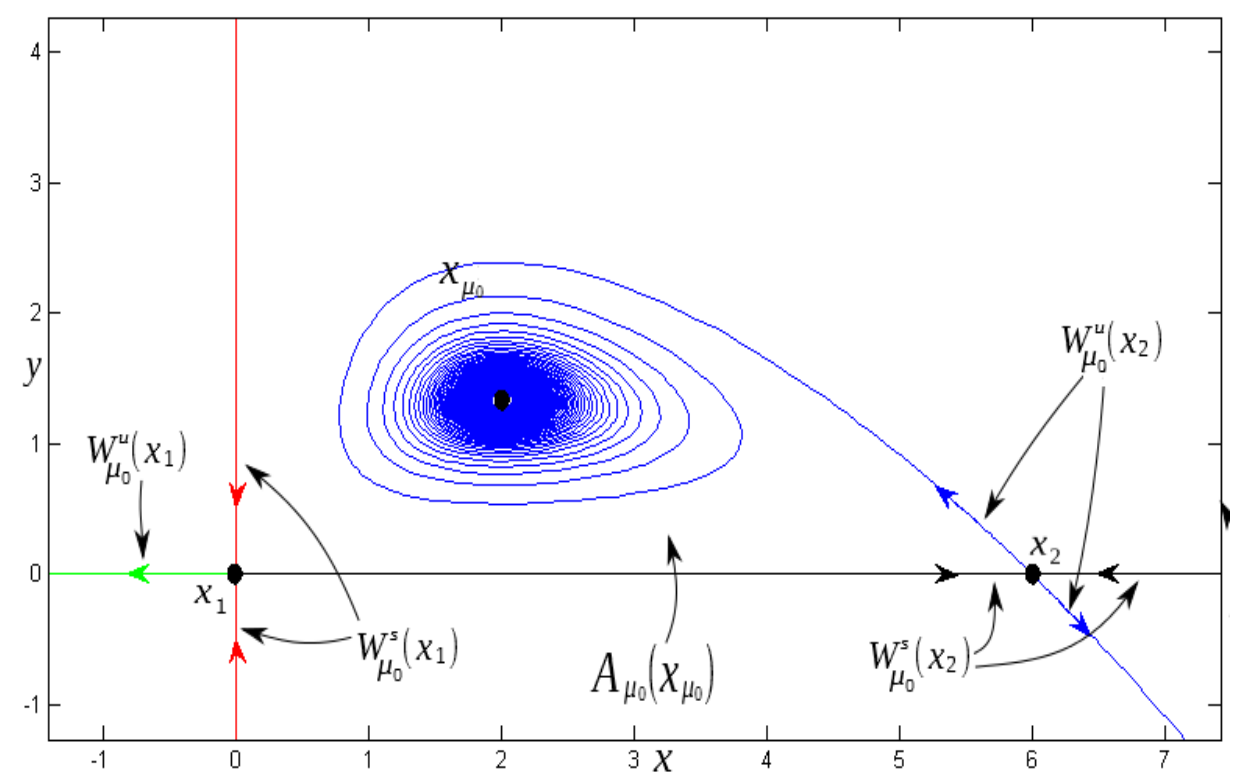

Figura 9.4: Retrato de fase do sistema (9.2) para $\mu_{0}=\frac{1}{6}$. A fronteira da região de estabilidade $\partial A_{\mu_{0}}\left(x_{\mu_{0}}\right)$ está contida na união da variedade estável $W_{\mu_{0}}^{s}\left(x_{1}\right)$ do ponto de equilíbrio hiperbólico do tipo-1 $x_{1}=(1,0)$, a curva em vermelho na Figura 9.4, com a variedade estável $W_{\mu_{0}}^{s}\left(x_{2}\right)$ do ponto de equilíbrio hiperbólico do tipo-1 $x_{2}=(6,0)$, a curva em preto na Figura 9.4.

Para $\mu=\frac{4}{10}$, o sistema (9.2) possui três pontos de equilíbrio, são eles: dois pontos de equilíbrio hiperbólicos do tipo- $1, x_{1}=(0,0)$ e $x_{2}=\left(\frac{5}{2}, 0\right)$, e um ponto de equilíbrio assintoticamente estável, $x_{\mu}^{H}=\left(2, \frac{2}{5}\right)$. O ponto de equilíbrio $x_{\mu}^{H}$ é originado do ponto de equilíbrio Hopf supercrítico do tipo-zero em uma bifurcação Hopf supercrítica do tipo-zero. Os pontos de equilíbrio hiperbólicos $x_{1}=(0,0)$ e $x_{2}=\left(\frac{5}{2}, 0\right)$ pertencem à fronteira da região de 
estabilidade $\partial A_{\mu}\left(x_{\mu}^{H}\right)$, de acordo com o Teorema 9.2.1, ver Figura 9.5.

Para $\mu=\frac{8}{100}$, o sistema (9.2) possui quatros elementos críticos, são eles: dois pontos de equilíbrio hiperbólicos do tipo-1, $x_{1}=(0,0)$ e $x_{2}=$ $\left(\frac{25}{2}, 0\right)$, um ponto de equilíbrio hiperbólico do tipo-2, $x_{\mu}^{H}=\left(2, \frac{42}{25}\right)$ e uma órbita periódica assintoticamente estável $\phi_{\mu}^{H}$. Os elementos críticos $x_{\mu}^{H}$ e $\phi_{\mu}^{H}$ foram originados do ponto de equilíbrio Hopf supercrítico do tipo-zero em uma bifurcação Hopf supercrítica do tipo-zero. Os pontos de equilíbrio hiperbólicos $x_{1}, x_{2}$ e $x_{\mu}^{H}$ pertencem à fronteira da região de estabilidade $\partial A_{\mu}\left(\phi_{\mu}^{H}\right)$, de acordo com o Teorema 9.2.1, ver Figura 9.6.

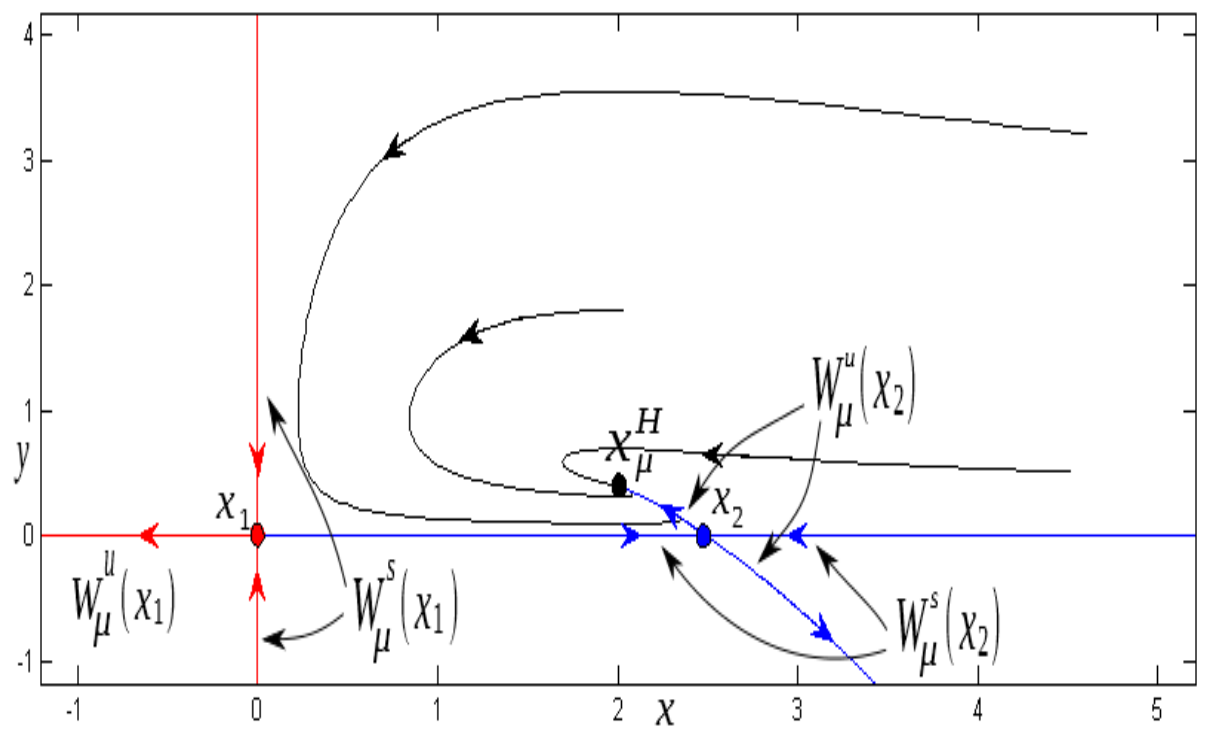

Figura 9.5: Retrato de fase do sistema (9.2) para $\mu=\frac{4}{10}$. A fronteira da região de estabilidade $\partial A_{\mu}\left(x_{\mu}^{s}\right)$ está contida na união da variedade estável $W_{\mu}^{s}\left(x_{1}\right)$ do ponto de equilibrio hiperbólico do tipo-1 $x_{1}=(0,0)$, a curva em vermelho na Figura 9.5, com a variedade estável $W_{\mu}^{s}\left(x_{2}\right)$ do ponto de equilíbrio hiperbólico do tipo-1 $x_{2}=\left(\frac{5}{2}, 0\right)$, a curva em azul na Figura 9.5. 


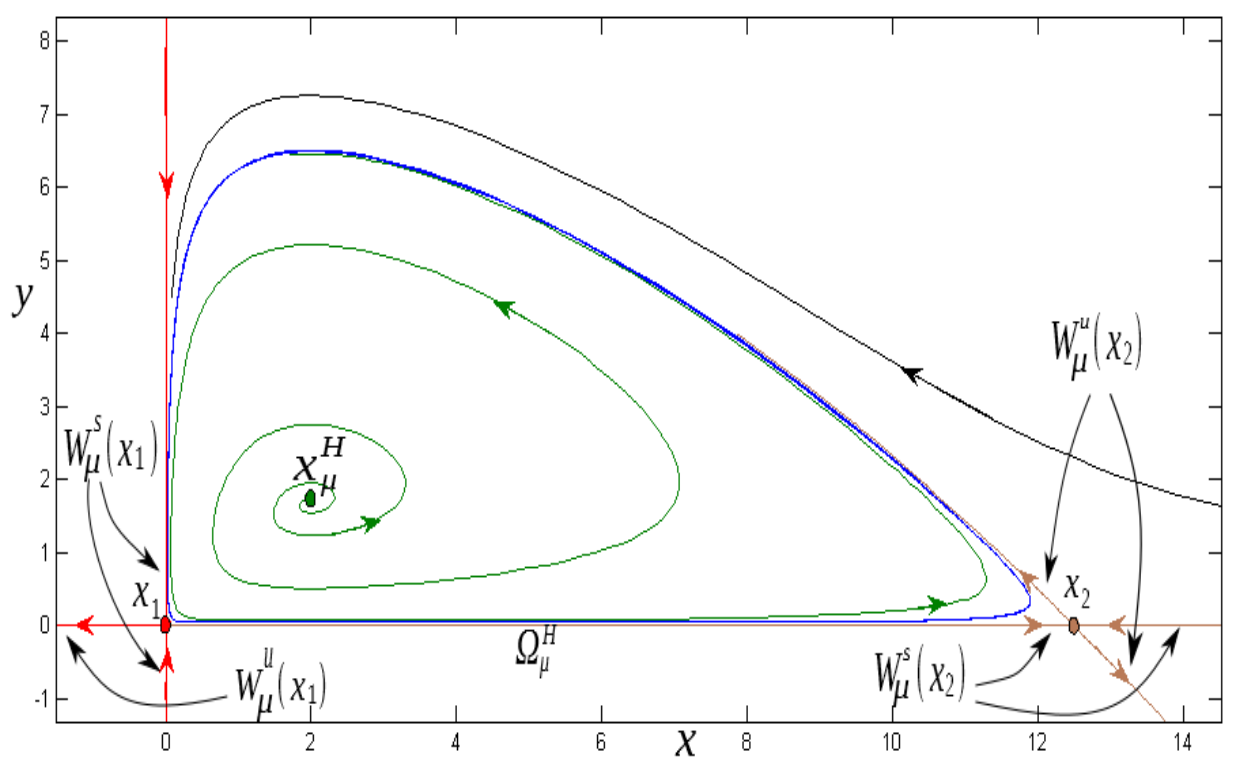

Figura 9.6: Retrato de fase do sistema (9.2) para $\mu=\frac{8}{100}$. A fronteira da região de estabilidade $\partial A_{\mu}\left(\phi_{\mu}^{H}\right)$ é formada pela união da variedade estável $W_{\mu}^{s}\left(x_{1}\right)$ do ponto de equilíbrio hiperbólico do tipo-1 $x_{1}=(0,0)$, a curva em vermelho na Figura 9.6, com a variedade estável $W_{\mu}^{s}\left(x_{2}\right)$ do ponto de equilíbrio hiperbólico do tipo-1 $x_{2}=\left(\frac{25}{2}, 0\right)$, a curva em marrom na Figura 9.6, e com a variedade estável $W_{\mu}^{s}\left(x_{\mu}^{H}\right)$ do ponto de equilíbrio hiperbólico do tipo-2 $x_{\mu}^{H}=\left(2, \frac{42}{25}\right)$, a curva em verde na Figura 9.6.

Exemplo 9.3.3. Considere o sistema extraído de [Mon06]

$$
\left\{\begin{array}{l}
\dot{x}=2 y \\
\dot{y}=2 x-3 x^{2}-y\left(x^{3}-x^{2}+y^{2}-\mu\right)
\end{array},\right.
$$

onde $(x, y) \in \mathbb{R}^{2}$ e $\mu \in \mathbb{R}$.

Para $\mu_{0}=-\frac{4}{27}$, vimos no Exemplo 7.5 que a fronteira da região de estabilidade fraca $\partial A_{\mu_{0}}\left(x_{\mu_{0}}\right)$ do ponto de equilíbrio supercrítico do tipo-0 $x_{\mu_{0}}=\left(\frac{2}{3}, 0\right)$ é formada pela variedade estável $W_{\mu_{0}}^{s}\left(x_{1}\right)$ do ponto de equilíbrio hiperbólico do tipo-1 $x_{1}=(0,0)$, ver Figura 9.7.

Para $\mu=-\frac{3}{10}$, o sistema (9.3) possui dois pontos de equilíbrio, são eles: um ponto de equilíbrio hiperbólico do tipo-1, $x_{1}=(0,0)$, e um ponto de equilíbrio assintoticamente estável, $x_{\mu}^{H}=\left(\frac{2}{3}, 0\right)$. O ponto de equilíbrio $x_{\mu}^{H}$ é originado do ponto de equilíbrio Hopf supercrítico do tipo-zero em uma bifurcação Hopf supercrítica do tipo-zero. O ponto de equilíbrio hiperbólico $x_{1}=(0,0)$ pertence à fronteira da região de estabilidade $\partial A_{\mu}\left(x_{\mu}^{H}\right)$, de acordo com o Teorema 9.2.1, ver Figura 9.8.

Para $\mu=-\frac{1}{10}$, o sistema (9.3) possui três elementos críticos, são eles: 
um ponto de equilíbrio hiperbólico do tipo-1, $x_{1 \mu}=(0,0)$, um ponto de equilíbrio hiperbólico do tipo-1, $x_{\mu}^{H}=\left(\frac{2}{3}, 0\right)$ e uma órbita periódica assintoticamente estável $\phi_{\mu}^{H}$. Os elementos críticos $x_{\mu}^{H}$ e $\phi_{\mu}^{H}$ foram originados do ponto de equilíbrio Hopf supercrítico do tipo-zero em uma bifurcação Hopf supercrítica do tipo-zero. Os pontos de equilíbrio hiperbólicos $x_{1 \mu}$ e $x_{\mu}^{H}$ pertencem à fronteira da região de estabilidade $\partial A_{\mu}\left(\phi_{\mu}^{H}\right)$, de acordo com o Teorema 9.2.1, ver Figura 9.9.

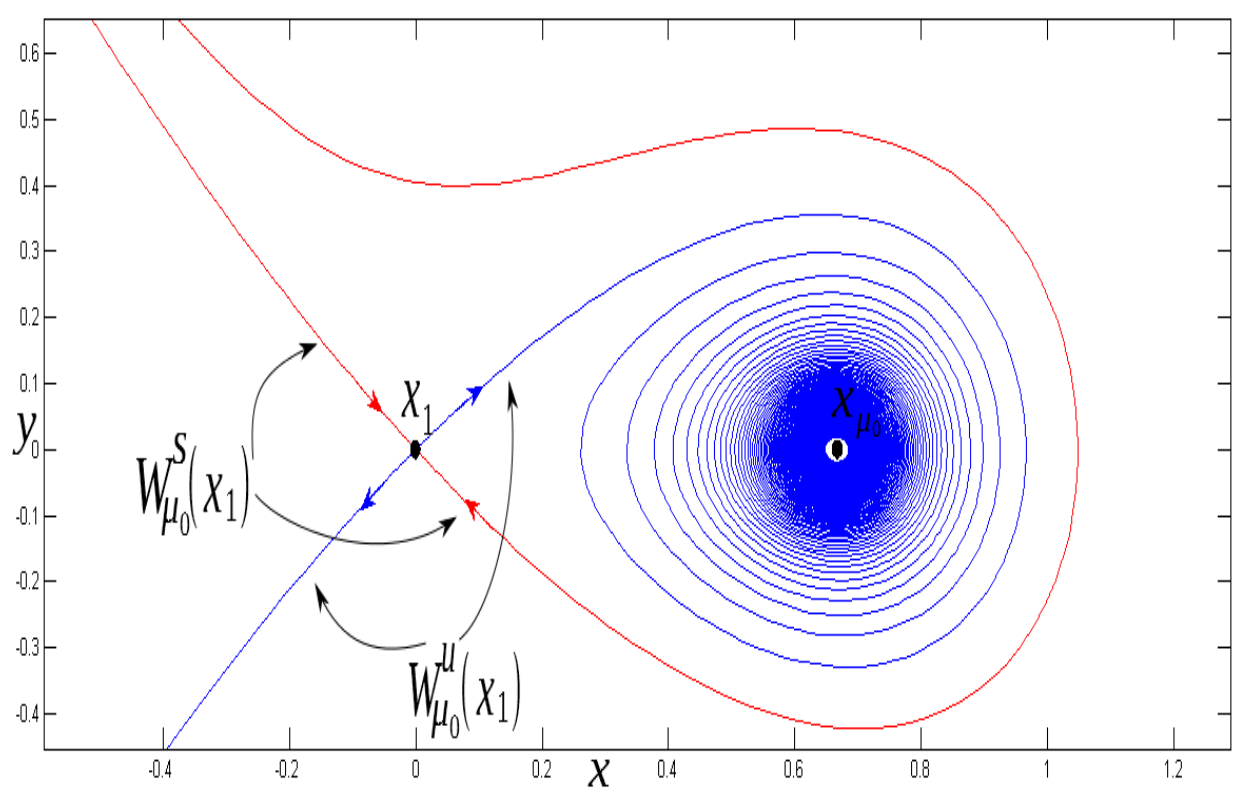

Figura 9.7: Retrato de fase do sistema (9.3) para $\mu_{0}=-\frac{4}{27}$. A fronteira da região de estabilidade $\partial A\left(x_{\mu_{0}}\right)$ é formada pela variedade estável $W_{\mu_{0}}^{s}\left(x_{1}\right)$ do ponto de equilíbrio hiperbólico do tipo-1 $x_{1}=(0,0)$, a curva em azul. 


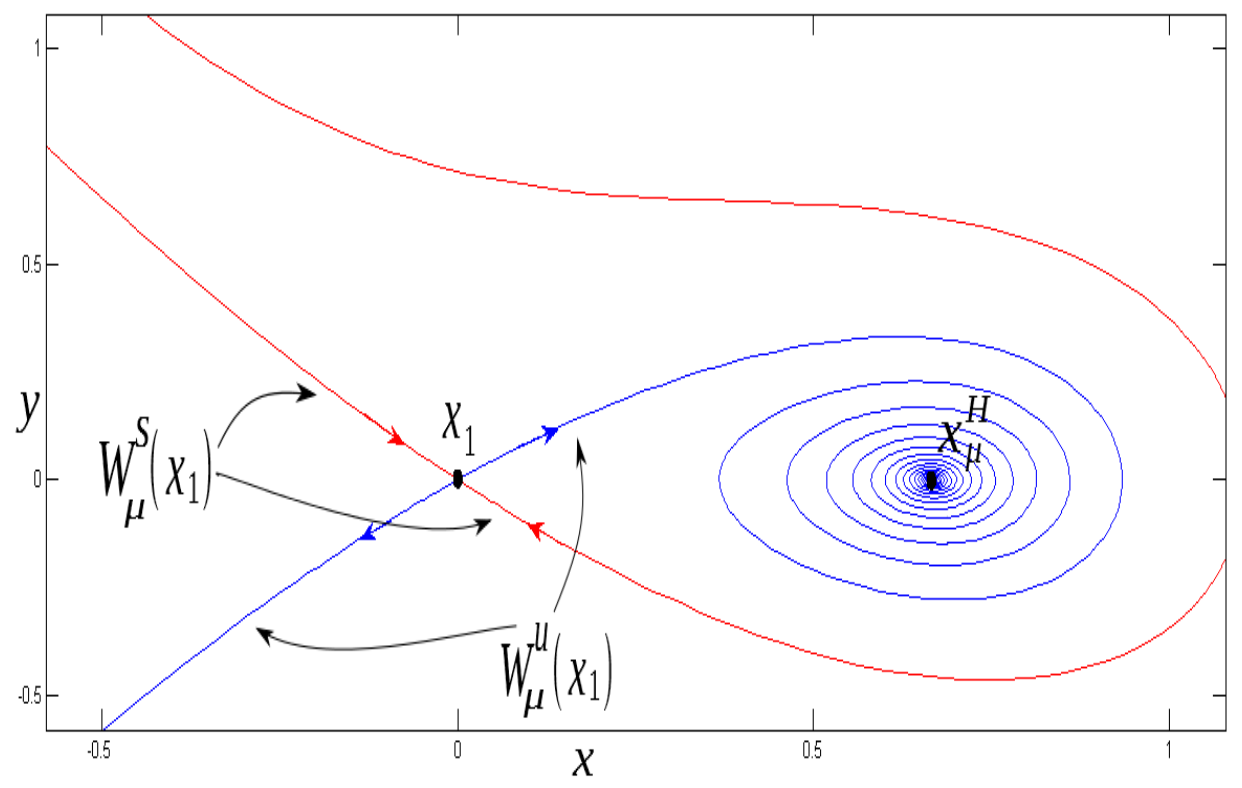

Figura 9.8: Retrato de fase do sistema (9.3) para $\mu=-\frac{3}{10}$. A fronteira da região de estabilidade $\partial A\left(x_{\mu}^{H}\right)$ é formada pela variedade estável $W_{\mu}^{s}\left(x_{1}\right)$ do ponto de equilibrio hiperbólico do tipo-1 $x_{1}=(1,0)$, a curva em vermelho.

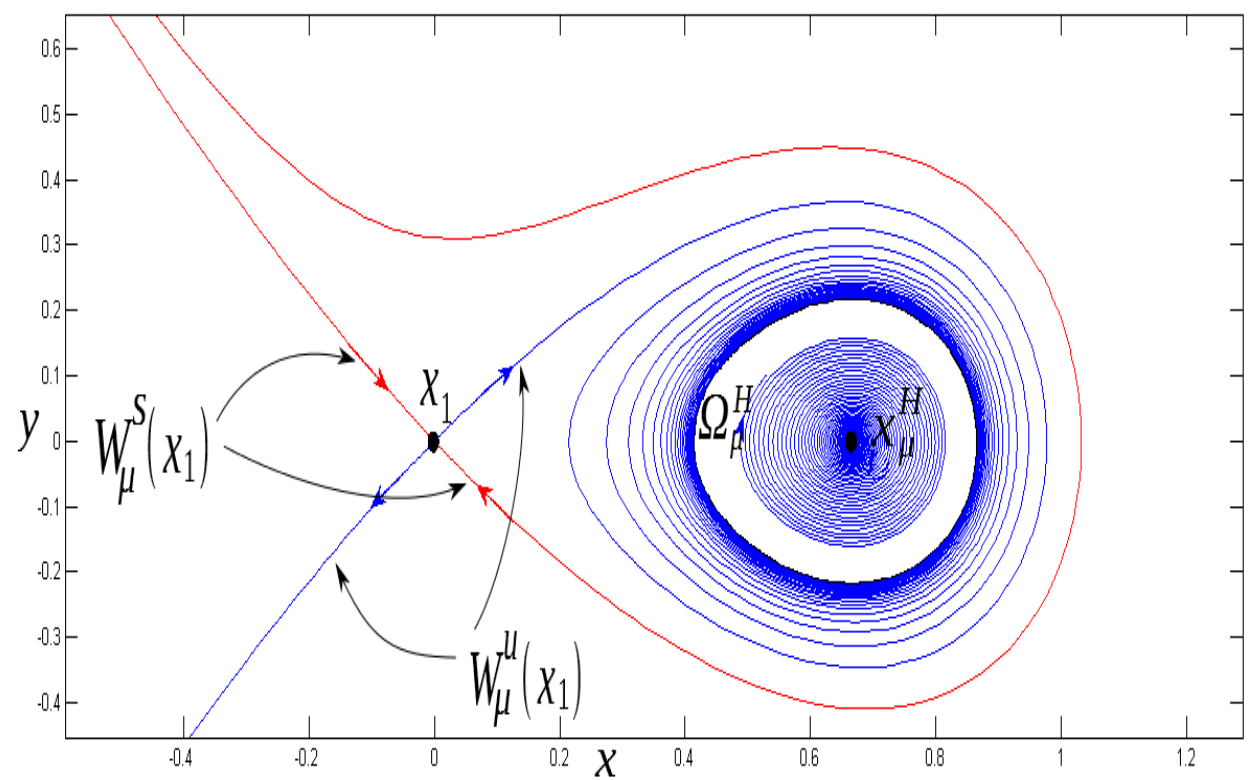

Figura 9.9: Retrato de fase do sistema (9.3) para $\mu=-\frac{1}{10}$. A fronteira da região de estabilidade $\partial A\left(\Omega_{\mu}^{H}\right)$ é formada pela união da variedade estável $W_{\mu}^{s}\left(x_{1}\right)$ do ponto de equilíbrio hiperbólico do tipo-1 $x_{1}=(1,0)$, a curva em vermelho, com a variedade estável $W_{\mu}^{s}\left(x_{\mu}^{H}\right)$ do ponto de equilíbrio hiperbólico do tipo-1 $x_{\mu}^{H}=\left(\frac{2}{3}, 0\right)$. 
Exemplo 9.3.4. Considere o sistema Predador-Presa em $\mathbb{R}^{3}$ extraído de [Vér13]

$$
\left\{\begin{array}{l}
\dot{x}=x(8-\mu-(3-\mu) x-3 y-2 z) \\
\dot{y}=y(3-\mu-2 x-(1-\mu) y) \\
\dot{z}=z(3-\mu-2 y-(1-\mu) z)
\end{array},\right.
$$

onde $(x, y, z) \in \mathbb{R}^{3}$ e $\mu \in \mathbb{R}$.

Para $\mu_{0}=0$, vimos no Exemplo 7.1.2 que a fronteira da região de estabilidade de $x_{\mu_{0}}^{s}=(0,0,3)$ está contida na união da variedade estável $W_{\mu_{0}}^{s}\left(x_{1}\right)$ do ponto de equilíbrio hiperbólico do tipo-1 $x_{1}=\left(\frac{8}{3}, 0,0\right)$, da variedade estável $W_{\mu_{0}}^{s}\left(x_{2}\right)$ do ponto de equilíbrio hiperbólico do tipo-1 $x_{2}=(2 / 3,0,3)$ com a variedade estável $W_{\mu_{0}}^{s}\left(x_{3}\right)$ do ponto de equilíbrio hiperbólico do tipo-1 $x_{3}=(1 / 3,7 / 3,0)$, ver Figura 9.10.

Para $\mu=-0.2$, o sistema (9.3.1) possui oitos pontos de equilíbrio, são eles: três pontos de equilíbrio hiperbólicos do tipo-1, $x_{1}=(2.5625,0,0)$, $x_{2}=(0.8958,0,2.6667)$ e $x_{3}=(0,2.6667,0)$, dois pontos de equilíbrio hiperbólicos do tipo-2, $x_{4}=(0,0,2.6667)$ e $x_{6}=(0,2.6667,-1.7778)$, um ponto de equilíbrio hiperbólico do tipo-3 $x_{5}=(0,0,0)$ e dois pontos de equilíbrio assintoticamente estáveis, $x_{\mu}^{s}=(-0.1111,2.8518,0)$ e $x_{\mu}^{H}=$ $(1,1,1)$. O ponto de equilíbrio $x_{\mu}^{H}$ é originado do ponto de equilíbrio Hopf supercrítico do tipo-0 em uma bifurcação Hopf supercrítica do tipo-0. Os pontos de equilíbrio hiperbólicos $x_{1}, x_{2}, x_{3}$ e $x_{4}$ pertencem à fronteira da região de estabilidade $\partial A_{\mu}\left(x_{\mu}^{H}\right)$, de acordo com o Teorema 9.1.6, ver Figura 9.11 .

Para $\mu=0.02$, o sistema (9.3.1) possui nove elementos críticos, são eles: três pontos de equilíbrio hiperbólicos do tipo-1, $x_{1}=(2.6778,0,0)$, $x_{2}=(0.6370,0,3.0408)$ e $x_{3}=(0.3635,2.2989,0)$, três pontos de equilíbrio hiperbólicos do tipo-2, $x_{4}=(0,0,3.0408), x_{6}=(0,3.0408,-3.1649) e$ $x_{\mu}^{H}=(1,1,1)$, um ponto de equilíbrio hiperbólico do tipo-3 $x_{5}=(0,0,0)$, um ponto de equilíbrio assintoticamente estável, $x_{\mu}^{s}=(0,3.0408,0)$ e uma órbita periódica assintoticamente estável $\phi_{\mu}^{H}$. Os elementos críticos $x_{\mu}^{H}$ e $\phi_{\mu}^{H}$ forma originados do ponto de equilíbrio Hopf supercrítico do tipo-0 em uma bifurcação Hopf supercrítica do tipo-0. Os pontos de equilíbrio hiperbólicos $x_{1}, x_{2}, x_{3}$ e $x_{\mu}^{H}$ pertencem à fronteira da região de estabilidade $\partial A_{\mu}\left(\phi_{\mu}^{H}\right)$, de acordo com o Teorema 9.1.6, ver Figura 9.12. 

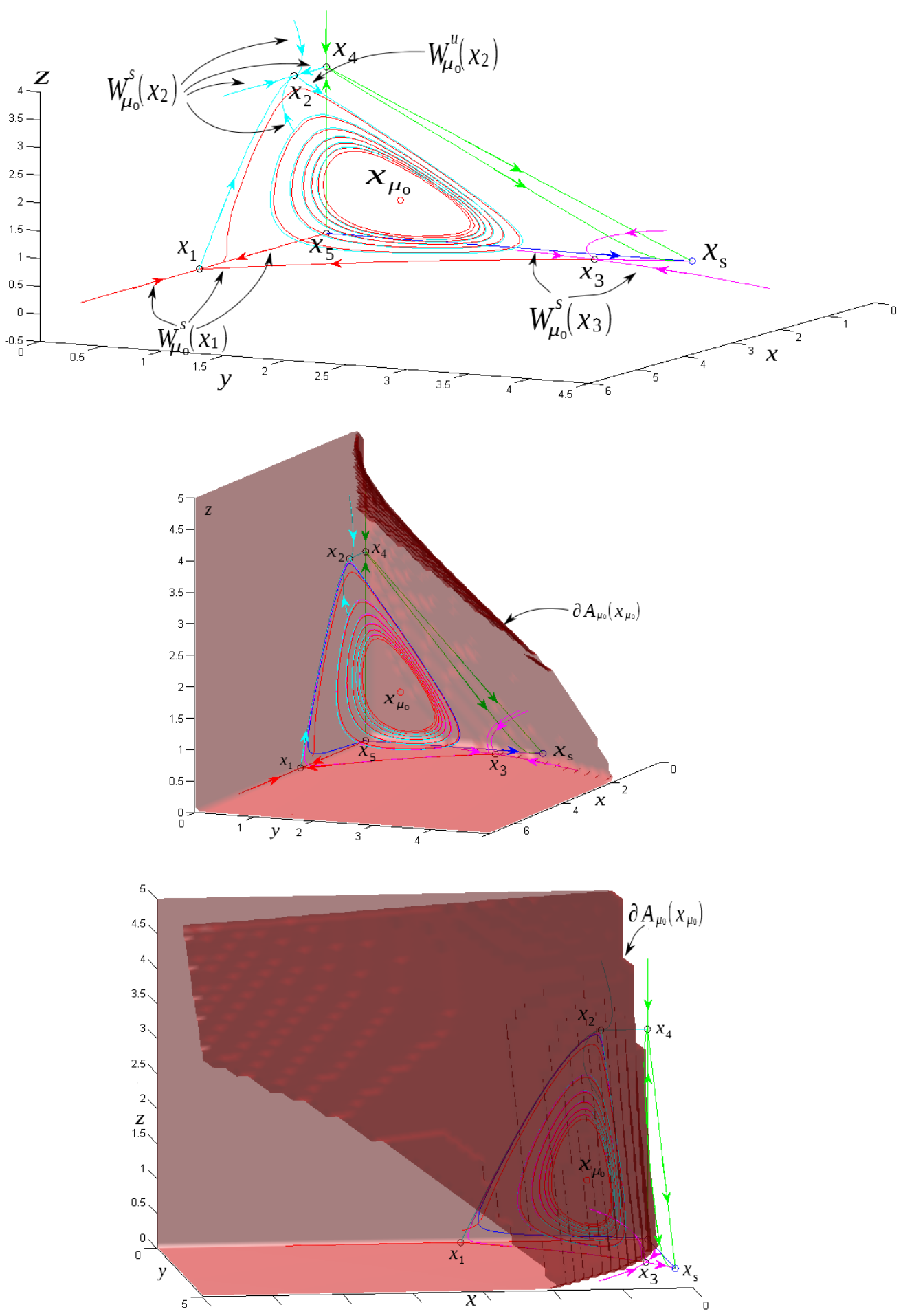

Figura 9.10: Retrato de fase do sistema (9.4) para $\mu_{0}=0$. A fronteira da região de estabilidade fraca $\partial A\left(x_{\mu_{0}}^{H}\right)$ está contida na união da variedade estável $W_{\mu_{0}}^{s}\left(x_{1}\right)$ do ponto de equilíbrio hiperbólico do tipo-1 $x_{1}=(8 / 3,0,0)$, da variedade estável $W_{\mu_{0}}^{s}\left(x_{2}\right)$ do ponto de equilíbrio hiperbólico do tipo- $1 x_{2}=(2 / 3,0,3)$ com a variedade estável $W_{\mu_{0}}^{s}\left(x_{3}\right)$ do ponto de equilíbrio hiperbólico do tipo-1 $x_{3}=(1 / 3,7 / 3,0)$. 

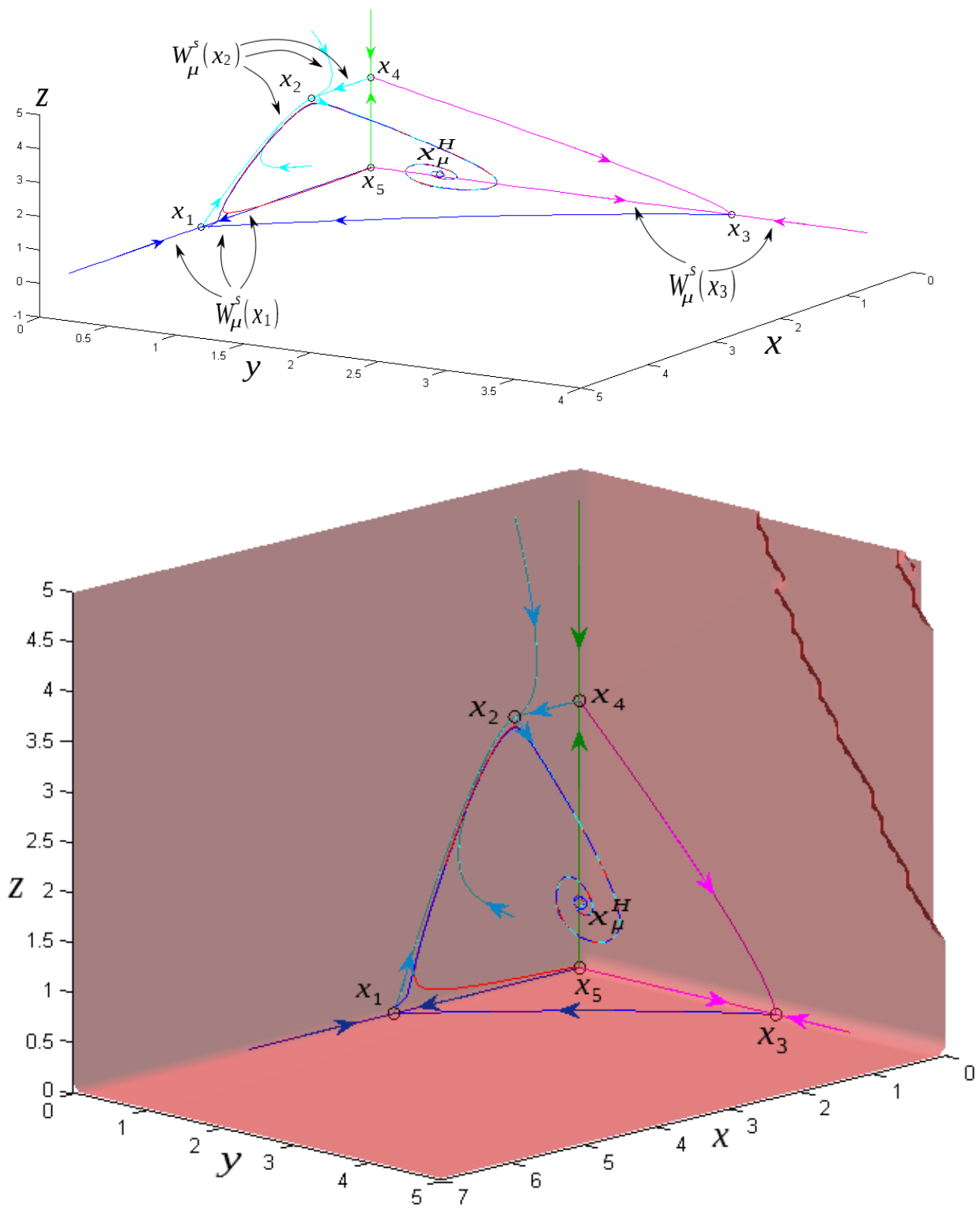

Figura 9.11: Retrato de fase do sistema (9.4) para $\mu=-0.2$. A fronteira da região de estabilidade $\partial A_{\mu}\left(x_{\mu}^{H}\right)$ está contida na união da variedade estável $W_{\mu}^{s}\left(x_{1}\right)$ do ponto de equilíbrio hiperbólico do tipo-1 $x_{1}=(2.5625,0,0)$, da variedade estável $W_{\mu}^{s}\left(x_{2}\right)$ do ponto de equilibrio hiperbólico do tipo- $1 x_{2}=(0.8958,0,2.6667)$, da variedade estável $W_{\mu}^{s}\left(x_{3}\right)$ do ponto de equilíbrio hiperbólico do tipo-1 $x_{3}=(0,2.6667,0)$ e com a variedade estável $W_{\mu}^{s}\left(x_{4}\right)$ do ponto de equilíbrio hiperbólico do tipo-2 $x_{4}=(0,0,2.6667)$. 

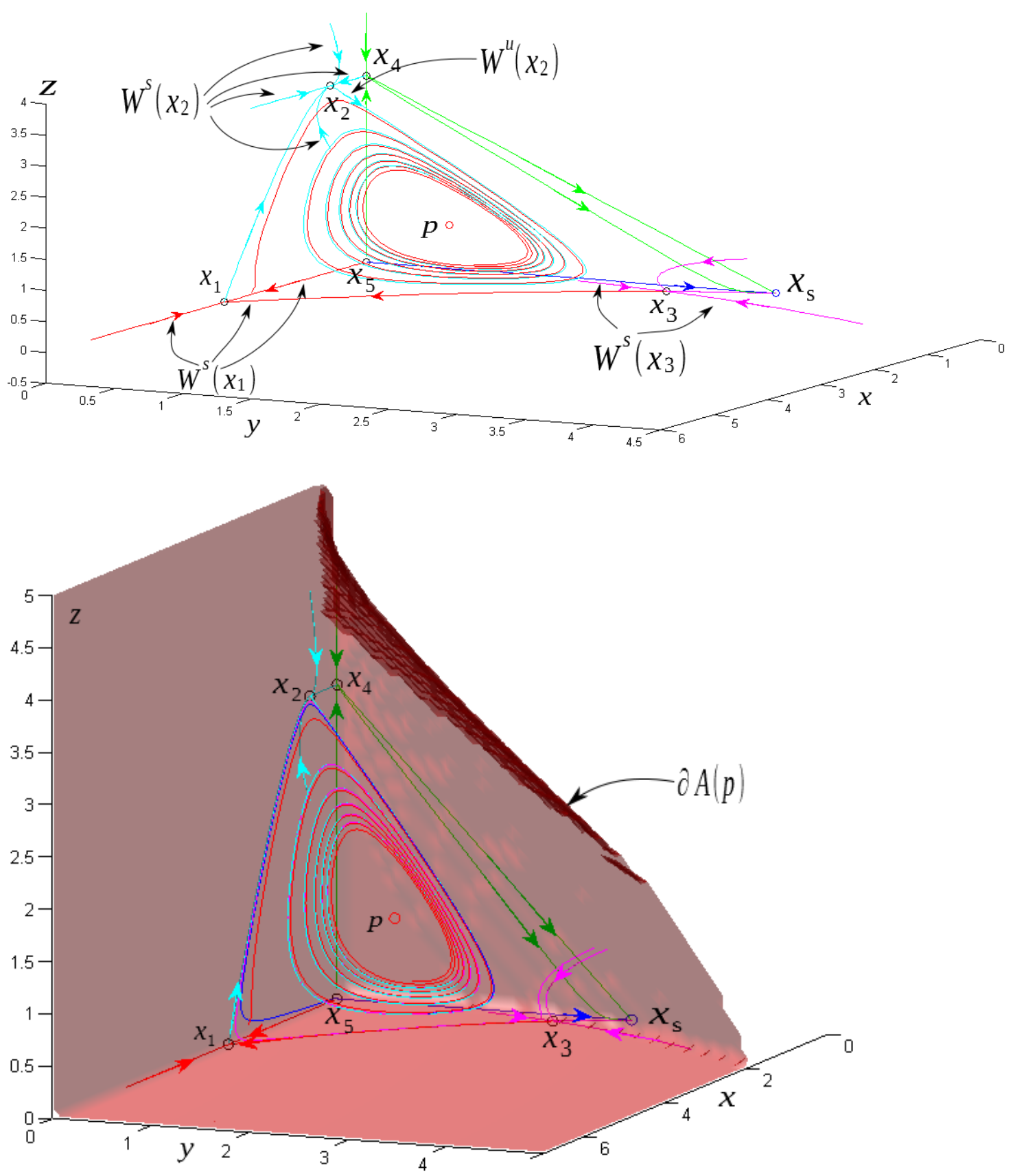

Figura 9.12: Retrato de fase do sistema (9.4) para $\mu_{0}=0.02$. A fronteira da região de estabilidade $\partial A_{\mu}\left(\phi_{\mu}^{H}\right)$ está contida na união da variedade estável $W_{\mu}^{s}\left(x_{1}\right)$ do ponto de equilíbrio hiperbólico do tipo-1 $x_{1}=(2.6778,0,0)$, da variedade estável $W_{\mu}^{s}\left(x_{2}\right)$ do ponto de equilíbrio hiperbólico do tipo-1 $x_{2}=(0.6370,0,3.0408)$, da variedade estável $W_{\mu}^{s}\left(x_{3}\right)$ do ponto de equilíbrio hiperbólico do tipo-1 $x_{3}=$ $(0.3635,2.2989,0)$ com a variedade estável $W_{\mu}^{s}\left(x_{\mu}^{H}\right)$ do ponto de equilíbrio hiperbólico do tipo-2 $x_{\mu}^{H}=(1,1,1)$. 
202 Capitulo 9. Bifurcação de Hopf na Fronteira da Região de Estabilidade 


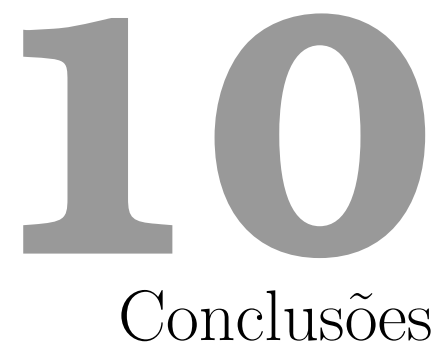

Este trabalho é parte de uma série de estudos com o objetivo de entender o comportamento da região de estabilidade de sistemas dinâmicos autônomos não lineares a variações de um parâmetro. Em particular, estudamos nesta tese como a fronteira da região de estabilidade de um ponto de equilíbrio assintoticamente estável se comporta quando surge uma Bifurcação de Hopf no sistema. Em outras palavras, estudamos o comportamento da fronteira da região de estabilidade ao variarmos o parâmetro numa vizinhança do parâmetro de bifurcação de Hopf.

O comportamento da região de estabilidade e de sua fronteira foi estudado em duas situações distintas. No primeiro caso, estudamos o comportamento da região de estabilidade quando uma bifurcação de Hopf ocorre na fronteira da região de estabilidade e no segundo caso, quando uma bifurcação de Hopf ocorre no atrator.

Para estudar o primeiro caso, caracterizamos a fronteira da região de estabilidade de um ponto de equilíbrio assintoticamente estável na presença de um ponto de equilíbrio Hopf e de elementos críticos hiperbólicos. Em seguida, analisamos a influência do conjunto de parâmetros sobre a região de atração. Exibimos resultados que estabelecem o comportamento da região de estabilidade e de sua fronteira quando ocorre uma bifurcação de Hopf na fronteira. Verificamos que à esquerda do valor crítico de bifurcação Hopf subcrítica, a fronteira da região de estabilidade do ponto de equilíbrio assintoticamente estável é a união das variedades estáveis dos elementos críticos que persistem na fronteira com as variedades estáveis do ponto de equilíbrio hiperbólico e da órbita fechada hiperbólica originados da bifurcação Hopf subcrítica. Após a órbita periódica coalescer com o ponto de equilíbrio hiperbólico, mostramos que a fronteira da região de estabilidade do ponto de 
equilíbrio assintoticamente estável como a união das variedades estáveis dos elementos críticos que persistem na fronteira mais a variedade estável do ponto de equilíbrio hiperbólico originado da bifurcação Hopf subcrítica.

Exibimos a fronteira da região de estabilidade do ponto de equilíbrio assintoticamente estável como a união das variedades estáveis dos elementos críticos que persistem na fronteira mais a variedade estável do ponto de equilíbrio hiperbólico originado da bifurcação à esquerda do valor crítico de bifurcação Hopf supercrítica. A órbita fechada hiperbólica surge quando o parâmetro cruza o eixo imaginário e neste caso, a fronteira da região de estabilidade do ponto de equilíbrio assintoticamente estável como a união das variedades estáveis dos elementos críticos que persistem na fronteira mais as variedades estáveis do ponto de equilíbrio hiperbólico e da órbita fechada hiperbólica originados da bifurcação Hopf supercrítica.

Concluímos que embora ocorra uma mudança topológica na caracterização da fronteira da região de estabilidade com a bifurcação de Hopf, não ocorre variações significativas ou abruptas no "tamanho " da região de estabilidade. Explorar estas caracterizações para obter estimativas da região de estabilidade na presença de um ponto de equilíbrio Hopf na fronteira e estudar o comportamento dessas estimativas na vizinhança de um parâmetro de bifurcação do tipo Hopf é um tema que poderá ser explorado em pesquisas futuras.

Para estudar o segundo caso, exploramos e apresentamos uma caracterização topológica da região de estabilidade fraca do ponto de equilíbrio Hopf supercrítico do tipo-zero e caracterizamos a fronteira da região de estabilidade fraca.

Mostramos o comportamento da fronteira da região de estabilidade na vizinhança de um valor de parâmetro crítico onde ocorre a bifurcação de Hopf supercrítica e as mudanças que surgem devido a essa bifurcação no atrator. Verificamos que o ponto de equilíbrio assintoticamente estável quando passa pelo parâmetro de bifurcação de Hopf supercrítica persiste mas pertence à fronteira da região de estabilidade da órbita periódica estável originada da bifurcação de Hopf supercrítica. Expomos a fronteira da região de estabilidade da órbita periódica estável originado da Bifurcação de Hopf supercrítica como a união das variedades estáveis dos elementos críticos que persistem na fronteira e do ponto de equilíbrio hiperbólico originado da bifurcação de Hopf supercrítica. Este equilíbrio pertence à interseção da fronteira de quase-estabilidade com o fecho da região de estabilidade da órbita periódica estável. E antes do parâmetro passar pelo parâmetro de bifurcação, a fronteira da região de estabilidade do ponto de equilíbrio assintoticamente estável originado da bifurcação Hopf supercrítica é a união das variedades estáveis dos elementos críticos que persistem na fronteira.

Caracterizar a fronteira de região de estabilidade na presença de um ponto de equilíbrio Hopf e estudar o comportamento da região de estabilidade e de sua fronteira na vizinhança de um parâmetro de bifurcação do tipo 
Hopf para outros tipos de sistemas, tais como sistemas discretos ou sistemas algébrico-diferenciais, poderá ser tema de futuras pesquisas nesta área.

\subsection{Publicações Relacionadas à Tese}

i. Gouveia Jr, Josaphat Ricardo Ribeiro, Fabíolo Moraes Amaral, and Luis Fernando Costa Alberto. "Stability boundary characterization of nonlinear autonomous dynamical systems in the presence of a supercritical Hopf equilibrium point." International Journal of Bifurcation and Chaos, vol. 23, n. 12, p. 1350196, 2013;

ii. Gouveia Jr, Josaphat Ricardo Ribeiro, Fabiolo Moraes Amaral, and Luis Fernando Costa Alberto. "Supercritical Hopf equilibrium points on the Boundary of the Stability Region." In: Decision and Control (CDC), 2013 IEEE 52nd Annual Conference on. IEEE, p. 5252-5257, 2013. 


\section{A \\ Algoritmo para o cálculo do primeiro coeficiente de Lyapunov para sistemas bidimensional}

Apresentaremos o algoritmo computacional desenvolvido para o software Maple para o cálculo do primeiro coeficiente de Lyapunov para sistemas dinâmico não lineares bidimensionais, ver [Kuz13], [Vér13] e [Gal12]. Considere o sistema dinâmico bidimensional

$$
\dot{x}=f(x, \mu), x \in \mathbb{R}^{2}, \mu \in \mathbb{R},
$$

O algoritmo está estruturado nas seguintes etapas:

Etapa 1 Encontrar o valor de bifurcação $\mu_{0}$ para o qual o sistema (B.1) possui um ponto de equilíbrio $x_{0}$ com autovalores $\lambda_{1,2}= \pm \omega_{0} i, \omega_{0}>0$. Este valor de bifurcação pode ser encontrado, resolvendo o sistema

$$
\left\{\begin{array}{l}
f(x, \mu)=0 \\
\operatorname{tr}(D f(x, \mu))=0
\end{array}\right.
$$

onde $\operatorname{tr}(A)$ é o traço da matriz $A$, e em seguida, verificar se $\operatorname{det} D f(x, \mu)=$ $\omega_{0}^{2}>0$, pois $\lambda_{1,2}=\frac{1}{2}\left(\operatorname{tr}(D f(x, \mu)) \pm \sqrt{(\operatorname{tr}(D f(x, \mu)))^{2}-4 \operatorname{det} D f(x, \mu)}\right)$.

Etapa 2 Calcular os autovetores complexos $q, p \in \mathbb{C}^{2}$,

$$
A q=i \omega_{0} q \text { e } A^{T} p=-i \omega_{0} p
$$


satisfazendo a condição de normalização $\langle p, q\rangle=1$, onde $A=D f\left(x_{0}, \mu_{0}\right)$ e $\langle p, q\rangle=\sum_{i=1}^{n} \bar{p}_{i} q_{i}$ é o produto escalar padrão em $\mathbb{C}^{2}$.

Etapa 3 Encontrar os coeficientes $g_{20}, g_{11}$ e $g_{21}$

$$
\begin{gathered}
g_{20}=\langle p, B(q, q)\rangle, g_{11}=\langle p, B(q, \bar{q})\rangle, \\
g_{21}=\langle p, C(q, q, \bar{q})\rangle
\end{gathered}
$$

onde as funções multilineares $B(x, y)$ e $C(x, y, z)$ são dadas por

$$
B_{i}(x, y)=\left.\sum_{j, k=1}^{n} \frac{\partial^{2} F_{i}(\xi)}{\partial \xi_{j} \partial \xi_{k}}\right|_{\xi=0} x_{j} y_{k}, i=1, \ldots, n
$$

e

$$
C_{i}(x, y, z)=\left.\sum_{j, k, l=1}^{n} \frac{\partial^{3} F_{i}(\xi)}{\partial \xi_{j} \partial \xi_{k} \partial \xi_{l}}\right|_{\xi=0} x_{j} y_{k} z_{l}, i=1, \ldots, n .
$$

Etapa 4 Calcular o primeiro coeficiente de Lyapunov dado por

$$
l_{1}=\frac{1}{2 \omega_{0}^{2}} \Re\left(i g_{20} g_{11}+\omega_{0} g_{21}\right)
$$

\section{A.1 Cálculo do coeficiente de Lyapunov do sis- tema (7.1)}

[> restart:

$[>$ with(linalg) $:$ with (student) $:$ with (Linear Algebra) :

[> with(Student $[$ Linear Algebra $])$ :

[> readlib(mtaylor) :

$[>$ readlib (coeftayl) :

Sistema

$\left[>F[1]:=X[1]-X[1] * X[2] /(1+0.5 * X[1])-X[1]^{2} / 6:\right.$

$[>F[2]:=-X[2] *(1-X[1] /(1+0.5 * X[1])):$

Pontos de Equilíbrios

[> solve $(F[1], F[2], X[1], X[2])$;

$$
\left\{X_{1}=0, X_{2}=0\right\},\left\{X_{1}=2, X_{2}=\frac{4}{3}\right\},\left\{X_{1}=6, X_{2}=0\right\}
$$

Matriz Jacobiana e Estabilidade dos Equilíbrios $[>J:=$ Matrix $(j a c o b i a n([F[1], F[2]],[X[1], X[2]]))$;

$$
J:=\left[\begin{array}{cc}
1-\frac{X_{2}}{1+0.5 X_{1}}+\frac{0.5 X_{1} X_{2}}{\left(1+0.5 X_{1}\right)^{2}}-\frac{X_{1}}{3} & \frac{-X 1}{1+0.5 X_{1}} \\
-X_{2}\left(-\frac{1}{1+0.5 X_{1}}+\frac{0.5 X_{1}}{\left(1+0.5 X_{1}\right)^{2}}\right) & -1+\frac{X_{1}}{1+0.5 X_{1}}
\end{array}\right]
$$


$[>J 1:=\operatorname{subs}(X[1]=0, X[2]=0, J) ;$

$$
J 1:=\left[\begin{array}{cc}
1 & 0 \\
0 & -1
\end{array}\right]
$$

$[>\operatorname{lambda}:=$ Eigenvalues $(J 1)$;

$$
\lambda:=\left[\begin{array}{c}
1 \\
-1
\end{array}\right]
$$

$[>J 2:=\operatorname{subs}(X[1]=6, X[2]=0, J) ;$

$$
J 2:=\left[\begin{array}{cc}
-1 & \frac{-3}{2} \\
0 & \frac{1}{2}
\end{array}\right]
$$

$[>\operatorname{lambda}:=$ Eigenvalues $(J 2) ;$

$$
\lambda:=\left[\begin{array}{c}
\frac{1}{2} \\
-1
\end{array}\right]
$$

$\left[>J 3:=\operatorname{subs}\left(X[1]=2, X[2]=\frac{4}{3}, J\right) ;\right.$

$$
J 3:=\left[\begin{array}{cc}
0 & -1 \\
\frac{1}{3} & 0
\end{array}\right]
$$

$[>\operatorname{lambda}:=$ Eigenvalues(J3);

$$
\lambda:=\left[\begin{array}{c}
\frac{1}{3} I \sqrt{3} \\
-\frac{1}{3} I \sqrt{3}
\end{array}\right]
$$

$[>$ omega $:=\operatorname{Im}(\operatorname{lambda}[1]) ;$

$$
\omega:=\frac{1}{3} \sqrt{3}
$$

Autovetores da Matriz J e da sua transposta $\left[>q 1:=\operatorname{eigenvects}\left(J 3{ }^{\prime}{ }^{\text {radical }}{ }^{\prime}\right)\right.$;

$$
q 1:=\left[\frac{1}{3} I \sqrt{3}, 1,\left\{\left[\begin{array}{ll}
I \sqrt{3} & 1
\end{array}\right]\right\}\right],\left[-\frac{1}{3} I \sqrt{3}, 1,\left\{\left[\begin{array}{ll}
-I \sqrt{3} & 1
\end{array}\right]\right\}\right]
$$

$[>q:=q 1[1][3][1]$

$$
q:=\left[\begin{array}{ll}
I \sqrt{3} & 1
\end{array}\right]
$$

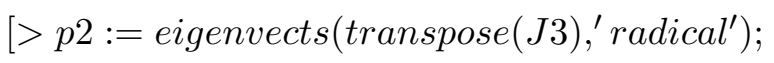

$$
p 2:=\left[\frac{1}{3} I \sqrt{3}, 1,\left\{\left[\begin{array}{ll}
-\frac{1}{3} I \sqrt{3} & 1
\end{array}\right]\right\}\right],\left[-\frac{1}{3} I \sqrt{3}, 1,\left\{\left[\begin{array}{ll}
\frac{1}{3} I \sqrt{3} & 1
\end{array}\right]\right\}\right]
$$


$[>p 1:=p 2[2][3][1]$

$$
p 1:=\left[\frac{1}{3} I \sqrt{3} \quad 1\right]
$$

$[>$ normalizacao $:=$ simplify $($ evalc $($ conjugate $(p 1[1]) * q[1]+$ conjugate ( $p 1[2]) * q[2]))$

$$
\text { normalizacao }:=2
$$

$[>$ fator $:=\operatorname{simplify}(\operatorname{evalc}(1 / \operatorname{conjugate}($ normalizacao $)))$

$$
\text { fator }:=\frac{1}{2}
$$

$[>p:=\operatorname{vector}([\operatorname{simplify}($ evalc $($ fator $* p 1[1]))$, simplify $($ evalc $($ fator $* p 1[2]$ )$)]$ );

$$
p:=\left[\begin{array}{ll}
\frac{1}{6} I \sqrt{3} & \frac{1}{2}
\end{array}\right]
$$

$[>\operatorname{simplify}($ evalc $($ conjugate $(p[1]) * q[1]+\operatorname{conjugate}(p[2]) * q[2]))$;

Deslocando o Equilibrio para a Origem

$$
\begin{gathered}
{\left[>X_{1}:=x_{1}+2: X_{2}:=x_{2}+4 / 3:\right.} \\
{\left[>F_{1}:=\operatorname{simplify}\left(X_{1}-X_{1} * X_{2} /\left(1+0.5 * X_{1}\right)-X_{1}^{2} / 6\right) ;\right.} \\
F_{1}:=-\frac{2 x_{1}^{2}+12 x_{1} x_{2}+24 x_{2}+x_{1}^{3}}{6\left(4+x_{1}\right)} \\
{\left[>F[2]:=\operatorname{simplify}\left(-X_{2} *\left(1-X_{1} /\left(1+0.5 * X_{1}\right)\right)\right) ;\right.} \\
F_{2}:=\frac{\left(3 x_{2}+4\right) x_{1}}{3\left(4+x_{1}\right)}
\end{gathered}
$$

Calculando as Funções Multilineares B(x,y) e C(x,y,z)

$[>B[1]:=2 * \operatorname{coeftayl}(F[1],[x[1], x[2]]=[0,0],[2,0]) * x i[1] * m u[1]+$ $\operatorname{coeftayl}(F[1],[x[1], x[2]]=[0,0],[1,1]) *(x i[1] * m u[2]+x i[2] * m u[1])+2$ $* \operatorname{coeftayl}(F[1],[x[1], x[2]]=[0,0],[0,2]) * x i[2] * m u[2]$;

$$
B[1]:=-\frac{1}{6} \xi_{1} \mu_{1}-\frac{1}{4} \xi_{1} \mu_{2}-\frac{1}{4} \xi_{2} \mu_{1}
$$

$[>B[2]:=2 * \operatorname{coeftayl}(F[2],[x[1], x[2]]=[0,0],[2,0]) * x i[1] * m u[1]+$ $\operatorname{coeftayl}(F[2],[x[1], x[2]]=[0,0],[1,1]) *(x i[1] * m u[2]+x i[2] * m u[1])+2$ $* \operatorname{coeftayl}(F[2],[x[1], x[2]]=[0,0],[0,2]) * x i[2] * m u[2]$;

$$
B[2]:=-\frac{1}{6} \xi_{1} \mu_{1}+\frac{1}{4} \xi_{1} \mu_{2}+\frac{1}{4} \xi_{2} \mu_{1}
$$


$[>C[1]:=6 * \operatorname{coeftayl}(F[1],[x[1], x[2]]=[0,0],[3,0]) * x i[1] * \operatorname{mu}[1] * \operatorname{eta}[1]$ $+2 * \operatorname{coeftayl}(F[1],[x[1], x[2]]=[0,0],[2,1]) *(x i[1] * m u[1] *$ eta $[2]+x i[1]$ $* m u[2] * \operatorname{eta}[1]+x i[2] * m u[1] * \operatorname{eta}[1])+2 * \operatorname{coeftayl}(F[1],[x[1], x[2]]=[0,0]$, $[1,2]) *(x i[1] * m u[2] * \operatorname{eta}[2]+x i[2] * m u[1] * \operatorname{eta}[2]+x i[2] * m u[2] *$ eta $[1])+6 * \operatorname{coeftayl}(F[1],[x[1], x[2]]=[0,0],[0,3]) * x i[2] * \operatorname{mu}[2] *$ eta $[2]$;

$$
C[1]:=-\frac{1}{8} \xi_{1} \mu_{1} \eta_{1}+\frac{1}{8} \xi_{1} \mu_{1} \eta_{2}+\frac{1}{8} \xi_{1} \mu_{2} \eta_{1}+\frac{1}{8} \xi_{2} \mu_{1} \eta_{1}
$$

$[>C[2]:=6 * \operatorname{coeftayl}(F[2],[x[1], x[2]]=[0,0],[3,0]) * x i[1] * m u[1] *$ eta $[1]$ $+2 * \operatorname{coeftayl}(F[2],[x[1], x[2]]=[0,0],[2,1]) *(x i[1] * m u[1] * \operatorname{eta}[2]+x i[1]$ $* m u[2] *$ eta $[1]+x i[2] * m u[1] *$ eta $[1])+2 * \operatorname{coeftayl}(F[2],[x[1], x[2]]=$ $[0,0],[1,2]) *(x i[1] * m u[2] *$ eta $[2]+x i[2] * m u[1] * \operatorname{eta}[2]+x i[2] * m u[2] *$ eta $[1])+6 * \operatorname{coeftayl}(F[2],[x[1], x[2]]=[0,0],[0,3]) * x i[2] * \operatorname{mu}[2] *$ eta $[2]$;

$$
C[2]:=\frac{1}{8} \xi_{1} \mu_{1} \eta_{1}-\frac{1}{8} \xi_{1} \mu_{1} \eta_{2}-\frac{1}{8} \xi_{1} \mu_{2} \eta_{1}-\frac{1}{8} \xi_{2} \mu_{1} \eta_{1}
$$

$[>B:=<B[1], B[2]>$ :

$[>C:=<C[1], C[2]>$ :

Calculando Primeiro Coeficiente de Lyapunov

$[>$ Cqqconjugateq $:=$ simplify $(\operatorname{subs}(x i[1]=q[1], x i[2]=q[2], m u[1]=q[1]$, $m u[2]=q[2]$, eta $[1]=$ conjugate $(q[1])$, eta $[2]=\operatorname{conjugate}(q[2]), C)) ;$

$$
\text { Cqqconjugateq }:=\left[\begin{array}{c}
-\frac{3}{8} I \sqrt{3}+\frac{3}{8} \\
\frac{3}{8} I \sqrt{3}-\frac{3}{8}
\end{array}\right]
$$

$[>r:=\operatorname{simplify}(\operatorname{conjugate}(p[1]) *$ Cqqconjugateq $[1]+\operatorname{conjugate}(p[2]) *$

Cqqconjugateq[2]);

$$
r:=-\frac{3}{8}+\frac{1}{8} I \sqrt{3}
$$

$[>B q q:=\operatorname{simplify}(\operatorname{subs}(x i[1]=q[1], x i[2]=q[2], m u[1]=q[1], m u[2]=q[2], B))$

$$
B q q:=\left[\begin{array}{c}
-\frac{1}{2} I \sqrt{3}+\frac{1}{2} \\
\frac{1}{2}+\frac{1}{2} I \sqrt{3}
\end{array}\right]
$$

$[>$ Bqconjugateq $:=$ simplify $(\operatorname{subs}(x i[1]=q[1], x i[2]=q[2], m u[1]=$ conjugate ( $q[1]), m u[2]=$ conjugate $(q[2]), B))$;

$$
\text { Bqconjugateq }:=\left[\begin{array}{l}
-\frac{1}{2} \\
-\frac{1}{2}
\end{array}\right]
$$

$[>s:=\operatorname{evalm}($ inverse(J3).Bqconjugateq $)$;

$$
s:=\left[\begin{array}{ll}
-\frac{3}{2} & \frac{1}{2}
\end{array}\right]
$$


$[>B q s:=\operatorname{simplify}(\operatorname{subs}(x i[1]=q[1], x i[2]=q[2], m u[1]=s[1], m u[2]=s[2]$, $B))$;

$$
B q s:=\left[\begin{array}{l}
\frac{1}{8} I \sqrt{3}+\frac{3}{8} \\
\frac{3}{8} I \sqrt{3}-\frac{3}{8}
\end{array}\right]
$$

$[>t:=\operatorname{simplify}($ conjugate $(p[1]) * B q s[1]+\operatorname{conjugate}(p[2]) * B q s[2]) ;$

$$
t:=-\frac{1}{8}+\frac{1}{8} I \sqrt{3}
$$

$[>M:=$ Matrix $(2,2$, shape $=$ identity $):$

$[>w:=\operatorname{simplify}($ evalm $($ inverse $(2 * I *$ omega $* M-J 3) . B q q))$;

$$
w:=\left[\begin{array}{ll}
-\frac{1}{2}+\frac{1}{6} I \sqrt{3} & -\frac{1}{6} I \sqrt{3}+\frac{5}{6}
\end{array}\right]
$$

$[>$ Bconjugateqw $:=\operatorname{simplify}(\operatorname{subs}(x i[1]=\operatorname{conjugate}(q[1]), x i[2]$

$=$ conjugate $(q[2]), m u[1]=w[1], m u[2]=w[2], B))$;

$$
\text { Bconjugateqw }:=\left[\begin{array}{c}
\frac{1}{12} I \sqrt{3}+\frac{1}{6} \\
-\frac{1}{4} I \sqrt{3}-\frac{1}{3}
\end{array}\right]
$$

$[>u:=\operatorname{simplify}(\operatorname{conjugate}(p[1]) *$ Bconjugateqw $[1]+\operatorname{conjugate}(p[2]) *$ Bconjugateqw $[2])$;

$$
\begin{gathered}
u:=-\frac{1}{8}-\frac{11}{72} I \sqrt{3} \\
{[>l[1]:=\operatorname{simplify}((1 /(2 * \text { omega })) * \operatorname{Re}(r-2 * t+u)) ;} \\
l_{1}:=-\frac{1}{8} I \sqrt{3}
\end{gathered}
$$

Bifuração de Hopf Supercrítica

\section{A.2 Cálculo do coeficiente de Lyapunov do sis- tema $(7.3)$}

[> restart:

$[>$ with(linalg) $:$ with (student) $:$ with (Linear Algebra) :

[> with (Student [Linear Algebra]) :

[> readlib(mtaylor) :

$[>$ readlib(coeftayl) :

Sistema

$[>F[1]:=2 * X[2]:$

$\left[>F[2]:=2 * X[1]-3 * X[1]^{2}-X[2] *\left(X[1]^{3}-X[1]^{2}+X[2]^{2}+4 / 27\right):\right.$ 
Pontos de Equilíbrios

[> solve $(F[1], F[2], X[1], X[2])$;

$$
\left\{X_{1}=0, X_{2}=0\right\},\left\{X_{1}=\frac{2}{3}, X_{2}=0\right\}
$$

Matriz Jacobiana e Estabilidade dos Equilíbrios

$[>J:=$ Matrix $(j a c o b i a n([F[1], F[2]],[X[1], X[2]]))$;

$$
\begin{gathered}
J:=\left[\begin{array}{cc}
0 & 2 \\
2-6 X_{1}-X_{2}\left(3 X_{1}^{2}-2 X[1]\right) & -X_{1}^{3}+X_{1}^{2}-3 X_{2}^{2}-.1481481481
\end{array}\right] \\
{[>J 1:=\operatorname{subs}(X[1]=0, X[2]=0, J)} \\
J 1:=\left[\begin{array}{cc}
0 & 2 \\
2 & -.1481481481
\end{array}\right]
\end{gathered}
$$

$[>$ lambda $:=$ Eigenvalues $(J 1)$

$$
\lambda:=\left[\begin{array}{c}
1.927297197 \\
-2.075445347
\end{array}\right]
$$

$\left[>J 2:=\operatorname{subs}\left(X[1]=\frac{2}{3}, X[2]=0, J\right) ;\right.$

$$
J 2:=\left[\begin{array}{cc}
0 & 2 \\
-2 & 10^{-10}
\end{array}\right]
$$

$[>\operatorname{lambda}:=$ Eigenvalues $(J 2)$;

$$
\lambda:=\left[\begin{array}{c}
2 I \\
-2 I
\end{array}\right]
$$

$[>$ omega $:=\operatorname{Im}(\operatorname{lambda}[1])$

$$
\omega:=2
$$

Autovetores da Matriz J e da sua transposta $\left[>q 1:=\operatorname{eigenvects}\left(J 2,{ }^{\prime}\right.\right.$ radical $\left.^{\prime}\right)$;

$$
q 1:=\left[2 I, 1,\left\{\left[\begin{array}{ll}
1 & I
\end{array}\right]\right\}\right],\left[-2 I, 1,\left\{\left[\begin{array}{ll}
1 & -I
\end{array}\right]\right\}\right]
$$

$[>q:=q 1[1][3][1]$

$$
q:=\left[\begin{array}{ll}
1 & I
\end{array}\right]
$$

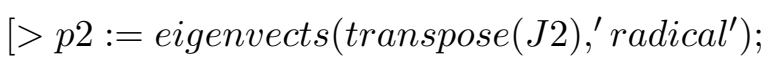

$$
p 2:=\left[2 I, 1,\left\{\left[\begin{array}{ll}
-1 & I
\end{array}\right]\right\}\right],\left[-2 I, 1,\left\{\left[\begin{array}{ll}
-1 & -I
\end{array}\right]\right\}\right]
$$


$[>p 1:=p 2[2][3][1]$

$$
p 1:=\left[\begin{array}{ll}
-1 & -I
\end{array}\right]
$$

$[>$ normalizacao $:=\operatorname{simplify}($ evalc(conjugate $(p 1[1]) * q[1]+$ conjugate ( $p 1[2]) * q[2]))$

normalizacao $:=-2$

$[>$ fator $:=\operatorname{simplify}(\operatorname{evalc}(1 / \operatorname{conjugate}($ normalizacao $)))$

$$
\text { fator }:=-0.5
$$

$[>p:=\operatorname{vector}([\operatorname{simplify}($ evalc $($ fator $* p 1[1]))$, simplify $($ evalc $($ fator $* p 1[2]$ )$)]$ );

$$
p:=\left[\begin{array}{ll}
0.5 & 0.5 I
\end{array}\right]
$$

$[>\operatorname{simplify}($ evalc $($ conjugate $(p[1]) * q[1]+\operatorname{conjugate}(p[2]) * q[2]))$;

Deslocando o Equilibrio para a Origem

$\left[>X_{1}:=x_{1}+2 / 3: X_{2}:=x_{2}:\right.$

$\left[>F_{1}:=\operatorname{simplify}(2 * X[2])\right.$;

$$
F_{1}:=2 x_{2}
$$

$\left[>F[2]:=\operatorname{simplify}\left(2 * X[1]-3 * X[1]^{2}-X[2] *\left(X[1]^{3}-X[1]^{2}+X[2]^{2}+4 / 27\right)\right)\right.$

$$
F_{2}:=-2 \cdot x_{1}-3 \cdot x_{1}^{2}-1 \cdot x_{2} x_{1}^{3}-1 \cdot x_{2} x_{1}^{2}-1 \cdot x_{2}^{3}
$$

Calculando as Funções Multilineares $\mathrm{B}(\mathrm{x}, \mathrm{y})$ e $\mathrm{C}(\mathrm{x}, \mathrm{y}, \mathrm{z})$

$[>B[1]:=2 * \operatorname{coeftayl}(F[1],[x[1], x[2]]=[0,0],[2,0]) * x i[1] * m u[1]+$ $\operatorname{coeftayl}(F[1],[x[1], x[2]]=[0,0],[1,1]) *(x i[1] * m u[2]+x i[2] * m u[1])+2$ $* \operatorname{coeftayl}(F[1],[x[1], x[2]]=[0,0],[0,2]) * x i[2] * m u[2] ;$

$$
B[1]:=0
$$

$[>B[2]:=2 * \operatorname{coeftayl}(F[2],[x[1], x[2]]=[0,0],[2,0]) * x i[1] * m u[1]+$ $\operatorname{coeftayl}(F[2],[x[1], x[2]]=[0,0],[1,1]) *(x i[1] * m u[2]+x i[2] * m u[1])+2$ $* \operatorname{coeftayl}(F[2],[x[1], x[2]]=[0,0],[0,2]) * x i[2] * m u[2]$;

$$
B[2]:=-6 \xi_{1} \mu_{1}
$$


$[>C[1]:=6 * \operatorname{coeftayl}(F[1],[x[1], x[2]]=[0,0],[3,0]) * x i[1] * \operatorname{mu}[1] * \operatorname{eta}[1]$ $+2 * \operatorname{coeftayl}(F[1],[x[1], x[2]]=[0,0],[2,1]) *(x i[1] * m u[1] * \operatorname{eta}[2]+x i[1]$ $* m u[2] * \operatorname{eta}[1]+x i[2] * m u[1] * \operatorname{eta}[1])+2 * \operatorname{coeftayl}(F[1],[x[1], x[2]]=[0,0]$, $[1,2]) *(x i[1] * m u[2] *$ eta $[2]+x i[2] * m u[1] *$ eta $[2]+x i[2] * m u[2] *$ eta $[1])+6 * \operatorname{coeftayl}(F[1],[x[1], x[2]]=[0,0],[0,3]) * x i[2] * \operatorname{mu}[2] *$ eta $[2]$;

$$
C[1]:=0
$$

$[>C[2]:=6 * \operatorname{coeftayl}(F[2],[x[1], x[2]]=[0,0],[3,0]) * x i[1] *$ mu $[1] * \operatorname{eta}[1]$ $+2 * \operatorname{coeftayl}(F[2],[x[1], x[2]]=[0,0],[2,1]) *(x i[1] * m u[1] *$ eta $[2]+x i[1]$ $* m u[2] *$ eta $[1]+x i[2] * m u[1] *$ eta $[1])+2 * \operatorname{coeftayl}(F[2],[x[1], x[2]]=$ $[0,0],[1,2]) *(x i[1] * m u[2] *$ eta $[2]+x i[2] * m u[1] * \operatorname{eta}[2]+x i[2] * m u[2] *$ eta $[1])+6 * \operatorname{coeftayl}(F[2],[x[1], x[2]]=[0,0],[0,3]) * x i[2] * \operatorname{mu}[2] *$ eta $[2]$;

$$
C[2]:=-2 \xi_{1} \mu_{1} \eta_{2}-2 \xi_{1} \mu_{2} \eta_{1}-2 \xi_{2} \mu_{1} \eta_{1}-6 \xi_{2} \mu_{2} \eta_{2}
$$

$[>B:=<B[1], B[2]>$ :

$[>C:=<C[1], C[2]>$;

Calculando Primeiro Coeficiente de Lyapunov

$[>$ Cqqconjugateq $:=$ simplify $(\operatorname{subs}(x i[1]=q[1], x i[2]=q[2], m u[1]=q[1]$, $m u[2]=q[2]$, eta $[1]=$ conjugate $(q[1])$, eta $[2]=$ conjugate $(q[2]), C))$;

$$
\text { Cqqconjugateq }:=\left[\begin{array}{c}
0 \\
\left.-1.010^{(}-10\right)-8 I
\end{array}\right]
$$

$[>r:=\operatorname{simplify}($ conjugate $(p[1]) *$ Cqqconjugateq $[1]+$ conjugate $(p[2]) *$ Cqqconjugateq[2]);

$$
\left.r:=-4+1.510^{(}-10\right) I
$$

$[>B q q:=\operatorname{simplify}(\operatorname{subs}(x i[1]=q[1], x i[2]=q[2], m u[1]=q[1], m u[2]=q[2], B))$

$$
B q q:=\left[\begin{array}{c}
0 \\
\left.-6-310^{(}-10\right) I
\end{array}\right]
$$

$[>$ Bqconjugateq $:=\operatorname{simplify}(\operatorname{subs}(x i[1]=q[1], x i[2]=q[2], m u[1]=$ conjugate ( $q[1]), m u[2]=$ conjugate $(q[2]), B))$;

$$
\text { Bqconjugateq }:=\left[\begin{array}{c}
0 \\
-6
\end{array}\right]
$$

$[>s:=\operatorname{evalm}($ inverse(J2).Bqconjugateq $)$;

$$
s:=\left[\begin{array}{ll}
3 & 0
\end{array}\right]
$$


$[>B q s:=\operatorname{simplify}(\operatorname{subs}(x i[1]=q[1], x i[2]=q[2], m u[1]=s[1], m u[2]=s[2]$, $B)$ );

$$
\begin{aligned}
& B q s:=\left[\begin{array}{c}
0 \\
-18-4.510(-10) I
\end{array}\right] \\
& {[>t:=\operatorname{simplify}(\text { conjugate }(p[1]) * B q s[1]+\operatorname{conjugate}(p[2]) * B q s[2]) ;} \\
& t:=9 I \\
& {[>M:=\text { Matrix }(2,2, \text { shape }=\text { identity }):} \\
& {[>w:=\operatorname{simplify}(\text { evalm }(\text { inverse }(2 * I * \text { omega } * M-J 2) . B q q)) \text {; }} \\
& w:=\left[\begin{array}{ll}
1.000000001 & 2.000000002 I
\end{array}\right] \\
& {[>\text { Bconjugateqw }:=\operatorname{simplify}(\operatorname{subs}(x i[1]=\operatorname{conjugate}(q[1]), x i[2]} \\
& =\operatorname{conjugate}(q[2]), m u[1]=w[1], m u[2]=w[2], B)) \text {; } \\
& \text { Bconjugateqw }:=\left[\begin{array}{c}
0 \\
-6.000000006
\end{array}\right] \\
& {[>u:=\operatorname{simplify}(\operatorname{conjugate}(p[1]) * \text { Bconjugateqw }[1]+\operatorname{conjugate}(p[2]) *} \\
& \text { Bconjugateqw[2]); } \\
& \left.u:=1.00000000210^{(}-10\right)+3.000000003 I \\
& {[>l[1]:=\operatorname{simplify}((1 /(2 * \text { omega })) * \operatorname{Re}(r-2 * t+u))} \\
& l_{1}:=-1
\end{aligned}
$$

Bifuração de Hopf Supercrítica 


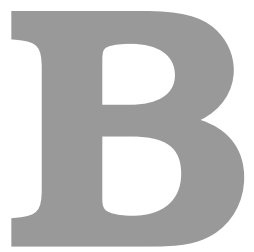

\section{Algoritmo para o cálculo do primeiro coeficiente de Lyapunov para sistemas $n$-dimensional}

Apresentaremos o algoritmo computacional desenvolvido para o software Maple para o cálculo do primeiro coeficiente de Lyapunov para sistemas dinâmico não lineares $n$-dimensional, com $n \geq 3$, ver [Kuz13], [Vér13] e [Gal12]. Considere o sistema dinâmico $n$-dimensional

$$
\dot{x}=f(x, \mu), x \in \mathbb{R}^{n}, \mu \in \mathbb{R},
$$

com $n \geq 3$. O algoritmo está estruturado nas seguintes etapas:

Etapa 1 Encontrar o valor de bifurcação $\mu_{0}$ para o qual o sistema (B.1) possui um ponto de equilíbrio $x_{0}$ com autovalores $\lambda_{1,2}= \pm \omega_{0} i, \omega_{0}>0$, e nenhum outro autovalor com parte real nula. Este valor de bifurcação pode ser encontrado, resolvendo o sistema

$$
\left\{\begin{array}{l}
f(x, \mu)=0 \\
\operatorname{det} M(x, \mu)=0
\end{array},\right.
$$

onde $M$ é a matriz

$$
\left(\begin{array}{ccc}
a_{22}+a_{11} & a_{23} & -a_{13} \\
a_{32} & a_{33}+a_{11} & a_{12} \\
-a_{31} & a_{21} & a_{33}+a_{22}
\end{array}\right)
$$


em que $\left(a_{i j}\right)$ são os elementos da matriz jacobiana $D f(x, \mu)$ do sistema (B.1). Observe que o determinante da matriz $M$ é a condição dada abaixo que garante a existência de um ponto de equilíbrio Hopf no sistema (B.1)

$$
b_{3}-b_{1} b_{2}=0
$$

onde $b_{i}, i=1,2,3$ são os coeficientes do polinômio característico $p(\lambda)=\lambda^{3}+$ $b_{1} \lambda^{2}+b_{2} \lambda+b_{3}$ da matriz jacobiana $D f(x, \mu)$ e $\lambda_{1}=\omega_{0} i$ é raiz do polinômio característico. Portanto, os autovalores $\lambda_{1,2}= \pm \omega_{0} i$ de $A=D f\left(x, \mu_{0}\right)$ são puramente imaginários, e $\lambda_{3} \neq 0$.

Etapa 2 Calcular os autovetores complexos $q, p \in \mathbb{C}^{n}$, tais que

$$
A q=i \omega_{0} q \text { e } A^{T} p=-i \omega_{0} p
$$

e satisfazendo a condição de normalização $\langle p, q\rangle=1$, onde $A=\operatorname{Df}\left(x_{0}, \mu_{0}\right)$ e $\langle p, q\rangle=\sum_{i=1}^{n} \bar{p}_{i} q_{i}$ é o produto escalar padrão em $\mathbb{C}^{n}$.

Etapa 3 Encontrar as expressões $s \in \mathbb{R}^{n}$ e $r \in \mathbb{C}^{n}$, tais que

$$
s=A^{-1} B(q, \bar{q}), r=(2 i \omega I-A)^{-1} B(q, q),
$$

onde $I_{n}$ é a matriz identidade de ordem $n$ e a função multilinear $B(x, y)$ é dada por

$$
B_{i}(x, y)=\left.\sum_{j, k=1}^{n} \frac{\partial^{2} F_{i}(\xi)}{\partial \xi_{j} \partial \xi_{k}}\right|_{\xi=0} x_{j} y_{k}, i=1, \ldots, n .
$$

Etapa 4 Calcular o primeiro coeficiente de Lyapunov dado por

$$
l_{1}=\frac{1}{2 \omega} \Re[\langle p, C(q, q, \bar{q})\rangle-2\langle p, B(q, s)\rangle+\langle p, B(\bar{q}, r)\rangle]
$$

onde a função $B(x, y)$ é definida como acima e a função multilinear $C(x, y, z)$ dada abaixo

$$
C_{i}(x, y, z)=\left.\sum_{j, k, l=1}^{n} \frac{\partial^{3} F_{i}(\xi)}{\partial \xi_{j} \partial \xi_{k} \partial \xi_{l}}\right|_{\xi=0} x_{j} y_{k} z_{l}, i=1, \ldots, n .
$$

\section{B.1 Cálculo do coeficiente de Lyapunov do sistema} (5.2)

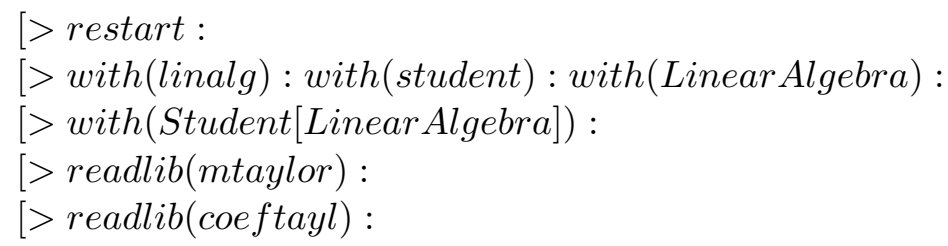




\section{Sistema}

$\left[>F[1]:=-X[1] * X[3]-X[2]-X[1] *\left(X[1]^{2}+X[2]^{2}\right):\right.$
$\left[>F[2]:=-X[2] * X[3]+X[1]-X[2] *\left(X[1]^{2}+X[2]^{2}\right):\right.$
$\left[>F[3]:=-0.1 *\left(X[3]+0.5 *\left(X[1]^{2}+X[2]^{2}\right)\right) *(X[3]-3) *(8-X[3]):\right.$

Pontos de Equilíbrio

[> solve $(F[1], F[2], F[3], X[1], X[2], X[3])$;

$$
\begin{gathered}
\left\{X_{1}=0, X_{2}=0, X_{3}=0\right\},\left\{\begin{array}{c}
\left.X_{1}=0, X_{2}=0, X_{3}=8\right\},\left\{X_{1}=0, X_{2}=\right. \\
\left.0, X_{3}=3\right\}
\end{array}\right.
\end{gathered}
$$

Matriz Jacobiana e Estabilidade dos Equilíbrios $[>J:=\operatorname{Matrix}(j a c o b i a n([F[1], F[2], F[3]],[X[1], X[2], X[3]]))$;

\section{$J:=$}

$\left[\left[-X_{3}-3 * X_{1}^{2}-X_{2}^{2},-1-2 * X_{1} * X_{2},-X_{1}\right],\left[1-2 * X_{1} * X_{2},-X_{3}-X_{1}^{2}-3 * X_{2}^{2}\right.\right.$,

$\left.-\mathrm{X}_{2}\right],\left[-0.1 * X_{1} *\left(X_{3}-3\right) *\left(8-X_{3}\right),-0.1 * X_{2} *\left(X_{3}-3\right) *(8-\right.$

$\left.X_{3}\right),-\left(0.1 *\left(X_{3}-3\right)\right) *\left(8-X_{3}\right)-\left(0.1 *\left(X_{3}+0.5 * X_{1}^{2}+0.5 * X_{2}^{2}\right)\right) *(8-$

$\left.\left.X_{3}\right)+\left(0.1 *\left(X_{3}+0.5 * X_{1}^{2}+0.5 * X_{2}^{2}\right)\right) *\left(X_{3}-3\right)\right]$

$[>J 1:=\operatorname{subs}(X[1]=0, X[2]=0, X[3]=3, J) ;$

$$
J 1:=\left[\begin{array}{ccc}
-3 & -1 & 0 \\
1 & -3 & 0 \\
0 & 0 & -1.5
\end{array}\right]
$$

$[>\operatorname{lambda}:=$ Eigenvalues $(J 1)$

$$
\lambda:=\left[\begin{array}{c}
-3+I \\
-3-I \\
-1.5
\end{array}\right]
$$

$[>J 2:=\operatorname{subs}(X[1]=0, X[2]=0, X[3]=8, J) ;$

$$
J 2:=\left[\begin{array}{ccc}
-8 & -1 & 0 \\
1 & -8 & 0 \\
0 & 0 & 4
\end{array}\right]
$$

$[>\operatorname{lambda}:=$ Eigenvalues $(J 2)$;

$$
\lambda:=\left[\begin{array}{c}
-8+I \\
-8-I \\
4
\end{array}\right]
$$

$[>J 3:=\operatorname{subs}(X[1]=0, X[2]=0, X[3]=0, J) ;$ 


$$
J 3:=\left[\begin{array}{ccc}
0 & -1 & 0 \\
1 & 0 & 0 \\
0 & 0 & 2.4
\end{array}\right]
$$

$[>$ lambda $:=$ Eigenvalues $(J 3)$;

$$
\lambda:=\left[\begin{array}{c}
I \\
-I \\
2.4
\end{array}\right]
$$

$[>$ omega $:=\operatorname{Im}(\operatorname{lambda}[1])$

$$
\omega:=1
$$

Autovetores da Matriz J e da sua transposta $\left[>q 1:=\right.$ eigenvects $\left(J 3,{ }^{\prime}\right.$ radical $\left.{ }^{\prime}\right)$;

$\left[-I, 1,\left\{\left[\begin{array}{lll}-1 & -I & 0\end{array}\right]\right\}\right],\left[I, 1,\left\{\left[\begin{array}{lll}-1 & I & 0\end{array}\right]\right\}\right],\left[2.4,1,\left\{\left[\begin{array}{lll}0 & 0 & 1\end{array}\right]\right\}\right]$ $[>q:=q 1[2][3][1]$

$$
q:=\left[I, 1,\left\{\left[\begin{array}{lll}
-1 & I & 0
\end{array}\right]\right.\right.
$$

$[>p 2:=$ eigenvects(transpose(J3),'radical' $)$;

$$
\left[-I, 1,\left\{\left[\begin{array}{lll}
1 & -I & 0
\end{array}\right]\right\}\right],\left[I, 1,\left\{\left[\begin{array}{lll}
1 & I & 0
\end{array}\right]\right\}\right],\left[2.4,1,\left\{\left[\begin{array}{lll}
0 & 0 & 1
\end{array}\right]\right\}\right]
$$

$[>p 1:=p 2[1][3][1]$

$$
p 1:=\left[\begin{array}{lll}
1 & -I & 0
\end{array}\right]
$$

$[>$ normalizacao $:=\operatorname{simplify}($ evalc $($ conjugate $(p 1[1]) * q[1]+$ conjugate ( $p 1[2]) * q[2]+$ conjugate $(p 1[3]) * q[3]))$;

$$
\text { normalizacao }:=-2
$$

$[>$ fator $:=\operatorname{simplify}(\operatorname{evalc}(1 /$ conjugate $($ normalizacao $))) ;$

$$
\text { fator }:=-0.5
$$

$[>p:=\operatorname{vector}([\operatorname{simplify}($ evalc $($ fator $* p 1[1]))$, simplify $($ evalc $($ fator $* p 1[2]$ )$)$, simplify $($ evalc $($ fator $* p 1[3]))])$;

$$
p:=\left[\begin{array}{cc}
-0.5 & 0.5 I \\
0 &
\end{array}\right]
$$


$[>\operatorname{simplify}($ evalc $($ conjugate $(p[1]) * q[1]+\operatorname{conjugate}(p[2]) * q[2]+$ conjugate ( $p[3]) * q[3]))$;

1

Deslocando o Equilibrio para a Origem

$$
\begin{aligned}
& {\left[>X_{1}:=x_{1}: X_{2}:=x_{2}: X_{3}:=x_{3}:\right.} \\
& {\left[>F_{1}:=\operatorname{simplify}\left(-X[1] * X[3]-X[2]-X[1] *\left(X[1]^{2}+X[2]^{2}\right)\right) ;\right.} \\
& F_{1}:=-x_{1} x_{3}-x_{2}-x_{1}^{3}-x_{1} x_{2}^{2} \\
& {\left[>F[2]:=\operatorname{simplify}\left(-X[2] * X[3]+X[1]-X[2] *\left(X[1]^{2}+X[2]^{2}\right)\right) ;\right.} \\
& F_{2}:=-x_{2} x_{3}+x_{1}-x_{2} x_{1}^{2}-x_{2}^{3} \\
& {\left[>F[3]:=\operatorname{simplify}\left(-0.1 *\left(X[3]+0.5 *\left(X[1]^{2}+X[2]^{2}\right)\right) *(X[3]-3) *(8-X[3])\right) ;\right.} \\
& F_{3}:=0.05\left(2 x_{3}+x_{1}^{2}+x_{2}^{2}\right)\left(x_{3}-3\right)\left(x_{3}-8\right)
\end{aligned}
$$

Calculando as Funções Multilineares B(x,y) e C(x,y,z)

$[>B[1]:=2 * \operatorname{coeftayl}(F[1],[x[1], x[2], x[3]]=[0,0,0],[2,0,0]) * x i[1] * m u[1]+$ $\operatorname{coeftayl}(F[1],[x[1], x[2], x[3]]=[0,0,0],[1,1,0]) *(x i[1] * m u[2]+x i[2] * m u[1])+$ $\operatorname{coeftayl}(F[1],[x[1], x[2], x[3]]=[0,0,0],[1,0,1]) *(x i[1] * m u[3]+x i[3] * m u[1])+$ $\operatorname{coeftayl}(F[1],[x[1], x[2], x[3]]=[0,0,0],[0,1,1]) *(x i[2] * m u[3]+x i[3] * m u[2])+$ $2 * \operatorname{coeftayl}(F[1],[x[1], x[2], x[3]]=[0,0,0],[0,2,0]) * x i[2] * m u[2]+2 *$ coeftayl ( $F[1],[x[1], x[2], x[3]]=[0,0,0],[0,0,2]) * x i[3] * m u[3] ;$

$$
\left.B[1]:=-\xi_{1} \mu_{3}\right]-\xi_{3} \mu_{1}
$$

$[>B[2]:=2 * \operatorname{coeftayl}(F[2],[x[1], x[2], x[3]]=[0,0,0],[2,0,0]) * x i[1] * m u[1]+$ $\operatorname{coeftayl}(F[2],[x[1], x[2], x[3]]=[0,0,0],[1,1,0]) *(x i[1] * m u[2]+x i[2] * m u[1])+$ $\operatorname{coeftayl}(F[2],[x[1], x[2], x[3]]=[0,0,0],[1,0,1]) *(x i[1] * m u[3]+x i[3] * m u[1])+$ $\operatorname{coeftayl}(F[2],[x[1], x[2], x[3]]=[0,0,0],[0,1,1]) *(x i[2] * m u[3]+x i[3] * m u[2])+$ $2 * \operatorname{coeftayl}(F[2],[x[1], x[2], x[3]]=[0,0,0],[0,2,0]) * x i[2] * m u[2]+2 *$ $\operatorname{coeftayl}(F[2],[x[1], x[2], x[3]]=[0,0,0],[0,0,2]) * x i[3] * m u[3]$;

$$
B[2]:=-\xi_{2} \mu_{3}-\xi_{3} \mu_{2}
$$

$[>B[3]:=2 * \operatorname{coeftayl}(F[2],[x[1], x[2], x[3]]=[0,0,0],[2,0,0]) * x i[1] * m u[1]+$ $2 * \operatorname{coeftayl}(F[3],[x[1], x[2], x[3]]=[0,0,0],[2,0,0]) * x i[1] *$ mu[1] + coeftayl ( $F[3],[x[1], x[2], x[3]]=[0,0,0],[1,1,0]) *(x i[1] * m u[2]+x i[2] * m u[1])+$ coeftayl ( $F[3],[x[1], x[2], x[3]]=[0,0,0],[1,0,1]) *(x i[1] * m u[3]+x i[3] * m u[1])+$ coeftayl ( $F[3],[x[1], x[2], x[3]]=[0,0,0],[0,1,1]) *(x i[2] * m u[3]+x i[3] * m u[2])+2 *$ $\operatorname{coeftayl}(F[3],[x[1], x[2], x[3]]=[0,0,0],[0,2,0]) * x i[2] * m u[2]+2 * \operatorname{coeftayl}($ $F[3],[x[1], x[2], x[3]]=[0,0,0],[0,0,2]) * x i[3] * m u[3]$; 


$$
B[3]:=2.4 \xi_{1} \mu_{1}+2.4 \xi_{2} \mu_{2}-2.2 \xi_{3} \mu_{3}
$$

$[>C[1]:=6 * \operatorname{coeftayl}(F[1],[x[1], x[2], x[3]]=[0,0,0],[3,0,0]) * x i[1] * m u[1] *$ eta $[1]+6 * \operatorname{coeftayl}(F[1],[x[1], x[2], x[3]]=[0,0,0],[0,3,0]) * x i[2] * m u[2] *$ eta $[2]+6 *$ coeftayl $(F[1],[x[1], x[2], x[3]]=[0,0,0],[0,0,3]) * x i[3] * m u[3] *$ eta $[3]+2 * \operatorname{coeftayl}(F[1],[x[1], x[2], x[3]]=[0,0,0],[2,1,0]) *(x i[1] * m u[1] *$ eta $[2]+x i[1] * m u[2] *$ eta $[1]+x i[2] * m u[1] * \operatorname{eta}[1])+2 * \operatorname{coeftayl}(F[1]$, $[x[1], x[2], x[3]]=[0,0,0],[2,0,1]) *(x i[1] * m u[1] *$ eta $[3]+x i[1] * m u[3] *$ eta $[1]+x i[3] * m u[1] * \operatorname{eta}[1])+2 * \operatorname{coeftayl}(F[1],[x[1], x[2], x[3]]=[0,0,0]$, $[1,2,0]) *(x i[1] * m u[2] *$ eta $[2]+x i[2] * m u[1] *$ eta $[2]+x i[2] *$ $m u[2] * \operatorname{eta}[1])+2 * \operatorname{coeftayl}(F[1],[x[1], x[2], x[3]]=[0,0,0],[0,2,1]) *$ $(x i[2] * m u[2] * \operatorname{eta}[3]+x i[2] * m u[3] * \operatorname{eta}[2]+x i[3] * m u[2] *$ eta $[2])+2 * \operatorname{coeftayl}(F[1],[x[1], x[2], x[3]]=[0,0,0],[1,0,2]) *(x i[1] *$ $m u[3] *$ eta $[3]+x i[3] * m u[1] *$ eta $[3]+x i[3] * m u[3] *$ eta $[1])+2 *$ $\operatorname{coeftayl}(F[1],[x[1], x[2], x[3]]=[0,0,0],[0,1,2]) *(x i[2] * m u[3] *$ eta $[3]+$ $x i[3] * m u[2] *$ eta $[3]+x i[3] * m u[3] *$ eta $[2])+\operatorname{coeftayl}(F[1],[x[1], x[2]$, $x[3]]=[0,0,0],[1,1,1]) *(x i[1] * m u[2] * \operatorname{eta}[3]+x i[1] * \operatorname{mu}[3] * \operatorname{eta}[2]$ $+x i[2] * m u[1] *$ eta $[3]+x i[2] * m u[3] *$ eta $[1]+x i[3] * m u[1] *$ eta $[2]+$ $x i[3] * m u[2] *$ eta $[1])$;

$$
C[1]:=-6 \xi_{1} \mu_{1} \eta_{1}-2 \xi_{1} \mu_{2} \eta_{2}-2 \xi_{2} \mu_{1} \eta_{2}-2 \xi_{2} \mu_{2} \eta_{1}
$$

$[>C[2]:=6 * \operatorname{coeftayl}(F[2],[x[1], x[2], x[3]]=[0,0,0],[3,0,0]) * x i[1] * m u[1]$ $*$ eta $[1]+6 * \operatorname{coeftayl}(F[2],[x[1], x[2], x[3]]=[0,0,0],[0,3,0]) * x i[2] *$ $m u[2] * \operatorname{eta}[2]+6 * \operatorname{coeftayl}(F[2],[x[1], x[2], x[3]]=[0,0,0],[0,0,3]) * x i[3]$ $* m u[3] * \operatorname{eta}[3]+2 * \operatorname{coeftayl}(F[2],[x[1], x[2], x[3]]=[0,0,0],[2,1,0]) *$ $(x i[1] * m u[1] *$ eta $[2]+x i[1] * m u[2] *$ eta $[1]+x i[2] * m u[1] *$ eta $[1])$ $+2 * \operatorname{coeftayl}(F[2],[x[1], x[2], x[3]]=[0,0,0],[2,0,1]) *(x i[1] * m u[1] *$ eta $[3]+x i[1] * m u[3] * \operatorname{eta}[1]+x i[3] * m u[1] * \operatorname{eta}[1])+2 * \operatorname{coeftayl}(F[2]$, $[x[1], x[2], x[3]]=[0,0,0],[1,2,0]) *(x i[1] * m u[2] * \operatorname{eta}[2]+x i[2] * m u[1]$ $* \operatorname{eta}[2]+x i[2] * m u[2] * \operatorname{eta}[1])+2 * \operatorname{coeftayl}(F[2],[x[1], x[2], x[3]]=[0,0$, $0],[0,2,1]) *(x i[2] * m u[2] *$ eta $[3]+x i[2] * m u[3] *$ eta $[2]+x i[3] *$ $m u[2] * \operatorname{eta}[2])+2 * \operatorname{coeftayl}(F[2],[x[1], x[2], x[3]]=[0,0,0],[1,0,2]) *(x i[1]$ $* m u[3] *$ eta $[3]+x i[3] * m u[1] *$ eta $[3]+x i[3] * m u[3] *$ eta $[1])+2 *$ $\operatorname{coeftayl}(F[2],[x[1], x[2], x[3]]=[0,0,0],[0,1,2]) *(x i[2] * m u[3] * \operatorname{eta}[3]+x i[3]$ $* m u[2] * \operatorname{eta}[3]+x i[3] * m u[3] * \operatorname{eta}[2])+\operatorname{coeftayl}(F[2],[x[1], x[2], x[3]]=[0,0$, $0],[1,1,1]) *(x i[1] * m u[2] * \operatorname{eta}[3]+x i[1] * m u[3] * \operatorname{eta}[2]+x i[2] * m u[1]$ $* e t a[3]+x i[2] * m u[3] *$ eta $[1]+x i[3] * m u[1] * \operatorname{eta}[2]+x i[3] * m u[2] *$ eta[1]);

$$
C[2]:=-6 \xi_{2} \mu_{2} \eta_{2}-2 \xi_{1} \mu_{1} \eta_{2}-2 \xi_{1} \mu_{2} \eta_{1}-2 \xi_{2} \mu_{1} \eta_{1}
$$

$[>C[3]:=6 * \operatorname{coeftayl}(F[3],[x[1], x[2], x[3]]=[0,0,0],[3,0,0]) * x i[1] * m u[1] *$ 
eta $[1]+6 * \operatorname{coeftayl}(F[3],[x[1], x[2], x[3]]=[0,0,0],[0,3,0]) * x i[2] * m u[2] *$ eta $[2]+6 * \operatorname{coeftayl}(F[3],[x[1], x[2], x[3]]=[0,0,0],[0,0,3]) * x i[3] * m u[3] *$ eta $[3]+2 * \operatorname{coeftayl}(F[3],[x[1], x[2], x[3]]=[0,0,0],[2,1,0]) *(x i[1] * m u[1] *$ eta $[2]+x i[1] * m u[2] *$ eta $[1]+x i[2] * m u[1] *$ eta $[1])+2 * \operatorname{coeftayl}(F[3],[x[1]$, $x[2], x[3]]=[0,0,0],[2,0,1]) *(x i[1] * m u[1] * \operatorname{eta}[3]+x i[1] * m u[3] *$ eta $[1]+$ $x i[3] * m u[1] *$ eta $[1])+2 * \operatorname{coeftayl}(F[3],[x[1], x[2], x[3]]=[0,0,0],[1,2,0]) *$ $(x i[1] * m u[2] *$ eta $[2]+x i[2] * m u[1] *$ eta $[2]+x i[2] * m u[2] *$ eta $[1])+2 *$ $\operatorname{coeftayl}(F[3],[x[1], x[2], x[3]]=[0,0,0],[0,2,1]) *(x i[2] * m u[2] * \operatorname{eta}[3]+x i[2] *$ $m u[3] * \operatorname{eta}[2]+x i[3] * m u[2] * \operatorname{eta}[2])+2 * \operatorname{coeftayl}(F[3],[x[1], x[2], x[3]]=[0,0,0]$, $[1,0,2]) *(x i[1] * m u[3] *$ eta $[3]+x i[3] * m u[1] *$ eta $[3]+x i[3] * m u[3] *$ eta $[1])$ $+2 * \operatorname{coeftayl}(F[3],[x[1], x[2], x[3]]=[0,0,0],[0,1,2]) *(x i[2] * m u[3] * \operatorname{eta}[3]+x i[3] *$ $m u[2] * \operatorname{eta}[3]+x i[3] * m u[3] * \operatorname{eta}[2])+\operatorname{coeftayl}(F[3],[x[1], x[2], x[3]]=[0,0,0]$, $[1,1,1]) *(x i[1] * m u[2] *$ eta $[3]+x i[1] * m u[3] *$ eta $[2]+x i[2] * m u[1] *$ eta $[3]+x i[2] * m u[3] *$ eta $[1]+x i[3] * m u[1] *$ eta $[2]+x i[3] * m u[2] *$ eta $[1])$;

$C[3]:=0.6 \xi_{3} \mu_{3} \eta_{3}-1.1 \xi_{1} \mu_{1} \eta_{3}-1.1 \xi_{1} \mu_{3} \eta_{1}-1.1 \xi_{3} \mu_{1} \eta_{1}-1.1 \xi_{2} \mu_{2} \eta_{3}-$

$1.1 \xi_{2} \mu_{3} \eta_{2}-1.1 \xi_{3} \mu_{2} \eta_{2}$

$[>B:=<B[1], B[2], B[3]>$ :

$[>C:=<C[1], C[2], C[3]>$ :

Calculando Primeiro Coeficiente de Lyapunov

$[>$ Cqqconjugateq $:=\operatorname{simplify}(\operatorname{subs}(x i[1]=q[1], x i[2]=q[2], x i[3]=q[3]$, $m u[1]=q[1], m u[2]=q[2], m u[3]=q[3]$, eta $[1]=\operatorname{conjugate}(q[1])$, eta $[2]=$ conjugate $(q[2])$, eta $[3]=$ conjugate $(q[3]), C))$;

$$
\text { Cqqconjugateq }:=\left[\begin{array}{c}
8 \\
-8 I \\
0
\end{array}\right]
$$

$[>r:=\operatorname{simplify}(\operatorname{conjugate}(p[1]) *$ Cqqconjugateq[1] + conjugate $(p[2]) *$ Cqqconjugateq $[2]+$ conjugate $(p[3]) *$ Cqqconjugateq $[3])$;

$$
r:=-8
$$

$[>B q q:=\operatorname{simplify}(\operatorname{subs}(x i[1]=q[1], x i[2]=q[2], x i[3]=q[3], m u[1]=q[1]$, $m u[2]=q[2], m u[3]=q[3], B)) ;$

$$
B q q:=\left[\begin{array}{l}
0 \\
0 \\
0
\end{array}\right]
$$

$[>$ Bqconjugateq $:=\operatorname{simplify}(\operatorname{subs}(x i[1]=q[1], x i[2]=q[2], x i[3]=q[3]$ $, m u[1]=\operatorname{conjugate}(q[1]), m u[2]=\operatorname{conjugate}(q[2]), m u[3]=\operatorname{conjugate}(q[3])$ $, B))$; 


$$
\text { Bqconjugateq }:=\left[\begin{array}{c}
0 \\
0 \\
4.8
\end{array}\right]
$$

$[>s:=\operatorname{evalm}($ inverse(J3).Bqconjugateq $) ;$

$$
s:=\left[\begin{array}{lll}
0 & 0 & 2
\end{array}\right]
$$

$[>B q s:=\operatorname{simplify}(\operatorname{subs}(x i[1]=q[1], x i[2]=q[2], x i[3]=q[3], m u[1]=s[1]$, $m u[2]=s[2], m u[3]=s[3], B))$;

$$
B q s:=\left[\begin{array}{c}
2 \\
-2 I \\
0
\end{array}\right]
$$

$[>t:=\operatorname{simplify}($ conjugate $(p[1]) *$ Bqs $[1]+$ conjugate $(p[2]) * B q s[2]+$ conjugate $(p[3]) * B q s[3])$;

$$
t:=-2
$$

$[>M:=$ Matrix $(3,3$, shape $=$ identity $):$

$[>w:=\operatorname{simplify}($ evalm $($ inverse $(2 * I *$ omega $* M-J 3) . B q q))$;

$$
w:=\left[\begin{array}{lll}
0 & 0 & 0
\end{array}\right]
$$

$[>$ Bconjugateqw $:=\operatorname{simplify}(\operatorname{subs}(x i[1]=\operatorname{conjugate}(q[1]), x i[2]$ $=\operatorname{conjugate}(q[2]), x i[3]=$ conjugate $(q[3]), m u[1]=w[1], m u[2]=$ $w[2], m u[3]=w[3], B))$;

$$
\text { Bconjugateqw }:=\left[\begin{array}{l}
0 \\
0 \\
0
\end{array}\right]
$$

$[>u:=\operatorname{simplify}(\operatorname{conjugate}(p[1]) *$ Bconjugateqw $[1]+\operatorname{conjugate}(p[2]) *$ Bconjugateqw $[2]+$ conjugate $(p[3]) *$ Bconjugateqw $[3])$;

$$
u:=0
$$

$[>l[1]:=\operatorname{simplify}((1 /(2 *$ omega $)) * \operatorname{Re}(r-2 * t+u))$

$$
l_{1}:=-2
$$

Bifuração de Hopf Supercrítica 


\section{B.2 Cálculo do coeficiente de Lyapunov do sistema (7.2)}

[> restart:

$[>$ with (linalg) : with(student) : with(Linear Algebra) :

[> with(Student [Linear Algebra]) :

[> readlib(mtaylor) :

$[>$ readlib(coeftayl) :

\section{Sistema}

$[>F[1]:=X[1] *(8-3 * X[1]-3 * X[2]-2 * X[3]):$

$[>F[2]:=X[2] *(3-2 * X[1]-X[2]):$

$[>F[3]:=X[3] *(3-2 * X[2]-X[3]):$

Pontos de Equilíbrio

[> solve $(F[1], F[2], F[3], X[1], X[2], X[3])$;

$\left\{X_{1}=0, X_{2}=0, X_{3}=0\right\},\left\{X_{1}=\frac{8}{3}, X_{2}=0, X_{3}=0\right\},\left\{X_{1}=0, X_{2}=\right.$ $\left.0, X_{3}=3\right\},\left\{X_{1}=\frac{2}{3}, X_{2}=0, X_{3}=3\right\},\left\{X_{1}=0, X_{2}=3, X_{3}=0\right\},\left\{X_{1}=\right.$ $\left.\frac{1}{3}, X_{2}=\frac{7}{3}, X_{3}=0\right\},\left\{X_{1}=0, X_{2}=3, X_{3}=-3\right\},\left\{X_{1}=1, X_{2}=1, X_{3}=1\right\}$

Matriz Jacobiana e Estabilidade dos Equilíbrios

$[>J:=$ Matrix $(j a c o b i a n([F[1], F[2], F[3]],[X[1], X[2], X[3]]))$;

$$
J:=\left[\begin{array}{ccc}
8-6 X_{1}-3 X_{2}-2 X_{3} & -3 X_{1} & -2 X_{1} \\
-2 X_{2} & 3-2 X_{1}-2 X_{2} & 0 \\
0 & -2 X_{3} & 3-2 X_{2}-2 X_{3}
\end{array}\right]
$$

$[>J 1:=\operatorname{subs}(X[1]=0, X[2]=0, X[3]=0, J)$;

$$
J 1:=\left[\begin{array}{ccc}
8 & 0 & 0 \\
0 & 3 & 0 \\
0 & 0 & 3
\end{array}\right]
$$

$[>l a m b d a:=$ Eigenvalues $(J 1)$

$$
\lambda:=\left[\begin{array}{l}
8 \\
3 \\
3
\end{array}\right]
$$

$\left[>J 2:=\operatorname{subs}\left(X[1]=\frac{8}{3}, X[2]=0, X[3]=0, J\right)\right.$;

$$
J 2:=\left[\begin{array}{ccc}
-8 & -8 & -\frac{16}{3} \\
0 & -\frac{7}{3} & 0 \\
0 & 0 & 3
\end{array}\right]
$$


$[>\operatorname{lambda}:=$ Eigenvalues(J2);

$$
\lambda:=\left[\begin{array}{c}
-\frac{7}{3} \\
-8 \\
3
\end{array}\right]
$$

$[>J 3:=\operatorname{subs}(X[1]=0, X[2]=3, X[3]=0, J)$;

$$
J 3:=\left[\begin{array}{ccc}
-1 & 0 & 0 \\
-6 & -3 & 0 \\
0 & 0 & -3
\end{array}\right]
$$

$[>\operatorname{lambda}:=$ Eigenvalues(J3);

$$
\lambda:=\left[\begin{array}{l}
-1 \\
-3 \\
-3
\end{array}\right]
$$

$\left[>J 4:=\operatorname{subs}\left(X[1]=\frac{2}{3}, X[2]=0, X[3]=3, J\right) ;\right.$

$$
J 4:=\left[\begin{array}{ccc}
-2 & -2 & -\frac{4}{3} \\
0 & \frac{5}{3} & 0 \\
0 & -6 & -3
\end{array}\right]
$$

$[>\operatorname{lambda}:=$ Eigenvalues $(J 4)$;

$$
\lambda:=\left[\begin{array}{c}
\frac{5}{3} \\
-3 \\
-2
\end{array}\right]
$$

$[>J 5:=\operatorname{subs}(X[1]=0, X[2]=0, X[3]=3, J)$;

$$
J 5:=\left[\begin{array}{ccc}
2 & 0 & 0 \\
0 & 3 & 0 \\
0 & -6 & -3
\end{array}\right]
$$

$[>l a m b d a:=$ Eigenvalues $(J 5)$;

$$
\lambda:=\left[\begin{array}{c}
2 \\
3 \\
-3
\end{array}\right]
$$

$\left[>J 6:=\operatorname{subs}\left(X[1]=\frac{1}{3}, X[2]=\frac{7}{3}, X[3]=0, J\right)\right.$;

$$
J 6:=\left[\begin{array}{ccc}
-1 & -1 & -0.67 \\
-4.67 & -2.33 & 0 \\
0 & 0 & -1.67
\end{array}\right]
$$


$[>\operatorname{lambda}:=$ Eigenvalues $(J 6)$

$$
\lambda:=\left[\begin{array}{c}
0.59 \\
-3.93 \\
-1.67
\end{array}\right]
$$

$[>J 7:=\operatorname{subs}(X[1]=0, X[2]=3, X[3]=-3, J)$

$$
J 7:=\left[\begin{array}{ccc}
5 & 0 & 0 \\
-6 & -3 & 0 \\
0 & 6 & 3
\end{array}\right]
$$

$[>$ lambda $:=$ Eigenvalues $(J 7)$

$$
\lambda:=\left[\begin{array}{c}
5 \\
-3 \\
3
\end{array}\right]
$$

$[>J 8:=\operatorname{subs}(X[1]=1, X[2]=1, X[3]=1, J)$

$$
J 8:=\left[\begin{array}{ccc}
-3 & -3 & -2 \\
-2 & -1 & 0 \\
0 & -2 & -1
\end{array}\right]
$$

$[>\operatorname{lambda}:=$ Eigenvalues $(J 8) ;$

$$
\lambda:=\left[\begin{array}{c}
-5 \\
I \\
-I
\end{array}\right]
$$

$[>$ omega $:=\operatorname{Im}(\operatorname{lambda}[2])$

$$
\omega:=1
$$

Autovetores da Matriz J e da sua transposta $\left[>q 1:=\operatorname{eigenvects}\left(J 8,{ }^{\prime}\right.\right.$ radical $\left.^{\prime}\right)$;

$q 1:=$

$\left[-5,1,\left\{\left[\begin{array}{lll}4 & 2 & 1\end{array}\right]\right\}\right],\left[I, 1,\left\{\left[\begin{array}{lll}-\frac{1}{2}-\frac{1}{2} I & 1 & -1+I\end{array}\right]\right\}\right],\left[-I, 1,\left\{\left[\begin{array}{lll}-\frac{1}{2}+\frac{1}{2} I & 1 & -1-I\end{array}\right]\right\}\right]$ $[>q:=q 1[2][3][1]$

$$
q:=\left[I, 1,\left\{\left[\begin{array}{lll}
-\frac{1}{2}-\frac{1}{2} I & 1 & -1+I
\end{array}\right]\right.\right.
$$

$\left[>p 2:=\right.$ eigenvects $\left(\right.$ transpose $(J 8),{ }^{\prime}$ radical $\left.^{\prime}\right)$;

$p 2:=$

$\left[I, 1,\left\{\left[\begin{array}{lll}-\frac{1}{2}-\frac{1}{2} I & \frac{1}{2}+I & 1\end{array}\right]\right\}\right],\left[-I, 1,\left\{\left[\begin{array}{lll}-\frac{1}{2}+\frac{1}{2} I & \frac{1}{2}-I & 1\end{array}\right]\right\}\right],\left[-5,1,\left\{\left[\begin{array}{lll}2 & 2 & 1\end{array}\right]\right\}\right]$ 
$[>p 1:=p 2[2][3][1]$

$$
p 1:=\left[\begin{array}{lll}
-\frac{1}{2}+\frac{1}{2} I & \frac{1}{2}-I & 1
\end{array}\right]
$$

$[>$ normalizacao $:=\operatorname{simplify}($ evalc $($ conjugate $(p 1[1]) * q[1]+$ conjugate ( $p 1[2]) * q[2]+$ conjugate $(p 1[3]) * q[3]))$;

$$
\text { normalizacao }:=-\frac{1}{2}+\frac{5}{2} I
$$

$[>$ fator $:=\operatorname{simplify}(\operatorname{evalc}(1 / \operatorname{conjugate}($ normalizacao $)))$;

$$
\text { fator }:=-\frac{1}{13}+\frac{5}{13} I
$$

$[>p:=\operatorname{vector}([\operatorname{simplify}($ evalc $($ fator $* p 1[1]))$, simplify $($ evalc $($ fator $* p 1[2]$ )$)$, simplify $($ evalc $($ fator $* p 1[3]))])$;

$$
p:=\left[\begin{array}{ll}
-\frac{2}{13}-\frac{3}{13} I & \frac{9}{26}+\frac{7}{26} I \\
-\frac{1}{13}+\frac{5}{13} I &
\end{array}\right]
$$

$[>\operatorname{simplify}($ evalc $($ conjugate $(p[1]) * q[1]+\operatorname{conjugate}(p[2]) * q[2]+$ conjugate ( $p[3]) * q[3]))$

Deslocando o Equilibrio para a Origem

$$
\begin{gathered}
{\left[>X_{1}:=x_{1}+1: X_{2}:=x_{2}+1: X_{3}:=x_{3}+1:\right.} \\
{\left[>F_{1}:=\operatorname{simplify}(X[1] *(8-3 * X[1]-3 * X[2]-2 * X[3])) ;\right.} \\
F_{1}:=-\left(x_{1}+1\right)\left(3 x_{1}+3 x_{2}+2 x_{3}\right) \\
{[>F[2]:=\operatorname{simplify}(X[2] *(3-2 * X[1]-X[2]))} \\
F_{2}:=-\left(x_{2}+1\right)\left(2 x_{1}+x_{2}\right) \\
{[>F[3]:=\operatorname{simplify}(X[3] *(3-2 * X[2]-X[3])) ;} \\
F_{3}:=-\left(x_{3}+1\right)\left(2 x_{2}+x_{3}\right)
\end{gathered}
$$

Calculando as Funções Multilineares B(x,y) e C(x,y,z)

$[>B[1]:=2 * \operatorname{coeftayl}(F[1],[x[1], x[2], x[3]]=[0,0,0],[2,0,0]) * x i[1] * m u[1]+$ $\operatorname{coeftayl}(F[1],[x[1], x[2], x[3]]=[0,0,0],[1,1,0]) *(x i[1] * m u[2]+x i[2] * m u[1])+$ $\operatorname{coeftayl}(F[1],[x[1], x[2], x[3]]=[0,0,0],[1,0,1]) *(x i[1] * m u[3]+x i[3] * m u[1])+$ $\operatorname{coeftayl}(F[1],[x[1], x[2], x[3]]=[0,0,0],[0,1,1]) *(x i[2] * m u[3]+x i[3] * m u[2])+$ $2 * \operatorname{coeftayl}(F[1],[x[1], x[2], x[3]]=[0,0,0],[0,2,0]) * x i[2] * m u[2]+2 *$ coeftayl ( $F[1],[x[1], x[2], x[3]]=[0,0,0],[0,0,2]) * x i[3] * m u[3]$; 


$$
B[1]:=-6 \xi_{1} \mu_{1}-3 \xi_{1} \mu_{2}-3 \xi_{2} \mu_{1}-2 \xi_{1} \mu_{3}-2 \xi_{3} \mu_{1}
$$

$[>B[2]:=2 * \operatorname{coeftayl}(F[2],[x[1], x[2], x[3]]=[0,0,0],[2,0,0]) * x i[1] * m u[1]+$ $\operatorname{coeftayl}(F[2],[x[1], x[2], x[3]]=[0,0,0],[1,1,0]) *(x i[1] * m u[2]+x i[2] * m u[1])+$ $\operatorname{coeftayl}(F[2],[x[1], x[2], x[3]]=[0,0,0],[1,0,1]) *(x i[1] * m u[3]+x i[3] * m u[1])+$ $\operatorname{coeftayl}(F[2],[x[1], x[2], x[3]]=[0,0,0],[0,1,1]) *(x i[2] * m u[3]+x i[3] * m u[2])+$ $2 * \operatorname{coeftayl}(F[2],[x[1], x[2], x[3]]=[0,0,0],[0,2,0]) * x i[2] * m u[2]+2 *$ $\operatorname{coeftayl}(F[2],[x[1], x[2], x[3]]=[0,0,0],[0,0,2]) * x i[3] * m u[3]$;

$$
B[2]:=-2 \xi_{1} \mu_{2}-2 \xi_{2} \mu_{1}-2 \xi_{2} \mu_{2}
$$

$[>B[3]:=2 * \operatorname{coeftayl}(F[2],[x[1], x[2], x[3]]=[0,0,0],[2,0,0]) * x i[1] * m u[1]+$ $2 * \operatorname{coeftayl}(F[3],[x[1], x[2], x[3]]=[0,0,0],[2,0,0]) * x i[1] *$ mu[1] + coeftayl ( $F[3],[x[1], x[2], x[3]]=[0,0,0],[1,1,0]) *(x i[1] * m u[2]+x i[2] * m u[1])+$ coeftayl ( $F[3],[x[1], x[2], x[3]]=[0,0,0],[1,0,1]) *(x i[1] * m u[3]+x i[3] * m u[1])+$ coeftayl ( $F[3],[x[1], x[2], x[3]]=[0,0,0],[0,1,1]) *(x i[2] * m u[3]+x i[3] * m u[2])+2 *$ $\operatorname{coeftayl}(F[3],[x[1], x[2], x[3]]=[0,0,0],[0,2,0]) * x i[2] * m u[2]+2 * \operatorname{coeftayl}($ $F[3],[x[1], x[2], x[3]]=[0,0,0],[0,0,2]) * x i[3] * m u[3]$;

$$
B[3]:=-2 \xi_{2} \mu_{3}-2 \xi_{3} \mu_{2}-2 \xi_{3} \mu_{3}
$$

$[>C[1]:=6 * \operatorname{coeftayl}(F[1],[x[1], x[2], x[3]]=[0,0,0],[3,0,0]) * x i[1] * m u[1] *$ eta $[1]+6 * \operatorname{coeftayl}(F[1],[x[1], x[2], x[3]]=[0,0,0],[0,3,0]) * x i[2] * m u[2] *$ eta $[2]+6 * \operatorname{coeftayl}(F[1],[x[1], x[2], x[3]]=[0,0,0],[0,0,3]) * x i[3] * m u[3] *$ eta $[3]+2 * \operatorname{coeftayl}(F[1],[x[1], x[2], x[3]]=[0,0,0],[2,1,0]) *(x i[1] * m u[1] *$ eta $[2]+x i[1] * m u[2] * \operatorname{eta}[1]+x i[2] * m u[1] * \operatorname{eta}[1])+2 * \operatorname{coeftayl}(F[1]$, $[x[1], x[2], x[3]]=[0,0,0],[2,0,1]) *(x i[1] * m u[1] * \operatorname{eta}[3]+x i[1] * m u[3] *$ eta $[1]+x i[3] * m u[1] * \operatorname{eta}[1])+2 * \operatorname{coeftayl}(F[1],[x[1], x[2], x[3]]=[0,0,0]$, $[1,2,0]) *(x i[1] * m u[2] *$ eta $[2]+x i[2] * m u[1] *$ eta $[2]+x i[2] *$ $m u[2] * \operatorname{eta}[1])+2 * \operatorname{coeftayl}(F[1],[x[1], x[2], x[3]]=[0,0,0],[0,2,1]) *$ $(x i[2] * m u[2] *$ eta $[3]+x i[2] * m u[3] *$ eta $[2]+x i[3] * m u[2] *$ eta $[2])+2 *$ coeftayl $(F[1],[x[1], x[2], x[3]]=[0,0,0],[1,0,2]) *(x i[1] *$ $m u[3] *$ eta $[3]+x i[3] * m u[1] *$ eta $[3]+x i[3] * m u[3] *$ eta $[1])+2 *$ $\operatorname{coeftayl}(F[1],[x[1], x[2], x[3]]=[0,0,0],[0,1,2]) *(x i[2] * m u[3] *$ eta $[3]+$ $x i[3] * m u[2] * \operatorname{eta}[3]+x i[3] * m u[3] *$ eta $[2])+\operatorname{coeftayl}(F[1],[x[1], x[2]$, $x[3]]=[0,0,0],[1,1,1]) *(x i[1] * m u[2] * \operatorname{eta}[3]+x i[1] * m u[3] *$ eta $[2]$ $+x i[2] * m u[1] * \operatorname{eta}[3]+x i[2] * m u[3] * \operatorname{eta}[1]+x i[3] * m u[1] *$ eta $[2]+$ $x i[3] * m u[2] *$ eta $[1])$;

$$
C[1]:=0
$$


$[>C[2]:=6 * \operatorname{coeftayl}(F[2],[x[1], x[2], x[3]]=[0,0,0],[3,0,0]) * x i[1] * m u[1]$ $* \operatorname{eta}[1]+6 * \operatorname{coeftayl}(F[2],[x[1], x[2], x[3]]=[0,0,0],[0,3,0]) * x i[2] *$ $m u[2] * \operatorname{eta}[2]+6 * \operatorname{coeftayl}(F[2],[x[1], x[2], x[3]]=[0,0,0],[0,0,3]) * x i[3]$ $* m u[3] * \operatorname{eta}[3]+2 * \operatorname{coeftayl}(F[2],[x[1], x[2], x[3]]=[0,0,0],[2,1,0]) *$ $(x i[1] * m u[1] *$ eta $[2]+x i[1] * m u[2] *$ eta $[1]+x i[2] * m u[1] *$ eta $[1])$ $+2 * \operatorname{coeftayl}(F[2],[x[1], x[2], x[3]]=[0,0,0],[2,0,1]) *(x i[1] * m u[1] *$ eta $[3]+x i[1] * m u[3] *$ eta $[1]+x i[3] * m u[1] * \operatorname{eta}[1])+2 * \operatorname{coeftayl}(F[2]$, $[x[1], x[2], x[3]]=[0,0,0],[1,2,0]) *(x i[1] * m u[2] * \operatorname{eta}[2]+x i[2] * m u[1]$ $* \operatorname{eta}[2]+x i[2] * m u[2] * \operatorname{eta}[1])+2 * \operatorname{coeftayl}(F[2],[x[1], x[2], x[3]]=[0,0$, $0],[0,2,1]) *(x i[2] * m u[2] *$ eta $[3]+x i[2] * m u[3] *$ eta $[2]+x i[3] *$ $m u[2] * \operatorname{eta}[2])+2 * \operatorname{coeftayl}(F[2],[x[1], x[2], x[3]]=[0,0,0],[1,0,2]) *(x i[1]$ $* m u[3] *$ eta $[3]+x i[3] * m u[1] *$ eta $[3]+x i[3] * m u[3] *$ eta $[1])+2 *$ $\operatorname{coeftayl}(F[2],[x[1], x[2], x[3]]=[0,0,0],[0,1,2]) *(x i[2] * m u[3] * \operatorname{eta}[3]+x i[3]$ $* m u[2] * \operatorname{eta}[3]+x i[3] * m u[3] * \operatorname{eta}[2])+\operatorname{coeftayl}(F[2],[x[1], x[2], x[3]]=[0,0$, $0],[1,1,1]) *(x i[1] * m u[2] * \operatorname{eta}[3]+x i[1] * m u[3] * \operatorname{eta}[2]+x i[2] * m u[1]$ $* e t a[3]+x i[2] * m u[3] *$ eta $[1]+x i[3] * m u[1] * \operatorname{eta}[2]+x i[3] * m u[2] *$ eta[1]);

$$
C[2]:=0
$$

$[>C[3]:=6 * \operatorname{coeftayl}(F[3],[x[1], x[2], x[3]]=[0,0,0],[3,0,0]) * x i[1] * m u[1] *$ eta $[1]+6 *$ coeftayl $(F[3],[x[1], x[2], x[3]]=[0,0,0],[0,3,0]) * x i[2] * m u[2] *$ eta $[2]+6 * \operatorname{coeftayl}(F[3],[x[1], x[2], x[3]]=[0,0,0],[0,0,3]) * x i[3] * m u[3] *$ eta $[3]+2 * \operatorname{coeftayl}(F[3],[x[1], x[2], x[3]]=[0,0,0],[2,1,0]) *(x i[1] * m u[1] *$ eta $[2]+x i[1] * m u[2] *$ eta $[1]+x i[2] * m u[1] * \operatorname{eta}[1])+2 * \operatorname{coeftayl}(F[3],[x[1]$, $x[2], x[3]]=[0,0,0],[2,0,1]) *(x i[1] * m u[1] *$ eta $[3]+x i[1] * m u[3] *$ eta $[1]+$ $x i[3] * \operatorname{mu}[1] * \operatorname{eta}[1])+2 * \operatorname{coeftayl}(F[3],[x[1], x[2], x[3]]=[0,0,0],[1,2,0]) *$ $(x i[1] * m u[2] *$ eta $[2]+x i[2] * m u[1] *$ eta $[2]+x i[2] * m u[2] *$ eta $[1])+2 *$ $\operatorname{coeftayl}(F[3],[x[1], x[2], x[3]]=[0,0,0],[0,2,1]) *(x i[2] * m u[2] * \operatorname{eta}[3]+x i[2] *$ $m u[3] * \operatorname{eta}[2]+x i[3] * m u[2] * \operatorname{eta}[2])+2 * \operatorname{coeftayl}(F[3],[x[1], x[2], x[3]]=[0,0,0]$, $[1,0,2]) *(x i[1] * m u[3] * \operatorname{eta}[3]+x i[3] * m u[1] * \operatorname{eta}[3]+x i[3] * m u[3] * \operatorname{eta}[1])$ $+2 * \operatorname{coeftayl}(F[3],[x[1], x[2], x[3]]=[0,0,0],[0,1,2]) *(x i[2] * \operatorname{mu}[3] * \operatorname{eta}[3]+x i[3] *$ $m u[2] * \operatorname{eta}[3]+x i[3] * m u[3] * \operatorname{eta}[2])+\operatorname{coeftayl}(F[3],[x[1], x[2], x[3]]=[0,0,0]$, $[1,1,1]) *(x i[1] * m u[2] *$ eta $[3]+x i[1] * m u[3] * \operatorname{eta}[2]+x i[2] * m u[1] *$ eta $[3]+x i[2] * m u[3] *$ eta $[1]+x i[3] * m u[1] *$ eta $[2]+x i[3] * m u[2] *$ eta $[1])$;

$$
C[3]:=0
$$

$[>B:=<B[1], B[2], B[3]>:$

$[>C:=<C[1], C[2], C[3]>$ :

Calculando Primeiro Coeficiente de Lyapunov

$[>$ Cqqconjugateq $:=\operatorname{simplify}(\operatorname{subs}(x i[1]=q[1], x i[2]=q[2], x i[3]=q[3]$, 
$m u[1]=q[1], m u[2]=q[2], m u[3]=q[3]$, eta $[1]=\operatorname{conjugate}(q[1])$, eta $[2]=$ conjugate $(q[2])$, eta $[3]=$ conjugate $(q[3]), C))$;

$$
\text { Cqqconjugateq }:=\left[\begin{array}{l}
0 \\
0 \\
0
\end{array}\right]
$$

$[>r:=\operatorname{simplify}($ conjugate $(p[1]) *$ Cqqconjugateq $[1]+\operatorname{conjugate}(p[2]) *$ Cqqconjugateq $[2]+$ conjugate $(p[3]) *$ Cqqconjugateq $[3]) ;$

$$
r:=0
$$

$[>B q q:=\operatorname{simplify}(\operatorname{subs}(x i[1]=q[1], x i[2]=q[2], x i[3]=q[3], m u[1]=q[1]$, $m u[2]=q[2], m u[3]=q[3], B))$;

$$
B q q:=\left[\begin{array}{c}
-1 \\
2 I \\
4
\end{array}\right]
$$

$[>$ Bqconjugateq $:=\operatorname{simplify}(\operatorname{subs}(x i[1]=q[1], x i[2]=q[2], x i[3]=q[3]$ $, m u[1]=\operatorname{conjugate}(q[1]), m u[2]=\operatorname{conjugate}(q[2]), m u[3]=\operatorname{conjugate}(q[3])$ $, B))$;

$$
\text { Bqconjugateq }:=\left[\begin{array}{l}
0 \\
0 \\
0
\end{array}\right]
$$

$[>s:=\operatorname{evalm}($ inverse(J8).Bqconjugateq $) ;$

$$
s:=\left[\begin{array}{lll}
0 & 0 & 0
\end{array}\right]
$$

$[>B q s:=\operatorname{simplify}(\operatorname{subs}(x i[1]=q[1], x i[2]=q[2], x i[3]=q[3], m u[1]=s[1]$, $m u[2]=s[2], m u[3]=s[3], B))$;

$$
B q s:=\left[\begin{array}{l}
0 \\
0 \\
0
\end{array}\right]
$$

$[>t:=\operatorname{simplify}($ conjugate $(p[1]) * B q s[1]+\operatorname{conjugate}(p[2]) * B q s[2]+$ conjugate $(p[3]) * B q s[3])$;

$$
t:=0
$$

$[>M:=$ Matrix $(3,3$, shape $=$ identity $):$

$[>w:=\operatorname{simplify}($ evalm $($ inverse $(2 * I *$ omega $* M-J 8) . B q q)) ;$ 


$$
w:=\left[\begin{array}{lll}
\frac{1}{87}+\frac{104}{87} I & -\frac{14}{87}-\frac{2}{29} I \quad \frac{80}{87}-\frac{148}{87} I
\end{array}\right]
$$

$[>$ Bconjugateqw $:=\operatorname{simplify}(\operatorname{subs}(x i[1]=\operatorname{conjugate}(q[1]), x i[2]$ $=\operatorname{conjugate}(q[2]), x i[3]=$ conjugate $(q[3]), m u[1]=w[1], m u[2]=$ $w[2], m u[3]=w[3], B))$;

$$
\text { Bconjugateqw }:=\left[\begin{array}{c}
\frac{8}{87}-\frac{3}{29} I \\
\frac{2}{29}-\frac{188}{87} I \\
\frac{280}{87}+\frac{40}{29} I
\end{array}\right]
$$

$[>u:=$ simplify $($ conjugate $(p[1]) *$ Bconjugateqw $[1]+$ conjugate $(p[2]) *$ Bconjugateqw $[2]+$ conjugate $(p[3]) *$ Bconjugateqw $[3])$;

$$
\begin{gathered}
u:=-\frac{100}{377}-\frac{2345}{1131} I \\
{[>l[1]:=\text { simplify }((1 /(2 * \text { omega })) * \operatorname{Re}(r-2 * t+u))} \\
l_{1}:=-\frac{50}{377}
\end{gathered}
$$

Bifuração de Hopf Supercrítica

\section{B.3 Cálculo do coeficiente de Lyapunov do sistema} (7.4)

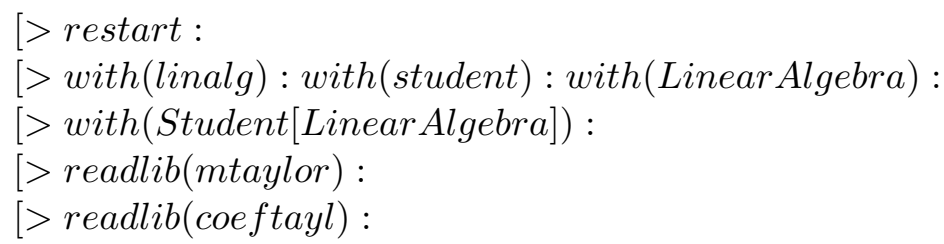

Sistema

$[>F[1]:=X[1]-X[2]:$

$[>F[2]:=5 * X[1]-X[2]-X[1] * X[3]:$

$[>F[3]:=X[1] * X[2]-13.93 * X[3]:$

Pontos de Equilíbrio

[> solve $(F[1], F[2], F[3], X[1], X[2], X[3])$;

$$
\begin{gathered}
\left\{X_{1}=0, X_{2}=0, X_{3}=0\right\},\left\{X_{1}=7.46, X_{2}=7.46, X_{3}=4\right\},\left\{X_{1}=\right. \\
\left.-7.46, X_{2}=-7.46, X_{3}=4\right\}
\end{gathered}
$$

Matriz Jacobiana e Estabilidade dos Equilíbrios $[>J:=\operatorname{Matrix}(j a c o b i a n([F[1], F[2], F[3]],[X[1], X[2], X[3]]))$; 


$$
J:=\left[\begin{array}{ccc}
1 & -1 & 0 \\
5-X_{3} & -1 & -X_{1} \\
X_{2} & X_{1} & -13.93
\end{array}\right]
$$

$[>J 1:=\operatorname{subs}(X[1]=7.46, X[2]=7.46, X[3]=4, J) ;$

$$
J 1:=\left[\begin{array}{ccc}
1 & -1 & 0 \\
1 & -1 & -7.46 \\
7.46 & 7.46 & -13.93
\end{array}\right]
$$

$[>\operatorname{lambda}:=$ Eigenvalues $(J 1)$

$$
\lambda:=\left[\begin{array}{c}
1.43 \\
-7.68+4.33 I \\
-7.68-4.33 I
\end{array}\right]
$$

$[>J 2:=\operatorname{subs}(X[1]=-7.46, X[2]=-7.46, X[3]=4, J)$;

$$
J 2:=\left[\begin{array}{ccc}
1 & -1 & 0 \\
1 & -1 & 7.46 \\
-7.46 & -7.46 & -13.93
\end{array}\right]
$$

$[>\operatorname{lambda}:=$ Eigenvalues $(J 2)$;

$$
\lambda:=\left[\begin{array}{c}
1.43 \\
-7.68+4.33 I \\
-7.68-4.33 I
\end{array}\right]
$$

$[>J 3:=\operatorname{subs}(X[1]=0, X[2]=0, X[3]=0, J)$;

$$
J 3:=\left[\begin{array}{ccc}
1 & -1 & 0 \\
5 & -1 & 0 \\
0 & 0 & -13.93
\end{array}\right]
$$

$[>$ lambda $:=$ Eigenvalues(J3);

$$
\lambda:=\left[\begin{array}{c}
2 I \\
-2 I \\
-13.93
\end{array}\right]
$$

$[>$ omega $:=\operatorname{Im}(\operatorname{lambda}[1])$

$$
\omega:=2
$$

Autovetores da Matriz J e da sua transposta $\left[>q 1:=\operatorname{eigenvects}\left(J 3{ }^{\prime}\right.\right.$ radical $\left.^{\prime}\right)$; 
$q 1:=$

$\left[-2 I, 1,\left\{\left[\begin{array}{lll}-0.4-0.2 I & -I & 0\end{array}\right]\right\}\right],\left[2 I, 1,\left\{\left[\begin{array}{lll}-0.4+0.2 I & I & 0\end{array}\right]\right\}\right]$, $\left[-13.93,1,\left\{\left[\begin{array}{lll}0 & 0 & 1\end{array}\right]\right\}\right]$

$[>q:=q 1[2][3][1]$

$$
q:=[I, 1,\{[-0.4+0.2 I \quad I \quad 0]
$$

$\left[>p 2:=\right.$ eigenvects(transpose $(J 3),{ }^{\prime}$ radical $\left.^{\prime}\right)$;

$$
p 2:=
$$

$\left[-2 I, 1,\left\{\left[\begin{array}{lll}2+I & -I & 0\end{array}\right]\right\}\right],\left[2 I, 1,\left\{\left[\begin{array}{lll}2-I & I & 0\end{array}\right]\right\}\right],\left[-13.93,1,\left\{\left[\begin{array}{lll}0 & 0 & 1\end{array}\right]\right\}\right]$ $[>p 1:=p 2[1][3][1]$

$$
p 1:=\left[\begin{array}{lll}
2+I & -I & 0
\end{array}\right]
$$

$[>$ normalizacao $:=$ simplify $($ evalc(conjugate $(p 1[1]) * q[1]+$ conjugate ( $p 1[2]) * q[2]+$ conjugate $(p 1[3]) * q[3]))$;

$$
\text { normalizacao }:=-1.6+0.8 I
$$

$[>$ fator $:=\operatorname{simplify}(\operatorname{evalc}(1 / \operatorname{conjugate}($ normalizacao $))) ;$

$$
\text { fator }:=-0.5+0.25 I
$$

$[>p:=\operatorname{vector}([\operatorname{simplify}($ evalc $($ fator $* p 1[1]))$, simplify $($ evalc $($ fator $* p 1[2]$ )$)$, simplify $($ evalc $($ fator $* p 1[3]))])$;

$$
p:=\left[\begin{array}{cc}
-1.25 & 0.25+0.5 I \\
0 &
\end{array}\right]
$$

$[>\operatorname{simplify}($ evalc $($ conjugate $(p[1]) * q[1]+\operatorname{conjugate}(p[2]) * q[2]+$ conjugate ( $p[3]) * q[3]))$;

1

Deslocando o Equilibrio para a Origem

$\left[>X_{1}:=x_{1}: X_{2}:=x_{2}: X_{3}:=x_{3}:\right.$

$\left[>F_{1}:=\operatorname{simplify}(X[1]-X[2])\right.$;

$$
F_{1}:=x_{1}-x_{2}
$$

$[>F[2]:=\operatorname{simplify}(5 * X[1]-X[2]-X[1] * X[3])$

$$
F_{2}:=5 x_{1}-x_{2}-x_{1} x_{3}
$$


$[>F[3]:=\operatorname{simplify}(X[1] * X[2]-13.93 * X[3])$

$$
F_{3}:=x_{1} x_{2}-13.93 x_{3}
$$

Calculando as Funções Multilineares $\mathrm{B}(\mathrm{x}, \mathrm{y})$ e $\mathrm{C}(\mathrm{x}, \mathrm{y}, \mathrm{z})$

$[>B[1]:=2 * \operatorname{coeftayl}(F[1],[x[1], x[2], x[3]]=[0,0,0],[2,0,0]) * x i[1] * m u[1]+$ $\operatorname{coeftayl}(F[1],[x[1], x[2], x[3]]=[0,0,0],[1,1,0]) *(x i[1] * m u[2]+x i[2] * m u[1])+$ $\operatorname{coeftayl}(F[1],[x[1], x[2], x[3]]=[0,0,0],[1,0,1]) *(x i[1] * m u[3]+x i[3] * m u[1])+$ $\operatorname{coeftayl}(F[1],[x[1], x[2], x[3]]=[0,0,0],[0,1,1]) *(x i[2] * m u[3]+x i[3] * m u[2])+$ $2 * \operatorname{coeftayl}(F[1],[x[1], x[2], x[3]]=[0,0,0],[0,2,0]) * x i[2] * m u[2]+2 *$ coeftayl ( $F[1],[x[1], x[2], x[3]]=[0,0,0],[0,0,2]) * x i[3] * m u[3]$;

$$
B[1]:=0
$$

$[>B[2]:=2 * \operatorname{coeftayl}(F[2],[x[1], x[2], x[3]]=[0,0,0],[2,0,0]) * x i[1] * m u[1]+$ $\operatorname{coeftayl}(F[2],[x[1], x[2], x[3]]=[0,0,0],[1,1,0]) *(x i[1] * m u[2]+x i[2] * m u[1])+$ $\operatorname{coeftayl}(F[2],[x[1], x[2], x[3]]=[0,0,0],[1,0,1]) *(x i[1] * m u[3]+x i[3] * m u[1])+$ $\operatorname{coeftayl}(F[2],[x[1], x[2], x[3]]=[0,0,0],[0,1,1]) *(x i[2] * m u[3]+x i[3] * m u[2])+$ $2 * \operatorname{coeftayl}(F[2],[x[1], x[2], x[3]]=[0,0,0],[0,2,0]) * x i[2] * m u[2]+2 *$ $\operatorname{coeftayl}(F[2],[x[1], x[2], x[3]]=[0,0,0],[0,0,2]) * x i[3] * m u[3] ;$

$$
B[2]:=-\xi_{1} \mu_{3}-\xi_{3} \mu_{1}
$$

$[>B[3]:=2 * \operatorname{coeftayl}(F[2],[x[1], x[2], x[3]]=[0,0,0],[2,0,0]) * x i[1] * m u[1]+$ $2 * \operatorname{coeftayl}(F[3],[x[1], x[2], x[3]]=[0,0,0],[2,0,0]) * x i[1] * m u[1]+\operatorname{coeftayl}($ $F[3],[x[1], x[2], x[3]]=[0,0,0],[1,1,0]) *(x i[1] * m u[2]+x i[2] * m u[1])+$ coeftayl ( $F[3],[x[1], x[2], x[3]]=[0,0,0],[1,0,1]) *(x i[1] * m u[3]+x i[3] * m u[1])+$ coeftayl ( $F[3],[x[1], x[2], x[3]]=[0,0,0],[0,1,1]) *(x i[2] * m u[3]+x i[3] * m u[2])+2 *$ $\operatorname{coeftayl}(F[3],[x[1], x[2], x[3]]=[0,0,0],[0,2,0]) * x i[2] * m u[2]+2 * \operatorname{coeftayl}($ $F[3],[x[1], x[2], x[3]]=[0,0,0],[0,0,2]) * x i[3] * m u[3]$;

$$
B[3]:=\xi_{1} \mu_{2}+\xi_{2} \mu_{1}
$$

$[>C[1]:=6 * \operatorname{coeftayl}(F[1],[x[1], x[2], x[3]]=[0,0,0],[3,0,0]) * x i[1] * m u[1] *$ eta $[1]+6 * \operatorname{coeftayl}(F[1],[x[1], x[2], x[3]]=[0,0,0],[0,3,0]) * x i[2] * m u[2] *$ eta $[2]+6 * \operatorname{coeftayl}(F[1],[x[1], x[2], x[3]]=[0,0,0],[0,0,3]) * x i[3] * m u[3] *$ eta $[3]+2 * \operatorname{coeftayl}(F[1],[x[1], x[2], x[3]]=[0,0,0],[2,1,0]) *(x i[1] * m u[1] *$ eta $[2]+x i[1] * m u[2] * \operatorname{eta}[1]+x i[2] * m u[1] * \operatorname{eta}[1])+2 * \operatorname{coeftayl}(F[1]$, $[x[1], x[2], x[3]]=[0,0,0],[2,0,1]) *(x i[1] * m u[1] *$ eta $[3]+x i[1] * m u[3] *$ eta $[1]+x i[3] * m u[1] *$ eta $[1])+2 * \operatorname{coeftayl}(F[1],[x[1], x[2], x[3]]=[0,0,0]$, $[1,2,0]) *(x i[1] * m u[2] *$ eta $[2]+x i[2] * m u[1] *$ eta $[2]+x i[2] *$ $m u[2] * \operatorname{eta}[1])+2 * \operatorname{coeftayl}(F[1],[x[1], x[2], x[3]]=[0,0,0],[0,2,1]) *$ 
$(x i[2] * m u[2] *$ eta $[3]+x i[2] * m u[3] *$ eta $[2]+x i[3] * m u[2] *$

eta[2]) $+2 *$ coeftayl $(F[1],[x[1], x[2], x[3]]=[0,0,0],[1,0,2]) *(x i[1] *$

$m u[3] *$ eta $[3]+x i[3] * m u[1] *$ eta $[3]+x i[3] * m u[3] *$ eta $[1])+2 *$

$\operatorname{coeftayl}(F[1],[x[1], x[2], x[3]]=[0,0,0],[0,1,2]) *(x i[2] * m u[3] *$ eta $[3]+$

$x i[3] * m u[2] *$ eta $[3]+x i[3] * m u[3] *$ eta $[2])+\operatorname{coeftayl}(F[1],[x[1], x[2]$,

$x[3]]=[0,0,0],[1,1,1]) *(x i[1] * \operatorname{mu}[2] * \operatorname{eta}[3]+x i[1] * m u[3] *$ eta $[2]$

$+x i[2] * m u[1] * \operatorname{eta}[3]+x i[2] * m u[3] * \operatorname{eta}[1]+x i[3] * m u[1] *$ eta $[2]+$

$x i[3] * m u[2] *$ eta $[1])$

$$
C[1]:=0
$$

$[>C[2]:=6 * \operatorname{coeftayl}(F[2],[x[1], x[2], x[3]]=[0,0,0],[3,0,0]) * x i[1] * m u[1]$ $* \operatorname{eta}[1]+6 * \operatorname{coeftayl}(F[2],[x[1], x[2], x[3]]=[0,0,0],[0,3,0]) * x i[2] *$ $m u[2] * \operatorname{eta}[2]+6 * \operatorname{coeftayl}(F[2],[x[1], x[2], x[3]]=[0,0,0],[0,0,3]) * x i[3]$ $* m u[3] * \operatorname{eta}[3]+2 * \operatorname{coeftayl}(F[2],[x[1], x[2], x[3]]=[0,0,0],[2,1,0]) *$ $(x i[1] * m u[1] *$ eta $[2]+x i[1] * m u[2] *$ eta $[1]+x i[2] * m u[1] *$ eta $[1])$ $+2 * \operatorname{coeftayl}(F[2],[x[1], x[2], x[3]]=[0,0,0],[2,0,1]) *(x i[1] * m u[1] *$ eta $[3]+x i[1] * m u[3] *$ eta $[1]+x i[3] * m u[1] *$ eta $[1])+2 * \operatorname{coeftayl}(F[2]$, $[x[1], x[2], x[3]]=[0,0,0],[1,2,0]) *(x i[1] * m u[2] * \operatorname{eta}[2]+x i[2] * m u[1]$ $* e t a[2]+x i[2] * m u[2] * \operatorname{eta}[1])+2 * \operatorname{coeftayl}(F[2],[x[1], x[2], x[3]]=[0,0$, $0],[0,2,1]) *(x i[2] * m u[2] * \operatorname{eta}[3]+x i[2] * m u[3] *$ eta $[2]+x i[3] *$ $m u[2] * \operatorname{eta}[2])+2 * \operatorname{coeftayl}(F[2],[x[1], x[2], x[3]]=[0,0,0],[1,0,2]) *(x i[1]$ $* m u[3] *$ eta $[3]+x i[3] * m u[1] *$ eta $[3]+x i[3] * m u[3] *$ eta $[1])+2 *$ $\operatorname{coeftayl}(F[2],[x[1], x[2], x[3]]=[0,0,0],[0,1,2]) *(x i[2] * m u[3] * \operatorname{eta}[3]+x i[3]$ $* m u[2] * \operatorname{eta}[3]+x i[3] * m u[3] * \operatorname{eta}[2])+\operatorname{coeftayl}(F[2],[x[1], x[2], x[3]]=[0,0$, $0],[1,1,1]) *(x i[1] * m u[2] *$ eta $[3]+x i[1] * m u[3] *$ eta $[2]+x i[2] * m u[1]$ $* e t a[3]+x i[2] * m u[3] *$ eta $[1]+x i[3] * m u[1] * \operatorname{eta}[2]+x i[3] * m u[2] *$ eta $[1])$;

$$
C[2]:=0
$$

$[>C[3]:=6 * \operatorname{coeftayl}(F[3],[x[1], x[2], x[3]]=[0,0,0],[3,0,0]) * x i[1] * m u[1] *$ eta $[1]+6 * \operatorname{coeftayl}(F[3],[x[1], x[2], x[3]]=[0,0,0],[0,3,0]) * x i[2] * m u[2] *$ eta $[2]+6 *$ coeftayl $(F[3],[x[1], x[2], x[3]]=[0,0,0],[0,0,3]) * x i[3] * m u[3] *$ eta $[3]+2 * \operatorname{coeftayl}(F[3],[x[1], x[2], x[3]]=[0,0,0],[2,1,0]) *(x i[1] * m u[1] *$ eta $[2]+x i[1] * m u[2] *$ eta $[1]+x i[2] * m u[1] * \operatorname{eta}[1])+2 * \operatorname{coeftayl}(F[3],[x[1]$, $x[2], x[3]]=[0,0,0],[2,0,1]) *(x i[1] * \operatorname{mu}[1] * \operatorname{eta}[3]+x i[1] * \operatorname{mu}[3] * \operatorname{eta}[1]+$ $x i[3] * m u[1] * \operatorname{eta}[1])+2 * \operatorname{coeftayl}(F[3],[x[1], x[2], x[3]]=[0,0,0],[1,2,0]) *$ $(x i[1] * m u[2] *$ eta $[2]+x i[2] * m u[1] *$ eta $[2]+x i[2] * m u[2] *$ eta $[1])+2 *$ $\operatorname{coeftayl}(F[3],[x[1], x[2], x[3]]=[0,0,0],[0,2,1]) *(x i[2] * m u[2] * \operatorname{eta}[3]+x i[2] *$ $m u[3] *$ eta $[2]+x i[3] * m u[2] * \operatorname{eta}[2])+2 * \operatorname{coeftayl}(F[3],[x[1], x[2], x[3]]=[0,0,0]$, $[1,0,2]) *(x i[1] * m u[3] *$ eta $[3]+x i[3] * m u[1] *$ eta $[3]+x i[3] * m u[3] *$ eta $[1])$ $+2 * \operatorname{coeftayl}(F[3],[x[1], x[2], x[3]]=[0,0,0],[0,1,2]) *(x i[2] * \operatorname{mu}[3] * \operatorname{eta}[3]+x i[3] *$ 
$m u[2] * \operatorname{eta}[3]+x i[3] * m u[3] * \operatorname{eta}[2])+\operatorname{coeftayl}(F[3],[x[1], x[2], x[3]]=[0,0,0]$, $[1,1,1]) *(x i[1] * m u[2] *$ eta $[3]+x i[1] * m u[3] * \operatorname{eta}[2]+x i[2] * m u[1] *$ $\eta[3]+x i[2] * m u[3]$ asteta $[1]+x i[3] * m u[1] *$ eta $[2]+x i[3] * m u[2] *$ eta[1] $)$;

$$
C[3]:=0
$$

$[>B:=<B[1], B[2], B[3]>$ :

$[>C:=<C[1], C[2], C[3]>$ :

Calculando Primeiro Coeficiente de Lyapunov

$[>$ Cqqconjugateq $:=\operatorname{simplify}(\operatorname{subs}(x i[1]=q[1], x i[2]=q[2], x i[3]=q[3]$, $m u[1]=q[1], m u[2]=q[2], m u[3]=q[3]$, eta $[1]=\operatorname{conjugate}(q[1])$, eta $[2]=$ conjugate $(q[2])$, eta $[3]=$ conjugate $(q[3]), C))$;

$$
\text { Cqqconjugateq }:=\left[\begin{array}{l}
0 \\
0 \\
0
\end{array}\right]
$$

$[>r:=\operatorname{simplify}($ conjugate $(p[1]) *$ Cqqconjugateq $[1]+\operatorname{conjugate}(p[2]) *$ Cqqconjugateq $[2]+$ conjugate $(p[3]) *$ Cqqconjugateq $[3])$;

$$
r:=0
$$

$[>B q q:=\operatorname{simplify}(\operatorname{subs}(x i[1]=q[1], x i[2]=q[2], x i[3]=q[3], m u[1]=q[1]$, $m u[2]=q[2], m u[3]=q[3], B)) ;$

$$
B q q:=\left[\begin{array}{c}
0 \\
0 \\
-0.4-0.8 I
\end{array}\right]
$$

$[>$ Bqconjugateq $:=$ simplify $($ subs $(x i[1]=q[1], x i[2]=q[2], x i[3]=q[3]$ $, m u[1]=\operatorname{conjugate}(q[1]), m u[2]=\operatorname{conjugate}(q[2]), m u[3]=\operatorname{conjugate}(q[3])$ $, B))$;

$$
\text { Bqconjugateq }:=\left[\begin{array}{c}
0 \\
0 \\
0.4
\end{array}\right]
$$

$[>s:=\operatorname{evalm}($ inverse(J3).Bqconjugateq $)$

$$
s:=\left[\begin{array}{lll}
0 & 0 & -0.03
\end{array}\right]
$$

$[>B q s:=\operatorname{simplify}(\operatorname{subs}(x i[1]=q[1], x i[2]=q[2], x i[3]=q[3], m u[1]=s[1]$, $m u[2]=s[2], m u[3]=s[3], B))$; 


$$
B q s:=\left[\begin{array}{c}
0 \\
-0.01+0.01 I \\
0
\end{array}\right]
$$

$[>t:=\operatorname{simplify}($ conjugate $(p[1]) * B q s[1]+\operatorname{conjugate}(p[2]) * B q s[2]+$ conjugate $(p[3]) *$ Bqs[3]);

$$
t:=0.01 I
$$

$[>M:=$ Matrix $(3,3$, shape $=$ identity $):$

$[>w:=\operatorname{simplify}($ evalm $($ inverse $(2 * I *$ omega $* M-J 3) . B q q)) ;$

$$
w:=\left[\begin{array}{lll}
0 & 0 & -0.04-0.05 I
\end{array}\right]
$$

$[>$ Bconjugateqw $:=\operatorname{simplify}(\operatorname{subs}(x i[1]=\operatorname{conjugate}(q[1]), x i[2]$ $=\operatorname{conjugate}(q[2]), x i[3]=$ conjugate $(q[3]), m u[1]=w[1], m u[2]=$ $w[2], m u[3]=w[3], B))$;

$$
\text { Bconjugateqw }:=\left[\begin{array}{c}
0 \\
-0.01-0.03 I \\
0
\end{array}\right]
$$

$[>u:=\operatorname{simplify}(\operatorname{conjugate}(p[1]) *$ Bconjugateqw $[1]+$ conjugate $(p[2]) *$ Bconjugateqw $[2]+$ conjugate $(p[3]) *$ Bconjugateqw $[3])$;

$$
u:=-0.02
$$

$[>l[1]:=\operatorname{simplify}((1 /(2 *$ omega $)) * \operatorname{Re}(r-2 * t+u))$

$$
l_{1}:=-0.0038
$$

Bifuração de Hopf Supercrítica

\section{B.4 Cálculo do coeficiente de Lyapunov do sistema}

\section{(7.5)}

[> restart:

$[>$ with $($ linalg $):$ with $($ student $):$ with (Linear Algebra $)$ :

[> with(Student [Linear Algebra]) :

[> readlib(mtaylor) :

[> readlib(coeftayl) :

\section{Sistema}

$[>F[1]:=X[2]:$

$[>F[2]:=X[3]:$

$\left[>F[3]:=-0.5 * X[3]-2 * X[2]-X[1]+X[1]^{2}:\right.$ 
Pontos de Equilíbrio

[> solve $(F[1], F[2], F[3], X[1], X[2], X[3])$;

$$
\left\{X_{1}=0, X_{2}=0, X_{3}=0\right\},\left\{X_{1}=1, X_{2}=0, X_{3}=0\right\}
$$

Matriz Jacobiana e Estabilidade dos Equilíbrios

$[>J:=\operatorname{Matrix}(j a c o b i a n([F[1], F[2], F[3]],[X[1], X[2], X[3]]))$;

$$
J:=\left[\begin{array}{ccc}
0 & 1 & 0 \\
0 & 0 & 1 \\
-1+2 X_{1} & -2 & -0.5
\end{array}\right]
$$

$[>J 1:=\operatorname{subs}(X[1]=1, X[2]=0, X[3]=0, J)$;

$$
J 1:=\left[\begin{array}{ccc}
0 & 1 & 0 \\
0 & 0 & 1 \\
1 & -2 & -0.5
\end{array}\right]
$$

$[>\operatorname{lambda}:=$ Eigenvalues $(J 1)$

$$
\lambda:=\left[\begin{array}{c}
-0.46+1.47 I \\
-0.46-1.47 I \\
0.42
\end{array}\right]
$$

$[>J 2:=\operatorname{subs}(X[1]=0, X[2]=0, X[3]=0, J) ;$

$$
J 2:=\left[\begin{array}{ccc}
0 & 1 & 0 \\
0 & 0 & 1 \\
-1 & -2 & -0.5
\end{array}\right]
$$

$[>\operatorname{lambda}:=$ Eigenvalues $(J 2)$;

$$
\lambda:=\left[\begin{array}{c}
1.41 I \\
-1.41 I \\
-0.5
\end{array}\right]
$$

$[>$ omega $:=\operatorname{Im}(\operatorname{lambda}[1])$

$$
\omega:=1.41
$$

Autovetores da Matriz J e da sua transposta

$\left[>q 1:=\operatorname{eigenvects}\left(J 2,{ }^{\prime}\right.\right.$ radical $\left.^{\prime}\right)$;

$q 1:=$

$[1.41 I, 1,\{[0.35-0.5 I, 0.71+0.5 I,-0.71+1.01 I]\}],[-1.41 I, 1,\{[0.35+0.5 I, 0.71-0.5 I$, $-0.71-1.01 \mathrm{I}\},[-0.5,1,\{[-0.87,0.44,-0.22]\}]$ 
$[>q:=q 1[1][3][1]$

$$
q:=\left[I, 1,\left\{\left[\begin{array}{lll}
0.35-0.5 I & 0.71+0.5 I & -0.71+1.01 I
\end{array}\right]\right.\right.
$$

$\left[>p 2:=\right.$ eigenvects $\left(\right.$ transpose $(J 2),{ }^{\prime}$ radical $\left.^{\prime}\right)$;

$p 2:=$

$[1.41 I, 1,\{[-0.33+0.39 I,-0.38+1.02 I, 0.56+0.47 I]\}],[-1.41 I, 1,\{[-0.33-0.39 I$, -0.38-1.02I, 0.56-0.47I $\},[-0.5,1,\{[-0.89,0,-0.45]\}]$

$[>p 1:=p 2[2][3][1]$

$$
p 1:=\left[\begin{array}{lll}
-0.33-0.39 I & -0.38-1.02 I & 0.56-0.47 I
\end{array}\right]
$$

$[>$ normalizacao $:=$ simplify $($ evalc $($ conjugate $(p 1[1]) * q[1]+$ conjugate ( $p 1[2]) * q[2]+$ conjugate $(p 1[3]) * q[3]))$;

$$
\text { normalizacao }:=-1.57+1.07 I
$$

$[>$ fator $:=\operatorname{simplify}(\operatorname{evalc}(1 / \operatorname{conjugate}($ normalizacao $))) ;$

$$
\text { fator }:=-0.44+0.3 I
$$

$[>p:=\operatorname{vector}([\operatorname{simplify}($ evalc $($ fator $* p 1[1]))$, simplify $($ evalc $($ fator $* p 1[2]$ )$)$, simplify $($ evalc $($ fator $* p 1[3]))])$;

$$
p:=\left[\begin{array}{cc}
0.26+0.07 I & 0.47+0.33 I \\
-0.1+0.37 I &
\end{array}\right]
$$

$[>\operatorname{simplify}($ evalc $($ conjugate $(p[1]) * q[1]+\operatorname{conjugate}(p[2]) * q[2]+$ conjugate ( $p[3]) * q[3]))$;

Deslocando o Equilibrio para a Origem

$\left[>X_{1}:=x_{1}: X_{2}:=x_{2}: X_{3}:=x_{3}:\right.$

$\left[>F_{1}:=\operatorname{simplify}(X[2])\right.$;

$$
F_{1}:=x_{2}
$$

$[>F[2]:=\operatorname{simplify}(X[3])$

$$
F_{2}:=x_{3}
$$

$\left[>F[3]:=\operatorname{simplify}\left(-0.5 * X[3]-2 * X[2]-X[1]+X[1]^{2}\right) ;\right.$ 


$$
F_{3}:=-0.5 x_{3}-2 x_{2}-x_{1}+x_{1}^{2}
$$

Calculando as Funções Multilineares $\mathrm{B}(\mathrm{x}, \mathrm{y})$ e $\mathrm{C}(\mathrm{x}, \mathrm{y}, \mathrm{z})$

$[>B[1]:=2 * \operatorname{coeftayl}(F[1],[x[1], x[2], x[3]]=[0,0,0],[2,0,0]) * x i[1] * m u[1]+$ $\operatorname{coeftayl}(F[1],[x[1], x[2], x[3]]=[0,0,0],[1,1,0]) *(x i[1] * m u[2]+x i[2] * m u[1])+$ $\operatorname{coeftayl}(F[1],[x[1], x[2], x[3]]=[0,0,0],[1,0,1]) *(x i[1] * m u[3]+x i[3] * m u[1])+$ $\operatorname{coeftayl}(F[1],[x[1], x[2], x[3]]=[0,0,0],[0,1,1]) *(x i[2] * m u[3]+x i[3] * m u[2])+$ $2 * \operatorname{coeftayl}(F[1],[x[1], x[2], x[3]]=[0,0,0],[0,2,0]) * x i[2] * m u[2]+2 *$ coeftayl ( $F[1],[x[1], x[2], x[3]]=[0,0,0],[0,0,2]) * x i[3] * m u[3] ;$

$$
B[1]:=0
$$

$[>B[2]:=2 * \operatorname{coeftayl}(F[2],[x[1], x[2], x[3]]=[0,0,0],[2,0,0]) * x i[1] * m u[1]+$ $\operatorname{coeftayl}(F[2],[x[1], x[2], x[3]]=[0,0,0],[1,1,0]) *(x i[1] * m u[2]+x i[2] * m u[1])+$ $\operatorname{coeftayl}(F[2],[x[1], x[2], x[3]]=[0,0,0],[1,0,1]) *(x i[1] * m u[3]+x i[3] * m u[1])+$ $\operatorname{coeftayl}(F[2],[x[1], x[2], x[3]]=[0,0,0],[0,1,1]) *(x i[2] * m u[3]+x i[3] * m u[2])+$ $2 * \operatorname{coeftayl}(F[2],[x[1], x[2], x[3]]=[0,0,0],[0,2,0]) * x i[2] * m u[2]+2 *$ $\operatorname{coeftayl}(F[2],[x[1], x[2], x[3]]=[0,0,0],[0,0,2]) * x i[3] * m u[3] ;$

$$
B[2]:=0
$$

$[>B[3]:=2 * \operatorname{coeftayl}(F[2],[x[1], x[2], x[3]]=[0,0,0],[2,0,0]) * x i[1] * m u[1]+$ $2 * \operatorname{coeftayl}(F[3],[x[1], x[2], x[3]]=[0,0,0],[2,0,0]) * x i[1] * m u[1]+\operatorname{coeftayl}(F[3]$, $[x[1], x[2], x[3]]=[0,0,0],[1,1,0]) *(x i[1] * m u[2]+x i[2] * m u[1])+\operatorname{coeftayl}($ $F[3],[x[1], x[2], x[3]]=[0,0,0],[1,0,1]) *(x i[1] * m u[3]+x i[3] * m u[1])+$ coeftayl ( $F[3],[x[1], x[2], x[3]]=[0,0,0],[0,1,1]) *(x i[2] * m u[3]+x i[3] * m u[2])+2 *$ $\operatorname{coeftayl}(F[3],[x[1], x[2], x[3]]=[0,0,0],[0,2,0]) * x i[2] * m u[2]+2 * \operatorname{coeftayl}($ $F[3],[x[1], x[2], x[3]]=[0,0,0],[0,0,2]) * x i[3] * m u[3]$;

$$
B[3]:=2 \xi_{1} \mu_{1}
$$

$[>C[1]:=6 * \operatorname{coeftayl}(F[1],[x[1], x[2], x[3]]=[0,0,0],[3,0,0]) * x i[1] * m u[1] *$ eta $[1]+6 * \operatorname{coeftayl}(F[1],[x[1], x[2], x[3]]=[0,0,0],[0,3,0]) * x i[2] * m u[2] *$ eta $[2]+6 * \operatorname{coeftayl}(F[1],[x[1], x[2], x[3]]=[0,0,0],[0,0,3]) * x i[3] * m u[3] *$ eta $[3]+2 * \operatorname{coeftayl}(F[1],[x[1], x[2], x[3]]=[0,0,0],[2,1,0]) *(x i[1] * m u[1] *$ eta $[2]+x i[1] * m u[2] * \operatorname{eta}[1]+x i[2] * m u[1] * \operatorname{eta}[1])+2 * \operatorname{coeftayl}(F[1]$, $[x[1], x[2], x[3]]=[0,0,0],[2,0,1]) *(x i[1] * m u[1] *$ eta $[3]+x i[1] * m u[3] *$ eta $[1]+x i[3] * m u[1] * \operatorname{eta}[1])+2 * \operatorname{coeftayl}(F[1],[x[1], x[2], x[3]]=[0,0,0]$, $[1,2,0]) *(x i[1] * m u[2] *$ eta $[2]+x i[2] * m u[1] *$ eta $[2]+x i[2] *$ $m u[2] * \operatorname{eta}[1])+2 * \operatorname{coeftayl}(F[1],[x[1], x[2], x[3]]=[0,0,0],[0,2,1]) *$ $(x i[2] * m u[2] *$ eta $[3]+x i[2] * m u[3] *$ eta $[2]+x i[3] * m u[2] *$ eta $[2])+2 * \operatorname{coeftayl}(F[1],[x[1], x[2], x[3]]=[0,0,0],[1,0,2]) *(x i[1] *$ 
$m u[3] * \operatorname{eta}[3]+x i[3] * m u[1] *$ eta $[3]+x i[3] * m u[3] *$ eta $[1])+2 *$ $\operatorname{coeftayl}(F[1],[x[1], x[2], x[3]]=[0,0,0],[0,1,2]) *(x i[2] * m u[3] *$ eta $[3]+$ $x i[3] * m u[2] * \operatorname{eta}[3]+x i[3] * m u[3] *$ eta $[2])+\operatorname{coeftayl}(F[1],[x[1], x[2]$, $x[3]]=[0,0,0],[1,1,1]) *(x i[1] * m u[2] * \operatorname{eta}[3]+x i[1] * \operatorname{mu}[3] * \operatorname{eta}[2]$ $+x i[2] * m u[1] *$ eta $[3]+x i[2] * m u[3] *$ eta $[1]+x i[3] * m u[1] *$ eta $[2]+$ $x i[3] * m u[2] * \operatorname{eta}[1])$;

$$
C[1]:=0
$$

$[>C[2]:=6 * \operatorname{coeftayl}(F[2],[x[1], x[2], x[3]]=[0,0,0],[3,0,0]) * x i[1] * m u[1]$ $* \operatorname{eta}[1]+6 * \operatorname{coeftayl}(F[2],[x[1], x[2], x[3]]=[0,0,0],[0,3,0]) * x i[2] *$ $m u[2] * \operatorname{eta}[2]+6 * \operatorname{coeftayl}(F[2],[x[1], x[2], x[3]]=[0,0,0],[0,0,3]) * x i[3]$ $* m u[3] * \operatorname{eta}[3]+2 * \operatorname{coeftayl}(F[2],[x[1], x[2], x[3]]=[0,0,0],[2,1,0]) *$ $(x i[1] * m u[1] *$ eta $[2]+x i[1] * m u[2] *$ eta $[1]+x i[2] * m u[1] *$ eta $[1])$ $+2 * \operatorname{coeftayl}(F[2],[x[1], x[2], x[3]]=[0,0,0],[2,0,1]) *(x i[1] * m u[1] *$ eta $[3]+x i[1] * m u[3] * \operatorname{eta}[1]+x i[3] * m u[1] * \operatorname{eta}[1])+2 * \operatorname{coeftayl}(F[2]$, $[x[1], x[2], x[3]]=[0,0,0],[1,2,0]) *(x i[1] * m u[2] * \operatorname{eta}[2]+x i[2] * m u[1]$ $* \operatorname{eta}[2]+x i[2] * m u[2] * \operatorname{eta}[1])+2 * \operatorname{coeftayl}(F[2],[x[1], x[2], x[3]]=[0,0$, $0],[0,2,1]) *(x i[2] * m u[2] *$ eta $[3]+x i[2] * m u[3] *$ eta $[2]+x i[3] *$ $m u[2] * \operatorname{eta}[2])+2 * \operatorname{coeftayl}(F[2],[x[1], x[2], x[3]]=[0,0,0],[1,0,2]) *(x i[1]$ $* m u[3] *$ eta $[3]+x i[3] * m u[1] *$ eta $[3]+x i[3] * m u[3] *$ eta $[1])+2 *$ $\operatorname{coeftayl}(F[2],[x[1], x[2], x[3]]=[0,0,0],[0,1,2]) *(x i[2] * m u[3] * \operatorname{eta}[3]+x i[3]$ $* m u[2] * \operatorname{eta}[3]+x i[3] * m u[3] * \operatorname{eta}[2])+\operatorname{coeftayl}(F[2],[x[1], x[2], x[3]]=[0,0$, $0],[1,1,1]) *(x i[1] * m u[2] * \operatorname{eta}[3]+x i[1] * m u[3] *$ eta $[2]+x i[2] * m u[1]$ $*$ eta $[3]+x i[2] * m u[3] *$ eta $[1]+x i[3] * m u[1] *$ eta $[2]+x i[3] * m u[2] *$ eta $[1])$;

$$
C[2]:=0
$$

$[>C[3]:=6 * \operatorname{coeftayl}(F[3],[x[1], x[2], x[3]]=[0,0,0],[3,0,0]) * x i[1] * m u[1] *$ eta $[1]+6 * \operatorname{coeftayl}(F[3],[x[1], x[2], x[3]]=[0,0,0],[0,3,0]) * x i[2] * m u[2] *$ eta $[2]+6 * \operatorname{coeftayl}(F[3],[x[1], x[2], x[3]]=[0,0,0],[0,0,3]) * x i[3] * m u[3] *$ eta $[3]+2 * \operatorname{coeftayl}(F[3],[x[1], x[2], x[3]]=[0,0,0],[2,1,0]) *(x i[1] * m u[1] *$ eta $[2]+x i[1] * m u[2] * \operatorname{eta}[1]+x i[2] * m u[1] * \operatorname{eta}[1])+2 * \operatorname{coeftayl}(F[3],[x[1]$, $x[2], x[3]]=[0,0,0],[2,0,1]) *(x i[1] * m u[1] *$ eta $[3]+x i[1] * m u[3] *$ eta $[1]+$ $x i[3] * m u[1] *$ eta $[1])+2 * \operatorname{coeftayl}(F[3],[x[1], x[2], x[3]]=[0,0,0],[1,2,0]) *$ $(x i[1] * m u[2] *$ eta $[2]+x i[2] * m u[1] *$ eta $[2]+x i[2] * m u[2] *$ eta $[1])+2 *$ $\operatorname{coeftayl}(F[3],[x[1], x[2], x[3]]=[0,0,0],[0,2,1]) *(x i[2] * \operatorname{mu}[2] * \operatorname{eta}[3]+x i[2] *$ $m u[3] * \operatorname{eta}[2]+x i[3] * m u[2] * \operatorname{eta}[2])+2 * \operatorname{coeftayl}(F[3],[x[1], x[2], x[3]]=[0,0,0]$, $[1,0,2]) *(x i[1] * m u[3] *$ eta $[3]+x i[3] * m u[1] *$ eta $[3]+x i[3] * m u[3] *$ eta $[1])$ $+2 * \operatorname{coeftayl}(F[3],[x[1], x[2], x[3]]=[0,0,0],[0,1,2]) *(x i[2] * \operatorname{mu}[3] * \operatorname{eta}[3]+x i[3] *$ $m u[2] * \operatorname{eta}[3]+x i[3] * m u[3] * \operatorname{eta}[2])+\operatorname{coeftayl}(F[3],[x[1], x[2], x[3]]=[0,0,0]$, $[1,1,1]) *(x i[1] * m u[2] *$ eta $[3]+x i[1] * m u[3] * \operatorname{eta}[2]+x i[2] * m u[1] *$ 


$$
\begin{gathered}
\text { eta }[3]+x i[2] * m u[3] * \operatorname{eta}[1]+x i[3] * m u[1] * \operatorname{eta}[2]+x i[3] * m u[2] * \operatorname{eta}[1]) \\
C[3]:=0
\end{gathered}
$$

$[>B:=<B[1], B[2], B[3]>$ :

$[>C:=<C[1], C[2], C[3]>$ :

Calculando Primeiro Coeficiente de Lyapunov

$[>$ Cqqconjugateq $:=\operatorname{simplify}(\operatorname{subs}(x i[1]=q[1], x i[2]=q[2], x i[3]=q[3]$, $m u[1]=q[1], m u[2]=q[2], m u[3]=q[3]$, eta $[1]=$ conjugate $(q[1])$, eta $[2]=$ conjugate $(q[2])$, eta $[3]=$ conjugate $(q[3]), C))$;

$$
\text { Cqqconjugateq }:=\left[\begin{array}{l}
0 \\
0 \\
0
\end{array}\right]
$$

$[>r:=\operatorname{simplify}($ conjugate $(p[1]) *$ Cqqconjugateq[1] + conjugate $(p[2]) *$ Cqqconjugateq $[2]+$ conjugate $(p[3]) *$ Cqqconjugateq[3]);

$$
r:=0
$$

$[>B q q:=\operatorname{simplify}(\operatorname{subs}(x i[1]=q[1], x i[2]=q[2], x i[3]=q[3], m u[1]=q[1]$, $m u[2]=q[2], m u[3]=q[3], B)) ;$

$$
B q q:=\left[\begin{array}{c}
0 \\
0 \\
-0.26-0.71 I
\end{array}\right]
$$

$[>$ Bqconjugateq $:=$ simplify $(\operatorname{subs}(x i[1]=q[1], x i[2]=q[2], x i[3]=q[3]$ $, m u[1]=\operatorname{conjugate}(q[1]), m u[2]=\operatorname{conjugate}(q[2]), m u[3]=\operatorname{conjugate}(q[3])$ $, B))$;

$$
\text { Bqconjugateq }:=\left[\begin{array}{c}
0 \\
0 \\
0.76
\end{array}\right]
$$

$[>s:=\operatorname{evalm}($ inverse(J2).Bqconjugateq);

$$
s:=\left[\begin{array}{lll}
-0.76 & 0 & 0
\end{array}\right]
$$

$[>B q s:=\operatorname{simplify}(\operatorname{subs}(x i[1]=q[1], x i[2]=q[2], x i[3]=q[3], m u[1]=s[1]$, $m u[2]=s[2], m u[3]=s[3], B))$;

$$
B q s:=\left[\begin{array}{c}
0 \\
0 \\
-0.54+0.76 I
\end{array}\right]
$$


$[>t:=\operatorname{simplify}($ conjugate $(p[1]) *$ Bqs $[1]+$ conjugate $(p[2]) * B q s[2]+$ conjugate $(p[3]) * B q s[3])$;

$$
t:=0.34+0.12 I
$$

$[>M:=$ Matrix $(3,3$, shape $=$ identity $):$

$[>w:=\operatorname{simplify}($ evalm $($ inverse $(2 * I *$ omega $* M-J 2) . B q q)) ;$

$$
w:=\left[\begin{array}{lll}
0.04-0.01 I & 0.02+0.12 I & -0.35+0.06 I
\end{array}\right]
$$

$[>$ Bconjugateqw $:=\operatorname{simplify}(\operatorname{subs}(x i[1]=\operatorname{conjugate}(q[1])$, xi $[2]$ $=$ conjugate $(q[2]), x i[3]=$ conjugate $(q[3]), m u[1]=w[1], m u[2]=$ $w[2], m u[3]=w[3], B))$;

$$
\text { Bconjugateqw }:=\left[\begin{array}{c}
0 \\
0 \\
0.04+0.04 I
\end{array}\right]
$$

$[>u:=\operatorname{simplify}(\operatorname{conjugate}(p[1]) *$ Bconjugateqw $[1]+\operatorname{conjugate}(p[2]) *$ Bconjugateqw $[2]+$ conjugate $(p[3]) *$ Bconjugateqw $[3])$;

$$
u:=0.01-0.02 I
$$

$[>l[1]:=\operatorname{simplify}((1 /(2 *$ omega $)) * \operatorname{Re}(r-2 * t+u))$

$$
l_{1}:=-0.23
$$

Bifuração de Hopf Supercrítica 


\section{Referências Bibliográficas}

[AAB10] Fabíolo Moraes Amaral, Luís Fernando Costa Alberto e Newton Geraldo Bretas. Stability boundary characterization of nonlinear autonomous dynamical systems in the presence of a typezero saddle-node equilibrium point. Trends in Applied and Computational Mathematics, 11(2):111-120, 2010. 76

$\left[\mathrm{AH}^{+} 71\right]$ Kenneth Joseph Arrow, Frank Hahn et al. General competitive analysis. 1971. 1

[Alb06] Luís Fernando Costa Alberto. Caracterização e estimativas da área de atração de sistemas dinâmicos não lineares. Tese de Doutorado, Universidade de São Paulo, 2006. 16

[Ama10] Fabíolo Moraes Amaral. Caracterização, estimativas e bifurcações da região de estabilidade de sistemas dinâmicos não lineares. Tese de Doutorado, Universidade de São Paulo, 2010. $\overline{3,4,16}, 52,53,55,57,58,59,62,67,75,76$

[BMAG03] Newton Geraldo Bretas, A. C. P. Martins, Luís Fernando Costa Alberto e Renato B. L. Guedes. Static simulation of voltage collapse considering the operational limits of the generators. Em Power Engineering Society General Meeting, 2003, IEEE, volume 4. IEEE, 2003. 3

[BS02] Nam Parshad Bhatia e Giorgio P Szegö. Stability theory of dynamical systems, volume 161. Springer Science \& Business Media, 2002. xviii, 20, 21, 24, 25, 53, 54, 55, 57, 58, 60, 61, 62, $63,64,65$

[CC95] Hsiao-Dong Chiang e Chia-Chi Chu. Theoretical foundation of the bcu method for direct stability analysis of network-reduction power system. models with small transfer conductances. Circuits and Systems I: Fundamental Theory and Applications, IEEE Transactions on, 42(5):252-265, 1995. 180

[CCC95] Hsiao-Dong Chang, Chia-Chi Chu e Gerry Cauley. Direct stability analysis of electric power systems using energy functions: 
theory, applications, and perspective. Proceedings of the IEEE, 83(11):1497-1529, 1995. 1

[CFA96] Hsiao-Dong Chiang e Lazhar Fekih-Ahmed. Quasi-stability regions of nonlinear dynamical systems: Theory. Circuits and Systems I: Fundamental Theory and Applications, IEEE Transactions on, 43(8):627-635, 1996. 132, 189

[CHW88] Hsiao-Dong Chiang, Morris W. Hirsch e Felix F. Wu. Stability regions of nonlinear autonomous dynamical systems. Automatic Control, IEEE Transactions on, 33(1):16-27, 1988. 2, 3, 4, 55, $67,68,69,70,71,76,85,87,91,94,97,105,106,107,109,112$, $115,182,185$

[CT89] Hsiao-Dong Chiang e James S. Thorp. The closest unstable equilibrium point method for power system dynamic security assessment. Circuits and Systems, IEEE Transactions on, 36(9):1187$1200,1989.3$

[CWV87] Hsiao-Dong Chiang, Felix F. Wu e Pravin P. Varaiya. Foundations of direct methods for power system transient stability analysis. Circuits and Systems, IEEE Transactions on, 34(2):160-173, 1987. 1,2

[CWV94] Hsiao-Dong Chiang, Felix F. Wu e Pravin P. Varaiya. A bcu method for direct analysis of power system transient stability. Power Systems, IEEE Transactions on, 9(3):1194-1208, 1994. 3

[DSR71] A. K. De Sarkar e N. Dharma Rao. Zubov's method and transient-stability problems of power systems. Electrical Engineers, Proceedings of the Institution of, 118(8):1035-1040, 1971. 2

[Gal12] Marluci Cristina Galindo. Comportamento caótico em sistemas dinâmicos e aplicação no estudo de tumores de câncer. Dissertação de Mestrado, 2012. 207, 217

[Gao04] Ying-hui Gao. Bifurcations and stability boundary of a power system. Acta Mathematicae Applicatae Sinica (English Series), 20(3):513-532, 2004. 3

[GH83] John Guckenheimer e Philip Holmes. Nonlinear oscillations, dynamical systems, and bifurcations of vector fields, volume 42 . New York Springer Verlag, 1983. 16, 141

[GJAA13] Josaphat Ricardo Ribeiro Gouveia Jr., Fabíolo Moraes Amaral e Luís Fernando Costa Alberto. Stability boundary characterization of nonlinear autonomous dynamical systems in the presence 
of a supercritical hopf equilibrium point. International Journal of Bifurcation and Chaos, 23(12):1350196, 2013. 99, 190

[GTV85] Roberto Genesio, Michele Tartaglia e Antonio Vicino. On the estimation of asymptotic stability regions: State of the art and new proposals. Automatic Control, IEEE Transactions on, 30(8):747-755, 1985. 1, 73

[Hal69] Jack K. Hale. Ordinary differential equations. 1969. 16, 141

[HPS70] Morris W. Hirsch, Charles C. Pugh e Michael Shub. Invariant manifolds. Bulletin of the American Mathematical Society, 76(5):1015-1019, 1970. 16, 37

[Kel75] John L. Kelley. General topology. Springer Science \& Business Media, 1975. 54

[KG96] Hassan K. Khalil e J. W. Grizzle. Nonlinear systems, volume 3. Prentice hall New Jersey, 1996. 145, 147

[Kuz13] Yuri A. Kuznetsov. Elements of applied bifurcation theory, volume 112. Springer Science \& Business Media, 2013. 41, 141, $148,155,157,158,160,161,162,164,165,166,171,174,176$, $177,207,217$

[LC00] Jaewook Lee e Hsiao-Dong Chiang. Stability regions of nonhyperbolic dynamical systems: theory and optimal estimation. Em Circuits and Systems, 2000. Proceedings. ISCAS 2000 Geneva. The 2000 IEEE International Symposium on, volume 2, páginas 200-203. IEEE, 2000. 1

[Lim81] Elon Lages Lima. Curso de Análise, v. 2. 1981. 7, 15, 29, 163

[Lim03] Elon Lages Lima. Espaços métricos, 3a edição. Projeto Euclides, Instituto de Matemática Pura e Aplicada, CNPq, 2003. 7, 8, 11, $12,15,55$

[Lim06] Elon Lages Lima. Álgebra linear. IMPA, 2006. 7

[LS76] J. P. La Salle. The Stability of Dynamical Systems. SIAM, 1976. 22,23

[May01] Robert McCredie May. Stability and complexity in model ecosystems, volume 6. Princeton University Press, 2001. 1

[Mon06] Luiz Henrique Alves Monteiro. Sistemas dinâmicos. Editora Livraria da Física, 2006. 71, 129, 130, 195

[Mun75] James R. Munkres. Topology: a first course. 1975. 13 
[Pal69] Jacob Palis. On morse-smale dynamical systems. Topology, 8(4):385-404, 1969. 45, 46, 47

[Per13] Lawrence Perko. Differential equations and dynamical systems, volume 7. Springer Science \& Business Media, 2013. 16, 30, 32, $33,34,35,141,177$

[Pon] L. S. Pontryagin. Ordinary differential equations. 1962. 165, 166

[SGAB09] Flávio H. J. R. Silva, Renato B. L. Guedes, Luís Fernando Costa Alberto e Newton Geraldo Bretas. Função energia generalizada de controle para estabilização de sistemas não lineares. Sba: Controle \& Automação Sociedade Brasileira de Automatica, 20(2):133-145, 2009. 1

[SHD03] Stephen Smale, Morris W. Hirsch e Robert L. Devaney. Differential equations, dynamical systems, and an introduction to chaos, volume 60. Academic Press, 2003. 16

[Shu13] Michael Shub. Global stability of dynamical systems. Springer Science \& Business Media, 2013. 16, 180

[Sma67] Stephen Smale. Differentiable dynamical systems. Bulletin of the American mathematical Society, 73(6):747-817, 1967.16

[Sot73] Jorge Sotomayor. Generic bifurcations of dynamical systems. Dynamical systems, 549, 1973. 182, 183, 185, 186, 188

[Sot79] Jorge Sotomayor. Licões de equacões diferenciais ordinárias, volume 11. Instituto de Matemática Pura e Aplicada, CNPq, 1979. $16,17,18,38,41$

[Vér13] Juliano Aparecido Vérri. Estabilidade global e bifurcação de hopf em um modelo de hiv baseado em sistemas do tipo lotkavolterra. Dissertação de Mestrado, 2013. 126, 193, 198, 207, 217

[Vid02] Mathukumalli Vidyasagar. Nonlinear systems analysis, volume 42. Siam, 2002. 16

[VWC85] Pravin P. Varaiya, Felix F. Wu e Rong-Liang Chen. Direct methods for transient stability analysis of power systems: Recent results. Proceedings of the IEEE, 73(12):1703-1715, 1985. 1

[Wig03] Stephen Wiggins. Introduction to applied nonlinear dynamical systems and chaos, 2003. 16, 49, 141 
[YV67] Yao-Nan Yu e Khien Vongsuriya. Nonlinear power system stability study by liapunov function and zubov's method. IEEE Transactions on Power Apparatus and Systems, 12(PAS86):1480-1485, 1967. 2 